This document was prepared in conjunction with work accomplished under Contract No. DE-AC09-96SR18500 with the U. S. Department of Energy.

\title{
DISCLAIMER
}

This report was prepared as an account of work sponsored by an agency of the United States Government. Neither the United States Government nor any agency thereof, nor any of their employees, nor any of their contractors, subcontractors or their employees, makes any warranty, express or implied, or assumes any legal liability or responsibility for the accuracy, completeness, or any third party's use or the results of such use of any information, apparatus, product, or process disclosed, or represents that its use would not infringe privately owned rights. Reference herein to any specific commercial product, process, or service by trade name, trademark, manufacturer, or otherwise, does not necessarily constitute or imply its endorsement, recommendation, or favoring by the United States Government or any agency thereof or its contractors or subcontractors. The views and opinions of authors expressed herein do not necessarily state or reflect those of the United States Government or any agency thereof. 


\section{Phase II of a Six Sigma Initiative to Study DWPF SME Analytical Turnaround Times: SRNL's Evaluation of Carbonate-Based Dissolution Methods}

Distribution

$\begin{array}{lllll}\text { C. J. } & \text { Bannochie } & \text { 773-42A } & \text { R. N. } \text { Mahannah } & \text { 704-28S } \\ \text { D. R. } & \text { Best } & \text { 786-1A } & \text { S. L. Marra } & \text { 999-W } \\ \text { N. E. } & \text { Bibler } & \text { SRNL } & \text { G. J. Matis } & \text { 210-S } \\ \text { L. M. } & \text { Chandler } & \text { SRNL } & \text { W. L. Melton } & \text { 704-28S } \\ \text { D. R. } & \text { Click } & \text { SRNL } & \text { M. S. Miller } & \text { 704-S } \\ \text { C. J. } & \text { Coleman } & \text { SRNL } & \text { D. G. Murdoch } & \text { 704-S } \\ \text { J. E. } & \text { Cook } & \text { 704-29S } & \text { J. E. Occhipinti } & \text { 704-S } \\ \text { R. E. Edwards } & \text { SRNL } & \text { A. B. Osteen } & \text { 210-S } \\ \text { T. B. } & \text { Edwards } & \text { 773-42A } & \text { P. C. Padezanin } & \text { 735-B } \\ \text { S. P. } & \text { Harris } & \text { 773-42A } & \text { P. M. Patel } & \text { 704-27S } \\ \text { C. C. Herman } & \text { 773-42A } & \text { D. K. Peeler } & \text { 999-W } \\ \text { K A. Hauer } & \text { 704-S } & \text { F. M. Pennebaker } & \text { SRNL } \\ \text { C. M. Hidlay } & \text { 704-29S } & \text { R. C. Tuckfield } & \text { 773-42A } \\ \text { R. M. Hoeppel } & \text { 704-27S } & \text { H. A. Woltermann } & \text { SRNL } \\ \text { C.M. Jantzen } & \text { SRNL } & \text { ADS Files } & \text { SRNL } \\ \text { O. G. Lien } & \text { 772-3F } & \text { SCS Files } & \text { 773-42A }\end{array}$


Westinghouse Savannah River Company

Savannah River National Laboratory

Analytical Development/Statistical Consulting Sections
WSRC-TR-2005-00396

Rev. 0

Page 2 of 140

REVIEWS AND APPROVALS

AUTHORS:

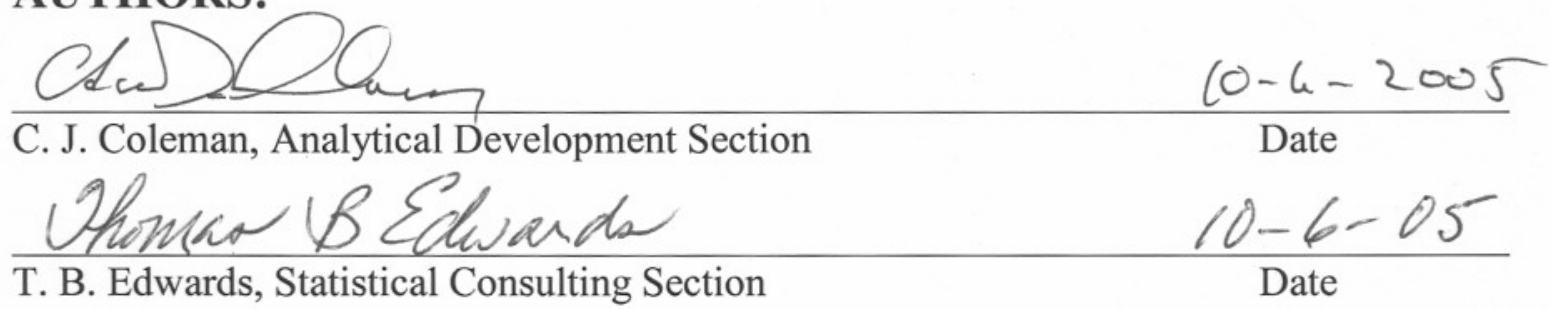

TECHNICAL REVIEWERS:

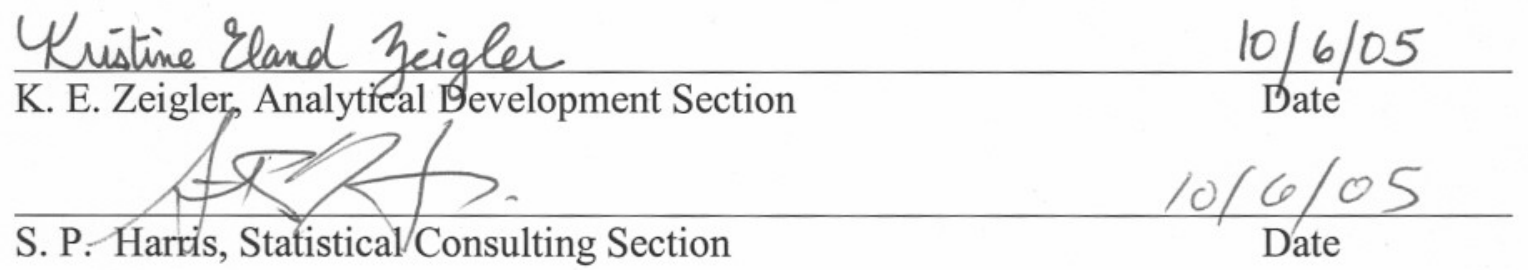

APPROVERS:

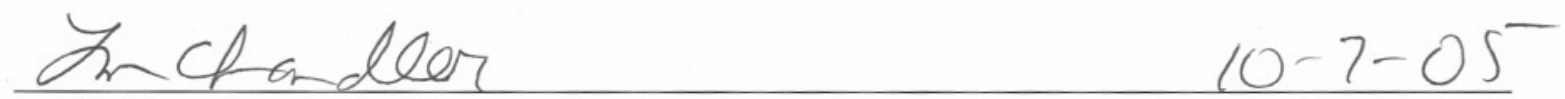

L. M. Chandler, Analytical Development Section

Date
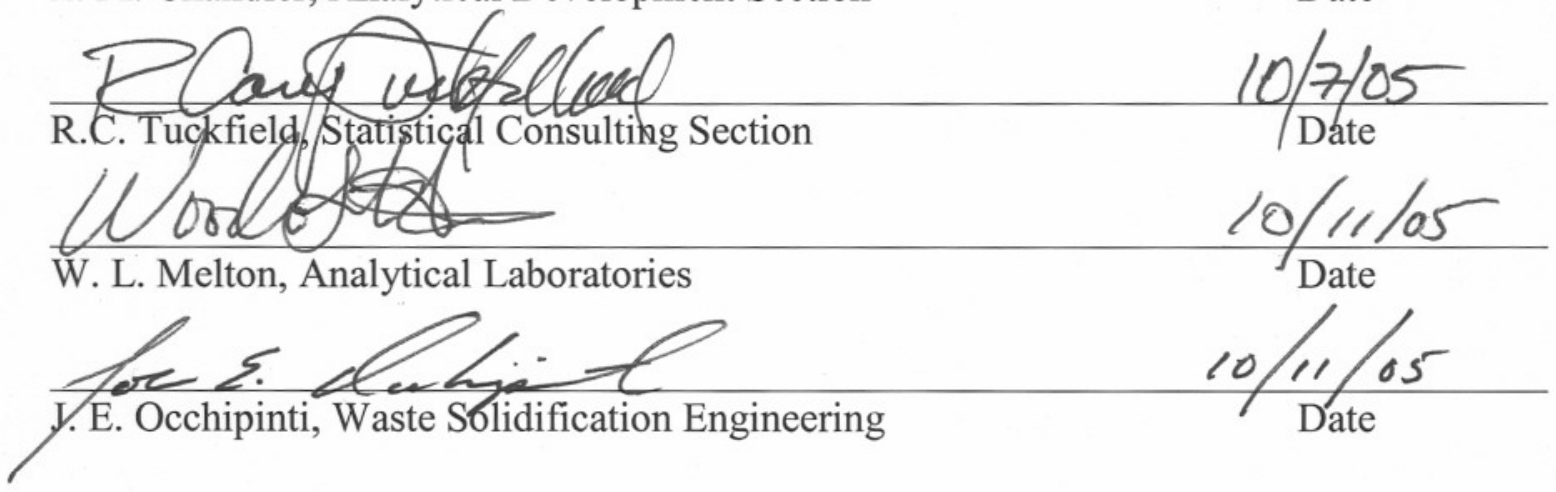


\section{TABLE OF CONTENTS}

1.0 Introduction 5

2.0 Summary of Recommendations 6

3.0 Summary of Experimental Results 7

4.0 Experimental Results 7

4.1 Preparation of Non-radioactive Glass Wafers for Digestion and Analysis 8

4.2 $\mathrm{Cs}_{2} \mathrm{CO}_{3}$ Fusion Digestions of Vitrified SME Simulant and ARG-1 Standard Glass

4.3 $\quad \mathrm{K}_{2} \mathrm{CO}_{3}$ Fusion Digestions of Vitrified SME Simulant and ARG-1 Standard Glass

4.4 $\mathrm{Na}_{2} \mathrm{O}_{2}-\mathrm{NaOH}$ Fusions of Vitrified SME Simulant and ARG-1

Standard Glass

4.5 Mixed Acid Digestions of Vitrified SME Simulant and ARG-1

Standard Glass

4.6 $\mathrm{Cs}_{2} \mathrm{CO}_{3}$ Fusion Digestions of DWPF Pour Stream Samples and ARG-1 Standard Glass

4.7 $\mathrm{Na}_{2} \mathrm{O}_{2}$ Fusion Digestions of DWPF Pour Stream Samples and ARG-1 Standard Glass (performed under guidance of ITS)

4.8 Mixed Acid Digestions of DWPF Pour Stream Samples and ARG-1 Standard Glass (performed under guidance of ITS) 10

5.0 Statistical Analyses

5.1 Comparisons of the Compositional Measurements of the Simulant Set of Samples

5.1.1 Measurements of Reagent Blanks in Simulant Set 12

5.1.2 Measurements of ARG-1 in Simulant Set 13

5.1.3 Measurements of Simulant Samples 16

5.2 Comparisons of the Compositional Measurements for the Radioactive Set of Samples

5.2.1 Measurements of Reagent Blanks in Radioactive Set 21

5.2.2 Measurements of ARG-1 in Radioactive Set 23

5.2.3 Measurements of Radioactive Samples 24

5.2.4 Acceptability of Radioactive Sample Measurements 28

6.0 References

Appendix A 
Westinghouse Savannah River Company

Savannah River National Laboratory

Analytical Development/Statistical Consulting Sections
WSRC-TR-2005-00396

Rev. 0

Page 4 of 140

This page intentionally left blank. 
October 5, 2005

Keywords: Six Sigma Initiative

Analytical Improvements Analytical Turnaround Times

Waste Acceptance

TO: $\quad$ L.M. Chandler, SRNL and R.C. Tuckfield, 773-42A

FROM: C.J. Coleman, SRNL and T.B. Edwards, 773-42A

\section{Phase II of a Six Sigma Initiative to Study DWPF SME Analytical Turnaround Times: SRNL's Evaluation of Carbonate-Based Dissolution Methods}

\subsection{Introduction}

The Analytical Development Section (ADS) and the Statistical Consulting Section (SCS) of the Savannah River National Laboratory (SRNL) are participating in a Six Sigma initiative to improve the Defense Waste Processing Facility (DWPF) Laboratory. The Six Sigma initiative has focused on reducing the analytical turnaround time of samples from the Slurry Mix Evaporator (SME) by developing streamlined sampling and analytical methods [1]. The objective of Phase I was to evaluate the sub-sampling of a larger sample bottle and the performance of a cesium carbonate $\left(\mathrm{Cs}_{2} \mathrm{CO}_{3}\right)$ digestion method. Successful implementation of the $\mathrm{Cs}_{2} \mathrm{CO}_{3}$ fusion method in the DWPF would have important time savings and convenience benefits because this single digestion would replace the dual digestion scheme now used. A single digestion scheme would result in more efficient operations in both the DWPF shielded cells and the inductively coupled plasma - atomic emission spectroscopy (ICP-AES) laboratory. By taking a small aliquot of SME slurry from a large sample bottle and dissolving the vitrified SME sample with carbonate fusion methods, an analytical turnaround time reduction from 27 hours to 9 hours could be realized in the DWPF. This analytical scheme has the potential for not only dramatically reducing turnaround times, but also streamlining operations to minimize wear and tear on critical shielded cell components that are prone to fail, including the Hydragard ${ }^{\mathrm{TM}}$ sampling valves and manipulators.

Favorable results from the Phase I tests [2] led to the recommendation for a Phase II effort as outlined in the DWPF Technical Task Request (TTR) [3]. There were three 
major tasks outlined in the TTR, and SRNL issued a Task Technical and QA Plan [4] with a corresponding set of three major task activities:

1) Compare weight percent (wt\%) total solids measurements of large volume samples versus peanut vial samples.

2) Evaluate $\mathrm{Cs}_{2} \mathrm{CO}_{3}$ and $\mathrm{K}_{2} \mathrm{CO}_{3}$ fusion methods using DWPF simulated glass (part 1) and (to the extent possible) using DWPF radioactive glass (part 2).

3) Recommend specific equipment to implement the new methods and work with SRNL's Equipment Engineering Section (EES) to ensure compatibility of the equipment with the DWPF shielded cells.

The focus of this report is major task activity \#2 (parts 1 and 2). The other two major task activities are to be addressed separately. The measurements supporting task activity \#1 are being conducted at the DWPF. EES is currently designing and fabricating the DWPF cell equipment needed for the new method as part of major task activity \#3.

As part of the discussion of the results in [2], it was noted that a potential drawback of using $\mathrm{Cs}_{2} \mathrm{CO}_{3}$ or any $\mathrm{Cs}$ salt to dissolve radioactive glass is that the large amount of stable Cs introduced would not be compatible with ion exchange techniques to remove ${ }^{137} \mathrm{Cs}$ from solutions to lower the whole body dose to personnel from ${ }^{137} \mathrm{Cs}$ gamma radiation. The DWPF has thus far processed only waste with relatively low amounts of ${ }^{137}$ Cs. However, beginning in 2007, the Modular Caustic Side Solvent Extraction Unit is scheduled to feed high ${ }^{137}$ Cs-bearing solutions to the DWPF. One possible remedy for the potential dose problems associated with ${ }^{137} \mathrm{Cs}$ is to use ion exchange techniques to remove the ${ }^{137} \mathrm{Cs}$ from dissolved samples before removing the solutions from the shielded cells for ICP-AES analysis. For this reason, and because it is unlikely that $\mathrm{K}$ determinations will be required for DWPF process control due to the low concentration of $\mathrm{K}$ in SRS waste, the analogous dissolution scheme using $\mathrm{K}_{2} \mathrm{CO}_{3}$ was added to the Phase II investigation scope.

The remainder of this report may be outlined as follows. Section 2 provides a summary of recommendations supported by the results of the Phase II tests. Section 3 provides a summary of the experimental results of the carbonate-based digestions. A discussion of the experimental details is provided in Section 4. Section 5 provides a discussion of the details of the statistical comparisons performed as part of this study. References are provided in Section 6. Appendix A and Appendix B provide the statistical details supporting the analyses of the simulant and radioactive sets of samples, respectively. Appendix C provides the details of the analytical procedures that help meet the Six Sigma team's goal of improved turnaround times and efficiency for the DWPF Lab.

\subsection{Summary of Recommendations}

- $\quad$ Proceed with testing the $\mathrm{Cs}_{2} \mathrm{CO}_{3}$ fusion digestion in the DWPF with the ultimate goal of establishing this method as the reference digestion method for SME samples. 
Additional refinements to the $\mathrm{Cs}_{2} \mathrm{CO}_{3}$ fusion methods are possible (such as lowering the fusion temperature and using $\mathrm{Cs}_{2} \mathrm{CO}_{3}$ fusion blanks as the substrate for preparing ICP-AES calibration standards). However, at this point in the testing program, method refinements are not required to initiate testing in the DWPF.

- Perform additional testing of the $\mathrm{K}_{2} \mathrm{CO}_{3}$ fusion digestion method at SRNL to determine if subtle method changes can provide results comparable to the $\mathrm{Cs}_{2} \mathrm{CO}_{3}$ fusion method. Possible modifications to the method include optimization of the flux time and temperature. Also, a mixture of $\mathrm{K}_{2} \mathrm{CO}_{3}$ and $\mathrm{KNO}_{3}$ should be investigated as a way to both lower the temperature of flux and increase the oxidizing power of the flux which could help to dissolve the most refractory oxides and metallic inclusions in the glass. Should this testing be successful, the solutions should be treated with an ion exchange material designed to remove ${ }^{137} \mathrm{Cs}$ to determine if this method would be viable for reducing the gamma dose from high ${ }^{137} \mathrm{Cs}$ solutions before elemental analysis.

\subsection{Summary of Experimental Results}

- The $\mathrm{Cs}_{2} \mathrm{CO}_{3}$ fusion digestion yielded elemental analyses on both simulated and actual DWPF glass samples that were comparable in both accuracy and precision to the combined results of the reference DWPF methods, the $\mathrm{Na}_{2} \mathrm{O}_{2}$ fusion and the mixedacid method. The Phase II experimental results strongly support the concept of using the $\mathrm{Cs}_{2} \mathrm{CO}_{3}$ fusion digestion as a single digestion method to replace the DWPF tandem digestion scheme for SME samples.

- The authors believe that the $\mathrm{Cs}_{2} \mathrm{CO}_{3}$ fusion may produce $\mathrm{B}$ determinations that are slightly biased low (5-10\%) due to B volatility at the $1100{ }^{\circ} \mathrm{C}$ fusion temperature coupled with a possible carrier effect from the volatility of cesium atoms. The authors feel it is prudent to declare this possibility, despite the fact that some data sets show no statistically significant difference in B determinations as a function of sample digestion technique. Should a negative bias in B determinations be confirmed by subsequent tests, possible solutions to this problem include modifying the flux conditions to minimize B loss or by bias corrections.

- The $\mathrm{K}_{2} \mathrm{CO}_{3}$ fusion digestion results were inferior in both precision and accuracy to the $\mathrm{Cs}_{2} \mathrm{CO}_{3}$ fusion. However, some of the individual analyses from the $\mathrm{K}_{2} \mathrm{CO}_{3}$ fusion were of high quality. This result suggests that method refinements could make the $\mathrm{K}_{2} \mathrm{CO}_{3}$ fusion a viable technique.

\subsection{Experimental Details}

Experimental details of how the simulant samples were prepared, dissolved, and analyzed are provided in Sections 4.1 through 4.5 while Sections 4.6 through 4.8 pertain to the digestion and analysis of the DWPF radioactive glass sample. Appendix C provides the 
integrated procedure for taking a slurry sample from a large sample bottle and measuring the wt. \% solids after drying at $115^{\circ} \mathrm{C}$ and after vitrifying the resulting powder at $1100{ }^{\circ} \mathrm{C}$, and then dissolving the resulting glass wafer with the $\mathrm{Cs}_{2} \mathrm{CO}_{3}$ fusion method.

\subsection{Preparation of Non-radioactive Glass Wafers for Digestion and Analysis}

The alkali fusion digestions $\left(\mathrm{Cs}_{2} \mathrm{CO}_{3}, \mathrm{~K}_{2} \mathrm{CO}_{3}\right.$, and $\left.\mathrm{Na}_{2} \mathrm{O}_{2}\right)$ of glass wafers eliminates the tedious glass crushing step needed to obtain the fine powdered sample that is required for effective digestions with the mixed acid method. A set of glass wafers was prepared from a large volume of SME simulant in a manner that would ensure, for all practical purposes, identical composition of each of the wafers. This technique eliminated the possibility that analytical differences could result from sub-sampling the SME slurry to create individual glass samples. Note that the technique used to make the glass wafers for dissolution in this study is different than will be used on actual SME samples in which small aliquots of SME slurry will be rapidly dried and vitrified. However, the form and weight (about $0.65 \mathrm{~g}$ on average) of the wafer approximate that of $1 \mathrm{~mL}$ of SME slurry converted to glass by heating at $1100{ }^{\circ} \mathrm{C}$.

The SME simulant (of Sludge Batch 2/3 Blend) used for digestion tests was obtained from M.E. Stone of the Immobilization Technology Section. The simulant was in a 2-liter bottle that was mixed overnight in a Toxicity Characteristic Leach Procedure (TCLP) vessel rotator at the rate of 30 rotations per minute. Approximately $35 \mathrm{~mL}$ of slurry was transferred to a $50 \mathrm{~mL}$ Pt-Au crucible. The slurry was first heated in a drying oven at $115^{\circ} \mathrm{C}$ to remove free water and form a powder. The powder was vitrified by heating at $1100{ }^{\circ} \mathrm{C}$ for three hours to form approximately $20 \mathrm{~g}$ of glass. The glass was crushed in a mechanical crusher equipped with an agate mortar and agate balls. The glass powder was sieved and the glass particles that passed through the 200 mesh sieve were collected. Approximately $0.65 \mathrm{~g}$ of glass powder was transferred to a $50 \mathrm{~mL}$ Pt-Au crucible and heated at $1100{ }^{\circ} \mathrm{C}$ for 10 minutes to form a thin glass wafer. This process was repeated until enough glass wafers were obtained to perform all the digestion experiments. Some of the -200 mesh powdered glass was not re-melted to form a wafer but set aside for tests with the mixed acid digestion method.

The ARG-1 glass standard was similarly heated at $1100{ }^{\circ} \mathrm{C}$ for 10 minutes to form glass wafers analogous to those prepared from the SME simulant. Approximately $0.65 \mathrm{~g}$ of ARG-1 glass standard (already in the form of a -200 mesh powder) was heated in Pt-Au crucibles to form the wafers. Note that should the proposed analytical scheme be adopted by the DWPF, in a matter of one day the DWPF Laboratory or SRNL could convert ARG-1 glass standard powder into enough glass wafers to last a year or longer. The glass wafers could be individually stored in wide-mouth plastic bottles (in a non-radiological area until ready to be used in the shielded cells) and then transferred into the Pt crucibles to obtain the weight of record prior to the digestion. The proposed analytical scheme completely eliminates the tedious operation of weighing glass powders in the shielded cell. 


\section{2 $\mathrm{Cs}_{2} \mathrm{CO}_{3}$ Fusion Digestions of Vitrified SME Simulant and ARG-1 Standard Glass}

The $\mathrm{Cs}_{2} \mathrm{CO}_{3}$ fusions were carried out in $50 \mathrm{~mL}$ Pt-Au alloy crucibles. A glass wafer was transferred to the Pt-Au crucible and the weight of the glass wafer obtained. Approximately 6 g of Alfa Aesar Puratronic ${ }^{\circledR}$ grade (99.994 \% -metals basis) $\mathrm{Cs}_{2} \mathrm{CO}_{3}$ was added to the crucible which was placed in the $1100{ }^{\circ} \mathrm{C}$ furnace and heated for 10 minutes after the furnace re-equilibrated to $1100{ }^{\circ} \mathrm{C}$. The crucible was allowed to cool for 5 minutes and then dropped into a $1000 \mathrm{~mL}$ wide-mouth bottle that was pre-charged with $100 \mathrm{~mL}$ of $3 \% \mathrm{H}_{2} \mathrm{O}_{2}$ prepared by diluting $10 \mathrm{~mL}$ of $30 \% \mathrm{H}_{2} \mathrm{O}_{2}$ to $100 \mathrm{~mL}$ in a plastic volumetric flask. The decomposition of $\mathrm{H}_{2} \mathrm{O}_{2}$ in the highly caustic solution created a warm, frothy solution that rapidly dissolved most of the flux residue. After 15 minutes, $250 \mathrm{~mL}$ of de-ionized water (measured in a $250 \mathrm{~mL}$ plastic volumetric flask) was added to dilute the solution and minimize the potential for silicates to precipitate upon addition of the acid solution. The final addition was $100 \mathrm{~mL}$ of a $50 \%$ solution prepared by diluting $50 \mathrm{~mL}$ of concentrated $\mathrm{HNO}_{3}$ to $100 \mathrm{~mL}$ with de-ionized water in a plastic volumetric flask. The acid solution was added slowly with occasional swirling of the $1000 \mathrm{~mL}$ bottle. Although in most cases the acid solution instantaneously dissolved the metal oxide and hydrous oxide residue, 30 minutes was conservatively allowed for complete dissolution. The volume of solution at this point was $450 \mathrm{~mL}$. A 20 -fold serial dilution of this solution with de-ionized water was performed prior to ICP-AES analysis to yield a final effective digestion volume of 9,000 $\mathrm{mL}$.

\section{3 $\mathrm{K}_{2} \mathrm{CO}_{3}$ Fusion Digestions of Vitrified SME Simulant and ARG-1 Standard Glass}

The $\mathrm{K}_{2} \mathrm{CO}_{3}$ fusions of vitrified SME simulant and ARG-1 glass standards were carried out analogously to the $\mathrm{Cs}_{2} \mathrm{CO}_{3}$ fusions. The $\mathrm{K}_{2} \mathrm{CO}_{3}$ was Alfa Aesar Puratronic $®$ grade (99.997 \% -metals basis).

\section{4 $\mathrm{Na}_{2} \mathrm{O}_{2}-\mathrm{NaOH}$ Fusions of Vitrified SME Simulant and ARG-1 Standard Glass}

$\mathrm{Na}_{2} \mathrm{O}_{2}-\mathrm{NaOH}$ fusions were carried out in zirconium crucibles to avoid the aggressive attack of $\mathrm{Na}_{2} \mathrm{O}_{2}$ and $\mathrm{NaOH}$ on Pt-Au crucibles. A mixture of $4.0 \mathrm{~g} \mathrm{Na}_{2} \mathrm{O}_{2}$ and $2.0 \mathrm{~g}$ $\mathrm{NaOH}$ was used to dissolve the glass wafers. The workup was analogous to that used for $\mathrm{Cs}_{2} \mathrm{CO}_{3}$ and $\mathrm{K}_{2} \mathrm{CO}_{3}$ fusions, with an initial dilution to $450 \mathrm{~mL}$ followed by a 20 -fold serial dilution with de-ionized water. $\mathrm{Na}$ and $\mathrm{Zr}$ were not measured from this digestion because these elements are introduced through the fusion reagents $\left(\mathrm{Na}_{2} \mathrm{O}_{2}\right.$ and $\left.\mathrm{NaOH}\right)$ or the crucible material ( $\mathrm{Zr}$ crucible).

\subsection{Mixed Acid Digestions of Vitrified SME Simulant and ARG-1 Standard Glass}

The mixed acid digestions were carried out on the -200 mesh powder in contrast to the alkali fusions that were carried out on the glass wafers. Approximately $0.65 \mathrm{~g}$ of -200 
mesh glass powder was transferred into a CEM Teflon pressure vessel. A mixture of 10 $\mathrm{mL}$ concentrated $\mathrm{HF}$ and $10 \mathrm{~mL}$ concentrated $\mathrm{HNO}_{3}$ was added and mixed with the powdered glass. The vessel was capped with the CEM capping station to maintain pressure and heated for 2 hours at $115^{\circ} \mathrm{C}$. After cooling, $80 \mathrm{~mL}$ of $0.6 \mathrm{M} \mathrm{H}_{3} \mathrm{BO}_{3}$ and 10 $\mathrm{mL}$ concentrated $\mathrm{HCl}$ were added and the solution heated for 1 hour at $75{ }^{\circ} \mathrm{C}$. This solution was diluted to $450 \mathrm{~mL}$. A 20-fold serial dilution with de-ionized water was made prior to ICP-AES determinations. Si and B were not measured from this preparation technique because Si determinations tend to be biased high from HF attack on the nebulizer/torch assembly and $\mathrm{B}$ is added with the $\mathrm{H}_{3} \mathrm{BO}_{3}$ reagent.

\section{6 $\mathrm{Cs}_{2} \mathrm{CO}_{3}$ Fusion Digestions of DWPF Pour Stream Samples and ARG-1 Standard Glass}

Since the DWPF glass pour stream sample had been crushed to a powder to support ITSdirected analyses of this sample, the $\mathrm{Cs}_{2} \mathrm{CO}_{3}$ fusions were carried out on this material "as-received" versus converting it to glass wafers by re-melting. However, there was only enough powder to perform four digestions. The last two digestions out of a total of six were performed on glass “chunks” that did not get adequately pulverized. This occurrence was fortuitous because it allowed comparison of elemental analysis data obtained on glass powder with that from glass chunks. The data revealed no significant difference as a function of glass particle size, further corroborating the efficacy of alkali fusions on glass chunks, wafers, shards, pellets, etc. Approximately $0.33-0.40 \mathrm{~g}$ of the glass (powder or chunk) and $4.0 \mathrm{~g}$ of $\mathrm{Cs}_{2} \mathrm{CO}_{3}$ were transferred into a Pt-Au crucible. The workup was identical to that used for the fusion digestions of non-radioactive simulated waste glass except that, because of the smaller sample weight, the serial dilution was 10fold rather than the 20 -fold used for simulants.

\section{7 $\mathrm{Na}_{2} \mathrm{O}_{2}$ Fusion Digestions of DWPF Pour Stream Samples and ARG-1 Standard Glass (performed under guidance of ITS)}

$0.25 \mathrm{~g}$ of crushed glass was heated at $675^{\circ} \mathrm{C}$ with a mixture of $1.5 \mathrm{~g} \mathrm{Na}_{2} \mathrm{O}_{2}$ and $1.0 \mathrm{~g}$ $\mathrm{NaOH}$ in a $\mathrm{Zr}$ crucible. The flux residue was diluted to $100 \mathrm{~mL}$ with water and $25 \mathrm{~mL}$ of concentrated $\mathrm{HCl}$. A serial dilution was performed before ICP-AES analysis.

\subsection{Mixed Acid Digestions of DWPF Pour Stream Samples and ARG-1 Standard Glass (performed under guidance of ITS)}

$0.25 \mathrm{~g}$ of crushed glass was heated with $5 \mathrm{~mL}$ concentrated $\mathrm{HF}$ and $5 \mathrm{~mL}$ concentrated $\mathrm{HNO}_{3}$ for 2 hours in a pressurized CEM Teflon vessel at $115^{\circ} \mathrm{C}$. After cooling, $40 \mathrm{~mL}$ of $0.6 \mathrm{M} \mathrm{H}_{3} \mathrm{BO}_{3}$ and $5 \mathrm{~mL}$ concentrated $\mathrm{HCl}$ were added, the vessel re-capped, and the solution re-heated at $115^{\circ} \mathrm{C}$ for 1 hour. The solution was then diluted to $100 \mathrm{~mL}$. A serial dilution was performed before ICP-AES analysis. 


\subsection{Statistical Analyses}

In this section, the data from the glass analyses of the Phase 2 study are presented and statistically evaluated. As described in the experimental section, these data include elemental measurements of glass samples made from a SME simulant, actual radioactive glass samples from the DWPF's pour stream, ARG-1 glass standards, and reagent blanks. The DWPF radioactive glass samples were available for this study from a separate task performed under the direction of ITS [5]. There was only enough radioactive glass to support a set of $\mathrm{Cs}_{2} \mathrm{CO}_{3}$ dissolutions. The elemental analyses of the radioactive glass from the $\mathrm{Cs}_{2} \mathrm{CO}_{3}$ fusion are compared to the $\mathrm{Na}_{2} \mathrm{O}_{2}$ and mixed acid results summarized in the ITS report [5] as part of the discussion that follows. The dissolved samples were analyzed with ICP-AES techniques under the guidance of an analytical plan ${ }^{1}$. For the radioactive glass, the available portions were dissolved by $\mathrm{Cs}_{2} \mathrm{CO}_{3}$. The set of elements analyzed was not identical for the simulant and radioactive glass samples. While both sets covered the major oxides typically in DWPF glasses (with the radioactive set adding uranium), there were some differences in the minor oxides. These differences are of no practical concern.

The elemental analyses are presented in the sections that follow and statistical comparisons are conducted, first for the simulant and then for the radioactive sample measurements, to assess the relative performances of the dissolution methods for each type of sample. The statistical comparisons were conducted using JMP Version 5.1.2 from SAS Institute, Inc. [6].

\subsection{Comparisons of the Compositional Measurements of the Simulant Set of Samples}

A primary objective of this test phase was the continued evaluation of the performance of the $\mathrm{Cs}_{2} \mathrm{CO}_{3}$ dissolution as well as an initial evaluation of the $\mathrm{K}_{2} \mathrm{CO}_{3}$ method. Table A1 in Appendix A provides the elemental concentration data generated for the simulant samples for the two carbonate-based preparations as well as the $\mathrm{Na}_{2} \mathrm{O}_{2}$ and mixed acid methods. In addition to the prepared glass samples of the simulant, samples of the ARG-1 standard glass and reagent blanks were included in the analytical plan that was used to guide the measurement of the chemical compositions of the simulant samples. The elemental concentrations were converted to oxide concentrations by multiplying the values for each element by the gravimetric factor for the corresponding oxide. In order to assign an oxide concentration for determinations below the detection limit, an arbitrary value of half the detection limit of the element was used.

\footnotetext{
1 The analytical plan was provided in the memorandum: Edwards, TB, “An Analytical Plan Supporting the DWPF Laboratory Six Sigma Process Improvement Project,” SRNL-SCS-2005-00030, June 13, 2005.
} 
Exhibit A1 in Appendix A provides a plot of the concentrations in weight percent (wt\%) of each oxide in analytical sequence. Exhibit A2 in Appendix A provides plots of each oxide’s concentration values by prep method grouped by type of sample.

\subsubsection{Measurements of Reagent Blanks in Simulant Set}

Table 1 provides a listing of the average measurement by oxide for the reagent blank samples for each of the preparation methods. Yellow shading is used to highlight averages that appear to be significantly higher than those generated by the other preparation methods. Specifically, note that the $\mathrm{Na}_{2} \mathrm{O}_{2}$ method yields $\mathrm{CaO}$ and $\mathrm{K}_{2} \mathrm{O}$ measurements for the blanks that are larger than the measurements from the other preparation methods, indicating contamination introduced by impurities in the $\mathrm{Na}_{2} \mathrm{O}_{2}$ reagent. 
Westinghouse Savannah River Company

WSRC-TR-2005-00396

Savannah River National Laboratory

Rev. 0

Analytical Development/Statistical Consulting Sections

Page 13 of 140

Table 1. Comparison of Measurement Averages for Blanks in Simulant Set

\begin{tabular}{|c|c|c|c|c|}
\hline $\begin{array}{c}\text { Preparation } \\
\text { Method }\end{array}$ & $\mathrm{Cs}_{2} \mathrm{CO}_{3}$ & $\mathrm{~K}_{2} \mathrm{CO}_{3}$ & $\begin{array}{c}\text { Mixed } \\
\text { Acid }\end{array}$ & $\mathrm{Na}_{2} \mathrm{O}_{2}$ \\
\hline Type of Sample & blank & blank & blank & blank \\
\hline N Rows & 2 & 2 & 2 & 2 \\
\hline $\mathrm{Ag}_{2} \mathrm{O}(\mathrm{wt} \%)$ & 0.018 & 0.018 & 0.018 & 0.018 \\
\hline $\mathrm{Al}_{2} \mathrm{O}_{3}(\mathrm{wt} \%)$ & 0.044 & 0.044 & 0.044 & 0.044 \\
\hline $\mathrm{B}_{2} \mathrm{O}_{3}(\mathrm{wt} \%)$ & 0.038 & 0.038 & & 0.072 \\
\hline BaO (wt\%) & 0.008 & 0.003 & 0.003 & 0.003 \\
\hline $\mathrm{CaO}$ (wt\%) & 0.011 & 0.011 & 0.011 & 0.199 \\
\hline CdO (wt\%) & 0.004 & 0.004 & 0.004 & 0.004 \\
\hline $\mathrm{Ce}_{2} \mathrm{O}_{3}(\mathrm{wt} \%)$ & 0.038 & 0.038 & 0.038 & 0.038 \\
\hline CoO (wt\%) & 0.007 & 0.007 & 0.007 & 0.011 \\
\hline $\mathrm{Cr}_{2} \mathrm{O}_{3}(\mathrm{wt} \%)$ & 0.009 & 0.009 & 0.009 & 0.009 \\
\hline $\mathrm{CuO}(\mathrm{wt} \%)$ & 0.003 & 0.003 & 0.003 & 0.003 \\
\hline $\mathrm{Fe}_{2} \mathrm{O}_{3}(\mathrm{wt} \%)$ & 0.006 & 0.006 & 0.006 & 0.006 \\
\hline $\mathrm{K}_{2} \mathrm{O}(\mathrm{wt} \%)$ & 0.169 & & 0.169 & 0.306 \\
\hline $\mathrm{La}_{2} \mathrm{O}_{3}(\mathrm{wt} \%)$ & 0.008 & 0.008 & 0.008 & 0.008 \\
\hline $\mathrm{Li}_{2} \mathrm{O}(\mathrm{wt} \%)$ & 0.012 & 0.012 & 0.012 & 0.012 \\
\hline MgO (wt\%) & 0.027 & 0.027 & 0.027 & 0.027 \\
\hline MnO (wt\%) & 0.003 & 0.003 & 0.003 & 0.003 \\
\hline $\mathrm{MoO}_{3}(\mathrm{w} \%)$ & 0.021 & 0.021 & 0.021 & 0.021 \\
\hline $\mathrm{Na}_{2} \mathrm{O}$ (wt\%) & 0.042 & 0.042 & 0.042 &. \\
\hline $\mathrm{Nb}_{2} \mathrm{O}_{5}(\mathrm{wt} \%)$ & 0.010 & 0.010 & 0.010 & 0.010 \\
\hline $\mathrm{Nd}_{2} \mathrm{O}_{3}(\mathrm{wt} \%)$ & 0.050 & 0.050 & 0.050 & 0.050 \\
\hline $\mathrm{NiO}(\mathrm{wt} \%)$ & 0.006 & 0.006 & 0.006 & 0.006 \\
\hline $\mathrm{P}_{2} \mathrm{O}_{5}(\mathrm{wt} \%)$ & 0.083 & 0.083 & 0.083 & 0.083 \\
\hline PbO (wt\%) & 0.022 & 0.022 & 0.022 & 0.022 \\
\hline $\mathrm{ReO}_{2}(\mathrm{wt} \%)$ & 0.008 & 0.008 & 0.008 & 0.008 \\
\hline $\mathrm{SO}_{4}(\mathrm{wt} \%)$ & 0.141 & 0.141 & 0.141 & 0.141 \\
\hline $\mathrm{SiO}_{2}(\mathrm{wt} \%)$ & 0.015 & 0.015 & - & 0.015 \\
\hline $\mathrm{SnO}_{2}(\mathrm{wt} \%)$ & 0.021 & 0.021 & 0.021 & 0.021 \\
\hline SrO (wt\%) & 0.003 & 0.003 & 0.003 & 0.003 \\
\hline $\mathrm{TiO}_{2}(\mathrm{wt} \%)$ & 0.001 & 0.001 & 0.001 & 0.001 \\
\hline $\mathrm{V}_{2} \mathrm{O}_{5}(\mathrm{wt} \%)$ & 0.021 & 0.021 & 0.021 & 0.021 \\
\hline ZnO (wt\%) & 0.003 & 0.003 & 0.003 & 0.003 \\
\hline $\mathrm{ZrO}_{2}(\mathrm{wt} \%)$ & 0.009 & 0.009 & 0.009 & \\
\hline
\end{tabular}

\subsubsection{Measurements of ARG-1 in Simulant Set}

Exhibit A3 provides comparisons across the preparation methods by oxide for the ARG-1 measurements. There are pairwise comparisons among the averages of the preparations as well as statistical tests for differences in the variances (i.e., precisions) of the preparation methods. Tables $2 \mathrm{a}$ and $2 \mathrm{~b}$ provide summary information of these measurements in the form of the average measurement, standard deviation, and \% relative standard deviation for each oxide by each preparation method. Yellow shading is used in Table $2 b$ to highlight the biases that are $5 \%$ or greater relative to the ARG-1 reference composition (also given in Table $2 \mathrm{~b}$ ). 
Table 2a. Measurement Averages, Standard Deviations, and \% Relative Standard Deviations for ARG-1 Samples in Simulant Set

\begin{tabular}{|c|c|c|c|c|c|c|c|c|c|c|c|c|}
\hline Preparation Method & \multicolumn{3}{|c|}{$\mathrm{Cs}_{2} \mathrm{CO}_{3}$} & \multicolumn{3}{|c|}{$\mathrm{K}_{2} \mathrm{CO}_{3}$} & \multicolumn{3}{|c|}{ Mixed Acid } & \multicolumn{3}{|c|}{$\mathrm{Na}_{2} \mathrm{O}_{2}$} \\
\hline N Rows (3) & Mean & Std Dev & $\begin{array}{c}\text { \% Rel Std } \\
\text { Dev }\end{array}$ & Mean & Std Dev & $\begin{array}{c}\text { \% Rel Std } \\
\text { Dev }\end{array}$ & Mean & Std Dev & $\begin{array}{c}\text { \% Rel Std } \\
\text { Dev }\end{array}$ & Mean & Std Dev & $\begin{array}{c}\text { \% Rel Std } \\
\text { Dev }\end{array}$ \\
\hline $\mathrm{Ag}_{2} \mathrm{O}(\mathrm{wt} \%)$ & 0.025 & 0.0131 & $52.7 \%$ & 0.017 & 0.0002 & $0.9 \%$ & 0.018 & 0.0002 & $1.1 \%$ & 0.017 & 0.0001 & $0.5 \%$ \\
\hline $\mathrm{Al}_{2} \mathrm{O}_{3}(\mathrm{wt} \%)$ & 4.654 & 0.0218 & $0.5 \%$ & 4.560 & 0.1796 & $3.9 \%$ & 4.434 & 0.0393 & $0.9 \%$ & 4.535 & 0.2146 & $4.7 \%$ \\
\hline $\mathrm{B}_{2} \mathrm{O}_{3}(\mathrm{wt} \%)$ & 7.964 & 0.1131 & $1.4 \%$ & 8.329 & 0.3547 & $4.3 \%$ & & & & 8.275 & 0.4186 & $5.1 \%$ \\
\hline $\mathrm{BaO}(\mathrm{wt} \%)$ & 0.098 & 0.0011 & $1.2 \%$ & 0.087 & 0.0036 & $4.1 \%$ & 0.089 & 0.0006 & $0.6 \%$ & 0.092 & 0.0061 & $6.7 \%$ \\
\hline $\mathrm{CaO}(\mathrm{wt} \%)$ & 1.445 & 0.0581 & $4.0 \%$ & 1.424 & 0.0963 & $6.8 \%$ & 1.455 & 0.0000 & $0.0 \%$ & 1.595 & 0.0740 & $4.6 \%$ \\
\hline $\mathrm{CdO}(\mathrm{wt} \%)$ & 0.004 & 0.0001 & $3.1 \%$ & 0.004 & 0.0000 & $1.0 \%$ & 0.004 & 0.0000 & $1.1 \%$ & 0.004 & 0.0000 & $0.5 \%$ \\
\hline $\mathrm{Ce}_{2} \mathrm{O}_{3}(\mathrm{wt} \%)$ & 0.037 & 0.0012 & $3.1 \%$ & 0.036 & 0.0004 & $1.0 \%$ & 0.038 & 0.0004 & $1.1 \%$ & 0.036 & 0.0002 & $0.5 \%$ \\
\hline $\mathrm{CoO}(\mathrm{wt} \%)$ & 0.009 & 0.0050 & $53.2 \%$ & 0.006 & 0.0001 & $1.0 \%$ & 0.014 & 0.0007 & $4.7 \%$ & 0.009 & 0.0049 & $53.2 \%$ \\
\hline $\mathrm{Cr}_{2} \mathrm{O}_{3}(\mathrm{wt} \%)$ & 0.100 & 0.0045 & $4.5 \%$ & 0.095 & 0.0049 & $5.2 \%$ & 0.097 & 0.0005 & $0.5 \%$ & 0.090 & 0.0036 & $4.0 \%$ \\
\hline $\mathrm{CuO}$ (wt\%) & 0.006 & 0.0050 & $81.2 \%$ & 0.003 & 0.0000 & $1.0 \%$ & 0.003 & 0.0000 & $1.0 \%$ & 0.003 & 0.0000 & $0.5 \%$ \\
\hline $\mathrm{Fe}_{2} \mathrm{O}_{3}(\mathrm{wt} \%)$ & 14.097 & 0.3362 & $2.4 \%$ & 13.692 & 0.7234 & $5.3 \%$ & 14.211 & 0.0378 & $0.3 \%$ & 13.811 & 0.5478 & $4.0 \%$ \\
\hline $\mathrm{K}_{2} \mathrm{O}(\mathrm{wt} \%)$ & 2.791 & 0.0502 & $1.8 \%$ & . & 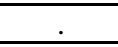 & & 2.879 & 0.0241 & $0.8 \%$ & 3.321 & 0.1840 & $5.5 \%$ \\
\hline $\mathrm{La}_{2} \mathrm{O}_{3}(\mathrm{wt} \%)$ & 0.007 & 0.0002 & $3.0 \%$ & 0.010 & 0.0044 & $45.1 \%$ & 0.007 & 0.0001 & $1.2 \%$ & 0.012 & 0.0091 & $73.0 \%$ \\
\hline $\mathrm{Li}_{2} \mathrm{O}(\mathrm{wt} \%)$ & 3.366 & 0.0448 & $1.3 \%$ & 3.251 & 0.0938 & $2.9 \%$ & 3.308 & 0.0124 & $0.4 \%$ & 3.186 & 0.1492 & $4.7 \%$ \\
\hline $\mathrm{MgO}(\mathrm{wt} \%)$ & 0.854 & 0.0152 & $1.8 \%$ & 0.835 & 0.0440 & $5.3 \%$ & 0.857 & 0.0085 & $1.0 \%$ & 0.812 & 0.0310 & $3.8 \%$ \\
\hline $\mathrm{MnO}(\mathrm{wt} \%)$ & 1.907 & 0.0325 & $1.7 \%$ & 1.877 & 0.0969 & $5.2 \%$ & 1.911 & 0.0000 & $0.0 \%$ & 1.825 & 0.0757 & $4.1 \%$ \\
\hline $\mathrm{MoO}_{3}(\mathrm{w} \%)$ & 0.021 & 0.0007 & $3.2 \%$ & 0.020 & 0.0002 & $0.9 \%$ & 0.021 & 0.0002 & $1.1 \%$ & 0.020 & 0.0001 & $0.4 \%$ \\
\hline $\mathrm{Na}_{2} \mathrm{O}(\mathrm{wt} \%)$ & 11.696 & 0.1364 & $1.2 \%$ & 11.319 & 0.3603 & $3.2 \%$ & 11.835 & 0.0404 & $0.3 \%$ & . & & \\
\hline $\mathrm{Nb}_{2} \mathrm{O}_{5}(\mathrm{wt} \%)$ & 0.081 & 0.0060 & $7.4 \%$ & 0.084 & 0.0093 & $11.1 \%$ & 0.093 & 0.0078 & $8.4 \%$ & 0.080 & 0.0118 & $14.7 \%$ \\
\hline $\mathrm{Nd}_{2} \mathrm{O}_{3}(\mathrm{wt} \%)$ & 0.049 & 0.0015 & $3.2 \%$ & 0.048 & 0.0005 & $1.0 \%$ & 0.049 & 0.0005 & $1.1 \%$ & 0.047 & 0.0002 & $0.5 \%$ \\
\hline $\mathrm{NiO}$ (wt\%) & 1.037 & 0.0288 & $2.8 \%$ & 0.780 & 0.0705 & $9.0 \%$ & 1.084 & 0.0078 & $0.7 \%$ & 1.022 & 0.0406 & $4.0 \%$ \\
\hline $\mathrm{P}_{2} \mathrm{O}_{5}(\mathrm{wt} \%)$ & 0.217 & 0.0335 & $15.4 \%$ & 0.231 & 0.0755 & $32.7 \%$ & 0.263 & 0.0138 & & 0.221 & 0.0147 & $6.6 \%$ \\
\hline $\mathrm{PbO}$ (wt\%) & 0.021 & 0.0007 & $3.2 \%$ & 0.021 & 0.0002 & $0.9 \%$ & 0.021 & 0.0002 & $1.0 \%$ & 0.021 & 0.0001 & $0.5 \%$ \\
\hline $\mathrm{ReO}_{2}(\mathrm{wt} \%)$ & 0.008 & 0.0002 & $2.9 \%$ & 0.008 & 0.0001 & $1.1 \%$ & 0.008 & 0.0001 & $1.1 \%$ & 0.008 & 0.0000 & $0.4 \%$ \\
\hline $\mathrm{SO}_{4}(\mathrm{wt} \%)$ & 0.139 & 0.0044 & $3.2 \%$ & 0.135 & 0.0014 & $1.0 \%$ & 0.140 & 0.0015 & $1.1 \%$ & 0.134 & 0.0006 & $0.5 \%$ \\
\hline $\mathrm{SiO}_{2}(\mathrm{wt} \%)$ & 49.703 & 0.6536 & $1.3 \%$ & 48.633 & 3.1173 & $6.4 \%$ & & - & & 47.207 & 2.1106 & $4.5 \%$ \\
\hline $\mathrm{SnO}_{2}$ (wt\%) & 0.020 & 0.0006 & $3.2 \%$ & 0.020 & 0.0002 & $1.0 \%$ & 0.021 & 0.0002 & $1.1 \%$ & 0.020 & 0.0001 & $0.5 \%$ \\
\hline SrO (wt\%) & 0.003 & 0.0001 & $3.2 \%$ & 0.003 & 0.0000 & $1.0 \%$ & 0.003 & 0.0000 & $1.1 \%$ & 0.007 & 0.0052 & $74.0 \%$ \\
\hline $\mathrm{TiO}_{2}(\mathrm{wt} \%)$ & 1.206 & 0.0058 & $0.5 \%$ & 1.172 & 0.0463 & $3.9 \%$ & 1.171 & 0.0042 & $0.4 \%$ & 1.145 & 0.0531 & $4.6 \%$ \\
\hline $\mathrm{V}_{2} \mathrm{O}_{5}(\mathrm{wt} \%)$ & 0.021 & 0.0007 & $3.2 \%$ & 0.020 & 0.0002 & $0.9 \%$ & 0.021 & 0.0002 & $1.1 \%$ & 0.020 & 0.0001 & $0.5 \%$ \\
\hline $\mathrm{ZnO}(\mathrm{wt} \%)$ & 0.023 & 0.0009 & $3.9 \%$ & 0.022 & 0.0020 & $8.9 \%$ & 0.024 & 0.0005 & $2.3 \%$ & 0.023 & 0.0014 & $6.3 \%$ \\
\hline $\mathrm{ZrO}_{2}(\mathrm{wt} \%)$ & 0.137 & 0.0016 & $1.1 \%$ & 0.129 & 0.0067 & $5.2 \%$ & 0.137 & 0.0030 & $2.2 \%$ & . & . & \\
\hline
\end{tabular}


Table 2b. Comparison of Measurement Averages for ARG-1 Samples in Simulant Set

\begin{tabular}{|c|c|c|c|c|c|c|c|c|c|}
\hline Preparation Method & $\mathrm{Cs}_{2} \mathrm{CO}_{3}$ & $\mathrm{~K}_{2} \mathrm{CO}_{3}$ & Mixed Acid & $\mathrm{Na}_{2} \mathrm{O}_{2}$ & Reference & & & & \\
\hline Type of Sample & ARG-1 & ARG-1 & ARG-1 & ARG-1 & Value & $\%$ Bias & \% Bias & $\%$ Bias & \% Bias \\
\hline N Rows & 3 & 3 & 3 & 3 & & $\mathrm{Cs}_{2} \mathrm{CO}_{3}$ & $\mathrm{~K}_{2} \mathrm{CO}_{3}$ & Mixed Acid & $\mathrm{Na}_{2} \mathrm{O}_{2}$ \\
\hline $\mathrm{Ag}_{2} \mathrm{O}(\mathrm{wt} \%)$ & 0.025 & 0.017 & 0.018 & 0.017 & & & & & \\
\hline $\mathrm{Al}_{2} \mathrm{O}_{3}(\mathrm{wt} \%)$ & 4.654 & 4.560 & 4.434 & 4.535 & 4.724 & $-1.5 \%$ & $-3.5 \%$ & $-6.1 \%$ & $-4.0 \%$ \\
\hline $\mathrm{B}_{2} \mathrm{O}_{3}(\mathrm{wt} \%)$ & 7.964 & 8.329 &. & 8.275 & 8.662 & $-8.1 \%$ & $-3.8 \%$ & & $-4.5 \%$ \\
\hline $\mathrm{BaO}(\mathrm{wt} \%)$ & 0.098 & 0.087 & 0.089 & 0.092 & 0.089 & $9.3 \%$ & $-3.0 \%$ & $0.1 \%$ & $2.8 \%$ \\
\hline $\mathrm{CaO}(\mathrm{wt} \%)$ & 1.445 & 1.424 & 1.455 & 1.595 & 1.427 & $1.2 \%$ & $-0.2 \%$ & $2.0 \%$ & $11.8 \%$ \\
\hline $\mathrm{CdO}(\mathrm{wt} \%)$ & 0.004 & 0.004 & 0.004 & 0.004 & & & & & \\
\hline $\mathrm{Ce}_{2} \mathrm{O}_{3}(\mathrm{wt} \%)$ & 0.037 & 0.036 & 0.038 & 0.036 & & & & & \\
\hline $\mathrm{CoO}(\mathrm{wt} \%)$ & 0.009 & 0.006 & 0.014 & 0.009 & & & & & \\
\hline $\mathrm{Cr}_{2} \mathrm{O}_{3}(\mathrm{wt} \%)$ & 0.100 & 0.095 & 0.097 & 0.090 & 0.088 & $14.3 \%$ & $8.1 \%$ & $11.0 \%$ & $2.8 \%$ \\
\hline $\mathrm{CuO}(\mathrm{wt} \%)$ & 0.006 & 0.003 & 0.003 & 0.003 & 0.0001 & & & & \\
\hline $\mathrm{Fe}_{2} \mathrm{O}_{3}(\mathrm{wt} \%)$ & 14.097 & 13.692 & 14.211 & 13.811 & 13.997 & $0.7 \%$ & $-2.2 \%$ & $1.5 \%$ & $-1.3 \%$ \\
\hline $\mathrm{K}_{2} \mathrm{O}(\mathrm{wt} \%)$ & 2.791 & . & 2.879 & 3.321 & 2.710 & $3.0 \%$ & & $6.2 \%$ & $22.5 \%$ \\
\hline $\mathrm{La}_{2} \mathrm{O}_{3}(\mathrm{wt} \%)$ & 0.007 & 0.010 & 0.007 & 0.012 & & & & & \\
\hline $\mathrm{Li}_{2} \mathrm{O}$ (wt\%) & 3.366 & 3.251 & 3.308 & 3.186 & 3.208 & $4.9 \%$ & $1.3 \%$ & $3.1 \%$ & $-0.7 \%$ \\
\hline $\mathrm{MgO}(\mathrm{wt} \%)$ & 0.854 & 0.835 & 0.857 & 0.812 & 0.862 & $-0.9 \%$ & $-3.1 \%$ & $-0.6 \%$ & $-5.8 \%$ \\
\hline $\mathrm{MnO}(\mathrm{wt} \%)$ & 1.907 & 1.877 & 1.911 & 1.825 & 1.885 & $1.1 \%$ & $-0.5 \%$ & $1.4 \%$ & $-3.2 \%$ \\
\hline $\mathrm{MoO}_{3}(\mathrm{w} \%)$ & 0.021 & 0.020 & 0.021 & 0.020 & & & & & \\
\hline $\mathrm{Na}_{2} \mathrm{O}(\mathrm{wt} \%)$ & 11.696 & 11.319 & 11.835 &. & 11.498 & $1.7 \%$ & $-1.6 \%$ & $2.9 \%$ & \\
\hline $\mathrm{Nb}_{2} \mathrm{O}_{5}(\mathrm{wt} \%)$ & 0.081 & 0.084 & 0.093 & 0.080 & & & & & \\
\hline $\mathrm{Nd}_{2} \mathrm{O}_{3}(\mathrm{wt} \%)$ & 0.049 & 0.048 & 0.049 & 0.047 & & & & & \\
\hline $\mathrm{NiO}$ (wt\%) & 1.037 & 0.780 & 1.084 & 1.022 & 1.056 & $-1.8 \%$ & $-26.1 \%$ & $2.6 \%$ & $-3.2 \%$ \\
\hline $\mathrm{P}_{2} \mathrm{O}_{5}(\mathrm{wt} \%)$ & 0.217 & 0.231 & 0.263 & 0.221 & 0.229 & $-5.2 \%$ & $0.7 \%$ & $14.7 \%$ & $-3.4 \%$ \\
\hline $\mathrm{PbO}$ (wt\%) & 0.021 & 0.021 & 0.021 & 0.021 & & & & & \\
\hline $\mathrm{ReO}_{2}(\mathrm{wt} \%)$ & 0.008 & 0.008 & 0.008 & 0.008 & & & & & \\
\hline $\mathrm{SO}_{4}(\mathrm{wt} \%)$ & 0.139 & 0.135 & 0.140 & 0.134 & & & & & \\
\hline $\mathrm{SiO}_{2}(\mathrm{wt} \%)$ & 49.703 & 48.633 & . & 47.207 & 47.899 & $3.8 \%$ & $1.5 \%$ & & $-1.4 \%$ \\
\hline $\mathrm{SnO}_{2}(\mathrm{wt} \%)$ & 0.020 & 0.020 & 0.021 & 0.020 & & & & & \\
\hline SrO (wt\%) & 0.003 & 0.003 & 0.003 & 0.007 & & & & & \\
\hline $\mathrm{TiO}_{2}(\mathrm{wt} \%)$ & 1.206 & 1.172 & 1.171 & 1.145 & 1.151 & $4.8 \%$ & $1.8 \%$ & $1.8 \%$ & $-0.5 \%$ \\
\hline $\mathrm{V}_{2} \mathrm{O}_{5}(\mathrm{wt} \%)$ & 0.021 & 0.020 & 0.021 & 0.020 & & & & & \\
\hline $\mathrm{ZnO}(\mathrm{wt} \%)$ & 0.023 & 0.022 & 0.024 & 0.023 & 0.012 & & & & \\
\hline $\mathrm{ZrO}_{2}(\mathrm{wt} \%)$ & 0.137 & 0.129 & 0.137 & . & 0.135 & $1.7 \%$ & $-4.4 \%$ & $1.7 \%$ & \\
\hline
\end{tabular}


While there are potentially a large number of observations that one might make regarding Tables 2a and $2 \mathrm{~b}$ and Exhibit A3, comments about $\mathrm{B}_{2} \mathrm{O}_{3}$ and $\mathrm{NiO}$ will be made to demonstrate the interpretation of the statistical tests of the exhibit. From Table 2a, the smallest average measurement for $\mathrm{B}_{2} \mathrm{O}_{3}$ was $7.964 \mathrm{wt} \%$ from the $\mathrm{Cs}_{2} \mathrm{CO}_{3}$ method while the largest average was $8.329 \mathrm{wt} \%$ from the $\mathrm{K}_{2} \mathrm{CO}_{3}$ method with the largest \% relative standard deviation for the measurements being $5.1 \%$ for the $\mathrm{Na}_{2} \mathrm{O}_{2}$ method and the smallest being $1.4 \%$ for the $\mathrm{Cs}_{2} \mathrm{CO}_{3}$ method. From Exhibit A3, the pairwise comparisons of the means indicate no statistically significant differences (since the letter associated with averages of all three methods is the same, "A" in the middle of the left-hand side of the exhibit). Also, the "Levene Test" for differences in the variances of the measurements indicates (by a "Prob $>F$ " value of 0.1810 , which is greater than 0.05 ) no statistically significant differences in the variances at the $5 \%$ significance level.

From Table 2a, the smallest average measurement for $\mathrm{NiO}$ was $0.780 \mathrm{wt} \%$ from the $\mathrm{K}_{2} \mathrm{CO}_{3}$ method while the largest average was $1.084 \mathrm{wt} \%$ from the mixed acid method with the largest \% relative standard deviation for the measurements being $9.0 \%$ for the $\mathrm{K}_{2} \mathrm{CO}_{3}$ method and the smallest being $0.7 \%$ for the mixed acid method. From Exhibit $\mathrm{A} 3$, the pairwise comparisons of the means indicate that the mean of the $\mathrm{K}_{2} \mathrm{CO}_{3}$ method is statistically significantly different (since the letter associated with its average is a " $\mathrm{B}$ " while the letter associated with the other averages is an " $A$ " in the middle of the left-hand side of the exhibit). The "Levene Test" for differences in the variances of the measurements indicates (by a "Prob $>F$ " value of 0.1278 , which is greater than 0.05 ) no statistically significant differences in the variances at the $5 \%$ significance level.

\subsubsection{Measurements of Simulant Samples}

Exhibit A4 in Appendix A provides comparisons across the preparation methods by oxide for the simulant sample measurements. There are pairwise comparisons among the averages of the preparations as well as statistical tests for differences in the variances (i.e., precisions) of the preparation methods. For those oxides at concentrations greater than $0.1 \mathrm{wt} \%$, a difference among the means is indicated for $\mathrm{CaO}\left(\mathrm{Na}_{2} \mathrm{O}_{2}\right.$ yielding a larger mean and $\mathrm{K}_{2} \mathrm{CO}_{3}$ a smaller), $\mathrm{Fe}_{2} \mathrm{O}_{3}\left(\mathrm{Cs}_{2} \mathrm{CO}_{3}\right.$ yielding a larger mean relative to that from $\left.\mathrm{K}_{2} \mathrm{CO}_{3}\right), \mathrm{K}_{2} \mathrm{O}\left(\mathrm{Na}_{2} \mathrm{O}_{2}\right.$ yielding a larger mean), $\mathrm{MgO}\left(\mathrm{Cs}_{2} \mathrm{CO}_{3}\right.$ yielding a larger mean relative to that from $\left.\mathrm{K}_{2} \mathrm{CO}_{3}\right), \mathrm{NiO}\left(\mathrm{K}_{2} \mathrm{CO}_{3}\right.$ yielding a smaller mean), $\mathrm{SO}_{4}$ (mixed acid yielding a smaller mean), $\mathrm{ZnO}\left(\mathrm{Cs}_{2} \mathrm{CO}_{3}\right.$ yielding a larger mean), and $\mathrm{ZrO}_{2}\left(\mathrm{~K}_{2} \mathrm{CO}_{3}\right.$ yielding a smaller mean).

Table 3 provides a summary of this information in the form of the average measurement, standard deviation, and \% relative standard deviation for each oxide by each preparation method. Table 4 provides pairwise comparisons of the average oxide concentrations across the four preparation methods. An entry in Table 4 that shows a pairwise comparison that is $5 \%$ or greater is highlighted. 
Table 3. Average Oxide Measurements, Standard Deviations, and \% Relative Standard Deviations for Simulant Samples by Preparation Method

\begin{tabular}{|c|c|c|c|c|c|c|c|c|c|c|c|c|}
\hline Preparation Method & \multicolumn{3}{|c|}{$\mathrm{Cs}_{2} \mathrm{CO}_{3}$} & \multicolumn{3}{|c|}{$\mathrm{K}_{2} \mathrm{CO}_{3}$} & \multicolumn{3}{|c|}{ Mixed Acid } & \multicolumn{3}{|c|}{$\mathrm{Na}_{2} \mathrm{O}_{2}$} \\
\hline N Rows (3) & Mean & Std Dev & $\begin{array}{c}\text { \% Rel Std } \\
\text { Dev }\end{array}$ & Mean & Std Dev & $\begin{array}{c}\text { \% Rel Std } \\
\text { Dev }\end{array}$ & Mean & Std Dev & $\begin{array}{c}\text { \% Rel Std } \\
\text { Dev }\end{array}$ & Mean & Std Dev & $\begin{array}{c}\text { \% Rel Std } \\
\text { Dev }\end{array}$ \\
\hline $\mathrm{Ag}_{2} \mathrm{O}(\mathrm{wt} \%)$ & 0.018 & 0.0004 & $2.2 \%$ & 0.018 & 0.0007 & $3.7 \%$ & 0.017 & 0.0005 & $2.6 \%$ & 0.018 & 0.0006 & $3.5 \%$ \\
\hline $\mathrm{Al}_{2} \mathrm{O}_{3}(\mathrm{wt} \%)$ & 6.333 & 0.0347 & $0.5 \%$ & 6.031 & 0.1565 & $2.6 \%$ & 6.012 & 0.3566 & $5.9 \%$ & 6.144 & 0.2400 & $3.9 \%$ \\
\hline $\mathrm{B}_{2} \mathrm{O}_{3}(\mathrm{wt} \%)$ & 5.066 & 0.0926 & $1.8 \%$ & 5.012 & 0.1464 & & 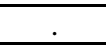 & & & 5.125 & 0.2207 & $4.3 \%$ \\
\hline $\mathrm{BaO}(\mathrm{wt} \%)$ & 0.075 & 0.0009 & $1.2 \%$ & 0.062 & 0.0020 & $3.2 \%$ & 0.064 & 0.0042 & $6.6 \%$ & 0.067 & 0.0065 & $9.7 \%$ \\
\hline $\mathrm{CaO}(\mathrm{wt} \%)$ & 1.388 & 0.0088 & $0.6 \%$ & 1.271 & 0.0493 & $3.9 \%$ & 1.319 & 0.0902 & $6.8 \%$ & 1.553 & 0.0680 & $4.4 \%$ \\
\hline $\mathrm{CdO}(\mathrm{wt} \%)$ & 0.004 & 0.0001 & $2.2 \%$ & 0.004 & 0.0001 & $3.7 \%$ & 0.004 & 0.0001 & $2.6 \%$ & 0.004 & 0.0001 & $3.5 \%$ \\
\hline $\mathrm{Ce}_{2} \mathrm{O}_{3}(\mathrm{wt} \%)$ & 0.038 & 0.0008 & $2.2 \%$ & 0.038 & 0.0014 & $3.7 \%$ & 0.037 & 0.0010 & $2.6 \%$ & 0.038 & 0.0014 & $3.5 \%$ \\
\hline $\mathrm{CoO}$ (wt\%) & 0.007 & 0.0001 & $2.1 \%$ & 0.007 & 0.0003 & $3.7 \%$ & 0.007 & 0.0002 & $2.5 \%$ & 0.007 & 0.0002 & $3.6 \%$ \\
\hline $\mathrm{Cr}_{2} \mathrm{O}_{3}(\mathrm{wt} \%)$ & 0.085 & 0.0019 & $2.3 \%$ & 0.077 & 0.0028 & $3.6 \%$ & 0.079 & 0.0055 & $6.9 \%$ & 0.076 & 0.0035 & $4.6 \%$ \\
\hline $\mathrm{CuO}$ (wt\%) & 0.060 & 0.0007 & $1.2 \%$ & 0.051 & 0.0033 & $6.4 \%$ & 0.059 & 0.0040 & $6.8 \%$ & 0.060 & 0.0031 & $5.2 \%$ \\
\hline $\mathrm{Fe}_{2} \mathrm{O}_{3}(\mathrm{wt} \%)$ & 14.535 & 0.0738 & $0.5 \%$ & 13.554 & 0.3870 & $2.9 \%$ & 13.801 & 0.8518 & $6.2 \%$ & 14.128 & 0.5943 & \\
\hline $\mathrm{K}_{2} \mathrm{O}(\mathrm{wt} \%)$ & 0.167 & 0.0037 & $2.2 \%$ & &. & & 0.199 & 0.0792 & $39.9 \%$ & 0.604 & 0.1794 & $29.7 \%$ \\
\hline $\mathrm{La}_{2} \mathrm{O}_{3}(\mathrm{wt} \%)$ & 0.007 & 0.0002 & $2.3 \%$ & 0.008 & 0.0003 & $3.6 \%$ & 0.007 & 0.0002 & $2.4 \%$ & 0.009 & 0.0041 & $43.7 \%$ \\
\hline $\mathrm{Li}_{2} \mathrm{O}$ (wt\%) & 5.206 & 0.0162 & $0.3 \%$ & 5.013 & 0.1456 & $2.9 \%$ & 5.088 & 0.2822 & $5.5 \%$ & 4.991 & 0.2116 & $4.2 \%$ \\
\hline $\mathrm{MgO}$ (wt\%) & 1.467 & 0.0102 & $0.7 \%$ & 1.371 & 0.0402 & $2.9 \%$ & 1.386 & 0.0924 & $6.7 \%$ & 1.387 & 0.0544 & $3.9 \%$ \\
\hline $\mathrm{MnO}$ (wt\%) & 2.154 & 0.0127 & $0.6 \%$ & 2.027 & 0.0554 & $2.7 \%$ & 2.034 & 0.1319 & $6.5 \%$ & 2.040 & 0.0880 & $4.3 \%$ \\
\hline $\mathrm{MoO}_{3}(\mathrm{w} \%)$ & 0.021 & 0.0005 & $2.2 \%$ & 0.021 & 0.0008 & $3.7 \%$ & 0.021 & 0.0005 & $2.6 \%$ & 0.021 & 0.0008 & \\
\hline $\mathrm{Na}_{2} \mathrm{O}(\mathrm{wt} \%)$ & 13.271 & 0.0433 & $0.3 \%$ & 12.802 & 0.3617 & $2.8 \%$ & 13.152 & 0.6975 & $5.3 \%$ & . & . & \\
\hline $\mathrm{Nb}_{2} \mathrm{O}_{5}(\mathrm{wt} \%)$ & 0.010 & 0.0002 & $2.2 \%$ & 0.010 & 0.0004 & $3.6 \%$ & 0.009 & 0.0003 & $2.7 \%$ & 0.010 & 0.0003 & $3.4 \%$ \\
\hline $\mathrm{Nd}_{2} \mathrm{O}_{3}(\mathrm{wt} \%)$ & 0.049 & 0.0011 & $2.2 \%$ & 0.050 & 0.0018 & $3.6 \%$ & 0.048 & 0.0013 & $2.6 \%$ & 0.050 & 0.0018 & $3.5 \%$ \\
\hline $\mathrm{NiO}(\mathrm{wt} \%)$ & 0.465 & 0.0051 & $1.1 \%$ & 0.373 & 0.0119 & $3.2 \%$ & 0.460 & 0.0287 & $6.2 \%$ & 0.462 & 0.0170 & $3.7 \%$ \\
\hline $\mathrm{P}_{2} \mathrm{O}_{5}(\mathrm{wt} \%)$ & 0.082 & 0.0018 & $2.2 \%$ & 0.084 & 0.0031 & & 0.081 & 0.0021 & & 0.084 & 0.0030 & $3.6 \%$ \\
\hline $\mathrm{PbO}(\mathrm{wt} \%)$ & 0.062 & 0.0137 & $21.9 \%$ & 0.045 & 0.0185 & $40.8 \%$ & 0.046 & 0.0125 & $27.1 \%$ & 0.038 & 0.0184 & $48.1 \%$ \\
\hline $\mathrm{ReO}_{2}(\mathrm{wt} \%)$ & 0.008 & 0.0002 & $2.2 \%$ & 0.008 & 0.0003 & $3.6 \%$ & 0.008 & 0.0002 & $2.7 \%$ & 0.008 & 0.0003 & $3.5 \%$ \\
\hline $\mathrm{SO}_{4}(\mathrm{wt} \%)$ & 0.646 & 0.0276 & $4.3 \%$ & 0.606 & 0.0503 & $8.3 \%$ & 0.469 & 0.0672 & $14.3 \%$ & 0.633 & 0.0835 & $13.2 \%$ \\
\hline $\mathrm{SiO}_{2}(\mathrm{wt} \%)$ & 50.024 & 1.2578 & $2.5 \%$ & 47.635 & 1.4551 & $3.1 \%$ & . &. & & 48.099 & 2.0178 & $4.2 \%$ \\
\hline $\mathrm{SnO}_{2}(\mathrm{wt} \%)$ & 0.020 & 0.0005 & $2.2 \%$ & 0.021 & 0.0008 & $3.7 \%$ & 0.020 & 0.0005 & $2.7 \%$ & 0.021 & 0.0007 & $3.6 \%$ \\
\hline SrO (wt\%) & 0.008 & 0.0001 & $0.8 \%$ & 0.007 & 0.0002 & $3.1 \%$ & 0.007 & 0.0006 & $8.0 \%$ & 0.010 & 0.0031 & $29.3 \%$ \\
\hline $\mathrm{TiO}_{2}(\mathrm{wt} \%)$ & 0.040 & 0.0004 & $1.0 \%$ & 0.037 & 0.0012 & $3.3 \%$ & 0.038 & 0.0014 & $3.6 \%$ & 0.038 & 0.0021 & $5.4 \%$ \\
\hline $\mathrm{V}_{2} \mathrm{O}_{5}(\mathrm{wt} \%)$ & 0.021 & 0.0005 & $2.2 \%$ & 0.022 & 0.0008 & $3.6 \%$ & 0.021 & 0.0005 & $2.6 \%$ & 0.022 & 0.0007 & $3.5 \%$ \\
\hline $\mathrm{ZnO}(\mathrm{wt} \%)$ & 0.149 & 0.0029 & $1.9 \%$ & 0.137 & 0.0044 & $3.2 \%$ & 0.138 & 0.0086 & $6.2 \%$ & 0.139 & 0.0056 & $4.0 \%$ \\
\hline $\mathrm{ZrO}_{2}(\mathrm{wt} \%)$ & 0.268 & 0.0046 & $1.7 \%$ & 0.253 & 0.0056 & $2.2 \%$ & 0.269 & 0.0118 & $4.4 \%$ & . & . & \\
\hline
\end{tabular}


Westinghouse Savannah River Company

WSRC-TR-2005-00396

Savannah River National Laboratory

Rev. 0

Analytical Development/Statistical Consulting Sections

Page 18 of 140

Table 4. Pairwise Measurement Comparisons of Preparation Methods by Oxide for Simulant Samples

\begin{tabular}{|c|c|c|c|c|c|c|c|c|c|}
\hline $\mathrm{Ag}_{2} \mathrm{O}(\mathrm{wt} \%)$ & $\mathrm{Cs}_{2} \mathrm{CO}_{3}$ & $\mathrm{~K}_{2} \mathrm{CO}_{3}$ & Mixed Acid & $\mathrm{Na}_{2} \mathrm{O}_{2}$ & $\mathrm{Ce}_{2} \mathrm{O}_{3}(\mathrm{wt} \%)$ & $\mathrm{Cs}_{2} \mathrm{CO}_{3}$ & $\mathrm{~K}_{2} \mathrm{CO}_{3}$ & Mixed Acid & $\mathrm{Na}_{2} \mathrm{O}_{2}$ \\
\hline $\mathrm{Cs}_{2} \mathrm{CO}_{3}$ & $0.0 \%$ & $-2.4 \%$ & $1.3 \%$ & $-2.3 \%$ & $\mathrm{Cs}_{2} \mathrm{CO}_{3}$ & $0.0 \%$ & $-2.5 \%$ & $1.3 \%$ & $-2.3 \%$ \\
\hline $\mathrm{K}_{2} \mathrm{CO}_{3}$ & $2.5 \%$ & $0.0 \%$ & $3.8 \%$ & $0.1 \%$ & $\mathrm{~K}_{2} \mathrm{CO}_{3}$ & $2.5 \%$ & $0.0 \%$ & $3.9 \%$ & $0.1 \%$ \\
\hline Mixed Acid & $-1.3 \%$ & $-3.7 \%$ & $0.0 \%$ & $-3.5 \%$ & Mixed Acid & $-1.3 \%$ & $-3.7 \%$ & $0.0 \%$ & $-3.6 \%$ \\
\hline $\mathrm{Na}_{2} \mathrm{O}_{2}$ & $2.3 \%$ & $-0.1 \%$ & $3.7 \%$ & $0.0 \%$ & $\mathrm{Na}_{2} \mathrm{O}_{2}$ & $2.4 \%$ & $-0.1 \%$ & $3.7 \%$ & $0.0 \%$ \\
\hline $\mathrm{Al}_{2} \mathrm{O}_{3}(\mathrm{wt} \%)$ & $\mathrm{Cs}_{2} \mathrm{CO}_{3}$ & $\mathrm{~K}_{2} \mathrm{CO}_{3}$ & Mixed Acid & $\mathrm{Na}_{2} \mathrm{O}_{2}$ & $\mathrm{CoO}(\mathrm{wt} \%)$ & $\mathrm{Cs}_{2} \mathrm{CO}_{3}$ & $\mathrm{~K}_{2} \mathrm{CO}_{3}$ & Mixed Acid & $\mathrm{Na}_{2} \mathrm{O}_{2}$ \\
\hline $\mathrm{Cs}_{2} \mathrm{CO}_{3}$ & $0.0 \%$ & $5.0 \%$ & $5.3 \%$ & $3.1 \%$ & $\mathrm{Cs}_{2} \mathrm{CO}_{3}$ & $0.0 \%$ & $-2.5 \%$ & $1.3 \%$ & $-2.3 \%$ \\
\hline $\mathrm{K}_{2} \mathrm{CO}_{3}$ & $-4.8 \%$ & $0.0 \%$ & $0.3 \%$ & $-1.8 \%$ & $\mathrm{~K}_{2} \mathrm{CO}_{3}$ & $2.6 \%$ & $0.0 \%$ & $3.9 \%$ & $0.2 \%$ \\
\hline Mixed Acid & $-5.1 \%$ & $-3.7 \%$ & $0.0 \%$ & $-3.6 \%$ & Mixed Acid & $-1.3 \%$ & $-3.8 \%$ & $0.0 \%$ & $-3.6 \%$ \\
\hline $\mathrm{Na}_{2} \mathrm{O}_{2}$ & $-3.0 \%$ & $1.9 \%$ & $2.2 \%$ & $0.0 \%$ & $\mathrm{Na}_{2} \mathrm{O}_{2}$ & $2.4 \%$ & $-0.2 \%$ & $3.7 \%$ & $0.0 \%$ \\
\hline $\mathrm{B}_{2} \mathrm{O}_{3}(\mathrm{wt} \%)$ & $\mathrm{Cs}_{2} \mathrm{CO}_{3}$ & $\mathrm{~K}_{2} \mathrm{CO}_{3}$ & Mixed Acid & $\mathrm{Na}_{2} \mathrm{O}_{2}$ & $\mathrm{Cr}_{2} \mathrm{O}_{3}(\mathrm{wt} \%)$ & $\mathrm{Cs}_{2} \mathrm{CO}_{3}$ & $\mathrm{~K}_{2} \mathrm{CO}_{3}$ & Mixed Acid & $\mathrm{Na}_{2} \mathrm{O}_{2}$ \\
\hline $\mathrm{Cs}_{2} \mathrm{CO}_{3}$ & $0.0 \%$ & $1.1 \%$ & & $-1.2 \%$ & $\mathrm{Cs}_{2} \mathrm{CO}_{3}$ & $0.0 \%$ & $10.0 \%$ & $7.7 \%$ & $12.2 \%$ \\
\hline $\mathrm{K}_{2} \mathrm{CO}_{3}$ & $-1.1 \%$ & $0.0 \%$ & & $-2.2 \%$ & $\mathrm{~K}_{2} \mathrm{CO}_{3}$ & $-9.1 \%$ & $0.0 \%$ & $-2.1 \%$ & $2.0 \%$ \\
\hline Mixed Acid & & & & & Mixed Acid & $-7.1 \%$ & $2.2 \%$ & $0.0 \%$ & $4.2 \%$ \\
\hline $\mathrm{Na}_{2} \mathrm{O}_{2}$ & $1.2 \%$ & $2.2 \%$ & & $0.0 \%$ & $\mathrm{Na}_{2} \mathrm{O}_{2}$ & $-10.9 \%$ & $-2.0 \%$ & $-4.1 \%$ & $0.0 \%$ \\
\hline & & & & & & & & & \\
\hline $\mathrm{BaO}(\mathrm{wt} \%)$ & $\mathrm{Cs}_{2} \mathrm{CO}_{3}$ & $\mathrm{~K}_{2} \mathrm{CO}_{3}$ & Mixed Acid & $\mathrm{Na}_{2} \mathrm{O}_{2}$ & $\mathrm{CuO}(\mathrm{wt} \%)$ & $\mathrm{Cs}_{2} \mathrm{CO}_{3}$ & $\mathrm{~K}_{2} \mathrm{CO}_{3}$ & Mixed Acid & $\mathrm{Na}_{2} \mathrm{O}_{2}$ \\
\hline $\mathrm{Cs}_{2} \mathrm{CO}_{3}$ & $0.0 \%$ & $20.7 \%$ & $18.1 \%$ & $11.6 \%$ & $\mathrm{Cs}_{2} \mathrm{CO}_{3}$ & $0.0 \%$ & $16.7 \%$ & $1.8 \%$ & $0.1 \%$ \\
\hline $\mathrm{K}_{2} \mathrm{CO}_{3}$ & $-17.1 \%$ & $0.0 \%$ & $-2.1 \%$ & $-7.5 \%$ & $\mathrm{~K}_{2} \mathrm{CO}_{3}$ & $-14.3 \%$ & $0.0 \%$ & $-12.8 \%$ & $-14.3 \%$ \\
\hline Mixed Acid & $-15.3 \%$ & $2.2 \%$ & $0.0 \%$ & $-5.5 \%$ & Mixed Acid & $-1.8 \%$ & $14.6 \%$ & $0.0 \%$ & $-1.7 \%$ \\
\hline $\mathrm{Na}_{2} \mathrm{O}_{2}$ & $-10.4 \%$ & $8.1 \%$ & $5.9 \%$ & $0.0 \%$ & $\mathrm{Na}_{2} \mathrm{O}_{2}$ & $-0.1 \%$ & $16.6 \%$ & $1.7 \%$ & $0.0 \%$ \\
\hline $\mathrm{CaO}(\mathrm{wt} \%)$ & $\mathrm{Cs}_{2} \mathrm{CO}_{3}$ & $\mathrm{~K}_{2} \mathrm{CO}_{3}$ & Mixed Acid & $\mathrm{Na}_{2} \mathrm{O}_{2}$ & $\mathrm{Fe}_{2} \mathrm{O}_{3}(\mathrm{wt} \%)$ & $\mathrm{Cs}_{2} \mathrm{CO}_{3}$ & $\mathrm{~K}_{2} \mathrm{CO}_{3}$ & Mixed Acid & $\mathrm{Na}_{2} \mathrm{O}_{2}$ \\
\hline $\mathrm{Cs}_{2} \mathrm{CO}_{3}$ & $0.0 \%$ & $9.2 \%$ & $5.3 \%$ & $-10.6 \%$ & $\mathrm{Cs}_{2} \mathrm{CO}_{3}$ & $0.0 \%$ & $7.2 \%$ & $5.3 \%$ & $2.9 \%$ \\
\hline $\mathrm{K}_{2} \mathrm{CO}_{3}$ & $-8.4 \%$ & $0.0 \%$ & $-3.6 \%$ & $-18.1 \%$ & $\mathrm{~K}_{2} \mathrm{CO}_{3}$ & $-6.8 \%$ & $0.0 \%$ & $-1.8 \%$ & $-4.1 \%$ \\
\hline Mixed Acid & $-5.0 \%$ & $3.7 \%$ & $0.0 \%$ & $-15.1 \%$ & Mixed Acid & $-5.0 \%$ & $1.8 \%$ & $0.0 \%$ & $-2.3 \%$ \\
\hline $\mathrm{Na}_{2} \mathrm{O}_{2}$ & $11.9 \%$ & $22.2 \%$ & $17.8 \%$ & $0.0 \%$ & $\mathrm{Na}_{2} \mathrm{O}_{2}$ & $-2.8 \%$ & $4.2 \%$ & $2.4 \%$ & $0.0 \%$ \\
\hline $\mathrm{CdO}(\mathrm{wt} \%)$ & $\mathrm{Cs}_{2} \mathrm{CO}_{3}$ & $\mathrm{~K}_{2} \mathrm{CO}_{3}$ & Mixed Acid & $\mathrm{Na}_{2} \mathrm{O}_{2}$ & $\mathrm{~K}_{2} \mathrm{O}(\mathrm{wt} \%)$ & $\mathrm{Cs}_{2} \mathrm{CO}_{3}$ & $\mathrm{~K}_{2} \mathrm{CO}_{3}$ & Mixed Acid & $\mathrm{Na}_{2} \mathrm{O}_{2}$ \\
\hline $\mathrm{Cs}_{2} \mathrm{CO}_{3}$ & $0.0 \%$ & $-2.5 \%$ & $1.2 \%$ & $-2.3 \%$ & $\mathrm{Cs}_{2} \mathrm{CO}_{3}$ & $0.0 \%$ & & $-15.7 \%$ & $-72.3 \%$ \\
\hline $\mathrm{K}_{2} \mathrm{CO}_{3}$ & $2.5 \%$ & $0.0 \%$ & $3.8 \%$ & $0.1 \%$ & $\mathrm{~K}_{2} \mathrm{CO}_{3}$ & & & & \\
\hline Mixed Acid & $-1.2 \%$ & $-3.6 \%$ & $0.0 \%$ & $-3.5 \%$ & Mixed Acid & $18.6 \%$ & & $0.0 \%$ & $-67.1 \%$ \\
\hline $\mathrm{Na}_{2} \mathrm{O}_{2}$ & $2.4 \%$ & $-0.1 \%$ & $3.6 \%$ & $0.0 \%$ & $\mathrm{Na}_{2} \mathrm{O}_{2}$ & $260.8 \%$ & & $204.1 \%$ & $0.0 \%$ \\
\hline
\end{tabular}


Table 4. Pairwise Measurement Comparisons of Preparation Methods by Oxide for Simulant Samples (continued)

\begin{tabular}{|c|c|c|c|c|c|c|c|c|c|}
\hline $\mathrm{La}_{2} \mathrm{O}_{3}(\mathrm{wt} \%)$ & $\mathrm{Cs}_{2} \mathrm{CO}_{3}$ & $\overline{\mathrm{K} \mathrm{K}_{2} \mathrm{CO}_{3}}$ & Mixed Acid & $\mathrm{CNa}_{2} \mathrm{O}_{2}$ & $\mathrm{Nb}_{2} \mathrm{O}_{5}(\mathrm{wt} \%)$ & $\mathrm{Cs}_{2} \mathrm{CO}_{3}$ & $\overline{\mathrm{K}} \mathrm{K}_{2} \mathrm{CO}_{3}$ & Mixed Acid & $\mathrm{Na}_{2} \mathrm{O}_{2}$ \\
\hline $\mathrm{Cs}_{2} \mathrm{CO}_{3}$ & $0.0 \%$ & $-2.2 \%$ & $1.5 \%$ & $-20.0 \%$ & $\mathrm{Cs}_{2} \mathrm{CO}_{3}$ & $0.0 \%$ & $-2.5 \%$ & $1.1 \%$ & $-2.4 \%$ \\
\hline $\mathrm{K}_{2} \mathrm{CO}_{3}$ & $2.2 \%$ & $0.0 \%$ & $3.7 \%$ & $-18.2 \%$ & $\mathrm{~K}_{2} \mathrm{CO}_{3}$ & $2.6 \%$ & $0.0 \%$ & $3.8 \%$ & $0.1 \%$ \\
\hline Mixed Acid & $-1.4 \%$ & $-3.6 \%$ & $0.0 \%$ & $-21.1 \%$ & Mixed Acid & $-1.1 \%$ & $-3.6 \%$ & $0.0 \%$ & $-3.5 \%$ \\
\hline $\mathrm{Na}_{2} \mathrm{O}_{2}$ & $24.9 \%$ & $22.2 \%$ & $26.8 \%$ & $0.0 \%$ & $\mathrm{Na}_{2} \mathrm{O}_{2}$ & $2.5 \%$ & $-0.1 \%$ & $3.6 \%$ & $0.0 \%$ \\
\hline $\mathrm{Li}_{2} \mathrm{O}(\mathrm{wt} \%)$ & $\mathrm{Cs}_{2} \mathrm{CO}_{3}$ & $\mathrm{~K}_{2} \mathrm{CO}_{3}$ & Mixed Acid & $\mathrm{Na}_{2} \mathrm{O}_{2}$ & $\mathrm{Nd}_{2} \mathrm{O}_{3}(\mathrm{wt} \%)$ & $\mathrm{Cs}_{2} \mathrm{CO}_{3}$ & $\mathrm{~K}_{2} \mathrm{CO}_{3}$ & Mixed Acid & $\mathrm{Na}_{2} \mathrm{O}_{2}$ \\
\hline $\mathrm{Cs}_{2} \mathrm{CO}_{3}$ & $0.0 \%$ & $3.9 \%$ & $2.3 \%$ & $4.3 \%$ & $\mathrm{Cs}_{2} \mathrm{CO}_{3}$ & $0.0 \%$ & $-2.5 \%$ & $1.3 \%$ & $-2.3 \%$ \\
\hline $\mathrm{K}_{2} \mathrm{CO}_{3}$ & $-3.7 \%$ & $0.0 \%$ & $-1.5 \%$ & $0.4 \%$ & $\mathrm{~K}_{2} \mathrm{CO}_{3}$ & $2.5 \%$ & $0.0 \%$ & $3.8 \%$ & $0.1 \%$ \\
\hline Mixed Acid & $-2.3 \%$ & $1.5 \%$ & $0.0 \%$ & $1.9 \%$ & Mixed Acid & $-1.3 \%$ & $-3.7 \%$ & $0.0 \%$ & $-3.6 \%$ \\
\hline $\mathrm{Na}_{2} \mathrm{O}_{2}$ & $-4.1 \%$ & $-0.4 \%$ & $-1.9 \%$ & $0.0 \%$ & $\mathrm{Na}_{2} \mathrm{O}_{2}$ & $2.4 \%$ & $-0.1 \%$ & $3.7 \%$ & $0.0 \%$ \\
\hline $\mathrm{MgO}(\mathrm{wt} \%)$ & $\mathrm{Cs}_{2} \mathrm{CO}_{3}$ & $\mathrm{~K}_{2} \mathrm{CO}_{3}$ & Mixed Acid & $\mathrm{Na}_{2} \mathrm{O}_{2}$ & $\mathrm{NiO}(\mathrm{wt} \%)$ & $\mathrm{Cs}_{2} \mathrm{CO}_{3}$ & $\mathrm{~K}_{2} \mathrm{CO}_{3}$ & Mixed Acid & $\mathrm{Na}_{2} \mathrm{O}_{2}$ \\
\hline $\mathrm{Cs}_{2} \mathrm{CO}_{3}$ & $0.0 \%$ & $7.0 \%$ & $5.8 \%$ & $5.7 \%$ & $\mathrm{Cs}_{2} \mathrm{CO}_{3}$ & $0.0 \%$ & $24.5 \%$ & $1.0 \%$ & $0.6 \%$ \\
\hline $\mathrm{K}_{2} \mathrm{CO}_{3}$ & $-6.6 \%$ & $0.0 \%$ & $-1.1 \%$ & $-1.2 \%$ & $\mathrm{~K}_{2} \mathrm{CO}_{3}$ & $-19.7 \%$ & $0.0 \%$ & $-18.9 \%$ & $-19.2 \%$ \\
\hline Mixed Acid & $-5.5 \%$ & $1.1 \%$ & $0.0 \%$ & $-0.1 \%$ & Mixed Acid & $-1.0 \%$ & $23.3 \%$ & $0.0 \%$ & $-0.4 \%$ \\
\hline $\mathrm{Na}_{2} \mathrm{O}_{2}$ & $-5.4 \%$ & $1.2 \%$ & $0.1 \%$ & $0.0 \%$ & $\mathrm{Na}_{2} \mathrm{O}_{2}$ & $-0.6 \%$ & $23.8 \%$ & $0.4 \%$ & $0.0 \%$ \\
\hline $\mathrm{MnO}(\mathrm{wt} \%)$ & $\mathrm{Cs}_{2} \mathrm{CO}_{3}$ & $\mathrm{~K}_{2} \mathrm{CO}_{3}$ & Mixed Acid & $\mathrm{Na}_{2} \mathrm{O}_{2}$ & $\mathrm{P}_{2} \mathrm{O}_{5}(\mathrm{wt} \%)$ & $\mathrm{Cs}_{2} \mathrm{CO}_{3}$ & $\mathrm{~K}_{2} \mathrm{CO}_{3}$ & Mixed Acid & $\mathrm{Na}_{2} \mathrm{O}_{2}$ \\
\hline $\mathrm{Cs}_{2} \mathrm{CO}_{3}$ & $0.0 \%$ & $6.3 \%$ & $5.9 \%$ & $5.6 \%$ & $\mathrm{Cs}_{2} \mathrm{CO}_{3}$ & $0.0 \%$ & $-2.4 \%$ & $1.3 \%$ & $-2.3 \%$ \\
\hline $\mathrm{K}_{2} \mathrm{CO}_{3}$ & $-5.9 \%$ & $0.0 \%$ & $-0.3 \%$ & $-0.6 \%$ & $\mathrm{~K}_{2} \mathrm{CO}_{3}$ & $2.5 \%$ & $0.0 \%$ & $3.9 \%$ & $0.1 \%$ \\
\hline Mixed Acid & $-5.6 \%$ & $0.3 \%$ & $0.0 \%$ & $-0.3 \%$ & Mixed Acid & $-1.3 \%$ & $-3.7 \%$ & $0.0 \%$ & $-3.6 \%$ \\
\hline $\mathrm{Na}_{2} \mathrm{O}_{2}$ & $-5.3 \%$ & $0.6 \%$ & $0.3 \%$ & $0.0 \%$ & $\mathrm{Na}_{2} \mathrm{O}_{2}$ & $2.4 \%$ & $-0.1 \%$ & $3.7 \%$ & $0.0 \%$ \\
\hline $\mathrm{MoO}_{3}(\mathrm{w} \%)$ & $\mathrm{Cs}_{2} \mathrm{CO}_{3}$ & $\mathrm{~K}_{2} \mathrm{CO}_{3}$ & Mixed Acid & $\mathrm{Na}_{2} \mathrm{O}_{2}$ & $\mathrm{PbO}$ (wt\%) & $\mathrm{Cs}_{2} \mathrm{CO}_{3}$ & $\mathrm{~K}_{2} \mathrm{CO}_{3}$ & Mixed Acid & $\mathrm{Na}_{2} \mathrm{O}_{2}$ \\
\hline $\mathrm{Cs}_{2} \mathrm{CO}_{3}$ & $0.0 \%$ & $-2.6 \%$ & $1.2 \%$ & $-2.4 \%$ & $\mathrm{Cs}_{2} \mathrm{CO}_{3}$ & $0.0 \%$ & $38.0 \%$ & $36.0 \%$ & $62.8 \%$ \\
\hline $\mathrm{K}_{2} \mathrm{CO}_{3}$ & $2.6 \%$ & $0.0 \%$ & $3.9 \%$ & $0.1 \%$ & $\mathrm{~K}_{2} \mathrm{CO}_{3}$ & $-27.5 \%$ & $0.0 \%$ & $-1.4 \%$ & $18.0 \%$ \\
\hline Mixed Acid & $-1.2 \%$ & $-3.7 \%$ & $0.0 \%$ & $-3.6 \%$ & Mixed Acid & $-26.5 \%$ & $1.5 \%$ & $0.0 \%$ & $19.7 \%$ \\
\hline $\mathrm{Na}_{2} \mathrm{O}_{2}$ & $2.5 \%$ & $-0.1 \%$ & $3.7 \%$ & $0.0 \%$ & $\mathrm{Na}_{2} \mathrm{O}_{2}$ & $-38.6 \%$ & $-15.3 \%$ & $-16.5 \%$ & $0.0 \%$ \\
\hline $\mathrm{Na}_{2} \mathrm{O}(\mathrm{wt} \%)$ & $\mathrm{Cs}_{2} \mathrm{CO}_{3}$ & $\mathrm{~K}_{2} \mathrm{CO}_{3}$ & Mixed Acid & $\mathrm{Na}_{2} \mathrm{O}_{2}$ & $\mathrm{ReO}_{2}(\mathrm{wt} \%)$ & $\mathrm{Cs}_{2} \mathrm{CO}_{3}$ & $\mathrm{~K}_{2} \mathrm{CO}_{3}$ & Mixed Acid & $\mathrm{Na}_{2} \mathrm{O}_{2}$ \\
\hline $\mathrm{Cs}_{2} \mathrm{CO}_{3}$ & $0.0 \%$ & $3.7 \%$ & $0.9 \%$ & & $\mathrm{Cs}_{2} \mathrm{CO}_{3}$ & $0.0 \%$ & $-2.5 \%$ & $1.3 \%$ & $-2.4 \%$ \\
\hline $\mathrm{K}_{2} \mathrm{CO}_{3}$ & $-3.5 \%$ & $0.0 \%$ & $-2.7 \%$ & & $\mathrm{~K}_{2} \mathrm{CO}_{3}$ & $2.6 \%$ & $0.0 \%$ & $3.9 \%$ & $0.1 \%$ \\
\hline Mixed Acid & $-0.9 \%$ & $2.7 \%$ & $0.0 \%$ & & Mixed Acid & $-1.3 \%$ & $-3.8 \%$ & $0.0 \%$ & $-3.7 \%$ \\
\hline $\mathrm{Na}_{2} \mathrm{O}_{2}$ & & & & & $\mathrm{Na}_{2} \mathrm{O}_{2}$ & $2.5 \%$ & $-0.1 \%$ & $3.8 \%$ & $0.0 \%$ \\
\hline
\end{tabular}


Westinghouse Savannah River Company

WSRC-TR-2005-00396

Savannah River National Laboratory

Rev. 0

Analytical Development/Statistical Consulting Sections

Page 20 of 140

Table 4. Pairwise Measurement Comparisons of Preparation Methods by Oxide for Simulant Samples (continued)

\begin{tabular}{|c|c|c|c|c|c|c|c|c|c|}
\hline $2 \mathrm{SO}_{4}(\mathrm{wt} \%)$ & $\mathrm{Cs}_{2} \mathrm{CO}_{3}$ & $\overline{\mathrm{K} \mathrm{K}_{2} \mathrm{CO}_{3}}$ & Mixed Acid & $\overline{\mathrm{N}} \mathrm{Na}_{2} \mathrm{O}_{2}$ & בnO (wt\%) & $\mathrm{Cs}_{2} \mathrm{CO}_{3}$ & $\overline{\mathrm{K} \mathrm{K}_{2} \mathrm{CO}_{3}}$ & Mixed Acid & $\mathrm{Na}_{2} \mathrm{O}_{2}$ \\
\hline $\mathrm{Cs}_{2} \mathrm{CO}_{3}$ & $0.0 \%$ & $6.6 \%$ & $37.8 \%$ & $2.1 \%$ & $\mathrm{Cs}_{2} \mathrm{CO}_{3}$ & $0.0 \%$ & $8.9 \%$ & $7.9 \%$ & $7.0 \%$ \\
\hline $\mathrm{K}_{2} \mathrm{CO}_{3}$ & $-6.2 \%$ & $0.0 \%$ & $29.3 \%$ & $-4.3 \%$ & $\mathrm{~K}_{2} \mathrm{CO}_{3}$ & $-8.2 \%$ & $0.0 \%$ & $-1.0 \%$ & $-1.8 \%$ \\
\hline Mixed Acid & $-27.4 \%$ & $-22.7 \%$ & $0.0 \%$ & $-25.9 \%$ & Mixed Acid & $-7.3 \%$ & $1.0 \%$ & $0.0 \%$ & $-0.8 \%$ \\
\hline $\mathrm{Na}_{2} \mathrm{O}_{2}$ & $-2.0 \%$ & $4.4 \%$ & $35.0 \%$ & $0.0 \%$ & $\mathrm{Na}_{2} \mathrm{O}_{2}$ & $-6.5 \%$ & $1.8 \%$ & $0.8 \%$ & $0.0 \%$ \\
\hline $\mathrm{SiO}_{2}(\mathrm{wt} \%)$ & $\mathrm{Cs}_{2} \mathrm{CO}_{3}$ & $\mathrm{~K}_{2} \mathrm{CO}_{3}$ & Mixed Acid & $\mathrm{Na}_{2} \mathrm{O}_{2}$ & $\mathrm{ZrO}_{2}(\mathrm{wt} \%)$ & $\mathrm{Cs}_{2} \mathrm{CO}_{3}$ & $\mathrm{~K}_{2} \mathrm{CO}_{3}$ & Mixed Acid & $\mathrm{Na}_{2} \mathrm{O}_{2}$ \\
\hline $\mathrm{Cs}_{2} \mathrm{CO}_{3}$ & $0.0 \%$ & $5.0 \%$ & & $4.0 \%$ & $\mathrm{Cs}_{2} \mathrm{CO}_{3}$ & $0.0 \%$ & $5.8 \%$ & $-0.5 \%$ & \\
\hline $\mathrm{K}_{2} \mathrm{CO}_{3}$ & $-4.8 \%$ & $0.0 \%$ & & $-1.0 \%$ & $\mathrm{~K}_{2} \mathrm{CO}_{3}$ & $-5.5 \%$ & $0.0 \%$ & $-5.9 \%$ & \\
\hline Mixed Acid & & & & & Mixed Acid & $0.5 \%$ & $6.3 \%$ & $0.0 \%$ & \\
\hline $\mathrm{Na}_{2} \mathrm{O}_{2}$ & $-3.8 \%$ & $1.0 \%$ & & $0.0 \%$ & $\mathrm{Na}_{2} \mathrm{O}_{2}$ & & & & \\
\hline & & & & & & & & & \\
\hline $\mathrm{SnO}_{2}(\mathrm{wt} \%)$ & $\mathrm{Cs}_{2} \mathrm{CO}_{3}$ & $\mathrm{~K}_{2} \mathrm{CO}_{3}$ & Mixed Acid & $\mathrm{Na}_{2} \mathrm{O}_{2}$ & & & & & \\
\hline $\mathrm{Cs}_{2} \mathrm{CO}_{3}$ & $0.0 \%$ & $-2.5 \%$ & $1.3 \%$ & $-2.3 \%$ & & & & & \\
\hline $\mathrm{K}_{2} \mathrm{CO}_{3}$ & $2.5 \%$ & $0.0 \%$ & $3.9 \%$ & $0.2 \%$ & & & & & \\
\hline Mixed Acid & $-1.3 \%$ & $-3.7 \%$ & $0.0 \%$ & $-3.6 \%$ & & & & & \\
\hline $\mathrm{Na}_{2} \mathrm{O}_{2}$ & $2.4 \%$ & $-0.2 \%$ & $3.7 \%$ & $0.0 \%$ & & & & & \\
\hline & & & & & & & & & \\
\hline $\mathrm{SrO}(\mathrm{wt} \%)$ & $\mathrm{Cs}_{2} \mathrm{CO}_{3}$ & $\mathrm{~K}_{2} \mathrm{CO}_{3}$ & Mixed Acid & $\mathrm{Na}_{2} \mathrm{O}_{2}$ & & & & & \\
\hline $\mathrm{Cs}_{2} \mathrm{CO}_{3}$ & $0.0 \%$ & $22.8 \%$ & $21.1 \%$ & $-18.8 \%$ & & & & & \\
\hline $\mathrm{K}_{2} \mathrm{CO}_{3}$ & $-18.6 \%$ & $0.0 \%$ & $-1.4 \%$ & $-33.9 \%$ & & & & & \\
\hline Mixed Acid & $-17.5 \%$ & $1.4 \%$ & $0.0 \%$ & $-33.0 \%$ & & & & & \\
\hline $\mathrm{Na}_{2} \mathrm{O}_{2}$ & $23.2 \%$ & $51.3 \%$ & $49.2 \%$ & $0.0 \%$ & & & & & \\
\hline & & & & & & & & & \\
\hline $\mathrm{TiO}_{2}(\mathrm{wt} \%)$ & $\mathrm{Cs}_{2} \mathrm{CO}_{3}$ & $\mathrm{~K}_{2} \mathrm{CO}_{3}$ & Mixed Acid & $\mathrm{Na}_{2} \mathrm{O}_{2}$ & & & & & \\
\hline $\mathrm{Cs}_{2} \mathrm{CO}_{3}$ & $0.0 \%$ & $7.6 \%$ & $3.8 \%$ & $4.4 \%$ & & & & & \\
\hline $\mathrm{K}_{2} \mathrm{CO}_{3}$ & $-7.0 \%$ & $0.0 \%$ & $-3.5 \%$ & $-3.0 \%$ & & & & & \\
\hline Mixed Acid & $-3.6 \%$ & $3.7 \%$ & $0.0 \%$ & $0.6 \%$ & & & & & \\
\hline $\mathrm{Na}_{2} \mathrm{O}_{2}$ & $-4.2 \%$ & $3.1 \%$ & $-0.6 \%$ & $0.0 \%$ & & & & & \\
\hline & & & & & & & & & \\
\hline $\mathrm{V}_{2} \mathrm{O}_{5}(\mathrm{wt} \%)$ & $\mathrm{Cs}_{2} \mathrm{CO}_{3}$ & $\mathrm{~K}_{2} \mathrm{CO}_{3}$ & Mixed Acid & $\mathrm{Na}_{2} \mathrm{O}_{2}$ & & & & & \\
\hline $\mathrm{Cs}_{2} \mathrm{CO}_{3}$ & $0.0 \%$ & $-2.4 \%$ & $1.4 \%$ & $-2.3 \%$ & & & & & \\
\hline $\mathrm{K}_{2} \mathrm{CO}_{3}$ & $2.5 \%$ & $0.0 \%$ & $3.9 \%$ & $0.1 \%$ & & & & & \\
\hline Mixed Acid & $-1.4 \%$ & $-3.8 \%$ & $0.0 \%$ & $-3.7 \%$ & & & & & \\
\hline $\mathrm{Na}_{2} \mathrm{O}_{2}$ & $2.4 \%$ & $-0.1 \%$ & $3.9 \%$ & $0.0 \%$ & & & & & \\
\hline
\end{tabular}




\subsection{Comparisons of the Compositional Measurements for the Radioactive Set of Measurements}

As described earlier, a limited amount of radioactive glass from the DWPF pour stream was available to support this study. Since there was not enough glass for both the $\mathrm{Cs}_{2} \mathrm{CO}_{3}$ and the $\mathrm{K}_{2} \mathrm{CO}_{3}$ methods to be investigated, only the $\mathrm{Cs}_{2} \mathrm{CO}_{3}$ method was performed. Table B1 in Appendix B provides the elemental concentration data generated for the radioactive samples as well as for samples of ARG-1 and for reagent blanks by the $\mathrm{Cs}_{2} \mathrm{CO}_{3}$ method. These chemical composition measurements were compared to the measurements generated by mixed acid and $\mathrm{Na}_{2} \mathrm{O}_{2}$ methods as reported by Bannochie and Bibler [5]. Tables B2 and B3 in Appendix B provide the elemental concentration data generated for radioactive samples, ARG-1 samples, and reagent blanks by the $\mathrm{Na}_{2} \mathrm{O}_{2}$ and mixed acid methods, respectively. The elemental concentrations were converted to oxide concentrations by multiplying the values for each element by the gravimetric factor for the corresponding oxide. During this process, an elemental concentration that was determined to be below the detection limit of the analytical procedures used by ADS was reduced to half of that detection limit as the oxide concentration was determined.

Exhibit B1 in Appendix B provides a plot of the concentrations in weight percent (wt\%) of each oxide in Laboratory Information Management System (LIMS) number sequence. Exhibit B2 in Appendix B provides plots of each oxide's concentration values by prep method grouped by type of sample.

\subsubsection{Measurements of Reagent Blanks in Radioactive Set}

Table 5 provides a listing of the average measurement by oxide for the reagent blank samples for each of the preparation methods. Yellow shading is used to highlight averages that appear to be significantly higher than those generated by the other preparation methods. For the $\mathrm{Cs}_{2} \mathrm{CO}_{3}$ fusion method, the level of $\mathrm{B}_{2} \mathrm{O}_{3}$ in the blank is notable because $\mathrm{B}$ was actually detected and measured in the ICP-AES analysis, as opposed to the blank level being calculated from the ICP-AES detection limit. Moreover, $\mathrm{B}$ is not normally detected in the blank. Since the $\mathrm{Cs}_{2} \mathrm{CO}_{3}$ came from the same chemical vendor lot as $\mathrm{Cs}_{2} \mathrm{CO}_{3}$ that had not shown $\mathrm{B}$ contamination and no detectable $\mathrm{B}$ was reported in the vendor's assay of this $\mathrm{Cs}_{2} \mathrm{CO}_{3}$, the source of the $\mathrm{B}$ is unknown. It is also not clear if $\mathrm{B}$ contamination affects the analysis of the DWPF pour stream sample and ARG-1 glass standard. The $\mathrm{B}$ values from the $\mathrm{Cs}_{2} \mathrm{CO}_{3}$ fusion were about $0.3 \mathrm{wt} \%$ higher versus the $\mathrm{Na}_{2} \mathrm{O}_{2}$ method on the ARG-1 glass standard (Table 6a) but there was no significant difference in B values on the DWPF pour stream glass (Table 6b) as a function of digestion method.

The relatively high levels of $\mathrm{K}_{2} \mathrm{O}$ and $\mathrm{PbO}$ shown in Table 5 are not the result of ICPAES measurement of $\mathrm{K}$ and $\mathrm{Pb}$, but rather the relatively high detection limits for these two elements. For example, the vendor reported a K concentration of 3 micrograms per gram of $\mathrm{Cs}_{2} \mathrm{CO}_{3}$. For 4 grams of $\mathrm{Cs}_{2} \mathrm{CO}_{3}$ used in fusions of radioactive glass, about 12 micrograms or 0.012 milligrams of $\mathrm{K}$ would be contributed by the $\mathrm{Cs}_{2} \mathrm{CO}_{3}$. Therefore, 
the actual contribution of $\mathrm{K}$ contamination from $\mathrm{Cs}_{2} \mathrm{CO}_{3}$ to the measured $\mathrm{K}$ concentration in a blank or sample analysis would be negligible (less than $0.01 \%$ ) for 330 milligram samples (to make blank measurements more meaningful, a similar sample weight factor was applied to the calculations of blanks even though no sample was involved so that the blank measurement was put on the same relative concentration basis as the actual samples). The vendor reported no detectable $\mathrm{Pb}$ in the assay of its high-purity $\mathrm{Cs}_{2} \mathrm{CO}_{3}$.

Table 5. Comparison of Measurement Averages for Blanks in Radioactive Set

\begin{tabular}{|c|c|c|c|}
\hline $\begin{array}{c}\text { Preparation } \\
\text { Method }\end{array}$ & $\mathrm{Cs}_{2} \mathrm{CO}_{3}$ & $\begin{array}{c}\text { Mixed } \\
\text { Acid }\end{array}$ & $\mathrm{Na}_{2} \mathrm{O}_{2}$ \\
\hline Type of Sample & blank & blank & blank \\
\hline N Rows & 2 & 1 & 1 \\
\hline $\mathrm{Ag}_{2} \mathrm{O}$ (wt\%) & 0.008 & 0.002 & 0.005 \\
\hline $\mathrm{Al}_{2} \mathrm{O}_{3}(\mathrm{wt} \%)$ & 0.054 & 0.016 & 0.032 \\
\hline $\mathrm{B}_{2} \mathrm{O}_{3}(\mathrm{wt} \%)$ & 0.335 & & 0.068 \\
\hline $\mathrm{BaO}(\mathrm{wt} \%)$ & 0.017 & 0.005 & 0.010 \\
\hline $\mathrm{CaO}$ (wt\%) & 0.009 & 0.003 & 0.014 \\
\hline CdO (wt\%) & 0.003 & 0.001 & 0.002 \\
\hline $\mathrm{Ce}_{2} \mathrm{O}_{3}(\mathrm{wt} \%)$ & 0.107 & 0.032 & 0.063 \\
\hline $\mathrm{Cr}_{2} \mathrm{O}_{3}(\mathrm{wt} \%)$ & 0.003 & 0.008 & 0.015 \\
\hline $\mathrm{CuO}(\mathrm{wt} \%)$ & 0.008 & 0.003 & 0.005 \\
\hline $\mathrm{Fe}_{2} \mathrm{O}_{3}(\mathrm{wt} \%)$ & 0.026 & 0.003 & 0.007 \\
\hline $\mathrm{K}_{2} \mathrm{O}(\mathrm{wt} \%)$ & 0.632 & 0.189 & 0.374 \\
\hline $\mathrm{La}_{2} \mathrm{O}_{3}(\mathrm{wt} \%)$ & 0.041 & 0.012 & 0.024 \\
\hline $\mathrm{Li}_{2} \mathrm{O}(\mathrm{wt} \%)$ & 0.070 & 0.021 & 0.041 \\
\hline MgO (wt\%) & 0.004 & 0.001 & 0.003 \\
\hline MnO (wt\%) & 0.002 & 0.001 & 0.001 \\
\hline $\mathrm{MoO}_{3}(\mathrm{wt} \%)$ & 0.135 & 0.040 & 0.080 \\
\hline $\mathrm{Na}_{2} \mathrm{O}(\mathrm{wt} \%)$ & 0.324 & 0.096 & \\
\hline NiO (wt\%) & 0.034 & 0.010 & 0.020 \\
\hline $\mathrm{P}_{2} \mathrm{O}_{5}(\mathrm{wt} \%)$ & 0.414 & 0.123 & 0.244 \\
\hline PbO (wt\%) & 0.555 & 0.165 & 0.326 \\
\hline $\mathrm{SO}_{4}(\mathrm{wt} \%)$ & 0.019 & 0.072 & 0.074 \\
\hline $\mathrm{SiO}_{2}(\mathrm{wt} \%)$ & 0.703 & & 0.414 \\
\hline $\mathrm{SnO}_{2}(\mathrm{wt} \%)$ & 0.257 & 0.698 & 0.152 \\
\hline SrO (wt\%) & 0.008 & 0.002 & 0.005 \\
\hline $\mathrm{TiO}_{2}(\mathrm{wt} \%)$ & 0.003 & 0.001 & 0.002 \\
\hline $\mathrm{U}_{3} \mathrm{O}_{8}(\mathrm{wt} \%)$ & 0.156 & 0.046 & 0.092 \\
\hline $\mathrm{V}_{2} \mathrm{O}_{5}(\mathrm{wt} \%)$ & 0.024 & 0.007 & 0.014 \\
\hline $\mathrm{ZnO}$ (wt\%) & 0.006 & 0.002 & 0.003 \\
\hline $\mathrm{ZrO}_{2}(\mathrm{wt} \%)$ & 0.007 & 0.002 & 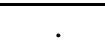 \\
\hline
\end{tabular}


Westinghouse Savannah River Company

WSRC-TR-2005-00396

Savannah River National Laboratory

Rev. 0

Analytical Development/Statistical Consulting Sections

Page 23 of 140

\subsubsection{Measurements of ARG-1 in Radioactive Set}

Exhibit B3 provides comparisons across the preparation methods by oxide for the ARG-1 measurements. There are pairwise comparisons among the averages of the preparations as well as statistical tests for differences in the variances (i.e., precisions) of the preparation methods.

Tables 6a and 6b provide summary information in the form of the average measurement for each oxide by each preparation method. Yellow shading is used to highlight biases that are $5 \%$ or greater relative to the ARG- 1 reference composition (also given in Table 6b).

Table 6a. Measurement Averages, Standard Deviations, and \% Relative Standard Deviations for ARG-1 Samples from Radioactive Set

(Concentrations in wt\%)

\begin{tabular}{|c|c|c|c|c|c|c|c|c|c|}
\hline Preparation & \multicolumn{3}{|c|}{$\mathrm{Cs}_{2} \mathrm{CO}_{3}$} & \multicolumn{3}{|c|}{ Mixed Acid } & \multicolumn{3}{|c|}{$\mathrm{Na}_{2} \mathrm{O}_{2}$} \\
\hline N Rows (3) & Mean & Std Dev & $\begin{array}{l}\% \text { Rel } \\
\text { Std Dev } \\
\end{array}$ & Mean & Std Dev & $\begin{array}{l}\text { \% Rel } \\
\text { Std Dev }\end{array}$ & Mean & Std Dev & $\begin{array}{l}\% \text { Rel } \\
\text { Std Dev }\end{array}$ \\
\hline $\mathrm{Ag}_{2} \mathrm{O}$ & 0.041 & 0.0589 & $142.0 \%$ & 0.002 & 0.0000 & $1.0 \%$ & 0.046 & 0.0075 & $16.3 \%$ \\
\hline $\mathrm{Al}_{2} \mathrm{O}_{3}$ & 4.062 & 0.0189 & $0.5 \%$ & 4.119 & 0.0500 & $1.2 \%$ & 4.069 & 0.0545 & $1.3 \%$ \\
\hline $\mathbf{B}_{2} \mathbf{O}_{3}$ & 8.125 & 0.1035 & $1.3 \%$ & & & & 7.835 & 0.1773 & $2.3 \%$ \\
\hline BaO & 0.089 & 0.0007 & $0.8 \%$ & 0.085 & 0.0009 & $1.0 \%$ & 0.105 & 0.0033 & $3.1 \%$ \\
\hline $\mathrm{CaO}$ & 1.383 & 0.0016 & $0.1 \%$ & 1.405 & 0.0137 & $1.0 \%$ & 1.665 & 0.0560 & $3.4 \%$ \\
\hline CdO & 0.003 & 0.0001 & $1.7 \%$ & 0.001 & 0.0000 & $1.2 \%$ & 0.002 & 0.0004 & $15.8 \%$ \\
\hline $\mathrm{Ce}_{2} \mathrm{O}_{3}$ & 0.101 & 0.0017 & $1.7 \%$ & 0.032 & 0.0003 & $1.0 \%$ & 0.823 & 0.0704 & $8.6 \%$ \\
\hline $\mathrm{Cr}_{2} \mathrm{O}_{3}$ & 0.084 & 0.0035 & $4.2 \%$ & 0.072 & 0.0062 & $8.5 \%$ & 0.060 & 0.0247 & $41.4 \%$ \\
\hline $\mathrm{CuO}$ & 0.008 & 0.0001 & $1.8 \%$ & 0.003 & 0.0000 & $1.1 \%$ & 0.006 & 0.0009 & $15.7 \%$ \\
\hline $\mathrm{Fe}_{2} \mathrm{O}_{3}$ & 13.978 & 0.0083 & $0.1 \%$ & 13.725 & 0.1271 & $0.9 \%$ & 14.097 & 0.1825 & $1.3 \%$ \\
\hline $\mathrm{K}_{2} \mathrm{O}$ & 1.763 & 1.1739 & $66.6 \%$ & 2.164 & 0.4394 & $20.3 \%$ & & & \\
\hline $\mathbf{L a}_{2} \mathbf{O}_{3}$ & 0.039 & 0.0006 & $1.7 \%$ & 0.012 & 0.0001 & $1.0 \%$ & 0.105 & 0.0096 & $9.1 \%$ \\
\hline $\mathbf{L i}_{2} \mathbf{O}$ & 3.064 & 0.0124 & $0.4 \%$ & 3.150 & 0.0249 & $0.8 \%$ & 3.473 & 0.0542 & $1.6 \%$ \\
\hline MgO & 0.824 & 0.0025 & $0.3 \%$ & 0.827 & 0.0075 & $0.9 \%$ & 0.826 & 0.0033 & $0.4 \%$ \\
\hline MnO & 1.855 & 0.0075 & $0.4 \%$ & 1.834 & 0.0129 & $0.7 \%$ & 1.885 & 0.0224 & $1.2 \%$ \\
\hline $\mathrm{MoO}_{3}$ & 0.127 & 0.0017 & $1.4 \%$ & 0.040 & 0.0004 & $1.0 \%$ & 0.128 & 0.0510 & $39.9 \%$ \\
\hline $\mathrm{Na}_{2} \mathrm{O}$ & 11.148 & 0.0934 & $0.8 \%$ & 11.040 & 0.1519 & $1.4 \%$ & & & \\
\hline $\mathrm{NiO}$ & 1.034 & 0.0193 & $1.9 \%$ & 1.054 & 0.0089 & $0.8 \%$ & 1.027 & 0.0115 & $1.1 \%$ \\
\hline $\mathbf{P}_{2} \mathbf{O}_{5}$ & 0.390 & 0.0060 & $1.5 \%$ & 0.123 & 0.0018 & $1.4 \%$ & 0.283 & 0.0450 & $15.9 \%$ \\
\hline PbO & 0.522 & 0.0087 & $1.7 \%$ & 0.165 & 0.0016 & $1.0 \%$ & 0.380 & 0.0600 & $15.8 \%$ \\
\hline $\mathrm{SO}_{4}$ & 0.170 & 0.1060 & $62.4 \%$ & 0.097 & 0.0516 & $53.1 \%$ & 0.086 & 0.0136 & $15.8 \%$ \\
\hline $\mathrm{SiO}_{2}$ & 48.776 & 0.9804 & $2.0 \%$ & & & & 49.347 & 0.6536 & $1.3 \%$ \\
\hline $\mathrm{SnO}_{2}$ & 0.242 & 0.0040 & $1.7 \%$ & 0.835 & 0.0097 & $1.2 \%$ & 0.176 & 0.0278 & $15.8 \%$ \\
\hline SrO & 0.197 & 0.0058 & $3.0 \%$ & 0.225 & 0.0030 & $1.3 \%$ & 0.341 & 0.0031 & $0.9 \%$ \\
\hline $\mathrm{TiO}_{2}$ & 1.168 & 0.0010 & $0.1 \%$ & 1.146 & 0.0121 & $1.1 \%$ & 1.145 & 0.0200 & $1.7 \%$ \\
\hline $\mathbf{U}_{3} \mathbf{O}_{8}$ & 0.147 & 0.0024 & $1.6 \%$ & 0.047 & 0.0005 & $1.0 \%$ & 0.539 & 0.1374 & $25.5 \%$ \\
\hline $\mathbf{V}_{2} \mathbf{O}_{5}$ & 0.064 & 0.0722 & $111.9 \%$ & 0.014 & 0.0121 & $85.2 \%$ & 0.017 & 0.0026 & $15.8 \%$ \\
\hline $\mathrm{ZnO}$ & 0.006 & 0.0001 & $1.7 \%$ & 0.010 & 0.0003 & $3.1 \%$ & 0.006 & 0.0031 & $50.7 \%$ \\
\hline $\mathrm{ZrO}_{2}$ & 0.122 & 0.0026 & $2.1 \%$ & 0.124 & 0.0016 & $1.3 \%$ & & & \\
\hline
\end{tabular}


Westinghouse Savannah River Company

WSRC-TR-2005-00396

Savannah River National Laboratory

Rev. 0

Analytical Development/Statistical Consulting Sections

Page 24 of 140

Table 6b. Comparison of Measurement Averages for ARG-1 Samples from Radioactive Set

\begin{tabular}{|c|c|c|c|c|c|c|c|}
\hline Preparation & $\mathrm{Cs}_{2} \mathrm{CO}_{3}$ & $\begin{array}{c}\text { Mixed } \\
\text { Acid }\end{array}$ & $\mathrm{Na}_{2} \mathrm{O}_{2}$ & & & & \\
\hline Type of Sample & ARG-1 & ARG-1 & ARG-1 & Reference & $\%$ Bias & $\%$ Bias & $\%$ Bias \\
\hline N Rows & 3 & 3 & 3 & Value & $\mathrm{Cs}_{2} \mathrm{CO}_{3}$ & Mixed Acid & $\mathrm{Na}_{2} \mathrm{O}_{2}$ \\
\hline $\mathrm{Ag}_{2} \mathrm{O}(\mathrm{wt} \%)$ & 0.041 & 0.002 & 0.046 & & & & \\
\hline $\mathrm{Al}_{2} \mathrm{O}_{3}(\mathrm{wt} \%)$ & 4.062 & 4.119 & 4.069 & 4.724 & $-14.0 \%$ & $-12.8 \%$ & $-13.9 \%$ \\
\hline $\mathrm{B}_{2} \mathrm{O}_{3}(\mathrm{wt} \%)$ & 8.125 & . & 7.835 & 8.662 & $-6.2 \%$ & & $-9.5 \%$ \\
\hline $\mathrm{BaO}$ (wt\%) & 0.089 & 0.085 & 0.105 & 0.089 & $-0.3 \%$ & $-4.3 \%$ & $17.5 \%$ \\
\hline $\mathrm{CaO}$ (wt\%) & 1.383 & 1.405 & 1.665 & 1.427 & $-3.1 \%$ & $-1.5 \%$ & $16.7 \%$ \\
\hline CdO (wt\%) & 0.003 & 0.001 & 0.002 & & & & \\
\hline $\mathrm{Ce}_{2} \mathrm{O}_{3}(\mathrm{wt} \%)$ & 0.101 & 0.032 & 0.823 & & & & \\
\hline $\mathrm{Cr}_{2} \mathrm{O}_{3}(\mathrm{wt} \%)$ & 0.084 & 0.072 & 0.060 & 0.088 & $-4.6 \%$ & $-17.6 \%$ & $-32.0 \%$ \\
\hline $\mathrm{CuO}$ (wt\%) & 0.008 & 0.003 & 0.006 & 0.0001 & $6266.7 \%$ & $1913.3 \%$ & $4526.7 \%$ \\
\hline $\mathrm{Fe}_{2} \mathrm{O}_{3}(\mathrm{wt} \%)$ & 13.978 & 13.725 & 14.097 & 13.997 & $-0.1 \%$ & $-1.9 \%$ & $0.7 \%$ \\
\hline $\mathrm{K}_{2} \mathrm{O}(\mathrm{wt} \%)$ & 1.763 & 2.164 & & 2.710 & $-34.9 \%$ & $-20.1 \%$ & \\
\hline $\mathrm{La}_{2} \mathrm{O}_{3}(\mathrm{wt} \%)$ & 0.039 & 0.012 & 0.105 & & & & \\
\hline $\mathrm{Li}_{2} \mathrm{O}$ (wt\%) & 3.064 & 3.150 & 3.473 & 3.208 & $-4.5 \%$ & $-1.8 \%$ & $8.3 \%$ \\
\hline MgO (wt \%) & 0.824 & 0.827 & 0.826 & 0.862 & $-4.5 \%$ & $-4.1 \%$ & $-4.2 \%$ \\
\hline MnO (wt\%) & 1.855 & 1.834 & 1.885 & 1.885 & $-1.6 \%$ & $-2.7 \%$ & $0.0 \%$ \\
\hline $\mathrm{MoO}_{3}(\mathrm{w} \%)$ & 0.127 & 0.040 & 0.128 & & & & \\
\hline $\mathrm{Na}_{2} \mathrm{O}(\mathrm{wt} \%)$ & 11.148 & 11.040 & & 11.498 & $-3.0 \%$ & $-4.0 \%$ & \\
\hline $\mathrm{NiO}$ (wt \%) & 1.034 & 1.054 & 1.027 & 1.056 & $-2.1 \%$ & $-0.2 \%$ & $-2.7 \%$ \\
\hline $\mathrm{P}_{2} \mathrm{O}_{5}$ (wt\%) & 0.390 & 0.123 & 0.283 & 0.229 & $70.0 \%$ & $-46.2 \%$ & $23.7 \%$ \\
\hline PbO (wt\%) & 0.522 & 0.165 & 0.380 & & & & \\
\hline $\mathrm{SO}_{4}(\mathrm{wt} \%)$ & 0.170 & 0.097 & 0.086 & & & & \\
\hline $\mathrm{SiO}_{2}(\mathrm{wt} \%)$ & 48.776 &. & 49.347 & 47.899 & $1.8 \%$ & & $3.0 \%$ \\
\hline $\mathrm{SnO}_{2}(\mathrm{wt} \%)$ & 0.242 & 0.835 & 0.176 & & & & \\
\hline SrO (wt\%) & 0.197 & 0.225 & 0.341 & & & & \\
\hline $\mathrm{TiO}_{2}(\mathrm{wt} \%)$ & 1.168 & 1.146 & 1.145 & 1.151 & $1.5 \%$ & $-0.4 \%$ & $-0.5 \%$ \\
\hline $\mathrm{U}_{3} \mathrm{O}_{8}(\mathrm{wt} \%)$ & 0.147 & 0.047 & 0.539 & & & & \\
\hline $\mathrm{V}_{2} \mathrm{O}_{5}(\mathrm{wt} \%)$ & 0.064 & 0.014 & 0.017 & & & & \\
\hline ZnO (wt\%) & 0.006 & 0.010 & 0.006 & 0.012 & $-53.8 \%$ & $-14.3 \%$ & $-49.2 \%$ \\
\hline $\mathrm{ZrO}_{2}(\mathrm{wt} \%)$ & 0.122 & 0.124 & & 0.135 & $-9.8 \%$ & $-7.9 \%$ & \\
\hline
\end{tabular}

\subsubsection{Measurements of Radioactive Samples}

Exhibit B4 in Appendix B provides comparisons across the three preparation methods by oxide for the radioactive sample measurements. There are pairwise comparisons among the averages of the preparations as well as statistical tests for differences in the variances (i.e., precisions) of the preparation methods.

Table 7 provides a summary of this information in the form of the average measurement, the standard deviation, and the \% relative standard deviation for each oxide by each 
Westinghouse Savannah River Company

WSRC-TR-2005-00396

Savannah River National Laboratory

Rev. 0

Analytical Development/Statistical Consulting Sections

Page 25 of 140

preparation method. Table 8 provides pairwise comparisons of the average oxide concentrations across the four preparation methods. Those entries in Table 8 that show differences greater than or equal to $5 \%$ are highlighted. Once again, to aid in the interpretation of the exhibit and tables, an example will be discussed. The example is $\mathrm{U}_{3} \mathrm{O}_{8}$. From Table 7, the smallest average measurement for $\mathrm{U}_{3} \mathrm{O}_{8}$ was $3.266 \mathrm{wt} \%$ from the $\mathrm{Cs}_{2} \mathrm{CO}_{3}$ method while the largest average was $3.664 \mathrm{wt} \%$ from the $\mathrm{Na}_{2} \mathrm{O}_{2}$ method with the largest \% relative standard deviation for the measurements being $5.5 \%$ for the $\mathrm{Na}_{2} \mathrm{O}_{2}$ method and the smallest being $1.0 \%$ for the mixed acid method. From Exhibit B4, the pairwise comparisons of the means indicate that the mean of the $\mathrm{Na}_{2} \mathrm{O}_{2}$ method is statistically significantly larger (since the letter associated with its average is an " $\mathrm{A}$ " while the letter associated with the other averages is a " $\mathrm{B}$ " in the middle of the right-hand side of the exhibit). The "Levene Test" for differences in the variances of the measurements indicates (by a "Prob $>F$ " value of 0.0220 , which is less than 0.05 ) that there is a statistically significant difference in the variances at the $5 \%$ significance level.

Table 7. Average Measurements, Standard Deviations, and \% Relative Standard Deviations for Each Oxide for Radioactive Samples by Preparation Method

\begin{tabular}{|c|c|c|c|c|c|c|c|c|c|}
\hline $\begin{array}{c}\text { Type of } \\
\text { Preparation }\end{array}$ & & $\mathrm{Cs}_{2} \mathrm{CO}_{3}$ & & & Mixed Ac & & & $\mathrm{Na}_{2} \mathrm{O}_{2}$ & \\
\hline N Rows $(6,4,4)$ & Mean & Std Dev & $\begin{array}{l}\% \text { Rel } \\
\text { Std Dev }\end{array}$ & Mean & Std Dev & $\begin{array}{l}\% \text { Rel } \\
\text { Std Dev }\end{array}$ & Mean & Std Dev & $\begin{array}{l}\text { \% Rel } \\
\text { Std Dev }\end{array}$ \\
\hline $\operatorname{Ag}_{2} \mathrm{O}(\mathrm{wt} \%)$ & 0.082 & 0.0625 & $76.1 \%$ & 0.002 & 0.0001 & $2.8 \%$ & 0.040 & 0.0032 & $8.1 \%$ \\
\hline $\mathrm{Al}_{2} \mathrm{O}_{3}(\mathrm{wt} \%)$ & 4.743 & 0.0902 & $1.9 \%$ & 4.823 & 0.0679 & $1.4 \%$ & 4.762 & 0.1282 & $2.7 \%$ \\
\hline $\mathrm{B}_{2} \mathrm{O}_{3}(\mathrm{wt} \%)$ & 4.476 & 0.2401 & $5.4 \%$ & & . & & 4.443 & 0.0872 & $2.0 \%$ \\
\hline $\mathrm{BaO}(\mathrm{wt} \%)$ & 0.050 & 0.0057 & $11.3 \%$ & 0.040 & 0.0005 & $1.2 \%$ & 0.055 & 0.0057 & $10.4 \%$ \\
\hline $\mathrm{CaO}(\mathrm{wt} \%)$ & 1.009 & 0.0041 & $0.4 \%$ & 1.031 & 0.0164 & $1.6 \%$ & 1.253 & 0.0263 & $2.1 \%$ \\
\hline CdO (wt\%) & 0.045 & 0.0061 & $13.6 \%$ & 0.070 & 0.0018 & $2.6 \%$ & 0.056 & 0.0028 & $4.9 \%$ \\
\hline $\mathrm{Ce}_{2} \mathrm{O}_{3}(\mathrm{wt} \%)$ & 0.169 & 0.1528 & $90.6 \%$ & 0.031 & 0.0009 & $2.9 \%$ & 0.692 & 0.1820 & $26.3 \%$ \\
\hline $\mathrm{Cr}_{2} \mathrm{O}_{3}(\mathrm{wt} \%)$ & 0.062 & 0.0076 & $12.2 \%$ & 0.059 & 0.0022 & $3.7 \%$ & 0.058 & 0.0094 & $16.2 \%$ \\
\hline $\mathrm{CuO}(\mathrm{wt} \%)$ & 0.009 & 0.0011 & $13.4 \%$ & 0.013 & 0.0004 & $3.1 \%$ & 0.005 & 0.0002 & $4.6 \%$ \\
\hline $\mathrm{Fe}_{2} \mathrm{O}_{3}(\mathrm{wt} \%)$ & 10.921 & 0.1352 & $1.2 \%$ & 10.619 & 0.1615 & $1.5 \%$ & 10.905 & 0.2483 & $2.3 \%$ \\
\hline $\mathrm{K}_{2} \mathrm{O}(\mathrm{wt} \%)$ & 0.637 & 0.0856 & $13.4 \%$ & 0.184 & 0.0053 & $2.9 \%$ & 1.447 & 1.1333 & \\
\hline $\mathrm{La}_{2} \mathrm{O}_{3}(\mathrm{wt} \%)$ & 0.041 & 0.0056 & $13.4 \%$ & 0.012 & 0.0003 & $2.9 \%$ & 0.099 & 0.0241 & $24.3 \%$ \\
\hline $\mathrm{Li}_{2} \mathrm{O}(\mathrm{wt} \%)$ & 4.679 & 0.0503 & $1.1 \%$ & 4.796 & 0.0711 & $1.5 \%$ & 5.124 & 0.1179 & $2.3 \%$ \\
\hline MgO (wt\%) & 1.166 & 0.0184 & $1.6 \%$ & 1.152 & 0.0174 & $1.5 \%$ & 1.174 & 0.0282 & $2.4 \%$ \\
\hline MnO (wt\%) & 2.109 & 0.0194 & $0.9 \%$ & 2.059 & 0.0307 & $1.5 \%$ & 2.111 & 0.0563 & $2.7 \%$ \\
\hline $\mathrm{MoO}_{3}(\mathrm{wt} \%)$ & 0.136 & 0.0180 & $13.3 \%$ & 0.039 & 0.0011 & $2.8 \%$ & 0.098 & 0.0354 & $36.0 \%$ \\
\hline $\mathrm{Na}_{2} \mathrm{O}(\mathrm{wt} \%)$ & 12.179 & 0.2925 & $2.4 \%$ & 11.869 & 0.1649 & $1.4 \%$ & . & $\dot{.}$ & \\
\hline $\mathrm{NiO}$ (wt\%) & 0.547 & 0.0264 & $4.8 \%$ & 0.568 & 0.0140 & $2.5 \%$ & 0.537 & 0.0218 & $4.1 \%$ \\
\hline $\mathrm{P}_{2} \mathrm{O}_{5}(\mathrm{wt} \%)$ & 0.416 & 0.0557 & $13.4 \%$ & 0.202 & 0.0970 & $48.0 \%$ & 0.242 & 0.0111 & $4.6 \%$ \\
\hline PbO (wt\%) & 0.556 & 0.0743 & $13.4 \%$ & 0.161 & 0.0046 & $2.9 \%$ & 0.325 & 0.0148 & $4.6 \%$ \\
\hline $\mathrm{SO}_{4}(\mathrm{wt} \%)$ & 0.513 & 0.0548 & $10.7 \%$ & 0.460 & 0.0468 & $10.2 \%$ & 0.436 & 0.0334 & $7.7 \%$ \\
\hline $\mathrm{SiO}_{2}(\mathrm{wt} \%)$ & 52.306 & 0.9827 & $1.9 \%$ & $\cdot$ & . & & 51.022 & 0.9958 & $2.0 \%$ \\
\hline $\mathrm{SnO}_{2}(\mathrm{wt} \%)$ & 0.259 & 0.0345 & $13.4 \%$ & 0.821 & 0.0334 & $4.1 \%$ & 0.189 & 0.0709 & $37.4 \%$ \\
\hline SrO (wt\%) & 0.159 & 0.0200 & $12.6 \%$ & 0.182 & 0.0025 & $1.4 \%$ & 0.282 & 0.0205 & $7.3 \%$ \\
\hline $\mathrm{TiO}_{2}(\mathrm{wt} \%)$ & 0.053 & 0.0014 & $2.6 \%$ & 0.063 & 0.0015 & $2.5 \%$ & 0.061 & 0.0014 & $2.2 \%$ \\
\hline $\mathrm{U}_{3} \mathrm{O}_{8}(\mathrm{wt} \%)$ & 3.266 & 0.1160 & $3.6 \%$ & 3.343 & 0.0340 & $1.0 \%$ & 3.664 & 0.2009 & $5.5 \%$ \\
\hline $\mathrm{V}_{2} \mathrm{O}_{5}(\mathrm{wt} \%)$ & 0.073 & 0.1199 & $164.3 \%$ & 0.014 & 0.0142 & $101.0 \%$ & 0.081 & 0.0790 & $97.9 \%$ \\
\hline $\mathrm{ZnO}(\mathrm{wt} \%)$ & 0.006 & 0.0008 & $13.4 \%$ & 0.008 & 0.0005 & $6.7 \%$ & 0.005 & 0.0028 & $57.2 \%$ \\
\hline $\mathrm{ZrO}_{2}(\mathrm{wt} \%)$ & 0.053 & 0.0014 & $2.7 \%$ & 0.054 & 0.0015 & $2.8 \%$ & . & . & \\
\hline
\end{tabular}


Westinghouse Savannah River Company

WSRC-TR-2005-00396

Savannah River National Laboratory

Rev. 0

Analytical Development/Statistical Consulting Sections

Page 26 of 140

Table 8. Pairwise Comparison of Average Oxide Measurements for Radioactive Samples Across Preparation Methods

\begin{tabular}{|c|c|c|c|c|c|c|c|}
\hline $\mathrm{Ag}_{2} \mathrm{O}(\mathrm{wt} \%)$ & $\mathrm{Cs}_{2} \mathrm{CO}_{3}$ & Mixed Acid & $\mathrm{Na}_{2} \mathrm{O}_{2}$ & $\mathrm{CuO}(\mathrm{wt} \%)$ & $\mathrm{Cs}_{2} \mathrm{CO}_{3}$ & Mixed Acid & $\mathrm{Na}_{2} \mathrm{O}_{2}$ \\
\hline $\mathrm{Cs}_{2} \mathrm{CO}_{3}$ & $0.0 \%$ & $3439.4 \%$ & $105.5 \%$ & $\mathrm{Cs}_{2} \mathrm{CO}_{3}$ & $0.0 \%$ & $-34.5 \%$ & $71.5 \%$ \\
\hline Mixed Acid & $-97.2 \%$ & $0.0 \%$ & $-94.2 \%$ & Mixed Acid & $52.6 \%$ & $0.0 \%$ & $161.8 \%$ \\
\hline $\mathrm{Na}_{2} \mathrm{O}_{2}$ & $-51.3 \%$ & $1622.2 \%$ & $0.0 \%$ & $\mathrm{Na}_{2} \mathrm{O}_{2}$ & $-41.7 \%$ & $-61.8 \%$ & $0.0 \%$ \\
\hline $\mathrm{Al}_{2} \mathrm{O}_{3}(\mathrm{wt} \%)$ & $\mathrm{Cs}_{2} \mathrm{CO}_{3}$ & Mixed Acid & $\mathrm{Na}_{2} \mathrm{O}_{2}$ & $\mathrm{Fe}_{2} \mathrm{O}_{3}(\mathrm{wt} \%)$ & $\mathrm{Cs}_{2} \mathrm{CO}_{3}$ & Mixed Acid & $\mathrm{Na}_{2} \mathrm{O}_{2}$ \\
\hline $\mathrm{Cs}_{2} \mathrm{CO}_{3}$ & $0.0 \%$ & $-1.7 \%$ & $-0.4 \%$ & $\mathrm{Cs}_{2} \mathrm{CO}_{3}$ & $0.0 \%$ & $2.8 \%$ & $0.1 \%$ \\
\hline Mixed Acid & $1.7 \%$ & $0.0 \%$ & $1.3 \%$ & Mixed Acid & $-2.8 \%$ & $0.0 \%$ & $-2.6 \%$ \\
\hline $\mathrm{Na}_{2} \mathrm{O}_{2}$ & $0.4 \%$ & $-1.3 \%$ & $0.0 \%$ & $\mathrm{Na}_{2} \mathrm{O}_{2}$ & $-0.1 \%$ & $2.7 \%$ & $0.0 \%$ \\
\hline $\mathrm{B}_{2} \mathrm{O}_{3}(\mathrm{wt} \%)$ & $\mathrm{Cs}_{2} \mathrm{CO}_{3}$ & Mixed Acid & $\mathrm{Na}_{2} \mathrm{O}_{2}$ & $\mathrm{~K}_{2} \mathrm{O}(\mathrm{wt} \%)$ & $\mathrm{Cs}_{2} \mathrm{CO}_{3}$ & Mixed Acid & $\mathrm{Na}_{2} \mathrm{O}_{2}$ \\
\hline $\mathrm{Cs}_{2} \mathrm{CO}_{3}$ & $0.0 \%$ & & $0.7 \%$ & $\mathrm{Cs}_{2} \mathrm{CO}_{3}$ & $0.0 \%$ & $245.9 \%$ & $-55.9 \%$ \\
\hline Mixed Acid & & & & Mixed Acid & $-71.1 \%$ & $0.0 \%$ & $-87.3 \%$ \\
\hline $\mathrm{Na}_{2} \mathrm{O}_{2}$ & $-0.7 \%$ & & $0.0 \%$ & $\mathrm{Na}_{2} \mathrm{O}_{2}$ & $127.0 \%$ & $685.1 \%$ & $0.0 \%$ \\
\hline $\mathrm{BaO}(\mathrm{wt} \%)$ & $\mathrm{Cs}_{2} \mathrm{CO}_{3}$ & Mixed Acid & $\mathrm{Na}_{2} \mathrm{O}_{2}$ & $\mathrm{La}_{2} \mathrm{O}_{3}(\mathrm{wt} \%)$ & $\mathrm{Cs}_{2} \mathrm{CO}_{3}$ & Mixed Acid & $\mathrm{Na}_{2} \mathrm{O}_{2}$ \\
\hline $\mathrm{Cs}_{2} \mathrm{CO}_{3}$ & $0.0 \%$ & $23.7 \%$ & $-8.5 \%$ & $\mathrm{Cs}_{2} \mathrm{CO}_{3}$ & $0.0 \%$ & $245.7 \%$ & $-58.3 \%$ \\
\hline Mixed Acid & $-19.2 \%$ & $0.0 \%$ & $-26.1 \%$ & Mixed Acid & $-71.1 \%$ & $0.0 \%$ & $-87.9 \%$ \\
\hline $\mathrm{Na}_{2} \mathrm{O}_{2}$ & & & & $\mathrm{Na}_{2} \mathrm{O}_{2}$ & $140.0 \%$ & $729.9 \%$ & $0.0 \%$ \\
\hline $\mathrm{CaO}(\mathrm{wt} \%)$ & $\mathrm{Cs}_{2} \mathrm{CO}_{3}$ & Mixed Acid & $\mathrm{Na}_{2} \mathrm{O}_{2}$ & $\mathrm{Li}_{2} \mathrm{O}(\mathrm{wt} \%)$ & $\mathrm{Cs}_{2} \mathrm{CO}_{3}$ & Mixed Acid & $\mathrm{Na}_{2} \mathrm{O}_{2}$ \\
\hline $\mathrm{Cs}_{2} \mathrm{CO}_{3}$ & $0.0 \%$ & $-2.2 \%$ & $-19.5 \%$ & $\mathrm{Cs}_{2} \mathrm{CO}_{3}$ & $0.0 \%$ & $-2.4 \%$ & $-8.7 \%$ \\
\hline Mixed Acid & $2.2 \%$ & $0.0 \%$ & $-17.7 \%$ & Mixed Acid & $2.5 \%$ & $0.0 \%$ & $-6.4 \%$ \\
\hline $\mathrm{Na}_{2} \mathrm{O}_{2}$ & $24.2 \%$ & $21.5 \%$ & $0.0 \%$ & $\mathrm{Na}_{2} \mathrm{O}_{2}$ & $9.5 \%$ & $6.8 \%$ & $0.0 \%$ \\
\hline CdO (wt\%) & $\mathrm{Cs}_{2} \mathrm{CO}_{3}$ & Mixed Acid & $\mathrm{Na}_{2} \mathrm{O}_{2}$ & MgO (wt\%) & $\mathrm{Cs}_{2} \mathrm{CO}_{3}$ & Mixed Acid & $\mathrm{Na}_{2} \mathrm{O}_{2}$ \\
\hline $\mathrm{Cs}_{2} \mathrm{CO}_{3}$ & $0.0 \%$ & $-36.3 \%$ & $-20.9 \%$ & $\mathrm{Cs}_{2} \mathrm{CO}_{3}$ & $0.0 \%$ & $1.3 \%$ & $-0.7 \%$ \\
\hline Mixed Acid & $56.9 \%$ & $0.0 \%$ & $24.1 \%$ & Mixed Acid & $-1.3 \%$ & $0.0 \%$ & $-1.9 \%$ \\
\hline $\mathrm{Na}_{2} \mathrm{O}_{2}$ & $26.4 \%$ & $-19.4 \%$ & $0.0 \%$ & $\mathrm{Na}_{2} \mathrm{O}_{2}$ & $0.7 \%$ & $2.0 \%$ & $0.0 \%$ \\
\hline $\mathrm{Ce}_{2} \mathrm{O}_{3}(\mathrm{wt} \%)$ & $\mathrm{Cs}_{2} \mathrm{CO}_{3}$ & Mixed Acid & $\mathrm{Na}_{2} \mathrm{O}_{2}$ & $\mathrm{MnO}(\mathrm{wt} \%)$ & $\mathrm{Cs}_{2} \mathrm{CO}_{3}$ & Mixed Acid & $\mathrm{Na}_{2} \mathrm{O}_{2}$ \\
\hline $\mathrm{Cs}_{2} \mathrm{CO}_{3}$ & $0.0 \%$ & $443.1 \%$ & $-75.6 \%$ & $\mathrm{Cs}_{2} \mathrm{CO}_{3}$ & $0.0 \%$ & $2.4 \%$ & $-0.1 \%$ \\
\hline Mixed Acid & $-81.6 \%$ & $0.0 \%$ & $-95.5 \%$ & Mixed Acid & $-2.3 \%$ & $0.0 \%$ & $-2.4 \%$ \\
\hline $\mathrm{Na}_{2} \mathrm{O}_{2}$ & $310.3 \%$ & $2128.3 \%$ & $0.0 \%$ & $\mathrm{Na}_{2} \mathrm{O}_{2}$ & $0.1 \%$ & $2.5 \%$ & $0.0 \%$ \\
\hline $\mathrm{Cr}_{2} \mathrm{O}_{3}(\mathrm{wt} \%)$ & $\mathrm{Cs}_{2} \mathrm{CO}_{3}$ & Mixed Acid & $\mathrm{Na}_{2} \mathrm{O}_{2}$ & $\mathrm{MoO}_{3}(\mathrm{wt} \%)$ & $\mathrm{Cs}_{2} \mathrm{CO}_{3}$ & Mixed Acid & $\mathrm{Na}_{2} \mathrm{O}_{2}$ \\
\hline $\mathrm{Cs}_{2} \mathrm{CO}_{3}$ & $0.0 \%$ & $4.7 \%$ & $7.1 \%$ & $\mathrm{Cs}_{2} \mathrm{CO}_{3}$ & $0.0 \%$ & $246.1 \%$ & $37.8 \%$ \\
\hline Mixed Acid & $-4.5 \%$ & $0.0 \%$ & $2.3 \%$ & Mixed Acid & $-71.1 \%$ & $0.0 \%$ & $-60.2 \%$ \\
\hline $\mathrm{Na}_{2} \mathrm{O}_{2}$ & $-6.7 \%$ & $-2.3 \%$ & $0.0 \%$ & $\mathrm{Na}_{2} \mathrm{O}_{2}$ & $-27.4 \%$ & $151.2 \%$ & $0.0 \%$ \\
\hline
\end{tabular}


Westinghouse Savannah River Company

WSRC-TR-2005-00396

Savannah River National Laboratory

Rev. 0

Analytical Development/Statistical Consulting Sections

Page 27 of 140

Table 8. Pairwise Comparison of Average Oxide Measurements for Radioactive Samples Across Preparation Methods (continued)

\begin{tabular}{|c|c|c|c|c|c|c|c|}
\hline $\mathrm{Na}_{2} \mathrm{O}$ (wt\%) & $\mathrm{Cs}_{2} \mathrm{CO}_{3}$ & Mixed Acid & $\mathrm{Na}_{2} \mathrm{O}_{2}$ & $\mathrm{TiO}_{2}(\mathrm{wt} \%)$ & $\mathrm{Cs}_{2} \mathrm{CO}_{3}$ & Mixed Acid & $\mathrm{Na}_{2} \mathrm{O}_{2}$ \\
\hline $\mathrm{Cs}_{2} \mathrm{CO}_{3}$ & $0.0 \%$ & $2.6 \%$ & & $\mathrm{Cs}_{2} \mathrm{CO}_{3}$ & $0.0 \%$ & $-15.1 \%$ & $-12.7 \%$ \\
\hline Mixed Acid & $-2.5 \%$ & $0.0 \%$ & & Mixed Acid & $17.7 \%$ & $0.0 \%$ & $2.8 \%$ \\
\hline $\mathrm{Na}_{2} \mathrm{O}_{2}$ & & & & $\mathrm{Na}_{2} \mathrm{O}_{2}$ & $14.5 \%$ & $-2.7 \%$ & $0.0 \%$ \\
\hline $\mathrm{NiO}$ (wt\%) & $\mathrm{Cs}_{2} \mathrm{CO}_{3}$ & Mixed Acid & $\mathrm{Na}_{2} \mathrm{O}_{2}$ & $\mathrm{U}_{3} \mathrm{O}_{8}(\mathrm{wt} \%)$ & $\mathrm{Cs}_{2} \mathrm{CO}_{3}$ & Mixed Acid & $\mathrm{Na}_{2} \mathrm{O}_{2}$ \\
\hline $\mathrm{Cs}_{2} \mathrm{CO}_{3}$ & $0.0 \%$ & $-3.8 \%$ & $1.7 \%$ & $\mathrm{Cs}_{2} \mathrm{CO}_{3}$ & $0.0 \%$ & $-2.3 \%$ & $-10.9 \%$ \\
\hline Mixed Acid & $3.9 \%$ & $0.0 \%$ & $5.7 \%$ & Mixed Acid & $2.3 \%$ & $0.0 \%$ & $-8.8 \%$ \\
\hline $\mathrm{Na}_{2} \mathrm{O}_{2}$ & $-1.7 \%$ & $-5.4 \%$ & $0.0 \%$ & $\mathrm{Na}_{2} \mathrm{O}_{2}$ & $12.2 \%$ & $9.6 \%$ & $0.0 \%$ \\
\hline $\mathrm{P}_{2} \mathrm{O}_{5}(\mathrm{wt} \%)$ & $\mathrm{Cs}_{2} \mathrm{CO}_{3}$ & Mixed Acid & $\mathrm{Na}_{2} \mathrm{O}_{2}$ & $\mathrm{~V}_{2} \mathrm{O}_{5}(\mathrm{wt} \%)$ & $\mathrm{Cs}_{2} \mathrm{CO}_{3}$ & Mixed Acid & $\mathrm{Na}_{2} \mathrm{O}_{2}$ \\
\hline $\mathrm{Cs}_{2} \mathrm{CO}_{3}$ & $0.0 \%$ & $105.5 \%$ & $71.5 \%$ & $\mathrm{Cs}_{2} \mathrm{CO}_{3}$ & $0.0 \%$ & $419.4 \%$ & $-9.5 \%$ \\
\hline Mixed Acid & $-51.3 \%$ & $0.0 \%$ & $-16.5 \%$ & Mixed Acid & $-80.7 \%$ & $0.0 \%$ & $-82.6 \%$ \\
\hline $\mathrm{Na}_{2} \mathrm{O}_{2}$ & $-41.7 \%$ & $19.8 \%$ & $0.0 \%$ & $\mathrm{Na}_{2} \mathrm{O}_{2}$ & $10.5 \%$ & $473.9 \%$ & $0.0 \%$ \\
\hline $\mathrm{PbO}(\mathrm{wt} \%)$ & $\mathrm{Cs}_{2} \mathrm{CO}_{3}$ & Mixed Acid & $\mathrm{Na}_{2} \mathrm{O}_{2}$ & $\mathrm{ZnO}(\mathrm{wt} \%)$ & $\mathrm{Cs}_{2} \mathrm{CO}_{3}$ & Mixed Acid & $\mathrm{Na}_{2} \mathrm{O}_{2}$ \\
\hline $\mathrm{Cs}_{2} \mathrm{CO}_{3}$ & $0.0 \%$ & $245.5 \%$ & $71.3 \%$ & $\mathrm{Cs}_{2} \mathrm{CO}_{3}$ & $0.0 \%$ & $-22.5 \%$ & $19.9 \%$ \\
\hline Mixed Acid & $-71.1 \%$ & $0.0 \%$ & $-50.4 \%$ & Mixed Acid & $29.0 \%$ & $0.0 \%$ & $54.6 \%$ \\
\hline $\mathrm{Na}_{2} \mathrm{O}_{2}$ & $-41.6 \%$ & $101.7 \%$ & $0.0 \%$ & $\mathrm{Na}_{2} \mathrm{O}_{2}$ & $-16.6 \%$ & $-35.3 \%$ & $0.0 \%$ \\
\hline $\mathrm{SO}_{4}(\mathrm{wt} \%)$ & $\mathrm{Cs}_{2} \mathrm{CO}_{3}$ & Mixed Acid & $\mathrm{Na}_{2} \mathrm{O}_{2}$ & $\mathrm{ZrO}_{2}(\mathrm{wt} \%)$ & $\mathrm{Cs}_{2} \mathrm{CO}_{3}$ & Mixed Acid & $\mathrm{Na}_{2} \mathrm{O}_{2}$ \\
\hline $\mathrm{Cs}_{2} \mathrm{CO}_{3}$ & $0.0 \%$ & $11.5 \%$ & $17.6 \%$ & $\mathrm{Cs}_{2} \mathrm{CO}_{3}$ & $0.0 \%$ & $-3.6 \%$ & \\
\hline Mixed Acid & $-10.3 \%$ & $0.0 \%$ & $5.5 \%$ & Mixed Acid & $3.8 \%$ & $0.0 \%$ & \\
\hline $\mathrm{Na}_{2} \mathrm{O}_{2}$ & $-15.0 \%$ & $-5.2 \%$ & $0.0 \%$ & $\mathrm{Na}_{2} \mathrm{O}_{2}$ & & & \\
\hline $\mathrm{SiO}_{2}(\mathrm{wt} \%)$ & $\mathrm{Cs}_{2} \mathrm{CO}_{3}$ & Mixed Acid & $\mathrm{Na}_{2} \mathrm{O}_{2}$ & & & & \\
\hline $\mathrm{Cs}_{2} \mathrm{CO}_{3}$ & $0.0 \%$ & & $2.5 \%$ & & & & \\
\hline \multicolumn{8}{|l|}{ Mixed Acid } \\
\hline $\mathrm{Na}_{2} \mathrm{O}_{2}$ & $-2.5 \%$ & & $0.0 \%$ & & & & \\
\hline $\mathrm{SnO}_{2}(\mathrm{wt} \%)$ & $\mathrm{Cs}_{2} \mathrm{CO}_{3}$ & Mixed Acid & $\mathrm{Na}_{2} \mathrm{O}_{2}$ & & & & \\
\hline $\mathrm{Cs}_{2} \mathrm{CO}_{3}$ & $0.0 \%$ & $-68.5 \%$ & $36.5 \%$ & & & & \\
\hline Mixed Acid & $217.7 \%$ & $0.0 \%$ & $333.5 \%$ & & & & \\
\hline $\mathrm{Na}_{2} \mathrm{O}_{2}$ & $-26.7 \%$ & $-76.9 \%$ & $0.0 \%$ & & & & \\
\hline $\mathrm{SrO}(\mathrm{wt} \%)$ & $\mathrm{Cs}_{2} \mathrm{CO}_{3}$ & Mixed Acid & $\mathrm{Na}_{2} \mathrm{O}_{2}$ & & & & \\
\hline $\mathrm{Cs}_{2} \mathrm{CO}_{3}$ & $0.0 \%$ & $-12.4 \%$ & $-43.5 \%$ & & & & \\
\hline Mixed Acid & $14.1 \%$ & $0.0 \%$ & $-35.6 \%$ & & & & \\
\hline $\mathrm{Na}_{2} \mathrm{O}_{2}$ & $77.1 \%$ & $55.2 \%$ & $0.0 \%$ & & & & \\
\hline
\end{tabular}




\subsubsection{Acceptability of Radioactive Sample Measurements}

Another point of interest for the radioactive samples is the assessment of their acceptability via DWPF's Process Composition Control System (PCCS). The acceptability process as described in [7] was conducted using the average compositions provided in Table 7. For the $\mathrm{Cs}_{2} \mathrm{CO}_{3}$ method, the composition data from this table were used directly while the averages of the mixed acid and $\mathrm{Na}_{2} \mathrm{O}_{2}$ composition data were used to represent the measurements from those methods. The critical predicted properties and measured concentrations derived from the compositions of Table 7 are listed in Table 9. These values were compared against the PCCS acceptance criteria at their Measurement Acceptance Region (MAR) as defined in [7]. All of the criteria were met with the exception of $\mathrm{Na}_{2} \mathrm{SO}_{4}$. For this criterion, both the $\mathrm{Cs}_{2} \mathrm{CO}_{3}$ and mixed acid/ $\mathrm{Na}_{2} \mathrm{O}_{2}$ results failed to satisfy the MAR. However, a special $\mathrm{SO}_{4}$ solubility study was conducted for the Sludge Batch 3 (SB3)/Frit 418 glass system [8], and the results from that study increased the limit for $\mathrm{SO}_{4}$ from $0.40 \mathrm{wt} \%$ to $0.60 \mathrm{wt} \%$, or in terms of $\mathrm{Na}_{2} \mathrm{SO}_{4}$, the limit was increased from $0.59 \mathrm{wt} \%$ to $0.88 \mathrm{wt} \%$. Using this limit for $\mathrm{Na}_{2} \mathrm{SO}_{4}$, the glass compositions for both the $\mathrm{Cs}_{2} \mathrm{CO}_{3}$ and the mixed acid/ $\mathrm{Na}_{2} \mathrm{O}_{2}$ dissolutions are acceptable at the MAR. As seen by these results, measurements from the $\mathrm{Cs}_{2} \mathrm{CO}_{3}$ and mixed acid/ $\mathrm{Na}_{2} \mathrm{O}_{2}$ dissolutions for the pour stream samples provide very consistent PCCS performance.

Table 9. PCCS Acceptance Criteria

\begin{tabular}{|c|c|c|c|}
\hline PCCS Criteria & $\mathrm{Cs}_{2} \mathrm{CO}_{3}$ & $\begin{array}{c}\text { Mixed } \\
\text { Acid/ } \\
\mathrm{Na}_{2} \mathrm{O}_{2} \\
\end{array}$ & $\begin{array}{c}\text { Acceptable } \\
\text { at the } \\
\text { MAR } \\
\end{array}$ \\
\hline $\mathrm{NL}[\mathrm{B}(\mathrm{g} / \mathrm{L})]$ & 0.96 & 1.06 & Yes \\
\hline $\mathrm{NL}[\mathrm{Li}(\mathrm{g} / \mathrm{L})]$ & 0.96 & 1.04 & Yes \\
\hline $\mathrm{NL}[\mathrm{Na}(\mathrm{g} / \mathrm{L})]$ & 0.95 & 1.04 & Yes \\
\hline $\mathrm{T}_{\mathrm{L}}$ Pred $\left({ }^{\mathrm{O}} \mathrm{C}\right)$ & 873.5 & 871.7 & Yes \\
\hline Visc Pred (P) & 66.1 & 58.4 & Yes \\
\hline Sum of Oxides & 99.7 & 98.4 & Yes \\
\hline $\mathrm{Al}_{2} \mathrm{O}_{3} \mathrm{wt} \%$ & 4.74 & 4.79 & Yes \\
\hline $\mathrm{Ti}_{2} \mathrm{O}$ wt $\%$ & 0.05 & 0.06 & Yes \\
\hline $\mathrm{NaCl} w \mathrm{t} \%$ & 0 & 0 & Yes \\
\hline $\mathrm{NaF}$ wt\% & 0 & 0 & Yes \\
\hline $\mathrm{Cr}_{2} \mathrm{O}_{3} \mathrm{wt} \%$ & 0.06 & 0.06 & Yes \\
\hline $\mathrm{Na}_{2} \mathrm{SO}_{4} \mathrm{wt} \%$ & 0.79 & 0.69 & No \\
\hline $\mathrm{Cu} w \mathrm{t} \%$ & 0.01 & 0.01 & Yes \\
\hline $\mathrm{R}_{2} \mathrm{O} w \mathrm{t} \%$ & 17.50 & 17.64 & Yes \\
\hline
\end{tabular}




\subsection{References}

[1] C.M. Hidlay, “DWPF Lab Sampling and Analytical Improvement” SS-PIP-20030070, January 13, 2004.

[2] Coleman, C.J., T.B. Edwards, and D.R. Click, "SRNL Evaluation of SubSampling Precision and Accuracy of DWPF Slurry Mix Evaporator Simulant," WSRC-TR-2004-00339, Revision 0, August 2004.

[3] Mahannah, R.N., “Technical Task Request: Phase II of 6 DWPF Sigma SME TAT,” HLW/TTR-2005-0008, Revision 0, April 2005.

[4] C. J. Coleman and T. B. Edwards, “Task Technical \& QA Plan: Phase II of Six Sigma DWPF SME Analytical Turnaround Time Study,” WSRC-RP-2005-01571, Revision 0, May 12, 2005.

[5] Bannochie, C. J. and N. E. Bibler, "Analysis of Sludge Batch 3 (Macrobatch 4) DWPF Pour Stream Glass Sample for Canister S02312,” WSRC-TR-2005-00354, Revision A, August 2005.

[6] SAS Institute, JMP®: Statistics and Graphics Guide, Version 5, SAS Institute, Inc., Cary, NC, 2002.

[7] Brown, K. G., R. L. Postles, and T. B. Edwards, "SME Acceptability Determination for DWPF Process Control,” WSRC-TR-95-0364, Revision 4, August 2002.

[8] Peeler, D. K., et al., "An Assessment of the Sulfate Solubility Limit for the Frit 418 - Sludge Batch 2/3 System,” WSRC-TR-2004-00081, Revision 0, February 2004. 
Westinghouse Savannah River Company

Savannah River National Laboratory

Analytical Development/Statistical Consulting Sections
WSRC-TR-2005-00396

Rev. 0

Page 30 of 140

This page intentionally left blank. 
Westinghouse Savannah River Company

Savannah River National Laboratory

Analytical Development/Statistical Consulting Sections
WSRC-TR-2005-00396

Rev. 0

Page 32 of 140

This page intentionally left blank. 
Table A1. Elemental Measurements ( $\mu \mathrm{g} / \mathrm{g}$ ) for DWPF Simulated Glass Prepared Using $\mathrm{Cs}_{2} \mathrm{CO}_{3}$ Digestions

\begin{tabular}{|c|c|c|c|c|c|c|c|c|c|c|c|}
\hline Sample ID & CB1 & CA3 & CB2 & CD2 & CD5 & CD1 & CA1 & $\begin{array}{l}\text { CD4 } \\
\end{array}$ & CA2 & CD6 & CD3 \\
\hline Type of Sample & blank & ARG-1 & blank & Simulant & Simulant & Simulant & ARG-1 & Simulant & ARG-1 & Simulant & Simulant \\
\hline LIMS \# & $219780 / 1.1$ & $219781 / 1.1$ & $219782 / 1.1$ & $219783 / 1.1$ & $219784 / 1.1$ & $219785 / 1.1$ & $219786 / 1.1$ & $219787 / 1.1$ & $219788 / 1.1$ & $219789 / 1.1$ & $219790 / 1.1$ \\
\hline $\mathrm{Ag}$ & $<331$ & $<314$ & $<331$ & $<332$ & $<318$ & $<334$ & 373 & $<319$ & $<332$ & $<333$ & $<327$ \\
\hline $\mathrm{Al}$ & $<467$ & 24700 & $<467$ & 33700 & 33300 & 33500 & 24500 & 33700 & 24700 & 33600 & 33300 \\
\hline $\mathrm{B}$ & $<238$ & 24700 & $<238$ & 15400 & 15900 & 15400 & 24400 & 15900 & 25100 & 16100 & 15700 \\
\hline $\mathrm{Ba}$ & 73.2 & 884 & 73.2 & 672 & 658 & 672 & 864 & 681 & 876 & 677 & 671 \\
\hline $\mathrm{Ca}$ & $<164$ & 10700 & $<164$ & 10000 & 9850 & 9910 & 9880 & 9910 & 10400 & 9990 & 9860 \\
\hline $\mathrm{Cd}$ & $<70.9$ & $<67.3$ & $<70.9$ & $<71.2$ & $<68.1$ & $<71.6$ & $<71.0$ & $<68.3$ & $<71.2$ & $<71.3$ & $<70.1$ \\
\hline $\mathrm{Ce}$ & $<648$ & $<615$ & $<648$ & $<650$ & $<622$ & $<654$ & $<649$ & $<624$ & $<650$ & $<651$ & $<641$ \\
\hline Co & $<105$ & $<100$ & $<105$ & $<106$ & $<101$ & $<106$ & 120 & $<102$ & $<106$ & $<106$ & $<104$ \\
\hline $\mathrm{Cr}$ & $<118$ & 718 & $<118$ & 582 & 574 & 569 & 656 & 602 & 684 & 574 & 595 \\
\hline $\mathrm{Cu}$ & $<53.6$ & $<50.9$ & $<53.6$ & 479 & 487 & 479 & 95.4 & 479 & $<53.8$ & 480 & 469 \\
\hline $\mathrm{Fe}$ & $<83.2$ & 101000 & $<83.2$ & 102000 & 101000 & 102000 & 96300 & 102000 & 98500 & 102000 & 101000 \\
\hline $\mathrm{K}$ & $<2810$ & 22700 & $<2810$ & $<2820$ & $<2700$ & $<2840$ & 23500 & $<2710$ & 23300 & $<2830$ & $<2780$ \\
\hline $\mathrm{La}$ & $<128$ & $<122$ & $<128$ & $<129$ & $<123$ & $<130$ & $<128$ & $<124$ & $<129$ & $<129$ & $<127$ \\
\hline $\mathrm{Li}$ & $<108$ & 15400 & $<108$ & 24200 & 24300 & 24200 & 15800 & 24200 & 15700 & 24100 & 24100 \\
\hline $\mathrm{Mg}$ & $<329$ & 5230 & $<329$ & 8920 & 8770 & 8820 & 5050 & 8920 & 5170 & 8820 & 8820 \\
\hline $\mathrm{Mn}$ & $<47.1$ & 15000 & $<47.1$ & 16800 & 16600 & 16800 & 14500 & 16700 & 14800 & 16600 & 16600 \\
\hline Mo & $<282$ & $<268$ & $<282$ & $<283$ & $<271$ & $<285$ & $<283$ & $<272$ & $<284$ & $<284$ & $<279$ \\
\hline $\mathrm{Na}$ & $<628$ & 85600 & $<628$ & 98300 & 98700 & 98900 & 87400 & 98500 & 87300 & 98300 & 98000 \\
\hline $\mathrm{Nb}$ & $<136$ & 609 & $<136$ & $<136$ & $<130$ & $<137$ & 525 & $<131$ & 566 & $<136$ & $<134$ \\
\hline $\mathrm{Nd}$ & $<852$ & $<809$ & $<852$ & $<855$ & $<818$ & $<860$ & $<854$ & $<821$ & $<856$ & $<857$ & $<843$ \\
\hline $\mathrm{Ni}$ & $<86.9$ & 8390 & $<86.9$ & 3650 & 3640 & 3610 & 7940 & 3730 & 8120 & 3650 & 3640 \\
\hline $\mathrm{P}$ & $<724$ & 1110 & $<724$ & $<727$ & $<696$ & $<731$ & 908 & $<698$ & 826 & $<729$ & $<717$ \\
\hline $\mathrm{Pb}$ & $<400$ & $<380$ & $<400$ & 543 & 453 & 498 & $<401$ & 523 & $<402$ & 672 & 791 \\
\hline $\operatorname{Re}$ & $<143$ & $<136$ & $<143$ & $<143$ & $<137$ & $<144$ & $<143$ & $<138$ & $<143$ & $<144$ & $<141$ \\
\hline$S$ & $<939$ & $<891$ & $<939$ & 2230 & 2220 & 2240 & $<940$ & 2160 & $<943$ & 2020 & 2070 \\
\hline Si & $<137$ & 235000 & $<137$ & 237000 & 223000 & 237000 & 229000 & 238000 & 233000 & 231000 & 237000 \\
\hline Sn & $<326$ & $<309$ & $<326$ & $<327$ & $<313$ & $<329$ & $<326$ & $<314$ & $<327$ & $<328$ & $<322$ \\
\hline $\mathrm{Sr}$ & $<47.4$ & $<45.0$ & $<47.4$ & 71.8 & 71 & $\begin{array}{l}71.7 \\
\end{array}$ & $<47.5$ & 71.8 & $<47.6$ & 71.8 & 72.8 \\
\hline $\mathrm{Ti}$ & $<12.1$ & 7250 & $<12.1$ & 241 & 237 & 236 & 7190 & 242 & 7250 & 237 & 240 \\
\hline $\mathrm{V}$ & $<238$ & $<226$ & $<238$ & $<239$ & $<229$ & $<241$ & $<239$ & $<230$ & $<239$ & $<240$ & $<236$ \\
\hline $\mathrm{Zn}$ & $<47.6$ & 197 & $<47.6$ & 1200 & 1240 & 1170 & 183 & 1190 & 186 & 1200 & 1190 \\
\hline $\mathrm{Zr}$ & $<126$ & 1010 & $<126$ & 2000 & 1920 & 1990 & 1010 & 2020 & 1030 & 1980 & 1990 \\
\hline
\end{tabular}


Table A2. Elemental Measurements ( $\mu \mathrm{g} / \mathrm{g}$ ) for DWPF Simulated Glass Prepared Using $\mathrm{K}_{2} \mathrm{CO}_{3}$ Digestions $^{2}$

\begin{tabular}{|c|c|c|c|c|c|c|c|c|c|c|c|}
\hline Sample ID & KA1 & KB1 & KD5 & KD6 & KB2 & KD4 & KD3 & KD1 & KA2 & KD2 & KA3 \\
\hline Type of Sample & ARG-1 & blank & Simulant & Simulant & blank & Simulant & Simulant & Simulant & ARG-1 & Simulant & ARG-1 \\
\hline LIMS \# & $219791 / 1.1$ & $219792 / 1.1$ & $219793 / 1.1$ & $219794 / 1.1$ & $219795 / 1.1$ & $219796 / 1.1$ & $219797 / 1.1$ & $219798 / 1.1$ & 219799/1.1 & $219800 / 1.1$ & $219801 / 1.1$ \\
\hline $\mathrm{Ag}$ & $<321$ & $<331$ & $<330$ & $<343$ & $<331$ & $<357$ & $<328$ & $<326$ & $<318$ & $<328$ & $<315$ \\
\hline $\mathrm{Al}$ & 24100 & $<467$ & 33100 & 31000 & $<467$ & 32200 & 31100 & 31600 & 23200 & 32500 & 25100 \\
\hline $\mathrm{B}$ & 25800 & $<238$ & 16000 & 15100 & $<238$ & 15500 & 15000 & 15700 & 24800 & 16100 & 27000 \\
\hline $\mathrm{Ba}$ & 774 & $<60.1$ & 586 & 553 & $<60.1$ & 557 & 533 & 548 & 745 & 564 & 809 \\
\hline $\mathrm{Ca}$ & 10100 & $<164$ & 9620 & 8890 & $<164$ & 9030 & 8690 & 8890 & 9530 & 9400 & 10900 \\
\hline $\mathrm{Cd}$ & $<68.8$ & $<70.9$ & $<70.8$ & $<73.5$ & $<70.9$ & $<76.5$ & $<70.4$ & $<69.8$ & $<68.1$ & $<70.2$ & $<67.5$ \\
\hline $\mathrm{Ce}$ & $<628$ & $<648$ & $<647$ & $<671$ & $<648$ & $<699$ & $<643$ & $<638$ & $<622$ & $<641$ & $<616$ \\
\hline Co & $<102$ & $<105$ & $<105$ & $<109$ & $<105$ & $<114$ & $<105$ & $<104$ & $<101$ & $<104$ & $<100$ \\
\hline $\mathrm{Cr}$ & 652 & $<118$ & 554 & 513 & $<118$ & 539 & 511 & 514 & 613 & 547 & 680 \\
\hline $\mathrm{Cu}$ & $<52.0$ & $<53.6$ & 407 & 411 & $<53.6$ & 427 & 360 & 425 & $<51.5$ & 431 & $<51.0$ \\
\hline $\mathrm{Fe}$ & 95400 & $<83.2$ & 98600 & 92800 & $<83.2$ & 95000 & 91200 & 94200 & 90900 & 97000 & 101000 \\
\hline $\mathrm{K}$ & 4920000 & 5140000 & 5160000 & 5010000 & 5070000 & 5160000 & 4760000 & 4710000 & 4680000 & 4860000 & 4750000 \\
\hline $\mathrm{La}$ & $<124$ & $<128$ & $<128$ & $<133$ & $<128$ & $<138$ & $<127$ & $<126$ & $<123$ & $<127$ & 127 \\
\hline $\mathrm{Li}$ & 15300 & $<108$ & 24100 & 22400 & $<108$ & 23800 & 22600 & 23200 & 14600 & 23600 & 15400 \\
\hline $\mathrm{Mg}$ & 5020 & $<329$ & 8660 & 8120 & $<329$ & 8330 & 7960 & 8160 & 4780 & 8360 & 5310 \\
\hline $\mathrm{Mn}$ & 14500 & $<47.1$ & 16300 & 15400 & $<47.1$ & 15800 & 15100 & 15600 & 13800 & 16000 & 15300 \\
\hline Mo & $<274$ & $<282$ & $<282$ & $<293$ & $<282$ & $<305$ & $<280$ & $<278$ & $<271$ & $<280$ & $<269$ \\
\hline $\mathrm{Na}$ & 85200 & $<628$ & 98300 & 91100 & $<628$ & 96600 & 92800 & 94500 & 80900 & 96500 & 85800 \\
\hline $\mathrm{Nb}$ & 575 & $<136$ & $<135$ & $<141$ & $<136$ & $<146$ & $<135$ & $<134$ & 531 & $<134$ & 659 \\
\hline $\mathrm{Nd}$ & $<827$ & $<852$ & $<851$ & $<883$ & $<852$ & $<919$ & $<845$ & $<839$ & $<818$ & $<844$ & $<811$ \\
\hline $\mathrm{Ni}$ & 6050 & $<86.9$ & 2940 & 2930 & $<86.9$ & 2880 & 2800 & 2970 & 5620 & 3080 & 6720 \\
\hline $\mathrm{P}$ & 756 & $<724$ & $<723$ & $<751$ & $<724$ & $<782$ & $<719$ & $<713$ & 884 & $<717$ & 1380 \\
\hline $\mathrm{Pb}$ & $<388$ & $<400$ & 484 & 474 & $<400$ & $<432$ & $<397$ & 601 & $<384$ & 549 & $<381$ \\
\hline $\operatorname{Re}$ & $<139$ & $<143$ & $<143$ & $<148$ & $<143$ & $<154$ & $<142$ & $<141$ & $<137$ & $<141$ & $<136$ \\
\hline$S$ & $<911$ & $<939$ & 1800 & 2270 & $<939$ & 2120 & 1880 & 2040 & $<901$ & 2030 & $<893$ \\
\hline $\mathrm{Si}$ & 229000 & $<137$ & 234000 & 219000 & $<137$ & 225000 & 214000 & 220000 & 212000 & 224000 & 241000 \\
\hline Sn & $<316$ & $<326$ & $<325$ & $<338$ & $<326$ & $<352$ & $<323$ & $<321$ & $<313$ & $<323$ & $<310$ \\
\hline $\mathrm{Sr}$ & $<46.0$ & $<47.4$ & 60.4 & 55.5 & $<47.4$ & 57.5 & 59.9 & 58.3 & $<45.5$ & 59.2 & $<45.1$ \\
\hline $\mathrm{Ti}$ & 7070 & $<12.1$ & 229 & 216 & $<12.1$ & 225 & 217 & 214 & 6730 & 231 & 7280 \\
\hline $\mathrm{V}$ & $<231$ & $<238$ & $<238$ & $<247$ & $<238$ & $<257$ & $<237$ & $<235$ & $<229$ & $<236$ & $<227$ \\
\hline $\mathrm{Zn}$ & 175 & $<47.6$ & 1140 & 1080 & $<47.6$ & 1080 & 1070 & 1080 & 167 & 1150 & 198 \\
\hline $\mathrm{Zr}$ & 946 & $<126$ & 1930 & 1830 & $<126$ & 1900 & 1830 & 1860 & 913 & 1900 & 1010 \\
\hline
\end{tabular}


Table A3. Elemental Measurements $(\mu \mathrm{g} / \mathrm{g})$ for DWPF Simulated Glass Prepared Using $\mathrm{Na}_{2} \mathrm{O}_{2}$ Digestions $^{3}$

\begin{tabular}{|c|c|c|c|c|c|c|c|c|c|c|c|}
\hline "Sample ID & ND3 & ND6 & ND5 & NA2 & ND2 & NA3 & ND1 & NA1 & NB2 & ND4 & NB1 \\
\hline Type of Sample & Simulant & Simulant & Simulant & ARG-1 & Simulant & ARG-1 & Simulant & ARG-1 & blank & Simulant & blank \\
\hline LIMS \# & $219802 / 1.1$ & $219803 / 1.1$ & $219804 / 1.1$ & $219805 / 1.1$ & $219806 / 1.1$ & $219807 / 1.1$ & $219808 / 1.1$ & 219809/1.1 & $219810 / 1.1$ & 219811/1.1 & $219812 / 1.1$ \\
\hline $\mathrm{Ag}$ & $<328$ & $<339$ & $<331$ & $<318$ & $<328$ & $<315$ & $<326$ & $<315$ & $<331$ & $<357$ & $<331$ \\
\hline $\mathrm{Al}$ & 34100 & 31300 & 31500 & 23200 & 31600 & 23500 & 34000 & 25300 & $<467$ & 32600 & $<467$ \\
\hline $\mathrm{B}$ & 16600 & 15300 & 15400 & 25000 & 15400 & 24900 & 16900 & 27200 & 329 & 15900 & $<238$ \\
\hline $\mathrm{Ba}$ & 615 & 570 & 567 & 767 & 570 & 877 & 716 & 823 & $<60.1$ & 575 & $<60.1$ \\
\hline $\mathrm{Ca}$ & 11600 & 10600 & 10800 & 11000 & 10800 & 11200 & 11800 & 12000 & 1540 & 11000 & 1310 \\
\hline $\mathrm{Cd}$ & $<70.4$ & $<72.7$ & $<71.0$ & $<68.1$ & $<70.2$ & $<67.5$ & $<69.8$ & $<67.6$ & $<70.9$ & $<76.5$ & $<70.9$ \\
\hline $\mathrm{Ce}$ & $<643$ & $<664$ & $<649$ & $<622$ & $<641$ & $<616$ & $<638$ & $<617$ & $<648$ & $<699$ & $<648$ \\
\hline Co & $<105$ & $<108$ & $<105$ & $<101$ & $<104$ & $<100$ & $<104$ & 117 & $<105$ & $<114$ & 122 \\
\hline $\mathrm{Cr}$ & 540 & 508 & 507 & 598 & 510 & 608 & 557 & 645 & $<118$ & 493 & $<118$ \\
\hline $\mathrm{Cu}$ & 503 & 456 & 458 & $<51.5$ & 464 & $<51.0$ & 515 & $<51.1$ & $<53.6$ & 474 & $<53.6$ \\
\hline $\mathrm{Fe}$ & 104000 & 95400 & 95500 & 94000 & 95700 & 94800 & 104000 & 101000 & $<83.2$ & 98300 & $<83.2$ \\
\hline $\mathrm{K}$ & 3690 & 5380 & 4150 & 25900 & 3460 & 27900 & 6300 & 28900 & $<2810$ & 7110 & 3680 \\
\hline $\mathrm{La}$ & $<127$ & $<132$ & $<128$ & $<123$ & $<127$ & 195 & 150 & $<122$ & $<128$ & $<138$ & $<128$ \\
\hline $\mathrm{Li}$ & 24200 & 22200 & 22400 & 14400 & 22500 & 14400 & 24500 & 15600 & $<108$ & 23300 & $<108$ \\
\hline $\mathrm{Mg}$ & 8800 & 8160 & 8160 & 4760 & 8050 & 4820 & 8760 & 5110 & $<329$ & 8270 & $<329$ \\
\hline $\mathrm{Mn}$ & 16600 & 15200 & 15300 & 13700 & 15300 & 13900 & 16700 & 14800 & $<47.1$ & 15700 & $<47.1$ \\
\hline Mo & $<280$ & $<290$ & $<283$ & $<271$ & $<280$ & $<269$ & $<278$ & $<269$ & $<282$ & $<305$ & $<282$ \\
\hline $\mathrm{Na}$ & 5330000 & 5090000 & 5000000 & 4900000 & 5120000 & 4810000 & 5370000 & 5160000 & 5330000 & 5700000 & 5130000 \\
\hline $\mathrm{Nb}$ & $<135$ & $<139$ & $<136$ & 467 & $<134$ & 606 & $<134$ & 613 & $<136$ & $<146$ & $<136$ \\
\hline $\mathrm{Nd}$ & $<845$ & $<874$ & $<853$ & $<818$ & $<844$ & $<811$ & $<839$ & $<812$ & $<852$ & $<919$ & $<852$ \\
\hline $\mathrm{Ni}$ & 3810 & 3520 & 3570 & 7820 & 3510 & 7880 & 3790 & 8400 & $<86.9$ & 3590 & $<86.9$ \\
\hline $\mathrm{P}$ & $<719$ & $<743$ & $<726$ & 1030 & $<717$ & 967 & $<713$ & 902 & $<724$ & $<782$ & $<724$ \\
\hline $\mathrm{Pb}$ & 443 & 504 & $<401$ & $<384$ & $<396$ & $<381$ & 576 & $<381$ & $<400$ & $<432$ & $<400$ \\
\hline $\mathrm{Re}$ & $<142$ & $<147$ & $<143$ & $<137$ & $<141$ & $<136$ & $<141$ & $<136$ & $<143$ & $<154$ & $<143$ \\
\hline $\mathrm{S}$ & 2400 & 1890 & 2120 & $<901$ & 1850 & $<893$ & 2500 & $<895$ & $<939$ & 1920 & $<939$ \\
\hline $\mathrm{Si}$ & 236000 & 216000 & 218000 & 214000 & 218000 & 216000 & 237000 & 232000 & $<137$ & 224000 & $<137$ \\
\hline Sn & $<323$ & $<334$ & $<326$ & $<313$ & $<323$ & $<310$ & $<321$ & $<311$ & $<326$ & $<352$ & $<326$ \\
\hline $\mathrm{Sr}$ & 82 & 75.7 & 78.1 & $<45.5$ & 73.1 & 109 & 141 & 48 & $<47.4$ & 80.8 & $<47.4$ \\
\hline $\mathrm{Ti}$ & 249 & 221 & 220 & 6660 & 217 & 6700 & 238 & 7230 & $<12.1$ & 228 & $<12.1$ \\
\hline $\mathrm{V}$ & $<237$ & $<245$ & $<239$ & $<229$ & $<236$ & $<227$ & $<235$ & $<227$ & $<238$ & $<257$ & $<238$ \\
\hline $\mathrm{Zn}$ & 1190 & 1080 & 1090 & 172 & 1090 & 178 & 1160 & 194 & $<47.6$ & 1110 & $<47.6$ \\
\hline $\mathrm{Zr}$ & 41300 & 33100 & 35000 & 31900 & 25000 & 32000 & 43000 & 59300 & 33800 & 74600 & 44900 \\
\hline
\end{tabular}

The $\mathrm{Na}$ and $\mathrm{Zr}$ values generated by this digestion method are ignored in this study. 
Westinghouse Savannah River Company

WSRC-TR-2005-00396

Savannah River National Laboratory

Rev. 0

Analytical Development/Statistical Consulting Sections

Page 36 of 140

Table A4. Elemental Measurements ( $\mu \mathrm{g} / \mathrm{g})$ for DWPF Simulated Glass Prepared Using Mixed Acid Digestions ${ }^{4}$

\begin{tabular}{|c|c|c|c|c|c|c|c|c|c|c|c|}
\hline Sample ID & "MA3 & MA2 & MD1 & MD6 & MD2 & MB1 & MD5 & MD4 & MD3 & MA1 & MB2 \\
\hline Type of Sample & ARG-1 & ARG-1 & Simulant & Simulant & Simulant & blank & Simulant & Simulant & Simulant & ARG-1 & blank \\
\hline LIMS \# & $219813 / 1.1$ & $219814 / 1.1$ & $219815 / 1.1$ & $219816 / 1.1$ & $219817 / 1.1$ & $219818 / 1.1$ & $219819 / 1.1$ & $219820 / 1.1$ & $219821 / 1.1$ & $219822 / 1.1$ & $219823 / 1.1$ \\
\hline $\mathrm{Ag}$ & $<326$ & $<328$ & $<316$ & $<334$ & $<333$ & $<331$ & $<314$ & $<320$ & $<321$ & $<333$ & $<331$ \\
\hline $\mathrm{Al}$ & 23400 & 23700 & 33000 & 28600 & 30400 & $<467$ & 32800 & 32,900 & 33200 & 23300 & $<467$ \\
\hline \multicolumn{12}{|l|}{$\mathrm{B}$} \\
\hline $\mathrm{Ba}$ & 798 & 807 & 595 & 505 & 542 & $<60.1$ & 586 & 586 & 599 & 798 & $<60.1$ \\
\hline $\mathrm{Ca}$ & 10400 & 10400 & 9840 & 8330 & 8950 & $<164$ & 9720 & 9,750 & 9960 & 10400 & $<164$ \\
\hline $\mathrm{Cd}$ & $<69.8$ & $<70.4$ & $<67.7$ & $<71.5$ & $<71.4$ & $<70.9$ & $<67.3$ & $<69$ & $<68.7$ & $<71.3$ & $<70.9$ \\
\hline $\mathrm{Ce}$ & $<637$ & $<643$ & $<619$ & $<653$ & $<652$ & $<648$ & $<614$ & $<626$ & $<628$ & $<651$ & $<648$ \\
\hline Co & 108 & 110 & $<101$ & $<106$ & $<106$ & $<105$ & $<99.9$ & $<102$ & $<102$ & 118 & $<105$ \\
\hline $\mathrm{Cr}$ & 670 & 664 & 555 & 485 & 505 & $<118$ & 555 & 570 & 577 & 664 & $<118$ \\
\hline $\mathrm{Cu}$ & $<52.8$ & $<53.2$ & 488 & 412 & 453 & $<53.6$ & 488 & 485 & 495 & $<53.9$ & $<53.6$ \\
\hline $\mathrm{Fe}$ & 99100 & 99500 & 100000 & 86300 & 92200 & $<83.2$ & 99800 & 99,900 & 101000 & 99600 & $<83.2$ \\
\hline $\mathrm{K}$ & 24100 & 23700 & $<2690$ & $<2840$ & $<2830$ & $<2810$ & 2990 & $<2,720$ & $<2730$ & 23900 & $<2810$ \\
\hline $\mathrm{La}$ & $<126$ & $<127$ & $<123$ & $<129$ & $<129$ & $<128$ & $<122$ & $<124$ & $<124$ & $<129$ & $<128$ \\
\hline $\mathrm{Li}$ & 15400 & 15400 & 24600 & 21400 & 22700 & $<108$ & 24100 & 24,300 & 24700 & 15300 & $<108$ \\
\hline $\mathrm{Mg}$ & 5180 & 5210 & 8660 & 7410 & 7940 & $<329$ & 8650 & 8,710 & 8780 & 5110 & $<329$ \\
\hline $\mathrm{Mn}$ & 14800 & 14800 & 16400 & 14000 & 15000 & $<47.1$ & 16300 & 16,300 & 16500 & 14800 & $<47.1$ \\
\hline Mo & $<278$ & $<280$ & $<270$ & $<285$ & $<284$ & $<282$ & $<268$ & $<273$ & $<274$ & $<284$ & $<282$ \\
\hline $\mathrm{Na}$ & 87800 & 88100 & 101000 & 88800 & 93600 & $<628$ & 101000 & 100,000 & 101000 & 87500 & $<628$ \\
\hline $\mathrm{Nb}$ & 709 & 635 & $<130$ & $<137$ & $<137$ & $<136$ & $<129$ & $<131$ & $<131$ & 603 & $<136$ \\
\hline $\mathrm{Nd}$ & $<839$ & $<846$ & $<814$ & $<859$ & $<858$ & $<852$ & $<808$ & $<824$ & $<826$ & $<857$ & $<852$ \\
\hline $\mathrm{Ni}$ & 8450 & 8530 & 3750 & 3240 & 3440 & $<86.9$ & 3710 & 3,750 & 3810 & 8570 & $<86.9$ \\
\hline $\mathrm{P}$ & 1140 & 1210 & $<692$ & $<731$ & $<729$ & $<724$ & $<687$ & $<700$ & $<702$ & 1090 & $<724$ \\
\hline $\mathrm{Pb}$ & $<394$ & $<397$ & 443 & $<403$ & 454 & $<400$ & 465 & 541 & 455 & $<402$ & $<400$ \\
\hline $\operatorname{Re}$ & $<141$ & $<142$ & $<136$ & $<144$ & $<144$ & $<143$ & $<136$ & $<138$ & $<138$ & $<144$ & $<143$ \\
\hline$S$ & $<924$ & $<932$ & 1680 & 1230 & 1730 & $<939$ & 1350 & 1,790 & 1610 & $<944$ & $<939$ \\
\hline \multicolumn{12}{|l|}{$\mathrm{Si}$} \\
\hline Sn & $<321$ & $<323$ & $<311$ & $<329$ & $<328$ & $<326$ & $<309$ & $<315$ & $<316$ & $<328$ & $<326$ \\
\hline $\mathrm{Sr}$ & $<46.7$ & $<47.1$ & 63.3 & 51.6 & 55.3 & $<47.4$ & 62.4 & 62 & 61.1 & $<47.7$ & $<47.4$ \\
\hline $\mathrm{Ti}$ & 7000 & 7050 & 232 & 218 & 225 & $<12.1$ & 239 & 239 & 228 & 7020 & $<12.1$ \\
\hline $\mathrm{V}$ & $<235$ & $<237$ & $<228$ & $<240$ & $<240$ & $<238$ & $<226$ & $<230$ & $<231$ & $<240$ & $<238$ \\
\hline $\mathrm{Zn}$ & 198 & 190 & 1150 & 994 & 1060 & $<47.6$ & 1140 & 1,150 & 1170 & 191 & $<47.6$ \\
\hline $\mathrm{Zr}$ & 1030 & 992 & 2050 & 1840 & 1940 & $<126$ & 2020 & 2,060 & 2050 & 1030 & $<126$ \\
\hline
\end{tabular}

The B and Si values generated by this digestion method were ignored in this study. 
Westinghouse Savannah River Company

WSRC-TR-2005-00396

Savannah River National Laboratory

Rev. 0

Analytical Development/Statistical Consulting Sections

Page 37 of 140

Exhibit A1. Chemical Composition Measurements in Analytical Sequence by Oxide for DWPF Simulated Glass

(Concentrations are in wt\%.)

\section{Legend}

$\begin{array}{ll}\text { Preparation Method } & \\ \text { - } \mathrm{Cs} 2 \mathrm{CO} 3 & \text { Type of Sample } \\ =\mathrm{K} 2 \mathrm{CO} 3 & \text { - ARG-1 } \\ \text { - Mixed Acid } & \text { + Simulant } \\ =\text { Na2O2 } & \text { x blank }\end{array}$

Individual Measurement of Ag2O (wt\%)

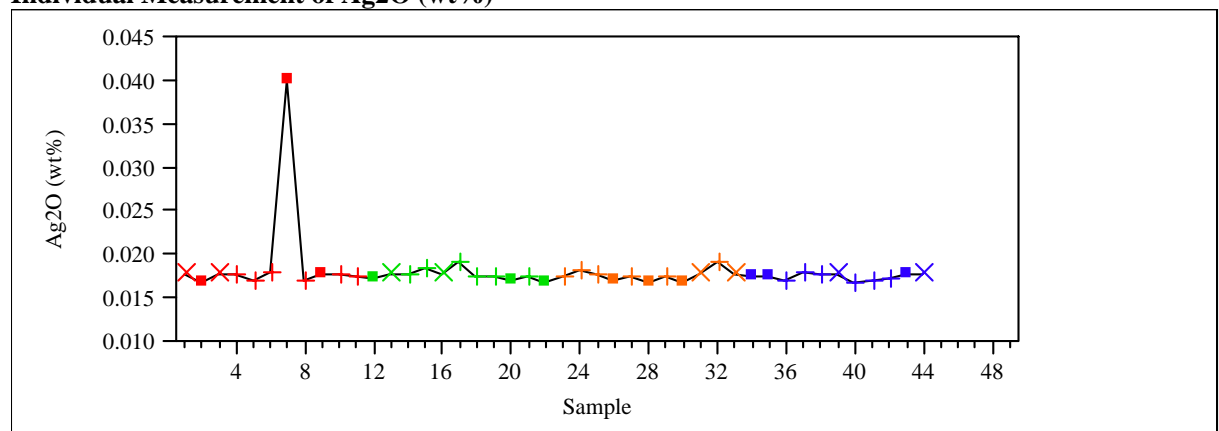

Individual Measurement of Al2O3 (wt\%)

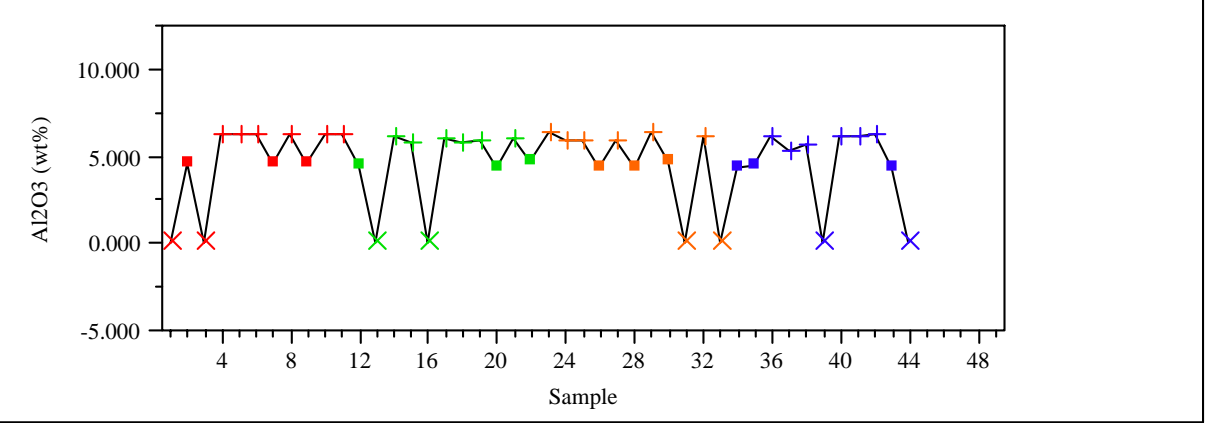

Individual Measurement of B2O3 (wt\%)

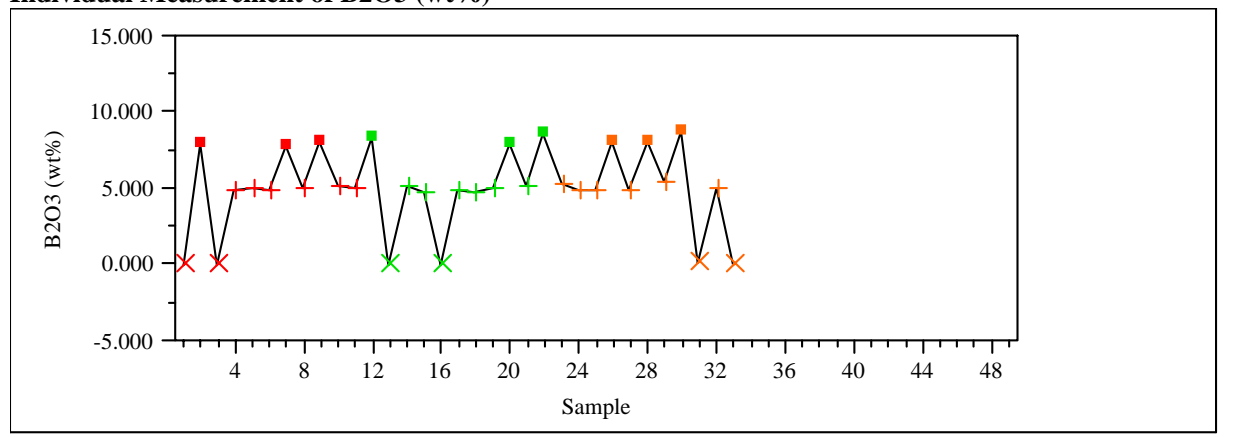


Westinghouse Savannah River Company

WSRC-TR-2005-00396

Savannah River National Laboratory

Rev. 0

Analytical Development/Statistical Consulting Sections

Page 38 of 140

Exhibit A1. Chemical Composition Measurements in Analytical Sequence by Oxide for DWPF Simulated Glass

(Concentrations are in wt\%.)

Individual Measurement of $\mathrm{BaO}$ (wt \%)

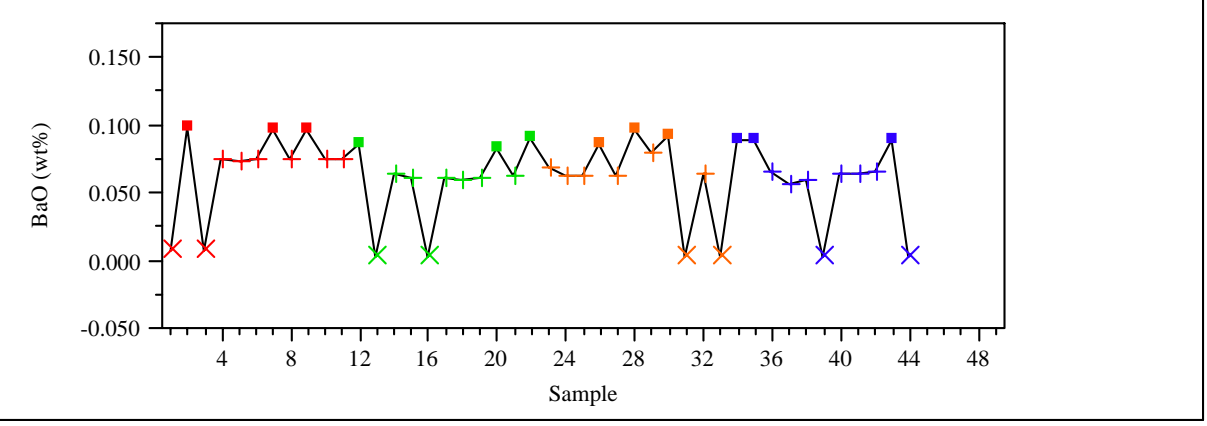

Individual Measurement of $\mathrm{CaO}$ (wt\%)

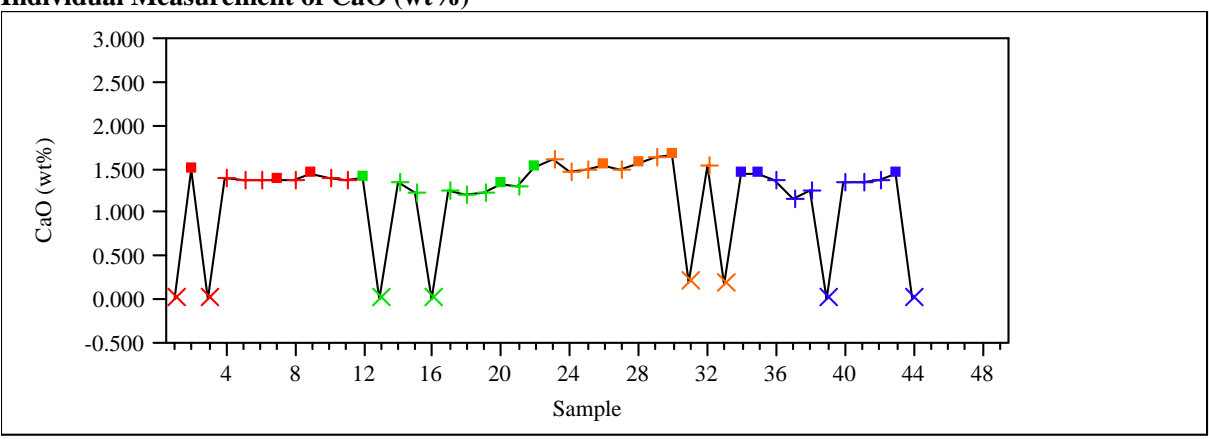

Individual Measurement of CdO (wt \%)

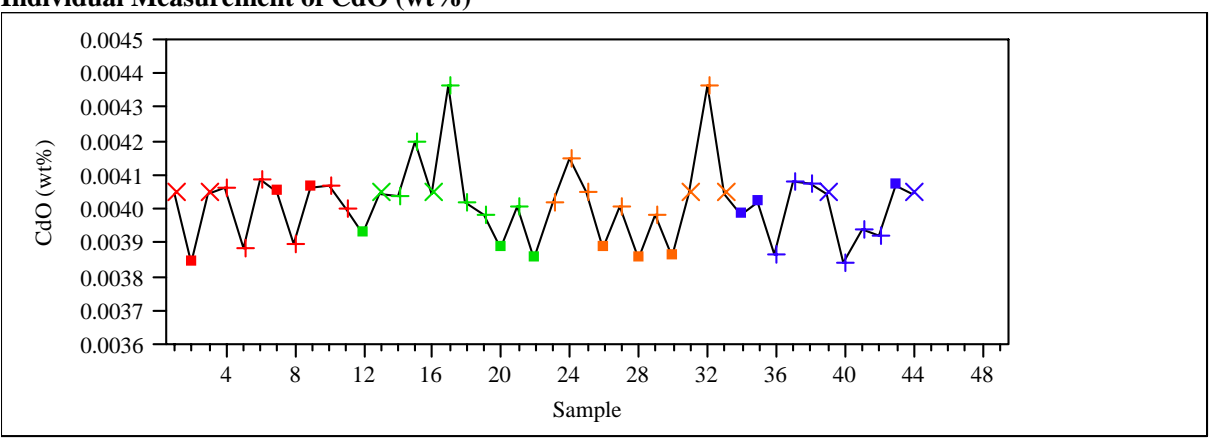

Individual Measurement of Ce2O3 (wt\%)

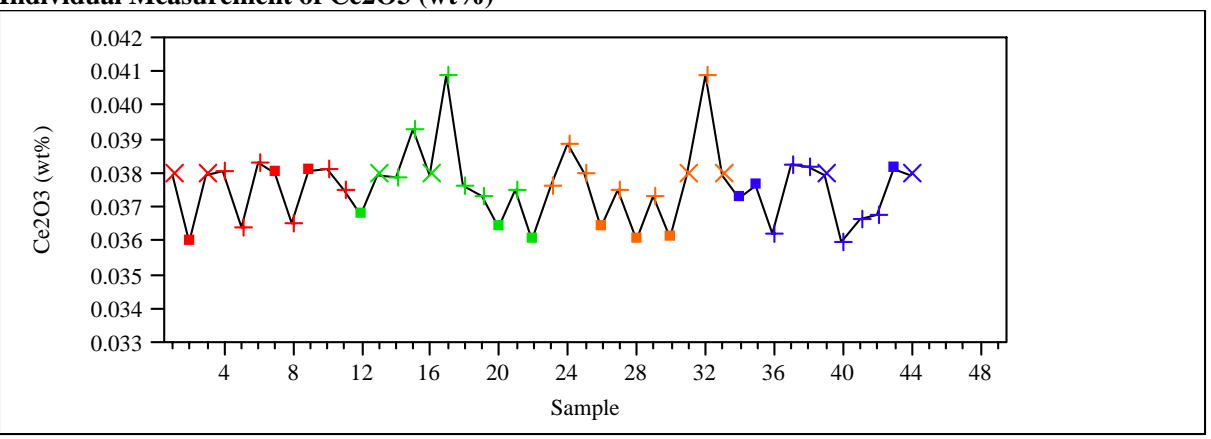


Westinghouse Savannah River Company

WSRC-TR-2005-00396

Savannah River National Laboratory

Rev. 0

Analytical Development/Statistical Consulting Sections

Page 39 of 140

Exhibit A1. Chemical Composition Measurements in Analytical Sequence by Oxide for DWPF Simulated Glass

(Concentrations are in wt\%.)

Individual Measurement of $\mathrm{CoO}$ (wt \%)

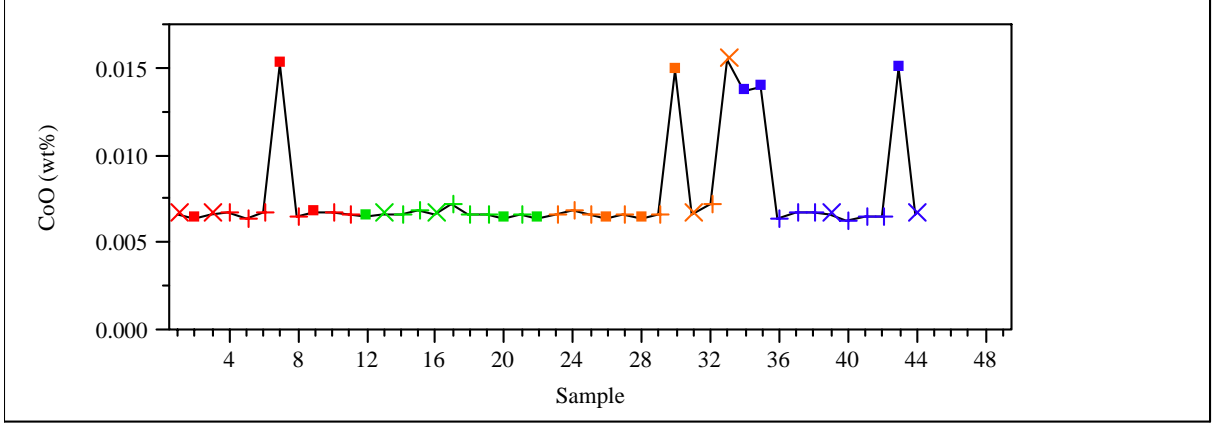

Individual Measurement of Cr2O3 (wt\%)

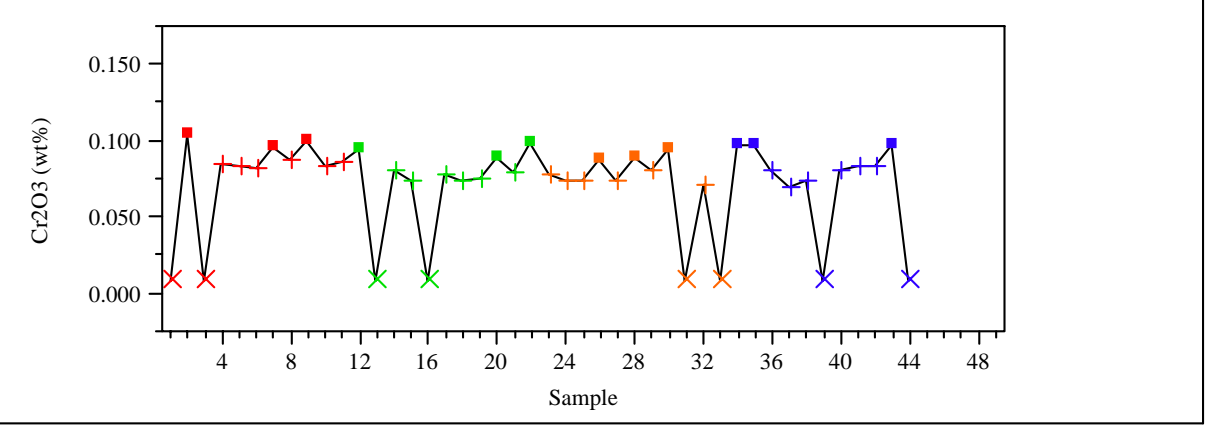

Individual Measurement of $\mathrm{CuO}$ (wt\%)

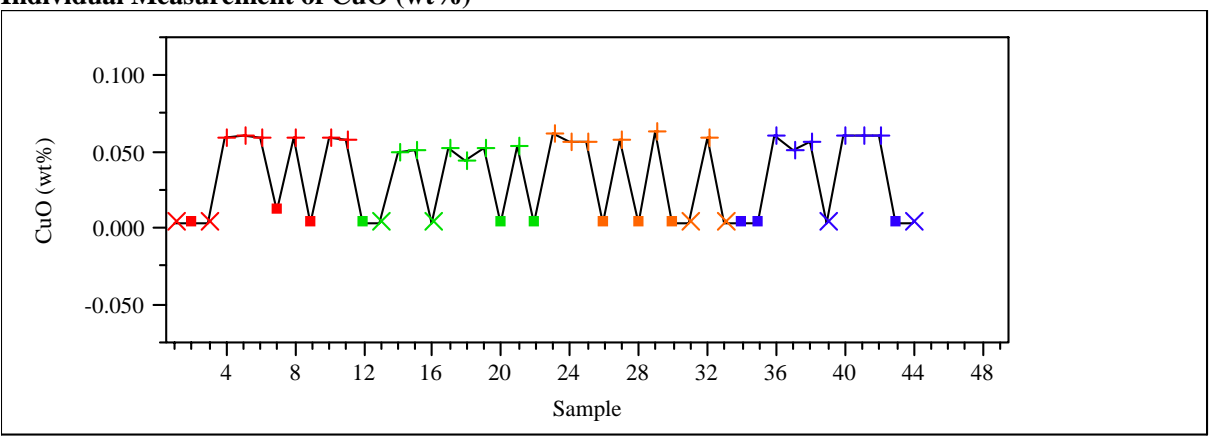

Individual Measurement of Fe2O3 (wt\%)

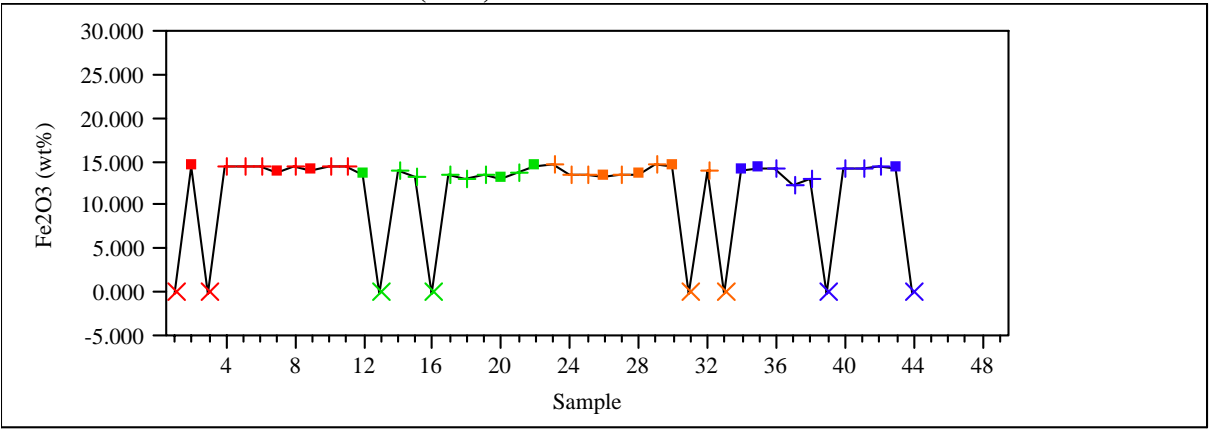


Westinghouse Savannah River Company

WSRC-TR-2005-00396

Savannah River National Laboratory

Rev. 0

Analytical Development/Statistical Consulting Sections

Page 40 of 140

Exhibit A1. Chemical Composition Measurements in Analytical Sequence by Oxide for DWPF Simulated Glass

\section{(Concentrations are in wt\%.)}

Individual Measurement of K2O (wt\%)

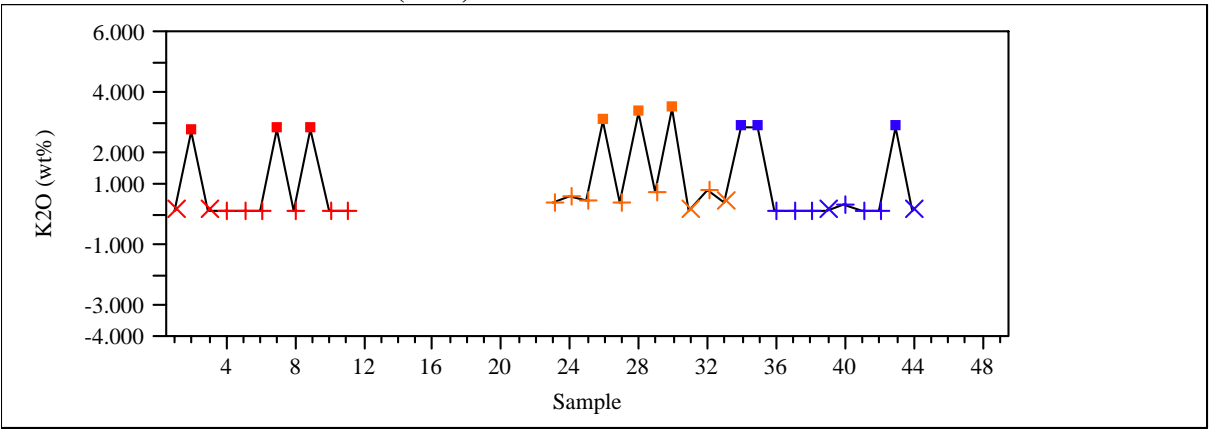

Individual Measurement of La2O3 (wt \%)

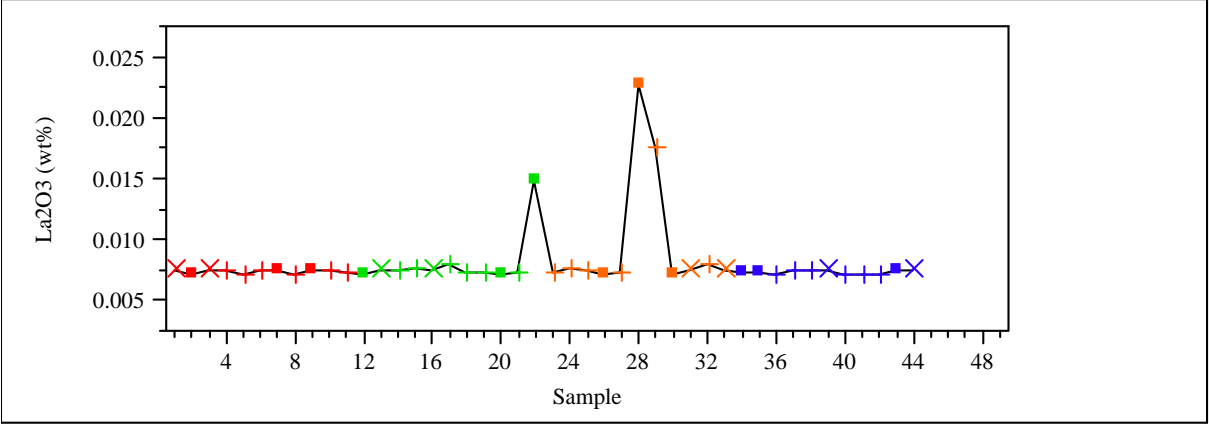

Individual Measurement of Li2O (wt \%)

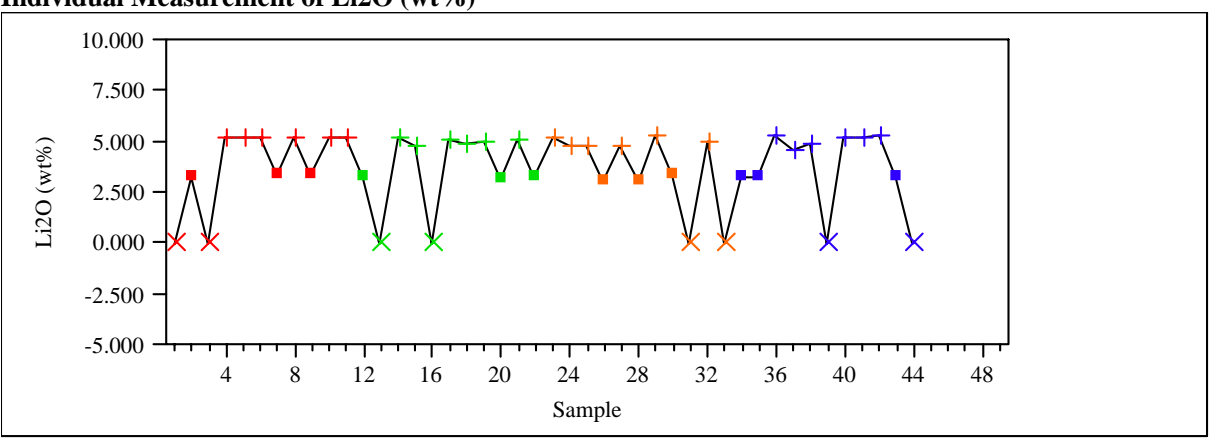

Individual Measurement of MgO (wt \%)

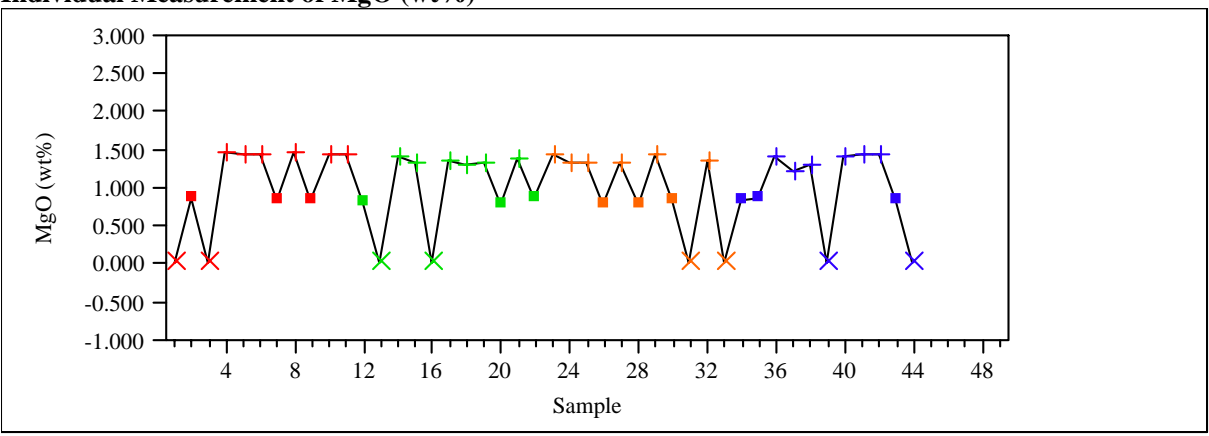


Westinghouse Savannah River Company

WSRC-TR-2005-00396

Savannah River National Laboratory

Rev. 0

Analytical Development/Statistical Consulting Sections

Page 41 of 140

Exhibit A1. Chemical Composition Measurements in Analytical Sequence by Oxide for DWPF Simulated Glass

(Concentrations are in wt\%.)

Individual Measurement of MnO (wt\%)

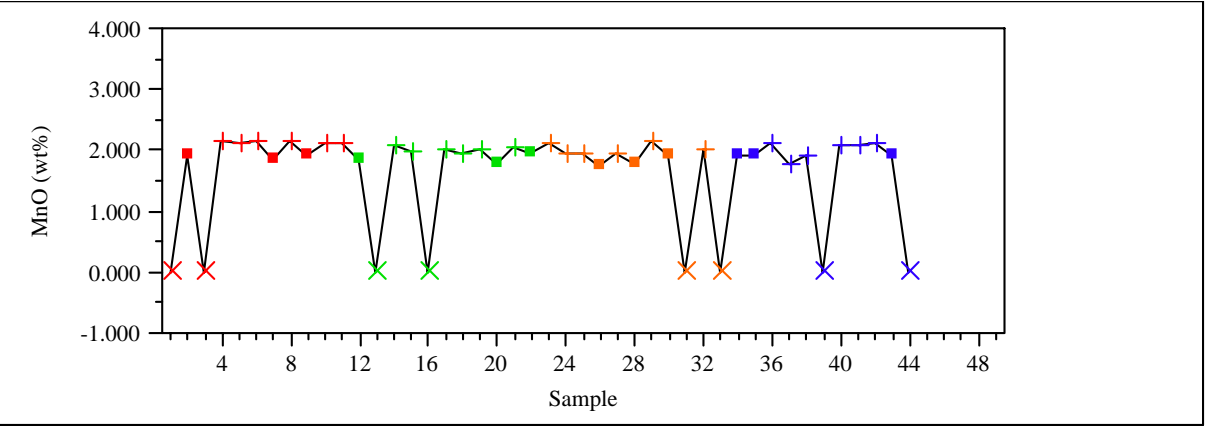

Individual Measurement of $\mathrm{MoO} 3(\mathrm{w} \%)$

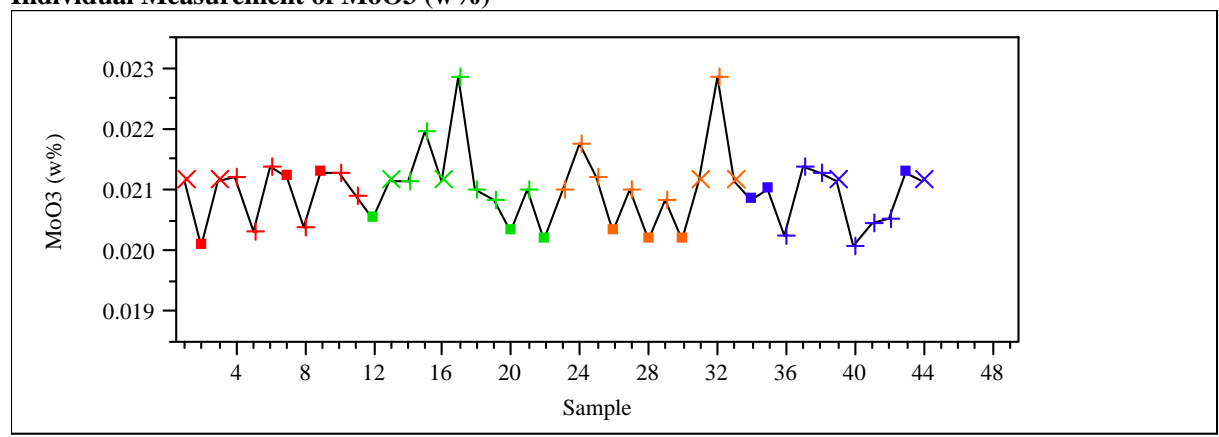

Individual Measurement of Na2O (wt\%)

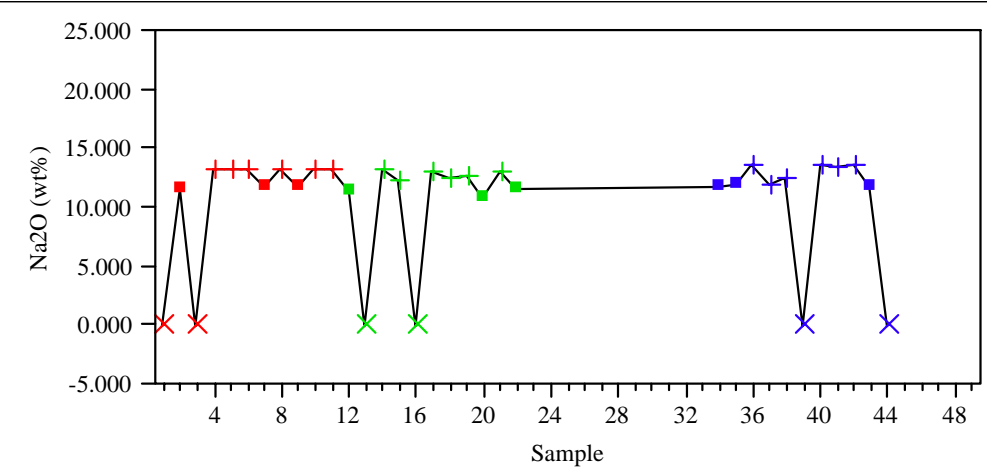


Westinghouse Savannah River Company

WSRC-TR-2005-00396

Savannah River National Laboratory

Rev. 0

Analytical Development/Statistical Consulting Sections

Page 42 of 140

Exhibit A1. Chemical Composition Measurements in Analytical Sequence by Oxide for DWPF Simulated Glass

(Concentrations are in wt\%.)

Individual Measurement of $\mathrm{Nb2O5}$ (wt\%)

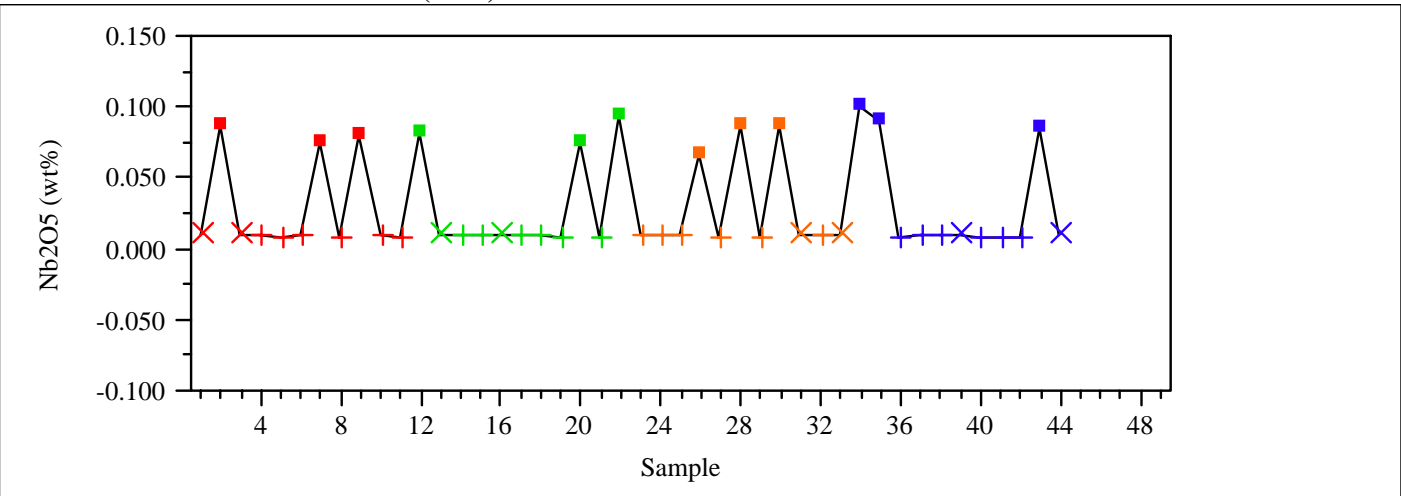

Individual Measurement of Nd2O3 (wt\%)

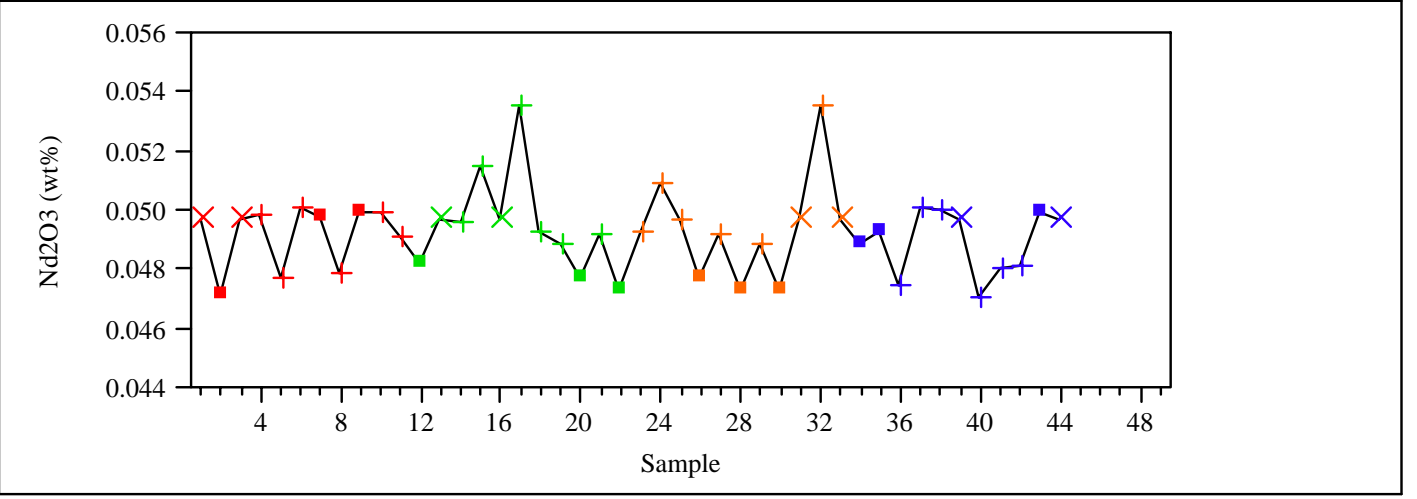

Individual Measurement of $\mathrm{NiO}$ (wt \%)

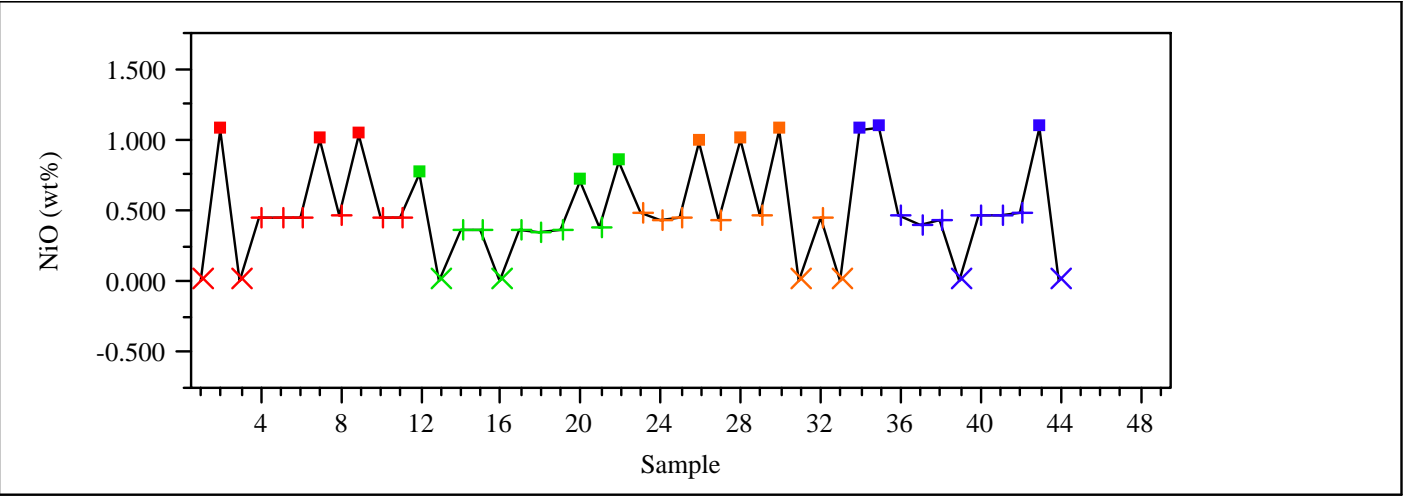


Westinghouse Savannah River Company

WSRC-TR-2005-00396

Savannah River National Laboratory

Rev. 0

Analytical Development/Statistical Consulting Sections

Page 43 of 140

Exhibit A1. Chemical Composition Measurements in Analytical Sequence by Oxide for DWPF Simulated Glass

(Concentrations are in wt\%.)

Individual Measurement of P2O5 (wt\%)

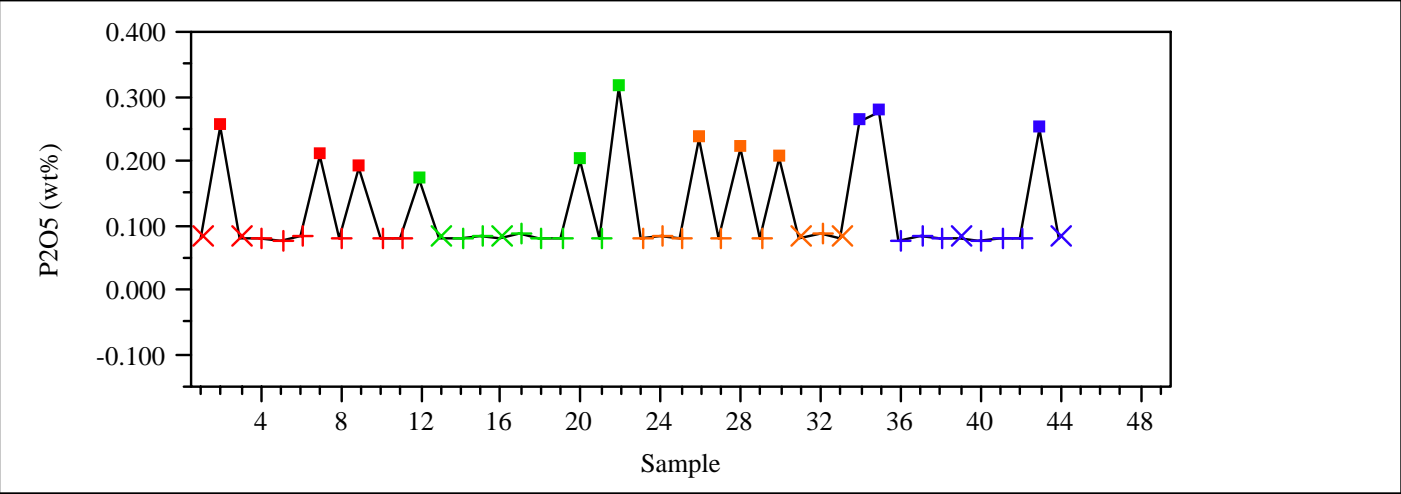

Individual Measurement of PbO (wt \%)

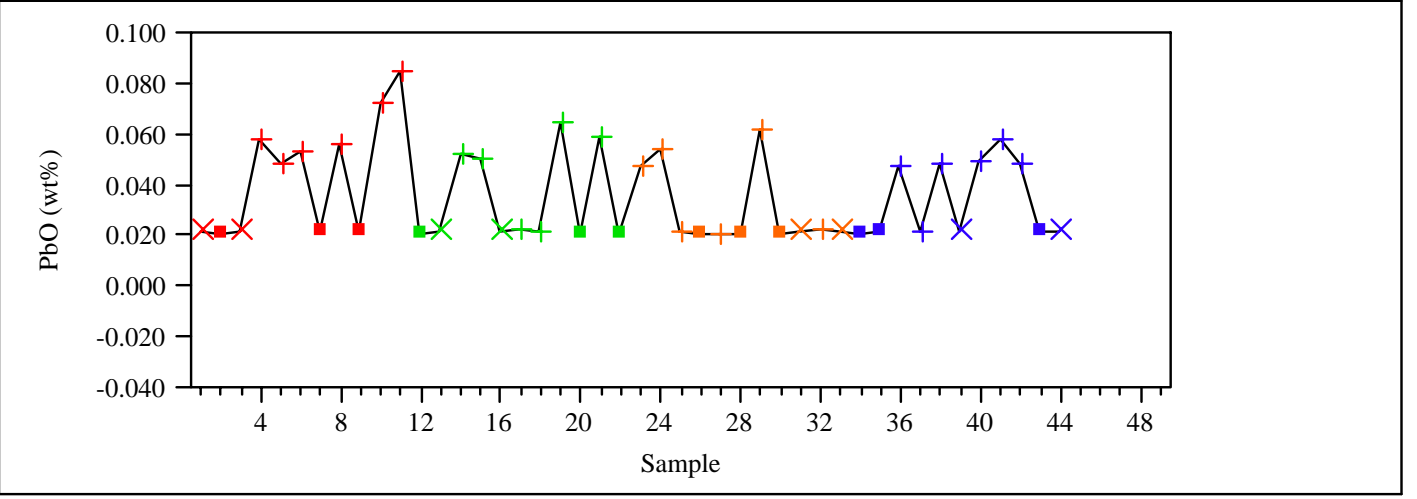

Individual Measurement of ReO2 (wt\%)

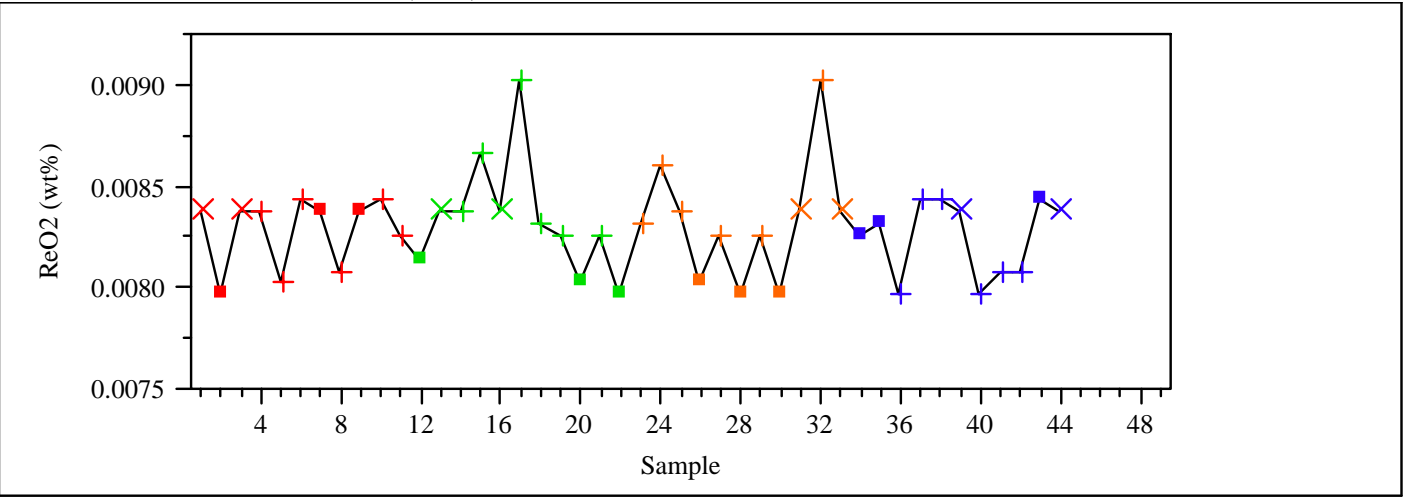


Westinghouse Savannah River Company

WSRC-TR-2005-00396

Savannah River National Laboratory

Rev. 0

Analytical Development/Statistical Consulting Sections

Page 44 of 140

Exhibit A1. Chemical Composition Measurements in Analytical Sequence by Oxide for DWPF Simulated Glass

(Concentrations are in wt\%.)

Individual Measurement of SO4 (wt \%)

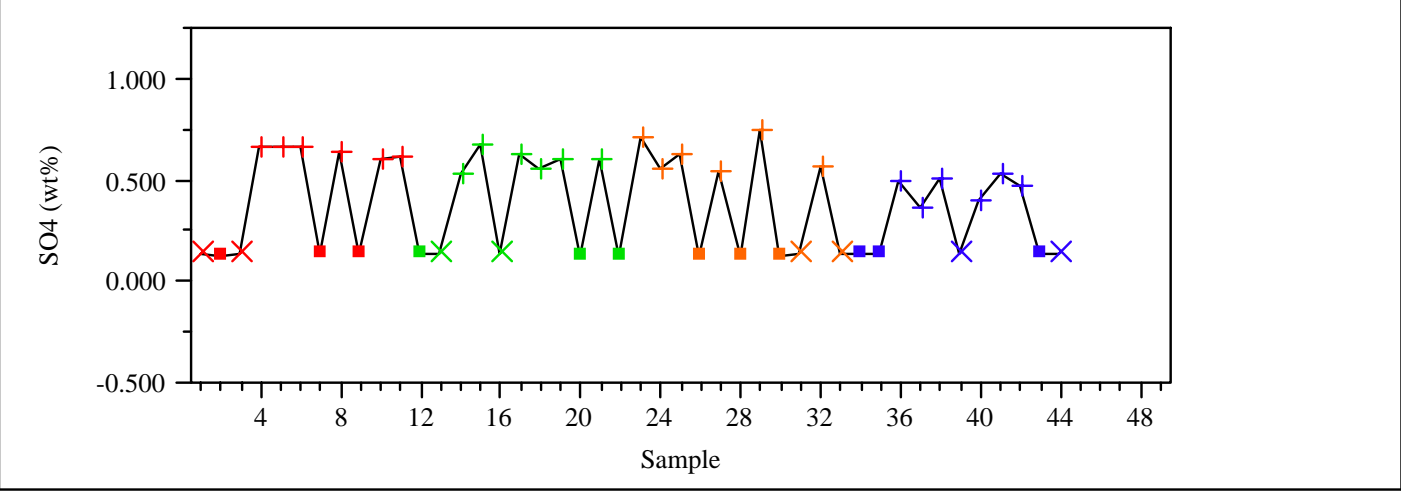

Individual Measurement of SiO2 (wt \%)

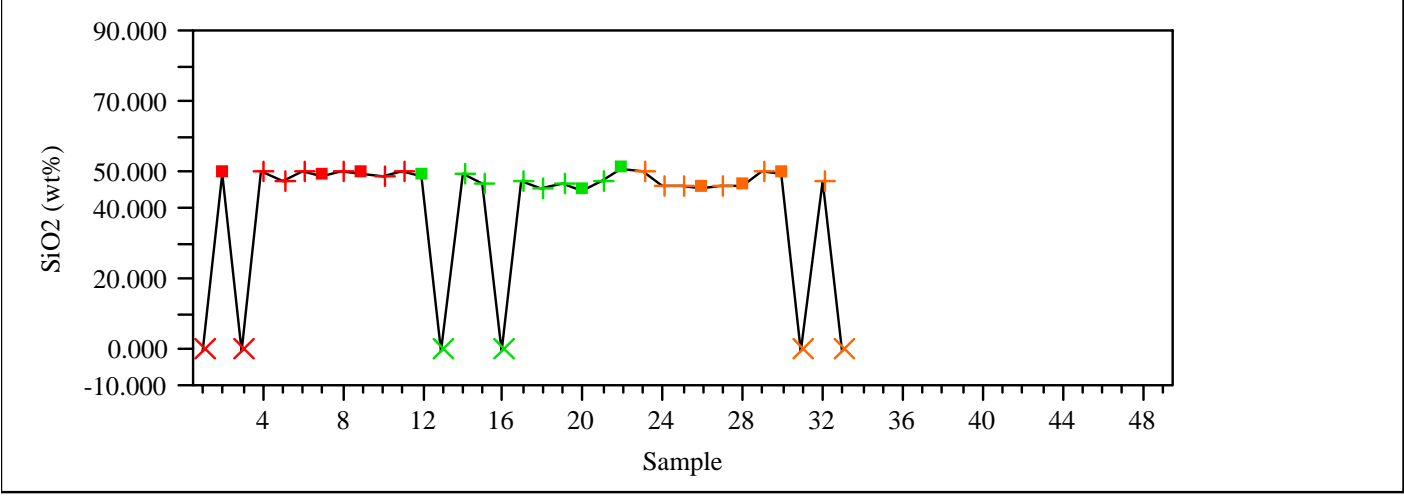

Individual Measurement of SnO2 (wt\%)

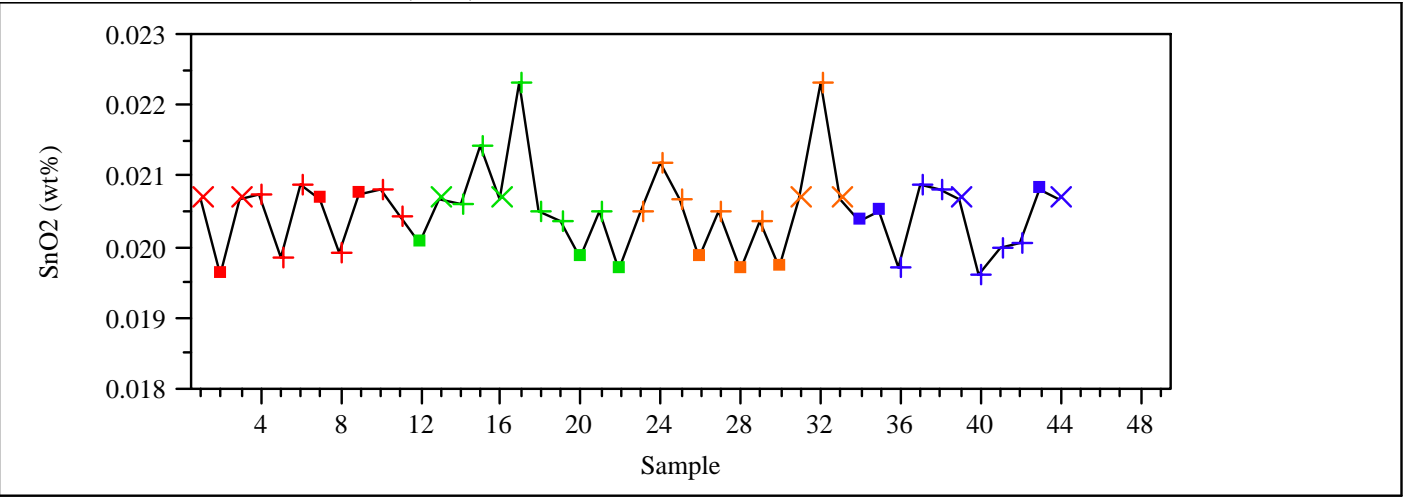


Westinghouse Savannah River Company

WSRC-TR-2005-00396

Savannah River National Laboratory

Rev. 0

Analytical Development/Statistical Consulting Sections

Page 45 of 140

Exhibit A1. Chemical Composition Measurements in Analytical Sequence by Oxide for DWPF Simulated Glass

(Concentrations are in wt\%.)

Individual Measurement of SrO (wt \%)

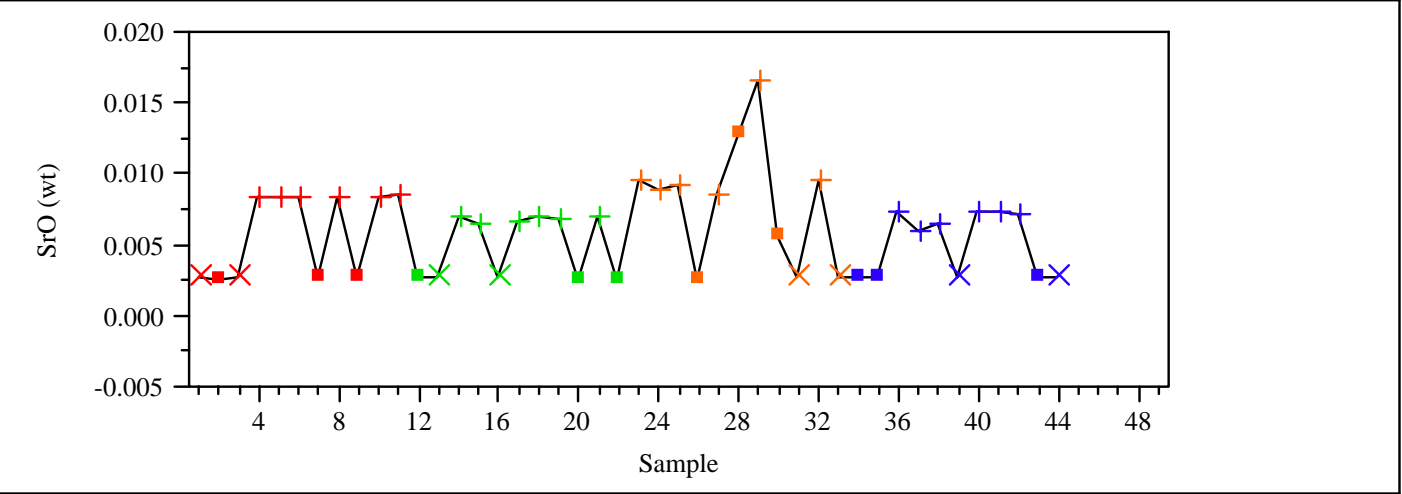

Individual Measurement of TiO2 (wt\%)

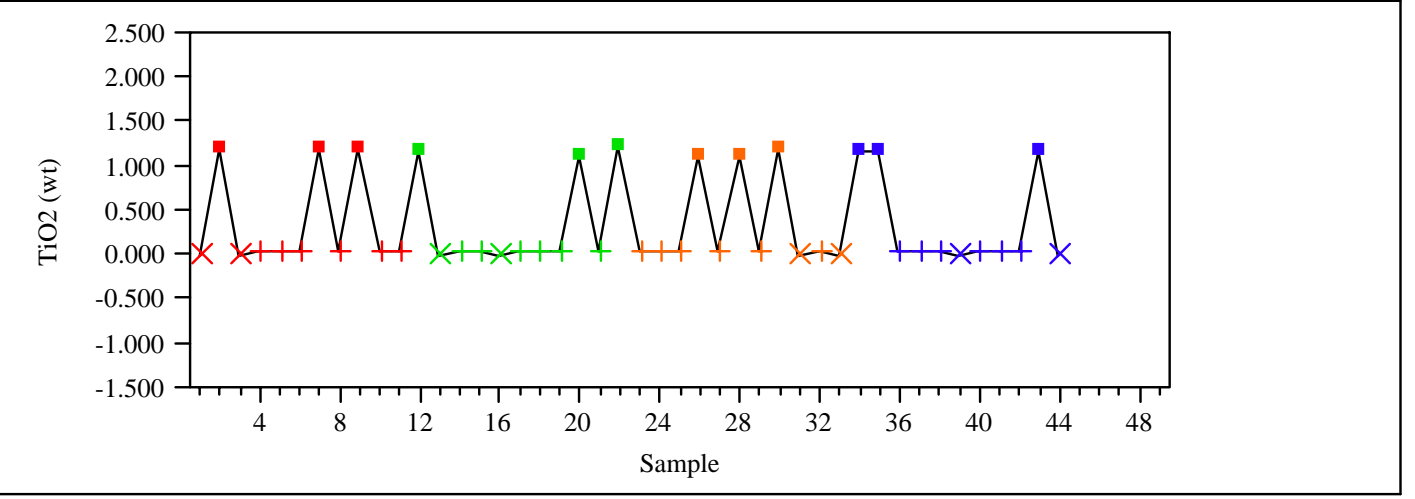

Individual Measurement of V2O5 (wt\%)

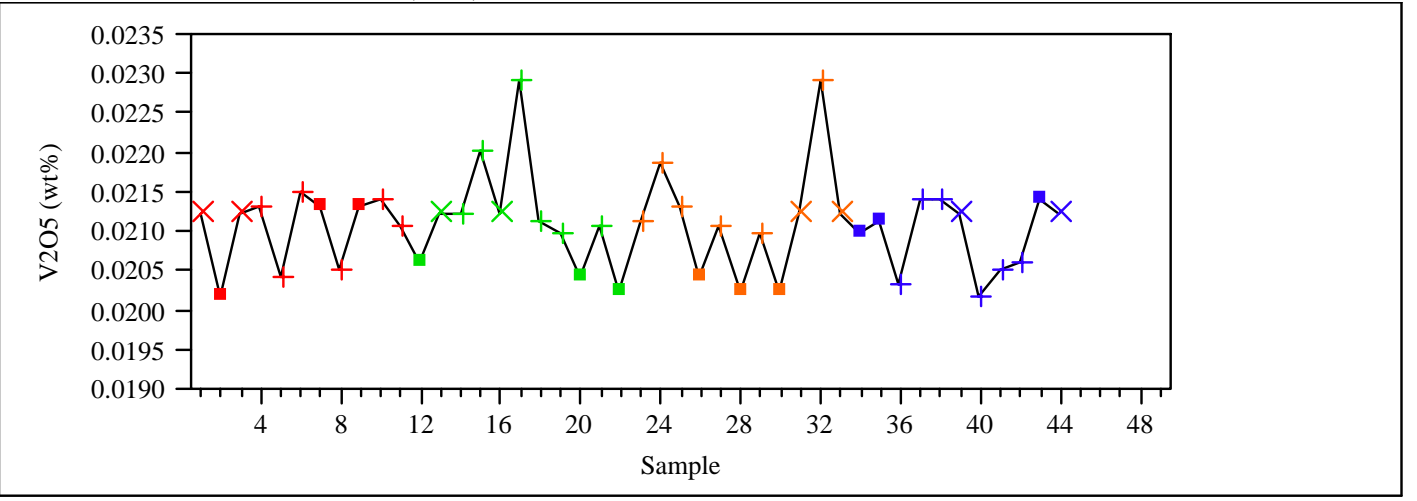


Westinghouse Savannah River Company

WSRC-TR-2005-00396

Savannah River National Laboratory

Rev. 0

Analytical Development/Statistical Consulting Sections

Page 46 of 140

Exhibit A1. Chemical Composition Measurements in Analytical Sequence by Oxide for DWPF Simulated Glass

(Concentrations are in wt\%.)

Individual Measurement of $\mathrm{ZnO}(\mathrm{wt} \%)$

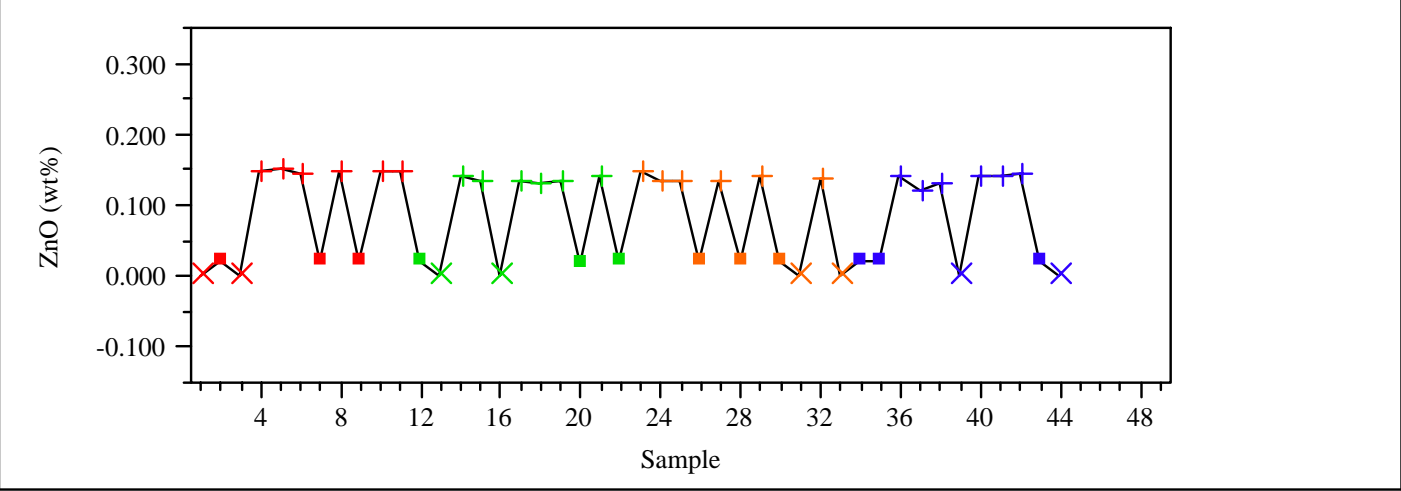

Individual Measurement of $\mathrm{ZrO} 2$ (wt \%)

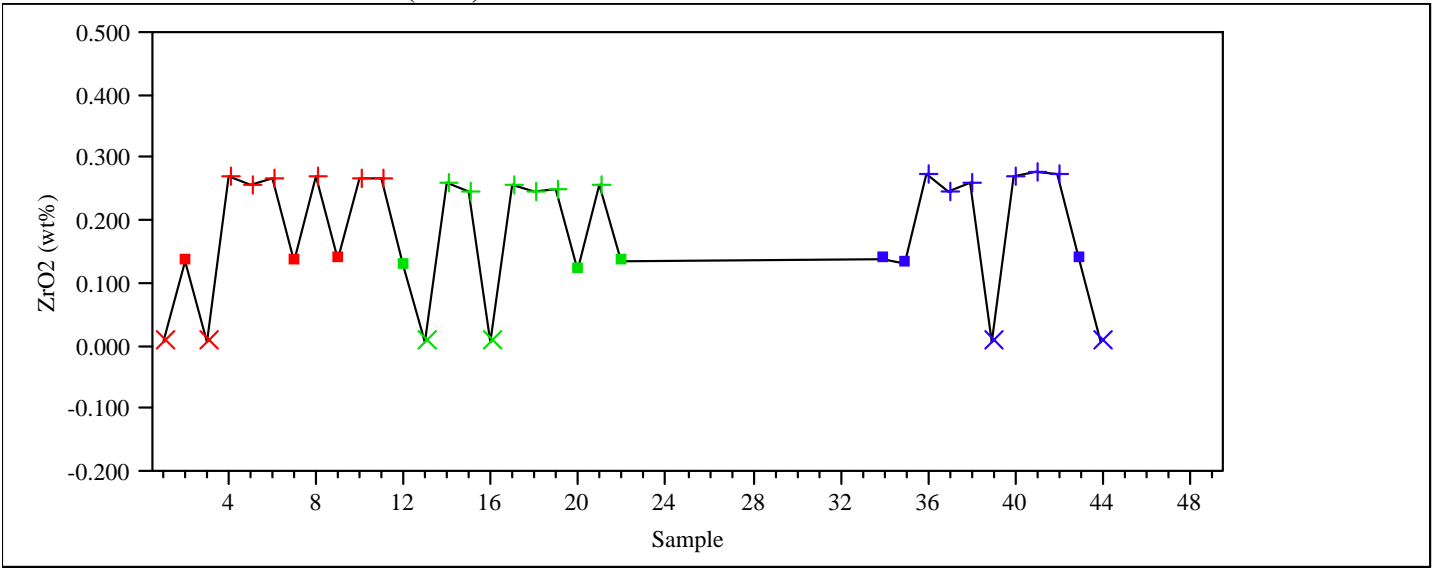




\section{Exhibit A2. Chemical Composition Measurements by Type of Prepared Sample by Oxide for DWPF Simulated Glass} (Concentrations are in wt\%.)

\section{Legend}

Preparation Method

- $\mathrm{Cs} 2 \mathrm{CO} 3$

- $\mathrm{K} 2 \mathrm{CO} 3$

Type of Sample

- - Mixed Ac

- ARG-1

- $\mathrm{Na} 2 \mathrm{O} 2$

Chart for Al2O3 (wt\%)

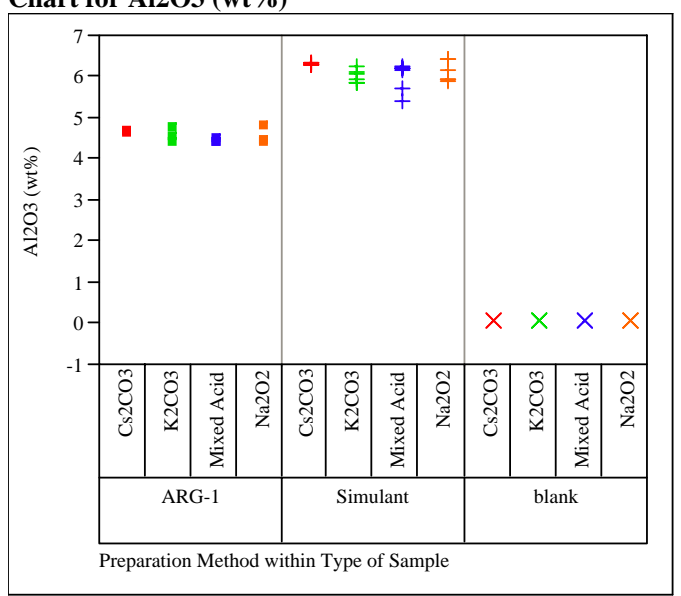

Chart for B2O3 (wt\%)

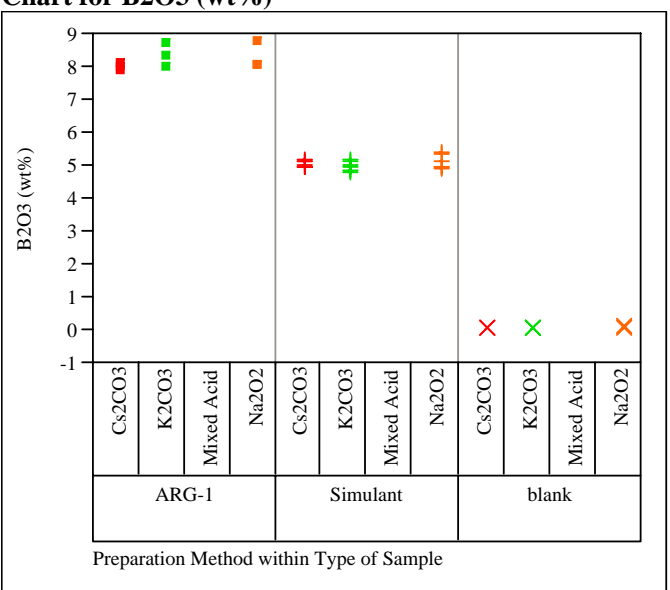

Chart for BaO (wt \%)

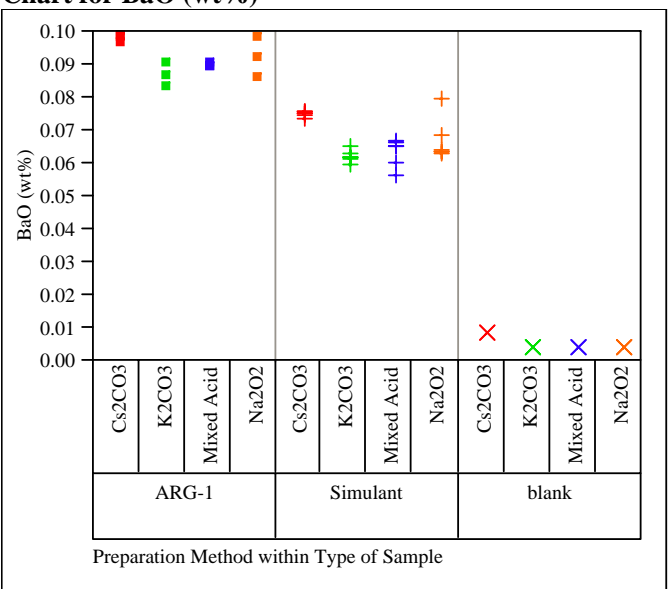

Chart for $\mathrm{CaO}$ (wt\%)

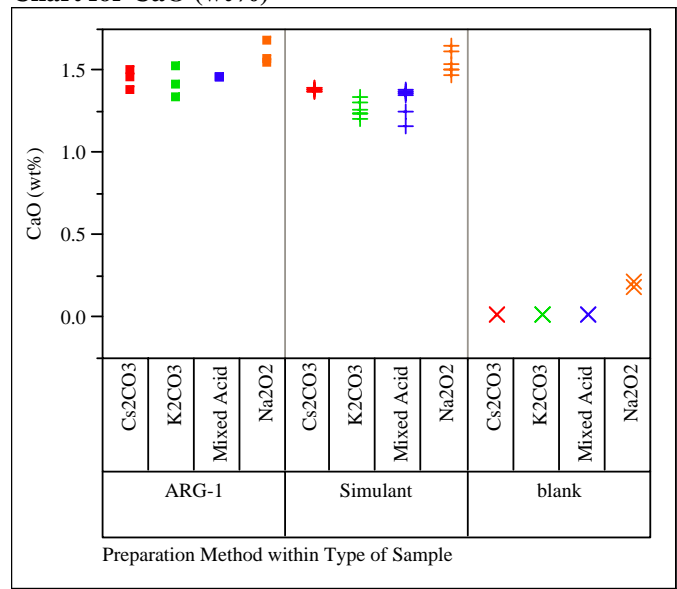

Chart for CdO (wt\%)

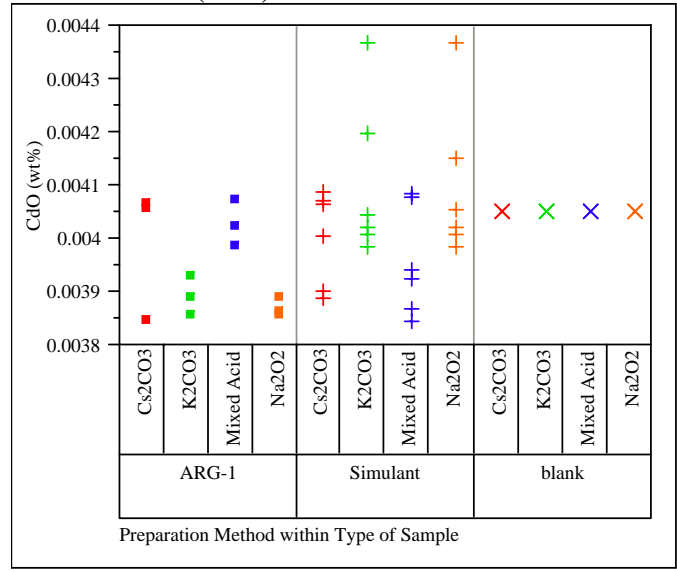


Exhibit A2. Chemical Composition Measurements by Type of Prepared Sample by Oxide for DWPF Simulated Glass (Concentrations are in wt\%.)
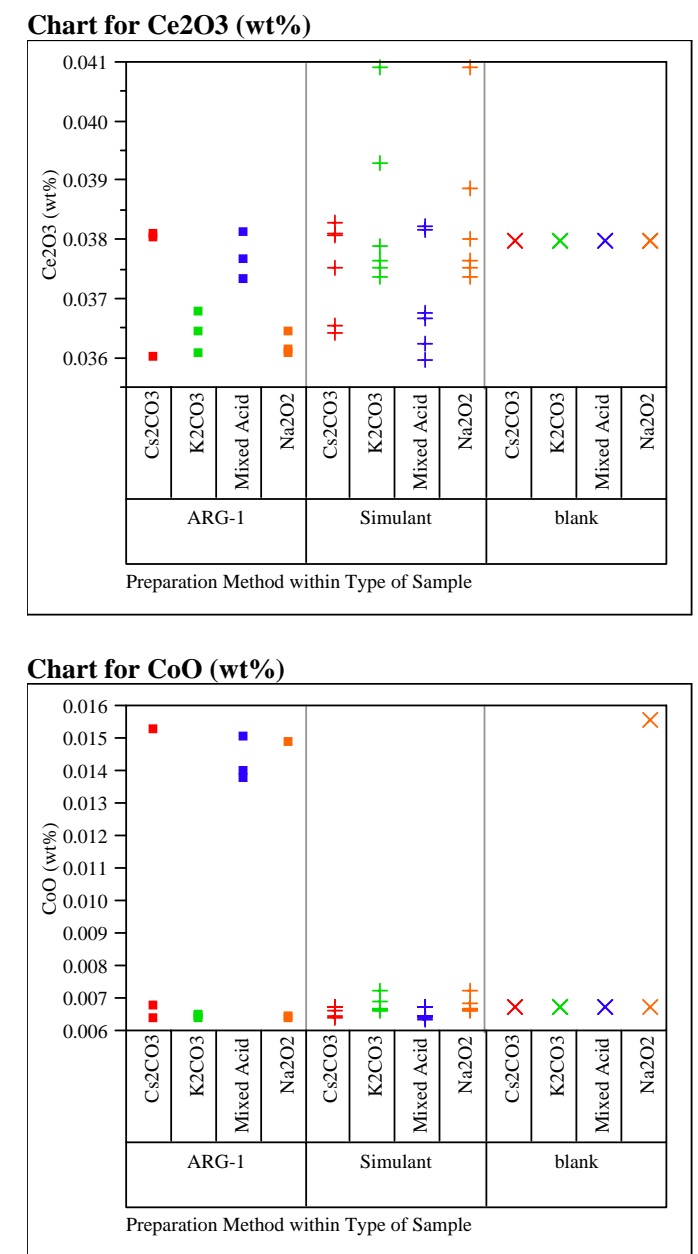

Chart for Cr2O3 (wt\%)

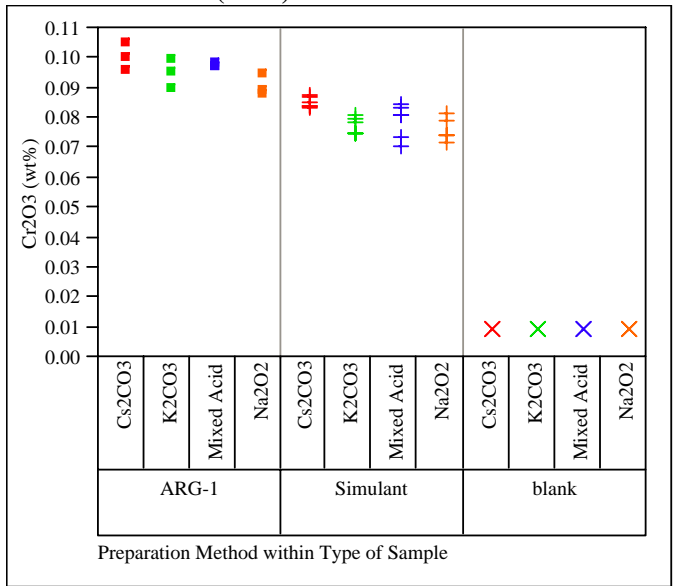

Chart for $\mathrm{CuO}(\mathrm{wt} \%)$

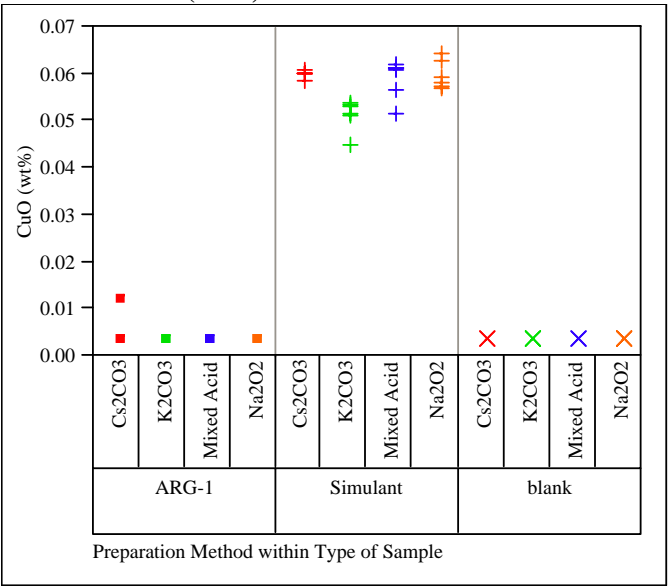

Chart for Fe2O3 (wt \%)

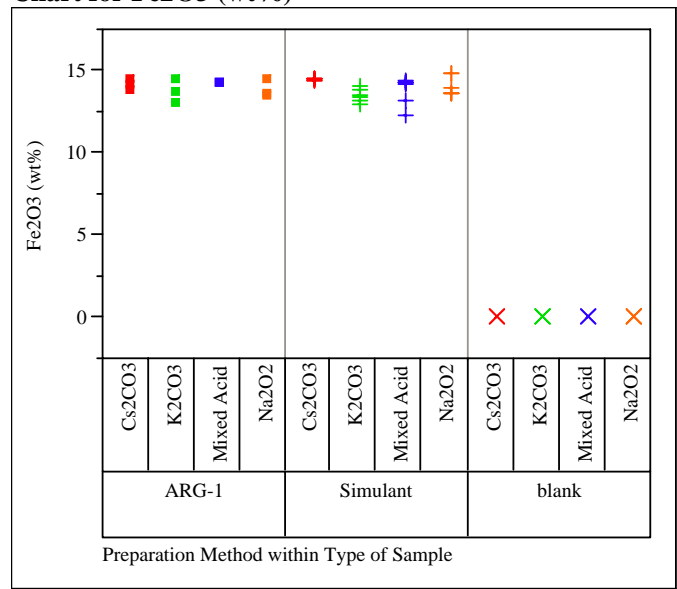

Chart for K2O (wt \%)

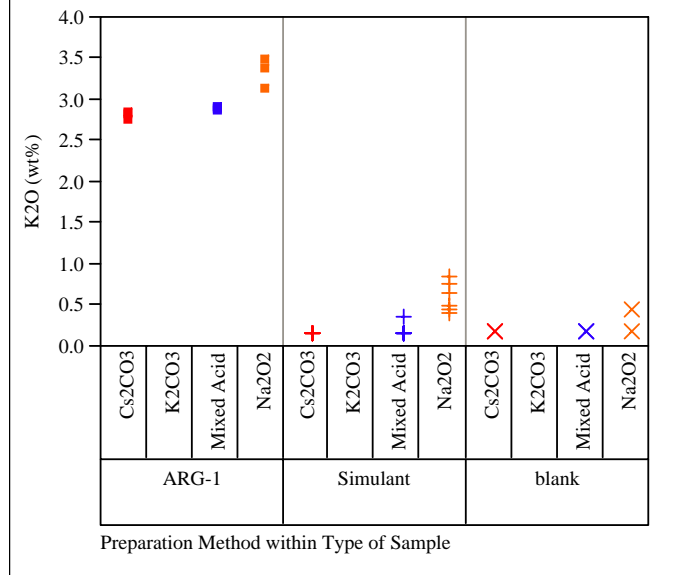


Exhibit A2. Chemical Composition Measurements by Type of Prepared Sample by Oxide for DWPF Simulated Glass (Concentrations are in wt\%.)

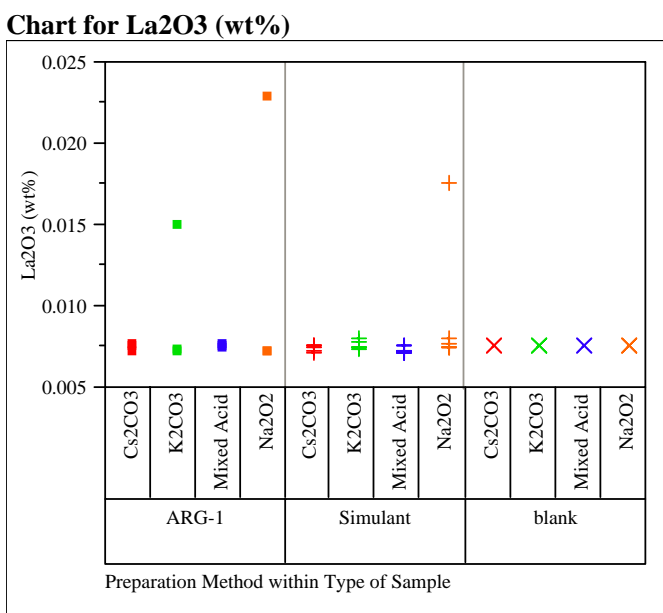

Chart for Li2O (wt\%)

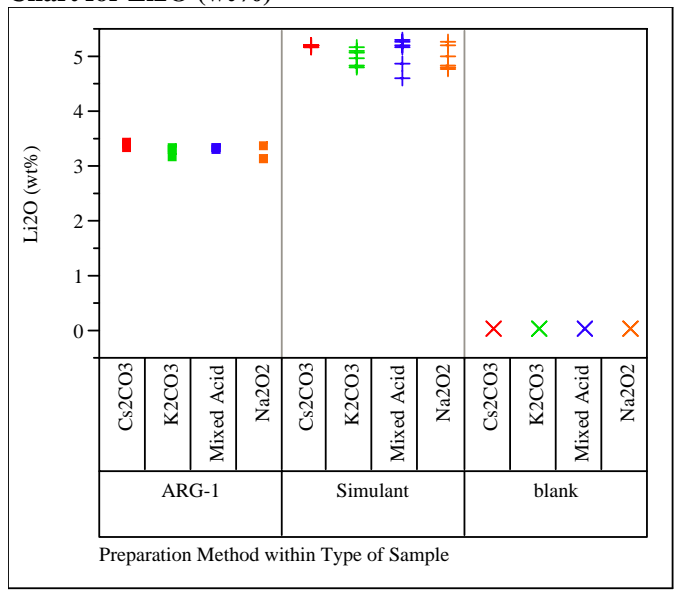

Chart for MgO (wt\%)

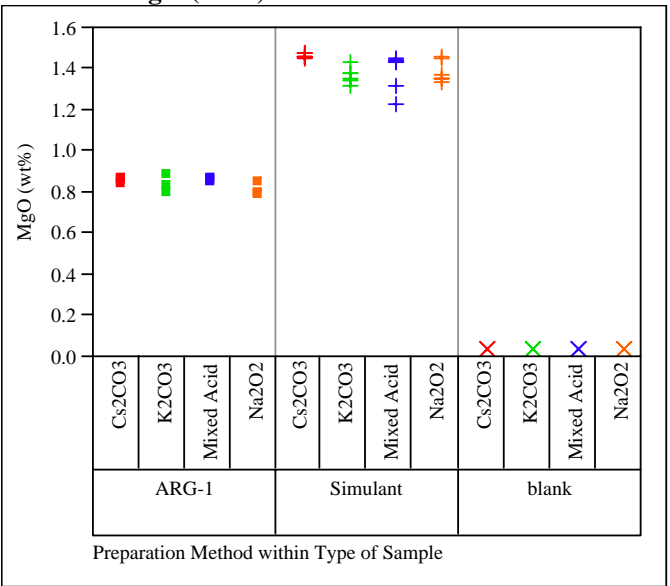

Chart for MnO (wt \%)

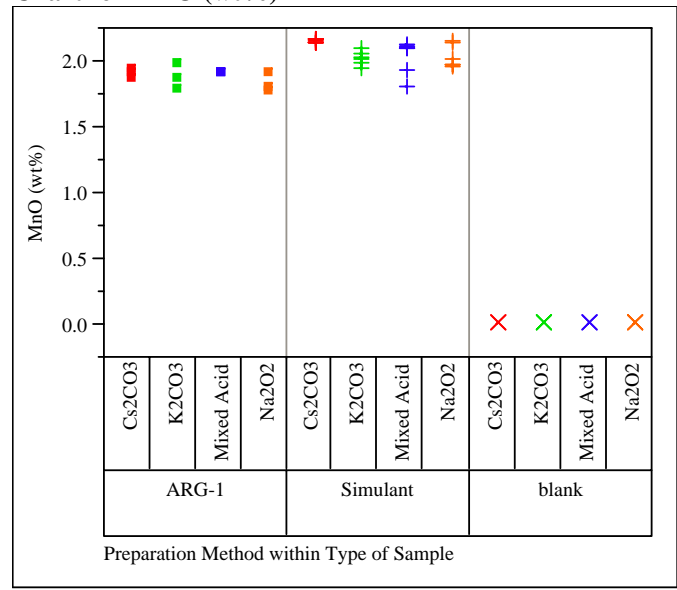

Chart for MoO3 (w\%)

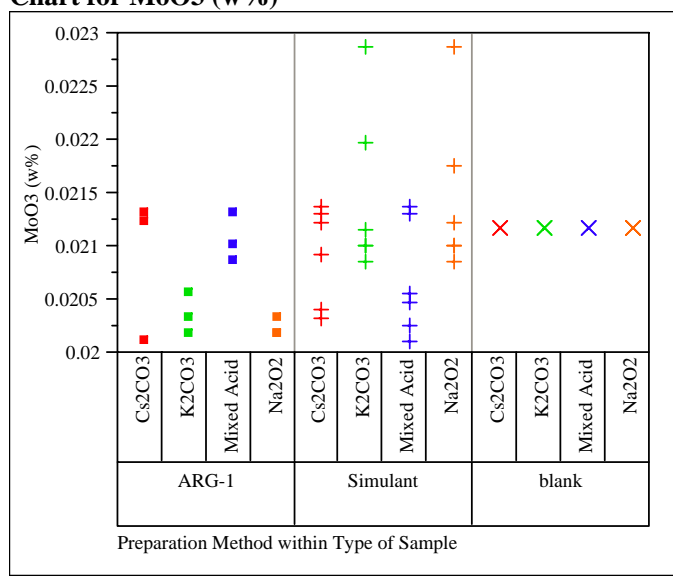

Chart for Na2O (wt\%)

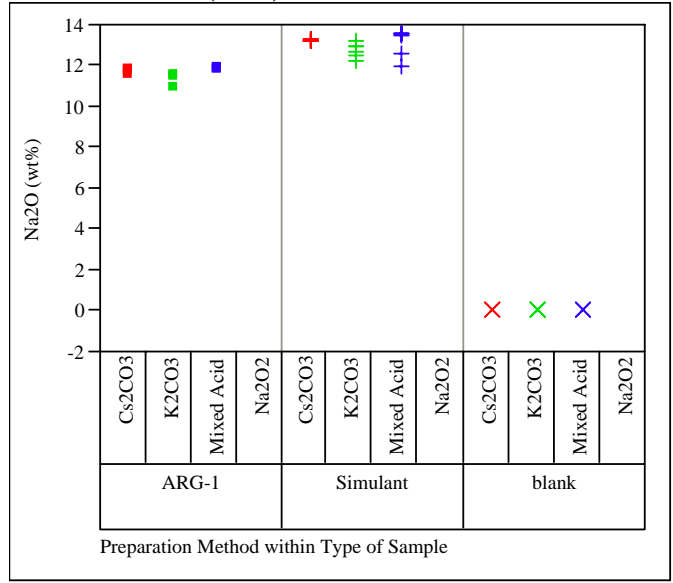


Exhibit A2. Chemical Composition Measurements by Type of Prepared Sample by Oxide for DWPF Simulated Glass (Concentrations are in wt\%.)

Chart for Nb2O5 (wt\%)

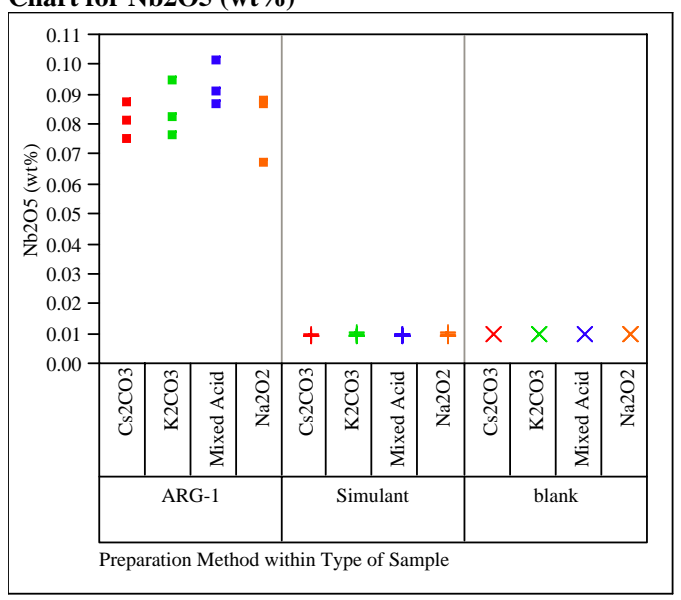

Chart for Nd2O3 (wt\%)

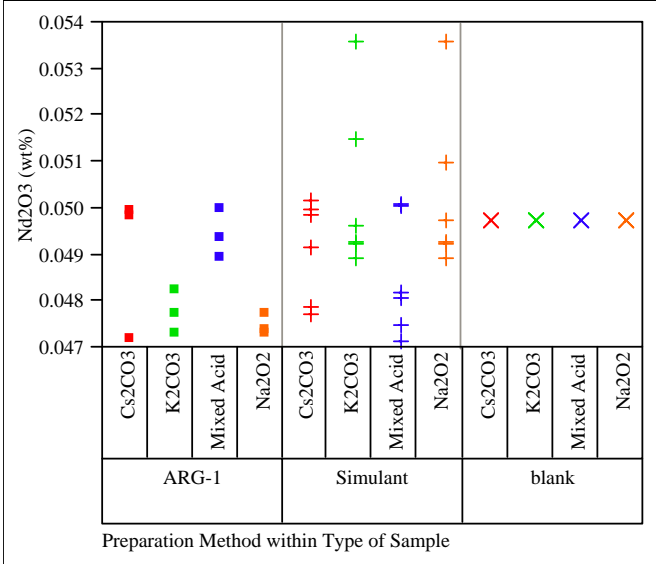

Chart for $\mathrm{NiO}(\mathrm{wt} \%)$

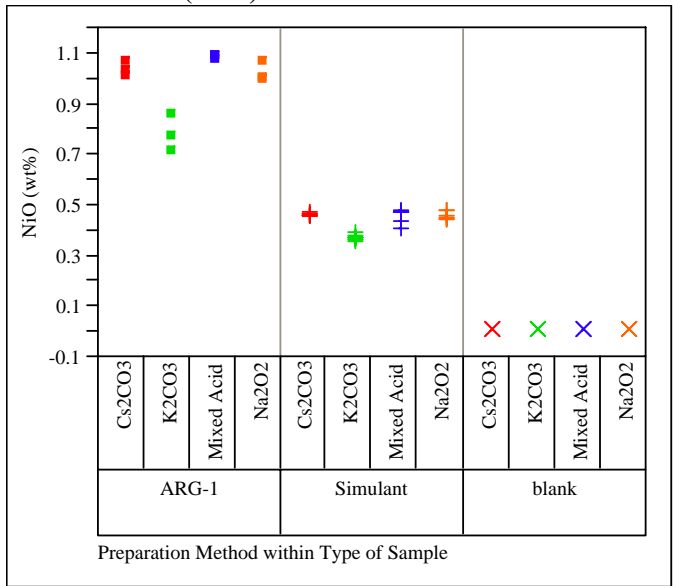

Chart for P2O5 (wt\%)

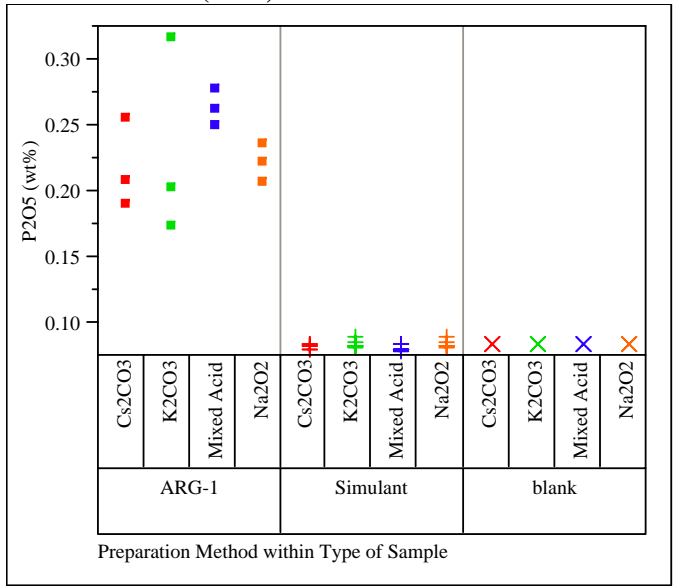

Chart for PbO (wt \%)

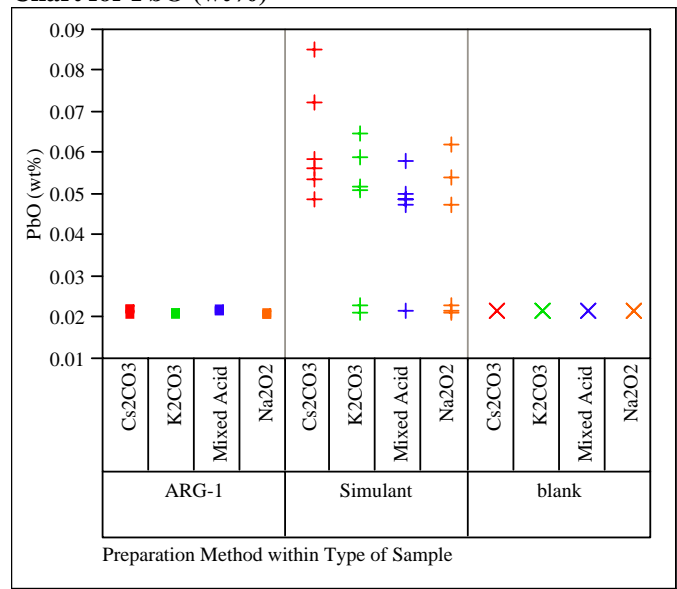

Chart for ReO2 (wt\%)

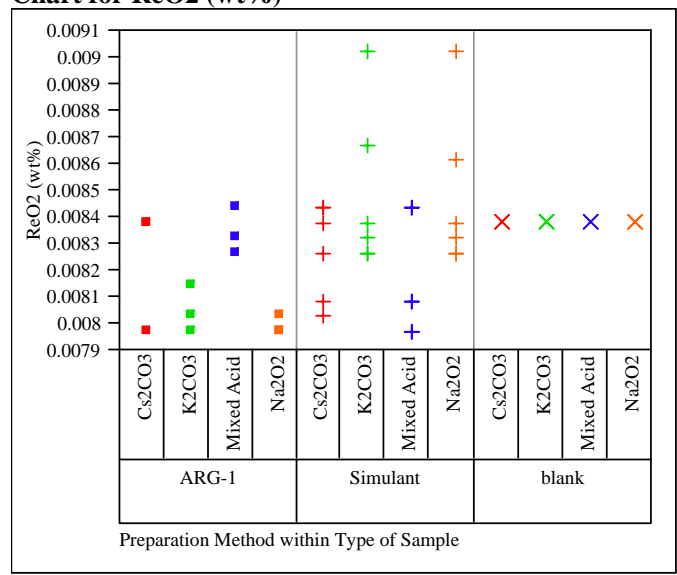


Exhibit A2. Chemical Composition Measurements by Type of Prepared Sample by Oxide for DWPF Simulated Glass (Concentrations are in wt\%.)

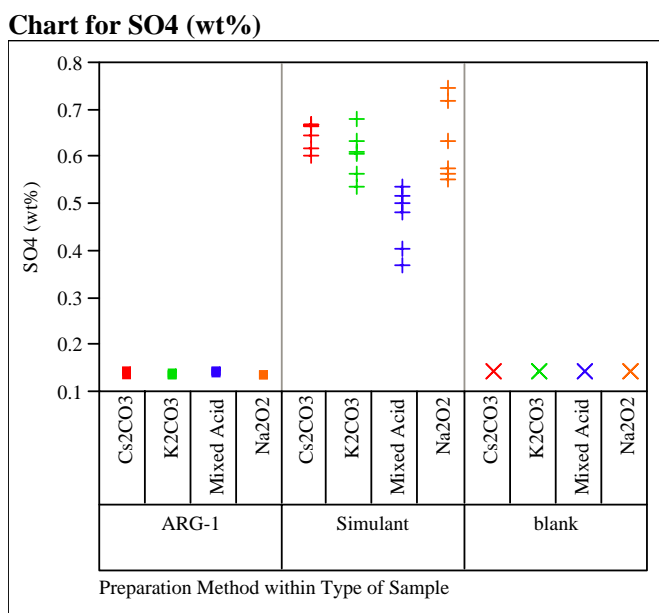

Chart for $\mathrm{SiO} 2(w \mathrm{w} \%)$

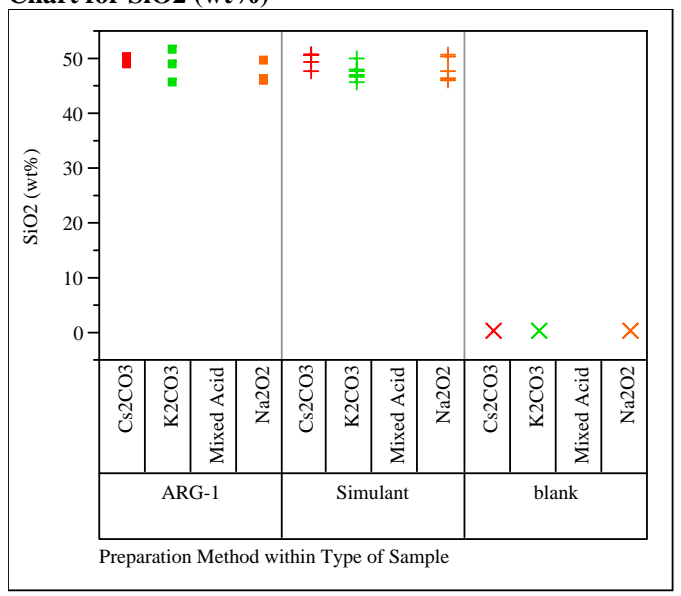

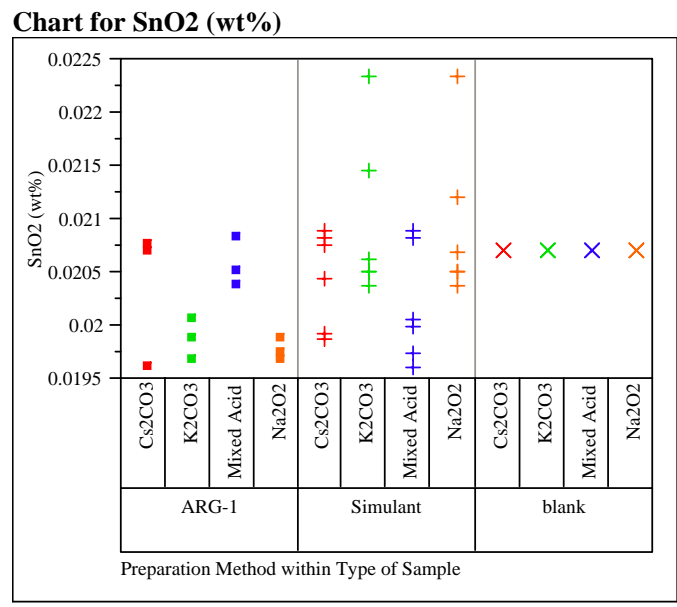

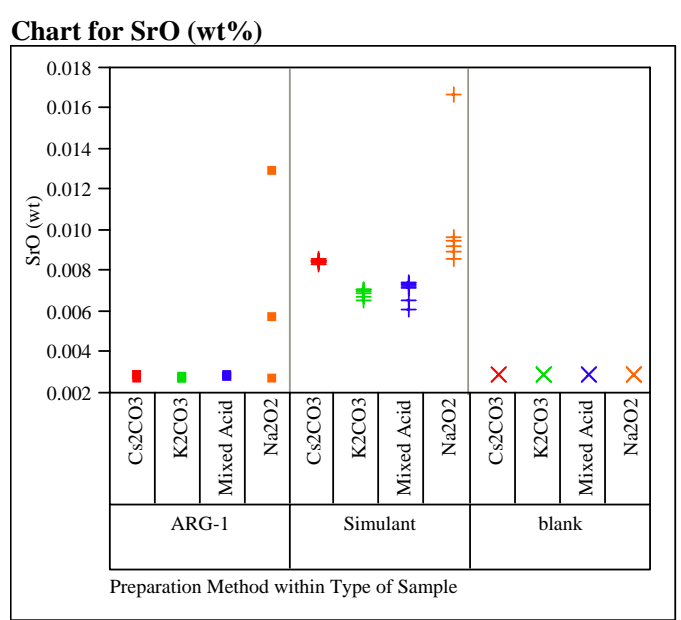

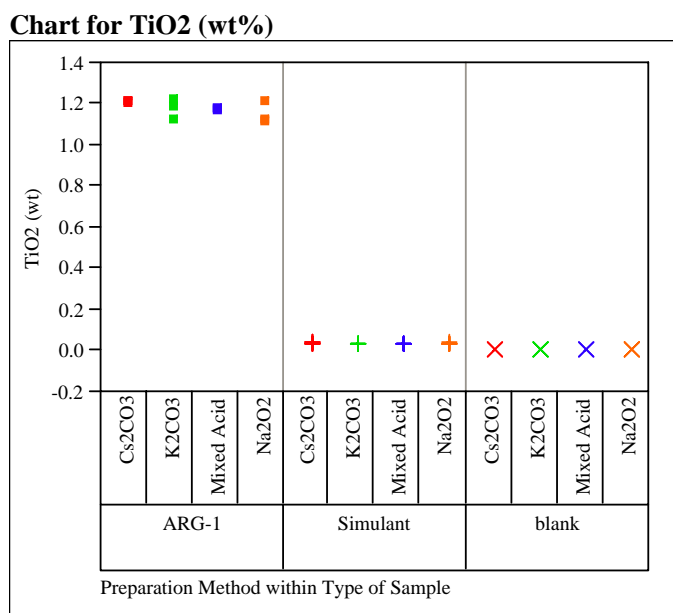

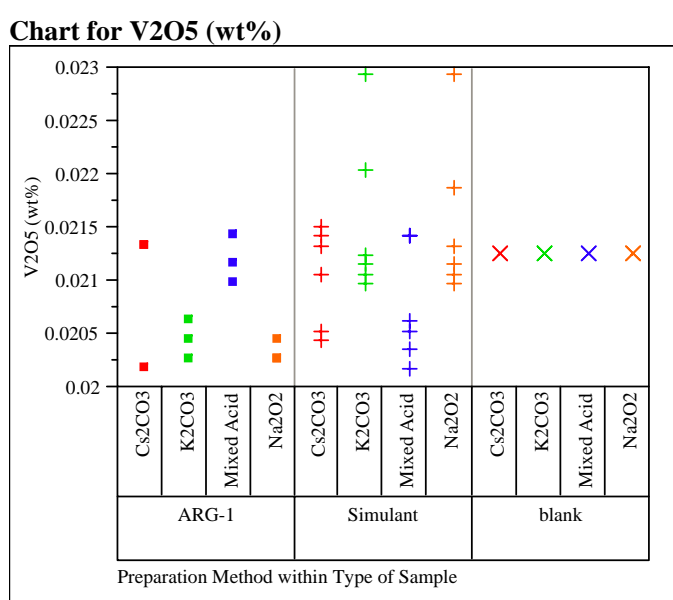


Exhibit A2. Chemical Composition Measurements by Type of Prepared Sample by Oxide for DWPF Simulated Glass (Concentrations are in wt\%.)

Chart for ZnO (wt \%)
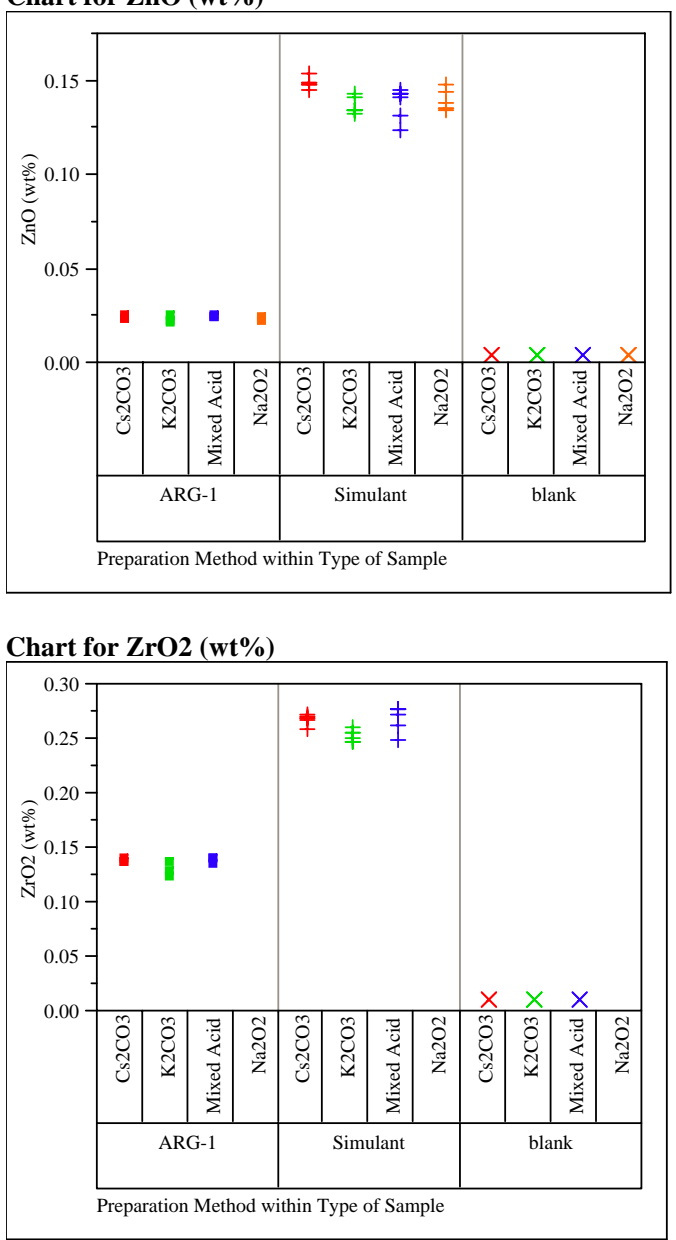


\section{Exhibit A3. Statistical Comparisons of Chemical Composition Measurements of ARG-1 Samples by Dissolution Method for Each Oxide (Concentrations in Wt\% Oxides)}

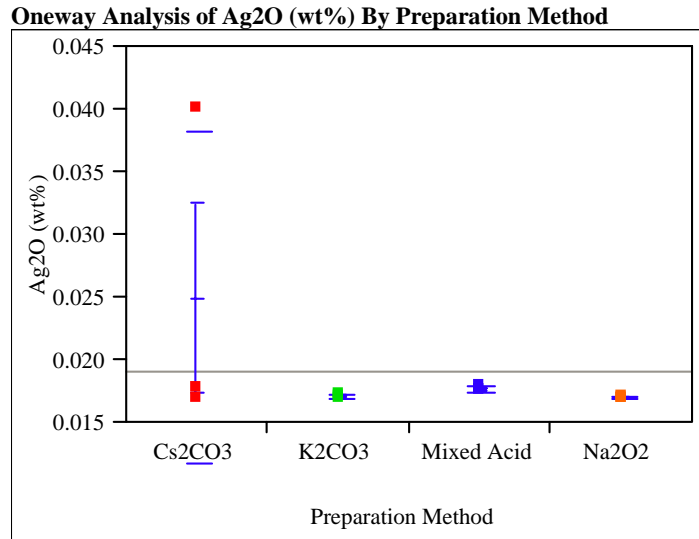

Means Comparisons

Comparisons for all pairs using Tukey-Kramer HSD

$$
\text { q* Alpha }
$$

Abs(Dif)-LSD Cs2CO3 Mixed Acid K2CO3 Na2O2

$\begin{array}{lllll}\mathrm{Cs} 2 \mathrm{CO} 3 & -0.01716 & -0.00991 & -0.00932 & -0.00921\end{array}$

$\begin{array}{lllll}\text { Mixed Acid } \quad-0.00991 & -0.01716 & -0.01657 & -0.01647\end{array}$

$\begin{array}{lllll}\mathrm{K} 2 \mathrm{CO} 3 & -0.00932 & -0.01657 & -0.01716 & -0.01706\end{array}$

$\begin{array}{lllll}\mathrm{Na} 2 \mathrm{O} 2 & -0.00921 & -0.01647 & -0.01706 & -0.01716\end{array}$

Positive values show pairs of means that are significantly different.

Level Mean

Cs2CO3 A 0.02492144

Mixed Acid A 0.01767059

K2CO3 A 0.01707978

$\mathrm{Na} 2 \mathrm{O} 2$ A 0.01697236

Levels not connected by same letter are significantly different Tests that the Variances are Equal

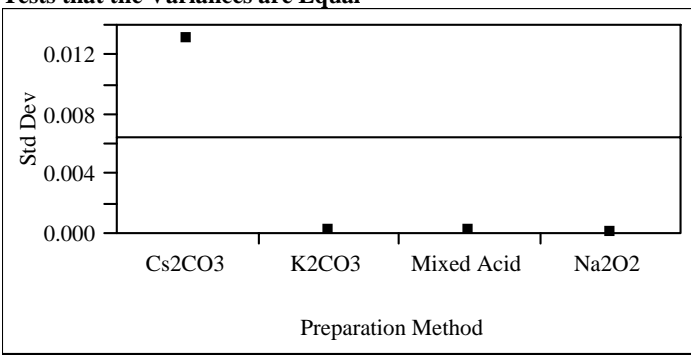

$\begin{array}{lrrrr}\text { Level } & \text { Count } & \text { Std Dev } & \begin{array}{r}\text { MeanAbsDif to } \\ \text { Mean }\end{array} & \begin{array}{r}\text { MeanAbsDif to } \\ \text { Median }\end{array} \\ \text { Cs2CO3 } & 3 & 0.0131259 & 0.0100975 & 0.0080565 \\ \text { K2CO3 } & 3 & 0.0001611 & 0.0001074 & 0.0001611 \\ \text { Mixed Acid } & 3 & 0.0001937 & 0.0001432 & 0.0001611 \\ \text { Na2O2 } & 3 & 0.0000930 & 0.0000716 & 0.0000537 \\ \text { Test } & \text { F Ratio } & \text { DFNum } & \text { DFDen } & \text { Prob > F } \\ \text { O'Brien[.5] } & 1.7773 & 3 & 8 & 0.2291 \\ \text { Brown- } & 1.2515 & 3 & 8 & 0.3539 \\ \text { Forsythe } & & & & \\ \text { Levene } & 15.4597 & 3 & 8 & 0.0011 \\ \text { Bartlett } & 11.9107 & 3 & . & <.0001 \\ \text { W } & 3 & & \end{array}$

Warning: Small sample sizes. Use Caution.

Welch Anova testing Means Equal, allowing Std Devs Not Equal

F Ratio DFNum DFDen Prob $>$ F

$\begin{array}{llll}8.2206 & 3 & 4.0389 & 0.0341\end{array}$

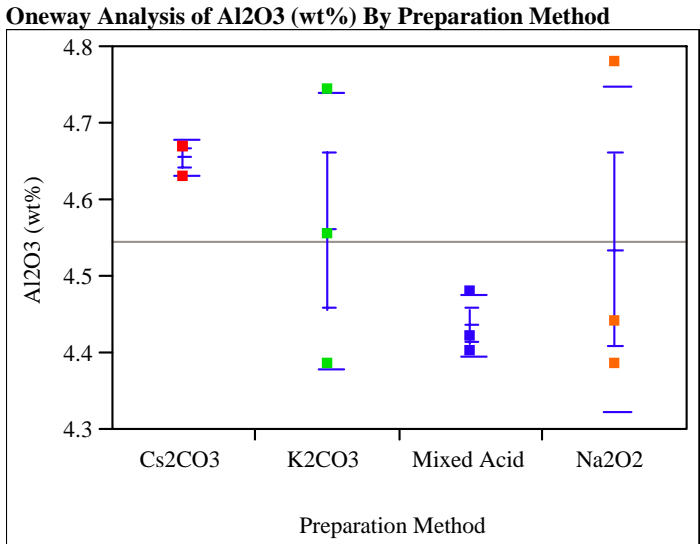

Means Comparisons

Comparisons for all pairs using Tukey-Kramer HSD

$\begin{array}{lrrrr}q^{*} & \text { Alpha } & & & \\ 3.20238 & 0.05 & & & \\ \text { Abs(Dif)-LSD } & \text { Cs2CO3 } & \text { K2CO3 } & \text { Na2O2 } & \text { Mixed Acid } \\ \text { 2CO3 } & -0.37054 & -0.27607 & -0.25087 & -0.15010 \\ \text { 2CO3 } & -0.27607 & -0.37054 & -0.34535 & -0.24457 \\ \text { 2O2 } & -0.25087 & -0.34535 & -0.37054 & -0.26977 \\ \text { ixed Acid } & -0.15010 & -0.24457 & -0.26977 & -0.37054\end{array}$

Positive values show pairs of means that are significantly different.

Level

K2CO3 A 4.5599933

$\mathrm{Na} 2 \mathrm{O} 2 \quad$ A 4.5348000

Mixed Acid A 4.4340267

Levels not connected by same letter are significantly different Tests that the Variances are Equal

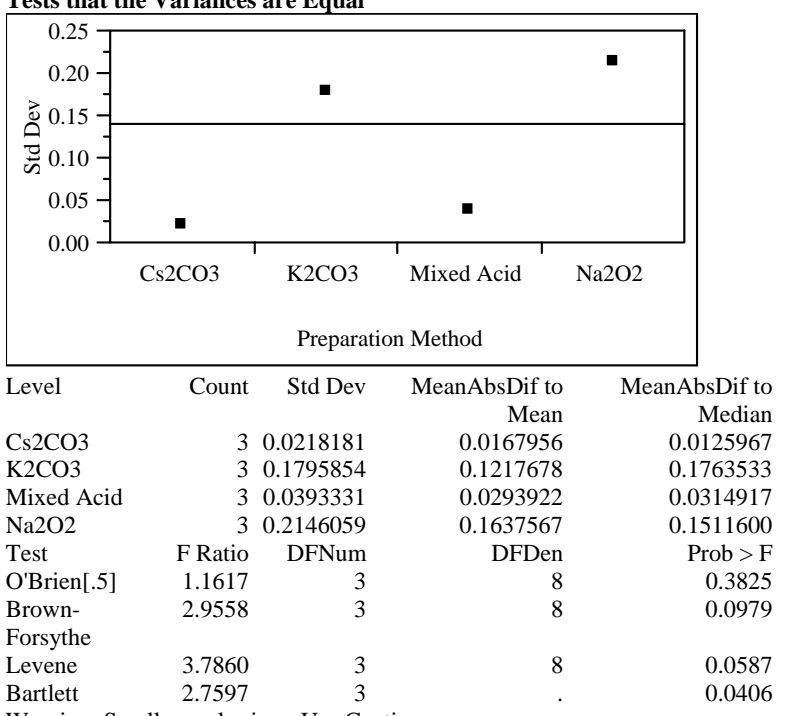

Warning: Small sample sizes. Use Caution.

Welch Anova testing Means Equal, allowing Std Devs Not Equal

F Ratio DFNum DFDen Prob $>$ F

$\begin{array}{llrl}17.9210 & 3 & 3.822 & 0.0101\end{array}$ 
Westinghouse Savannah River Company

WSRC-TR-2005-00396

Savannah River National Laboratory

Rev. 0

Analytical Development/Statistical Consulting Sections

Page 54 of 140

\section{Exhibit A3. Statistical Comparisons of Chemical Composition Measurements of ARG-1 Samples by Dissolution Method for Each Oxide \\ (Concentrations in Wt\% Oxides)}

Oneway Analysis of B2O3 (wt\%) By Preparation Method

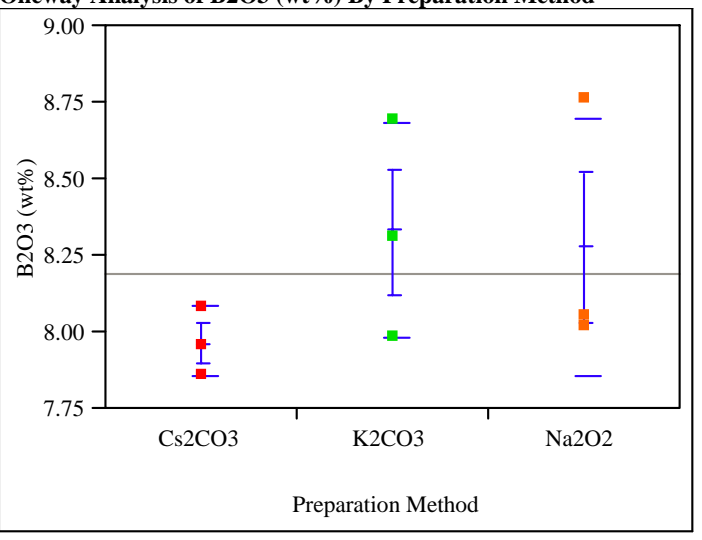

Missing Rows

3Means Comparisons

Comparisons for all pairs using Tukey-Kramer HSD $\begin{array}{rr}\text { q* Alpha } \\ 3.06815 & 0.05\end{array}$

Abs(Dif)-LSD $\quad \mathrm{K} 2 \mathrm{CO} 3 \quad \mathrm{Na} 2 \mathrm{O} 2 \mathrm{Cs} 2 \mathrm{CO} 3$

$\begin{array}{llll}\mathrm{K} 2 \mathrm{CO} 3 & -0.81021 & -0.75654 & -0.44528\end{array}$

$\mathrm{Na} 2 \mathrm{O} 2 \quad-0.75654-0.81021-0.49895$

$\begin{array}{llll}\text { Cs2CO3 } & -0.44528 & -0.49895 & -0.81021\end{array}$

Positive values show pairs of means that are significantly different.

Level Mean

K2CO3 A 8.3288080

$\mathrm{Na} 2 \mathrm{O} 2$ A 8.2751430

Cs2CO3 A 7.9638860

Levels not connected by same letter are significantly different

Tests that the Variances are Equal

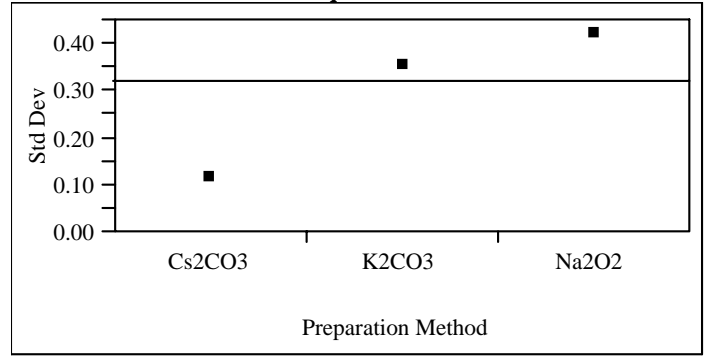

\begin{tabular}{|c|c|c|c|c|}
\hline Level & Count & Std Dev & $\begin{array}{r}\text { MeanAbsDif to } \\
\text { Mean }\end{array}$ & $\begin{array}{r}\text { MeanAbsDif to } \\
\text { Median }\end{array}$ \\
\hline Cs2CO3 & 3 & 0.1130792 & 0.0787087 & 0.1073300 \\
\hline K2CO3 & 3 & 0.3546765 & 0.2432813 & 0.3434560 \\
\hline $\mathrm{Na} 2 \mathrm{O} 2$ & 3 & 0.4185870 & 0.3219900 & 0.2575920 \\
\hline Test & F Ratio & DFNum & DFDen & Prob $>F$ \\
\hline O'Brien[.5] & 0.7919 & 2 & 6 & 0.4952 \\
\hline $\begin{array}{l}\text { Brown- } \\
\text { Forsythe }\end{array}$ & 0.8341 & 2 & 6 & 0.4790 \\
\hline Levene & 2.3036 & 2 & 6 & 0.18 \\
\hline Bartlett & 1.1465 & 2 & . & 0.31 \\
\hline
\end{tabular}

Warning: Small sample sizes. Use Caution.

Welch Anova testing Means Equal, allowing Std Devs Not Equal

F Ratio DFNum DFDen Prob $>$ F

$\begin{array}{llll}1.6927 & 2 & 3.072 & 0.3195\end{array}$

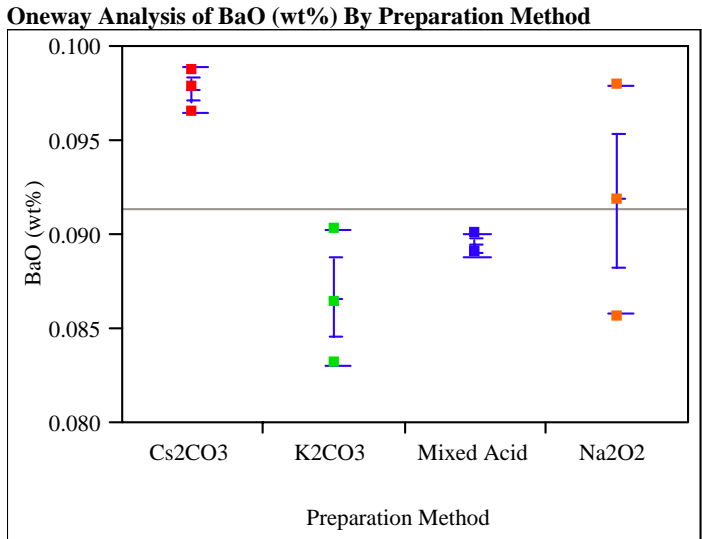

Means Comparisons

Comparisons for all pairs using Tukey-Kramer HSD $\mathrm{q}^{*}$ Alpha

$\begin{array}{rrr}3.20238 & 0.05 & \\ \text { Na2O } & \text { Mixed Acid } & \text { K2CO3 }\end{array}$

$\begin{array}{lllll}\text { Cs2CO3 } & -0.00944 & -0.00359 & -0.00121 & 0.00158\end{array}$

$\begin{array}{lllll}\mathrm{Na} 2 \mathrm{O} 2 & -0.00359 & -0.00944 & -0.00706 & -0.00426\end{array}$

$\begin{array}{lllll}\text { Mixed Acid } \quad-0.00121 & -0.00706 & -0.00944 & -0.00665\end{array}$

$\begin{array}{lllll}\mathrm{K} 2 \mathrm{CO} 3 & 0.00158 & -0.00426 & -0.00665 & -0.00944\end{array}$

Positive values show pairs of means that are significantly different.

Level

$\mathrm{Cs} 2 \mathrm{CO} 3$ A 0.09765653

$\mathrm{Na} 2 \mathrm{O} 2 \quad$ A $\quad$ B 0.09181352

Mixed Acid A B 0.08943165

K2CO3 B 0.08664040

Levels not connected by same letter are significantly different Tests that the Variances are Equal

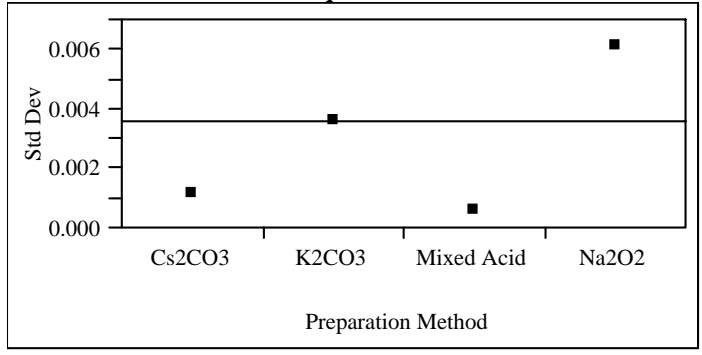

\begin{tabular}{|c|c|c|c|c|}
\hline Level & Count & Std Dev & $\begin{array}{r}\text { MeanAbsDif to } \\
\text { Mean }\end{array}$ & $\begin{array}{r}\text { MeanAbsDif to } \\
\text { Median }\end{array}$ \\
\hline Cs2CO3 & 3 & 0.0011239 & 0.0007940 & 0.001042 \\
\hline K2CO3 & 3 & 0.0035780 & 0.0024563 & 0.00346 \\
\hline Mixed Acid & 3 & 0.0005802 & 0.0004466 & 0.00033 \\
\hline $\mathrm{Na} 2 \mathrm{O} 2$ & 3 & 0.0061411 & 0.0041186 & 0.00610 \\
\hline Test & F Ratio & DFNum & DFDen & Prob \\
\hline O'Brien[.5] & 1.3568 & 3 & 8 & 0.32 \\
\hline $\begin{array}{l}\text { Brown- } \\
\text { Forsythe }\end{array}$ & 144.0487 & 3 & 8 & $<.0$ \\
\hline Levene & 2.0900 & 3 & 8 & 0.17 \\
\hline Bartlett & 2.7279 & 3 & . & 0.0 \\
\hline
\end{tabular}

Warning: Small sample sizes. Use Caution.

Welch Anova testing Means Equal, allowing Std Devs Not Equal

F Ratio DFNum DFDen Prob $>$ F

$\begin{array}{llll}32.4688 & 3 & 3.7986 & 0.0036\end{array}$ 


\section{Exhibit A3. Statistical Comparisons of Chemical Composition Measurements of ARG-1 Samples by Dissolution Method for Each Oxide (Concentrations in Wt\% Oxides)}

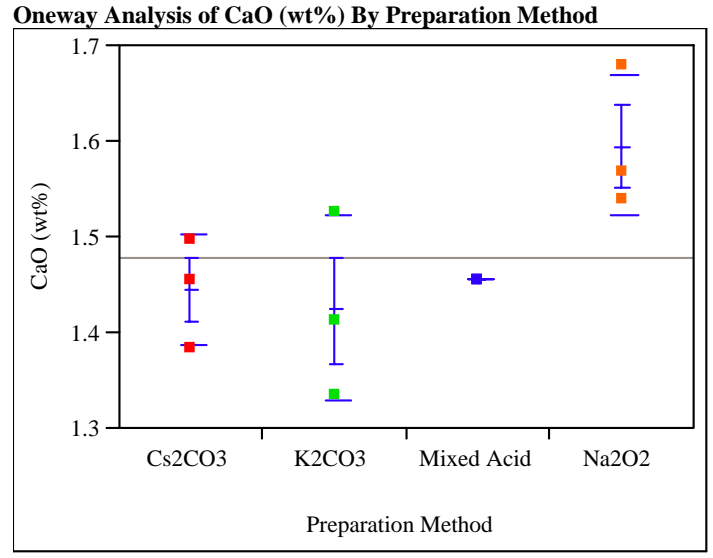

Means Comparisons

Comparisons for all pairs using Tukey-Kramer HSD

$$
\begin{array}{rr}
\text { q* }^{*} & \text { Alpha } \\
3.20238 & 0.05
\end{array}
$$

Abs(Dif)-LSD Na2O2 Mixed Acid Cs2CO3 K2CO3

Na2O2 $\quad-0.17601 \quad-0.03609-0.02583-0.00484$

$\begin{array}{lllll}\text { Mixed Acid } \quad-0.03609 & -0.17601 & -0.16575 & -0.14476\end{array}$

$\begin{array}{lllll}\mathrm{Cs} 2 \mathrm{CO} 3 & -0.02583 & -0.16575 & -0.17601 & -0.15502\end{array}$

$\begin{array}{lllll}\mathrm{K} 2 \mathrm{CO} 3 & -0.00484 & -0.14476 & -0.15502 & -0.17601\end{array}$

Positive values show pairs of means that are significantly different.

Level

Mixed Acid A 1.4551680

Cs2CO3 A 1.4449072

K2CO3 A 1.4239192

Levels not connected by same letter are significantly different Tests that the Variances are Equal

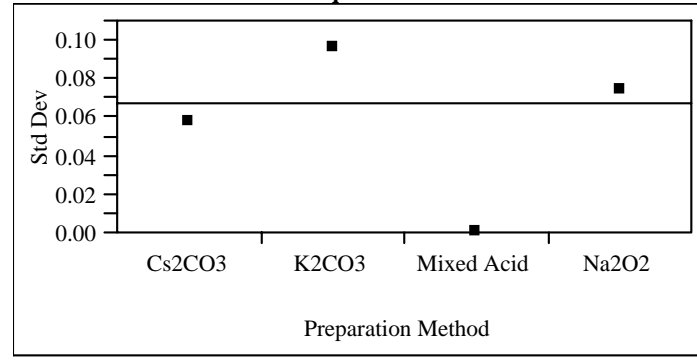

$\begin{array}{lrrrr}\text { Level } & \text { Count } & \text { Std Dev } & \begin{array}{r}\text { MeanAbsDif to } \\ \text { Mean }\end{array} & \begin{array}{r}\text { MeanAbsDif to } \\ \text { Median }\end{array} \\ \text { Cs2CO3 } & 3 & 0.0580513 & 0.0416651 & 0.0522368 \\ \text { K2CO3 } & 3 & 0.0962944 & 0.0674725 & 0.0904816 \\ \text { Mixed Acid } & 3 & 2.719 \mathrm{e}-16 & 2.22 \mathrm{e}-16 & 0.0000000 \\ \text { Na2O2 } & 3 & 0.0740387 & 0.0559680 & 0.0559680 \\ \text { Test } & \text { F Ratio } & \text { DFNum } & \text { DFDen } & \text { Prob }>\text { F } \\ \text { O'Brien[.5] } & 0.8423 & 3 & 8 & 0.5081 \\ \text { Brown- } & 5.5527 & 3 & 8 & 0.0234 \\ \text { Forsythe } & & & & \\ \text { Levene } & 2.6106 & 3 & 8 & 0.1236 \\ \text { Bartlett } & 36.2342 & 3 & . & <.0001 \\ \text { Wart } & & 3 & & \end{array}$

Warning: Small sample sizes. Use Caution.

Welch Anova testing Means Equal, allowing Std Devs Not Equal

F Ratio DFNum DFDen Prob $>$ F

$\begin{array}{lrrr}2.6486 & 3 & 3.3333 & 0.2080\end{array}$

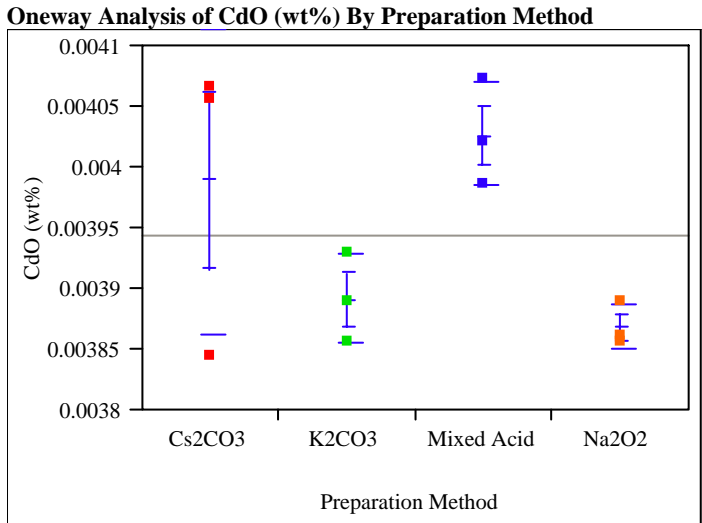

Means Comparisons

Comparisons for all pairs using Tukey-Kramer HSD

$$
\begin{array}{rr}
q^{*} & \text { Alpha } \\
3.20238 & 0.05
\end{array}
$$

Abs(Dif)-LSD Mixed Acid Cs2CO3 $\quad$ K2CO3 Na2O2

Mixed Acid $\quad-0.00018-0.00014-0.00005-0.00002$

$\begin{array}{lllll}\mathrm{Cs} 2 \mathrm{CO} 3 & -0.00014 & -0.00018 & -0.00008 & -0.00006\end{array}$

$\begin{array}{lllll}\mathrm{K} 2 \mathrm{CO} 3 & -0.00005 & -0.00008 & -0.00018 & -0.00016\end{array}$

$\begin{array}{lllll}\mathrm{Na} 2 \mathrm{O} 2 & -0.00002 & -0.00006 & -0.00016 & -0.00018\end{array}$

Positive values show pairs of means that are significantly different.

$$
\begin{array}{lrr}
\text { Level } & & \text { Mean } \\
\text { Mixed Acid } & \text { A } & 0.00402661 \\
\text { Cs2CO3 } & \text { A } & 0.00398853 \\
\text { K2CO3 } & \text { A } & 0.00389144 \\
\text { Na2O2 } & \text { A } & 0.00386859
\end{array}
$$

Levels not connected by same letter are significantly different Tests that the Variances are Equal

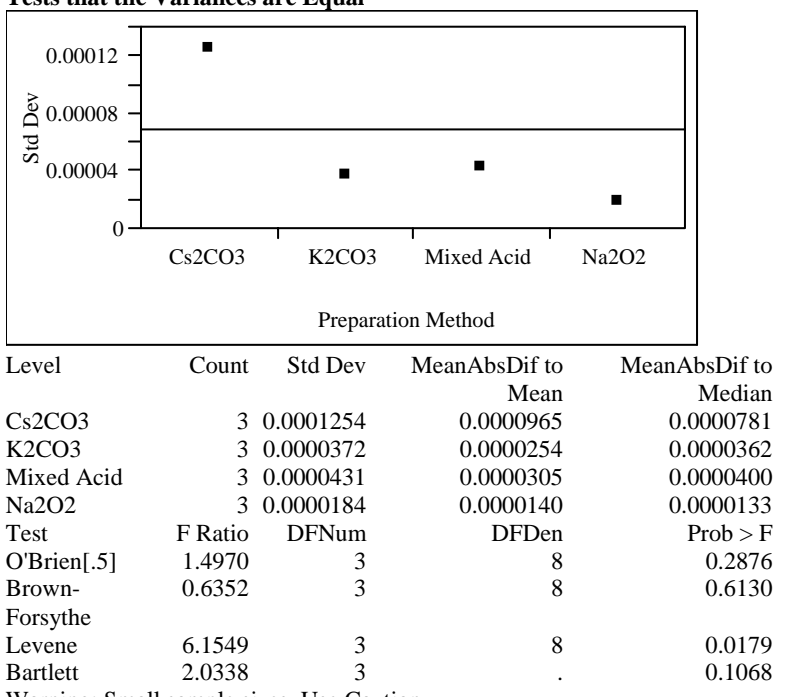

Warning: Small sample sizes. Use Caution.

Welch Anova testing Means Equal, allowing Std Devs Not Equal

F Ratio DFNum DFDen Prob $>$ F

$\begin{array}{llll}8.9393 & 3 & 3.9649 & 0.0307\end{array}$ 


\section{Exhibit A3. Statistical Comparisons of Chemical Composition Measurements of ARG-1 Samples by Dissolution Method for Each Oxide (Concentrations in Wt\% Oxides)}

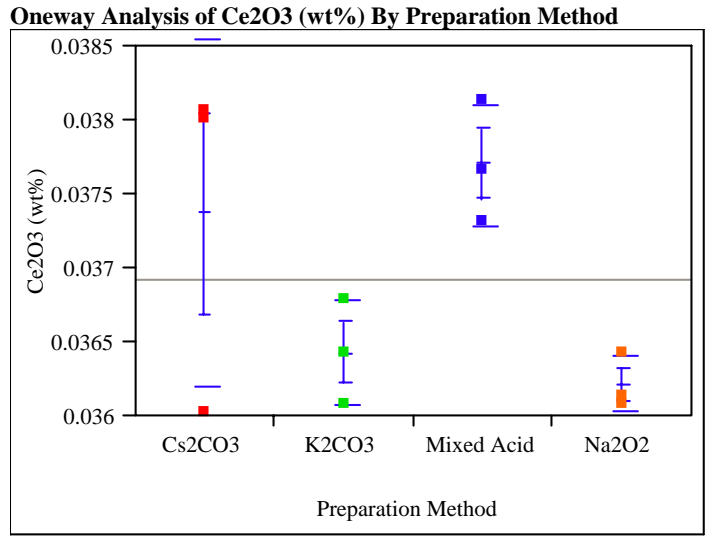

Means Comparisons

Comparisons for all pairs using Tukey-Kramer HSD

$$
\begin{array}{rr}
\mathrm{q}^{*} & \text { Alpha } \\
3.20238 & 0.05
\end{array}
$$

Abs(Dif)-LSD Mixed Acid Cs2CO3 K2CO3 Na2O2

$\begin{array}{lllll}\text { Mixed Acid } & -0.00170 & -0.00137 & -0.00043 & -0.00022\end{array}$

$\begin{array}{lllll}\mathrm{Cs} 2 \mathrm{CO} 3 & -0.00137 & -0.00170 & -0.00076 & -0.00055\end{array}$

K2CO3 $\quad-0.00043-0.00076-0.00170-0.00148$

$\begin{array}{lllll}\mathrm{Na} 2 \mathrm{O} 2 & -0.00022 & -0.00055 & -0.00148 & -0.00170\end{array}$

Positive values show pairs of means that are significantly different.

Level Mean

Mixed Acid A 0.03769634

Cs2CO3 A 0.03736447

K2CO3 A 0.03642743

$\mathrm{Na} 2 \mathrm{O} 2$ A 0.03621269

Levels not connected by same letter are significantly different Tests that the Variances are Equal

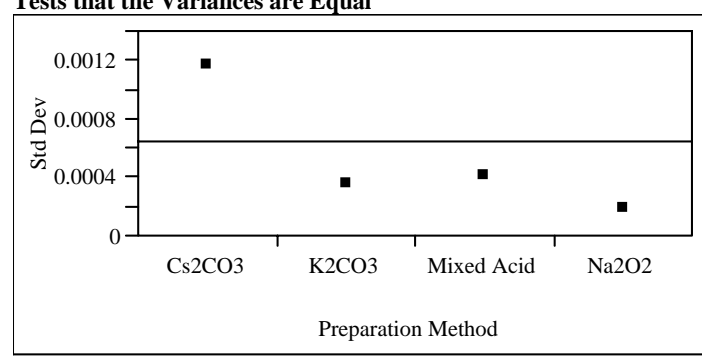

\begin{tabular}{|c|c|c|c|c|}
\hline Level & Count & Std Dev & $\begin{array}{r}\text { MeanAbsDif to } \\
\text { Mean }\end{array}$ & $\begin{array}{r}\text { MeanAbsDif to } \\
\text { Median }\end{array}$ \\
\hline Cs2CO3 & 3 & 0.0011669 & 0.0008980 & 0.0007028 \\
\hline K2CO3 & 3 & 0.0003514 & 0.0002343 & 0.0003514 \\
\hline Mixed Acid & 3 & 0.0004113 & 0.0002863 & 0.0003904 \\
\hline $\mathrm{Na} 2 \mathrm{O} 2$ & 3 & 0.0001883 & 0.0001432 & 0.0001367 \\
\hline Test & F Ratio & DFNum & DFDen & Prob $>$ F \\
\hline O'Brien[.5] & 1.4795 & 3 & 8 & 0.2918 \\
\hline $\begin{array}{l}\text { Brown- } \\
\text { Forsythe }\end{array}$ & 0.5155 & 3 & 8 & 0.6831 \\
\hline Levene & 5.7995 & 3 & 8 & $0.02 C$ \\
\hline Bartlett & 1.9050 & 3 & . & $0.12 €$ \\
\hline
\end{tabular}

Warning: Small sample sizes. Use Caution.

Welch Anova testing Means Equal, allowing Std Devs Not Equal

F Ratio DFNum DFDen Prob $>$ F

$\begin{array}{llll}8.5290 & 3 & 4.0223 & 0.0323\end{array}$

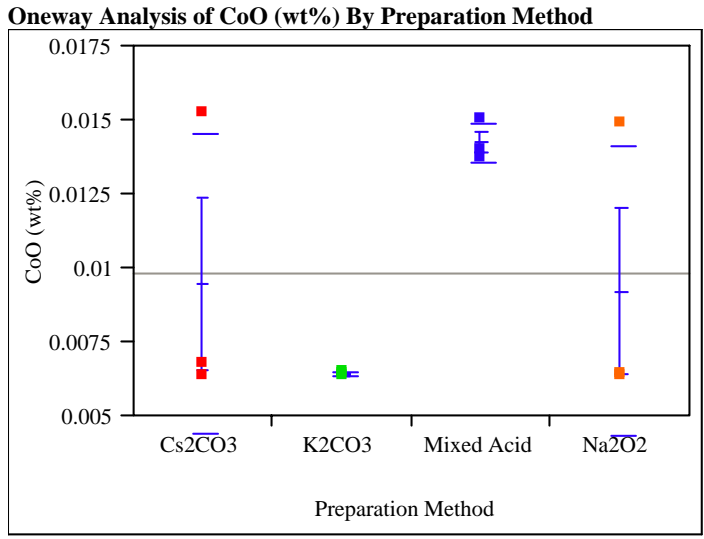

Means Comparisons

Comparisons for all pairs using Tukey-Kramer HSD

$$
\begin{array}{rr}
\mathrm{q}^{*} & \text { Alpha } \\
3.20238 & 0.05
\end{array}
$$

Abs(Dif)-LSD Mixed Acid Cs2CO3 Na2O2 K2CO3

$\begin{array}{lllll}\text { Mixed Acid } & -0.00923 & -0.00444 & -0.00420 & -0.00141\end{array}$

$\begin{array}{lllll}\mathrm{Cs} 2 \mathrm{CO} 3 & -0.00444 & -0.00923 & -0.00899 & -0.00619\end{array}$

Na2O2 $\quad-0.00420 \quad-0.00899 \quad-0.00923 \quad-0.00643$

$\begin{array}{lllll}\mathrm{K} 2 \mathrm{CO} 3 & -0.00141 & -0.00619 & -0.00643 & -0.00923\end{array}$

Positive values show pairs of means that are significantly different.

Level

Mixed Acid A 0.01424080

Cs2CO3 A 0.00945148

$\mathrm{Na} 2 \mathrm{O} 2 \quad$ A 0.00921838

K2CO3 A 0.00642108

Levels not connected by same letter are significantly different Tests that the Variances are Equal

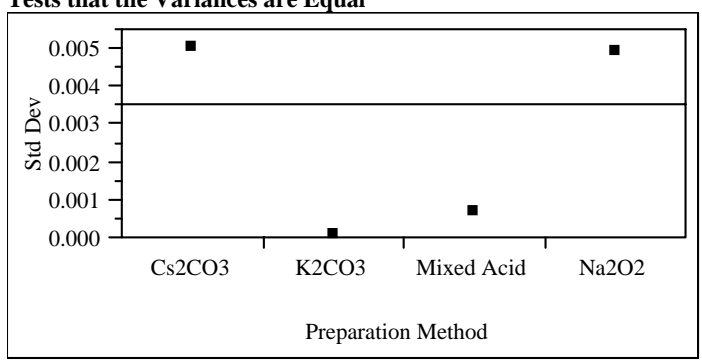

$\begin{array}{lrrrr}\text { Level } & \text { Count } & \text { Std Dev } & \begin{array}{r}\text { MeanAbsDif to } \\ \text { Mean }\end{array} & \begin{array}{r}\text { MeanAbsDif to } \\ \text { Median }\end{array} \\ \text { Cs2CO3 } & 3 & 0.0050322 & 0.0038710 & 0.0030940 \\ \text { K2CO3 } & 3 & 0.0000636 & 0.0000424 & 0.0000636 \\ \text { Mixed Acid } & 3 & 0.0006728 & 0.0005086 & 0.0005086 \\ \text { Na2O2 } & 3 & 0.0049002 & 0.0037721 & 0.0028609 \\ \text { Test } & \text { F Ratio } & \text { DFNum } & \text { DFDen } & \text { Prob > F } \\ \text { O'Brien[.5] } & 1.1642 & 3 & 8 & 0.3817 \\ \text { Brown- } & 0.6444 & 3 & 8 & 0.6079 \\ \text { Forsythe } & & & & \\ \text { Levene } & 9.0939 & 3 & 8 & 0.0059 \\ \text { Bartlett } & 5.5060 & 3 & . & 0.0009\end{array}$

Warning: Small sample sizes. Use Caution.

Welch Anova testing Means Equal, allowing Std Devs Not Equal

F Ratio DFNum DFDen Prob $>$ F

$\begin{array}{rrrr}96.2674 & 3 & 3.3537 & 0.0010\end{array}$ 


\section{Exhibit A3. Statistical Comparisons of Chemical Composition Measurements of ARG-1 Samples by Dissolution Method for Each Oxide (Concentrations in Wt\% Oxides)}

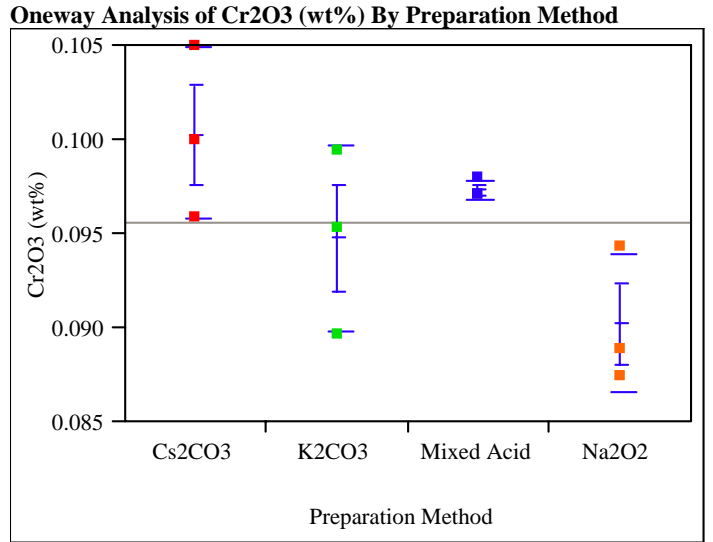

Means Comparisons

Comparisons for all pairs using Tukey-Kramer HSD

$$
\text { q* Alpha }
$$

Abs(Dif)-LSD Cs2CO3 Mixed Acid K2CO3 Na2O2

$\begin{array}{lllll}\mathrm{Cs} 2 \mathrm{CO} 3 & -0.00997 & -0.00704 & -0.00446 & 0.00012\end{array}$

$\begin{array}{lllll}\text { Mixed Acid } & -0.00704 & -0.00997 & -0.00739 & -0.00281\end{array}$

$\begin{array}{lllll}\mathrm{K} 2 \mathrm{CO} 3 & -0.00446 & -0.00739 & -0.00997 & -0.00539\end{array}$

$\begin{array}{lllll}\mathrm{Na} 2 \mathrm{O} 2 & 0.00012 & -0.00281 & -0.00539 & -0.00997\end{array}$

Positive values show pairs of means that are significantly different.

$$
\begin{array}{lrrr}
\text { Level } & & \text { Mean } \\
\text { Cs2CO3 } & \text { A } & & 0.10026576 \\
\text { Mixed Acid } & \text { A } & \text { B } & 0.09734256 \\
\text { K2CO3 } & \text { A } & \text { B } & 0.09476040 \\
\text { Na2O2 } & & \text { B } & 0.09018072
\end{array}
$$

\begin{tabular}{|c|c|c|c|c|}
\hline Level & Count & Std Dev & $\begin{array}{r}\text { MeanAbsDif to } \\
\text { Mean }\end{array}$ & $\begin{array}{r}\text { MeanAbsDif to } \\
\text { Median }\end{array}$ \\
\hline Cs2CO3 & 3 & 0.0045380 & 0.0031181 & 0.0043848 \\
\hline K2CO3 & 3 & 0.0049183 & 0.0034429 & 0.0046284 \\
\hline Mixed Acid & 3 & 0.0005063 & 0.0003898 & 0.0002923 \\
\hline $\mathrm{Na} 2 \mathrm{O} 2$ & 3 & 0.0036188 & 0.0027283 & 0.0027770 \\
\hline Test & F Ratio & DFNum & DFDen & Prob $>F$ \\
\hline O'Brien[.5] & 0.6742 & 3 & 8 & 0.5916 \\
\hline $\begin{array}{l}\text { Brown- } \\
\text { Forsythe }\end{array}$ & 7.2798 & 3 & 8 & 0.0113 \\
\hline Levene & 1.5998 & 3 & 8 & 0.26 \\
\hline Bartlett & 1.8118 & 3 & . & 0.14 \\
\hline
\end{tabular}

Levels not connected by same letter are significantly different Tests that the Variances are Equal

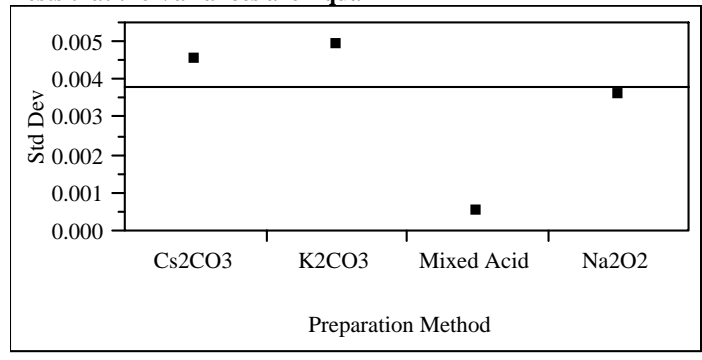

Warning: Small sample sizes. Use Caution.

Welch Anova testing Means Equal, allowing Std Devs Not Equal

F Ratio DFNum DFDen Prob $>$ F

$\begin{array}{llll}3.2698 & 3 & 3.4241 & 0.1603\end{array}$

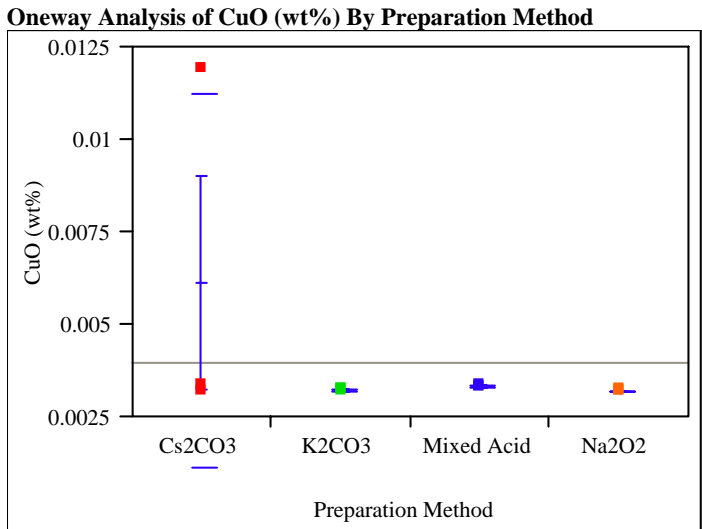

Means Comparisons

Comparisons for all pairs using Tukey-Kramer HSD

$$
\begin{array}{rr}
\text { q }^{*} & \text { Alpha } \\
3.20238 & 0.05
\end{array}
$$

Abs(Dif)-LSD Cs2CO3 Mixed Acid K2CO3 Na2O2

$\begin{array}{lllll}\text { Cs2CO3 } & -0.00654 & -0.00371 & -0.00360 & -0.00358\end{array}$

$\begin{array}{lllll}\text { Mixed Acid } & -0.00371 & -0.00654 & -0.00643 & -0.00641\end{array}$

$\begin{array}{lllll}\mathrm{K} 2 \mathrm{CO} 3 & -0.00360 & -0.00643 & -0.00654 & -0.00652\end{array}$

$\begin{array}{lllll}\mathrm{Na} 2 \mathrm{O} 2 & -0.00358 & -0.00641 & -0.00652 & -0.00654\end{array}$

Positive values show pairs of means that are significantly different.

Level

\begin{tabular}{|c|c|c|c|c|}
\hline Level & Count & Std Dev & $\begin{array}{r}\text { MeanAbsDif to } \\
\text { Mean }\end{array}$ & $\begin{array}{r}\text { MeanAbsDif to } \\
\text { Median }\end{array}$ \\
\hline Cs2CO3 & 3 & 0.0050039 & 0.0038514 & 0.0029793 \\
\hline K2CO3 & 3 & 0.0000313 & 0.0000209 & 0.0000313 \\
\hline Mixed Acid & 3 & 0.0000348 & 0.0000250 & 0.0000313 \\
\hline $\mathrm{Na} 2 \mathrm{O} 2$ & 3 & 0.0000166 & 0.0000125 & 0.0000125 \\
\hline Test & F Ratio & DFNum & DFDen & Prob $>F$ \\
\hline O'Brien[.5] & 1.7777 & 3 & 8 & 0.2291 \\
\hline $\begin{array}{l}\text { Brown- } \\
\text { Forsythe }\end{array}$ & 1.1150 & 3 & 8 & 0.3985 \\
\hline Levene & 15.7883 & 3 & 8 & 0.0010 \\
\hline Bartlett & 14.3228 & 3 & . & $<.0001$ \\
\hline
\end{tabular}

0.00616511

$\mathrm{K} 2 \mathrm{CO} 3$

$\mathrm{Na} 2 \mathrm{O} 2 \quad$ A 0.00320461

Levels not connected by same letter are significantly different Tests that the Variances are Equal

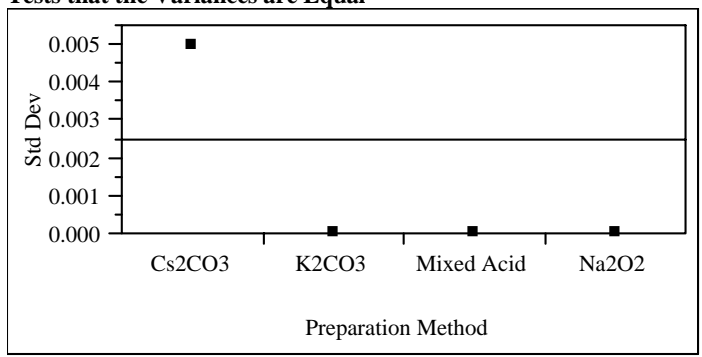

Warning: Small sample sizes. Use Caution.

Welch Anova testing Means Equal, allowing Std Devs Not Equal

F Ratio DFNum DFDen Prob $>$ F

$\begin{array}{rrrr}8.9825 & 3 & 4.0031 & 0.0299\end{array}$ 
Westinghouse Savannah River Company

WSRC-TR-2005-00396

Savannah River National Laboratory

Rev. 0

Analytical Development/Statistical Consulting Sections

Page 58 of 140

\section{Exhibit A3. Statistical Comparisons of Chemical Composition Measurements of ARG-1 Samples by Dissolution Method for Each Oxide (Concentrations in Wt\% Oxides)}

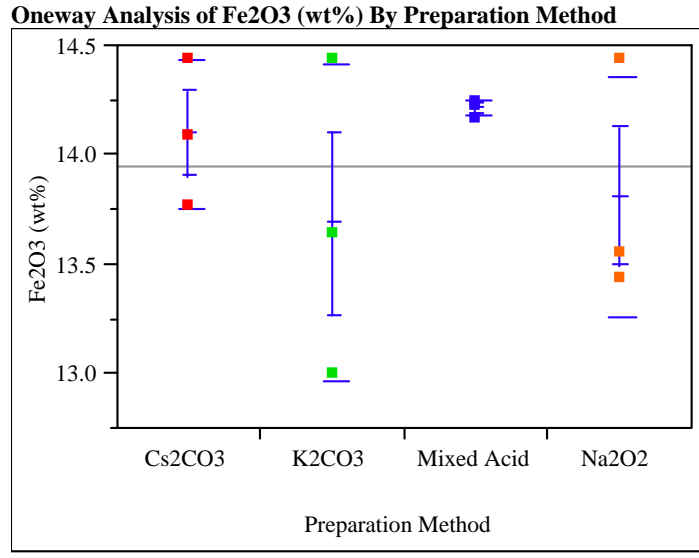

Means Comparisons

Comparisons for all pairs using Tukey-Kramer HSD

$$
\begin{array}{rr}
\mathrm{q}^{*} & \text { Alpha } \\
3.20238 & 0.05
\end{array}
$$

Abs(Dif)-LSD Mixed Acid Cs2CO3 Na2O2 K2CO3

Mixed Acid $\quad-1.2661 \quad-1.1517 \quad-0.8658 \quad-0.7466$

$\begin{array}{lllll}\text { Cs2CO3 } & -1.1517 & -1.2661 & -0.9802 & -0.8610\end{array}$

$\mathrm{Na2O} 2 \quad-0.8658 \quad-0.9802 \quad-1.2661 \quad-1.1470$

K2CO3 $\quad-0.7466 \quad-0.8610-1.1470 \quad-1.2661$

Positive values show pairs of means that are significantly different.

Level

Mean

Mixed Acid A 14.211218

Cs2CO3 A 14.096842

$\mathrm{Na} 2 \mathrm{O} 2 \quad$ A 13.810902

K2CO3 A 13.691760

Levels not connected by same letter are significantly different Tests that the Variances are Equal

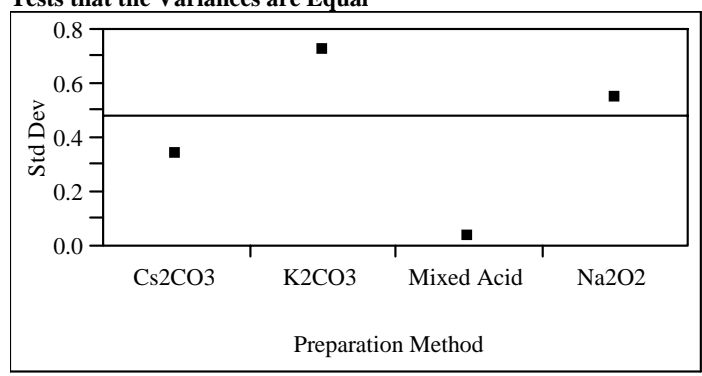

$\begin{array}{lrrrr}\text { Level } & \text { Count } & \text { Std Dev } & \begin{array}{r}\text { MeanAbsDif to } \\ \text { Mean }\end{array} & \begin{array}{r}\text { MeanAbsDif to } \\ \text { Median }\end{array} \\ \text { Cs2CO3 } & 3 & 0.3362076 & 0.2287520 & 0.3288310 \\ \text { K2CO3 } & 3 & 0.7234244 & 0.4988064 & 0.6957873 \\ \text { Mixed Acid } & 3 & 0.0378263 & 0.0285940 & 0.0285940 \\ \text { Na2O2 } & 3 & 0.5477822 & 0.4193787 & 0.3717220 \\ \text { Test } & \text { F Ratio } & \text { DFNum } & \text { DFDen } & \text { Prob }>\text { F } \\ \text { O'Brien[.5] } & 0.9867 & 3 & 8 & 0.4464 \\ \text { Brown- } & 4.2975 & 3 & 8 & 0.0440 \\ \text { Forsythe } & & & & \\ \text { Levene } & 2.3943 & 3 & 8 & 0.1439 \\ \text { Bartlett } & 2.6367 & 3 & . & 0.0479 \\ \text { Warne } & & 3 & & \end{array}$

Warning: Small sample sizes. Use Caution.

Welch Anova testing Means Equal, allowing Std Devs Not Equal

F Ratio DFNum DFDen Prob $>$ F

$\begin{array}{llll}0.8243 & 3 & 3.3772 & 0.5542\end{array}$

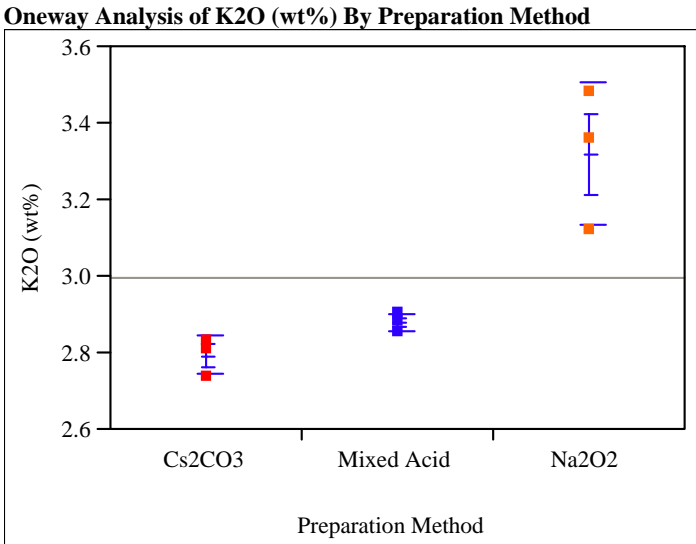

Missing Rows

3Means Comparisons

Comparisons for all pairs using Tukey-Kramer HSD

$\begin{array}{rr}\mathrm{q}^{*} & \text { Alpha } \\ 3.06815 & 0.05\end{array}$

Abs(Dif)-LSD Na2O2 Mixed Acid Cs2CO3

$\begin{array}{llll}\mathrm{Na} 2 \mathrm{O} 2 & -0.27804 & 0.16365 & 0.25199\end{array}$

Mixed Acid $\quad 0.16365 \quad-0.27804-0.18970$

$\begin{array}{llll}\text { Cs2CO3 } & 0.25199 & -0.18970 & -0.27804\end{array}$

Positive values show pairs of means that are significantly different

Level

Mixed Acid B 2.8789940

Cs2CO3 B 2.7906567

Levels not connected by same letter are significantly different Tests that the Variances are Equal

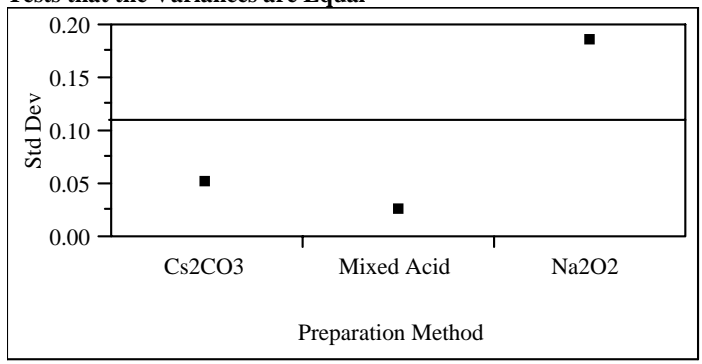

$\begin{array}{lrrrr}\text { Level } & \text { Count } & \text { Std Dev } & \begin{array}{r}\text { MeanAbsDif to } \\ \text { Mean }\end{array} & \begin{array}{r}\text { MeanAbsDif to } \\ \text { Median }\end{array} \\ \text { Cs2CO3 } & 3 & 0.0501515 & 0.0374764 & 0.0401533 \\ \text { Mixed Acid } & 3 & 0.0240920 & 0.0160613 & 0.0240920 \\ \text { Na2O2 } & 3 & 0.1840057 & 0.1338444 & 0.1606133 \\ \text { Test } & \text { F Ratio } & \text { DFNum } & \text { DFDen } & \text { Prob > F } \\ \text { O'Brien[.5] } & 1.6139 & 2 & 6 & 0.2749 \\ \text { Brown- } & 8.9310 & 2 & 6 & 0.0159 \\ \text { Forsythe } & & & & \\ \text { Levene } & 4.6686 & 2 & 6 & 0.0599 \\ \text { Bartlett } & 2.9722 & 2 & . & 0.0512\end{array}$

Warning: Small sample sizes. Use Caution.

Welch Anova testing Means Equal, allowing Std Devs Not Equal

F Ratio DFNum DFDen Prob $>$ F

$\begin{array}{llll}10.7118 & 2 & 3.1812 & 0.0386\end{array}$ 
Westinghouse Savannah River Company

WSRC-TR-2005-00396

Savannah River National Laboratory

Rev. 0

Analytical Development/Statistical Consulting Sections

Page 59 of 140

\section{Exhibit A3. Statistical Comparisons of Chemical Composition Measurements of ARG-1 Samples by Dissolution Method for Each Oxide (Concentrations in Wt\% Oxides)}

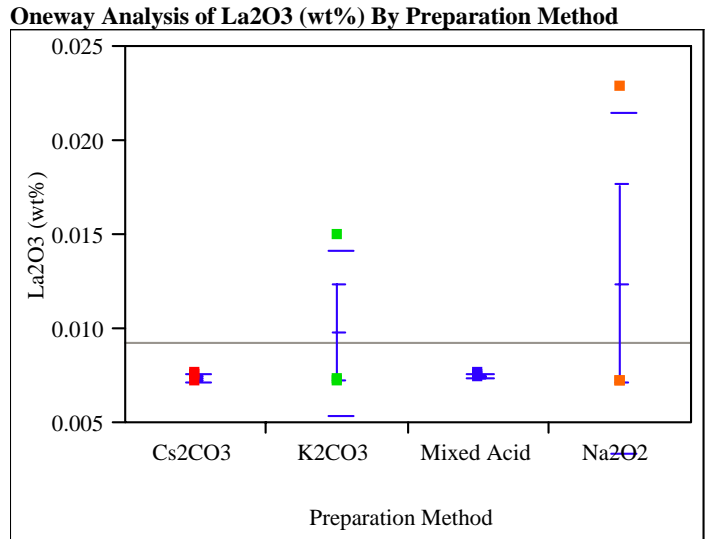

Means Comparisons

Comparisons for all pairs using Tukey-Kramer HSD

q* Alpha

Abs(Dif)-LSD Na2O2 K2CO3 Mixed Acid Cs2CO3

$\begin{array}{lllll}\mathrm{Na} 2 \mathrm{O} 2 & -0.01318 & -0.01056 & -0.00823 & -0.00817\end{array}$

$\begin{array}{lllll}\mathrm{K} 2 \mathrm{CO} 3 & -0.01056 & -0.01318 & -0.01085 & -0.01079\end{array}$

$\begin{array}{lllll}\text { Mixed Acid } \quad-0.00823 & -0.01085 & -0.01318 & -0.01312\end{array}$

$\begin{array}{lllll}\text { Cs2CO3 } & -0.00817 & -0.01079 & -0.01312 & -0.01318\end{array}$

Positive values show pairs of means that are significantly different.

$\begin{array}{lrr}\text { Level } & & \text { Mean } \\ \text { Na2O2 } & \text { A } & 0.01241213 \\ \text { K2CO3 } & \text { A } & 0.00979288 \\ \text { Mixed Acid } & \text { A } & 0.00746683 \\ \text { Cs2CO3 } & \text { A } & 0.00740819\end{array}$

Levels not connected by same letter are significantly different Tests that the Variances are Equal

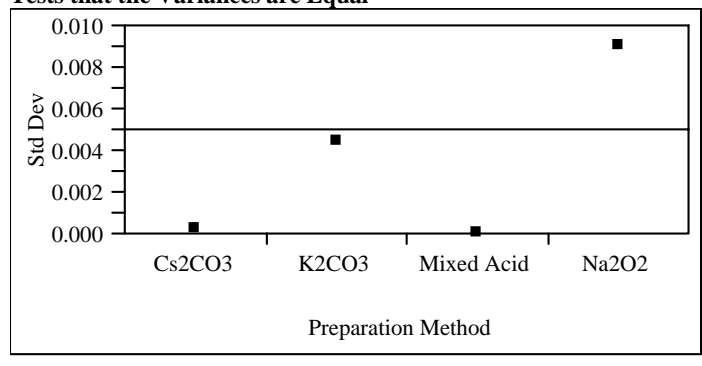

$\begin{array}{lrrrr}\text { Level } & \text { Count } & \text { Std Dev } & \begin{array}{r}\text { MeanAbsDif to } \\ \text { Mean }\end{array} & \begin{array}{r}\text { MeanAbsif to } \\ \text { Median }\end{array} \\ \text { Cs2CO3 } & 3 & 0.0002220 & 0.0001694 & 0.0001564 \\ \text { K2CO3 } & 3 & 0.0044183 & 0.0034011 & 0.0025802 \\ \text { Mixed Acid } & 3 & 0.0000896 & 0.0000652 & 0.0000782 \\ \text { Na2O2 } & 3 & 0.0090565 & 0.0069716 & 0.0052581 \\ \text { Test } & \text { F Ratio } & \text { DFNum } & \text { DFDen } & \text { Prob }>\text { F } \\ \text { O'Brien[.5] } & 1.5098 & 3 & 8 & 0.2846 \\ \text { Brown- } & 0.7202 & 3 & 8 & 0.5674 \\ \text { Forsythe } & & & & \\ \text { Levene } & 11.3625 & 3 & 8 & 0.0030 \\ \text { Bartlett } & 7.3909 & 3 & . & <.0001 \\ \text { Warning }\end{array}$

Warning: Small sample sizes. Use Caution.

Welch Anova testing Means Equal, allowing Std Devs Not Equal

F Ratio DFNum DFDen Prob $>$ F

$\begin{array}{llll}0.4665 & 3 & 3.6251 & 0.7230\end{array}$

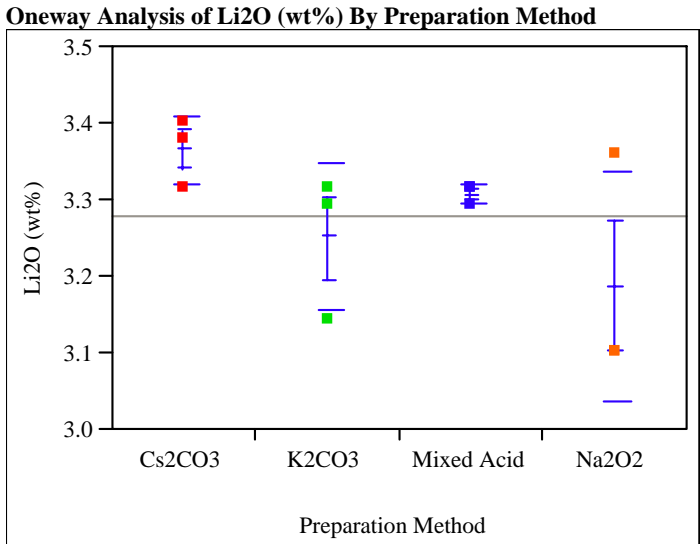

Means Comparisons

Comparisons for all pairs using Tukey-Kramer HSD

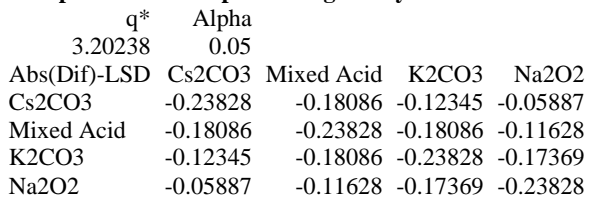

Positive values show pairs of means that are significantly different

Level

Mean

Cs2CO3 A 3.3657003

Mixed Acid A 3.3082897

K2CO3 A 3.2508790

$\mathrm{Na} 2 \mathrm{O} 2$ A 3.1862920

Levels not connected by same letter are significantly different Tests that the Variances are Equal

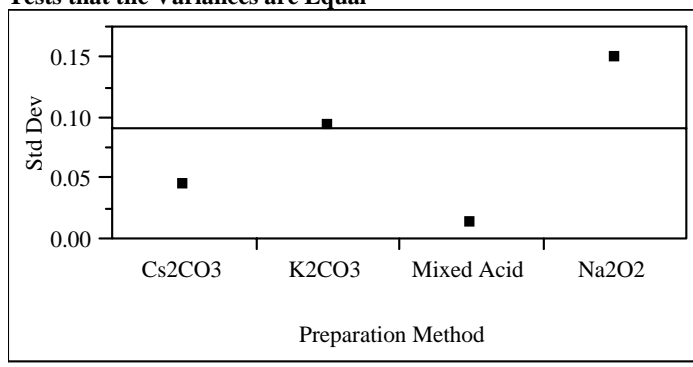

$\begin{array}{lrrrr}\text { Level } & \text { Count } & \text { Std Dev } & \begin{array}{r}\text { MeanAbsDif to } \\ \text { Mean }\end{array} & \begin{array}{r}\text { MeanAbsDif to } \\ \text { Median }\end{array} \\ \text { Cs2CO3 } & 3 & 0.0448162 & 0.0334896 & 0.0358817 \\ \text { K2CO3 } & 3 & 0.0938427 & 0.0717633 & 0.0645870 \\ \text { Mixed Acid } & 3 & 0.0124298 & 0.0095684 & 0.0071763 \\ \text { Na2O2 } & 3 & 0.1491573 & 0.1148213 & 0.0861160 \\ \text { Test } & \text { F Ratio } & \text { DFNum } & \text { DFDen } & \text { Prob > F } \\ \text { O'Brien[.5] } & 1.2363 & 3 & 8 & 0.3586 \\ \text { Brown- } & 0.4955 & 3 & 8 & 0.6954 \\ \text { Forsythe } & & & & \\ \text { Levene } & 6.5316 & 3 & 8 & 0.0152 \\ \text { Bartlett } & 2.4053 & 3 & . & 0.0653 \\ \text { Want } & & 3 & & \end{array}$

Warning: Small sample sizes. Use Caution.

Welch Anova testing Means Equal, allowing Std Devs Not Equal

F Ratio DFNum DFDen Prob $>$ F

$\begin{array}{llll}1.9210 & 3 & 3.5342 & 0.2821\end{array}$ 


\section{Exhibit A3. Statistical Comparisons of Chemical Composition Measurements of ARG-1 Samples by Dissolution Method for Each Oxide (Concentrations in Wt\% Oxides)}

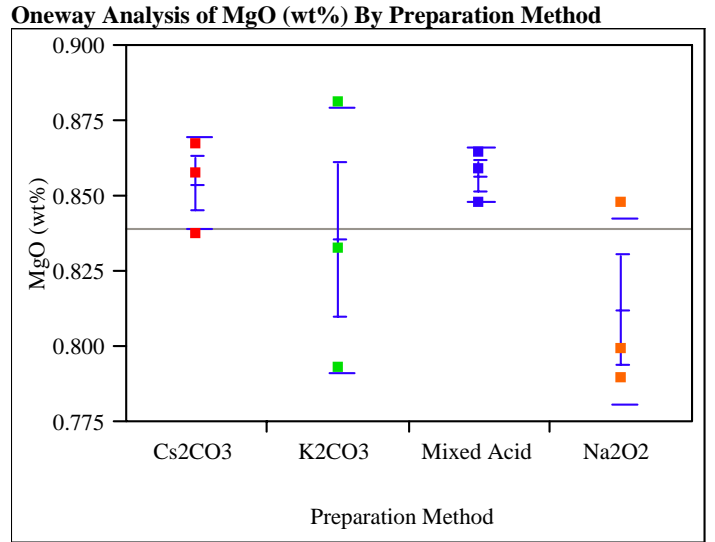

Means Comparisons

Comparisons for all pairs using Tukey-Kramer HSD

$$
\begin{array}{rr}
q^{*} & \text { Alpha } \\
3.20238 & 0.05
\end{array}
$$

Abs(Dif)-LSD Mixed Acid Cs2CO3 K2CO3 Na2O2

Mixed Acid $\quad-0.07400-0.07123-0.05244-0.02922$

Cs2CO3 $\quad-0.07123-0.07400-0.05520-0.03199$

$\begin{array}{lllll}\mathrm{K} 2 \mathrm{CO} 3 & -0.05244 & -0.05520 & -0.07400 & -0.05078\end{array}$

$\mathrm{Na2O} 2 \quad-0.02922-0.03199-0.05078-0.07400$

Positive values show pairs of means that are significantly different.

Level Mean

Mixed Acid A 0.85678833

Cs2CO3 A 0.85402450

K2CO3 A 0.83523043

$\mathrm{Na} 2 \mathrm{O} 2$ A 0.81201423

Levels not connected by same letter are significantly different Tests that the Variances are Equal

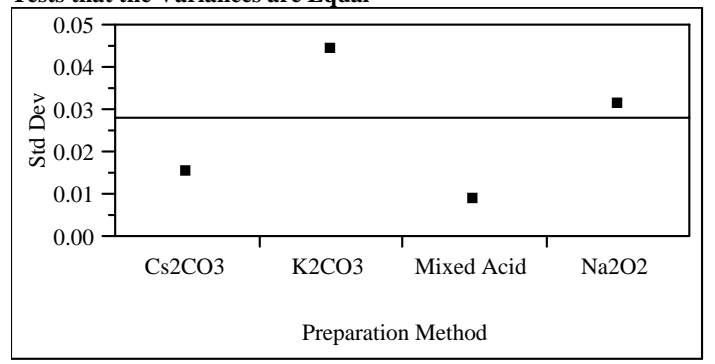

$\begin{array}{lrrrr}\text { Level } & \text { Count } & \text { Std Dev } & \begin{array}{r}\text { MeanAbsDif to } \\ \text { Mean }\end{array} & \begin{array}{r}\text { MeanAbsDif to } \\ \text { Median }\end{array} \\ \text { Cs2CO3 } & 3 & 0.0151986 & 0.0110553 & 0.0132664 \\ \text { K2CO3 } & 3 & 0.0440101 & 0.0302179 & 0.0425630 \\ \text { Mixed Acid } & 3 & 0.0085097 & 0.0062647 & 0.0071860 \\ \text { Na2O2 } & 3 & 0.0310387 & 0.0235847 & 0.0226634 \\ \text { Test } & \text { F Ratio } & \text { DFNum } & \text { DFDen } & \text { Prob }>\text { F } \\ \text { O'Brien[.5] } & 1.0867 & 3 & 8 & 0.4085 \\ \text { Brown- } & 5.1678 & 3 & 8 & 0.0282 \\ \text { Forsythe } & & & & \\ \text { Levene } & 1.9339 & 3 & 8 & 0.2027 \\ \text { Bartlett } & 1.4229 & 3 & . & 0.2339 \\ \text { Want } & 3 & & \end{array}$

Warning: Small sample sizes. Use Caution.

Welch Anova testing Means Equal, allowing Std Devs Not Equal

F Ratio DFNum DFDen Prob > F

$\begin{array}{llll}1.5742 & 3 & 3.9315 & 0.3293\end{array}$

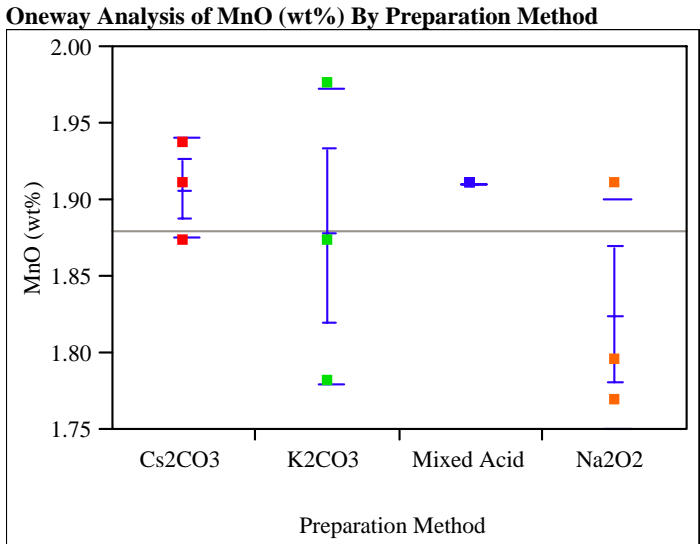

Means Comparisons

Comparisons for all pairs using Tukey-Kramer HSD

$$
\begin{array}{rr}
q^{*} & \text { Alpha } \\
3.20238 & 0.05
\end{array}
$$

Abs(Dif)-LSD Mixed Acid $\quad \mathrm{Cs} 2 \mathrm{CO} 3 \quad \mathrm{~K} 2 \mathrm{CO} 3 \quad \mathrm{Na} 2 \mathrm{O} 2$

$\begin{array}{lllll}\text { Mixed Acid } & -0.16626 & -0.16195 & -0.13182 & -0.08018\end{array}$

$\begin{array}{lllll}\text { Cs2CO3 } & -0.16195 & -0.16626 & -0.13613 & -0.08448\end{array}$

$\begin{array}{lllll}\mathrm{K} 2 \mathrm{CO} 3 & -0.13182 & -0.13613 & -0.16626 & -0.11461\end{array}$

$\begin{array}{lllll}\mathrm{Na} 2 \mathrm{O} 2 & -0.08018 & -0.08448 & -0.11461 & -0.16626\end{array}$

Positive values show pairs of means that are significantly different

Level

Mean

Mixed Acid A 1.9109760

Cs2CO3 A 1.9066720

K2CO3 A 1.8765440

$\mathrm{Na} 2 \mathrm{O} 2$ A 1.8248960

Levels not connected by same letter are significantly different Tests that the Variances are Equal

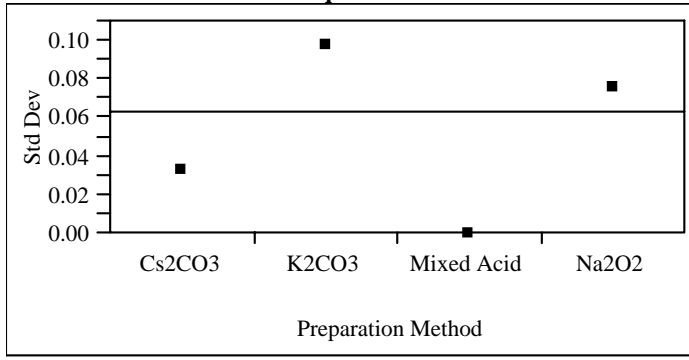

$\begin{array}{lrrrr}\text { Level } & \text { Count } & \text { Std Dev } & \begin{array}{r}\text { MeanAbsDif to } \\ \text { Mean }\end{array} & \begin{array}{r}\text { MeanAbsDif to } \\ \text { Median }\end{array} \\ \text { Cs2CO3 } & 3 & 0.0324945 & 0.0229547 & 0.0301280 \\ \text { K2CO3 } & 3 & 0.0969117 & 0.0659947 & 0.0946880 \\ \text { Mixed Acid } & 3 & 0.0000000 & 0.0000000 & 0.0000000 \\ \text { Na2O2 } & 3 & 0.0756574 & 0.0573867 & 0.0559520 \\ \text { Test } & \text { F Ratio } & \text { DFNum } & \text { DFDen } & \text { Prob > F } \\ \text { O'Brien[.5] } & 1.1010 & 3 & 8 & 0.4034 \\ \text { Brown- } & 6.8235 & 3 & 8 & 0.0135 \\ \text { Forsythe } & & & & \\ \text { Levene } & 2.8878 & 3 & 8 & 0.1024 \\ \text { Bartlett } & . & 3 & . & 0.0000 \\ \text { Warn }\end{array}$

Warning: Small sample sizes. Use Caution.

Welch Anova testing Means Equal, allowing Std Devs Not Equal

F Ratio DFNum DFDen Prob $>$ F 


\section{Exhibit A3. Statistical Comparisons of Chemical Composition Measurements of ARG-1 Samples by Dissolution Method for Each Oxide (Concentrations in Wt\% Oxides)}

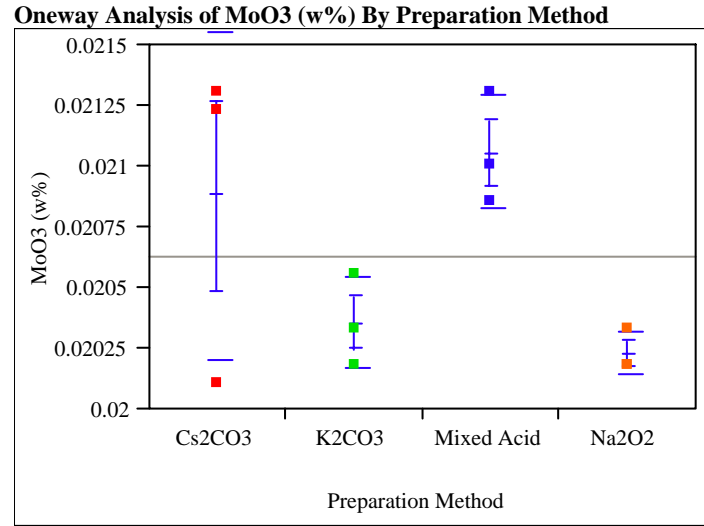

Means Comparisons

Comparisons for all pairs using Tukey-Kramer HSD

$$
\begin{array}{rr}
q^{*} & \text { Alpha } \\
3.20238 & 0.05
\end{array}
$$

Abs(Dif)-LSD Mixed Acid $\mathrm{Cs} 2 \mathrm{CO} 3 \quad \mathrm{~K} 2 \mathrm{CO} 3 \quad \mathrm{Na} 2 \mathrm{O} 2$

$\begin{array}{lllll}\text { Mixed Acid } & -0.00097 & -0.00079 & -0.00027 & -0.00014\end{array}$

$\begin{array}{lllll}\mathrm{Cs} 2 \mathrm{CO} 3 & -0.00079 & -0.00097 & -0.00044 & -0.00032\end{array}$

$\begin{array}{lllll}\mathrm{K} 2 \mathrm{CO} 3 & -0.00027 & -0.00044 & -0.00097 & -0.00084\end{array}$

$\begin{array}{lllll}\mathrm{Na} 2 \mathrm{O} 2 & -0.00014 & -0.00032 & -0.00084 & -0.00097\end{array}$

Positive values show pairs of means that are significantly different.

$$
\begin{array}{lrr}
\text { Level } & & \text { Mean } \\
\text { Mixed Acid } & \text { A } & 0.02105421 \\
\text { Cs2CO3 } & \text { A } & 0.02087918 \\
\text { K2CO3 } & \text { A } & 0.02035407 \\
\text { Na2O2 } & \text { A } & 0.02022905
\end{array}
$$

Levels not connected by same letter are significantly different

Tests that the Variances are Equal

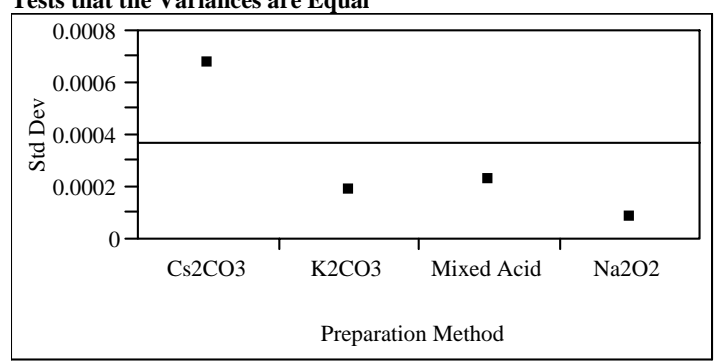

\begin{tabular}{lrrrr}
\hline Level & Count & Std Dev & $\begin{array}{r}\text { MeanAbsDif to } \\
\text { Mean }\end{array}$ & $\begin{array}{r}\text { MeanAbsDif to } \\
\text { Median }\end{array}$ \\
Cs2CO3 & 3 & 0.0006724 & 0.0005168 & 0.0004251 \\
K2CO3 & 3 & 0.0001888 & 0.0001334 & 0.0001750 \\
Mixed Acid & 3 & 0.0002292 & 0.0001667 & 0.0002000 \\
Na2O2 & 3 & 0.0000866 & 0.0000667 & 0.0000500 \\
Test & F Ratio & DFNum & DFDen & Prob $>$ F \\
O'Brien[.5] & 1.5175 & 3 & 8 & 0.2828 \\
Brown- & 0.7610 & 3 & 8 & 0.5468 \\
Forsythe & & & & \\
Levene & 6.7630 & 3 & 8 & 0.0138 \\
Bartlett & 2.2147 & 3 &. & 0.0842
\end{tabular}

Warning: Small sample sizes. Use Caution.

Welch Anova testing Means Equal, allowing Std Devs Not Equal

F Ratio DFNum DFDen Prob > F $\begin{array}{llll}8.9403 & 3 & 3.8949 & 0.0319\end{array}$

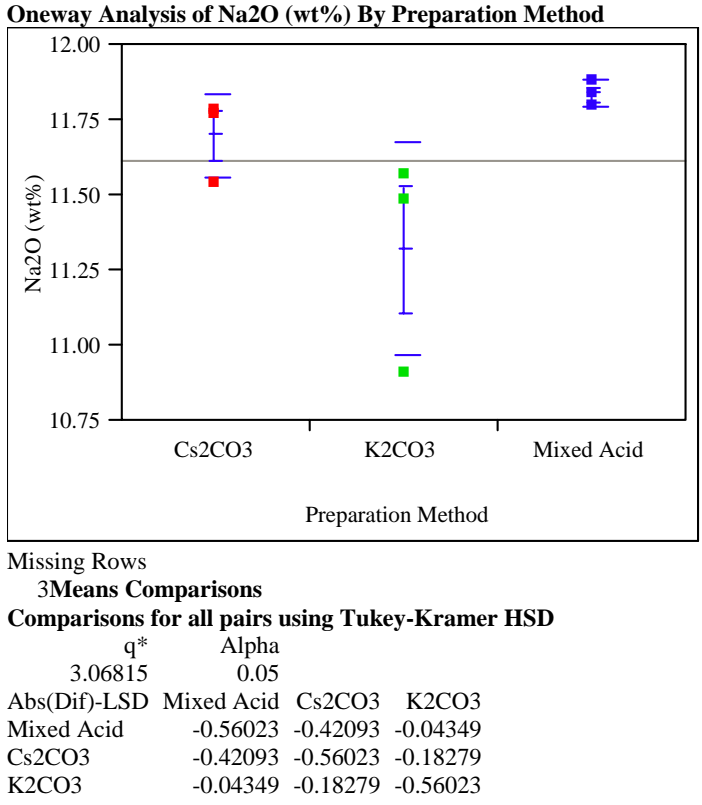

Positive values show pairs of means that are significantly different.

$$
\begin{array}{lrr}
\text { Level } & & \text { Mean } \\
\text { Mixed Acid } & \text { A } & 11.835440 \\
\text { Cs2CO3 } & \text { A } & 11.696147 \\
\text { K2CO3 } & \text { A } & 11.318707
\end{array}
$$

Levels not connected by same letter are significantly different Tests that the Variances are Equal

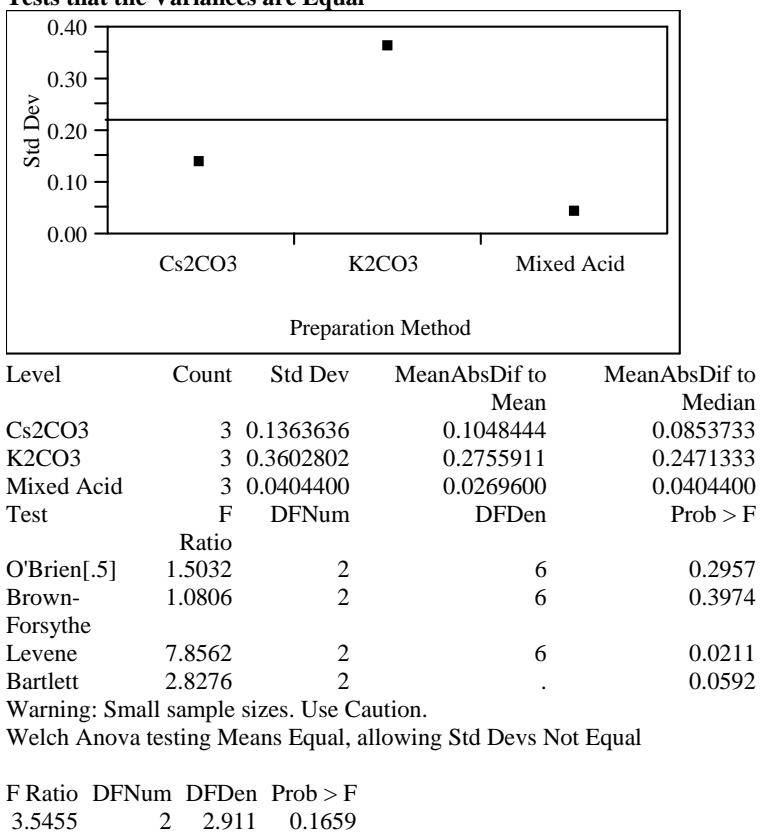




\section{Exhibit A3. Statistical Comparisons of Chemical Composition Measurements of ARG-1 Samples by Dissolution Method for Each Oxide (Concentrations in Wt\% Oxides)}

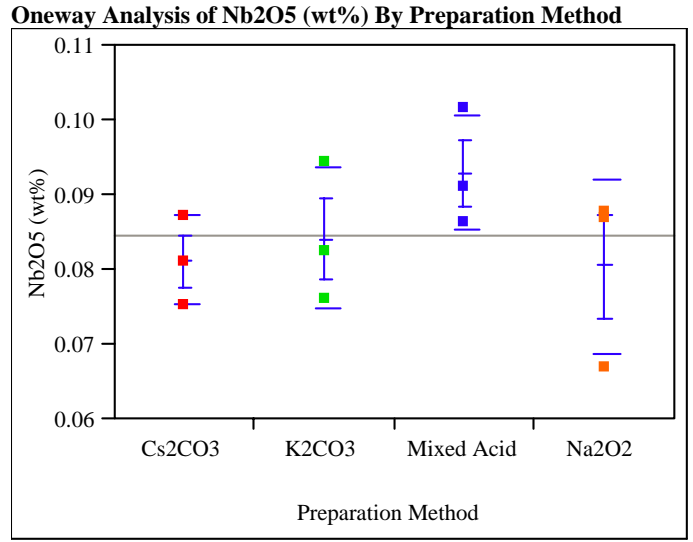

Means Comparisons

Comparisons for all pairs using Tukey-Kramer HSD

$$
3.20238 \quad 0.05
$$

Abs(Dif)-LSD Mixed Acid $\quad$ K2CO3 Cs2CO3 Na2O2

$\begin{array}{lllll}\text { Mixed Acid } & -0.02346 & -0.01478 & -0.01168 & -0.01101\end{array}$

$\begin{array}{lllll}\mathrm{K} 2 \mathrm{CO} 3 & -0.01478 & -0.02346 & -0.02036 & -0.01969\end{array}$

$\begin{array}{llllll}\mathrm{Cs} 2 \mathrm{CO} 3 & -0.01168 & -0.02036 & -0.02346 & -0.02279\end{array}$

$\mathrm{Na2O} 2 \quad-0.01101-0.01969-0.02279-0.02346$

Positive values show pairs of means that are significantly different.

Level Mean

Mixed Acid A 0.09283945

K2CO3 A 0.08416108

Cs2CO3 A 0.08106167

$\mathrm{Na} 2 \mathrm{O} 2$ A 0.08039410

Levels not connected by same letter are significantly different Tests that the Variances are Equal

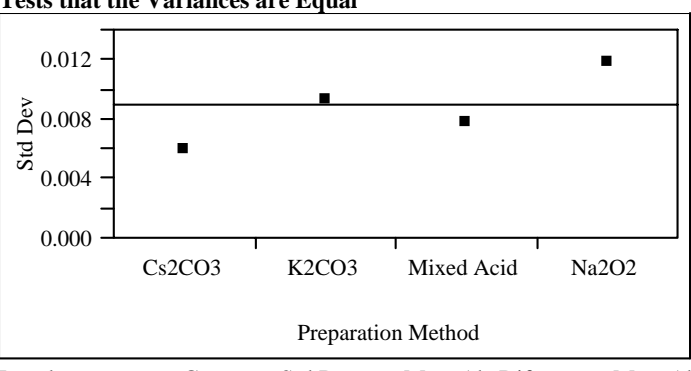

$\begin{array}{lrrrr}\text { Level } & \text { Count } & \text { Std Dev } & \begin{array}{r}\text { MeanAbsDif to } \\ \text { Mean }\end{array} & \begin{array}{r}\text { MeanAbsDif to } \\ \text { Median }\end{array} \\ \text { Cs2CO3 } & 3 & 0.0060087 & 0.0040372 & 0.0059604 \\ \text { K2CO3 } & 3 & 0.0093030 & 0.0067392 & 0.0082015 \\ \text { Mixed Acid } & 3 & 0.0077775 & 0.0057220 & 0.0065803 \\ \text { Na2O2 } & 3 & 0.0117797 & 0.0090598 & 0.0072956 \\ \text { Test } & \text { F Ratio } & \text { DFNum } & \text { DFDen } & \text { Prob }>\text { F } \\ \text { O'Brien[.5] } & 0.4338 & 3 & 8 & 0.7347 \\ \text { Brown- } & 0.0786 & 3 & 8 & 0.9698 \\ \text { Forsythe } & & & & \\ \text { Levene } & 0.9270 & 3 & 8 & 0.4708 \\ \text { Bartlett } & 0.2590 & 3 & . & 0.8549 \\ \text { Wann } & & 3 & & \end{array}$

Warning: Small sample sizes. Use Caution.

Welch Anova testing Means Equal, allowing Std Devs Not Equal

F Ratio DFNum DFDen Prob $>$ F

$$
\begin{array}{lrrr}
1.2224 & 3 & 4.331 & 0.4038
\end{array}
$$

Oneway Analysis of Nd2O3 (wt \%) By Preparation Method

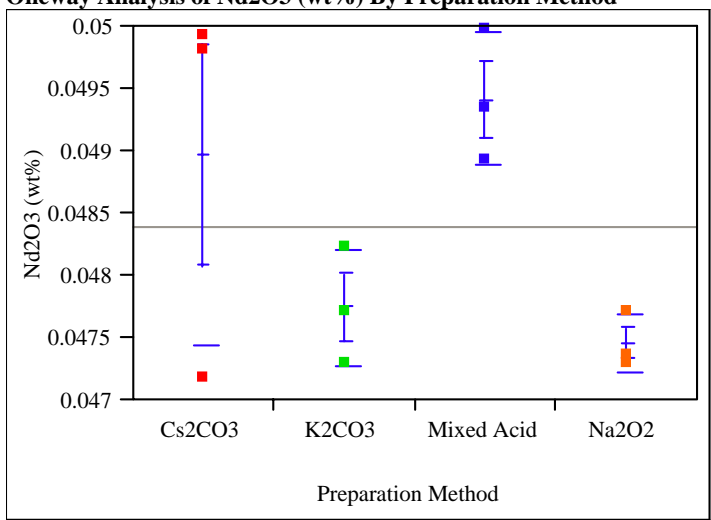

Means Comparisons

Comparisons for all pairs using Tukey-Kramer HSD

$$
\begin{array}{rr}
\text { q* }^{*} & \text { Alpha } \\
3.20238 & 0.05
\end{array}
$$

Abs(Dif)-LSD Mixed Acid Cs2CO3 K2CO3 Na2O2

$\begin{array}{lllll}\text { Mixed Acid } & -0.00225 & -0.00180 & -0.00057 & -0.00028\end{array}$

$\begin{array}{lllll}\mathrm{Cs} 2 \mathrm{CO} 3 & -0.00180 & -0.00225 & -0.00102 & -0.00073\end{array}$

K2CO3 $\quad-0.00057-0.00102-0.00225-0.00195$

$\begin{array}{lllll}\mathrm{Na} 2 \mathrm{O} 2 & -0.00028 & -0.00073 & -0.00195 & -0.00225\end{array}$

Positive values show pairs of means that are significantly different.

Level Mean

Mixed Acid A 0.04941648

Cs2CO3 A 0.04896936

K2CO3 A 0.04774464

$\mathrm{Na} 2 \mathrm{O} 2$ A 0.04745304

Levels not connected by same letter are significantly different

\begin{tabular}{|c|c|c|c|c|}
\hline Level & Count & Std Dev & $\begin{array}{r}\text { MeanAbsDif to } \\
\text { Mean }\end{array}$ & $\begin{array}{r}\text { MeanAbsDif to } \\
\text { Median }\end{array}$ \\
\hline Cs2CO3 & 3 & 0.0015500 & 0.0011923 & 0.0009526 \\
\hline K2CO3 & 3 & 0.0004678 & 0.0003240 & 0.0004471 \\
\hline Mixed Acid & 3 & 0.0005292 & 0.0003758 & 0.0004860 \\
\hline $\mathrm{Na} 2 \mathrm{O} 2$ & 3 & 0.0002208 & 0.0001685 & 0.0001555 \\
\hline Test & F Ratio & DFNum & DFDen & Prob $>F$ \\
\hline O'Brien[.5] & 1.4963 & 3 & 8 & 0.2878 \\
\hline $\begin{array}{l}\text { Brown- } \\
\text { Forsythe }\end{array}$ & 0.6074 & 3 & 8 & 0.6287 \\
\hline Levene & 6.2588 & 3 & 8 & 0.0171 \\
\hline Bartlett & 2.0519 & 3 & . & 0.1043 \\
\hline
\end{tabular}
Tests that the Variances are Equal

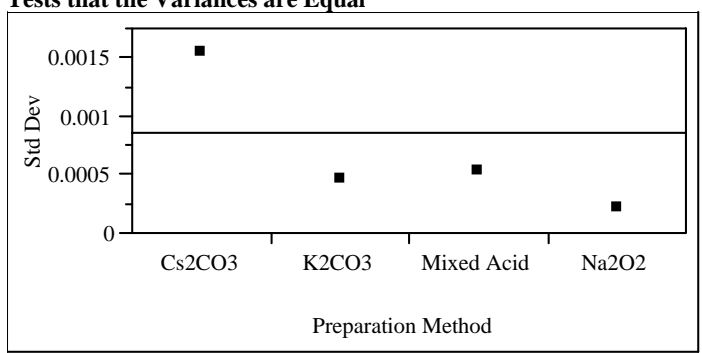

Warning: Small sample sizes. Use Caution.

Welch Anova testing Means Equal, allowing Std Devs Not Equal

F Ratio DFNum DFDen Prob $>$ F

$\begin{array}{lrrr}9.2209 & 3 & 3.9402 & 0.0295\end{array}$ 


\section{Exhibit A3. Statistical Comparisons of Chemical Composition Measurements of ARG-1 Samples by Dissolution Method for Each Oxide (Concentrations in Wt\% Oxides)}

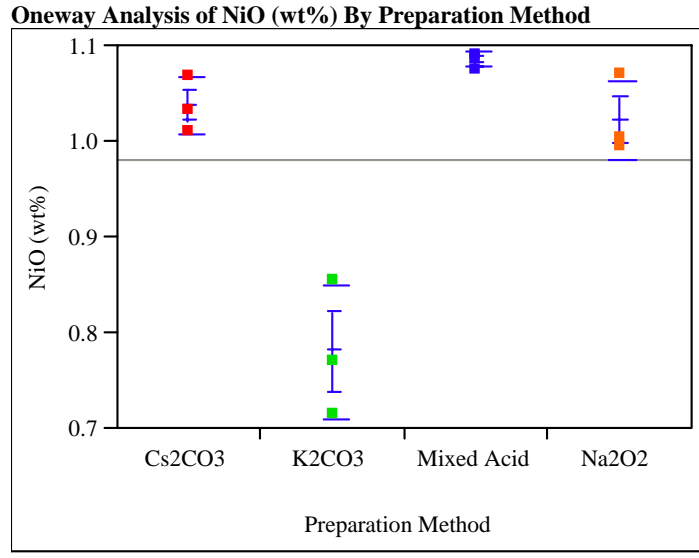

Means Comparisons

Comparisons for all pairs using Tukey-Kramer HSD

$$
\begin{array}{rr}
\mathrm{q}^{*} & \text { Alpha } \\
3.20238 & 0.05
\end{array}
$$

Abs(Dif)-LSD Mixed Acid Cs2CO3 Na2O2 K2CO3

$\begin{array}{lllll}\text { Mixed Acid } \quad-0.11333 & -0.06667 & -0.05183 & 0.19037\end{array}$

$\begin{array}{lllll}\mathrm{Cs} 2 \mathrm{CO} 3 & -0.06667 & -0.11333 & -0.09848 & 0.14371\end{array}$

$\begin{array}{lllll}\mathrm{Na} 2 \mathrm{O} 2 & -0.05183 & -0.09848 & -0.11333 & 0.12887\end{array}$

$\begin{array}{lllll}\text { K2CO3 } & 0.19037 & 0.14371 & 0.12887 & -0.11333\end{array}$

Positive values show pairs of means that are significantly different.

Level Mean

Mixed Acid A 1.0837458

Cs2CO3 A 1.0370875

$\mathrm{Na} 2 \mathrm{O} 2 \quad \mathrm{~A} \quad 1.0222417$

K2CO3 B 0.7800425

Levels not connected by same letter are significantly different Tests that the Variances are Equal

\begin{tabular}{l|rrrr|}
\hline & & & \\
\end{tabular}

Warning: Small sample sizes. Use Caution.

Welch Anova testing Means Equal, allowing Std Devs Not Equal

F Ratio DFNum DFDen Prob $>$ F

$\begin{array}{rrrr}16.1399 & 3 & 3.5711 & 0.0148\end{array}$
Oneway Analysis of P2O5 (wt\%) By Preparation Method

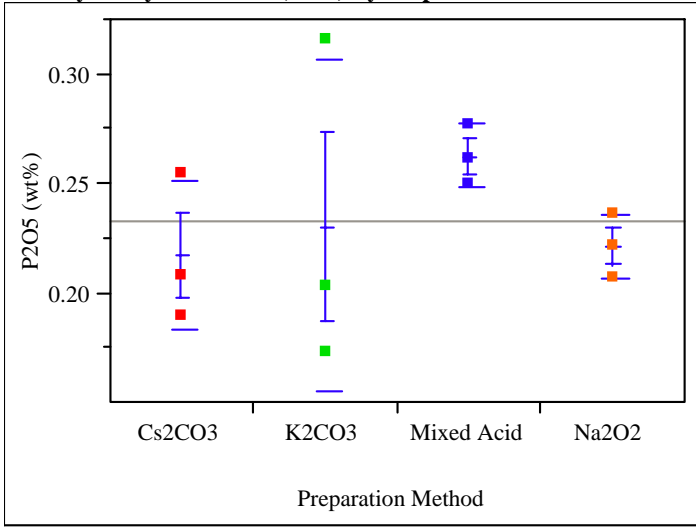

Means Comparisons

Comparisons for all pairs using Tukey-Kramer HSD

$$
\begin{array}{rr}
\text { q* }^{*} & \text { Alpha } \\
3.20238 & 0.05
\end{array}
$$

Abs(Dif)-LSD Mixed Acid $\quad$ K2CO3 Na2O2 Cs2CO3

Mixed Acid $\quad-0.11117-0.07909-0.06985-0.06565$

$\begin{array}{lllll}\text { K2CO3 } & -0.07909 & -0.11117 & -0.10193 & -0.09773\end{array}$

$\begin{array}{lllll}\mathrm{Na} 2 \mathrm{O} 2 & -0.06985 & -0.10193 & -0.11117 & -0.10697\end{array}$

$\begin{array}{lllll}\text { Cs2CO3 } & -0.06565 & -0.09773 & -0.10697 & -0.11117\end{array}$

Positive values show pairs of means that are significantly different.

Level

Mixed Acid A 0.26274720

K2CO3 A 0.23066760

$\mathrm{Na2O} 2$ A 0.22142562

Cs2CO3 A 0.21722472

Levels not connected by same letter are significantly different Tests that the Variances are Equal

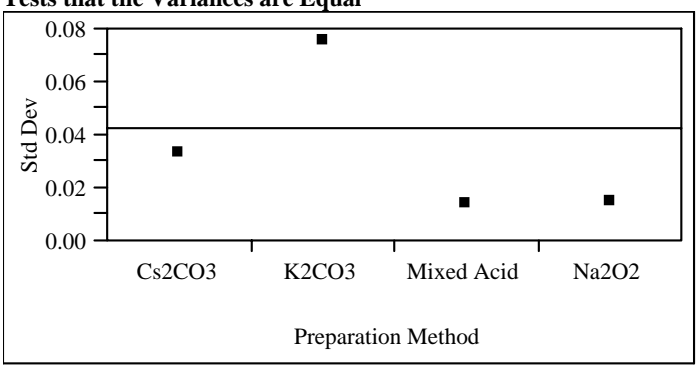

$\begin{array}{lrrrr}\text { Level } & \text { Count } & \text { Std Dev } & \begin{array}{r}\text { MeanAbsDif to } \\ \text { Mean }\end{array} & \begin{array}{r}\text { MeanAbsDif to } \\ \text { Median }\end{array} \\ \text { Cs2CO3 } & 3 & 0.0334921 & 0.0247471 & 0.0279551 \\ \text { K2CO3 } & 3 & 0.0755222 & 0.0570304 & 0.0574378 \\ \text { Mixed Acid } & 3 & 0.0138119 & 0.0096748 & 0.0129846 \\ \text { Na2O2 } & 3 & 0.0146656 & 0.0098276 & 0.0145886 \\ \text { Test } & \text { F Ratio } & \text { DFNum } & \text { DFDen } & \text { Prob > F } \\ \text { O'Brien[.5] } & 1.4556 & 3 & 8 & 0.2977 \\ \text { Brown- } & 1.9347 & 3 & 8 & 0.2026 \\ \text { Forsythe } & & & & \\ \text { Levene } & 5.1914 & 3 & 8 & 0.0278 \\ \text { Bartlett } & 2.0446 & 3 & . & 0.1053 \\ \text { Wan }\end{array}$

Warning: Small sample sizes. Use Caution.

Welch Anova testing Means Equal, allowing Std Devs Not Equal

F Ratio DFNum DFDen Prob $>$ F

$\begin{array}{llll}3.6179 & 3 & 4.1395 & 0.1192\end{array}$ 


\section{Exhibit A3. Statistical Comparisons of Chemical Composition Measurements of ARG-1 Samples by Dissolution Method for Each Oxide (Concentrations in Wt\% Oxides)}

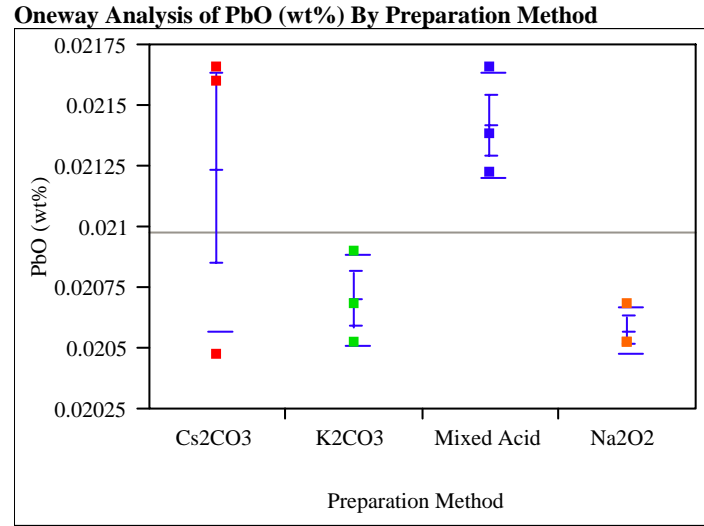

Means Comparisons

Comparisons for all pairs using Tukey-Kramer HSD

$$
\begin{array}{rr}
q^{*} & \text { Alpha } \\
3.20238 & 0.05
\end{array}
$$

Abs(Dif)-LSD Mixed Acid $\mathrm{Cs2CO} \quad \mathrm{K} 2 \mathrm{CO} 3 \quad \mathrm{Na} 2 \mathrm{O} 2$

Mixed Acid $\quad-0.00096-0.00078-0.00024-0.00012$

$\begin{array}{lllll}\mathrm{Cs} 2 \mathrm{CO} 3 & -0.00078 & -0.00096 & -0.00042 & -0.00030\end{array}$

$\begin{array}{lllll}\mathrm{K} 2 \mathrm{CO} 3 & -0.00024 & -0.00042 & -0.00096 & -0.00083\end{array}$

$\begin{array}{lllll}\mathrm{Na} 2 \mathrm{O} 2 & -0.00012 & -0.00030 & -0.00083 & -0.00096\end{array}$

Positive values show pairs of means that are significantly different.

$$
\begin{array}{lrr}
\text { Level } & & \text { Mean } \\
\text { Mixed Acid } & \text { A } & 0.02141833 \\
\text { Cs2CO3 } & \text { A } & 0.02123879 \\
\text { K2CO3 } & \text { A } & 0.02070019 \\
\text { Na2O2 } & \text { A } & 0.02057452
\end{array}
$$

Levels not connected by same letter are significantly different

Tests that the Variances are Equal

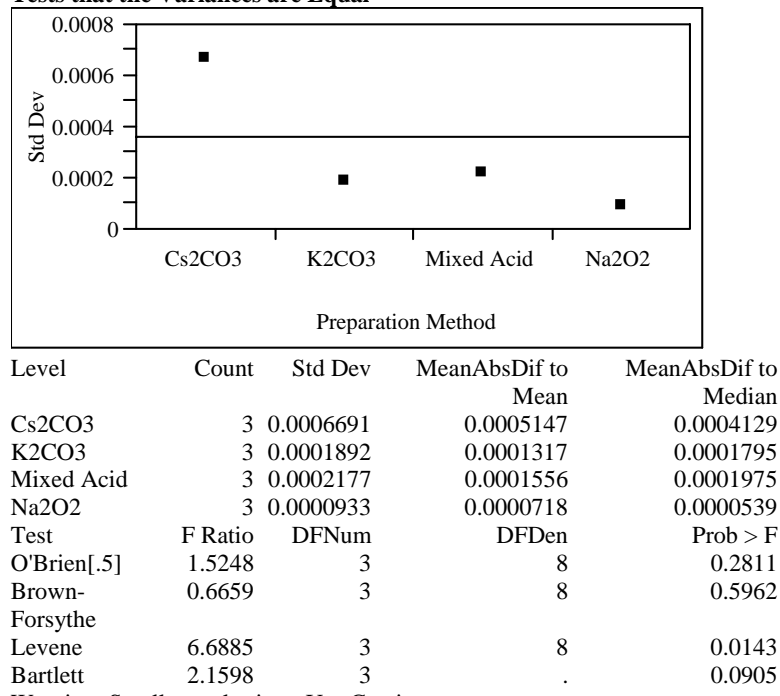

Warning: Small sample sizes. Use Caution.

Welch Anova testing Means Equal, allowing Std Devs Not Equal

F Ratio DFNum DFDen Prob $>$ F

$\begin{array}{llll}9.9712 & 3 & 3.9641 & 0.0255\end{array}$

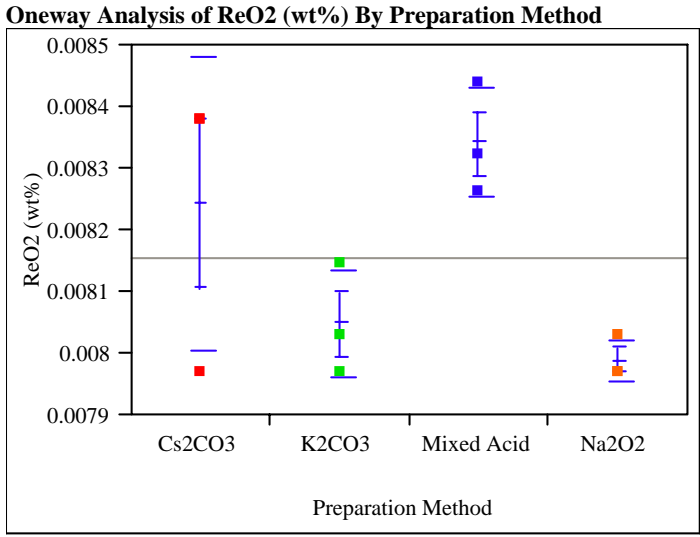

Means Comparisons

Comparisons for all pairs using Tukey-Kramer HSD

$$
\begin{array}{rr}
q^{*} & \text { Alpha } \\
3.20238 & 0.05
\end{array}
$$

Abs(Dif)-LSD Mixed Acid Cs2CO3 K2CO3 Na2O2

Mixed Acid $\quad-0.00035-0.00026-0.00006-0.00000$

$\begin{array}{lllll}\mathrm{Cs} 2 \mathrm{CO} 3 & -0.00026 & -0.00035 & -0.00016 & -0.00010\end{array}$

$\begin{array}{lllll}\mathrm{K} 2 \mathrm{CO} 3 & -0.00006 & -0.00016 & -0.00035 & -0.00030\end{array}$

$\begin{array}{lllll}\mathrm{Na} 2 \mathrm{O} 2 & -0.00000 & -0.00010 & -0.00030 & -0.00035\end{array}$

Positive values show pairs of means that are significantly different.

Level

Mixed Acid A 0.00834002

Cs2CO3 A 0.00824236

K2CO3 A 0.00804705

$\mathrm{Na2O} 2$ A 0.00798845

Levels not connected by same letter are significantly different Tests that the Variances are Equal

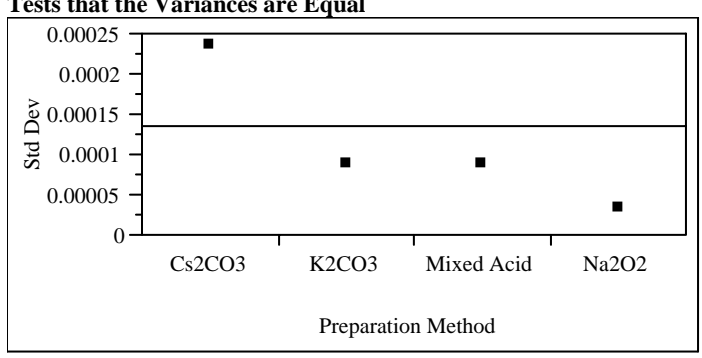

$\begin{array}{lrrrr}\text { Level } & \text { Count } & \text { Std Dev } & \begin{array}{r}\text { MeanAbsDif to } \\ \text { Mean }\end{array} & \begin{array}{r}\text { MeanAbsif to } \\ \text { Median }\end{array} \\ \text { Cs2CO3 } & 3 & 0.0002368 & 0.0001823 & 0.0001367 \\ \text { K2CO3 } & 3 & 0.0000895 & 0.0000651 & 0.0000781 \\ \text { Mixed Acid } & 3 & 0.0000895 & 0.0000651 & 0.0000781 \\ \text { Na2O2 } & 3 & 0.0000338 & 0.0000260 & 0.0000195 \\ \text { Test } & \text { F Ratio } & \text { DFNum } & \text { DFDen } & \text { Prob > F } \\ \text { O'Brien[.5] } & 1.3995 & 3 & 8 & 0.3120 \\ \text { Brown- } & 0.4615 & 3 & 8 & 0.7168 \\ \text { Forsythe } & & & & \\ \text { Levene } & 5.6842 & 3 & 8 & 0.0221 \\ \text { Bartlett } & 1.8244 & 3 & . & 0.1403 \\ \text { Wan } & & 3 & & \end{array}$

Warning: Small sample sizes. Use Caution.

Welch Anova testing Means Equal, allowing Std Devs Not Equal

F Ratio DFNum DFDen Prob $>$ F

$\begin{array}{llll}10.5973 & 3 & 3.8309 & 0.0249\end{array}$ 


\section{Exhibit A3. Statistical Comparisons of Chemical Composition Measurements of ARG-1 Samples by Dissolution Method for Each Oxide (Concentrations in Wt\% Oxides)}

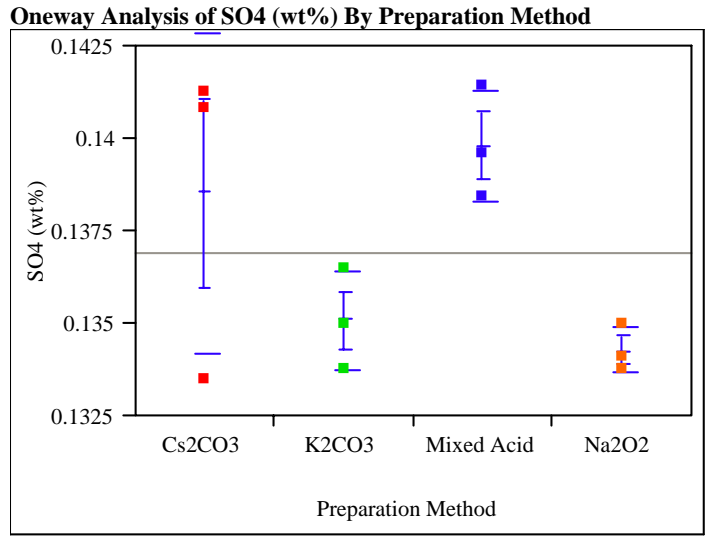

Means Comparisons

Comparisons for all pairs using Tukey-Kramer HSD

$$
\begin{array}{rr}
q^{*} & \text { Alpha } \\
3.20238 & 0.05
\end{array}
$$

Abs(Dif)-LSD Mixed Acid Cs2CO3 K2CO3 Na2O2

$\begin{array}{lllll}\text { Mixed Acid } & -0.00635 & -0.00505 & -0.00161 & -0.00081\end{array}$

$\begin{array}{lllll}\mathrm{Cs} 2 \mathrm{CO} 3 & -0.00505 & -0.00635 & -0.00291 & -0.00211\end{array}$

K2CO3 $\quad-0.00161-0.00291-0.00635-0.00555$

$\mathrm{Na2O} \quad-0.00081-0.00211-0.00555-0.00635$

Positive values show pairs of means that are significantly different.

Level Mean

Mixed Acid A 0.13980867

Cs2CO3 A 0.13851044

K2CO3 A 0.13506516

$\mathrm{Na} 2 \mathrm{O} 2$ A 0.13426625

Levels not connected by same letter are significantly different Tests that the Variances are Equal

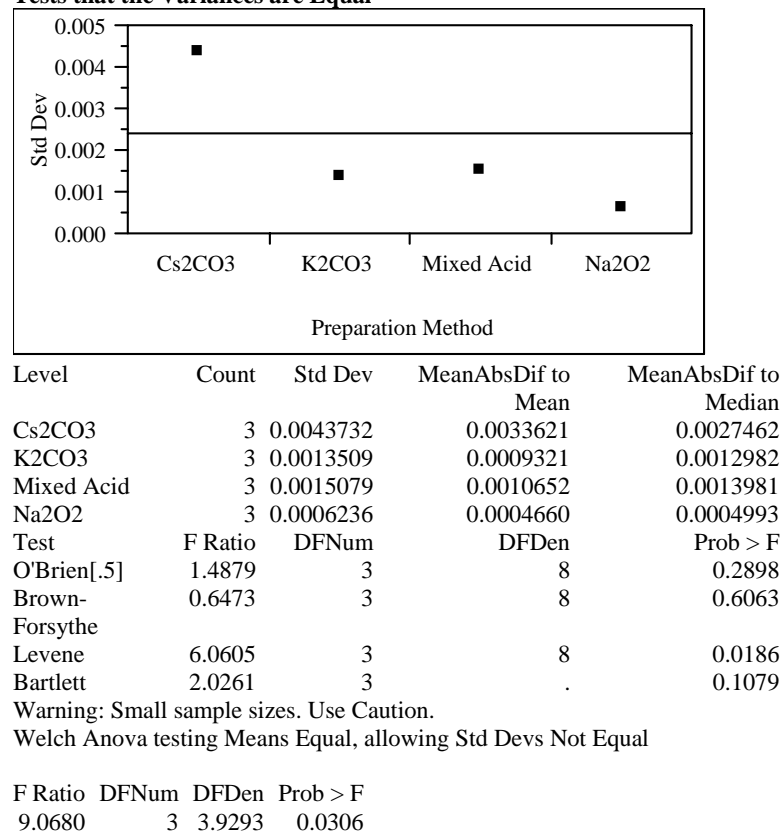

Oneway Analysis of SiO2 (wt\%) By Preparation Method

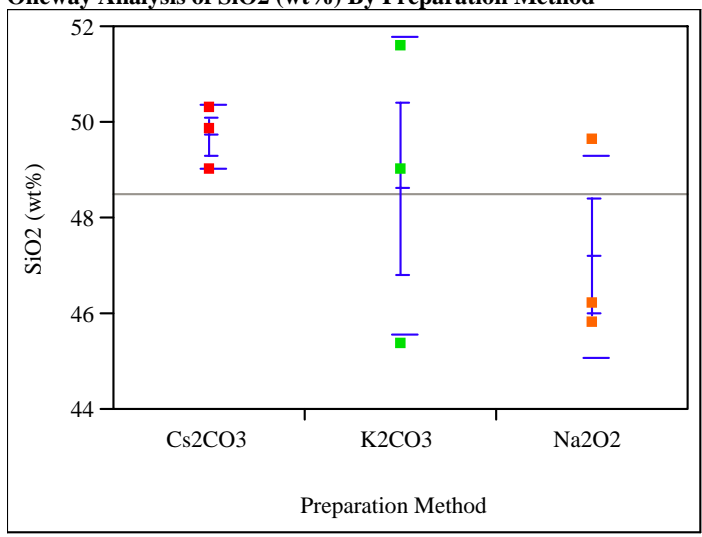

Missing Rows

3Means Comparisons

Comparisons for all pairs using Tukey-Kramer HSD q* Alpha

s(Dif)-LSD Cs2CO3

K2CO3 $\mathrm{Na} 2 \mathrm{O} 2$

Cs2CO3 $\quad-5.5263-4.4567 \quad-3.0305$

K2CO3 $\quad-4.4567-5.5263-4.1001$

$\mathrm{Na2O} 2 \quad-3.0305-4.1001-5.5263$

Positive values show pairs of means that are significantly different

Level

Mean

Cs2CO3 A 49.703070

$\mathrm{K} 2 \mathrm{CO} 3$ A 48.633420

$\mathrm{Na} 2 \mathrm{O} 2$ A 47.207220

Levels not connected by same letter are significantly different Tests that the Variances are Equal

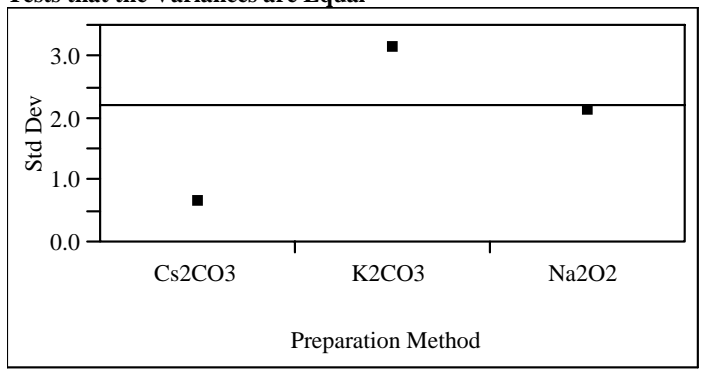

\begin{tabular}{|c|c|c|c|c|}
\hline Level & Count & Std Dev & $\begin{array}{r}\text { MeanAbsDif to } \\
\text { Mean }\end{array}$ & $\begin{array}{r}\text { MeanAbsDif to } \\
\text { Median }\end{array}$ \\
\hline Cs2CO3 & 3 & 0.653567 & 0.475400 & 0.570480 \\
\hline K2CO3 & 3 & 3.117316 & 2.186840 & 2.923710 \\
\hline $\mathrm{Na} 2 \mathrm{O} 2$ & 3 & 2.110583 & 1.616360 & 1.426200 \\
\hline Test & F Ratio & DFNum & DFDen & Prob $>F$ \\
\hline O'Brien[.5] & 1.0114 & 2 & 6 & 0.4183 \\
\hline $\begin{array}{l}\text { Brown- } \\
\text { Forsythe }\end{array}$ & 3.7200 & 2 & 6 & 0.0890 \\
\hline Levene & 2.1573 & 2 & 6 & 0.1968 \\
\hline Bartlett & 1.4971 & 2 & . & 0.2238 \\
\hline
\end{tabular}

Warning: Small sample sizes. Use Caution.

Welch Anova testing Means Equal, allowing Std Devs Not Equal

F Ratio DFNum DFDen Prob $>$ F

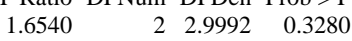




\section{Exhibit A3. Statistical Comparisons of Chemical Composition Measurements of ARG-1 Samples by Dissolution Method for Each Oxide (Concentrations in Wt\% Oxides)}

Oneway Analysis of SnO2 (wt \%) By Preparation Method

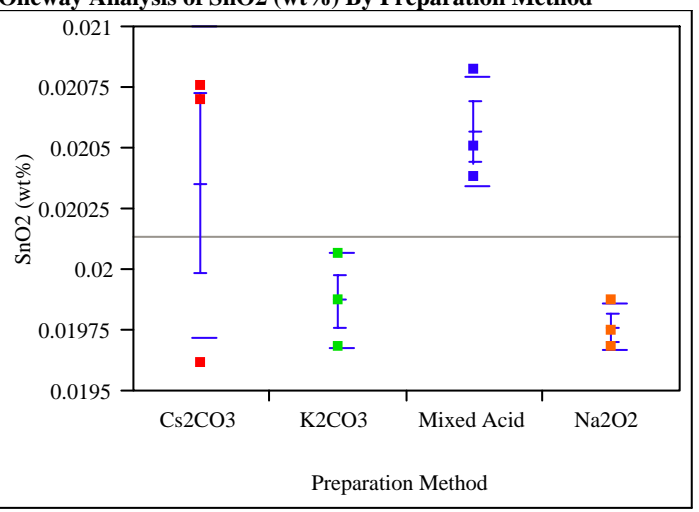

Means Comparisons

Comparisons for all pairs using Tukey-Kramer HSD

$$
\begin{array}{rr}
q^{*} & \text { Alpha } \\
3.20238 & 0.05
\end{array}
$$

Abs(Dif)-LSD Mixed Acid Cs2CO3 $\quad \mathrm{K} 2 \mathrm{CO} 3 \quad \mathrm{Na} 2 \mathrm{O} 2$

$\begin{array}{lllll}\text { Mixed Acid } & -0.00093 & -0.00072 & -0.00024 & -0.00013\end{array}$

$\begin{array}{lllll}\mathrm{Cs} 2 \mathrm{CO} 3 & -0.00072 & -0.00093 & -0.00045 & -0.00034\end{array}$

$\begin{array}{lllll}\mathrm{K} 2 \mathrm{CO} 3 & -0.00024 & -0.00045 & -0.00093 & -0.00083\end{array}$

$\begin{array}{lllll}\mathrm{Na} 2 \mathrm{O} 2 & -0.00013 & -0.00034 & -0.00083 & -0.00093\end{array}$

Positive values show pairs of means that are significantly different.

Level Mean

Mixed Acid A 0.02056752

Cs2CO3 A 0.02035592

K2CO3 A 0.01986924

$\mathrm{Na} 2 \mathrm{O} 2 \quad$ A 0.01976344

Levels not connected by same letter are significantly different

Tests that the Variances are Equal

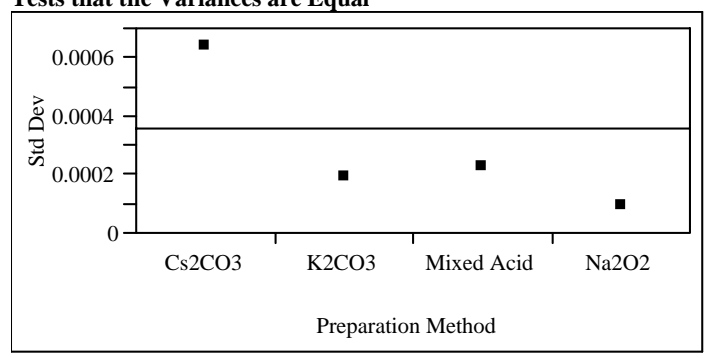

$\begin{array}{lrrrr}\text { Level } & \text { Count } & \text { Std Dev } & \begin{array}{r}\text { MeanAbsDif to } \\ \text { Mean }\end{array} & \begin{array}{r}\text { MeanAbsDif to } \\ \text { Median }\end{array} \\ \text { Cs2CO3 } & 3 & 0.0006422 & 0.0004937 & 0.0004020 \\ \text { K2CO3 } & 3 & 0.0001904 & 0.0001270 & 0.0001904 \\ \text { Mixed Acid } & 3 & 0.0002289 & 0.0001693 & 0.0001904 \\ \text { Na2O2 } & 3 & 0.0000970 & 0.0000705 & 0.0000846 \\ \text { Test } & \text { F Ratio } & \text { DFNum } & \text { DFDen } & \text { Prob > F } \\ \text { O'Brien[.5] } & 1.4841 & 3 & 8 & 0.2907 \\ \text { Brown- } & 0.5952 & 3 & 8 & 0.6356 \\ \text { Forsythe } & & & & \\ \text { Levene } & 6.1816 & 3 & 8 & 0.0177 \\ \text { Bartlett } & 1.9770 & 3 & . & 0.1150 \\ \text { Waring }\end{array}$

Warning: Small sample sizes. Use Caution.

Welch Anova testing Means Equal, allowing Std Devs Not Equal

F Ratio DFNum DFDen Prob $>$ F

$\begin{array}{llll}8.2409 & 3 & 3.9763 & 0.0350\end{array}$

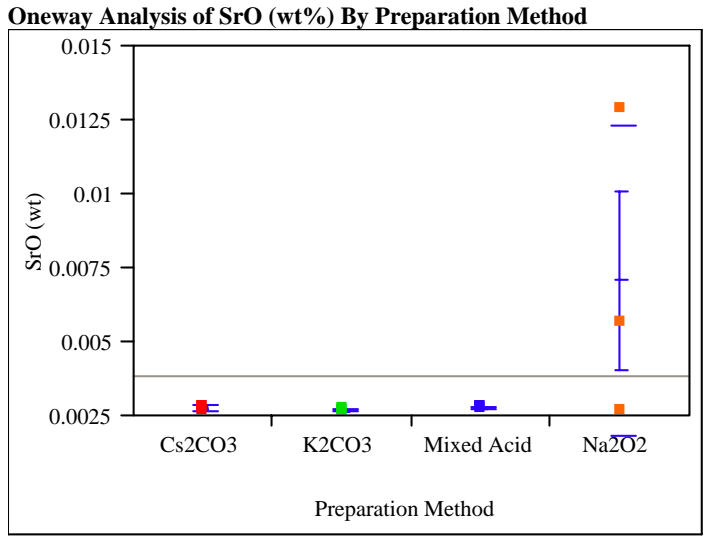

Means Comparisons

Comparisons for all pairs using Tukey-Kramer HSD

$$
\begin{array}{rr}
q^{*} & \text { Alpha } \\
3.20238 & 0.05
\end{array}
$$

Abs(Dif)-LSD Na2O2 Mixed Acid Cs2CO3 K2CO3

$\begin{array}{lllll}\mathrm{Na} 2 \mathrm{O} 2 & -0.00686 & -0.00256 & -0.00253 & -0.00246\end{array}$

$\begin{array}{lllll}\text { Mixed Acid } \quad-0.00256 & -0.00686 & -0.00683 & -0.00676\end{array}$

$\begin{array}{lllll}\mathrm{Cs} 2 \mathrm{CO} 3 & -0.00253 & -0.00683 & -0.00686 & -0.00679\end{array}$

$\begin{array}{lllll}\mathrm{K} 2 \mathrm{CO} 3 & -0.00246 & -0.00676 & -0.00679 & -0.00686\end{array}$

Positive values show pairs of means that are significantly different.
Level
Mixed Acid A 0.00278897
Cs2CO3 A 0.00276137
K2CO3 A 0.00269239

Levels not connected by same letter are significantly different Tests that the Variances are Equal

\begin{tabular}{|c|c|c|c|c|}
\hline Level & Count & Std Dev & $\begin{array}{r}\text { MeanAbsDif to } \\
\text { Mean }\end{array}$ & $\begin{array}{r}\text { MeanAbsDif to } \\
\text { Median }\end{array}$ \\
\hline Cs2CO3 & 3 & 0.0000871 & 0.0000670 & 0.0000532 \\
\hline K2CO3 & 3 & 0.0000267 & 0.0000184 & 0.0000256 \\
\hline Mixed Acid & 3 & 0.0000298 & 0.0000210 & 0.0000276 \\
\hline $\mathrm{Na} 2 \mathrm{O} 2$ & 3 & 0.0052440 & 0.0038697 & 0.0043953 \\
\hline Test & F Ratio & DFNum & DFDen & Prob $>$ F \\
\hline O'Brien[.5] & 1.7774 & 3 & 8 & 0.2291 \\
\hline $\begin{array}{l}\text { Brown- } \\
\text { Forsythe }\end{array}$ & 9.5605 & 3 & 8 & 0.0051 \\
\hline Levene & 8.7552 & 3 & 8 & 0.0066 \\
\hline Bartlett & 12.9975 & 3 & . & $<.0001$ \\
\hline
\end{tabular}

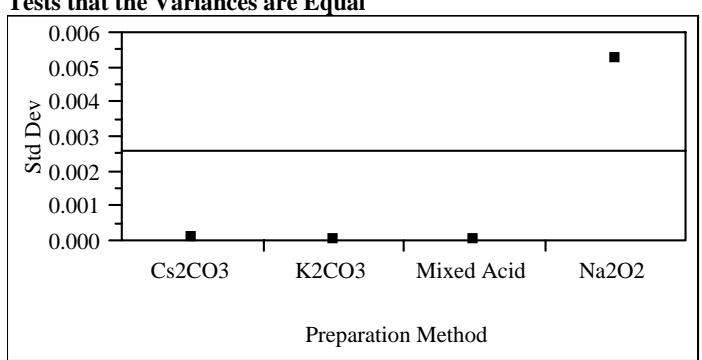

Warning: Small sample sizes. Use Caution.

Welch Anova testing Means Equal, allowing Std Devs Not Equal

F Ratio DFNum DFDen Prob $>$ F

$\begin{array}{rrrr}4.9808 & 3 & 4.0656 & 0.0759\end{array}$ 


\section{Exhibit A3. Statistical Comparisons of Chemical Composition Measurements of ARG-1 Samples by Dissolution Method for Each Oxide (Concentrations in Wt\% Oxides)}

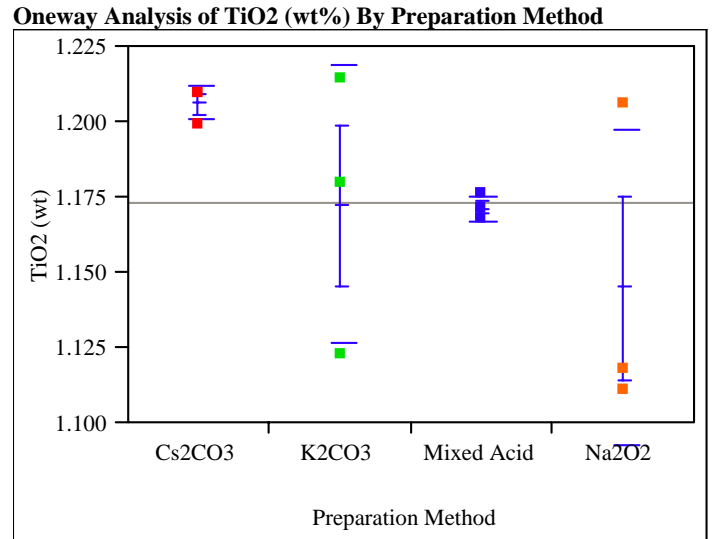

Means Comparisons

Comparisons for all pairs using Tukey-Kramer HSD$$
\text { q* Alpha }
$$

Abs(Dif)-LSD Cs2CO3 K2CO3 Mixed Acid Na2O2

$\begin{array}{lllll}\mathrm{Cs} 2 \mathrm{CO} 3 & -0.09254 & -0.05863 & -0.05807 & -0.03138\end{array}$

$\begin{array}{lllll}\mathrm{K} 2 \mathrm{CO} 3 & -0.05863 & -0.09254 & -0.09199 & -0.06530\end{array}$

Mixed Acid $\quad-0.05807 \quad-0.09199 \quad-0.09254-0.06586$

$\begin{array}{lllll}\mathrm{Na} 2 \mathrm{O} 2 & -0.03138 & -0.06530 & -0.06586 & -0.09254\end{array}$

Positive values show pairs of means that are significantly different.

$$
\begin{array}{lrr}
\text { Level } & & \text { Mean } \\
\text { Cs2CO3 } & \text { A } & 1.2059640 \\
\text { K2CO3 } & \text { A } & 1.1720480 \\
\text { Mixed Acid } & \text { A } & 1.1714920 \\
\text { Na2O2 } & \text { A } & 1.1448040
\end{array}
$$

\begin{tabular}{|c|c|c|c|c|}
\hline Level & Count & Std Dev & $\begin{array}{r}\text { MeanAbsDif to } \\
\text { Mean }\end{array}$ & $\begin{array}{r}\text { MeanAbsDif to } \\
\text { Median }\end{array}$ \\
\hline $\mathrm{Cs} 2 \mathrm{CO} 3$ & 3 & 0.0057781 & 0.0044480 & 0.0033360 \\
\hline K2CO3 & 3 & 0.0462951 & 0.0329893 & 0.0422560 \\
\hline Mixed Acid & 3 & 0.0041977 & 0.0029653 & 0.0038920 \\
\hline $\mathrm{Na} 2 \mathrm{O} 2$ & 3 & 0.0530711 & 0.0407733 & 0.0339160 \\
\hline Test & F Ratio & DFNum & DFDen & Prob $>F$ \\
\hline O'Brien[.5] & 1.1827 & 3 & 8 & 0.375 \\
\hline $\begin{array}{l}\text { Brown- } \\
\text { Forsythe }\end{array}$ & 2.0238 & 3 & 8 & 0.1892 \\
\hline Levene & 5.3789 & 3 & 8 & \\
\hline Bartlett & 3.6092 & 3 & & 0.0 \\
\hline
\end{tabular}

Levels not connected by same letter are significantly different Tests that the Variances are Equal

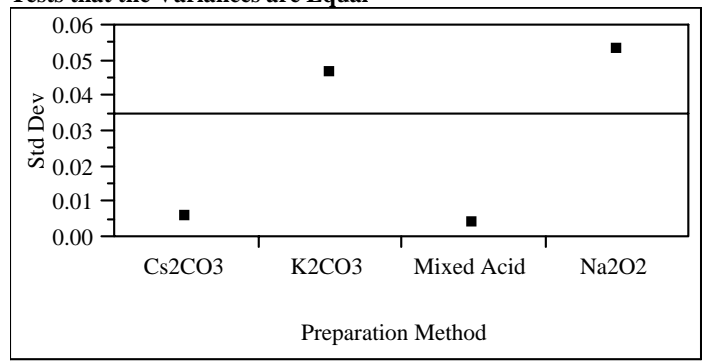

Warning: Small sample sizes. Use Caution.

Welch Anova testing Means Equal, allowing Std Devs Not Equal

F Ratio DFNum DFDen Prob $>$ F

$\begin{array}{llll}17.8444 & 3 & 3.9408 & 0.0093\end{array}$

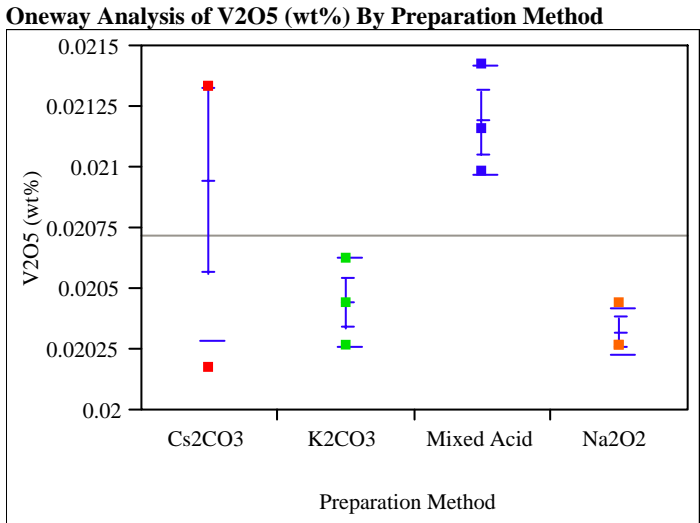

Means Comparisons

Comparisons for all pairs using Tukey-Kramer HSD

$$
\begin{array}{rr}
q^{*} & \text { Alpha } \\
3.20238 & 0.05
\end{array}
$$

Abs(Dif)-LSD Mixed Acid Cs2CO3 K2CO3 Na2O2

Mixed Acid $\quad-0.00096-0.00072 \quad-0.00022-0.00010$

$\begin{array}{lllll}\mathrm{Cs} 2 \mathrm{CO} 3 & -0.00072 & -0.00096 & -0.00046 & -0.00034\end{array}$

$\begin{array}{lllll}\mathrm{K} 2 \mathrm{CO} 3 & -0.00022 & -0.00046 & -0.00096 & -0.00084\end{array}$

$\mathrm{Na2O} 2 \quad-0.00010-0.00034 \quad-0.00084-0.00096$

Positive values show pairs of means that are significantly different.

$$
\begin{array}{lrr}
\text { Level } & & \text { Mean } \\
\text { Mixed Acid } & \text { A } & 0.02118437 \\
\text { Cs2CO3 } & \text { A } & 0.02094635 \\
\text { K2CO3 } & \text { A } & 0.02044054 \\
\text { Na2O2 } & \text { A } & 0.02032153
\end{array}
$$

Levels not connected by same letter are significantly different Tests that the Variances are Equal

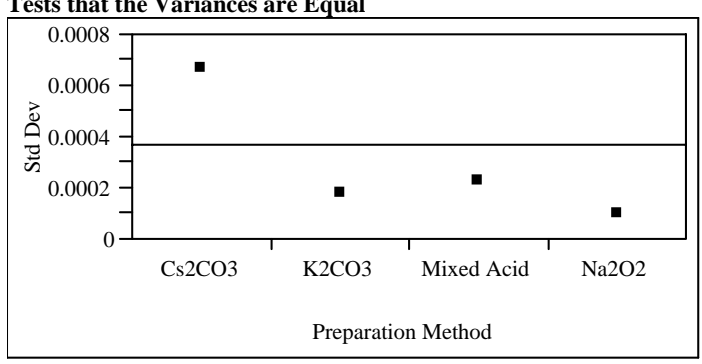

$\begin{array}{lrrrr}\text { Level } & \text { Count } & \text { Std Dev } & \begin{array}{r}\text { MeanAbsDif to } \\ \text { Mean }\end{array} & \begin{array}{r}\text { MeanAbsDif to } \\ \text { Median }\end{array} \\ \text { Cs2CO3 } & 3 & 0.0006699 & 0.0005157 & 0.0003868 \\ \text { K2CO3 } & 3 & 0.0001785 & 0.0001190 & 0.0001785 \\ \text { Mixed Acid } & 3 & 0.0002246 & 0.0001587 & 0.0002083 \\ \text { Na2O2 } & 3 & 0.0001031 & 0.0000793 & 0.0000595 \\ \text { Test } & \text { F Ratio } & \text { DFNum } & \text { DFDen } & \text { Prob > F } \\ \text { O'Brien[.5] } & 1.5224 & 3 & 8 & 0.2816 \\ \text { Brown- } & 0.4751 & 3 & 8 & 0.7082 \\ \text { Forsythe } & & & & \\ \text { Levene } & 6.5185 & 3 & 8 & 0.0153 \\ \text { Bartlett } & 2.0865 & 3 & . & 0.0996\end{array}$

Warning: Small sample sizes. Use Caution.

Welch Anova testing Means Equal, allowing Std Devs Not Equal

F Ratio DFNum DFDen Prob $>$ F

$9.5689-3 \quad 4.0478-0.0262$ 


\section{Exhibit A3. Statistical Comparisons of Chemical Composition Measurements of ARG-1 Samples by Dissolution Method for Each Oxide (Concentrations in Wt\% Oxides)}

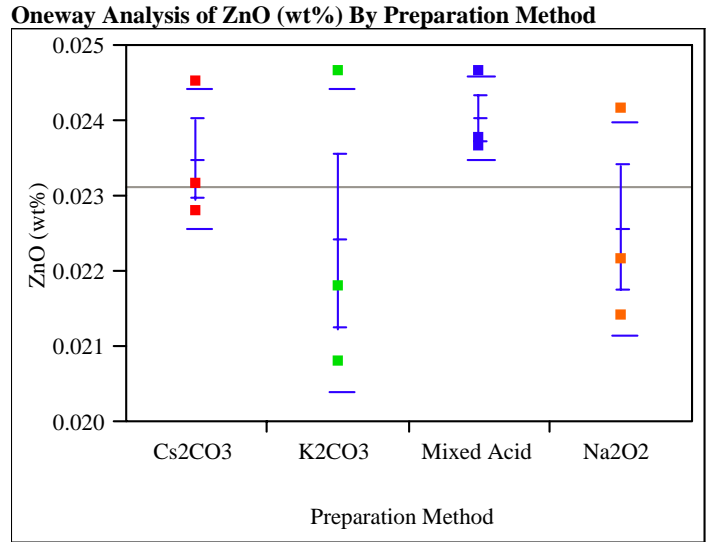

Means Comparisons

Comparisons for all pairs using Tukey-Kramer HSD

$$
\begin{array}{rr}
q^{*} & \text { Alpha } \\
3.20238 & 0.05
\end{array}
$$

Abs(Dif)-LSD Mixed Acid Cs2CO3 Na2O2 K2CO3

Mixed Acid $\quad-0.00350-0.00296-0.00204-0.00188$

Cs2CO3 $\quad-0.00296-0.00350-0.00258-0.00242$

$\begin{array}{lllll}\mathrm{Na} 2 \mathrm{O} 2 & -0.00204 & -0.00258 & -0.00350 & -0.00333\end{array}$

$\begin{array}{lllll}\mathrm{K} 2 \mathrm{CO} 3 & -0.00188 & -0.00242 & -0.00333 & -0.00350\end{array}$

Positive values show pairs of means that are significantly different.

Level Mean

Mixed Acid A 0.02402464

Cs2CO3 A 0.02348523

$\mathrm{Na} 2 \mathrm{O} 2$ A 0.02257237

K2CO3 A 0.02240640

Levels not connected by same letter are significantly different Tests that the Variances are Equal

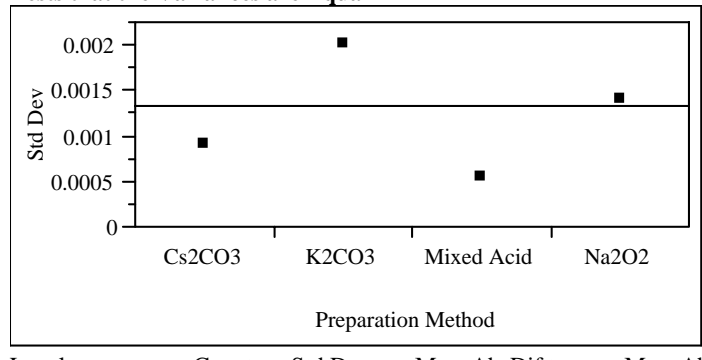

\begin{tabular}{|c|c|c|c|c|}
\hline Level & Count & Std Dev & $\begin{array}{r}\text { MeanAbsDif to } \\
\text { Mean }\end{array}$ & $\begin{array}{r}\text { MeanAbsDif to } \\
\text { Median }\end{array}$ \\
\hline $\mathrm{Cs} 2 \mathrm{CO} 3$ & 3 & 0.0009176 & 0.0006916 & 0.0007054 \\
\hline $\mathrm{K} 2 \mathrm{CO} 3$ & 3 & 0.0020033 & 0.0014938 & 0.0016182 \\
\hline Mixed Acid & 3 & 0.0005426 & 0.0004149 & 0.0003734 \\
\hline $\mathrm{Na} 2 \mathrm{O} 2$ & 3 & 0.0014156 & 0.0010512 & 0.0011618 \\
\hline Test & F Ratio & DFNum & DFDen & Prob $>$ F \\
\hline O'Brien[.5] & 0.9206 & 3 & 8 & 0.4735 \\
\hline $\begin{array}{l}\text { Brown- } \\
\text { Forsythe }\end{array}$ & 1.6086 & 3 & 8 & 0.2624 \\
\hline Levene & 2.2272 & 3 & 8 & 0.1 \\
\hline Bartlett & 0.9023 & 3 & t & 0.43 \\
\hline
\end{tabular}

Warning: Small sample sizes. Use Caution.

Welch Anova testing Means Equal, allowing Std Devs Not Equal

F Ratio DFNum DFDen Prob $>$ F

$\begin{array}{rrrr}1.1017 & 3 & 4.0547 & 0.4447\end{array}$

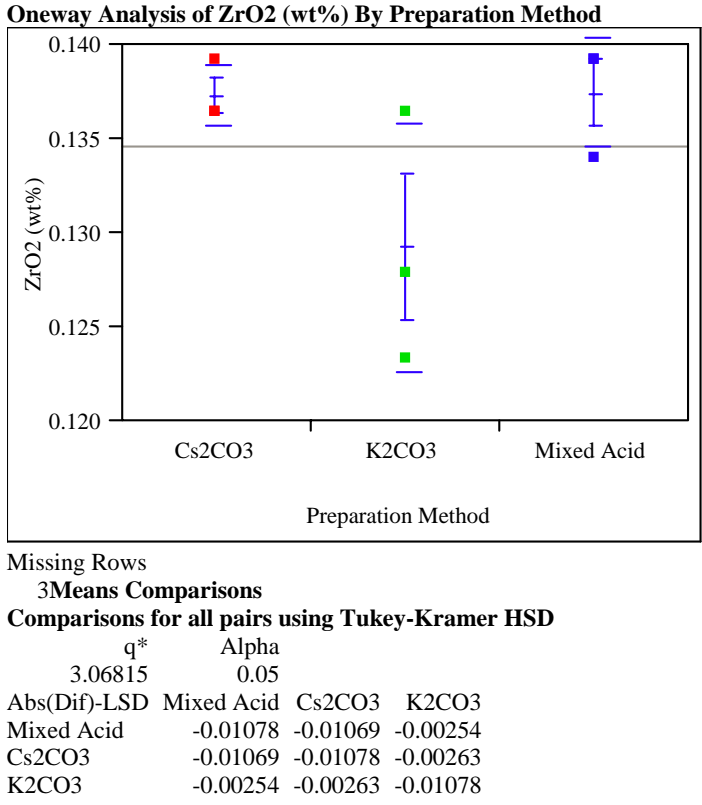

Positive values show pairs of means that are significantly different.

$\begin{array}{lrr}\text { Level } & & \text { Mean } \\ \text { Mixed Acid } & \text { A } & 0.13742139 \\ \text { Cs2CO3 } & \text { A } & 0.13733133 \\ \text { K2CO3 } & \text { A } & 0.12918151\end{array}$

Levels not connected by same letter are significantly different Tests that the Variances are Equal

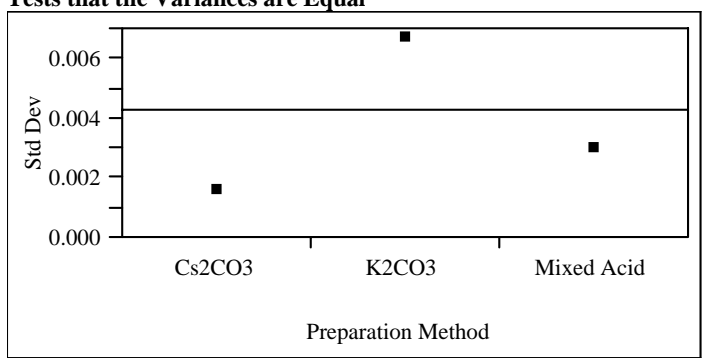

$\begin{array}{lrrrr}\text { Level } & \text { Count } & \text { Std Dev } & \begin{array}{r}\text { MeanAbsDif to } \\ \text { Mean }\end{array} & \begin{array}{r}\text { MeanAbsDif to } \\ \text { Median }\end{array} \\ \text { Cs2CO3 } & 3 & 0.0015598 & 0.0012007 & 0.0009005 \\ \text { K2CO3 } & 3 & 0.0066620 & 0.0048329 & 0.0058535 \\ \text { Mixed Acid } & 3 & 0.0029636 & 0.0022814 & 0.0017110 \\ \text { Test } & \text { F Ratio } & \text { DFNum } & \text { DFDen } & \text { Prob > F } \\ \text { O'Brien[.5] } & 1.3282 & 2 & 6 & 0.3330 \\ \text { Brown- } & 3.7234 & 2 & 6 & 0.0888 \\ \text { Forsythe } & & & & \\ \text { Levene } & 2.9553 & 2 & 6 & 0.1278 \\ \text { Bartlett } & 1.5577 & 2 & . & 0.2106\end{array}$

Warning: Small sample sizes. Use Caution.

Welch Anova testing Means Equal, allowing Std Devs Not Equal

F Ratio DFNum DFDen Prob $>$ F

$\begin{array}{rrrr}1.8016 & 2 & 3.3152 & 0.2954\end{array}$ 
Westinghouse Savannah River Company

WSRC-TR-2005-00396

Savannah River National Laboratory

Rev. 0

Analytical Development/Statistical Consulting Sections

Page 69 of 140

\section{Exhibit A4. Statistical Comparisons of Chemical Composition Measurements of Simulant Samples Across Preparation Methods by Oxide \\ (Concentrations in Wt\% Oxides)}

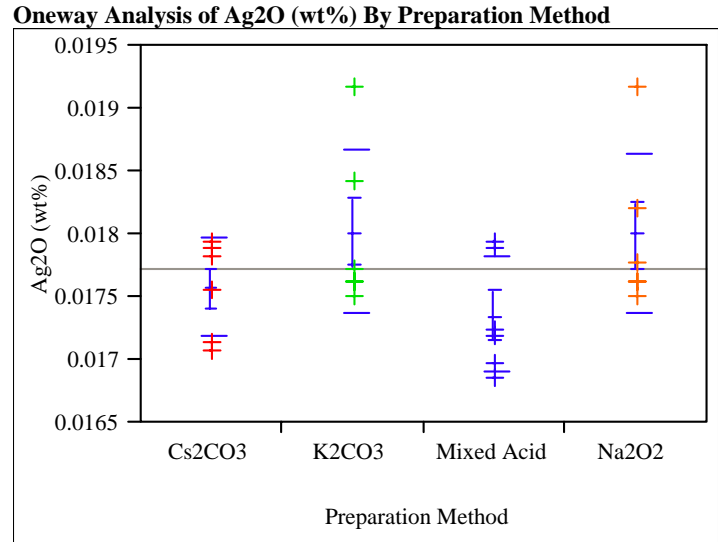

Means Comparisons

Comparisons for all pairs using Tukey-Kramer HSD

$$
\text { q* Alpha }
$$

Abs(Dif)-LSD K2CO3 Na2O2 Cs2CO3 Mixed Acid

$\begin{array}{lllll}\mathrm{K} 2 \mathrm{CO} 3 & -0.00088 & -0.00086 & -0.00044 & -0.00022\end{array}$

$\begin{array}{lllll}\mathrm{Na} 2 \mathrm{O} 2 & -0.00086 & -0.00088 & -0.00047 & -0.00025\end{array}$

$\begin{array}{lllll}\mathrm{Cs} 2 \mathrm{CO} 3 & -0.00044 & -0.00047 & -0.00088 & -0.00066\end{array}$

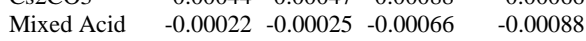

Positive values show pairs of means that are significantly different.

$$
\begin{array}{lrr}
\text { Level } & & \text { Mean } \\
\text { K2CO3 } & \text { A } & 0.01801075 \\
\text { Na2O2 } & \text { A } & 0.01798390 \\
\text { Cs2CO3 } & \text { A } & 0.01757212
\end{array}
$$

Mixed Acid A 0.01734833

Levels not connected by same letter are significantly different Tests that the Variances are Equal

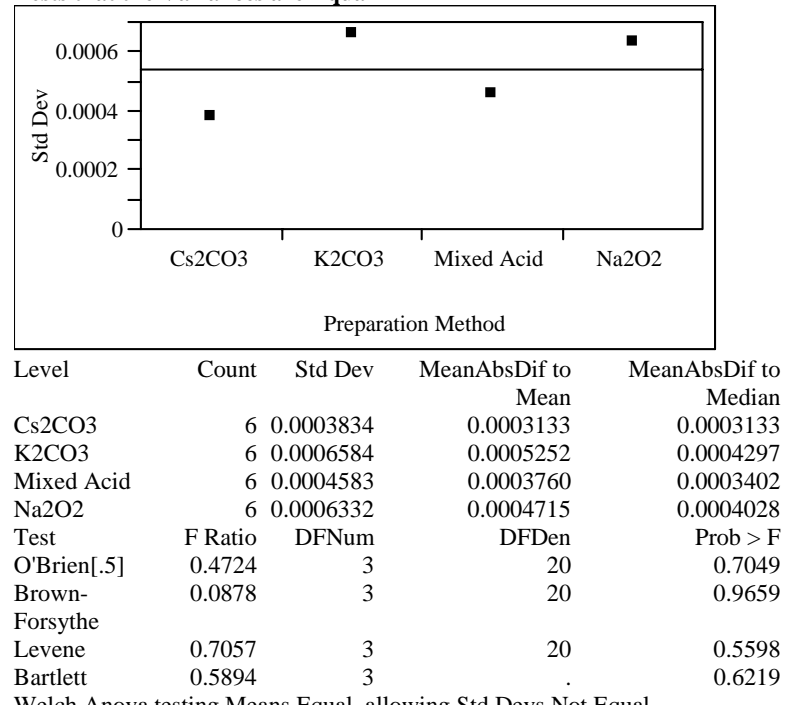

Oneway Analysis of Al2O3 (wt \%) By Preparation Method

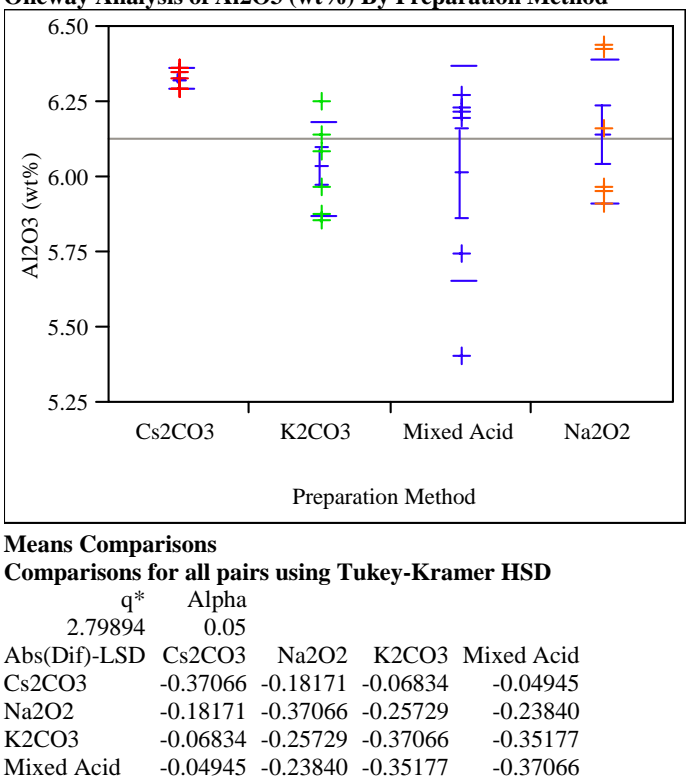

Positive values show pairs of means that are significantly different.

Level

$\mathrm{Na} 2 \mathrm{O} 2 \quad$ A 6.1440242

K2CO3 A 6.0306542

Mixed Acid A 6.0117592

Levels not connected by same letter are significantly different Tests that the Variances are Equal

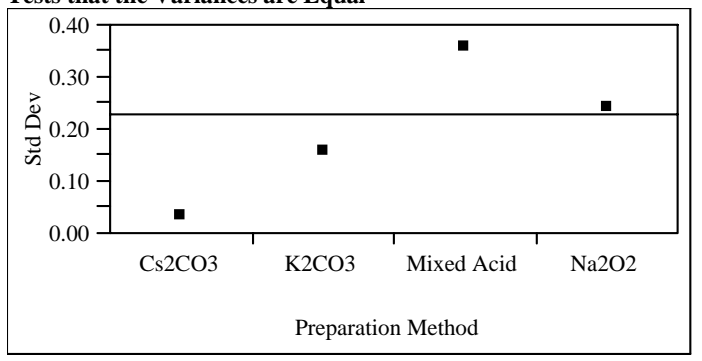

$\begin{array}{lrrrr}\text { Level } & \text { Count } & \text { Std Dev } & \begin{array}{r}\text { MeanAbsDif to } \\ \text { Mean }\end{array} & \begin{array}{r}\text { MeanAbsDif to } \\ \text { Median }\end{array} \\ \text { Cs2CO3 } & 6 & 0.0346695 & 0.0283425 & 0.0283425 \\ \text { K2CO3 } & 6 & 0.1564600 & 0.1291158 & 0.1291158 \\ \text { Mixed Acid } & 6 & 0.3565936 & 0.2918228 & 0.2298892 \\ \text { Na2O2 } & 6 & 0.2400235 & 0.1983975 & 0.1983975 \\ \text { Test } & \text { F Ratio } & \text { DFNum } & \text { DFDen } & \text { Prob > F } \\ \text { O'Brien[.5] } & 2.1655 & 3 & 20 & 0.1239 \\ \text { Brown- } & 1.4483 & 3 & 20 & 0.2586 \\ \text { Forsythe } & & & & \\ \text { Levene } & 7.4144 & 3 & 20 & 0.0016 \\ \text { Bartlett } & 5.4937 & 3 & . & 0.0009\end{array}$

Welch Anova testing Means Equal, allowing Std Devs Not Equal

F Ratio DFNum DFDen Prob $>$ F

$\begin{array}{llll}8.3251 & 3 & 8.7389 & 0.0062\end{array}$

F Ratio DFNum DFDen Prob $>$ F

$\begin{array}{rrrr}1.8658 & 3 & 10.867 & 0.1945\end{array}$ 


\section{Exhibit A4. Statistical Comparisons of Chemical Composition Measurements of Simulant Samples Across Preparation Methods by Oxide \\ (Concentrations in Wt\% Oxides)}

Oneway Analysis of B2O3 (wt\%) By Preparation Method

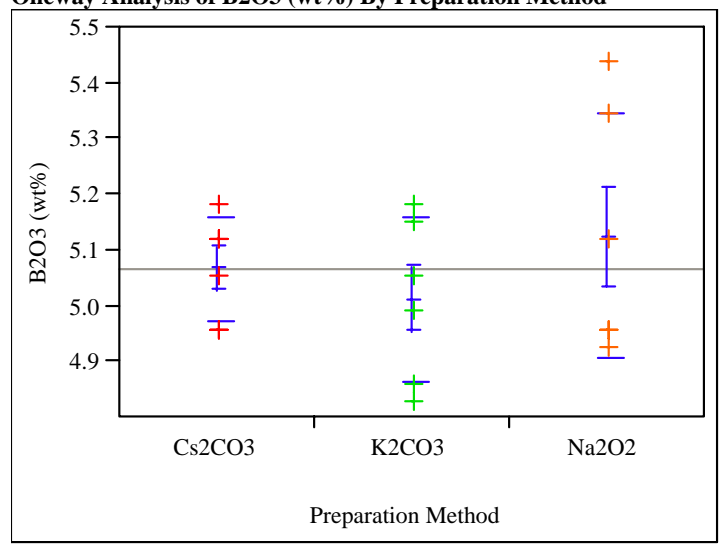

Missing Rows

6Means Comparisons

Comparisons for all pairs using Tukey-Kramer HSD

$$
\begin{array}{rr}
q^{*} & \text { Alpha } \\
2.59747 & 0.05
\end{array}
$$

Abs(Dif)-LSD Na2O2 Cs2CO3 K2CO3

Na2O2 $\quad-0.24288-0.18385-0.13018$

Cs2CO3 $\quad-0.18385-0.24288-0.18922$

$\begin{array}{llll}\mathrm{K} 2 \mathrm{CO} 3 & -0.13018 & -0.18922 & -0.24288\end{array}$

Positive values show pairs of means that are significantly different.

$$
\begin{array}{lrr}
\text { Level } & & \text { Mean } \\
\mathrm{Na} 2 \mathrm{O} 2 & \text { A } & 5.1250075 \\
\mathrm{Cs} 2 \mathrm{CO} 3 & \text { A } & 5.0659760 \\
\text { K2CO3 } & \text { A } & 5.0123110
\end{array}
$$

Levels not connected by same letter are significantly different Tests that the Variances are Equal

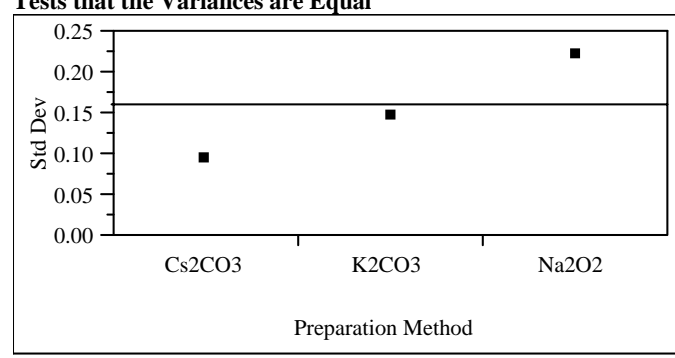

$\begin{array}{lrrrr}\text { Level } & \text { Count } & \text { Std Dev } & \begin{array}{r}\text { MeanAbsDif to } \\ \text { Mean }\end{array} & \begin{array}{r}\text { MeanAbsDif to } \\ \text { Median }\end{array} \\ \text { Cs2CO3 } & 6 & 0.0925780 & 0.0751310 & 0.0751310 \\ \text { K2CO3 } & 6 & 0.1463786 & 0.1180630 & 0.1180630 \\ \text { Na2O2 } & 6 & 0.2206669 & 0.1788833 & 0.1770945 \\ \text { Test } & \text { F } & \text { DFNum } & \text { DFDen } & \text { Prob }>\text { F } \\ & \text { Ratio } & & & \\ \text { O'Brien[.5] } & 3.0809 & 2 & 15 & 0.0757 \\ \text { Brown- } & 1.7411 & 2 & 15 & 0.2089 \\ \text { Forsythe } & & & & \\ \text { Levene } & 2.9133 & 2 & 15 & 0.0853 \\ \text { Bartlett } & 1.6122 & 2 & . & 0.1994 \\ \text { Went Ans }\end{array}$

Welch Anova testing Means Equal, allowing Std Devs Not Equal

F Ratio DFNum DFDen Prob $>$ F

$\begin{array}{llll}0.5500 & 2 & 9.0121 & 0.5952\end{array}$

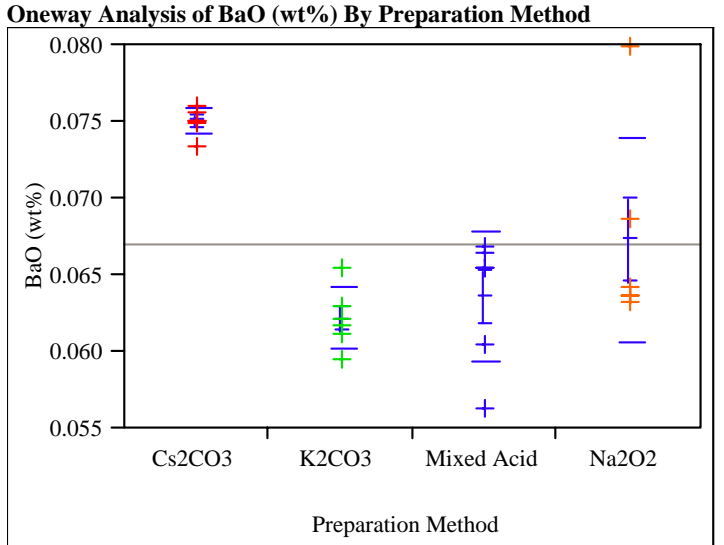

Means Comparisons

Comparisons for all pairs using Tukey-Kramer HSD

$$
\begin{array}{rr}
\text { q*}^{*} & \text { Alpha } \\
2.79894 & 0.05
\end{array}
$$

Abs(Dif)-LSD Cs2CO3 Na2O2 Mixed Acid K2CO3

$\begin{array}{lllll}\text { Cs2CO3 } & -0.00651 & 0.00127 & 0.00499 & 0.00633\end{array}$

$\mathrm{Na2O} 2 \quad 0.00127 \quad-0.00651 \quad-0.00278-0.00145$

$\begin{array}{lllll}\text { Mixed Acid } \quad 0.00499 & -0.00278 & -0.00651 & -0.00517\end{array}$

$\begin{array}{lllll}\text { K2CO3 } & 0.00633 & -0.00145 & -0.00517 & -0.00651\end{array}$

Positive values show pairs of means that are significantly different.

$$
\begin{array}{lrr}
\text { Level } & & \text { Mean } \\
\text { Cs2CO3 } & \text { A } & 0.07501019 \\
\text { Na2O2 } & \text { B } & 0.06723191 \\
\text { Mixed Acid } & \text { B } & 0.06351024 \\
\text { K2CO3 } & \text { B } & 0.06217044
\end{array}
$$

Levels not connected by same letter are significantly different Tests that the Variances are Equal

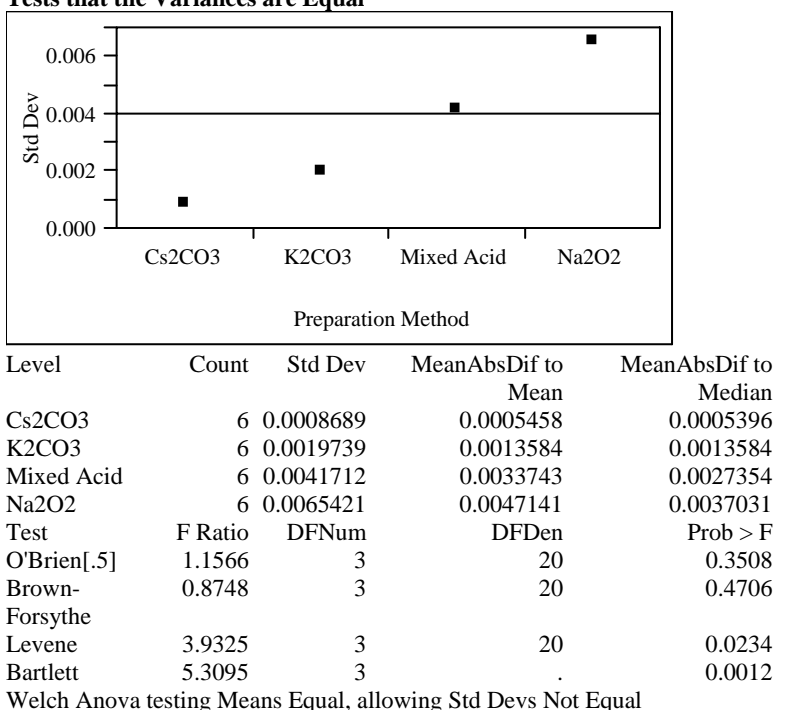

F Ratio DFNum DFDen Prob $>$ F

$\begin{array}{rrr}71.8507 & 3 & 9.3973<.0001\end{array}$ 


\section{Exhibit A4. Statistical Comparisons of Chemical Composition Measurements of Simulant Samples Across Preparation Methods by Oxide \\ (Concentrations in Wt\% Oxides)}

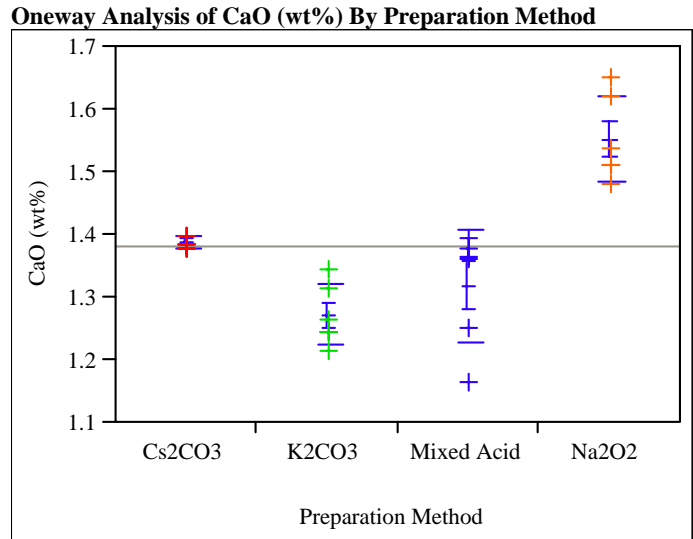

Means Comparisons

Comparisons for all pairs using Tukey-Kramer HSD

$$
\text { q* Alpha }
$$

Abs(Dif)-LSD Na2O2 Cs2CO3 Mixed Acid K2CO3

$\begin{array}{lllll}\mathrm{Na} 2 \mathrm{O} 2 & -0.09980 & 0.06531 & 0.13457 & 0.18191\end{array}$

$\begin{array}{lllll}\mathrm{Cs} 2 \mathrm{CO} 3 & 0.06531 & -0.09980 & -0.03054 & 0.01680\end{array}$

$\begin{array}{lllll}\text { Mixed Acid } \quad 0.13457 & -0.03054 & -0.09980 & -0.05246\end{array}$

$\begin{array}{lllll}\mathrm{K} 2 \mathrm{CO} 3 & 0.18191 & 0.01680 & -0.05246 & -0.09980\end{array}$

Positive values show pairs of means that are significantly different.

\begin{tabular}{|c|c|c|c|}
\hline Level & & & Mean \\
\hline $\mathrm{Na} 2 \mathrm{O} 2$ & A & & 1.5531120 \\
\hline Cs2CO3 & B & & 1.3880064 \\
\hline Mixed Acid & B & $\mathrm{C}$ & 1.3187460 \\
\hline K2CO3 & & C & 1.2714064 \\
\hline
\end{tabular}

Levels not connected by same letter are significantly different Tests that the Variances are Equal

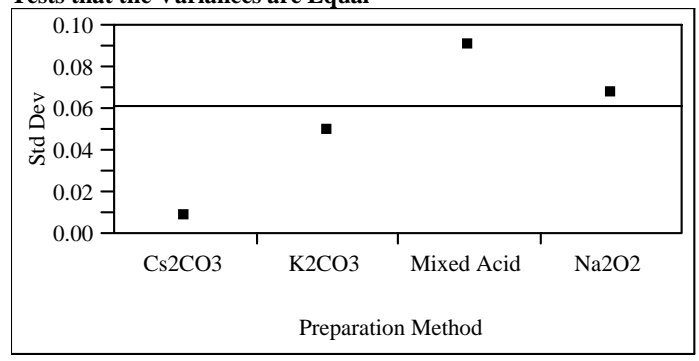

\begin{tabular}{|c|c|c|c|c|}
\hline Level & Count & Std Dev & $\begin{array}{r}\text { MeanAbsDif to } \\
\text { Mean }\end{array}$ & $\begin{array}{r}\text { MeanAbsDif to } \\
\text { Median }\end{array}$ \\
\hline Cs2CO3 & 6 & 0.0088493 & 0.0069960 & 0.0065296 \\
\hline $\mathrm{K} 2 \mathrm{CO} 3$ & 6 & 0.0492921 & 0.0394885 & 0.0368456 \\
\hline Mixed Acid & 6 & 0.0901534 & 0.0732248 & 0.0594660 \\
\hline $\mathrm{Na} 2 \mathrm{O} 2$ & 6 & 0.0679729 & 0.0559680 & 0.0513040 \\
\hline Test & F Ratio & DFNum & DFDen & Prob > \\
\hline O'Brien[.5] & 1.8217 & 3 & 20 & 0.175 \\
\hline $\begin{array}{l}\text { Brown- } \\
\text { Forsythe }\end{array}$ & 1.3360 & 3 & 20 & 0.2908 \\
\hline Levene & 6.0952 & 3 & 20 & 0.00 \\
\hline Bartlett & 5.2127 & 3 & & 0.001 \\
\hline
\end{tabular}

Welch Anova testing Means Equal, allowing Std Devs Not Equal

F Ratio DFNum DFDen Prob $>$ F

$\begin{array}{llll}20.8850 & 3 & 8.6408 & 0.0003\end{array}$

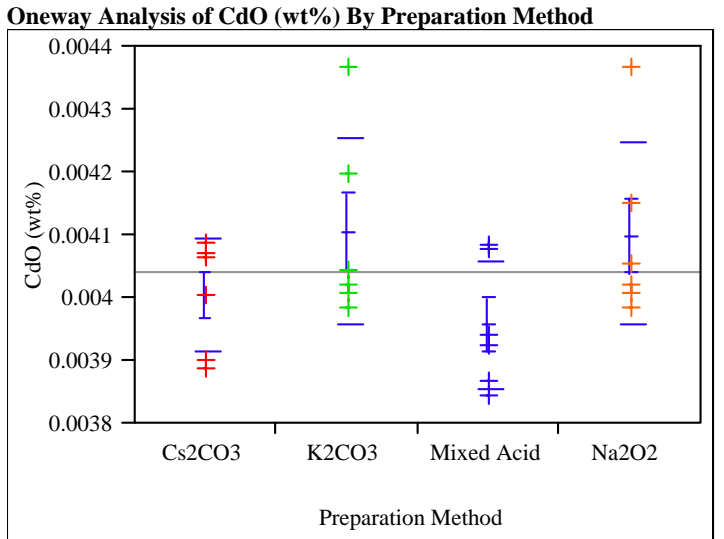

Means Comparisons

Comparisons for all pairs using Tukey-Kramer HSD

$$
\text { q* Alpha }
$$$$
2.79894 \quad 0.05
$$

Abs(Dif)-LSD K2CO3 Na2O2 Cs2CO3 Mixed Acid

K2CO3 $\quad-0.00020-0.00020-0.00010 \quad-0.00005$

$\mathrm{Na2O} 2 \quad-0.00020-0.00020-0.00011 \quad-0.00006$

$\begin{array}{lllll}\mathrm{Cs} 2 \mathrm{CO} 3 & -0.00010 & -0.00011 & -0.00020 & -0.00015\end{array}$

$\begin{array}{llll}\text { Mixed Acid } \quad-0.00005 & -0.00006 & -0.00015 & -0.00020\end{array}$

Positive values show pairs of means that are significantly different.

Level

K2CO3 A 0.00410466

$\mathrm{Na} 2 \mathrm{O} 2$ A 0.00409895

Cs2CO3 A 0.00400376

Mixed Acid A 0.00395617

Levels not connected by same letter are significantly different Tests that the Variances are Equal

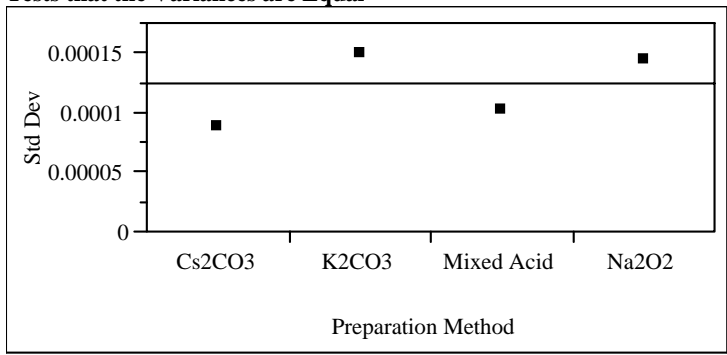

$\begin{array}{lrrrr}\text { Level } & \text { Count } & \text { Std Dev } & \begin{array}{r}\text { MeanAbsDif to } \\ \text { Mean }\end{array} & \begin{array}{r}\text { MeanAbsif to } \\ \text { Median }\end{array} \\ \text { Cs2CO3 } & 6 & 0.0000890 & 0.0000723 & 0.0000723 \\ \text { K2CO3 } & 6 & 0.0001500 & 0.0001193 & 0.0000990 \\ \text { Mixed Acid } & 6 & 0.0001030 & 0.0000831 & 0.0000781 \\ \text { Na2O2 } & 6 & 0.0001446 & 0.0001079 & 0.0000933 \\ \text { Test } & \text { F Ratio } & \text { DFNum } & \text { DFDen } & \text { Prob > F } \\ \text { O'Brien[.5] } & 0.4761 & 3 & 20 & 0.7024 \\ \text { Brown- } & 0.0973 & 3 & 20 & 0.9606 \\ \text { Forsythe } & & & & \\ \text { Levene } & 0.6913 & 3 & 20 & 0.5680 \\ \text { Bartlett } & 0.5763 & 3 & . & 0.6306\end{array}$

Welch Anova testing Means Equal, allowing Std Devs Not Equal

6306

F Ratio DFNum DFDen Prob $>$ F

$\begin{array}{llll}1.8413 & 3 & 10.882 & 0.1986\end{array}$ 


\section{Exhibit A4. Statistical Comparisons of Chemical Composition Measurements of Simulant Samples Across Preparation Methods by Oxide \\ (Concentrations in Wt\% Oxides)}

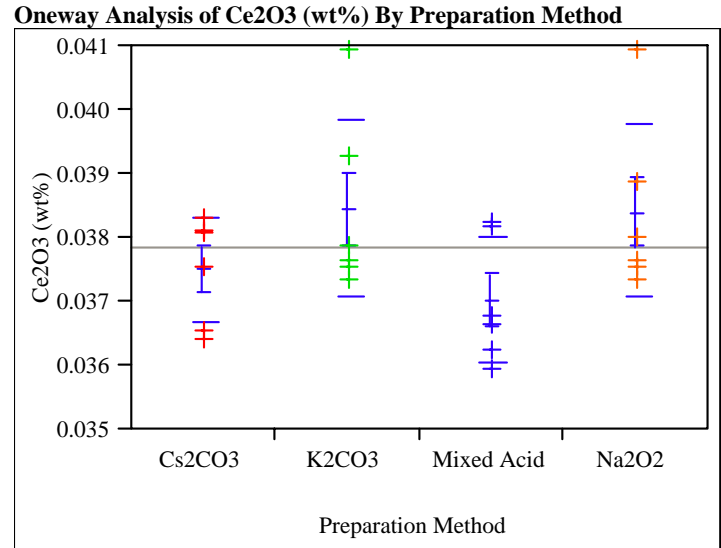

Means Comparisons

Comparisons for all pairs using Tukey-Kramer HSD

$\begin{array}{lrrrr}\mathrm{q}^{*} & \text { Alpha } & & & \\ 2.79894 & 0.05 & & & \\ \text { s(Dif)-LSD } & \text { K2CO3 } & \text { Na2O2 } & \text { Cs2CO3 } & \text { Mixed Acid } \\ 2 \mathrm{CO} 3 & -0.00188 & -0.00184 & -0.00094 & -0.00045 \\ 2 \mathrm{O} 2 & -0.00184 & -0.00188 & -0.00099 & -0.00050 \\ 2 \mathrm{CO} 3 & -0.00094 & -0.00099 & -0.00188 & -0.00140 \\ \text { ixed Acid } & -0.00045 & -0.00050 & -0.00140 & -0.00188\end{array}$

Positive values show pairs of means that are significantly different.

$$
\begin{array}{lrr}
\text { Level } & & \text { Mean } \\
\text { K2CO3 } & \text { A } & 0.03844792 \\
\text { Na2O2 } & \text { A } & 0.03839912 \\
\text { Cs2CO3 } & \text { A } & 0.03750112 \\
\text { Mixed Acid } & \text { A } & 0.03701308
\end{array}
$$

Levels not connected by same letter are significantly different Tests that the Variances are Equal

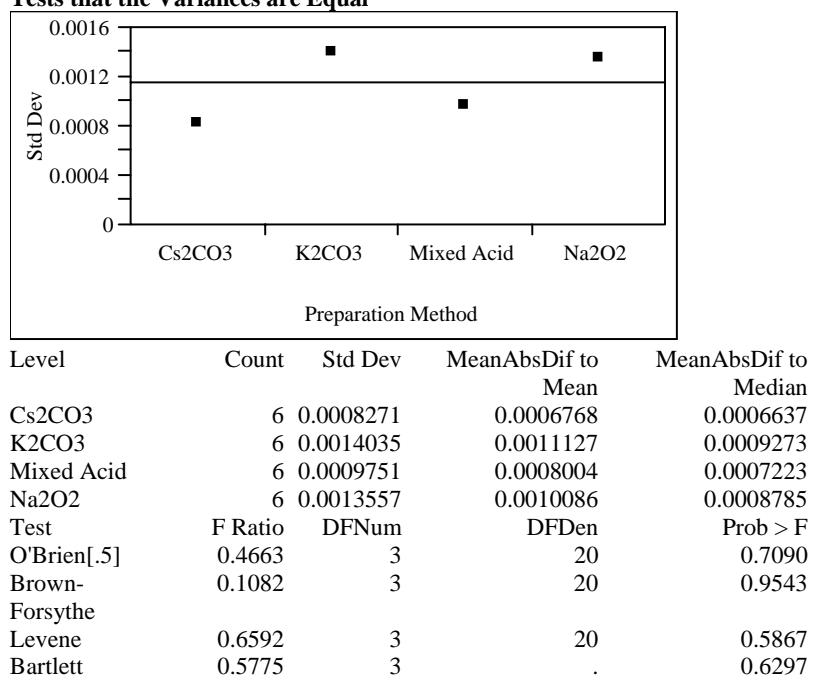

Oneway Analysis of $\mathrm{CoO}$ (wt\%) By Preparation Method

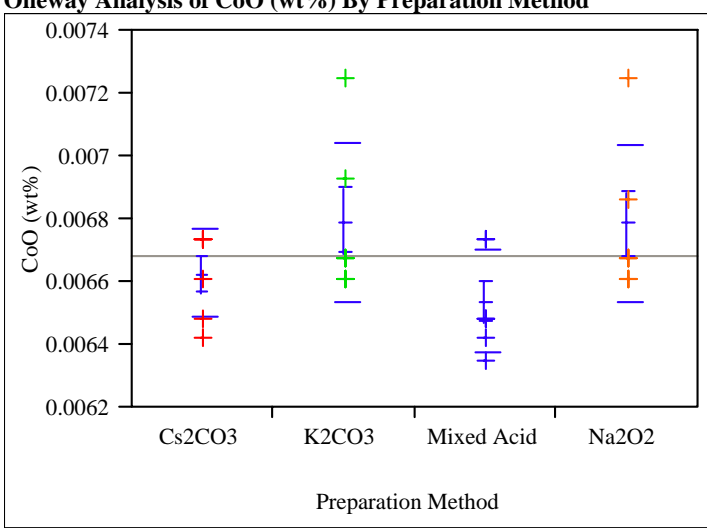

Means Comparisons

Comparisons for all pairs using Tukey-Kramer HSD q* Alpha

$\begin{array}{rrr}\text { Abs(Dif)-LSD } & \mathrm{K} 2 \mathrm{CO} 3 \mathrm{Na} 2 \mathrm{O} 2 & \mathrm{Cs} 2 \mathrm{CO} 3 \\ \text { Mixed Acid }\end{array}$

$\begin{array}{lllll}\text { K2CO3 } & -0.00033 & -0.00032 & -0.00017 & -0.00008\end{array}$

$\mathrm{Na2O} 2 \quad-0.00032-0.00033-0.00018 \quad-0.00009$

$\begin{array}{lllll}\mathrm{Cs} 2 \mathrm{CO} 3 & -0.00017 & -0.00018 & -0.00033 & -0.00025\end{array}$

$\begin{array}{llll}\text { Mixed Acid } \quad-0.00008 & -0.00009 & -0.00025 & -0.00033\end{array}$

Positive values show pairs of means that are significantly different.

Level

K2CO3 A 0.00679193

$\mathrm{Na} 2 \mathrm{O} 2$ A 0.00678133

Cs2CO3 A 0.00662240

Mixed Acid A 0.00653657

Levels not connected by same letter are significantly different Tests that the Variances are Equal

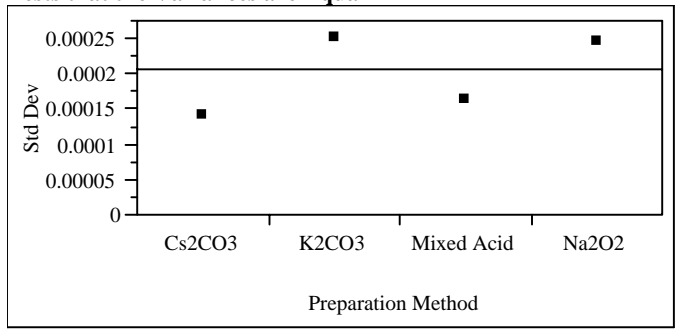

$\begin{array}{lrrrr}\text { Level } & \text { Count } & \text { Std Dev } & \begin{array}{r}\text { MeanAbsDif to } \\ \text { Mean }\end{array} & \begin{array}{r}\text { MeanAbsDif to } \\ \text { Median }\end{array} \\ \text { Cs2CO3 } & 6 & 0.0001417 & 0.0001166 & 0.0001166 \\ \text { K2CO3 } & 6 & 0.0002524 & 0.0001978 & 0.0001589 \\ \text { Mixed Acid } & 6 & 0.0001643 & 0.0001349 & 0.0001176 \\ \text { Na2O2 } & 6 & 0.0002468 & \begin{array}{r}0.0001837 \\ \text { DFDen }\end{array} & 0.0001483 \\ \text { Test } & \text { F Ratio } & \text { DFNum } & 20 & \text { Prob }>\text { F } \\ \text { O'Brien[.5] } & 0.5145 & 3 & 20 & 0.6770 \\ \text { Brown- } & 0.0941 & 3 & & 0.9624 \\ \text { Forsythe } & & & 20 & 0.5195 \\ \text { Levene } & 0.7789 & 3 & \text {. } & 0.5302 \\ \text { Bartlett } & 0.7363 & 3 & \end{array}$

F Ratio DFNum DFDen Prob $>$ F

$\begin{array}{llll}1.9620 & 3 & 10.829 & 0.1791\end{array}$

F Ratio DFNum DFDen Prob $>$ F $\begin{array}{llll}1.9349 & 3 & 10.876 & 0.1831\end{array}$ 


\section{Exhibit A4. Statistical Comparisons of Chemical Composition Measurements of Simulant Samples Across Preparation Methods by Oxide \\ (Concentrations in Wt\% Oxides)}

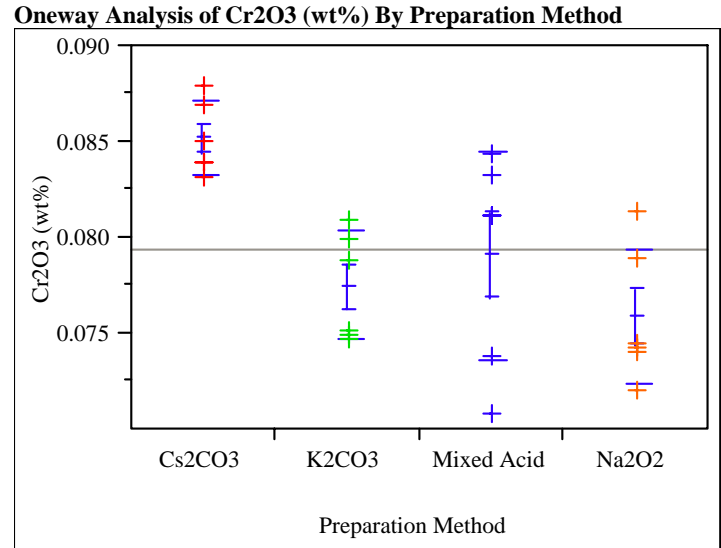

Means Comparisons

Comparisons for all pairs using Tukey-Kramer HSD

$$
\begin{array}{rr}
q^{*} & \text { Alpha } \\
2.79894 & 0.05
\end{array}
$$

Abs(Dif)-LSD Cs2CO3 Mixed Acid K2CO3 Na2O2

$\begin{array}{lllll}\mathrm{Cs} 2 \mathrm{CO} 3 & -0.00592 & 0.00014 & 0.00182 & 0.00336\end{array}$

$\begin{array}{lllll}\text { Mixed Acid } \quad 0.00014 & -0.00592 & -0.00424 & -0.00271\end{array}$

$\begin{array}{lllll}\text { K2CO3 } & 0.00182 & -0.00424 & -0.00592 & -0.00439\end{array}$

$\begin{array}{lllll}\mathrm{Na} 2 \mathrm{O} 2 & 0.00336 & -0.00271 & -0.00439 & -0.00592\end{array}$

Positive values show pairs of means that are significantly different.

$$
\begin{array}{lrr}
\text { Level } & \text { Mean } \\
\text { Cs2CO3 } & \text { A } & 0.08516256 \\
\text { Mixed Acid } & \text { B } & 0.07909692 \\
\text { K2CO3 } & \text { B } & 0.07741608 \\
\text { Na2O2 } & \text { B } & 0.07588140
\end{array}
$$

Levels not connected by same letter are significantly different Tests that the Variances are Equal

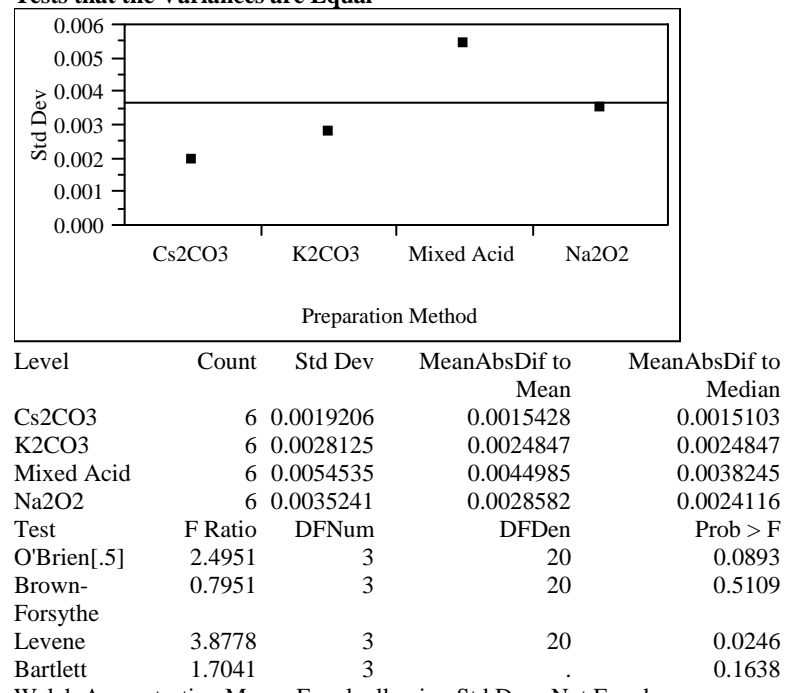

Welch Anova testing Means Equal, allowing Std Devs Not Equal

F Ratio DFNum DFDen Prob $>$ F

$\begin{array}{rrrr}15.0473 & 3 & 10.545 & 0.0004\end{array}$

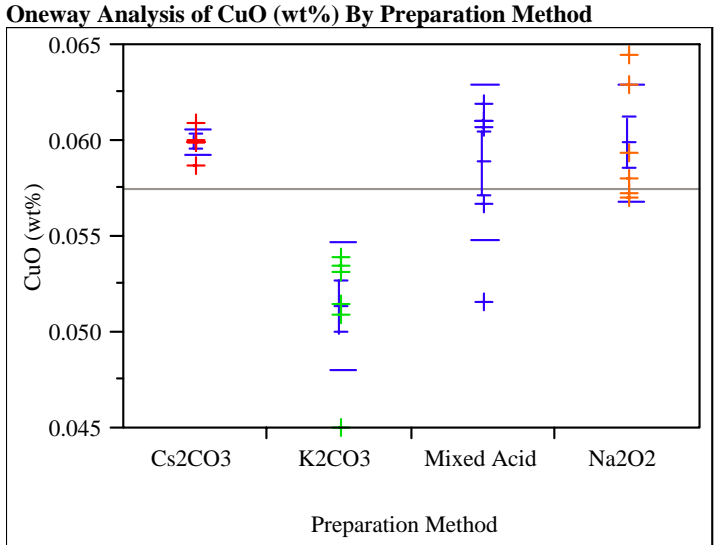

Means Comparisons

Comparisons for all pairs using Tukey-Kramer HSD

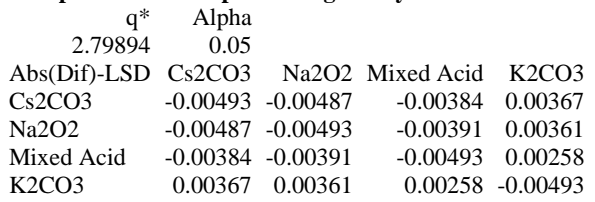

Positive values show pairs of means that are significantly different.

$$
\begin{aligned}
& \text { Level Mean } \\
& \text { Cs2CO3 A } 0.05994036 \\
& \mathrm{Na} 2 \mathrm{O} 2 \quad \text { A } 0.05987777 \\
& \text { Mixed Acid A } 0.05885546 \\
& \text { K2CO3 B } 0.05134466
\end{aligned}
$$

Levels not connected by same letter are significantly different Tests that the Variances are Equal

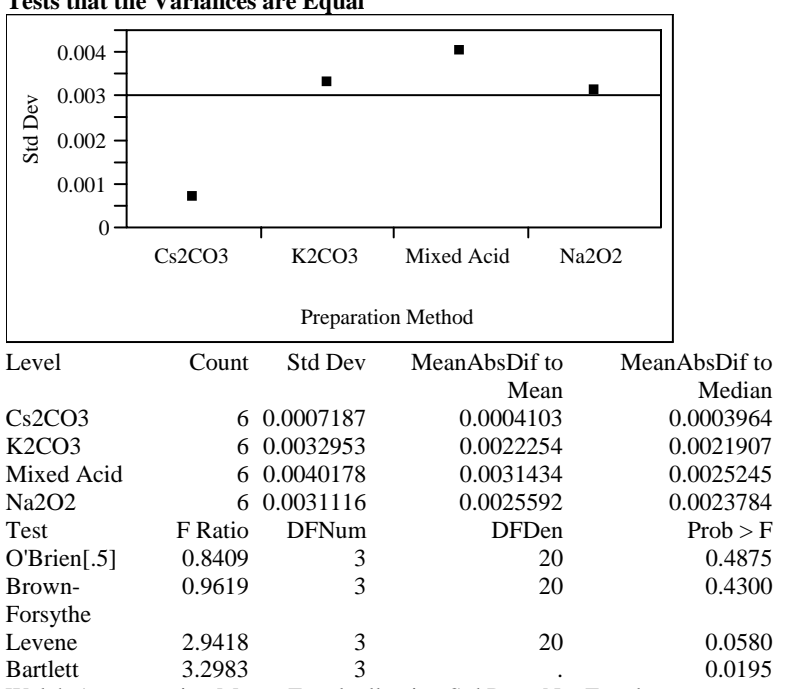

Welch Anova testing Means Equal, allowing Std Devs Not Equal

F Ratio DFNum DFDen Prob $>$ F

$\begin{array}{llll}11.3824 & 3 & 8.9803 & 0.0021\end{array}$ 
Westinghouse Savannah River Company

WSRC-TR-2005-00396

Savannah River National Laboratory

Rev. 0

Analytical Development/Statistical Consulting Sections

Page 74 of 140

\section{Exhibit A4. Statistical Comparisons of Chemical Composition Measurements of Simulant Samples Across Preparation Methods by Oxide \\ (Concentrations in Wt\% Oxides)}

Oneway Analysis of Fe2O3 (wt \%) By Preparation Method

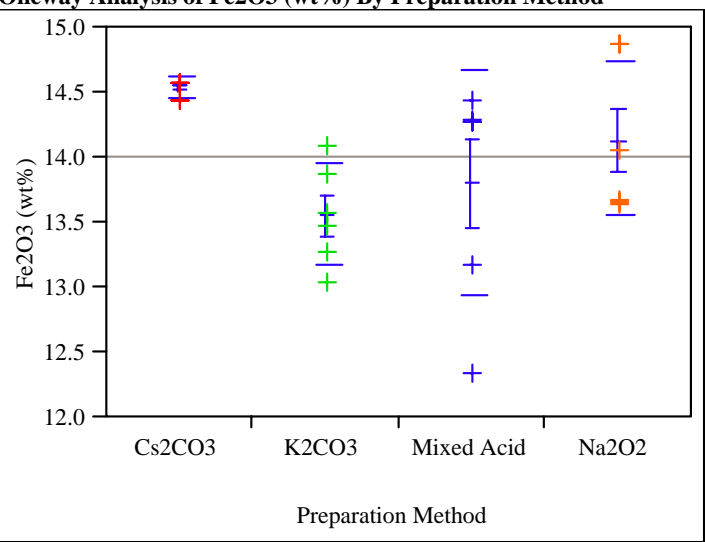

Means Comparisons

Comparisons for all pairs using Tukey-Kramer HSD

Abs(Dif)-LSD Cs2CO3 Na2O2 Mixed Acid K2CO3

$\begin{array}{lllll}\mathrm{Cs} 2 \mathrm{CO} 3 & -0.89753 & -0.49006 & -0.16361 & 0.08420\end{array}$

$\mathrm{Na2O} 2 \quad-0.49006-0.89753 \quad-0.57108-0.32326$

Mixed Acid $\quad-0.16361-0.57108 \quad-0.89753-0.64971$

$\begin{array}{lrrrr}\mathrm{K} 2 \mathrm{CO} 3 & 0.08420 & -0.32326 & -0.64971 & -0.89753\end{array}$

Positive values show pairs of means that are significantly different.

Level Mean

Cs2CO3 A 14.535283

$\mathrm{Na2O} 2 \quad$ A B 14.127819

Mixed Acid A B 13.801371

K2CO3 B 13.553556

Levels not connected by same letter are significantly different Tests that the Variances are Equal

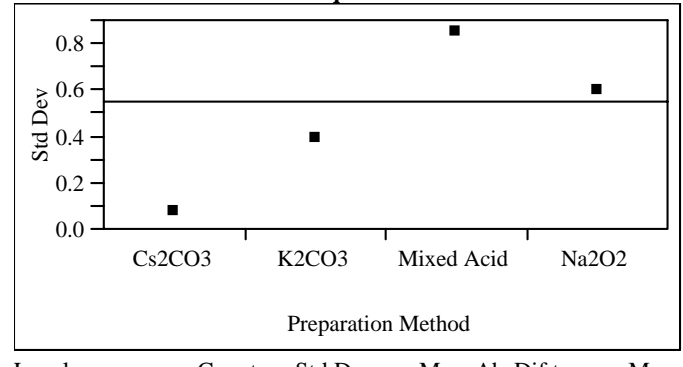

\begin{tabular}{|c|c|c|c|c|}
\hline Level & Count & Std Dev & $\begin{array}{r}\text { MeanAbsDif to } \\
\text { Mean }\end{array}$ & $\begin{array}{r}\text { MeanAbsDif to } \\
\text { Median }\end{array}$ \\
\hline $\mathrm{Cs} 2 \mathrm{CO} 3$ & 6 & 0.0738294 & 0.0635422 & 0.0476567 \\
\hline K2CO3 & 6 & 0.3870238 & 0.2954713 & 0.2954713 \\
\hline Mixed Acid & 6 & 0.8517781 & 0.6941988 & 0.5385203 \\
\hline $\mathrm{Na} 2 \mathrm{O} 2$ & 6 & 0.5942750 & 0.4940408 & 0.4694182 \\
\hline Test & F Ratio & DFNum & DFDen & Prob $>$ F \\
\hline O'Brien[.5] & 2.0252 & 3 & 20 & 0.1427 \\
\hline $\begin{array}{l}\text { Brown- } \\
\text { Forsythe }\end{array}$ & 1.3226 & 3 & 20 & 0.2949 \\
\hline Levene & 6.9160 & 3 & 20 & 0.002 \\
\hline Bartlett & 5.7967 & 3 & & $0.00 c$ \\
\hline
\end{tabular}

Welch Anova testing Means Equal, allowing Std Devs Not Equal

F Ratio DFNum DFDen Prob $>$ F

$\begin{array}{llll}12.5719 & 3 & 8.6429 & 0.0016\end{array}$
Oneway Analysis of K2O (wt\%) By Preparation Method

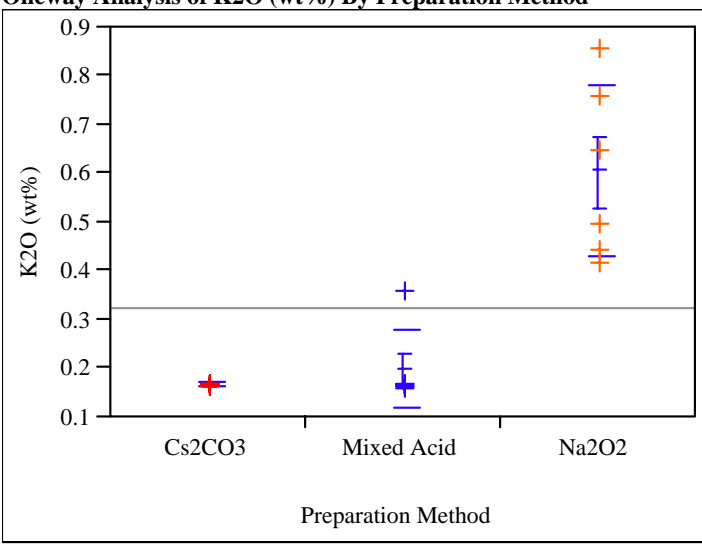

Missing Rows

6Means Comparisons

Comparisons for all pairs using Tukey-Kramer HSD

$$
\begin{array}{rr}
\mathrm{q}^{*} & \text { Alpha } \\
2.59747 & 0.05
\end{array}
$$

Abs(Dif)-LSD Na2O2 Mixed Acid Cs2CO3

$\begin{array}{llll}\mathrm{Na} 2 \mathrm{O} 2 & -0.16986 & 0.23559 & 0.26681\end{array}$

Mixed Acid $\quad 0.23559 \quad-0.16986-0.13864$

$\begin{array}{llll}\mathrm{Cs} 2 \mathrm{CO} 3 & 0.26681 & -0.13864 & -0.16986\end{array}$

Positive values show pairs of means that are significantly different.

$$
\begin{array}{lrr}
\text { Level } & & \text { Mean } \\
\text { Na2O2 } & \text { A } & 0.60410690 \\
\text { Mixed Acid } & \text { B } & 0.19865862 \\
\text { Cs2CO3 } & \text { B } & 0.16743940
\end{array}
$$

Levels not connected by same letter are significantly different Tests that the Variances are Equal

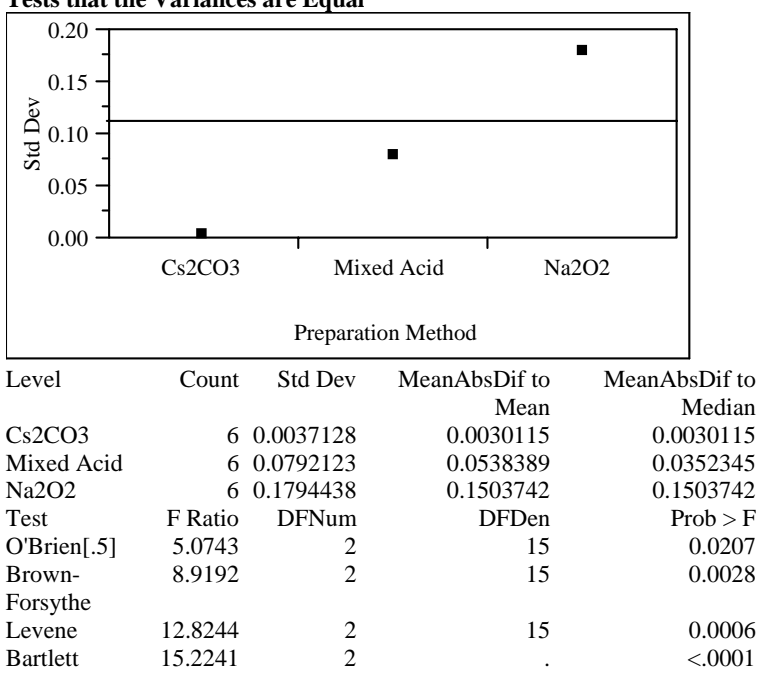

Welch Anova testing Means Equal, allowing Std Devs Not Equal

F Ratio DFNum DFDen Prob $>$ F

$\begin{array}{llll}16.5647 & 2 & 6.6841 & 0.0026\end{array}$ 


\section{Exhibit A4. Statistical Comparisons of Chemical Composition Measurements of Simulant Samples Across Preparation Methods by Oxide \\ (Concentrations in Wt\% Oxides)}

Oneway Analysis of La2O3 (wt\%) By Preparation Method

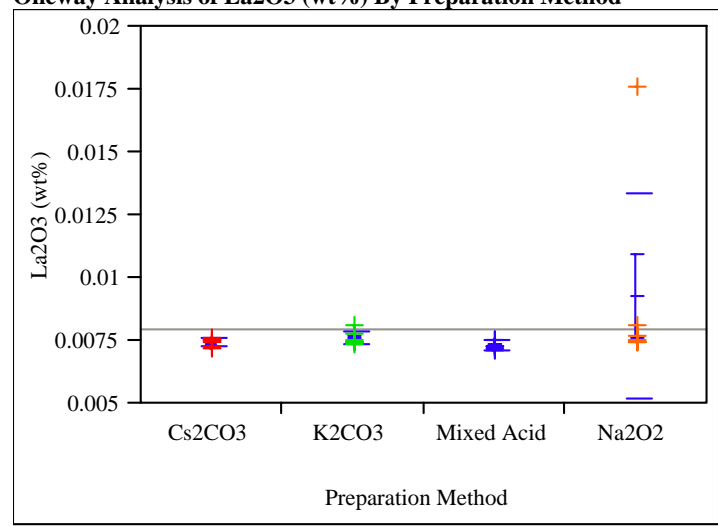

Means Comparisons

Comparisons for all pairs using Tukey-Kramer HSD

q* Alpha

0.05

Abs(Dif)-LSD Na2O2 K2CO3 Cs2CO3 Mixed Acid

$\begin{array}{lllll}\mathrm{Na} 2 \mathrm{O} 2 & -0.00330 & -0.00161 & -0.00144 & -0.00134\end{array}$

$\begin{array}{lllll}\mathrm{K} 2 \mathrm{CO} 3 & -0.00161 & -0.00330 & -0.00313 & -0.00303\end{array}$

$\begin{array}{lllll}\mathrm{Cs} 2 \mathrm{CO} 3 & -0.00144 & -0.00313 & -0.00330 & -0.00319\end{array}$

$\begin{array}{llll}\text { Mixed Acid } \quad-0.00134 & -0.00303 & -0.00319 & -0.00330\end{array}$

Positive values show pairs of means that are significantly different.

$\begin{array}{lrr}\text { Level } & & \text { Mean } \\ \text { Na2O2 } & \text { A } & 0.00930421 \\ \text { K2CO3 } & \text { A } & 0.00761343 \\ \text { Cs2CO3 } & \text { A } & 0.00744728 \\ \text { Mixed Acid } & \text { A } & 0.00733977\end{array}$

Levels not connected by same letter are significantly different Tests that the Variances are Equal

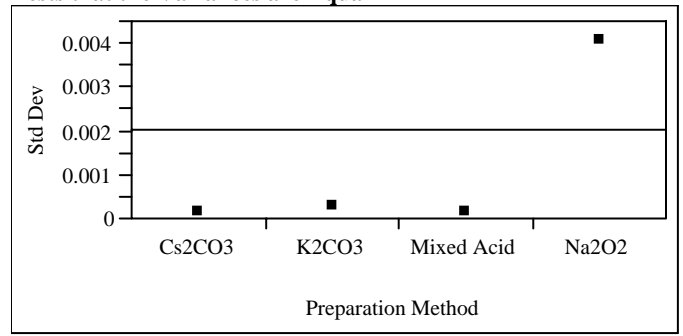

$\begin{array}{lrrrr}\text { Level } & \text { Count } & \text { Std Dev } & \begin{array}{r}\text { MeanAbsDif to } \\ \text { Mean }\end{array} & \begin{array}{r}\text { MeanAbsif to } \\ \text { Median }\end{array} \\ \text { Cs2CO3 } & 6 & 0.0001700 & 0.0001368 & 0.0001368 \\ \text { K2CO3 } & 6 & 0.0002761 & 0.0002215 & 0.0001857 \\ \text { Mixed Acid } & 6 & 0.0001795 & 0.0001499 & 0.0001271 \\ \text { Na2O2 } & 6 & 0.0040677 & 0.0027626 & 0.0018374 \\ \text { Test } & \text { F Ratio } & \text { DFNum } & \text { DFDen } & \text { Prob }>\text { F } \\ \text { O'Brien[.5] } & 1.2382 & 3 & 20 & 0.3221 \\ \text { Brown- } & 1.0707 & 3 & 20 & 0.3838 \\ \text { Forsythe } & & & & \\ \text { Levene } & 5.4444 & 3 & 20 & 0.0067 \\ \text { Bartlett } & 19.1699 & 3 & . & <.0001 \\ \text { Wenth } & & 3 & & \end{array}$

Welch Anova testing Means Equal, allowing Std Devs Not Equal

F Ratio DFNum DFDen Prob $>$ F

$\begin{array}{llll}1.6251 & 3 & 10.524 & 0.2423\end{array}$
Oneway Analysis of Li2O (wt\%) By Preparation Method

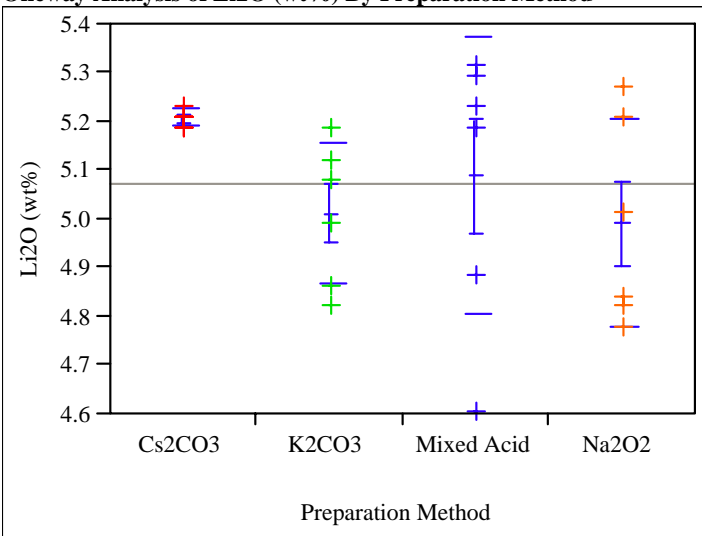

Means Comparisons

Comparisons for all pairs using Tukey-Kramer HSD q* Alpha

$2.79894 \quad 0.05$

Abs(Dif)-LSD Cs2CO3 Mixed Acid K2CO3 Na2O2

$\begin{array}{lllll}\text { Cs2CO3 } & -0.30862 & -0.19021 & -0.11486 & -0.09333\end{array}$

$\begin{array}{lllll}\text { Mixed Acid } & -0.19021 & -0.30862 & -0.23327 & -0.21174\end{array}$

$\begin{array}{lllll}\mathrm{K} 2 \mathrm{CO} 3 & -0.11486 & -0.23327 & -0.30862 & -0.28709\end{array}$

$\begin{array}{lllll}\mathrm{Na} 2 \mathrm{O} 2 & -0.09333 & -0.21174 & -0.28709 & -0.30862\end{array}$

Positive values show pairs of means that are significantly different.

Level Mean

Cs2CO3 A 5.2064298

Mixed Acid A 5.0880203

K2CO3 A 5.0126688

$\mathrm{Na} 2 \mathrm{O} 2$ A 4.9911398

Levels not connected by same letter are significantly different Tests that the Variances are Equal

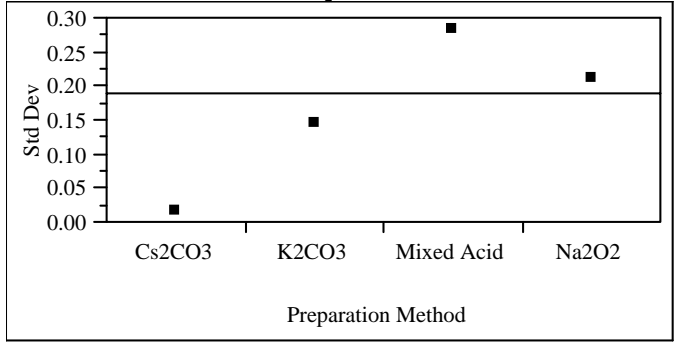

$\begin{array}{lrrrr}\text { Level } & \text { Count } & \text { Std Dev } & \begin{array}{r}\text { MeanAbsDif to } \\ \text { Mean }\end{array} & \begin{array}{r}\text { MeanAbsDif to } \\ \text { Median }\end{array} \\ \text { Cs2CO3 } & 6 & 0.0162064 & 0.0119606 & 0.0107645 \\ \text { K2CO3 } & 6 & 0.1456460 & 0.1184095 & 0.1184095 \\ \text { Mixed Acid } & 6 & 0.2822407 & 0.2272506 & 0.1937610 \\ \text { Na2O2 } & 6 & 0.2115619 & 0.1758202 & 0.1758202 \\ \text { Test } & \text { F Ratio } & \text { DFNum } & \text { DFDen } & \text { Prob > F } \\ \text { O'Brien[.5] } & 1.9805 & 3 & 20 & 0.1494 \\ \text { Brown- } & 2.3358 & 3 & 20 & 0.1045 \\ \text { Forsythe } & & & & \\ \text { Levene } & 6.8600 & 3 & 20 & 0.0023 \\ \text { Bartlett } & 6.9071 & 3 & . & 0.0001\end{array}$

Welch Anova testing Means Equal, allowing Std Devs Not Equal

F Ratio DFNum DFDen Prob $>$ F

$\begin{array}{llll}5.0444 & 3 & 8.4504 & 0.0277\end{array}$ 


\section{Exhibit A4. Statistical Comparisons of Chemical Composition Measurements of Simulant Samples Across Preparation Methods by Oxide \\ (Concentrations in Wt\% Oxides)}

Oneway Analysis of MgO (wt\%) By Preparation Method

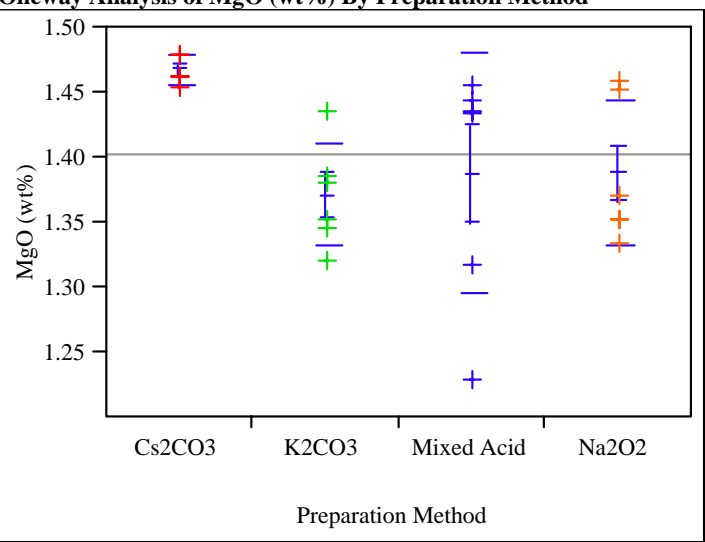

Means Comparisons

Comparisons for all pairs using Tukey-Kramer HSD $\mathrm{q}^{*} \quad$ Alpha

Abs(Dif)-LSD Cs2CO3 Na2O2 Mixed Acid K2CO3

$\begin{array}{lllll}\mathrm{Cs} 2 \mathrm{CO} 3 & -0.09288 & -0.01356 & -0.01217 & 0.00330\end{array}$

$\mathrm{Na2O} 2 \quad-0.01356-0.09288-0.09150 \quad-0.07602$

Mixed Acid $\quad-0.01217 \quad-0.09150 \quad-0.09288-0.07740$

$\begin{array}{lllll}\mathrm{K} 2 \mathrm{CO} 3 & 0.00330 & -0.07602 & -0.07740 & -0.09288\end{array}$

Positive values show pairs of means that are significantly different.

Level Mean

Cs2CO3 A 1.4667664

$\mathrm{Na} 2 \mathrm{O} 2 \quad$ A B 1.3874443

Mixed Acid A B 1.3860624

K2CO3 B 1.3705850

Levels not connected by same letter are significantly different Tests that the Variances are Equal

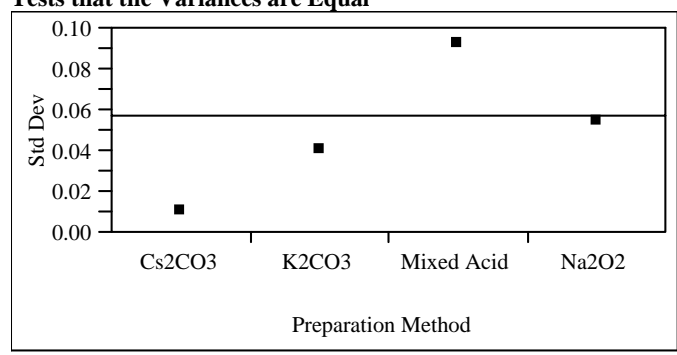

$\begin{array}{lrrrr}\text { Level } & \text { Count } & \text { Std Dev } & \begin{array}{r}\text { MeanAbsDif to } \\ \text { Mean }\end{array} & \begin{array}{r}\text { MeanAbsDif to } \\ \text { Median }\end{array} \\ \text { Cs2CO3 } & 6 & 0.0101550 & 0.0082915 & 0.0069096 \\ \text { K2CO3 } & 6 & 0.0402356 & 0.0306786 & 0.0306786 \\ \text { Mixed Acid } & 6 & 0.0923863 & 0.0755448 & 0.0594224 \\ \text { Na2O2 } & 6 & 0.0543727 & 0.0456954 & 0.0403520 \\ \text { Test } & \text { F Ratio } & \text { DFNum } & \text { DFDen } & \text { Prob > F } \\ \text { O'Brien[.5] } & 2.1623 & 3 & 20 & 0.1243 \\ \text { Brown- } & 1.2010 & 3 & 20 & 0.3349 \\ \text { Forsythe } & & & & \\ \text { Levene } & 7.2160 & 3 & 20 & 0.0018 \\ \text { Bartlett } & 5.1411 & 3 & . & 0.0015 \\ \text { Wenth } & & & & \end{array}$

Welch Anova testing Means Equal, allowing Std Devs Not Equal

F Ratio DFNum DFDen Prob > F

$\begin{array}{llll}13.4859 & 3 & 8.8808 & 0.0012\end{array}$
Oneway Analysis of MnO (wt\%) By Preparation Method

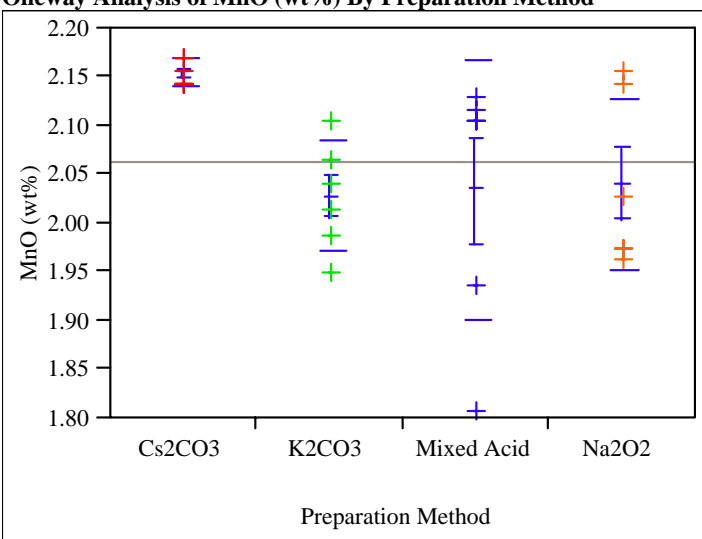

Means Comparisons

Comparisons for all pairs using Tukey-Kramer HSD $q^{*} \quad$ Alpha

$2.79894 \quad 0.05$

Abs(Dif)-LSD Cs2CO3 Na2O2 Mixed Acid K2CO3

$\begin{array}{lllll}\text { Cs2CO3 } & -0.13605 & -0.02200 & -0.01554 & -0.00908\end{array}$

$\begin{array}{lllll}\mathrm{Na} 2 \mathrm{O} 2 & -0.02200 & -0.13605 & -0.12960 & -0.12314\end{array}$

$\begin{array}{llll}\text { Mixed Acid } \quad-0.01554 & -0.12960 & -0.13605 & -0.12960\end{array}$

$\begin{array}{lllll}\text { K2CO3 } & -0.00908 & -0.12314 & -0.12960 & -0.13605\end{array}$

Positive values show pairs of means that are significantly different.

Level Mean

Cs2CO3 A 2.1541520

$\mathrm{Na} 2 \mathrm{O} 2$ A 2.0400960

Mixed Acid A 2.0336400

K2CO3 A 2.0271840

Levels not connected by same letter are significantly different Tests that the Variances are Equal

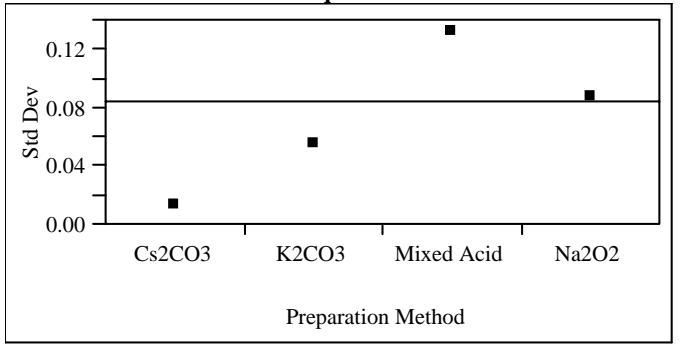

\begin{tabular}{|c|c|c|c|c|}
\hline Level & Count & Std Dev & $\begin{array}{r}\text { MeanAbsDif to } \\
\text { Mean }\end{array}$ & $\begin{array}{r}\text { MeanAbsDif to } \\
\text { Median }\end{array}$ \\
\hline Cs2CO3 & 6 & 0.0126950 & 0.0107600 & 0.0107600 \\
\hline K2CO3 & 6 & 0.0553863 & 0.0430400 & 0.0430400 \\
\hline Mixed Acid & 6 & 0.1318669 & 0.1076000 & 0.0839280 \\
\hline $\mathrm{Na} 2 \mathrm{O} 2$ & 6 & 0.0879534 & 0.0731680 & 0.0688640 \\
\hline Test & F Ratio & DFNum & DFDen & Prob $>F$ \\
\hline O'Brien[.5] & 2.1062 & 3 & 20 & 0.1315 \\
\hline $\begin{array}{l}\text { Brown- } \\
\text { Forsythe }\end{array}$ & 1.2511 & 3 & 20 & 0.3178 \\
\hline Levene & 7.2527 & 3 & 20 & 0.0018 \\
\hline Bartlett & 5.5947 & 3 & . & 0.0008 \\
\hline
\end{tabular}

Welch Anova testing Means Equal, allowing Std Devs Not Equal

F Ratio DFNum DFDen Prob $>$ F

$\begin{array}{llll}12.4617 & 3 & 8.7536 & 0.0016\end{array}$ 
Westinghouse Savannah River Company

WSRC-TR-2005-00396

Savannah River National Laboratory

Rev. 0

Analytical Development/Statistical Consulting Sections

Page 77 of 140

\section{Exhibit A4. Statistical Comparisons of Chemical Composition Measurements of Simulant Samples Across Preparation Methods by Oxide \\ (Concentrations in Wt\% Oxides)}

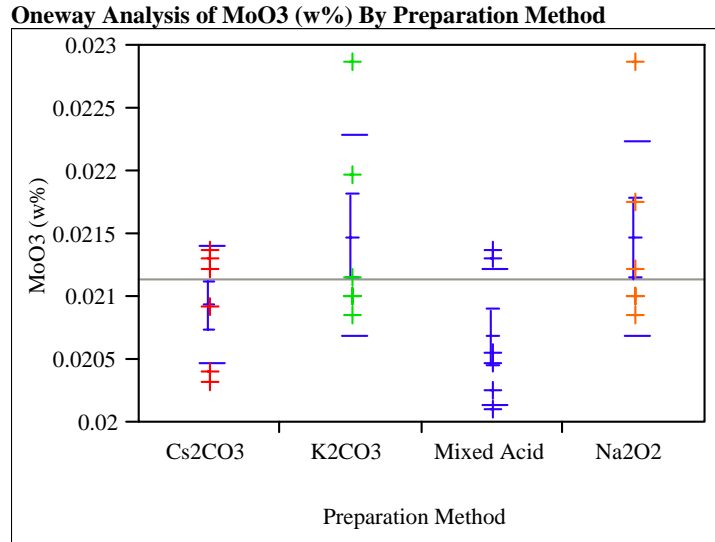

Means Comparisons

Comparisons for all pairs using Tukey-Kramer HSD

$$
\text { q* Alpha }
$$

Abs(Dif)-LSD K2CO3 Na2O2 Cs2CO3 Mixed Acid

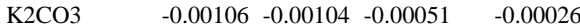

$\begin{array}{lllll}\mathrm{Na} 2 \mathrm{O} 2 & -0.00104 & -0.00106 & -0.00054 & -0.00029\end{array}$

$\begin{array}{lllll}\mathrm{Cs} 2 \mathrm{CO} 3 & -0.00051 & -0.00054 & -0.00106 & -0.00081\end{array}$

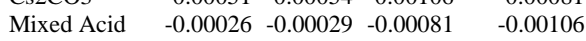

Positive values show pairs of means that are significantly different.

$$
\begin{array}{lrr}
\text { Level } & & \text { Mean } \\
\text { K2CO3 } & \text { A } & 0.02147929 \\
\mathrm{Na} 2 \mathrm{O} 2 & \text { A } & 0.02145429 \\
\mathrm{Cs} 2 \mathrm{CO} 3 & \text { A } & 0.02092918
\end{array}
$$

Mixed Acid A 0.02067913

Levels not connected by same letter are significantly different Tests that the Variances are Equal

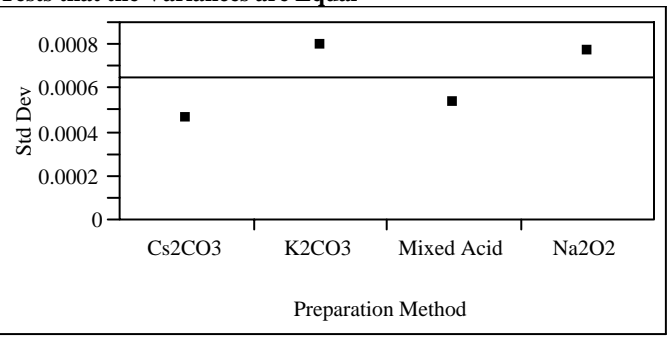

$\begin{array}{lrrrr}\text { Level } & \text { Count } & \text { Std Dev } & \text { MAD to Mean } & \text { MeanAbsDif to Median } \\ \text { Cs2CO3 } & 6 & 0.0004624 & 0.0003751 & 0.0003751 \\ \text { K2CO3 } & 6 & 0.0007948 & 0.0006335 & 0.0005251 \\ \text { Mixed Acid } & 6 & 0.0005382 & 0.0004418 & 0.0004001 \\ \text { Na2O2 } & 6 & 0.0007665 & 0.0005751 & 0.0005001 \\ \text { Test } & \text { F-ratio } & \text { DFNum } & \text { DFDen } & \text { Prob }>\text { F } \\ \text { O'Brien[.5] } & 0.5087 & 3 & 20 & 0.6808 \\ \text { Brwn-Frsthe } & 0.1163 & 3 & 20 & 0.9495 \\ \text { Levene } & 0.7667 & 3 & 20 & 0.5260 \\ \text { Bartlett } & 0.6204 & 3 & . & 0.6017\end{array}$

Welch Anova testing Means Equal, allowing Std Devs Not Equal

F Ratio DFNum DFDen Prob $>$ F

$\begin{array}{llll}1.9473 & 3 & 10.866 & 0.1812\end{array}$

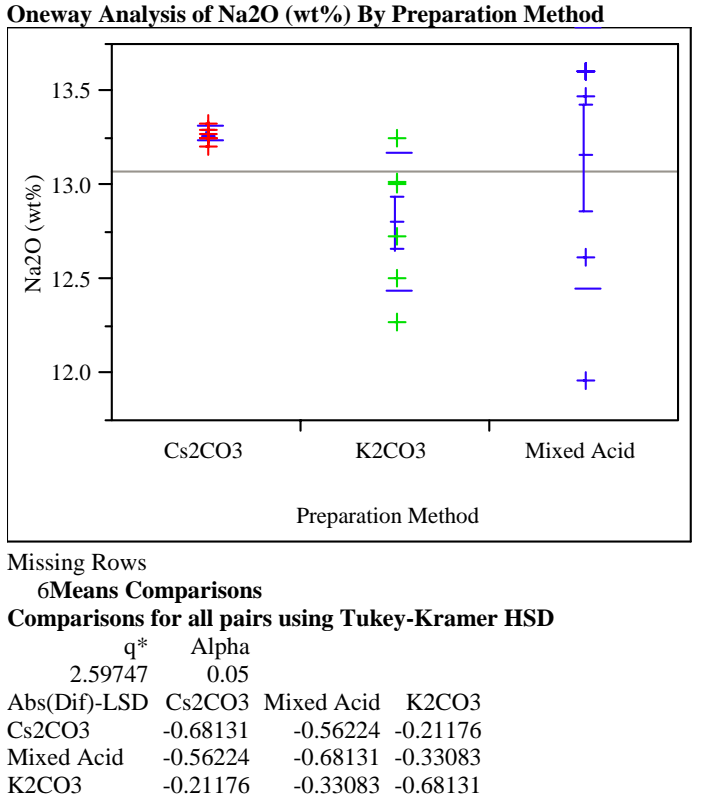

Positive values show pairs of means that are significantly different.

\begin{tabular}{|c|c|c|c|c|}
\hline Level & Count & Std Dev & $\begin{array}{r}\text { MeanAbsDif to } \\
\text { Mean }\end{array}$ & $\begin{array}{r}\text { MeanAbsDif to } \\
\text { Median }\end{array}$ \\
\hline Cs2CO3 & 6 & 0.0432622 & 0.0337000 & 0.0337000 \\
\hline K2CO3 & 6 & 0.3616729 & 0.2920667 & 0.2920667 \\
\hline Mixed Acid & 6 & 0.6975124 & 0.5721511 & 0.4628133 \\
\hline Test & F Ratio & DFNum & DFDen & Prob $>$ F \\
\hline O'Brien[.5] & 2.4897 & 2 & 15 & 0.1165 \\
\hline $\begin{array}{l}\text { Brown- } \\
\text { Forsythe }\end{array}$ & 1.8574 & 2 & 15 & 0.1902 \\
\hline Levene & 10.6434 & 2 & 15 & 0.0013 \\
\hline Bartlett & 9.8762 & 2 & & $<.0001$ \\
\hline
\end{tabular}

$\begin{array}{lrr}\text { Level } & & \text { Mean } \\ \text { Cs2CO3 } & \text { A } & 13.271060 \\ \text { Mixed Acid } & \text { A } & 13.151987 \\ \text { K2CO3 } & \text { A } & 12.801507\end{array}$

Levels not connected by same letter are significantly different Tests that the Variances are Equal

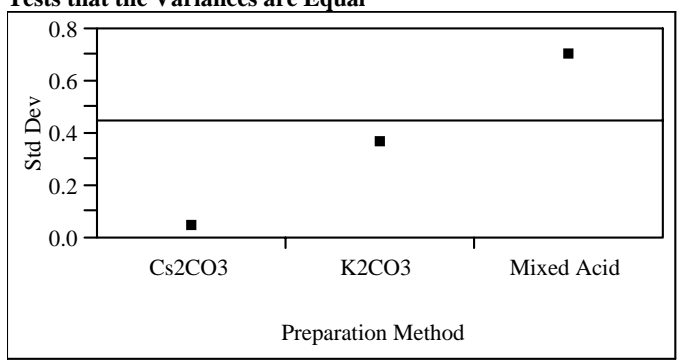

F Ratio DFNum DFDen Prob $>$ F

$\begin{array}{rrrr}4.6100 & 2 & 6.7859 & 0.0544\end{array}$ 


\section{Exhibit A4. Statistical Comparisons of Chemical Composition Measurements of Simulant Samples Across Preparation Methods by Oxide \\ (Concentrations in Wt\% Oxides)}

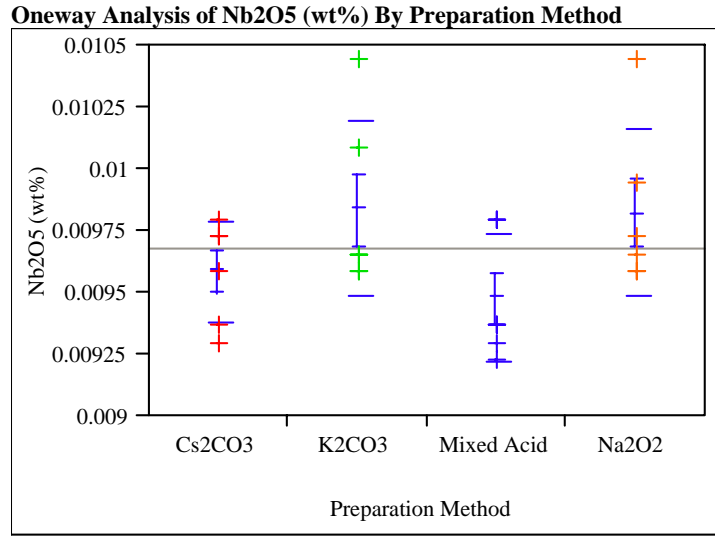

Means Comparison

Comparisons for all pairs using Tukey-Kramer HSD

$$
\begin{array}{rr}
q^{*} & \text { Alpha } \\
2.79894 & 0.05
\end{array}
$$

Abs(Dif)-LSD K2CO3 Na2O2 Cs2CO3 Mixed Acid

$\begin{array}{lllll}\mathrm{K} 2 \mathrm{CO} 3 & -0.00047 & -0.00046 & -0.00022 & -0.00011\end{array}$

$\begin{array}{lllll}\mathrm{Na} 2 \mathrm{O} 2 & -0.00046 & -0.00047 & -0.00023 & -0.00013\end{array}$

$\begin{array}{lllll}\mathrm{Cs} 2 \mathrm{CO} 3 & -0.00022 & -0.00023 & -0.00047 & -0.00037\end{array}$

$\begin{array}{lllll}\text { Mixed Acid } \quad-0.00011 & -0.00013 & -0.00037 & -0.00047\end{array}$

Positive values show pairs of means that are significantly different.

Level Mean

K2CO3 A 0.00983469

$\mathrm{Na} 2 \mathrm{O} 2$ A 0.00982277

Cs2CO3 A 0.00958435

Mixed Acid A 0.00947706

Levels not connected by same letter are significantly different Tests that the Variances are Equal

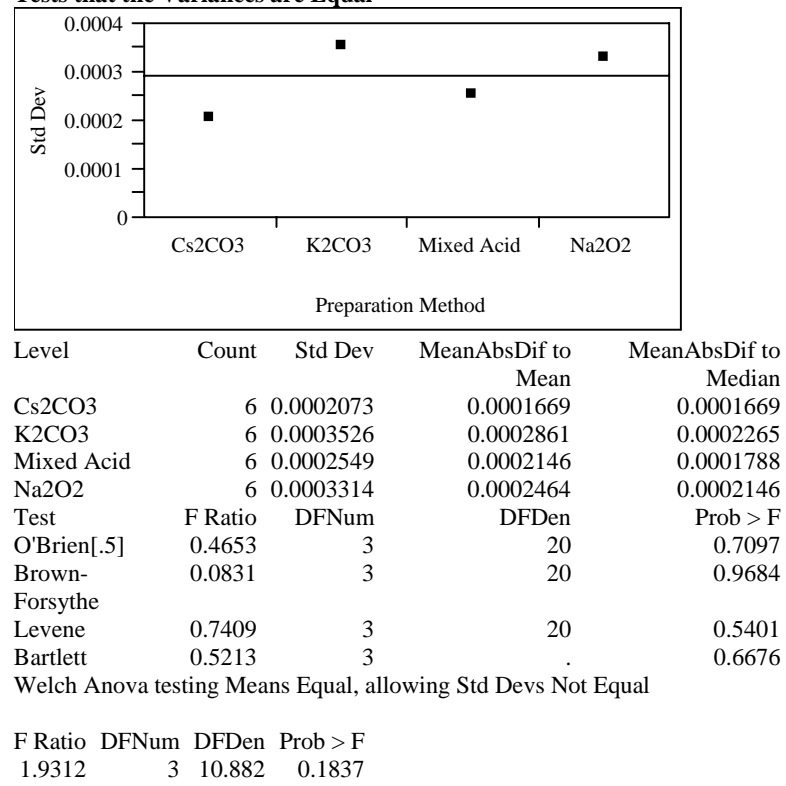

Oneway Analysis of Nd2O3 (wt\%) By Preparation Method

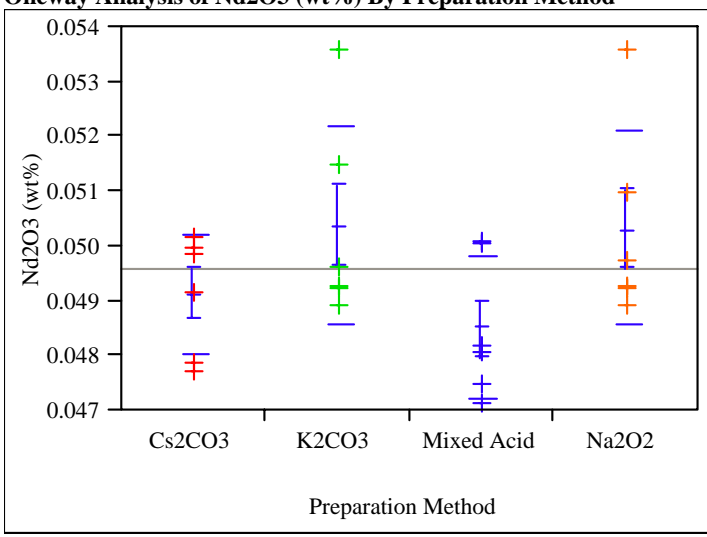

Means Comparisons

Comparisons for all pairs using Tukey-Kramer HSD

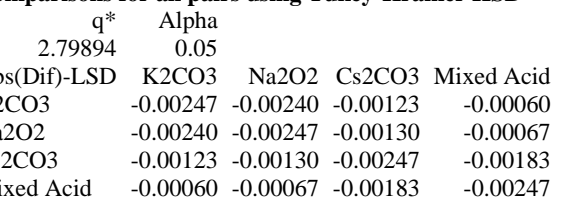

Positive values show pairs of means that are significantly different.

Level

K2CO3 A 0.05035932

$\mathrm{Na} 2 \mathrm{O} 2$ A 0.05029128

Cs2CO3 A 0.04912488

Mixed Acid A 0.04849308

Levels not connected by same letter are significantly different Tests that the Variances are Equal

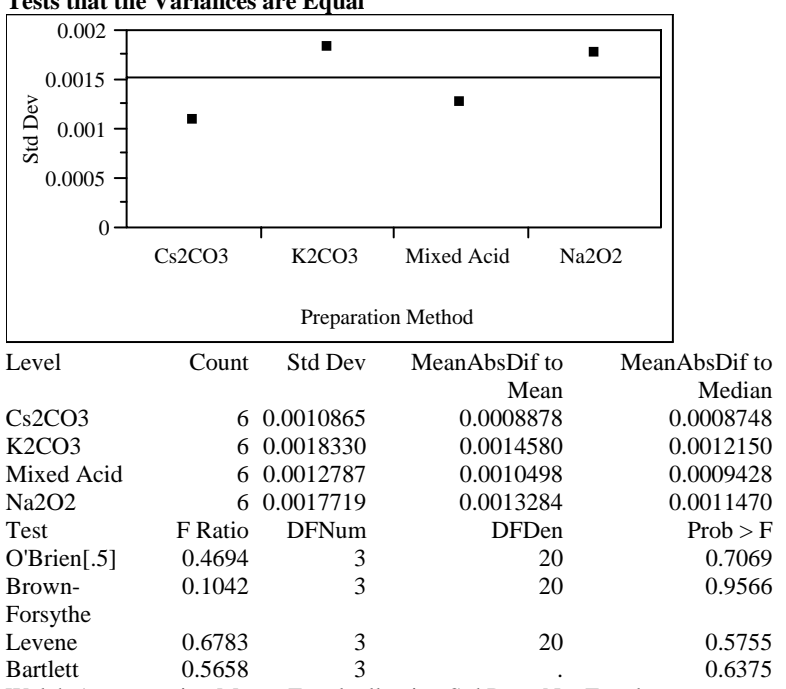

Welch Anova testing Means Equal, allowing Std Devs Not Equal

F Ratio DFNum DFDen Prob $>$ F

$\begin{array}{llll}1.9084 & 3 & 10.88 & 0.1873\end{array}$ 


\section{Exhibit A4. Statistical Comparisons of Chemical Composition Measurements of Simulant Samples Across Preparation Methods by Oxide \\ (Concentrations in Wt\% Oxides)}

Oneway Analysis of NiO (wt\%) By Preparation Method

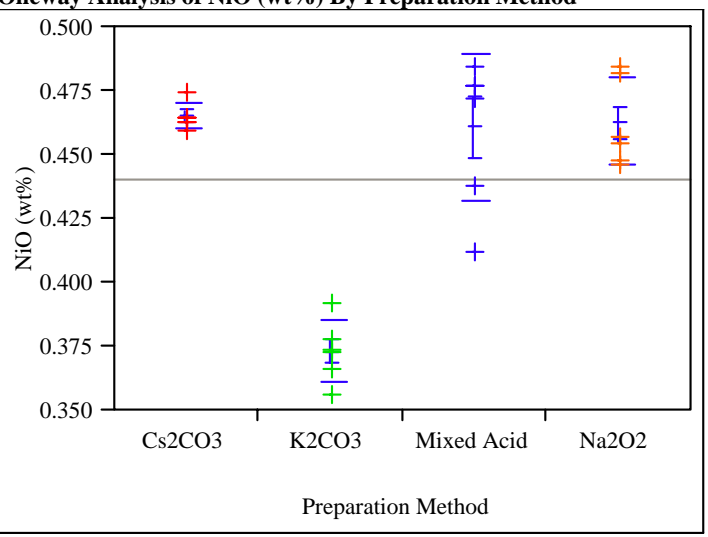

Means Comparisons

Comparisons for all pairs using Tukey-Kramer HSD

$$
\begin{array}{rr}
q^{*} & \text { Alpha } \\
2.79894 & 0.05
\end{array}
$$

Abs(Dif)-LSD Cs2CO3 Na2O2 Mixed Acid $\mathrm{K} 2 \mathrm{CO} 3$

$\begin{array}{lllll}\mathrm{Cs} 2 \mathrm{CO} 3 & -0.02894 & -0.02618 & -0.02427 & 0.06268\end{array}$

$\begin{array}{lllll}\mathrm{Na} 2 \mathrm{O} 2 & -0.02618 & -0.02894 & -0.02703 & 0.05992\end{array}$

$\begin{array}{lllll}\text { Mixed Acid } \quad-0.02427 & -0.02703 & -0.02894 & 0.05802\end{array}$

$\begin{array}{lllll}\text { K2CO3 } & 0.06268 & 0.05992 & 0.05802 & -0.02894\end{array}$

Positive values show pairs of means that are significantly different.

$$
\begin{array}{lrr}
\text { Level } & & \text { Mean } \\
\text { Cs2CO3 } & \text { A } & 0.46488667 \\
\text { Na2O2 } & \text { A } & 0.46212958 \\
\text { Mixed Acid } & \text { A } & 0.46022083 \\
\text { K2CO3 } & \text { B } & 0.37326667
\end{array}
$$

Levels not connected by same letter are significantly different Tests that the Variances are Equal

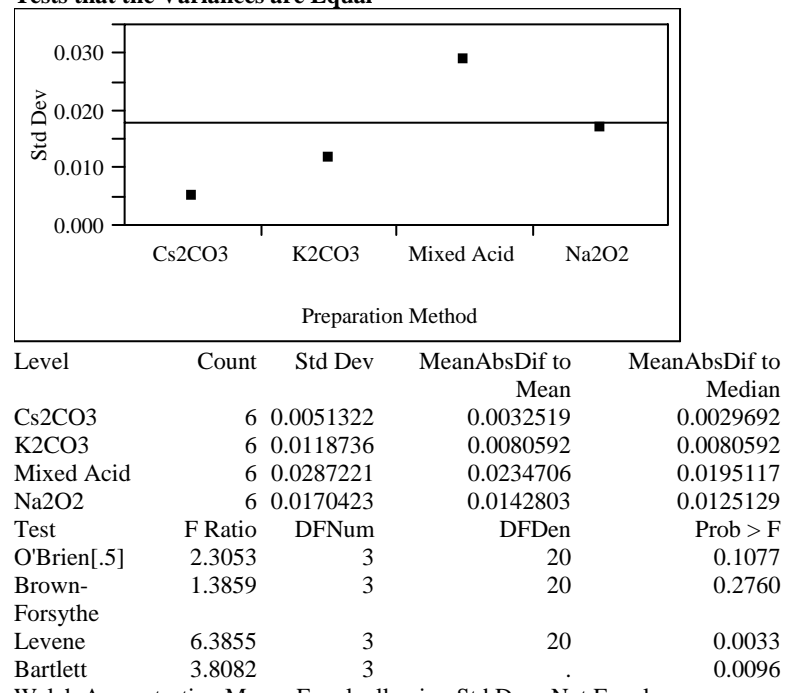

Welch Anova testing Means Equal, allowing Std Devs Not Equal

F Ratio DFNum DFDen Prob $>$ F

$\begin{array}{rrrr}89.0095 & 3 & 9.5824 & <.0001\end{array}$

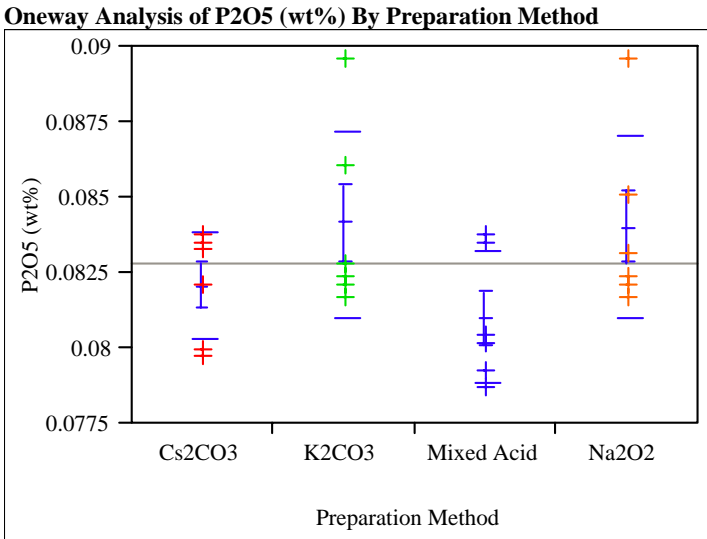

Means Comparisons

Comparisons for all pairs using Tukey-Kramer HSD

q* Alpha

bs(Dif)-LSD K2CO3 Na2O2 Cs2CO3 Mixed Acid

$\begin{array}{lllll}\text { K2CO3 } & -0.00415 & -0.00406 & -0.00211 & -0.00102\end{array}$

$\begin{array}{lllll}\mathrm{Na} 2 \mathrm{O} 2 & -0.00406 & -0.00415 & -0.00221 & -0.00112\end{array}$

$\begin{array}{lllll}\text { Cs2CO3 } & -0.00211 & -0.00221 & -0.00415 & -0.00307\end{array}$

$\begin{array}{llll}\text { Mixed Acid } \quad-0.00102 & -0.00112 & -0.00307 & -0.00415\end{array}$

Positive values show pairs of means that are significantly different.

Level

Mean

K2CO3 A 0.08411347

$\mathrm{Na} 2 \mathrm{O} 2$ A 0.08401800

Cs2CO3 A 0.08207031

Mixed Acid A 0.08098189

Levels not connected by same letter are significantly different Tests that the Variances are Equal

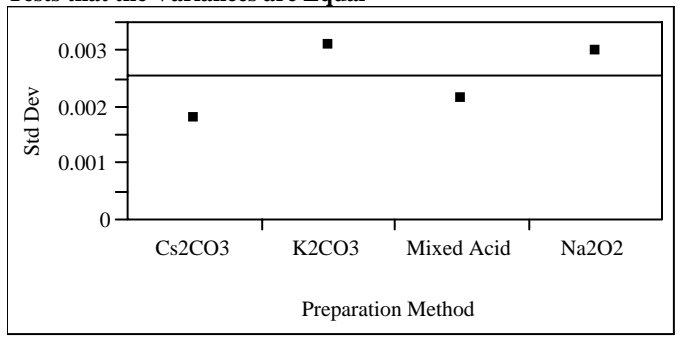

$\begin{array}{lrrrr}\text { Level } & \text { Count } & \text { Std Dev } & \begin{array}{r}\text { MeanAbsDif to } \\ \text { Mean }\end{array} & \begin{array}{r}\text { MeanAbsDif to } \\ \text { Median }\end{array} \\ \text { Cs2CO3 } & 6 & 0.0018038 & 0.0014767 & 0.0014512 \\ \text { K2CO3 } & 6 & 0.0031035 & 0.0024696 & 0.0020432 \\ \text { Mixed Acid } & 6 & 0.0021488 & 0.0017695 & 0.0015849 \\ \text { Na2O2 } & 6 & 0.0029882 & 0.0022277 & 0.0019477 \\ \text { Test } & \text { F Ratio } & \text { DFNum } & \text { DFDen } & \text { Prob > F } \\ \text { O'Brien[.5] } & 0.4863 & 3 & 20 & 0.6956 \\ \text { Brown- } & 0.1139 & 3 & 20 & 0.9509 \\ \text { Forsythe } & & & & \\ \text { Levene } & 0.7059 & 3 & 20 & 0.5597 \\ \text { Bartlett } & 0.5987 & 3 & . & 0.6158 \\ \text { Well } & & 3 & & \end{array}$

Welch Anova testing Means Equal, allowing Std Devs Not Equal

1.8948 DFNum DFDen Prob > F 


\section{Exhibit A4. Statistical Comparisons of Chemical Composition Measurements of Simulant Samples Across Preparation Methods by Oxide \\ (Concentrations in Wt\% Oxides)}

Oneway Analysis of PbO (wt \%) By Preparation Method

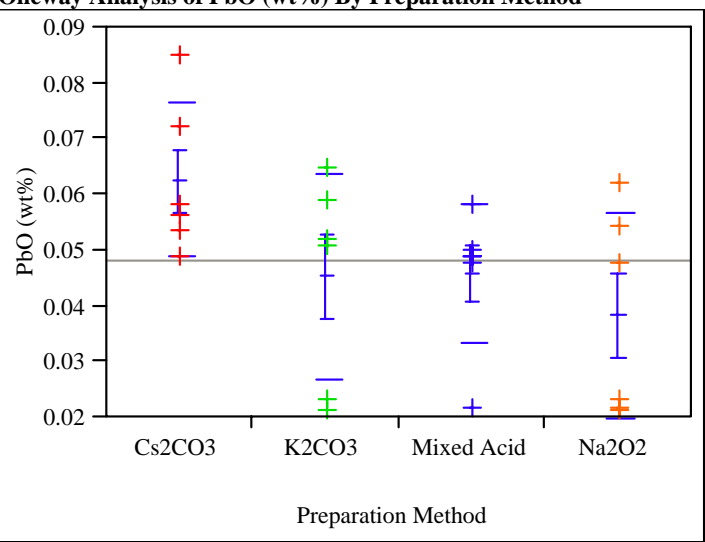

Means Comparisons

Comparisons for all pairs using Tukey-Kramer HSD q* Alpha

Abs(Dif)-LSD Cs2CO3 Mixed Acid K2CO3 Na2O2

$\begin{array}{lllll}\text { Cs2CO3 } & -0.02585 & -0.00933 & -0.00866 & -0.00175\end{array}$

$\begin{array}{lllll}\text { Mixed Acid } \quad-0.00933 & -0.02585 & -0.02519 & -0.01828\end{array}$

$\begin{array}{lllll}\mathrm{K} 2 \mathrm{CO} 3 & -0.00866 & -0.02519 & -0.02585 & -0.01894\end{array}$

$\begin{array}{lllll}\mathrm{Na} 2 \mathrm{O} 2 & -0.00175 & -0.01828 & -0.01894 & -0.02585\end{array}$

Positive values show pairs of means that are significantly different.

Level Mean

Cs2CO3 A 0.06247760

Mixed Acid A 0.04595156

K2CO3 A 0.04528728

$\mathrm{Na} 2 \mathrm{O} 2$ A 0.03837525

Levels not connected by same letter are significantly different Tests that the Variances are Equal

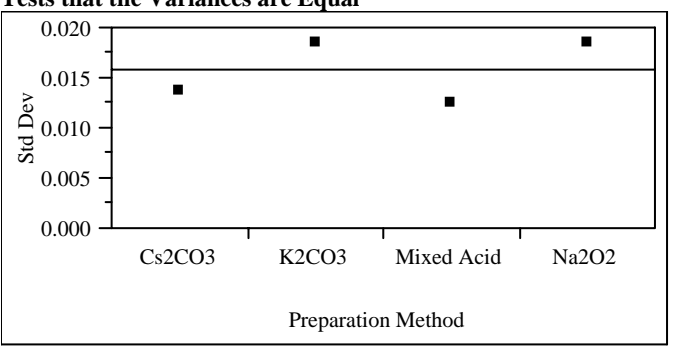

\begin{tabular}{|c|c|c|c|c|}
\hline Level & Count & Std Dev & $\begin{array}{r}\text { MeanAbsDif to } \\
\text { Mean }\end{array}$ & $\begin{array}{r}\text { MeanAbsDif to } \\
\text { Median }\end{array}$ \\
\hline $\mathrm{Cs} 2 \mathrm{CO} 3$ & 6 & 0.0136644 & 0.0108797 & 0.0095512 \\
\hline K2CO3 & 6 & 0.0184759 & 0.0153082 & 0.0133842 \\
\hline Mixed Acid & 6 & 0.0124749 & 0.0080820 & 0.0065081 \\
\hline $\mathrm{Na} 2 \mathrm{O} 2$ & 6 & 0.0184460 & 0.0163106 & 0.0163106 \\
\hline Test & F Ratio & DFNum & DFDen & Prob $>$ \\
\hline O'Brien[.5] & 0.7824 & 3 & 20 & 0.5177 \\
\hline $\begin{array}{l}\text { Brown- } \\
\text { Forsythe }\end{array}$ & 1.0334 & 3 & 20 & 0.3991 \\
\hline Levene & 1.7517 & 3 & 20 & 0.1 \\
\hline Bartlett & 0.3706 & 3 & & 0.7 \\
\hline
\end{tabular}

Welch Anova testing Means Equal, allowing Std Devs Not Equal

F Ratio DFNum DFDen Prob $>$ F

$\begin{array}{llll}2.4937 & 3 & 10.965 & 0.1144\end{array}$

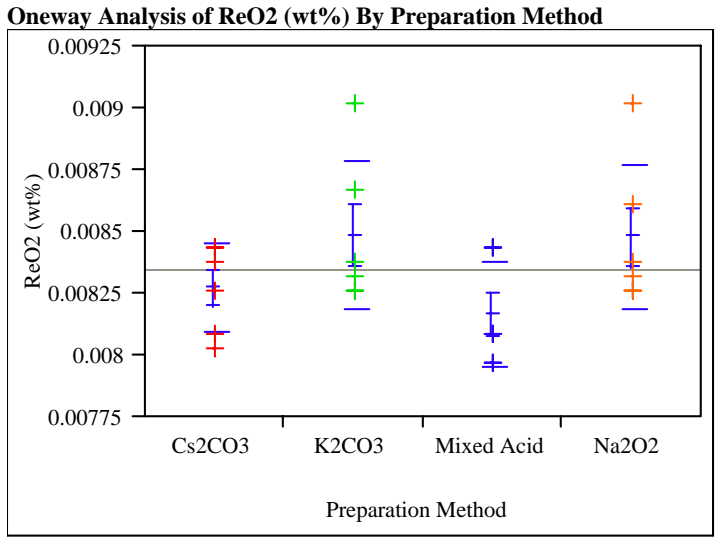

Means Comparisons

Comparisons for all pairs using Tukey-Kramer HSD $\begin{array}{rr}q^{*} & \text { Alpha } \\ 2.79894 & 0.05\end{array}$

Abs(Dif)-LSD K2CO3 Na2O2 Cs2CO3 Mixed Acid

$\begin{array}{lllll}\mathrm{K} 2 \mathrm{CO} 3 & -0.00041 & -0.00040 & -0.00020 & -0.00009\end{array}$

$\mathrm{Na2O} 2 \quad-0.00040-0.00041-0.00021-0.00010$

$\begin{array}{lllll}\mathrm{Cs} 2 \mathrm{CO} 3 & -0.00020 & -0.00021 & -0.00041 & -0.00031\end{array}$

$\begin{array}{llll}\text { Mixed Acid } \quad-0.00009 & -0.00010 & -0.00031 & -0.00041\end{array}$

Positive values show pairs of means that are significantly different.

$\begin{array}{lrr}\text { Level } & & \text { Mean } \\ \text { K2CO3 } & \text { A } & 0.00848651 \\ \mathrm{Na} 2 \mathrm{O} 2 & \text { A } & 0.00847674 \\ \text { Cs2CO3 } & \text { A } & 0.00827166\end{array}$

$\begin{array}{lll}\text { Cs2CO3 A } & 0.00827166 \\ \text { Mixed Acid } & \text { A } & 0.00816424\end{array}$

Levels not connected by same letter are significantly different Tests that the Variances are Equal

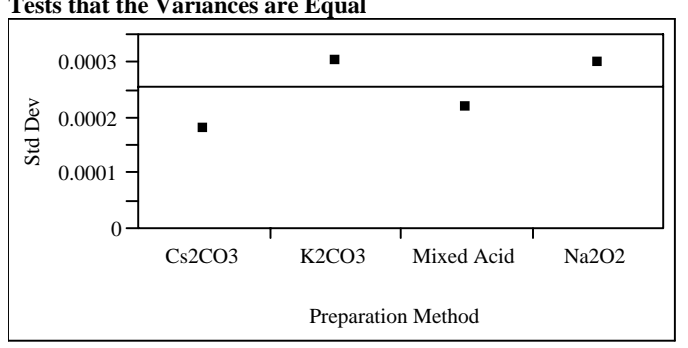

$\begin{array}{lrrrr}\text { Level } & \text { Count } & \text { Std Dev } & \begin{array}{r}\text { MeanAbsDif to } \\ \text { Mean }\end{array} & \begin{array}{r}\text { MeanAbsDif to } \\ \text { Median }\end{array} \\ \text { Cs2CO3 } & 6 & 0.0001793 & 0.0001465 & 0.0001465 \\ \text { K2CO3 } & 6 & 0.0003043 & 0.0002409 & 0.0002051 \\ \text { Mixed Acid } & 6 & 0.0002182 & 0.0001823 & 0.0001563 \\ \text { Na2O2 } & 6 & 0.0002980 & 0.0002279 & 0.0001953 \\ \text { Test } & \text { F Ratio } & \text { DFNum } & \text { DFDen } & \text { Prob > F } \\ \text { O'Brien[.5] } & 0.4818 & 3 & 20 & 0.6986 \\ \text { Brown- } & 0.1226 & 3 & 20 & 0.9457 \\ \text { Forsythe } & & & & \\ \text { Levene } & 0.7075 & 3 & 20 & 0.5588 \\ \text { Bartlett } & 0.5647 & 3 & . & 0.6382\end{array}$

Welch Anova testing Means Equal, allowing Std Devs Not Equal

F Ratio DFNum DFDen Prob > F

$\begin{array}{llll}2.0283 & 3 & 10.868 & 0.1690\end{array}$ 


\section{Exhibit A4. Statistical Comparisons of Chemical Composition Measurements of Simulant Samples Across Preparation Methods by Oxide \\ (Concentrations in Wt\% Oxides)}

Oneway Analysis of SO4 (wt \%) By Preparation Method

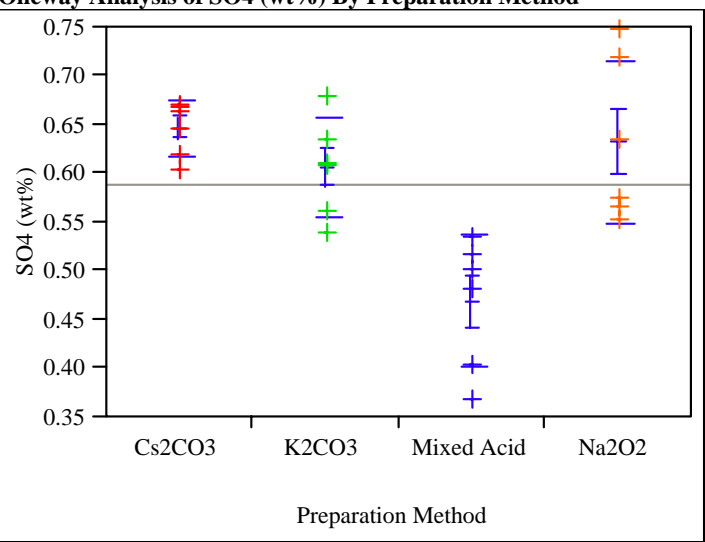

Means Comparisons

Comparisons for all pairs using Tukey-Kramer HSD$$
79894 \quad 0.05
$$

Abs(Dif)-LSD Cs2CO3 Na2O2 K2CO3 Mixed Acid

$\begin{array}{lllll}\mathrm{Cs} 2 \mathrm{CO} 3 & -0.09823 & -0.08525 & -0.05828 & 0.07903\end{array}$

$\begin{array}{lllll}\mathrm{Na} 2 \mathrm{O} 2 & -0.08525 & -0.09823 & -0.07127 & 0.06605\end{array}$

$\begin{array}{lllll}\mathrm{K} 2 \mathrm{CO} 3 & -0.05828 & -0.07127 & -0.09823 & 0.03908\end{array}$

$\begin{array}{lllll}\text { Mixed Acid } \quad 0.07903 & 0.06605 & 0.03908 & -0.09823\end{array}$

Positive values show pairs of means that are significantly different.

$$
\begin{aligned}
& \text { Level Mean } \\
& \text { Cs2CO3 A } 0.64611577 \\
& \mathrm{Na} 2 \mathrm{O} 2 \quad \text { A } 0.63313353 \\
& \text { K2CO3 A } 0.60617043 \\
& \text { Mixed Acid } \quad \text { B } 0.46885835
\end{aligned}
$$

Levels not connected by same letter are significantly different Tests that the Variances are Equal

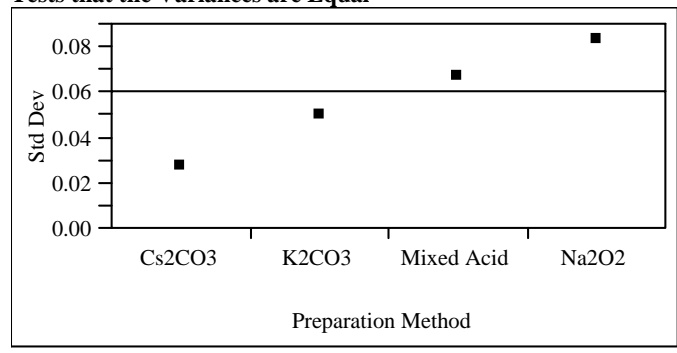

$\begin{array}{lrrrr}\text { Level } & \text { Count } & \text { Std Dev } & \begin{array}{r}\text { MeanAbsDif to } \\ \text { Mean }\end{array} & \begin{array}{r}\text { MeanAbsDif to } \\ \text { Median }\end{array} \\ \text { Cs2CO3 } & 6 & 0.0276317 & 0.0223028 & 0.0219699 \\ \text { K2CO3 } & 6 & 0.0502978 & 0.0366166 & 0.0359508 \\ \text { Mixed Acid } & 6 & 0.0671977 & 0.0549248 & 0.0504310 \\ \text { Na2O2 } & 6 & 0.0834920 & 0.0679071 & 0.0679071 \\ \text { Test } & \text { F Ratio } & \text { DFNum } & \text { DFDen } & \text { Prob > F } \\ \text { O'Brien[.5] } & 2.4324 & 3 & 20 & 0.0950 \\ \text { Brown- } & 1.5970 & 3 & 20 & 0.2215 \\ \text { Forsythe } & & & & \\ \text { Levene } & 2.8253 & 3 & 20 & 0.0648 \\ \text { Bartlett } & 1.7235 & 3 & . & 0.1597 \\ \text { Wenth } & & 3 & & \end{array}$

esting Means Equal, allowing Std Devs Not Equal

F Ratio DFNum DFDen Prob $>$ F

$\begin{array}{llll}10.6977 & 3 & 10.191 & 0.0017\end{array}$
Oneway Analysis of SiO2 (wt\%) By Preparation Method

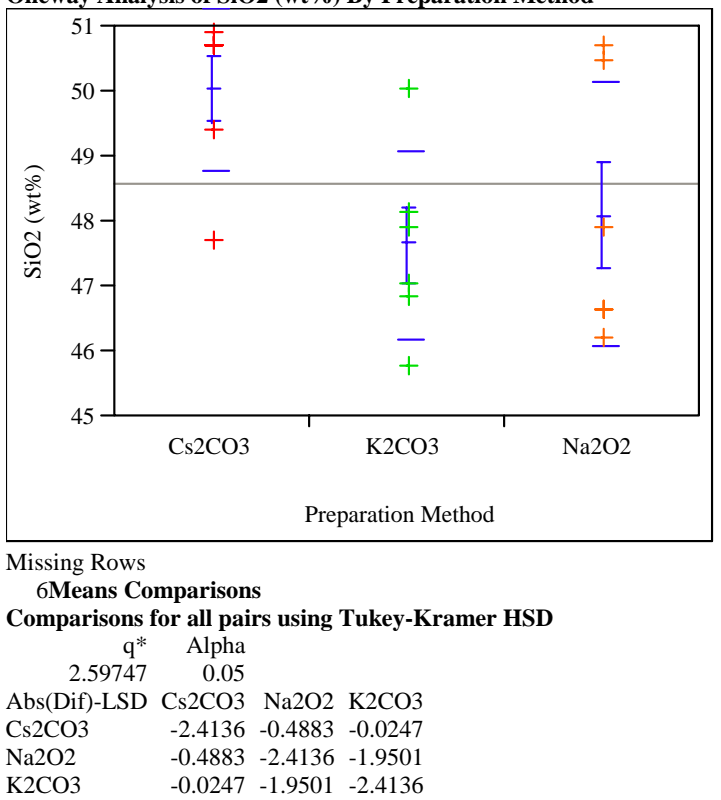

Positive values show pairs of means that are significantly different.

Level Mean

Cs2CO3 A 50.023965

$\mathrm{Na} 2 \mathrm{O} 2$ A 48.098595

\begin{tabular}{|c|c|c|c|c|}
\hline Level & Count & Std Dev & $\begin{array}{r}\text { MeanAbsDif to } \\
\text { Mean }\end{array}$ & $\begin{array}{r}\text { MeanAbsDif to } \\
\text { Median }\end{array}$ \\
\hline Cs2CO3 & 6 & 1.257768 & 0.974570 & 0.748755 \\
\hline K2CO3 & 6 & 1.455143 & 1.069650 & 1.069650 \\
\hline $\mathrm{Na} 2 \mathrm{O} 2$ & 6 & 2.017834 & 1.663900 & 1.604475 \\
\hline Test & F Ratio & DFNum & DFDen & Prob $>F$ \\
\hline O'Brien[.5] & 1.0690 & 2 & 15 & 0.3681 \\
\hline $\begin{array}{l}\text { Brown- } \\
\text { Forsythe }\end{array}$ & 0.8371 & 2 & 15 & 0.4522 \\
\hline Levene & 1.2975 & 2 & 15 & 0.3022 \\
\hline Bartlett & 0.5567 & 2 & . & 0.5731 \\
\hline
\end{tabular}

K2CO3 A 47.635080

Levels not connected by same letter are significantly different Tests that the Variances are Equal

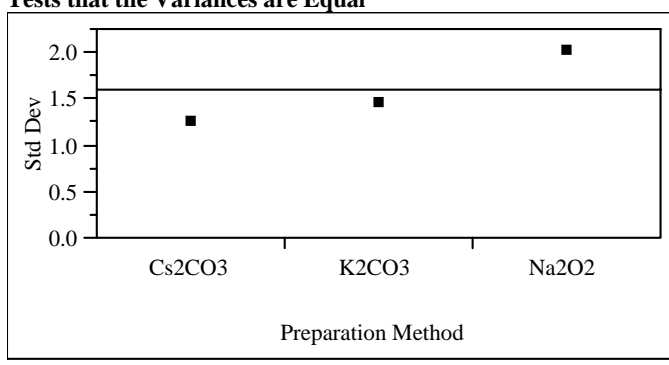

Welch Anova testing Means Equal, allowing Std Devs Not Equal

F Ratio DFNum DFDen Prob $>$ F

$\begin{array}{llll}4.7909 & 2 & 9.6989 & 0.0357\end{array}$ 


\section{Exhibit A4. Statistical Comparisons of Chemical Composition Measurements of Simulant Samples Across Preparation Methods by Oxide \\ (Concentrations in Wt\% Oxides)}

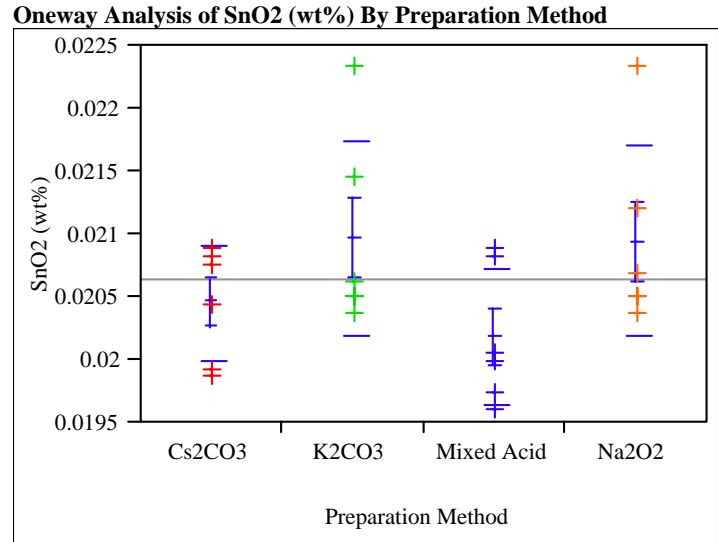

Means Comparisons

Comparisons for all pairs using Tukey-Kramer HSD$$
\text { q* Alpha }
$$

Abs(Dif)-LSD K2CO3 Na2O2 Cs2CO3 Mixed Acid

K2CO3 $\quad-0.00104-0.00101-0.00052-0.00026$

$\begin{array}{lllll}\mathrm{Na} 2 \mathrm{O} 2 & -0.00101 & -0.00104 & -0.00056 & -0.00029\end{array}$

$\begin{array}{lllll}\mathrm{Cs} 2 \mathrm{CO} 3 & -0.00052 & -0.00056 & -0.00104 & -0.00078\end{array}$

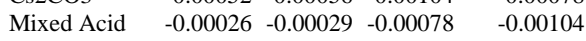

Positive values show pairs of means that are significantly different.

$\begin{array}{lrr}\text { Level } & & \text { Mean } \\ \text { K2CO3 } & \text { A } & 0.02096956 \\ \mathrm{Na} 2 \mathrm{O} 2 & \text { A } & 0.02093782 \\ \text { Cs2CO3 } & \text { A } & 0.02045114 \\ \text { Mixed Acid } & \text { A } & 0.02018664\end{array}$

Mixed Acid A 0.02018664

Levels not connected by same letter are significantly different

Tests that the Variances are Equal

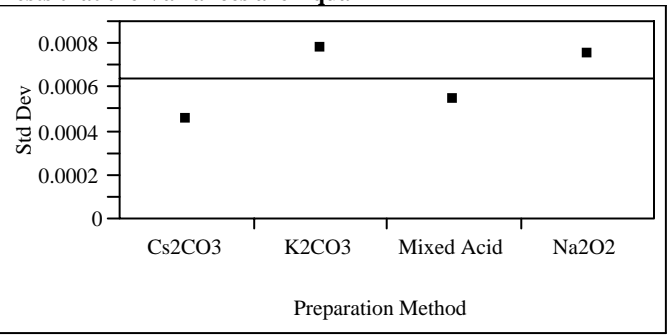

$\begin{array}{lrrrr}\text { Level } & \text { Count } & \text { Std Dev } & \begin{array}{r}\text { MeanAbsDif to } \\ \text { Mean }\end{array} & \begin{array}{r}\text { MeanAbsDif to } \\ \text { Median }\end{array} \\ \text { Cs2CO3 } & 6 & 0.0004532 & 0.0003703 & 0.0003703 \\ \text { K2CO3 } & 6 & 0.0007782 & 0.0006207 & 0.0005078 \\ \text { Mixed Acid } & 6 & 0.0005416 & 0.0004444 & 0.0004020 \\ \text { Na2O2 } & 6 & 0.0007483 & 0.0005572 & 0.0004761 \\ \text { Test } & \text { F Ratio } & \text { DFNum } & \text { DFDen } & \text { Prob }>\text { F } \\ \text { O'Brien[.5] } & 0.4724 & 3 & 20 & 0.7049 \\ \text { Brown- } & 0.0878 & 3 & 20 & 0.9659 \\ \text { Forsythe } & & & & \\ \text { Levene } & 0.7057 & 3 & 20 & 0.5598 \\ \text { Bartlett } & 0.5894 & 3 & . & 0.6219 \\ \text { Welt } & & 3 & & \end{array}$

Welch Anova testing Means Equal, allowing Std Devs Not Equal

F Ratio DFNum DFDen Prob $>$ F

$\begin{array}{llll}1.8658 & 3 & 10.867 & 0.1945\end{array}$

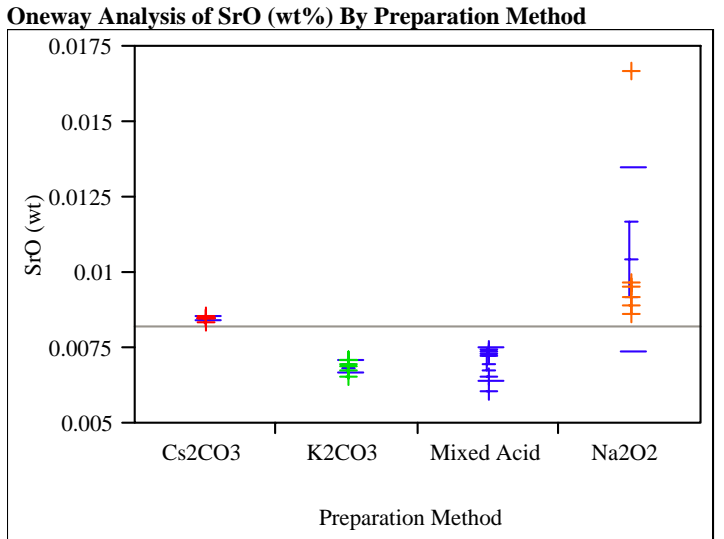

Means Comparisons

Comparisons for all pairs using Tukey-Kramer HSD

q* Alpha

Abs(Dif)-LSD Na2O2 Cs2CO3 Mixed Acid K2CO3

$\begin{array}{lllll}\mathrm{Na} 2 \mathrm{O} 2 & -0.00253 & -0.00056 & 0.00092 & 0.00102\end{array}$

$\begin{array}{lllll}\mathrm{Cs} 2 \mathrm{CO} 3 & -0.00056 & -0.00253 & -0.00104 & -0.00095\end{array}$

Mixed Acid $\quad 0.00092-0.00104 \quad-0.00253-0.00243$

$\begin{array}{lllll}\mathrm{K} 2 \mathrm{CO} 3 & 0.00102 & -0.00095 & -0.00243 & -0.00253\end{array}$

Positive values show pairs of means that are significantly different.

$\begin{array}{lrrr}\text { Level } & & & \text { Mean } \\ \text { Na2O2 } & \text { A } & & 0.01046010 \\ \text { Cs2CO3 } & \text { A } & \text { B } & 0.00849304 \\ \text { Mixed Acid } & & \text { B } & 0.00701085 \\ \text { K2CO3 } & \text { B } & 0.00691427\end{array}$

Levels not connected by same letter are significantly different Tests that the Variances are Equal

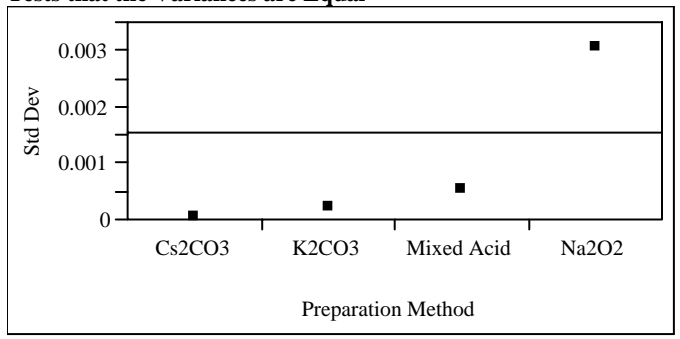

$\begin{array}{lrrrr}\text { Level } & \text { Count } & \text { Std Dev } & \begin{array}{r}\text { MeanAbsDif to } \\ \text { Mean }\end{array} & \begin{array}{r}\text { MeanAbsDif to } \\ \text { Median }\end{array} \\ \text { Cs2CO3 } & 6 & 0.0000679 & 0.0000388 & 0.0000374 \\ \text { K2CO3 } & 6 & 0.0002122 & 0.0001616 & 0.0001616 \\ \text { Mixed Acid } & 6 & 0.0005583 & 0.0004599 & 0.0003883 \\ \text { Na2O2 } & 6 & 0.0030688 & 0.0020715 & 0.0015157 \\ \text { Test } & \text { F Ratio } & \text { DFNum } & \text { DFDen } & \text { Prob }>\text { F } \\ \text { O'Brien[.5] } & 1.2473 & 3 & 20 & 0.3191 \\ \text { Brown- } & 1.3276 & 3 & 20 & 0.2934 \\ \text { Forsythe } & & & & \\ \text { Levene } & 4.9089 & 3 & 20 & 0.0102 \\ \text { Bartlett } & 16.8910 & 3 & . & <.0001\end{array}$

Welch Anova testing Means Equal, allowing Std Devs Not Equal

F Ratio DFNum DFDen Prob $>$ F

$\begin{array}{llll}98.0264 & 3 & 8.8998<.0001\end{array}$ 


\section{Exhibit A4. Statistical Comparisons of Chemical Composition Measurements of Simulant Samples Across Preparation Methods by Oxide \\ (Concentrations in Wt\% Oxides)}

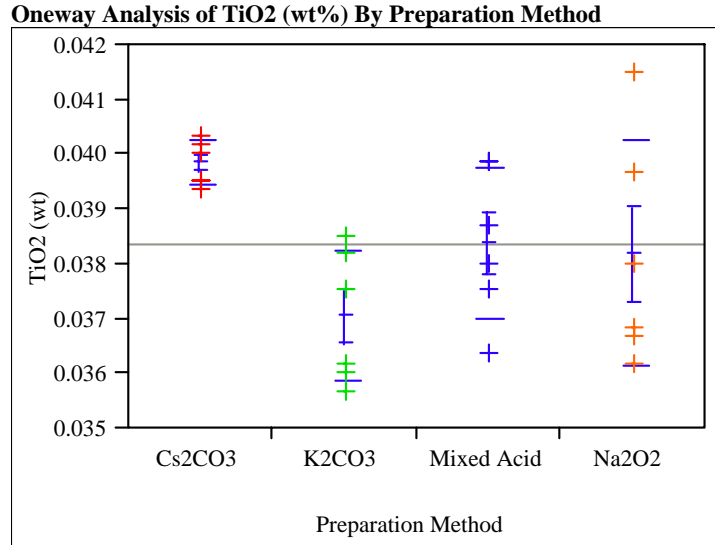

Means Comparisons

Comparisons for all pairs using Tukey-Kramer HSD

$$
\begin{array}{rr}
q^{*} & \text { Alpha } \\
2.79894 & 0.05
\end{array}
$$

Abs(Dif)-LSD Cs2CO3 Mixed Acid Na2O2 K2CO3

$\begin{array}{lllll}\mathrm{Cs} 2 \mathrm{CO} 3 & -0.00226 & -0.00081 & -0.00059 & 0.00055\end{array}$

$\begin{array}{lllll}\text { Mixed Acid } \quad-0.00081 & -0.00226 & -0.00204 & -0.00090\end{array}$

$\begin{array}{lllll}\mathrm{Na} 2 \mathrm{O} 2 & -0.00059 & -0.00204 & -0.00226 & -0.00112\end{array}$

$\begin{array}{lllll}\text { K2CO3 } & 0.00055 & -0.00090 & -0.00112 & -0.00226\end{array}$

Positive values show pairs of means that are significantly different.

Level Mean

Cs2CO3 A 0.03983740

Mixed Acid A B 0.03839180

$\mathrm{Na} 2 \mathrm{O} 2$ A $\quad$ B 0.03816940

K2CO3 $\quad$ B 0.03702960

Levels not connected by same letter are significantly different

Tests that the Variances are Equal

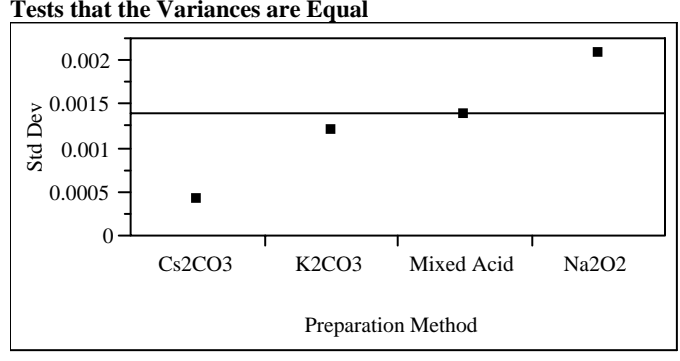

\begin{tabular}{|c|c|c|c|c|}
\hline Level & Count & Std Dev & $\begin{array}{r}\text { MeanAbsDif to } \\
\text { Mean }\end{array}$ & $\begin{array}{r}\text { MeanAbsDif to } \\
\text { Median }\end{array}$ \\
\hline Cs2CO3 & 6 & 0.0004142 & 0.0003614 & 0.0003614 \\
\hline $\mathrm{K} 2 \mathrm{CO} 3$ & 6 & 0.0012120 & 0.0010564 & 0.0010564 \\
\hline Mixed Acid & 6 & 0.0013731 & 0.0010842 & 0.0010842 \\
\hline $\mathrm{Na} 2 \mathrm{O} 2$ & 6 & 0.0020711 & 0.0016309 & 0.0015846 \\
\hline Test & F Ratio & DFNum & DFDen & Prob $>$ F \\
\hline O'Brien[.5] & 2.0600 & 3 & 20 & 0.1378 \\
\hline $\begin{array}{l}\text { Brown- } \\
\text { Forsythe }\end{array}$ & 2.3681 & 3 & 20 & 0.1012 \\
\hline Levene & 3.7825 & 3 & 20 & $0.02 €$ \\
\hline Bartlett & 3.0262 & 3 & & 0.02 \\
\hline
\end{tabular}

Welch Anova testing Means Equal, allowing Std Devs Not Equal

F Ratio DFNum DFDen Prob $>$ F

$\begin{array}{rrrr}10.2132 & 3 & 9.4078 & 0.0026\end{array}$
Oneway Analysis of V2O5 (wt\%) By Preparation Method

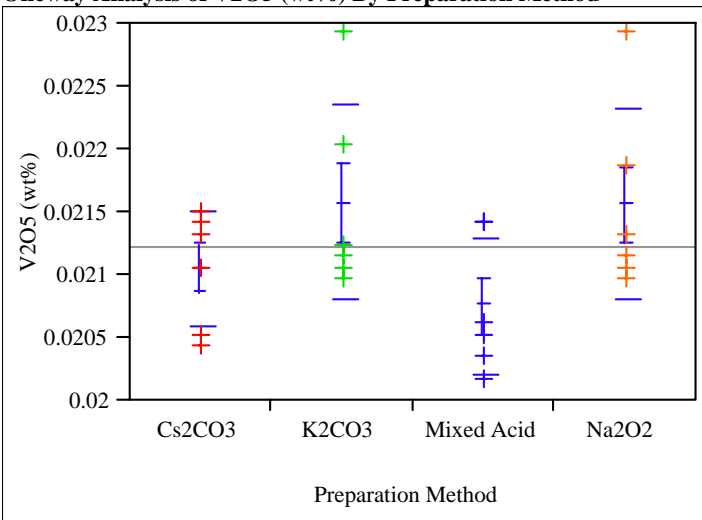

Means Comparisons

Comparisons for all pairs using Tukey-Kramer HSD

$$
\text { q* Alpha }
$$

$2.79894 \quad 0.05$

Abs(Dif)-LSD K2CO3 Na2O2 Cs2CO3 Mixed Acid

$\begin{array}{lllll}\text { K2CO3 } & -0.00104 & -0.00103 & -0.00052 & -0.00022\end{array}$

$\begin{array}{lllll}\mathrm{Na} 2 \mathrm{O} 2 & -0.00103 & -0.00104 & -0.00054 & -0.00024\end{array}$

$\begin{array}{lllll}\mathrm{Cs} 2 \mathrm{CO} 3 & -0.00052 & -0.00054 & -0.00104 & -0.00075\end{array}$

$\begin{array}{llll}\text { Mixed Acid } \quad-0.00022 & -0.00024 & -0.00075 & -0.00104\end{array}$

Positive values show pairs of means that are significantly different.

$\begin{array}{lrr}\text { Level } & & \text { Mean } \\ \text { K2CO3 } & \text { A } & 0.02157117 \\ \text { Na2O2 } & \text { A } & 0.02155629 \\ \text { Cs2CO3 } & \text { A } & 0.02105048 \\ \text { Mixed Acid } & \text { A } & 0.02075295\end{array}$

Levels not connected by same letter are significantly different Tests that the Variances are Equal

\begin{tabular}{|c|c|c|c|c|}
\hline Level & Count & Std Dev & $\begin{array}{r}\text { MeanAbsDif to } \\
\text { Mean }\end{array}$ & $\begin{array}{r}\text { MeanAbsDif to } \\
\text { Median }\end{array}$ \\
\hline Cs2CO3 & 6 & 0.0004635 & 0.0003769 & 0.0003719 \\
\hline K2CO3 & 6 & 0.0007734 & 0.0006149 & 0.0005058 \\
\hline Mixed Acid & 6 & 0.0005407 & 0.0004463 & 0.0004017 \\
\hline $\mathrm{Na} 2 \mathrm{O} 2$ & 6 & 0.0007484 & 0.0005653 & 0.0004909 \\
\hline Test & F Ratio & DFNum & DFDen & Prob $>$ F \\
\hline O'Brien[.5] & 0.4676 & 3 & 20 & 0.7081 \\
\hline $\begin{array}{l}\text { Brown- } \\
\text { Forsythe }\end{array}$ & 0.0984 & 3 & 20 & 0.9600 \\
\hline Levene & 0.6784 & 3 & 20 & 0.5755 \\
\hline Bartlett & 0.5481 & 3 & & 0.6494 \\
\hline
\end{tabular}

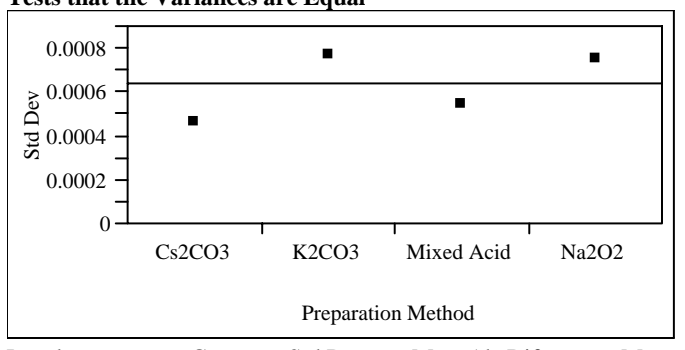

Welch Anova testing Means Equal, allowing Std Devs Not Equal

F Ratio DFNum DFDen Prob > F

$\begin{array}{llll}2.0727 & 3 & 10.889 & 0.1626\end{array}$ 


\section{Exhibit A4. Statistical Comparisons of Chemical Composition Measurements of Simulant Samples Across Preparation Methods by Oxide \\ (Concentrations in Wt\% Oxides)}

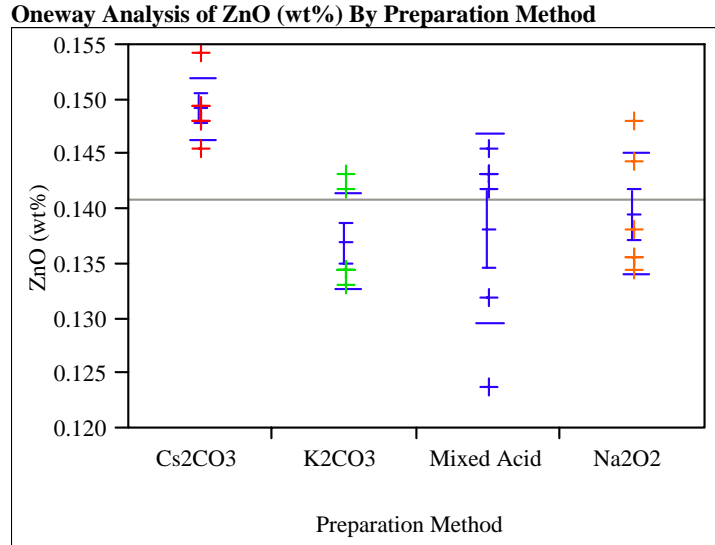

Means Comparisons

Comparisons for all pairs using Tukey-Kramer HSD

$$
\begin{array}{rr}
q^{*} & \text { Alpha } \\
2.79894 & 0.05
\end{array}
$$

Abs(Dif)-LSD Cs2CO3 Na2O2 Mixed Acid K2CO3

$\begin{array}{lllll}\mathrm{Cs} 2 \mathrm{CO} 3 & -0.00928 & 0.00047 & 0.00164 & 0.00296\end{array}$

$\begin{array}{lllll}\mathrm{Na} 2 \mathrm{O} 2 & 0.00047 & -0.00928 & -0.00812 & -0.00679\end{array}$

$\begin{array}{lllll}\text { Mixed Acid } \quad 0.00164 & -0.00812 & -0.00928 & -0.00795\end{array}$

$\begin{array}{lllll}\text { K2CO3 } & 0.00296 & -0.00679 & -0.00795 & -0.00928\end{array}$

Positive values show pairs of means that are significantly different.

$$
\begin{array}{lrr}
\text { Level } & & \begin{array}{r}
\text { Mean } \\
\text { Cs2CO3 }
\end{array} \\
\text { Na2O2 } & \text { B } & 0.14916853 \\
\text { Mixed Acid } & \text { B } & 0.138255776 \\
\text { K2CO3 } & \text { B } & 0.13692800
\end{array}
$$

Levels not connected by same letter are significantly different Tests that the Variances are Equal

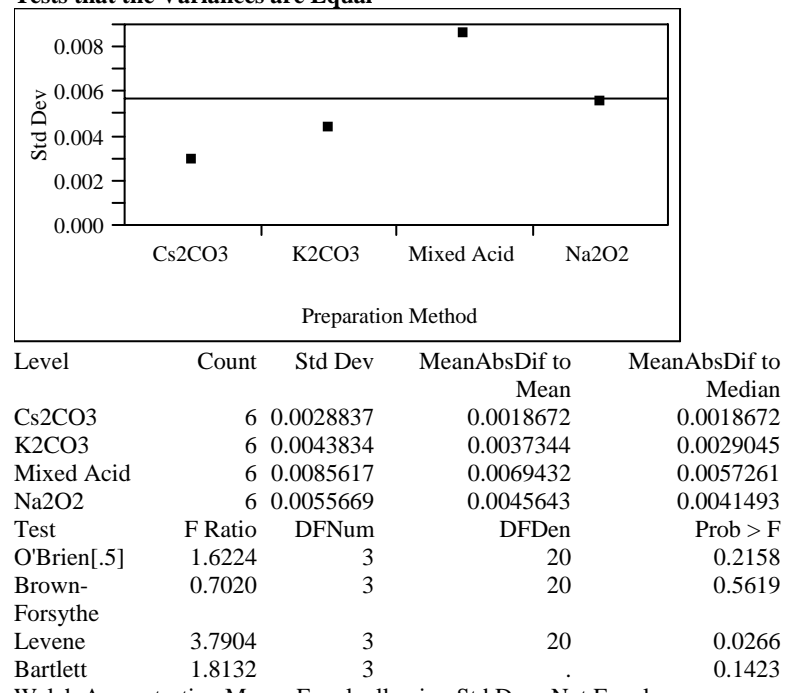

Welch Anova testing Means Equal, allowing Std Devs Not Equal

F Ratio DFNum DFDen Prob $>\mathrm{F}$

$\begin{array}{lrrr}12.3123 & 3 & 10.476 & 0.0009\end{array}$

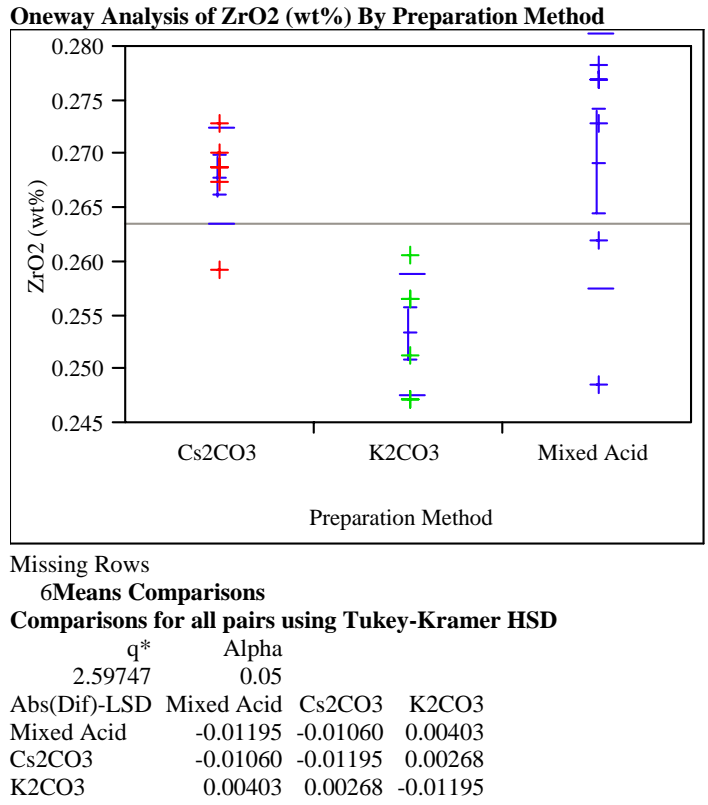

Positive values show pairs of means that are significantly different.

$$
\begin{array}{lrr}
\text { Level } & & \text { Mean } \\
\text { Mixed Acid } & \text { A } & 0.26925947 \\
\text { Cs2CO3 } & \text { A } & 0.26790867 \\
\text { K2CO3 } & \text { B } & 0.25327500
\end{array}
$$

Levels not connected by same letter are significantly different Tests that the Variances are Equal

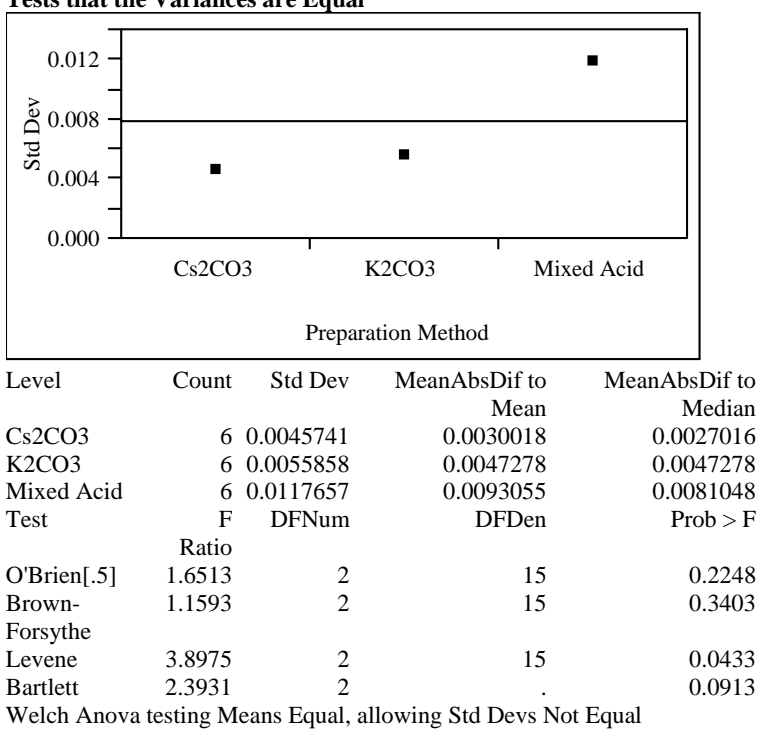

F Ratio DFNum DFDen Prob $>$ F

$\begin{array}{rrrr}12.4627 & 2 & 9.2355 & 0.0024\end{array}$ 


\section{APPENDIX B.}

\section{SUPPLEMENTAL TABLES AND EXHIBITS FOR DWPF RADIOACTIVE GLASS RESULTS}


Table B1. Elemental Measurements ( $\mu \mathrm{g} / \mathrm{g})$ for DWPF Radioactive Glass Prepared Using $\mathrm{Cs}_{2} \mathrm{CO}_{3}$ Digestions

\begin{tabular}{|c|c|c|c|c|c|c|c|c|c|c|c|}
\hline $\begin{array}{l}\text { Sample ID } \\
\end{array}$ & CB1 & CA3 & CB2 & $\begin{array}{ll}\text { CD2 } \\
\end{array}$ & CD5 & $\begin{array}{ll}\text { CD1 } \\
\end{array}$ & CA1 & CD4 & CA2 & CD6 & CD3 \\
\hline Type of Sample & blank & ARG-1 & blank & $\begin{array}{c}\text { Pour } \\
\text { Stream }\end{array}$ & $\begin{array}{c}\text { Pour } \\
\text { Stream }\end{array}$ & $\begin{array}{c}\text { Pour } \\
\text { Stream }\end{array}$ & ARG-1 & $\begin{array}{c}\text { Pour } \\
\text { Stream }\end{array}$ & ARG-1 & $\begin{array}{c}\text { Pour } \\
\text { Stream }\end{array}$ & $\begin{array}{c}\text { Pour } \\
\text { Stream }\end{array}$ \\
\hline LIMS & $\begin{array}{c}220611 \\
10 \mathrm{x}\end{array}$ & $\begin{array}{c}220612 \\
10 x\end{array}$ & $\begin{array}{c}220613 \\
10 x\end{array}$ & $\begin{array}{c}220614 \\
10 \mathrm{x}\end{array}$ & $\begin{array}{c}220615 \\
10 x\end{array}$ & $\begin{array}{c}220616 \\
10 \mathrm{x}\end{array}$ & $\begin{array}{c}220617 \\
10 x\end{array}$ & $\begin{array}{c}220618 \\
10 \mathrm{x}\end{array}$ & $\begin{array}{c}220619 \\
10 x\end{array}$ & $\begin{array}{c}220620 \\
10 x\end{array}$ & $\begin{array}{c}220621 \\
10 \mathrm{x}\end{array}$ \\
\hline $\mathrm{Ag}$ & $<149$ & $<139$ & $<149$ & 1570 & $<158$ & 1340 & 1020 & 416 & $<139$ & 415 & 767 \\
\hline $\mathrm{Al}$ & $<<68$ & 21500 & $<568$ & 24500 & 25400 & 25200 & 21600 & 25500 & 21400 & 25500 & 24500 \\
\hline $\mathrm{B}$ & 1290 & 25000 & 793 & 14600 & 13700 & 14300 & 25600 & 14600 & 25100 & 12700 & 13500 \\
\hline $\mathrm{Ba}$ & $<311$ & 798 & $<311$ & 547 & 438 & 447 & 791 & 406 & 804 & 424 & 423 \\
\hline $\mathrm{Be}$ & $<20.9$ & $<19.5$ & $<20.9$ & $<21.8$ & $<22.3$ & $<21.8$ & $<20.1$ & $<22.7$ & $<19.5$ & $<15.3$ & $<22.2$ \\
\hline $\mathrm{Ca}$ & $<122$ & 9880 & $<122$ & 7240 & 7190 & 7220 & 9900 & 7230 & 9880 & 7210 & 7160 \\
\hline $\mathrm{Cd}$ & $<58.1$ & $<54.2$ & $<58.1$ & 312 & 373 & 396 & $<55.8$ & 368 & $<54.2$ & 463 & 433 \\
\hline $\mathrm{Ce}$ & $<1820$ & $<1700$ & $<1820$ & 4090 & $<1940$ & $<1900$ & $<1750$ & $<1980$ & $<1700$ & $<1340$ & $<1930$ \\
\hline $\mathrm{Cr}$ & $<38.6$ & 558 & $<38.6$ & 382 & 408 & 372 & 559 & 515 & 600 & 445 & 427 \\
\hline $\mathrm{Cu}$ & $<135$ & $<126$ & $<135$ & $<141$ & $<144$ & $<141$ & $<130$ & $<147$ & $<126$ & $<98.9$ & $<143$ \\
\hline $\mathrm{Fe}$ & 288 & 97800 & $<162$ & 75200 & 76700 & 77100 & 97700 & 77600 & 97800 & 76300 & 75400 \\
\hline Gd & $<230$ & $<214$ & $<230$ & 262 & $<244$ & $<240$ & $<220$ & $<249$ & $<214$ & 171 & $<244$ \\
\hline $\bar{K}$ & $<10500$ & $<9820$ & $<10500$ & $<11000$ & $<11200$ & $<11000$ & 24400 & $<11400$ & 14600 & $<7700$ & $<11200$ \\
\hline $\mathrm{La}$ & $<703$ & $<655$ & $<703$ & $<734$ & $<748$ & $<734$ & $<674$ & $<762$ & $<655$ & $<514$ & $<745$ \\
\hline $\mathrm{Li}$ & $<649$ & 14300 & $<649$ & 21500 & 21900 & 21800 & 14200 & 22000 & 14200 & 21800 & 21400 \\
\hline $\mathrm{Mg}$ & $<54.1$ & 4980 & $<54.1$ & 6850 & 7090 & 7110 & 4970 & 7110 & 4950 & 7100 & 6940 \\
\hline $\mathrm{Mn}$ & $<27.0$ & 14400 & $<27.0$ & 16200 & 16400 & 16400 & 14300 & 16500 & 14400 & 16400 & 16100 \\
\hline Mo & $<1800$ & $<1680$ & $<1800$ & $<1880$ & $<1910$ & $<1880$ & $<1720$ & $<1950$ & $<1680$ & $<1320$ & $<1910$ \\
\hline $\mathrm{Na}$ & $<<810$ & 81900 & $<4810$ & 89500 & 93400 & 91700 & 83100 & 91400 & 83100 & 88000 & 88100 \\
\hline $\mathrm{Ni}$ & $<541$ & 8230 & $<541$ & 4200 & 4610 & 4370 & 7950 & 4280 & 8190 & 4330 & 3980 \\
\hline$P$ & $<3610$ & $<3370$ & $<3610$ & $<3770$ & $<3840$ & $<3770$ & $<3460$ & $<3910$ & $<3370$ & $<2640$ & $<3830$ \\
\hline $\mathrm{Pb}$ & $<10300$ & $<9590$ & $<10300$ & $<10700$ & $<10900$ & $<10700$ & $<9870$ & $<11200$ & $<9590$ & $<7530$ & $<10900$ \\
\hline $\mathrm{S}$ & $<128$ & 430 & $<128$ & 1630 & 1510 & 2030 & 302 & 1710 & 969 & 1600 & 1790 \\
\hline $\mathrm{Sb}$ & $<1570$ & $<1460$ & $<1570$ & $<1640$ & $<1670$ & $<1640$ & $<1500$ & $<1700$ & $<1460$ & $<1150$ & $<1660$ \\
\hline $\mathrm{Si}$ & $<<6570$ & 223000 & $<6570$ & 239000 & 244000 & 244000 & 232000 & 253000 & 229000 & 244000 & 243000 \\
\hline Sn & $<4050$ & $<3780$ & $<4050$ & $<4230$ & $<4310$ & $<4230$ & $<3890$ & $<4400$ & $<3780$ & $<2970$ & $<4300$ \\
\hline $\mathrm{Sr}$ & $<135$ & 1720 & $<135$ & 1670 & 1270 & 1290 & 1640 & 1190 & 1630 & 1370 & 1280 \\
\hline $\mathrm{Ti}$ & $<40.5$ & 7000 & $<40.5$ & 324 & 318 & 325 & 7010 & 312 & 7000 & 333 & 312 \\
\hline $\mathrm{U}$ & $<2650$ & $<2470$ & $<2650$ & 29600 & 27700 & 26900 & $<2540$ & 27400 & $<2470$ & 27600 & 27000 \\
\hline $\mathrm{V}$ & $<270$ & 828 & $<270$ & $<282$ & $<288$ & 1780 & $<259$ & $<293$ & $<252$ & $<198$ & $<287$ \\
\hline $\mathrm{Zn}$ & $<94.6$ & $<88.2$ & $<94.6$ & $<98.7$ & $<101$ & $<98.7$ & $<90.8$ & $<103$ & $<88.2$ & $<69.2$ & $<100$ \\
\hline $\mathrm{Zr}$ & $<108$ & 881 & $<108$ & 401 & 389 & 401 & 903 & 384 & 919 & 381 & 376 \\
\hline
\end{tabular}


Table B2. Elemental Measurements $(\mu \mathrm{g} / \mathrm{g})$ for DWPF Radioactive Glass Prepared Using $\mathrm{Na}_{2} \mathrm{O}_{2}$ Digestions

\begin{tabular}{|c|c|c|c|c|c|c|c|c|}
\hline Sample ID & PR1 & $\begin{array}{l}\text { PR2 } \\
\end{array}$ & PR3 & PR4 & ARG-1 (PF) & ARG-2 (PF) & ARG-3 (PF) & Blank \\
\hline Type of Sample & Pour Stream & Pour Stream & Pour Stream & Pour Stream & ARG-1 & ARG-1 & ARG-1 & blank \\
\hline LIMS & 3-220491 & 3-220492 & 3-220493 & 3-220494 & 3-220495 & 3-220496 & 3-220497 & $3-220498$ \\
\hline $\mathrm{Ag}$ & 331 & 368 & 393 & 396 & 391 & 509 & 385 & $<87.6$ \\
\hline $\mathrm{Al}$ & 25500 & 24200 & 25400 & 25700 & 21700 & 21700 & 21200 & $<335$ \\
\hline $\mathrm{B}$ & 13900 & 13400 & 13900 & 14000 & 24300 & 24900 & 23800 & $<422$ \\
\hline $\mathrm{Ba}$ & 426 & 480 & 503 & 548 & 911 & 939 & 970 & $<183$ \\
\hline $\mathrm{Be}$ & 10.8 & 88 & 40.6 & 86.7 & 28.9 & 114 & 71.1 & $<35.1$ \\
\hline $\mathrm{Ca}$ & 9110 & 8680 & 9010 & 9010 & 11900 & 12300 & 11500 & 98.6 \\
\hline $\mathrm{Cd}$ & 501 & 471 & 479 & 525 & $<38.9$ & $<46.5$ & $<34.0$ & $<34.3$ \\
\hline $\mathrm{Ce}$ & 4310 & 5140 & 6270 & 7900 & 6440 & 6990 & 7640 & $<1076$ \\
\hline $\mathrm{Cr}$ & 434 & 448 & 399 & 305 & 487 & 523 & 214 & $<207$ \\
\hline $\mathrm{Cu}$ & $<79.7$ & $<82.6$ & $<80.3$ & $<74.1$ & $<90.5$ & $<108$ & $<79.1$ & $<79.7$ \\
\hline $\mathrm{Fe}$ & 77000 & 73700 & 76900 & 77500 & 98300 & 100000 & 97500 & $<95.6$ \\
\hline $\mathrm{Gd}$ & 426 & 539 & 617 & 691 & 464 & 568 & 604 & $<135$ \\
\hline $\mathrm{K}$ & 6930 & $<6440$ & 24700 & 13200 & & & & $<6210$ \\
\hline $\mathrm{La}$ & 670 & 723 & 867 & 1130 & 831 & 864 & 986 & $<414$ \\
\hline $\mathrm{Li}$ & 24100 & 23000 & 23900 & 24200 & 16100 & 16400 & 15900 & $<382$ \\
\hline $\mathrm{Mg}$ & 7160 & 6830 & 7140 & 7200 & 4980 & 5000 & 4960 & $<31.9$ \\
\hline $\mathrm{Mn}$ & 16500 & 15700 & 16600 & 16600 & 14500 & 14800 & 14500 & $<15.9$ \\
\hline Mo & $<1060$ & $<1100$ & $<1070$ & 1010 & $<1200$ & $<1440$ & 1240 & $<1060$ \\
\hline \multicolumn{9}{|l|}{$\mathrm{Na}$} \\
\hline $\mathrm{Ni}$ & 4300 & 3990 & 4210 & 4390 & 8110 & 8140 & 7970 & $<319$ \\
\hline $\mathrm{P}$ & $<2130$ & $<2210$ & $<2140$ & $<1980$ & $<2420$ & $<2890$ & $<2110$ & $<2130$ \\
\hline $\mathrm{Pb}$ & $<6060$ & $<6290$ & $<6110$ & $<5640$ & $<6890$ & $<8230$ & $<6020$ & $<6060$ \\
\hline S & 1450 & 1300 & 1550 & 1520 & $<561$ & $<670$ & $<490$ & $<494$ \\
\hline $\mathrm{Sb}$ & $<924$ & 1050 & 1180 & 1020 & 1270 & 1280 & 1390 & $<924$ \\
\hline $\mathrm{Si}$ & 234000 & 235000 & 242000 & 243000 & 230000 & 234000 & 228000 & $<3870$ \\
\hline Sn & $<2390$ & $<2480$ & $<2410$ & 2330 & $<2710$ & $<3240$ & $<2370$ & $<2390$ \\
\hline $\mathrm{Sr}$ & 2280 & 2220 & 2420 & 2610 & 2850 & 2900 & 2890 & $<79.7$ \\
\hline $\mathrm{Ti}$ & 373 & 355 & 370 & 371 & 6860 & 6990 & 6750 & $<23.9$ \\
\hline $\mathrm{U}$ & 30100 & 29200 & 32200 & 32800 & 3410 & 4550 & 5740 & $<1560$ \\
\hline $\mathrm{V}$ & 946 & $<165$ & 705 & $<148$ & $<181$ & $<216$ & $<158$ & $<159$ \\
\hline $\mathrm{Zn}$ & $<55.8$ & $<57.9$ & $<56.2$ & 73.7 & $<63.3$ & $<75.7$ & 77.4 & $<55.8$ \\
\hline $\mathrm{Zr}$ & 36700 & 29800 & 27600 & 17400 & 49100 & 64100 & 29800 & 3730 \\
\hline
\end{tabular}


Table B3. Elemental Measurements ( $\mu \mathrm{g} / \mathrm{g}$ ) for DWPF Radioactive Glass Prepared Using Mixed Acid Digestions

\begin{tabular}{|c|c|c|c|c|c|c|c|c|}
\hline Sample ID & AR1 & AR2 & AR3 & AR4 & ARG-1 (MA) & ARG-2 (MA) & ARG-3 (MA) & Blank \\
\hline Type of Sample & Pour Stream & Pour Stream & Pour Stream & Pour Stream & ARG-1 & ARG-1 & ARG-1 & blank \\
\hline LIMS & $3-220481$ & $3-220482$ & $3-220483$ & $3-220484$ & $3-220485$ & $3-220486$ & $3-220487$ & $3-220488$ \\
\hline $\mathrm{Ag}$ & $<44.2$ & $<41.5$ & $<44.0$ & $<43.1$ & $<44.7$ & $<43.8$ & $<44.4$ & $<44.2$ \\
\hline $\mathrm{Al}$ & 25000 & 25700 & 25800 & 25600 & 22000 & 21500 & 21900 & $<169$ \\
\hline $\mathrm{B}$ & 920000 & 887000 & 970000 & 918000 & 956000 & 946000 & 957000 & 960000 \\
\hline $\mathrm{Ba}$ & 356 & 363 & 362 & 366 & 772 & 757 & 768 & $<92.4$ \\
\hline $\mathrm{Be}$ & $<6.22$ & $<5.84$ & $<6.19$ & $<6.07$ & 17.9 & 17 & 16.3 & $<6.22$ \\
\hline $\mathrm{Ca}$ & 7210 & 7430 & 7480 & 7360 & 10100 & 9930 & 10100 & $<36.1$ \\
\hline $\mathrm{Cd}$ & 595 & 615 & 634 & 609 & $<<17.5$ & $<17.1$ & $<17.3$ & $<17.3$ \\
\hline $\mathrm{Ce}$ & $<542$ & $<509$ & $<540$ & $<529$ & $<549$ & $<538$ & $<544$ & $<542$ \\
\hline $\mathrm{Cr}$ & 401 & 399 & 428 & 395 & 450 & 534 & 499 & $<104$ \\
\hline $\mathrm{Cu}$ & 105 & 99.5 & 107 & 103 & $<40.7$ & $<39.8$ & $<40.3$ & $<40.2$ \\
\hline $\mathrm{Fe}$ & 72600 & 75000 & 74900 & 74600 & 96300 & 95000 & 96700 & $<48.2$ \\
\hline $\mathrm{Gd}$ & 119 & 121 & 117 & 121 & $<69.1$ & $<67.7$ & $<68.5$ & $<68.3$ \\
\hline $\mathrm{K}$ & $<3130$ & $<2940$ & $<3120$ & $<3050$ & 22000 & 14900 & 17000 & $<3130$ \\
\hline $\mathrm{La}$ & $<209$ & $<196$ & $<208$ & $<204$ & $<211$ & $<207$ & $<210$ & $<209$ \\
\hline $\mathrm{Li}$ & 21800 & 22500 & 22500 & 22300 & 14700 & 14500 & 14700 & $<193$ \\
\hline $\mathrm{Mg}$ & 6790 & 7010 & 7010 & 6970 & 4990 & 4940 & 5030 & $<16.1$ \\
\hline $\mathrm{Mn}$ & 15600 & 16100 & 16100 & 16000 & 14200 & 14100 & 14300 & $<8.03$ \\
\hline Mo & $<534$ & $<502$ & $<532$ & $<522$ & $<541$ & $<530$ & $<536$ & $<534$ \\
\hline $\mathrm{Na}$ & 86400 & 88700 & 89200 & 87900 & 82500 & 80600 & 82600 & $<1430$ \\
\hline $\mathrm{Ni}$ & 4330 & 4520 & 4420 & 4580 & 8200 & 8310 & 8330 & $<161$ \\
\hline $\mathrm{P}$ & 1320 & $<1010$ & $<1070$ & 1170 & $<1090$ & $<1060$ & $<1080$ & $<1070$ \\
\hline $\mathrm{Pb}$ & $<3060$ & $<2870$ & $<3040$ & $<2980$ & $<3090$ & $<3030$ & $<3070$ & $<3060$ \\
\hline $\mathrm{S}$ & 1760 & 1480 & 1500 & 1400 & 515 & 179 & 280 & 241 \\
\hline $\mathrm{Sb}$ & $<466$ & $<438$ & $<464$ & $<455$ & $<472$ & $<462$ & $<468$ & $<466$ \\
\hline $\mathrm{Si}$ & 193000 & 202000 & 207000 & 203000 & 166000 & 189000 & 189000 & 2950 \\
\hline Sn & 6510 & 6130 & 6770 & 6470 & 6490 & 6590 & 6640 & 5500 \\
\hline $\mathrm{Sr}$ & 1510 & 1530 & 1560 & 1540 & 1910 & 1880 & 1930 & $<40.2$ \\
\hline $\mathrm{Ti}$ & 368 & 378 & 390 & 374 & 6910 & 6790 & 6920 & $<12.0$ \\
\hline $\mathrm{U}$ & 28000 & 28700 & 28400 & 28300 & $<797$ & $<781$ & $<790$ & $<787$ \\
\hline $\mathrm{V}$ & 198 & $<75.5$ & $<80.0$ & $<78.4$ & $<81.3$ & 158 & $<80.6$ & $<80.3$ \\
\hline $\mathrm{Zn}$ & 56.9 & 66.5 & 62.4 & 59.5 & 80.7 & 81.6 & 85.5 & $<28.1$ \\
\hline $\mathrm{Zr}$ & 387 & 404 & 411 & 411 & 931 & 908 & 923 & $<32.1$ \\
\hline
\end{tabular}


Exhibit B1. Chemical Composition Measurements in LIMS Order by Oxide for DWPF Radioactive Glass Set

Legend

Preparation Method

- Cs2CO3

- Mixed Acid

- $\mathrm{Na} 2 \mathrm{O} 2$
Type of Sample

- ARG-1

+ Pour Stream

x blank

Individual Measurement of Ag2O (wt\%)

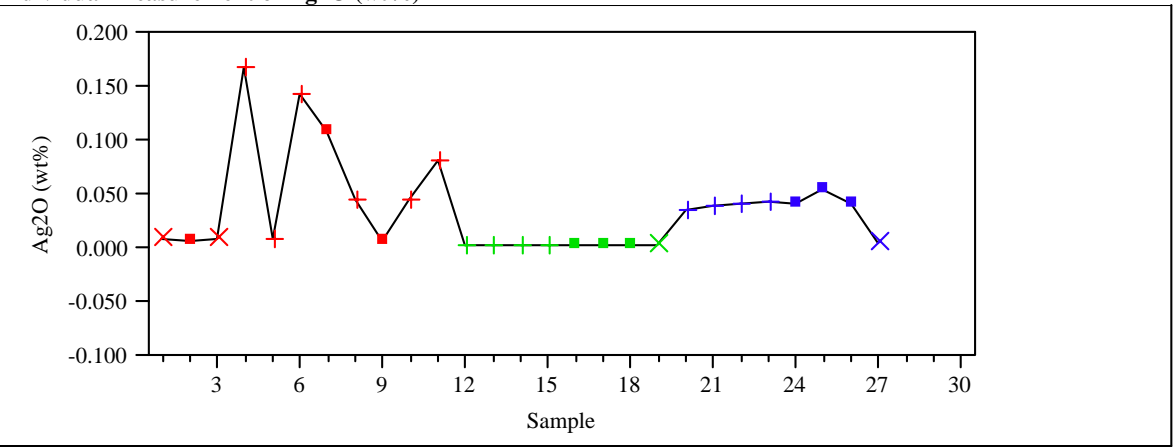

Individual Measurement of Al2O3 (wt\%)

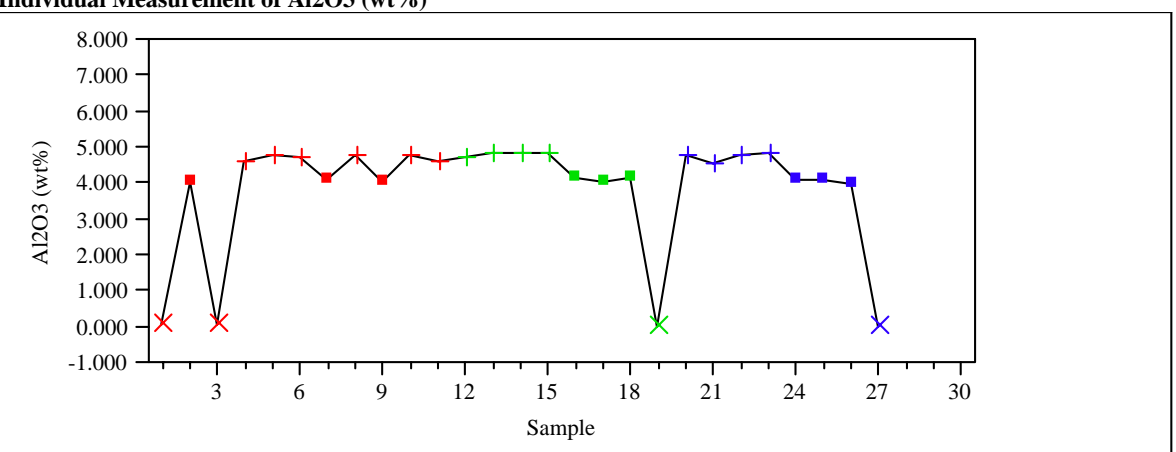

Individual Measurement of B2O3 (wt\%)

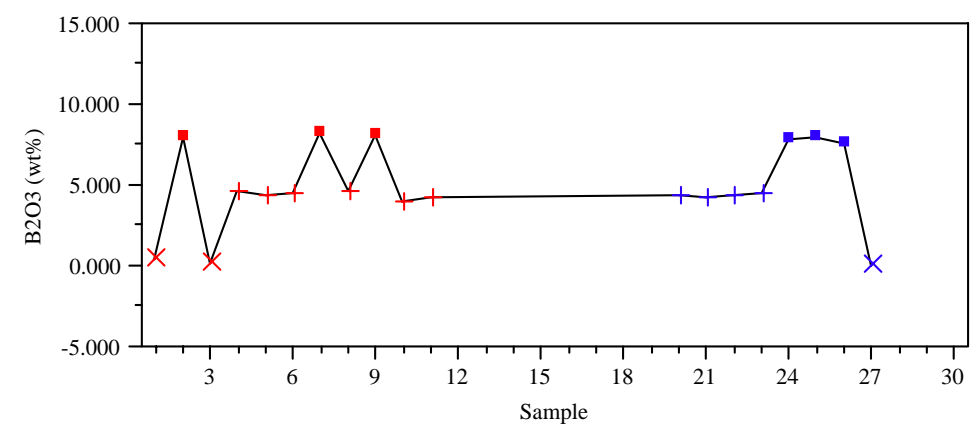


Exhibit B1. Chemical Composition Measurements in LIMS Order by Oxide for DWPF Radioactive Glass Set

Individual Measurement of $\mathrm{BaO}(\mathrm{wt} \%)$
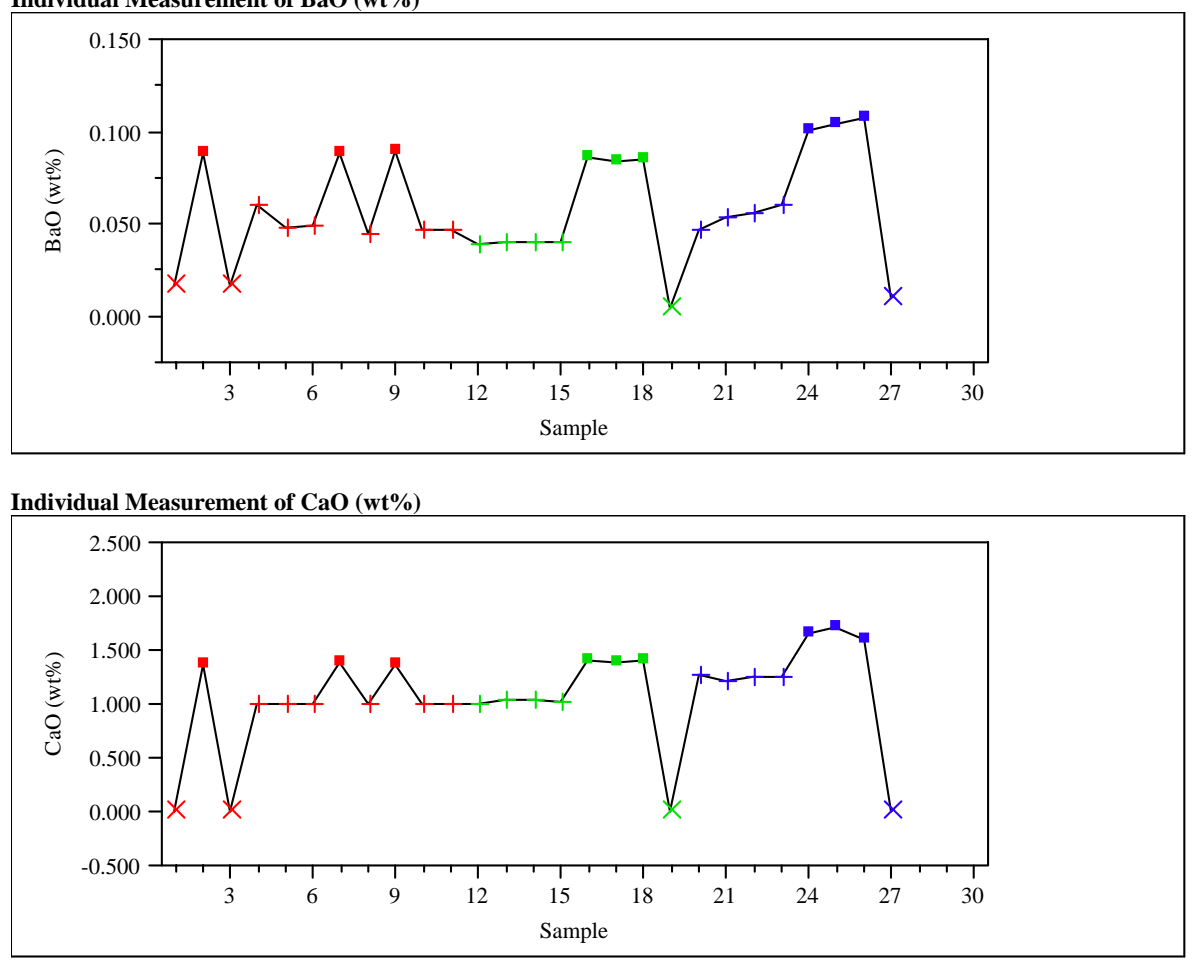

Individual Measurement of CdO (wt\%)

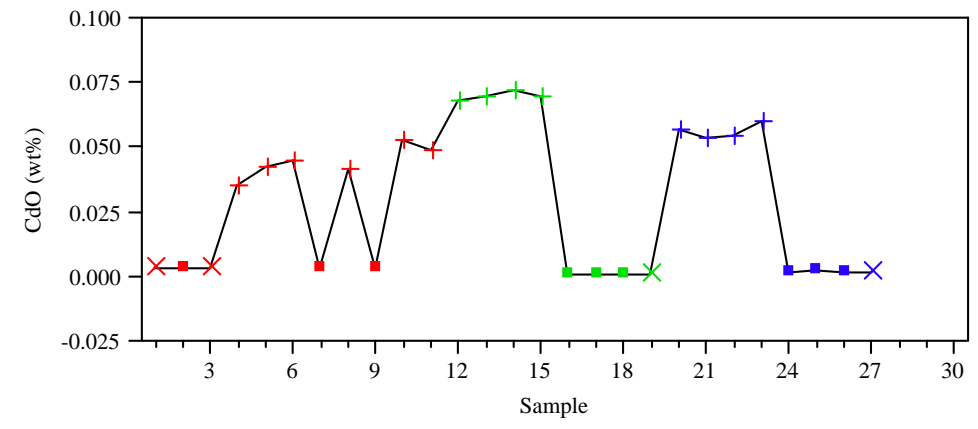

Individual Measurement of Ce2O3 (wt\%)

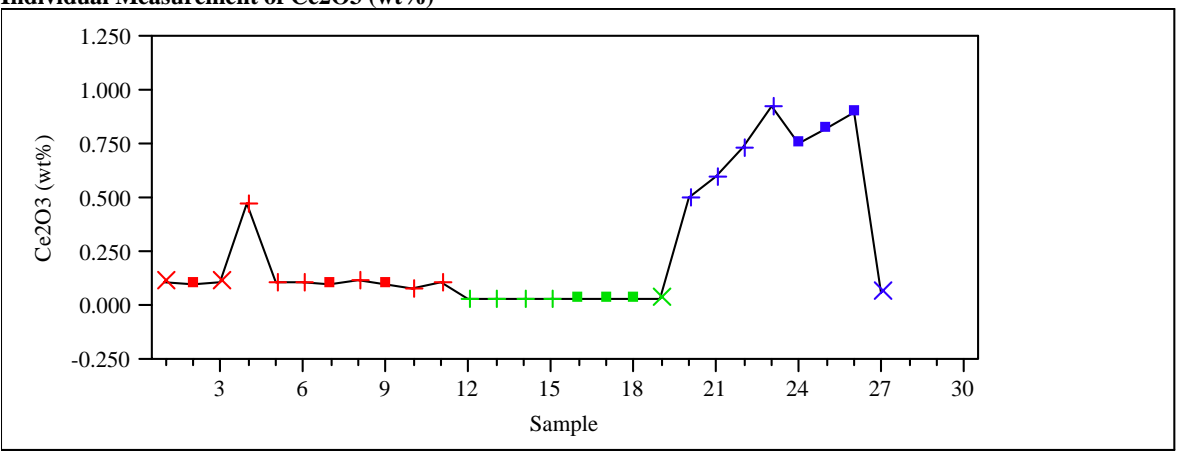


Westinghouse Savannah River Company

WSRC-TR-2005-00396

Savannah River National Laboratory

Rev. 0

Analytical Development/Statistical Consulting Sections

Page 92 of 140

Exhibit B1. Chemical Composition Measurements in LIMS Order by Oxide for DWPF Radioactive Glass Set

Individual Measurement of Cr2O3 (wt\%)
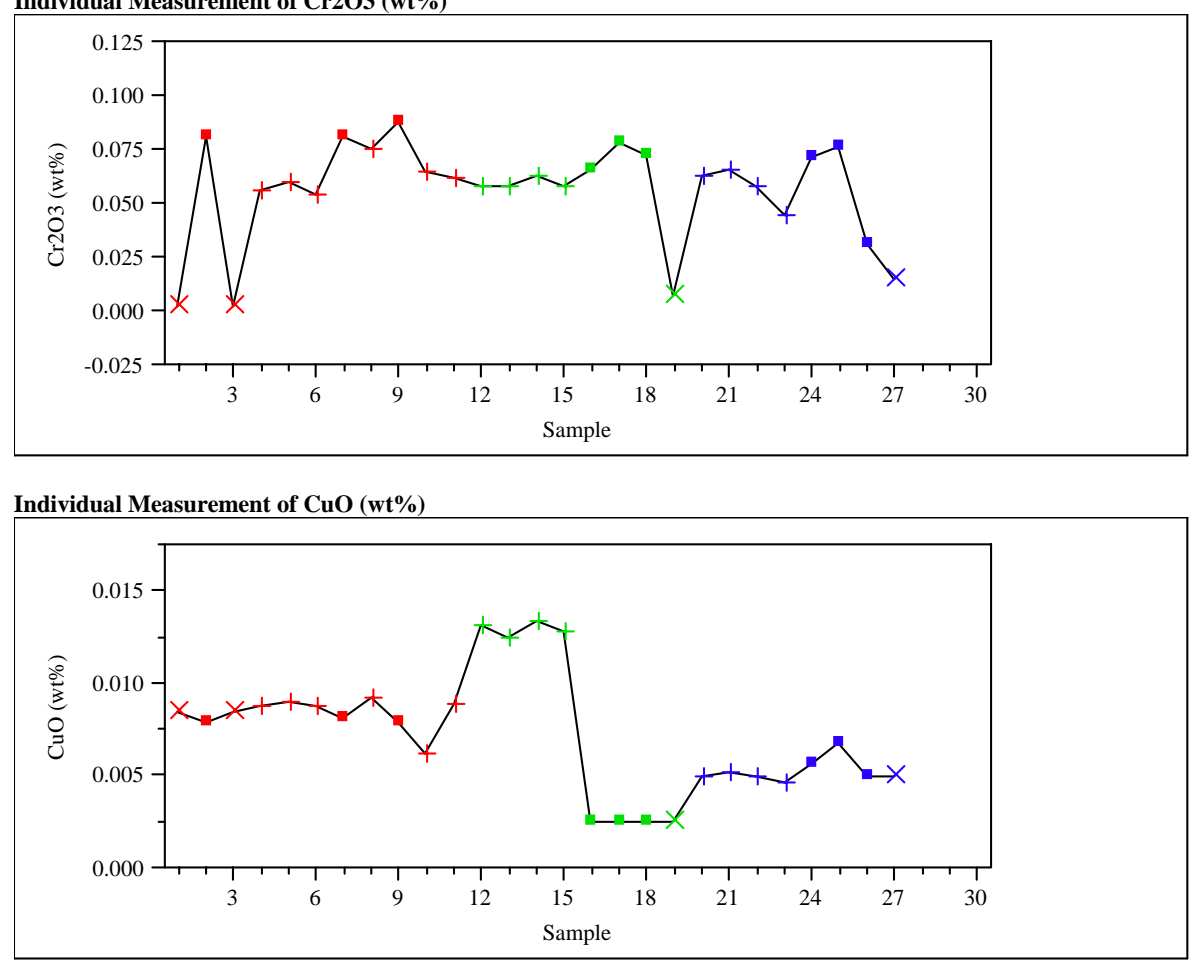

Individual Measurement of Fe2O3 (wt \%)

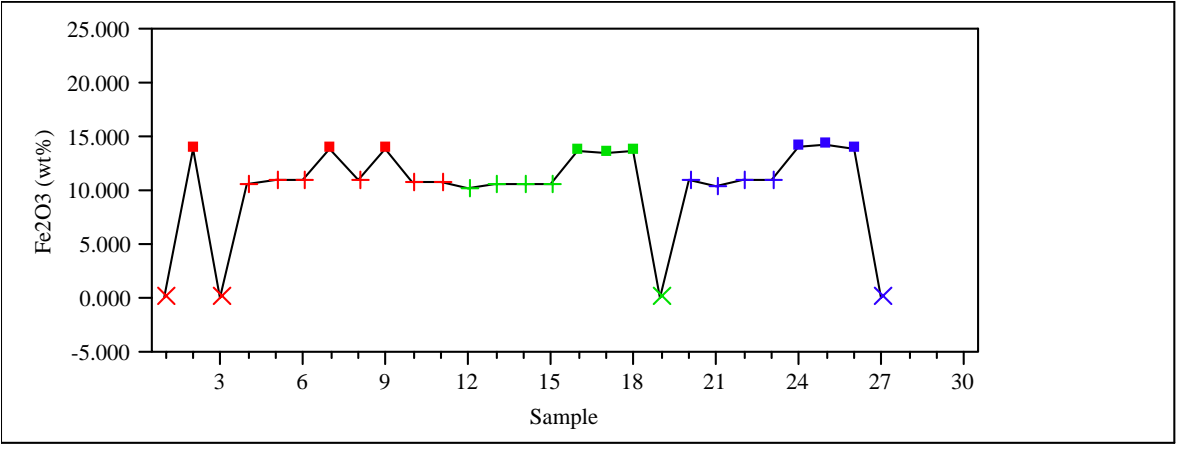

Individual Measurement of K2O (wt \%)

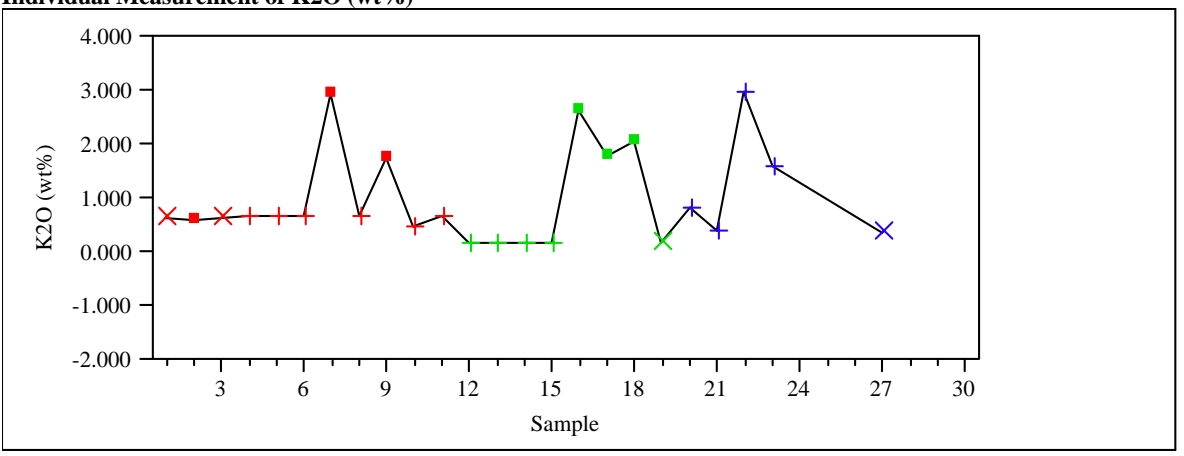


Exhibit B1. Chemical Composition Measurements in LIMS Order by Oxide for DWPF Radioactive Glass Set

Individual Measurement of La2O3 (wt\%)
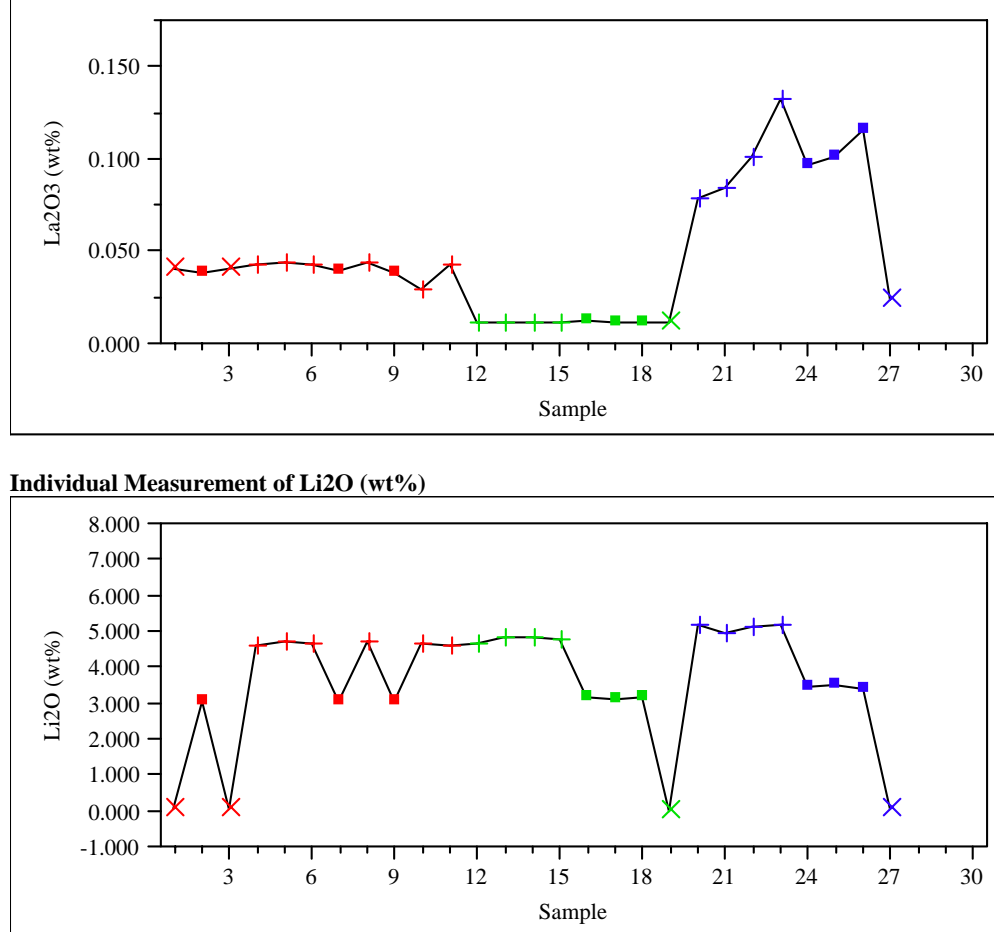

Individual Measurement of MgO (wt\%)

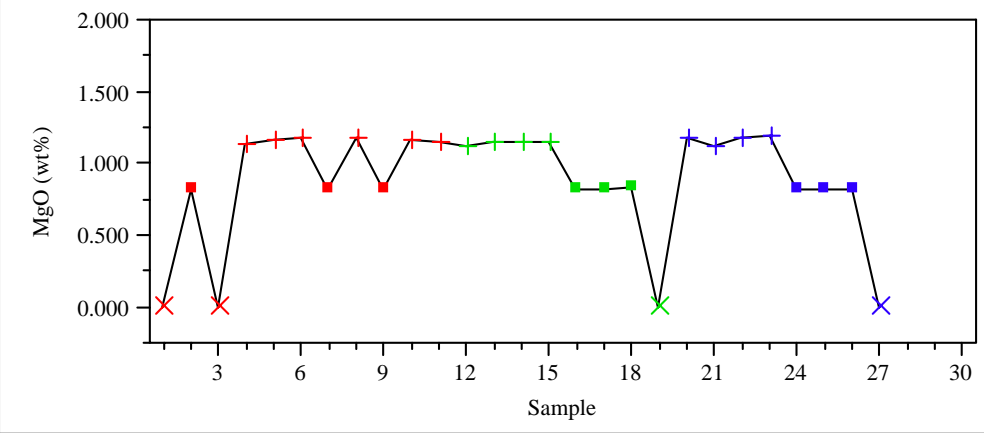

Individual Measurement of $\mathrm{MnO}$ (wt\%)

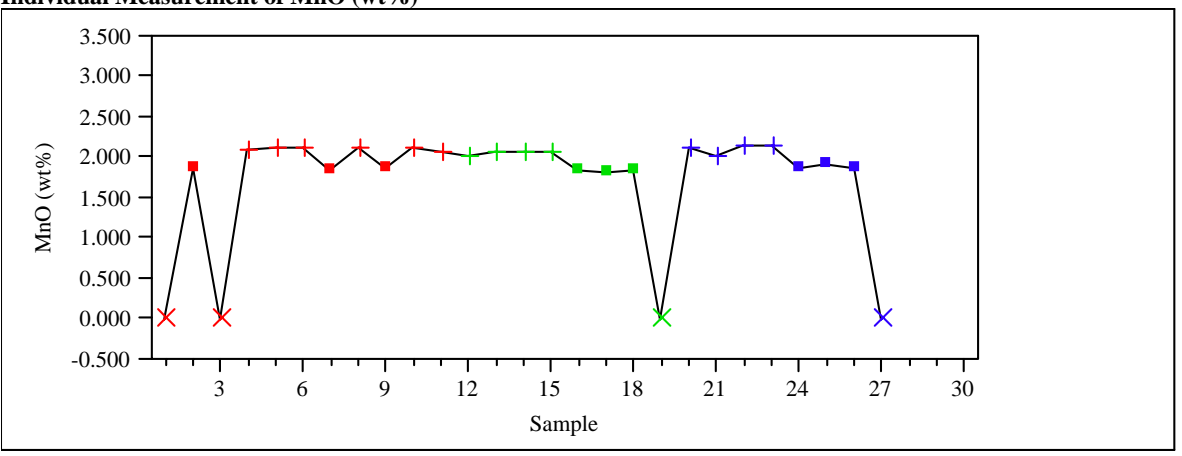


Exhibit B1. Chemical Composition Measurements in LIMS Order by Oxide for DWPF Radioactive Glass Set

Individual Measurement of $\mathrm{MoO} 3$ (wt\%)
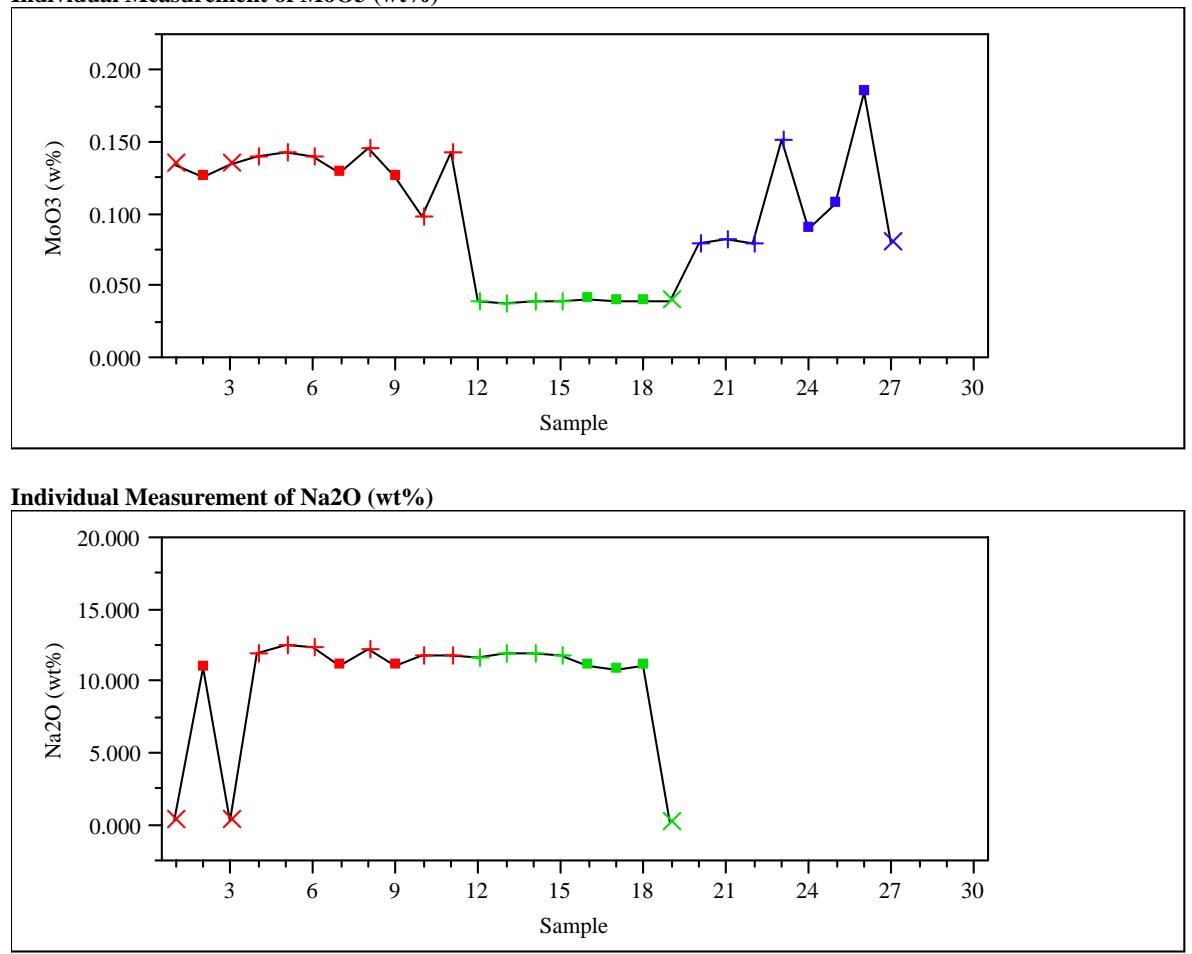

Individual Measurement of $\mathrm{NiO}$ (wt\%)

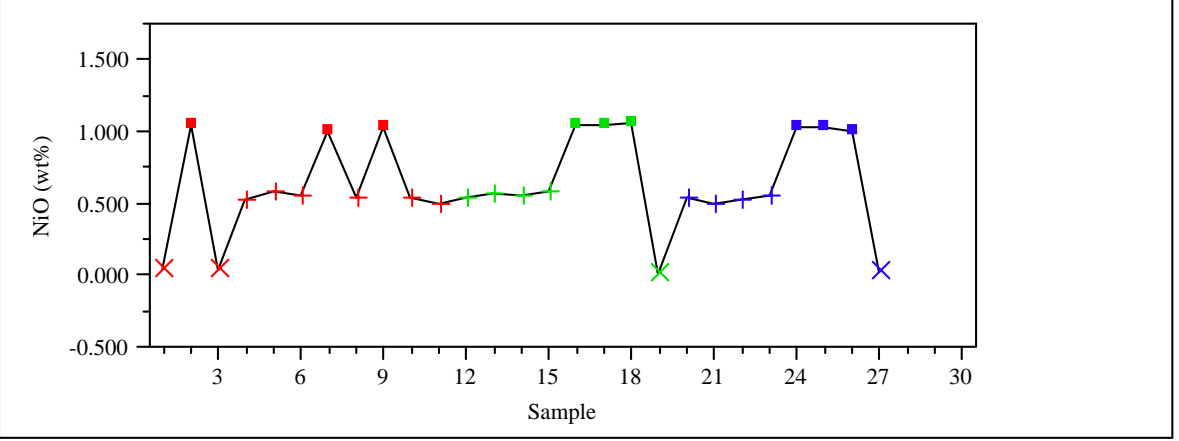

Individual Measurement of P2O5 (wt \%)

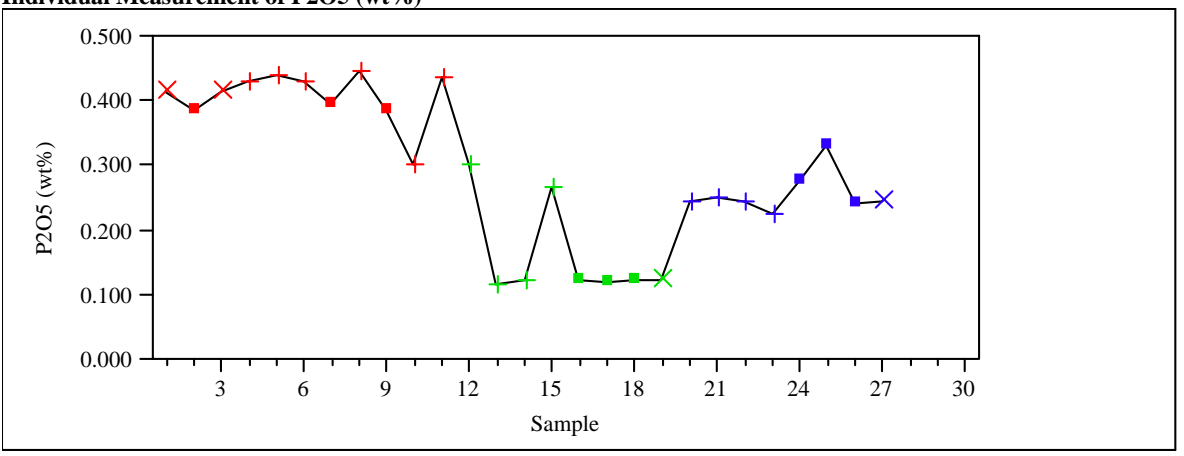


Exhibit B1. Chemical Composition Measurements in LIMS Order by Oxide for DWPF Radioactive Glass Set

Individual Measurement of PbO (wt \%)
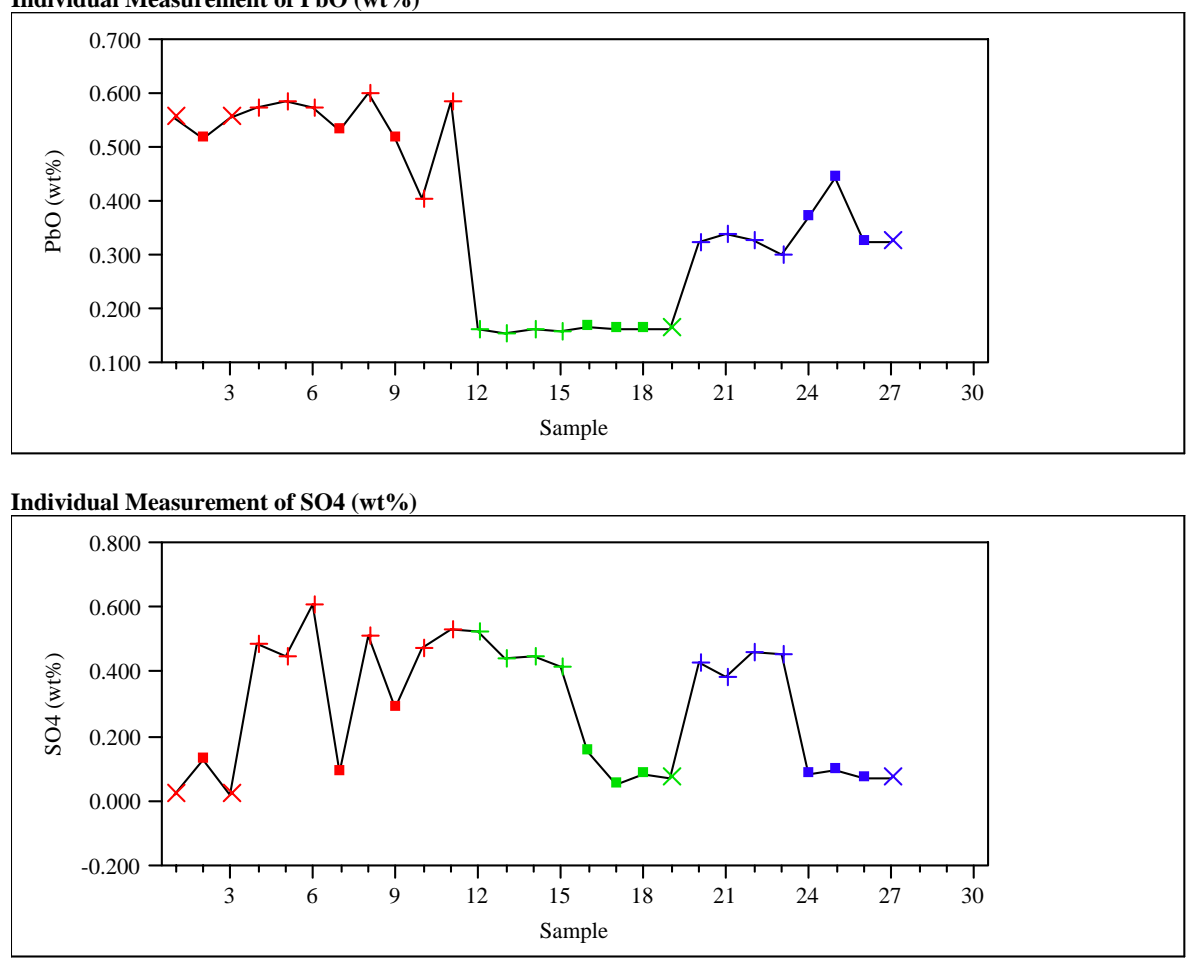

Individual Measurement of $\mathrm{SiO} 2(\mathrm{wt} \%)$

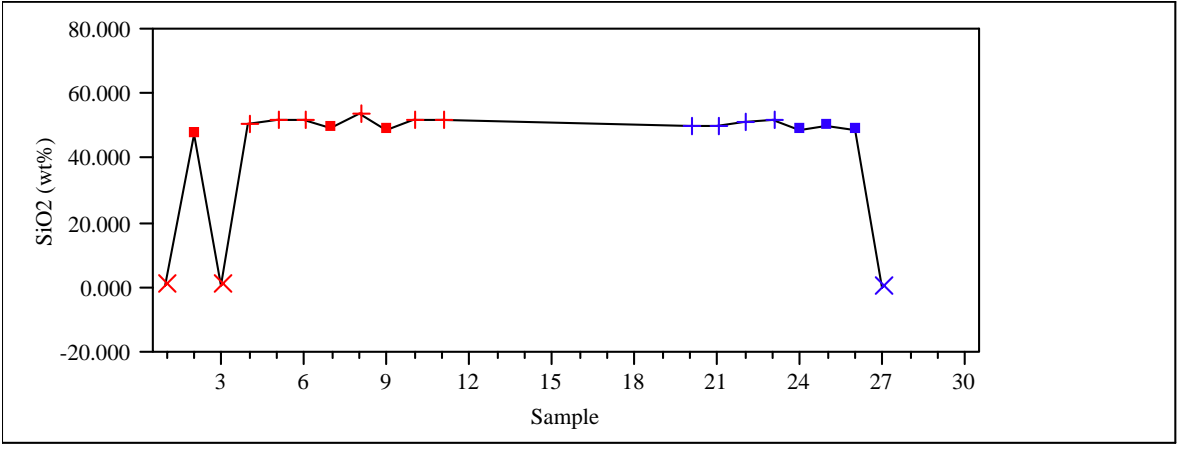

Individual Measurement of SnO2 (wt \%)

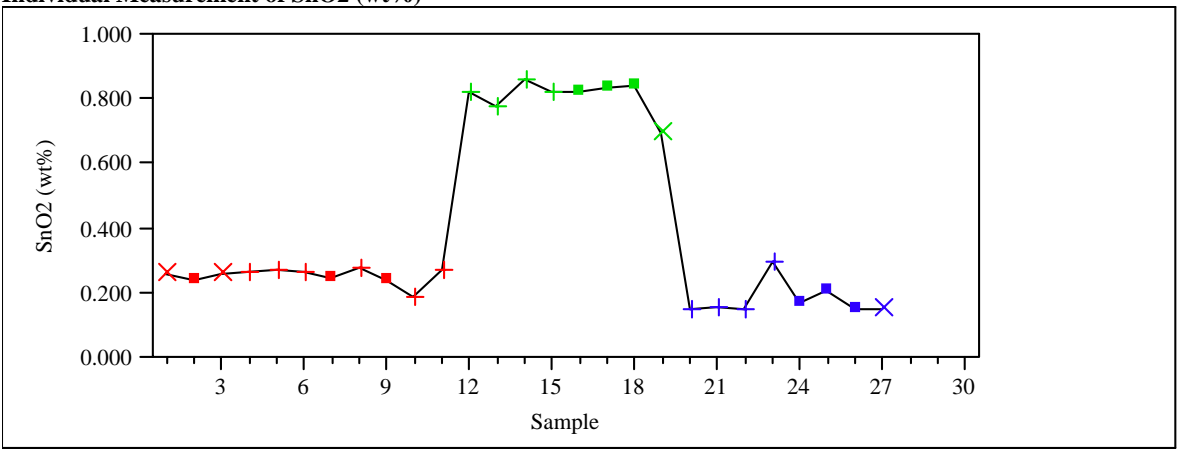


Exhibit B1. Chemical Composition Measurements in LIMS Order by Oxide for DWPF Radioactive Glass Set

Individual Measurement of SrO (wt\%)
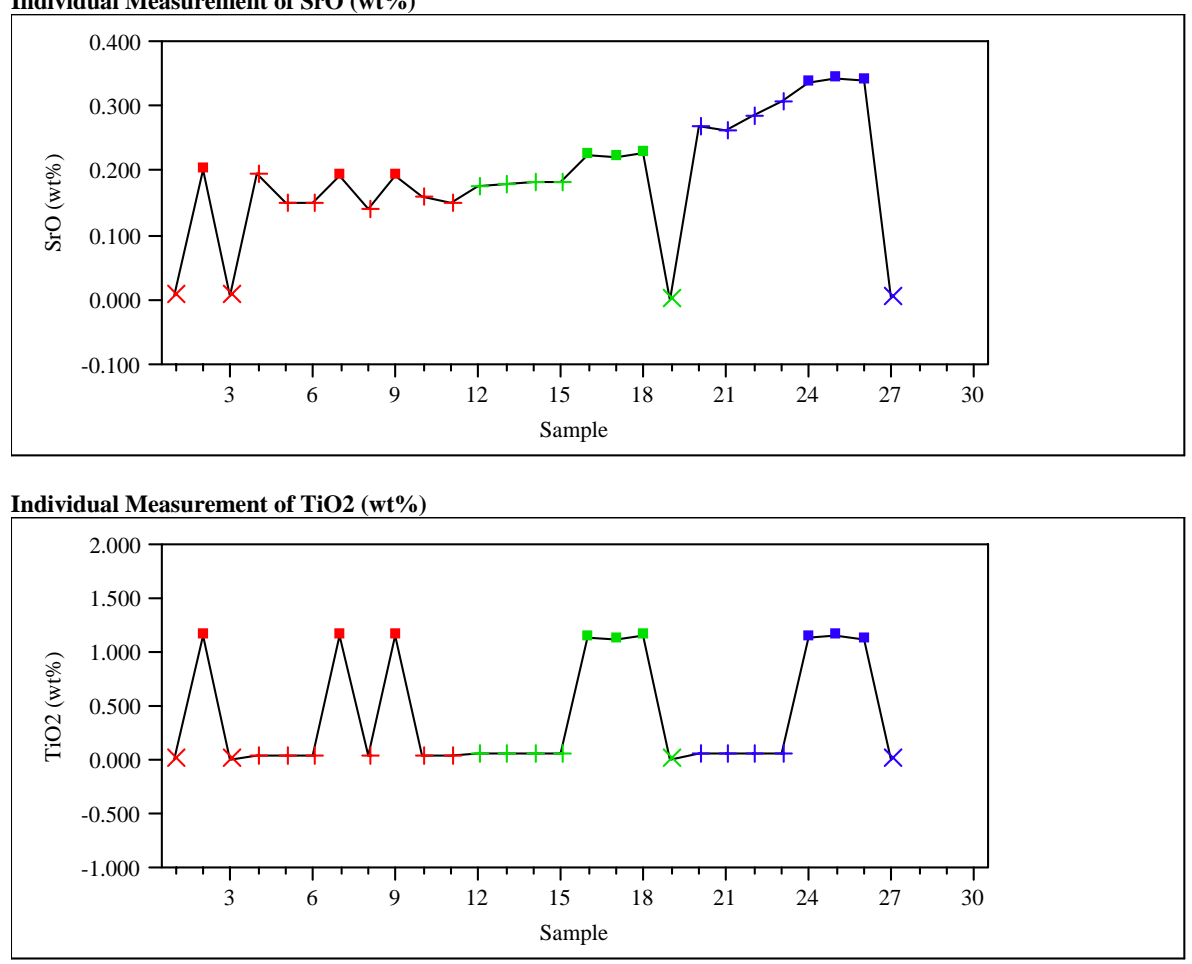

Individual Measurement of U3O8 (wt \%)

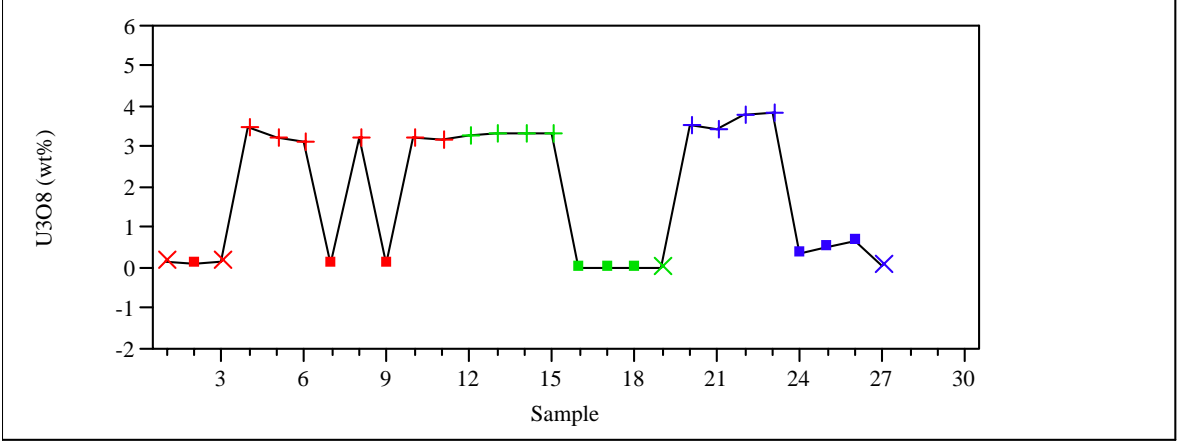

Individual Measurement of V2O5 (wt \%)

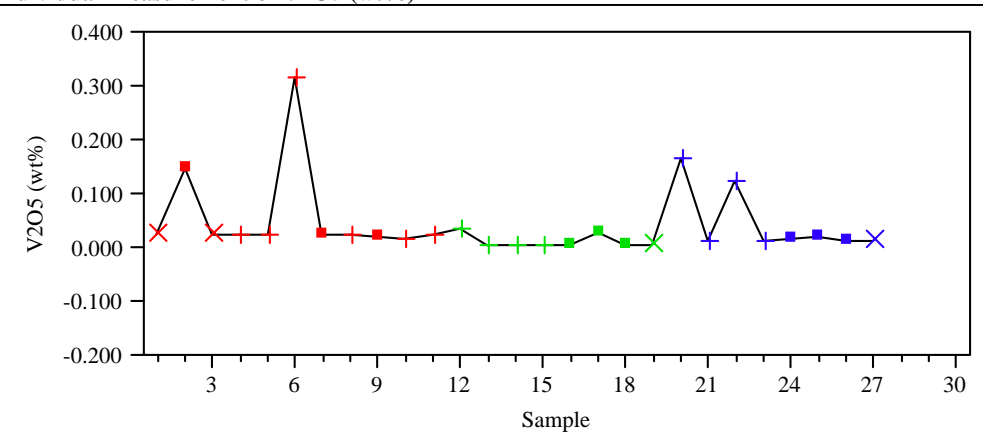


Exhibit B1. Chemical Composition Measurements in LIMS Order by Oxide for DWPF Radioactive Glass Set

Individual Measurement of $\mathrm{ZnO}$ (wt\%)
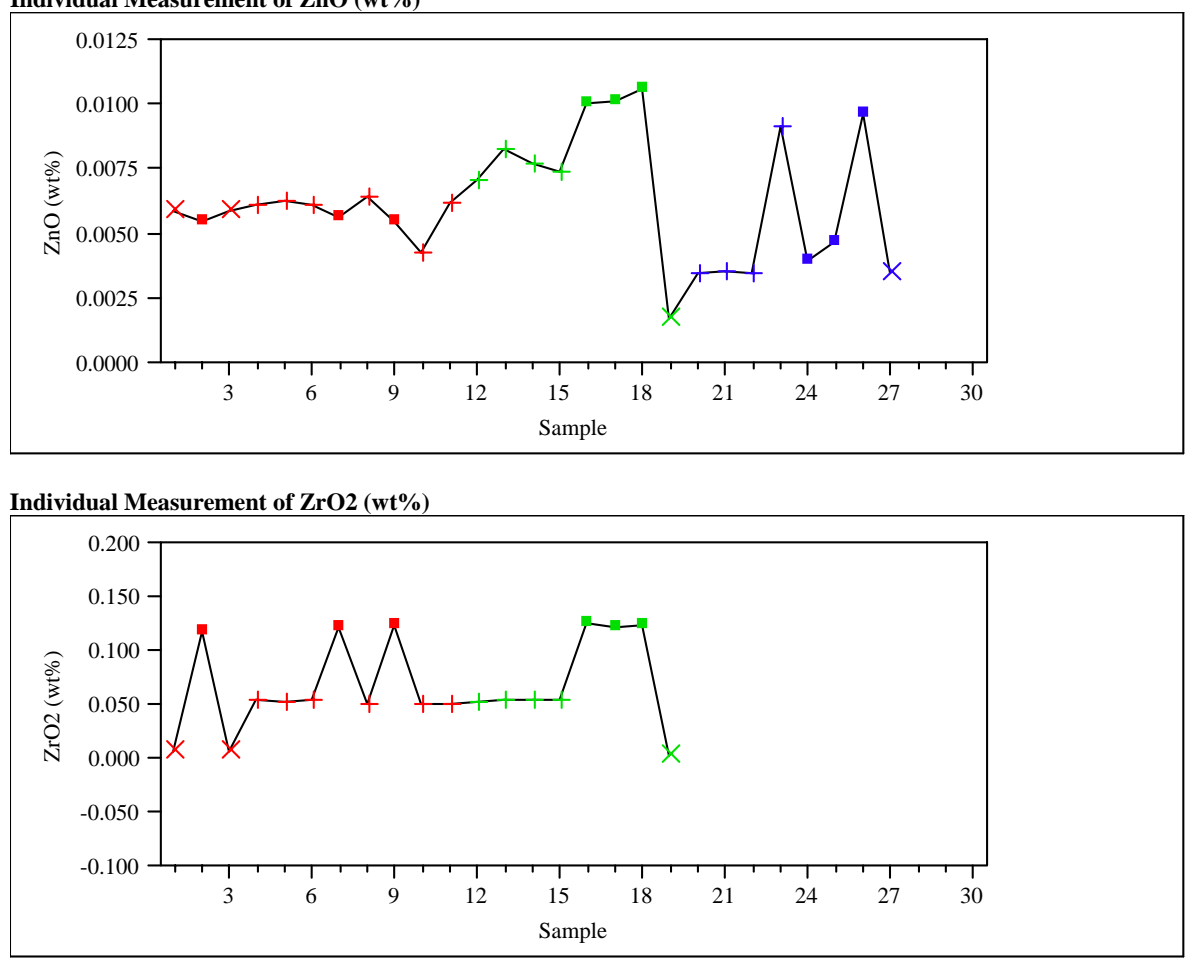
Westinghouse Savannah River Company

WSRC-TR-2005-00396

Savannah River National Laboratory

Rev. 0

Analytical Development/Statistical Consulting Sections

Page 98 of 140

Exhibit B2. Chemical Composition Measurements by Type of Prepared Sample by Oxide for DWPF Radioactive Glass

(Concentrations are in wt\%.)

\section{Legend}

Preparation Method

- Cs2CO3

- Mixed Acid

- $\mathrm{Na} 2 \mathrm{O} 2$

Type of Sample

- ARG-1

+ Pour Stream

x blank

Chart for Ag2O (wt\%)

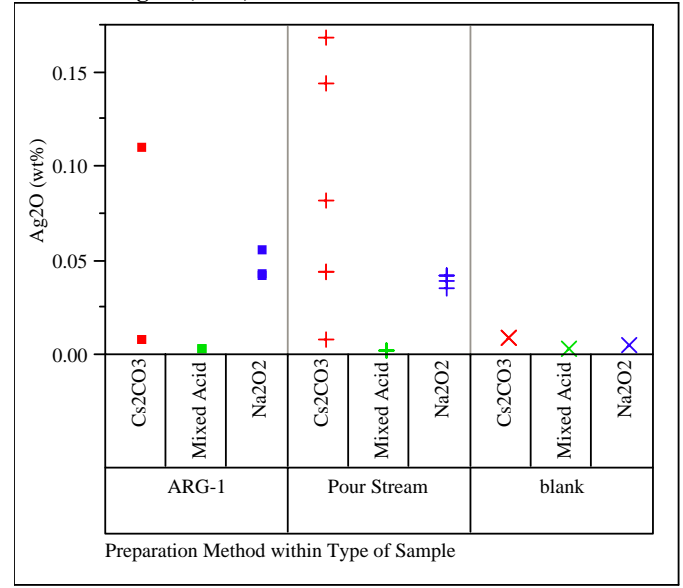

Chart for Al2O3 (wt\%)

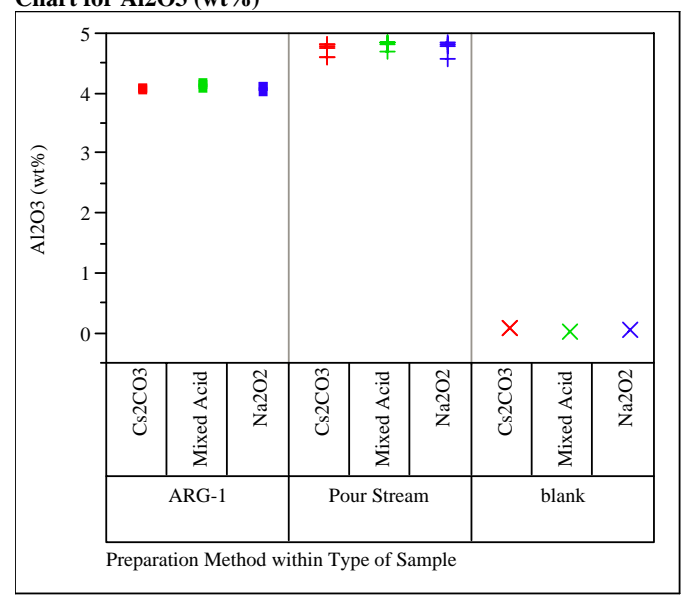

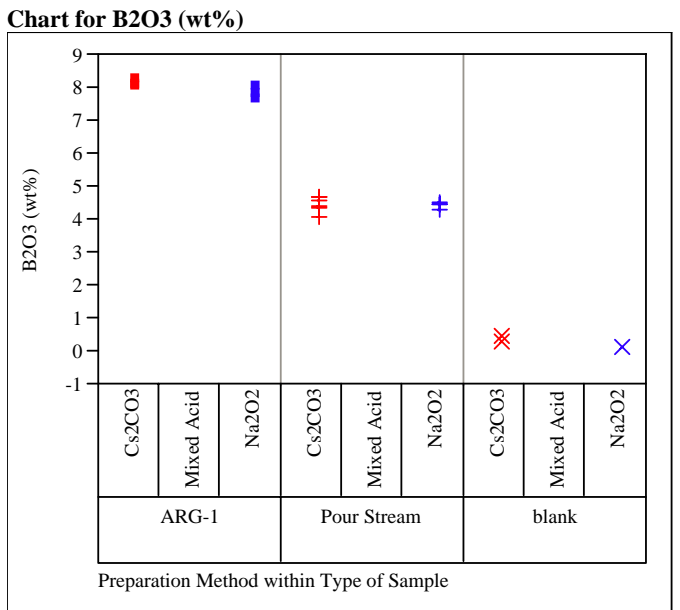

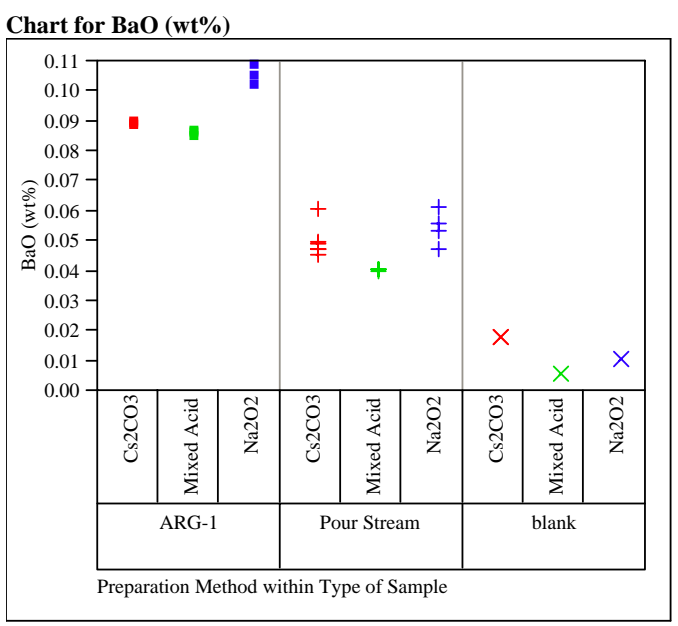

Chart for $\mathrm{CaO}(w \mathrm{t} \%)$

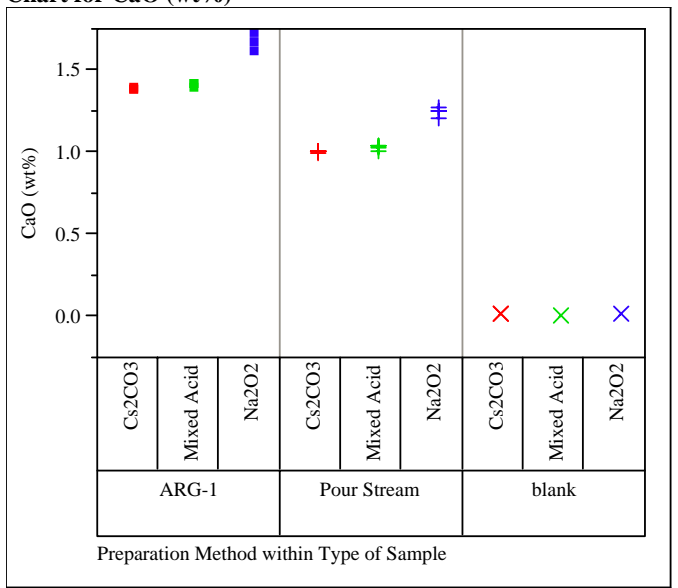


Westinghouse Savannah River Company

WSRC-TR-2005-00396

Savannah River National Laboratory

Rev. 0

Analytical Development/Statistical Consulting Sections

Page 99 of 140

Exhibit B2. Chemical Composition Measurements by Type of Prepared Sample by Oxide for DWPF Radioactive Glass

(Concentrations are in wt\%.)

Chart for CdO (wt\%)

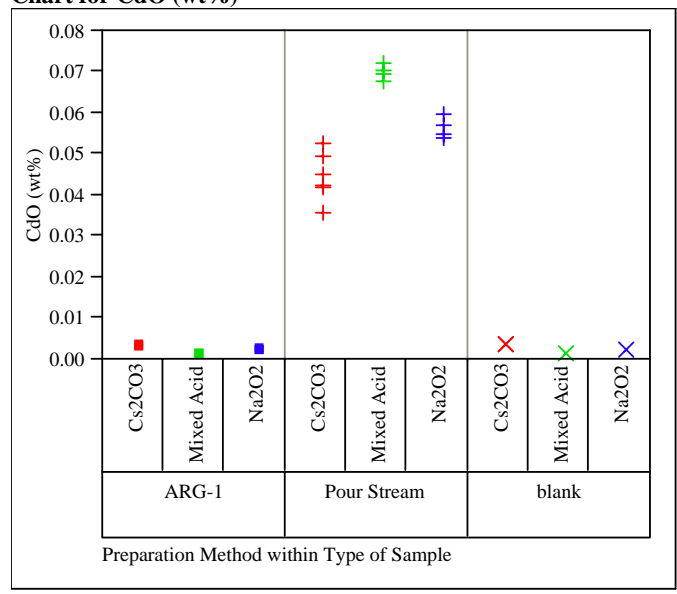

Chart for Ce2O3 (wt \%)

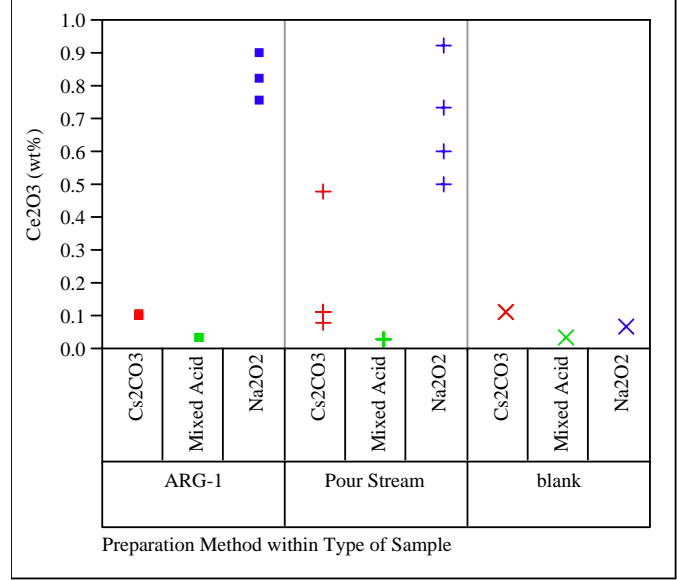

Chart for Cr2O3 (wt \%)

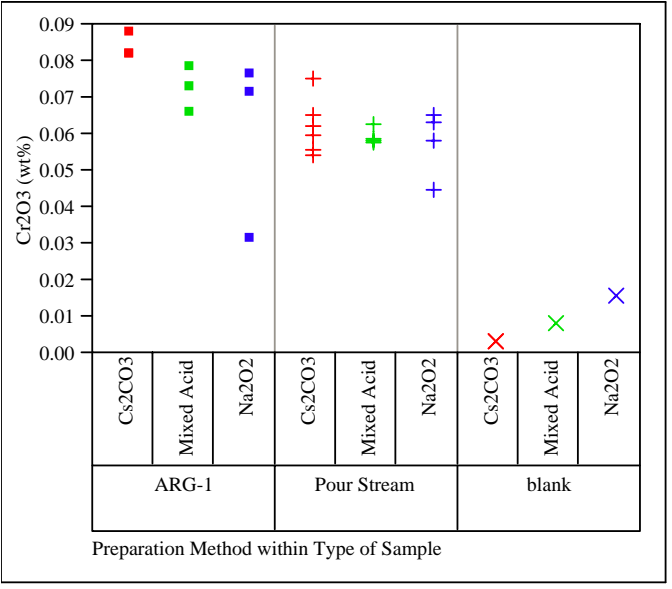

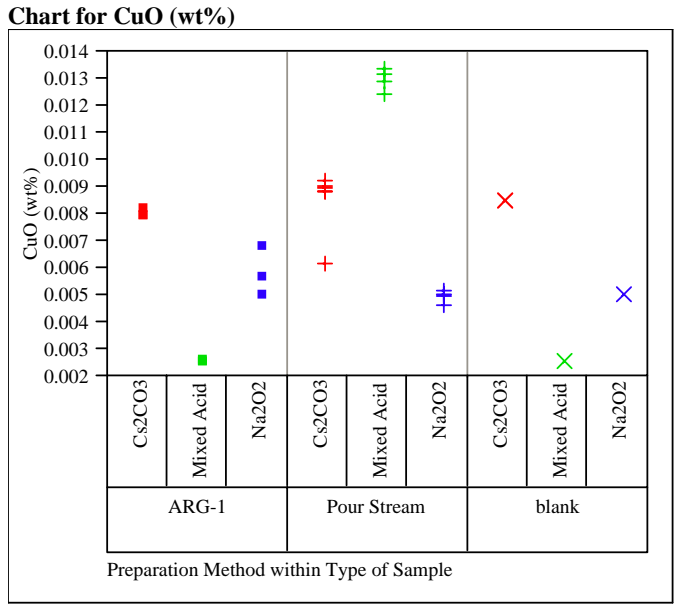

Chart for Fe2O3 (wt \%)

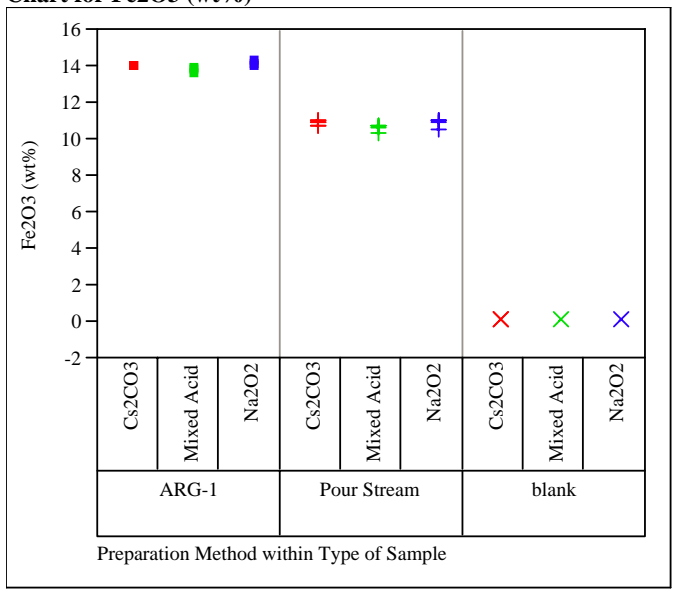

Chart for K2O (wt \%)

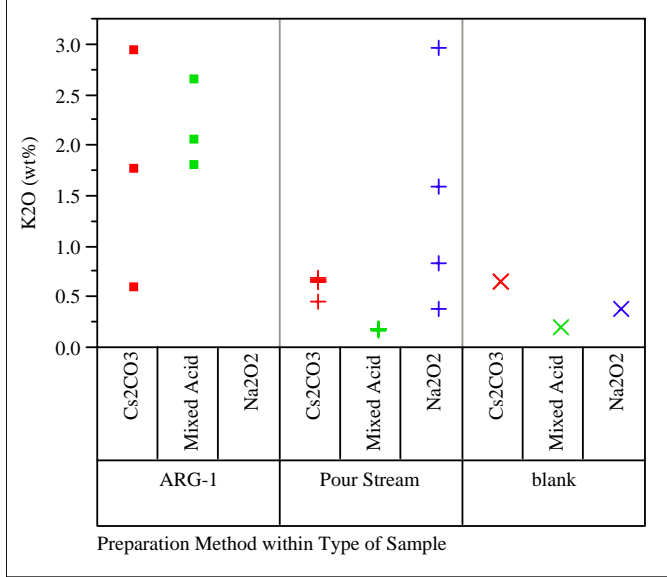


Westinghouse Savannah River Company

WSRC-TR-2005-00396

Savannah River National Laboratory

Rev. 0

Analytical Development/Statistical Consulting Sections

Page 100 of 140

Exhibit B2. Chemical Composition Measurements by Type of Prepared Sample by Oxide for DWPF Radioactive Glass

(Concentrations are in wt\%.)

Chart for La2O3 (wt \%)

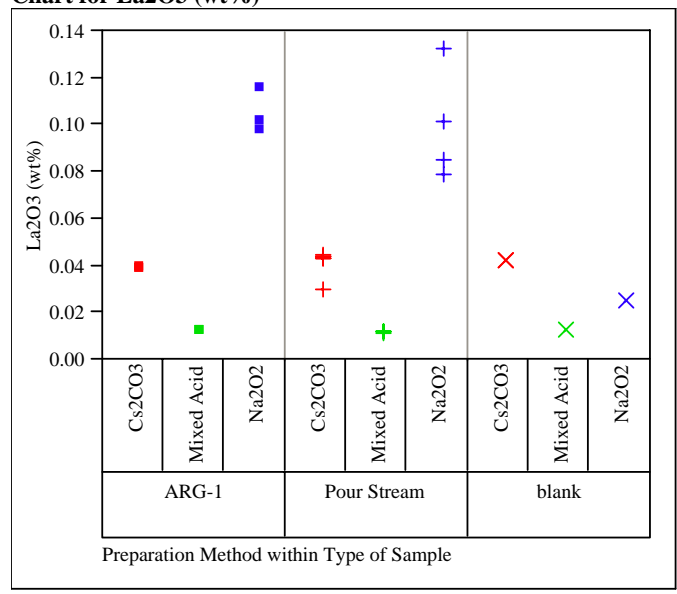

Chart for Li2O (wt\%)

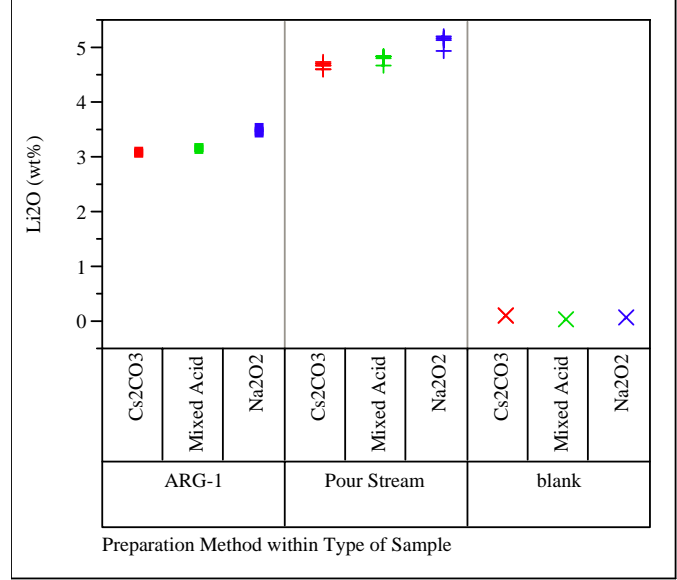

Chart for MgO (wt\%)

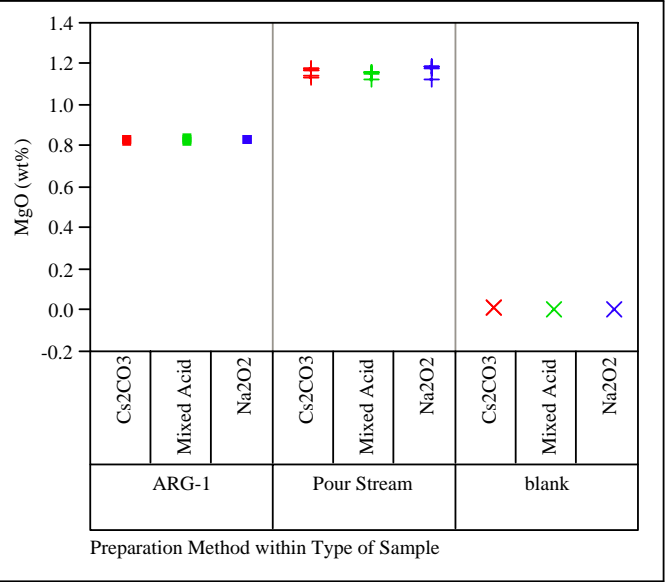

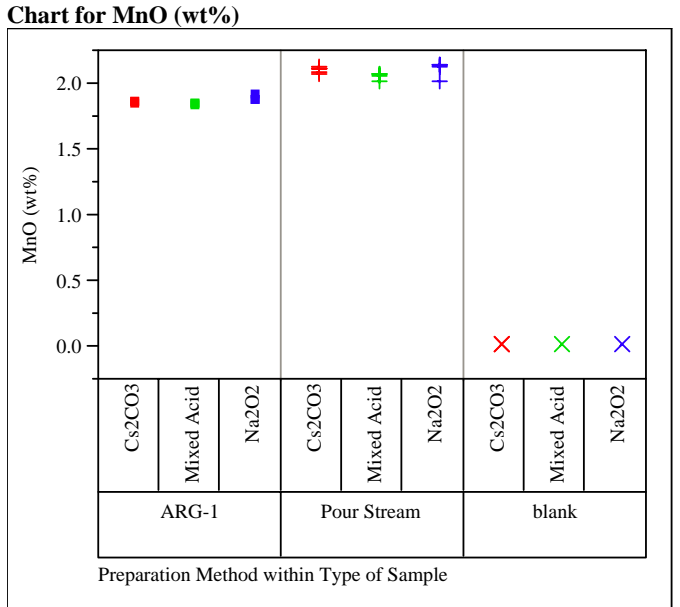

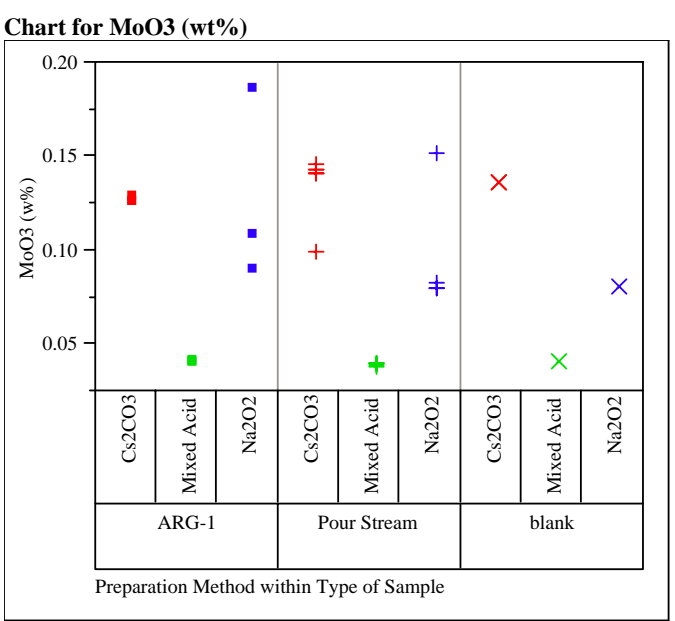

Chart for Na2O (wt\%)

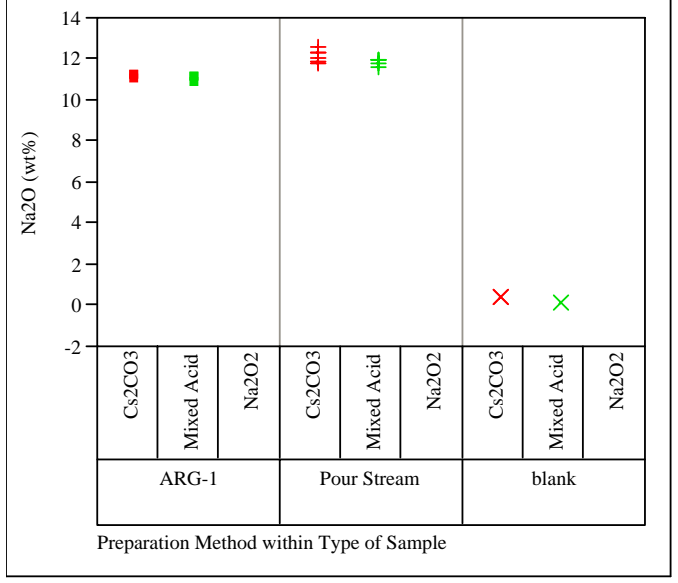


Westinghouse Savannah River Company

WSRC-TR-2005-00396

Savannah River National Laboratory

Rev. 0

Analytical Development/Statistical Consulting Sections

Page 101 of 140

Exhibit B2. Chemical Composition Measurements by Type of Prepared Sample by Oxide for DWPF Radioactive Glass

(Concentrations are in wt\%.)
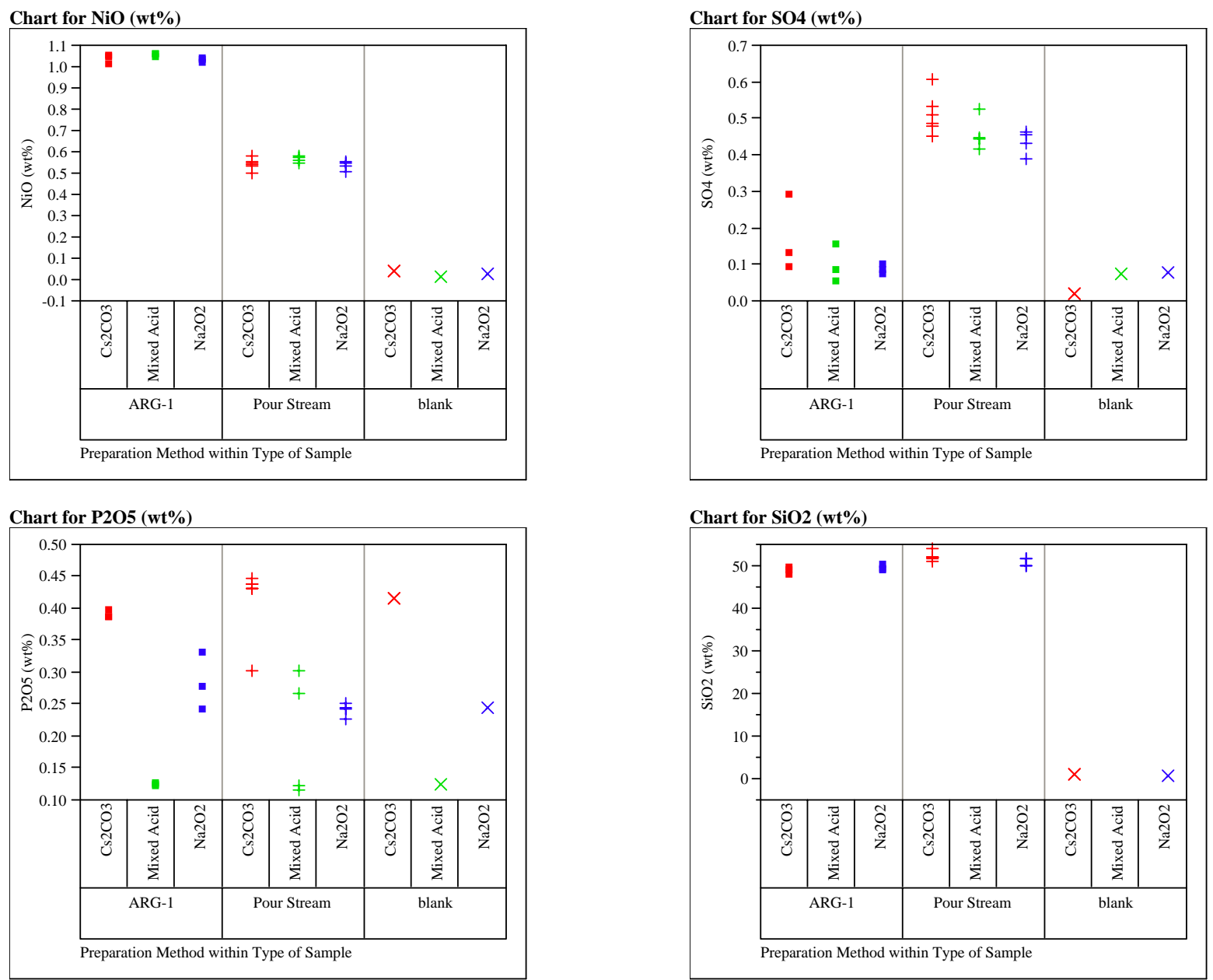

Chart for PbO (wt\%)
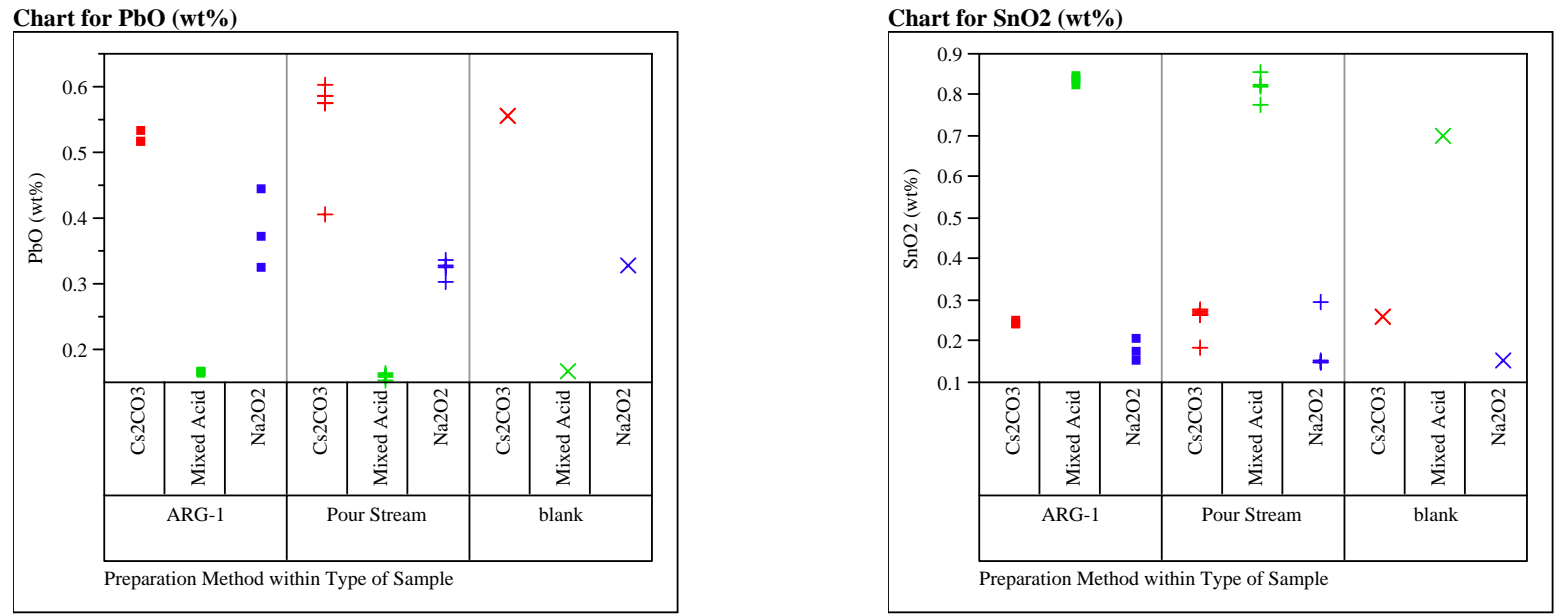
Westinghouse Savannah River Company

WSRC-TR-2005-00396

Savannah River National Laboratory

Rev. 0

Analytical Development/Statistical Consulting Sections

Page 102 of 140

Exhibit B2. Chemical Composition Measurements by Type of Prepared Sample by Oxide for DWPF Radioactive Glass

(Concentrations are in wt\%.)
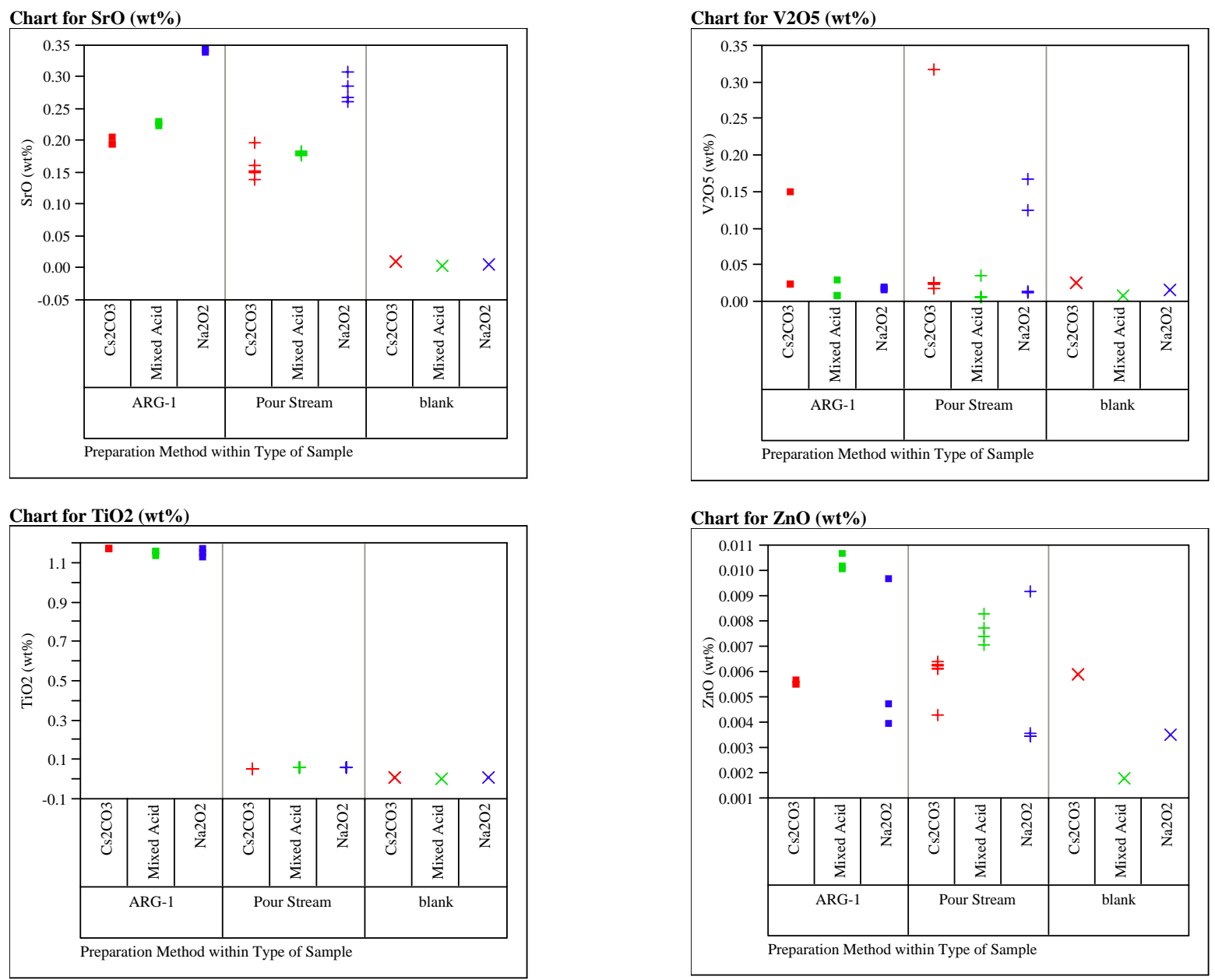

Chart for U3O8 (wt\%)
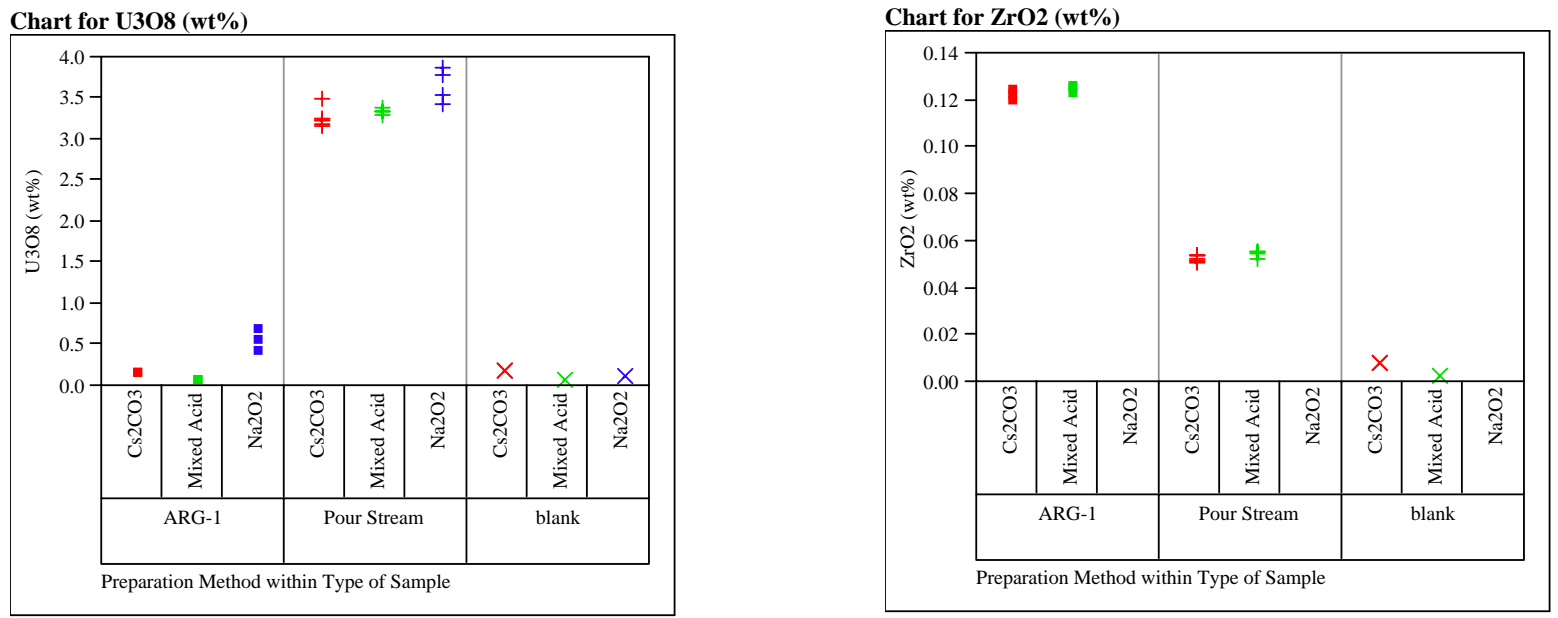


\section{Exhibit B3. Statistical Comparisons of Chemical Composition Measurements of ARG-1 Samples in Radioactive Set by Dissolution Method for Each Oxide (Concentrations in Wt\% Oxides)}

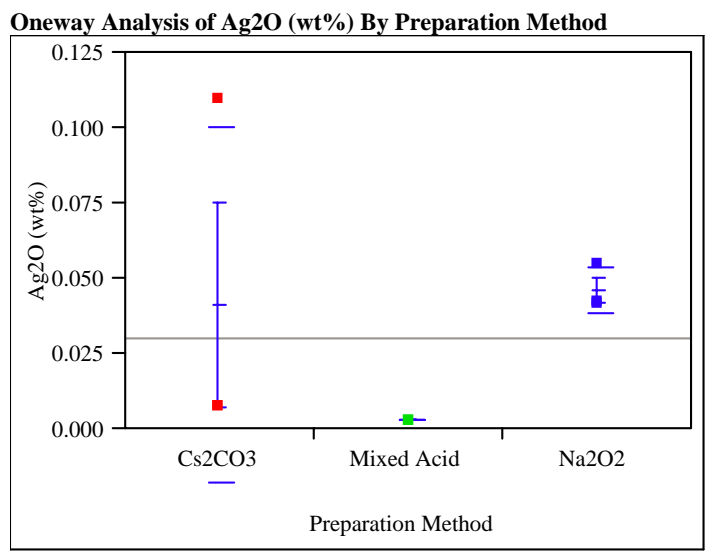

Means Comparisons

Comparisons for all pairs using Tukey-Kramer HSD

$$
\begin{array}{rr}
\text { q* } & \text { Alpha } \\
3.06815 & 0.05
\end{array}
$$

Abs(Dif)-LSD Na2O2 Cs2CO3 Mixed Acid

$\begin{array}{llll}\mathrm{Na} 2 \mathrm{O} 2 & -0.08595 & -0.08144 & -0.04232\end{array}$

$\begin{array}{llll}\mathrm{Cs} 2 \mathrm{CO} 3 & -0.08144 & -0.08595 & -0.04683\end{array}$

Mixed Acid $\quad-0.04232 \quad-0.04683 \quad-0.08595$

Positive values show pairs of means that are significantly different.

Level Mean

$\mathrm{Na} 2 \mathrm{O} 2$ A 0.04601157

Cs2CO3 A 0.04149993

Mixed Acid A 0.00237935

Levels not connected by same letter are significantly different Tests that the Variances are Equal

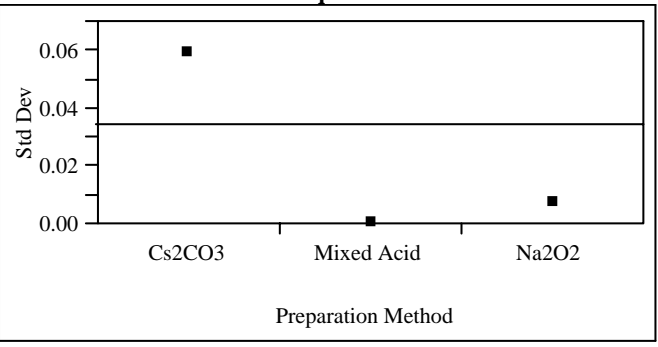

$\begin{array}{lrrrr}\text { Level } & \text { Count } & \text { Std Dev } & \begin{array}{r}\text { MeanAbsDif to } \\ \text { Mean }\end{array} & \begin{array}{r}\text { MeanAbsDif to } \\ \text { Median }\end{array} \\ \text { Cs2CO3 } & 3 & 0.0589490 & 0.0453790 & 0.0340342 \\ \text { Mixed Acid } & 3 & 0.0000246 & 0.0000179 & 0.0000215 \\ \text { Na2O2 } & 3 & 0.0075112 & 0.0057768 & 0.0046549 \\ \text { Test } & \text { F Ratio } & \text { DFNum } & \text { DFDen } & \text { Prob > F } \\ \text { O'Brien[.5] } & 1.7489 & 2 & 6 & 0.2521 \\ \text { Brown- } & 0.8692 & 2 & 6 & 0.4661 \\ \text { Forsythe } & & & & \\ \text { Levene } & 13.9850 & 2 & 6 & 0.0055 \\ \text { Bartlett } & 13.4471 & 2 & . & <.0001\end{array}$

Warning: Small sample sizes. Use Caution.

Welch Anova testing Means Equal, allowing Std Devs Not Equal

F Ratio DFNum DFDen Prob $>$ F

$\begin{array}{llll}41.0207 & 2 & 2.6667 & 0.0099\end{array}$

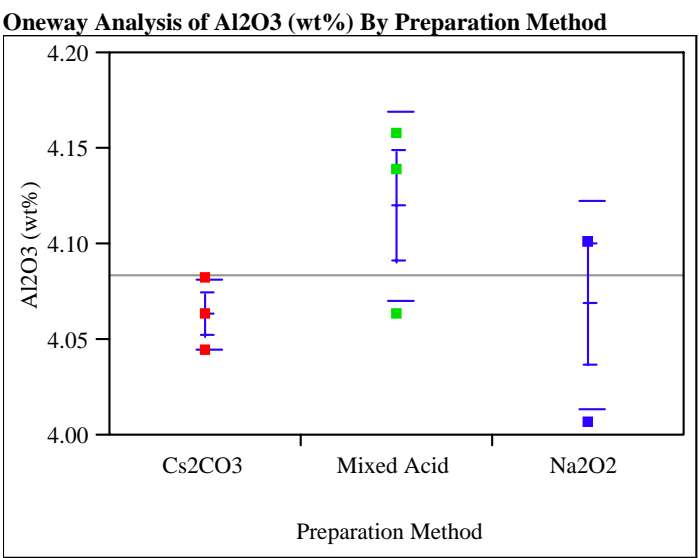

Means Comparisons

Comparisons for all pairs using Tukey-Kramer HSD q* Alpha

Abs(Dif)-LSD Mixed Acid $\mathrm{Na} 2 \mathrm{O} 2 \mathrm{Cs} 2 \mathrm{CO} 3$

$\begin{array}{llll}\text { Mixed Acid } & -0.11045 & -0.06006 & -0.05376\end{array}$

$\mathrm{Na2O} \quad-0.06006-0.11045-0.10415$

Cs2CO3 $\quad-0.05376-0.10415-0.11045$

Positive values show pairs of means that are significantly different.

Level

Mean

Mixed Acid A 4.1191100

$\mathrm{Na} 2 \mathrm{O} 2$ A 4.0687233

Cs2CO3 A 4.0624250

Levels not connected by same letter are significantly different Tests that the Variances are Equal

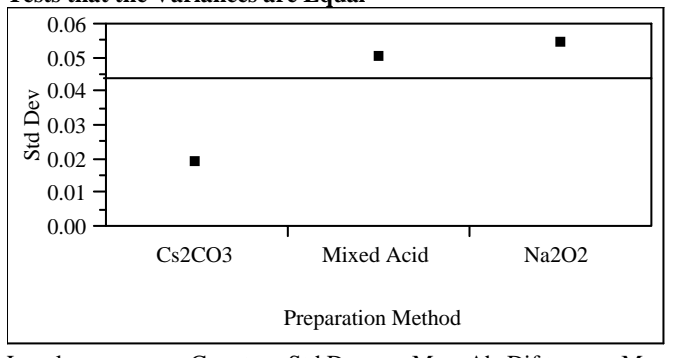

\begin{tabular}{|c|c|c|c|c|}
\hline Level & Count & Std Dev & $\begin{array}{r}\text { MeanAbsDif to } \\
\text { Mean }\end{array}$ & $\begin{array}{r}\text { MeanAbsDif to } \\
\text { Median }\end{array}$ \\
\hline Cs2CO3 & 3 & 0.0188950 & 0.0125967 & 0.0188950 \\
\hline Mixed Acid & 3 & 0.0499915 & 0.0377900 & 0.0377900 \\
\hline $\mathrm{Na} 2 \mathrm{O} 2$ & 3 & 0.0545452 & 0.0419889 & 0.0314917 \\
\hline Test & F Ratio & DFNum & DFDen & Prob $>F$ \\
\hline O'Brien[.5] & 0.6813 & 2 & 6 & 0.5412 \\
\hline $\begin{array}{l}\text { Brown- } \\
\text { Forsythe }\end{array}$ & 0.2059 & 2 & 6 & 0.81 \\
\hline Levene & 2.8197 & 2 & 6 & 0.1 \\
\hline Bartlett & 0.8326 & 2 & & 0.43 \\
\hline
\end{tabular}

Warning: Small sample sizes. Use Caution.

Welch Anova testing Means Equal, allowing Std Devs Not Equal

F Ratio DFNum DFDen Prob > F

$\begin{array}{rrrr}1.3994 & 2 & 3.2346 & 0.3649\end{array}$ 


\section{Exhibit B3. Statistical Comparisons of Chemical Composition Measurements of ARG-1 Samples in Radioactive Set by Dissolution Method for Each Oxide (Concentrations in Wt\% Oxides)}

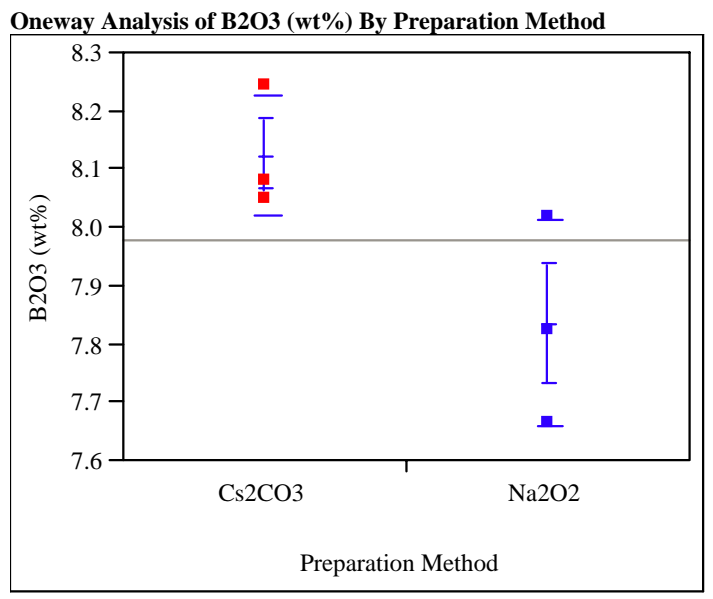

Missing Rows

3Means Comparisons

Comparisons for all pairs using Tukey-Kramer HSD

$$
\begin{aligned}
& \mathrm{q}^{*} \text { Alpha } \\
& \text { Abs(Dif)-LSD Cs2CO3 Na2O2 } \\
& \text { Cs2CO3 }-0.32915-0.03936
\end{aligned}
$$$$
\mathrm{Na} 2 \mathrm{O} 2 \quad-0.03936-0.32915
$$

Positive values show pairs of means that are significantly different.

Level - Level Difference Lower CL Upper CL

\begin{tabular}{|c|c|c|c|c|}
\hline \multirow{4}{*}{ 送 } & \multicolumn{3}{|r|}{ a } & \\
\hline & \multicolumn{3}{|l|}{ - } & \\
\hline & Cs2C & & $\mathrm{Na} 2 \mathrm{O} 2$ & \\
\hline & \multicolumn{3}{|c|}{ Preparation Method } & \\
\hline Level & Count & Std Dev & $\begin{array}{r}\text { MeanAbsDif to } \\
\text { Mean }\end{array}$ & $\begin{array}{r}\text { MeanAbsDif to } \\
\text { Median }\end{array}$ \\
\hline Cs2CO3 & 3 & 0.1035053 & 0.0787087 & 0.0751310 \\
\hline $\mathrm{Na} 2 \mathrm{O} 2$ & 3 & 0.1773383 & 0.1216407 & 0.1717280 \\
\hline Test & F Ratio & DFNum & DFDen & Prob $>$ F \\
\hline O'Brien[.5] & 0.6925 & 1 & 4 & 0.4521 \\
\hline $\begin{array}{l}\text { Brown- } \\
\text { Forsythe }\end{array}$ & 4.7647 & 1 & 4 & 0.0944 \\
\hline Levene & 0.5180 & 1 & 4 & 0.5115 \\
\hline Bartlett & 0.4430 & 1 & . & 0.5057 \\
\hline F Test 2-sided & 2.9355 & 2 & 2 & 0.5082 \\
\hline
\end{tabular}

Cs2CO3 Na2O2 $0.2897910 \quad-0.0393580 .6189396$

Tests that the Variances are Equal

Warning: Small sample sizes. Use Caution.

Welch Anova testing Means Equal, allowing Std Devs Not Equal

F Ratio DFNum DFDen Prob $>$ F

$\begin{array}{rrrr}5.9754 & 1 & 3.2209 & 0.0863\end{array}$

t Test

2.4445

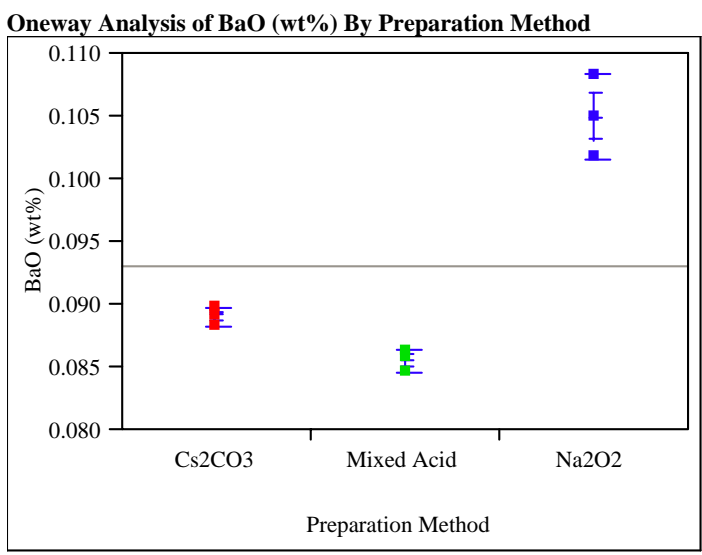

Means Comparisons

Comparisons for all pairs using Tukey-Kramer HSD

$$
\begin{array}{rr}
\text { q*}^{*} & \text { Alpha } \\
3.06815 & 0.05
\end{array}
$$

Abs(Dif)-LSD Na2O2 Cs2CO3 Mixed Acid

$\begin{array}{llll}\mathrm{Na} 2 \mathrm{O} 2 & -0.00504 & 0.01085 & 0.01443\end{array}$

$\begin{array}{llll}\mathrm{Cs} 2 \mathrm{CO} 3 & 0.01085 & -0.00504 & -0.00147\end{array}$

Mixed Acid $\quad 0.01443 \quad-0.00147 \quad-0.00504$

Positive values show pairs of means that are significantly different

$\begin{array}{lrr}\text { Level } & & \text { Mean } \\ \text { Na2O2 } & \text { A } & 0.10495100 \\ \text { Cs2CO3 } & \text { B } & 0.08905948 \\ \text { Mixed Acid } & \text { B } & 0.08548668\end{array}$

Levels not connected by same letter are significantly different Tests that the Variances are Equal

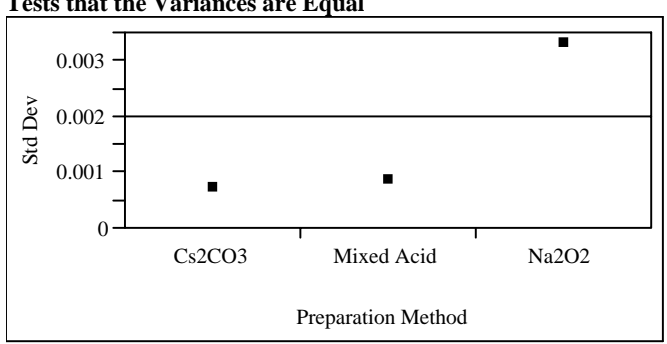

$\begin{array}{lrrrr}\text { Level } & \text { Count } & \text { Std Dev } & \begin{array}{r}\text { MeanAbsDif to } \\ \text { Mean }\end{array} & \begin{array}{r}\text { MeanAbsDif to } \\ \text { Median }\end{array} \\ \text { Cs2CO3 } & 3 & 0.0007264 & 0.0004962 & 0.0007071 \\ \text { Mixed Acid } & 3 & 0.0008672 & 0.0006451 & 0.0007071 \\ \text { Na2O2 } & 3 & 0.0032951 & 0.0022330 & 0.0032379 \\ \text { Test } & \text { F Ratio } & \text { DFNum } & \text { DFDen } & \text { Prob > F } \\ \text { O'Brien[.5] } & 1.5638 & 2 & 6 & 0.2840 \\ \text { Brown- } & 78.3729 & 2 & 6 & <.0001 \\ \text { Forsythe } & & & & \\ \text { Levene } & 2.2759 & 2 & 6 & 0.1839 \\ \text { Bartlett } & 2.2355 & 2 & . & 0.1069\end{array}$

Warning: Small sample sizes. Use Caution.

Welch Anova testing Means Equal, allowing Std Devs Not Equal

F Ratio DFNum DFDen Prob $>$ F

$\begin{array}{lrrr}46.7639 & 2 & 3.5844 & 0.0027\end{array}$ 


\section{Exhibit B3. Statistical Comparisons of Chemical Composition Measurements of ARG-1 Samples in Radioactive Set by Dissolution Method for Each Oxide (Concentrations in Wt\% Oxides)}

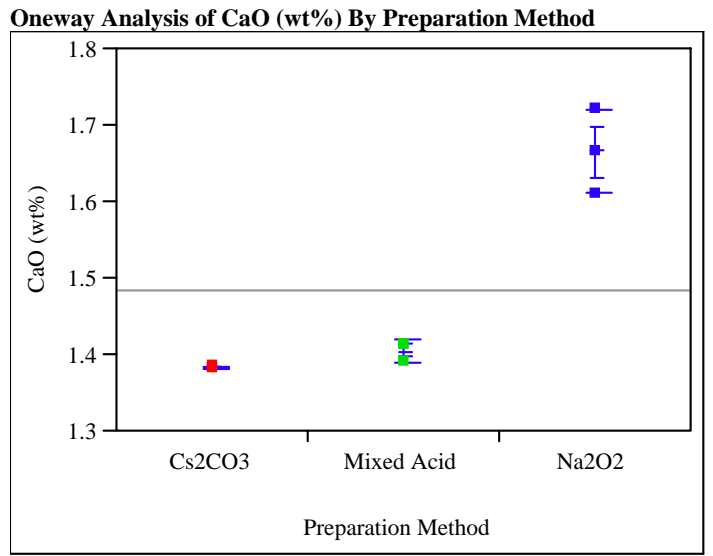

Means Comparisons

Comparisons for all pairs using Tukey-Kramer HSD q Alpha

Abs(Dif)-LSD Na2O2 Mixed Acid Cs2CO3

$\mathrm{Na} 2 \mathrm{O} 2$ $-0.08338$

$0.17640 \quad 0.19832$

Mixed Acid

0.17640

$-0.08338-0.06146$

Cs2CO3

0.19832

$-0.06146-0.08338$

Positive values show pairs of means that are significantly different.

$\begin{array}{lrr}\text { Level } & & \text { Mean } \\ \text { Na2O2 } & \text { A } & 1.6650480 \\ \text { Mixed Acid } & \text { B } & 1.4052632 \\ \text { Cs2CO3 } & \text { B } 1.3833424\end{array}$

Levels not connected by same letter are significantly different

Tests that the Variances are Equal

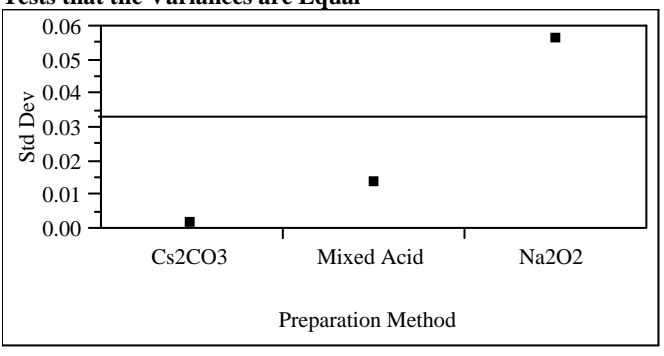

\begin{tabular}{|c|c|c|c|c|}
\hline Level & Count & Std Dev & $\begin{array}{r}\text { MeanAbsDif to } \\
\text { Mean }\end{array}$ & $\begin{array}{r}\text { MeanAbsDif t } \\
\text { Media }\end{array}$ \\
\hline Cs2CO3 & 3 & 0.0016157 & 0.0012437 & 0.00093 \\
\hline Mixed Acid & 3 & 0.0137331 & 0.0105717 & 0.00792 \\
\hline $\mathrm{Na} 2 \mathrm{O} 2$ & 3 & 0.0559680 & 0.0373120 & 0.05596 \\
\hline Test & F Ratio & DFNum & DFDen & Prob \\
\hline O'Brien[.5] & 1.6696 & 2 & 6 & 0.26 \\
\hline $\begin{array}{l}\text { Brown- } \\
\text { Forsythe }\end{array}$ & 42.2491 & 2 & 6 & 0.00 \\
\hline Levene & 2.9609 & 2 & 6 & \\
\hline Bartlett & 5.5489 & 2 & . & 0.00 \\
\hline
\end{tabular}

Warning: Small sample sizes. Use Caution.

Welch Anova testing Means Equal, allowing Std Devs Not Equal

F Ratio DFNum DFDen Prob $>$ F

$\begin{array}{rrrr}33.4229 & 2 & 2.7053 & 0.0124\end{array}$

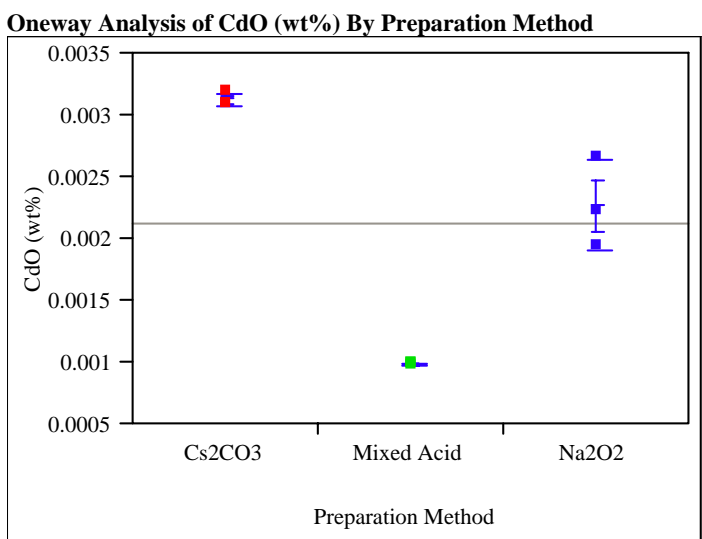

Means Comparisons

Comparisons for all pairs using Tukey-Kramer HSD

$$
\text { q* Alpha }
$$

Abs(Dif)-LSD Cs2CO3 Na2O2 Mixed Acid

$\begin{array}{llll}\mathrm{Cs} 2 \mathrm{CO} 3 & -0.00053 & 0.00033 & 0.00161\end{array}$

$\begin{array}{llll}\mathrm{Na} 2 \mathrm{O} 2 & 0.00033 & -0.00053 & 0.00076\end{array}$

$\begin{array}{llr}\text { Mixed Acid } \quad 0.00161 & 0.00076 & -0.00053\end{array}$

Positive values show pairs of means that are significantly different.

\begin{tabular}{|c|c|c|}
\hline Level & & Mean \\
\hline Cs2CO3 & A & 0.00312609 \\
\hline $\mathrm{Na} 2 \mathrm{O} 2$ & B & 0.00227318 \\
\hline Acid & & 0.0009 \\
\hline
\end{tabular}

Levels not connected by same letter are significantly different Tests that the Variances are Equal

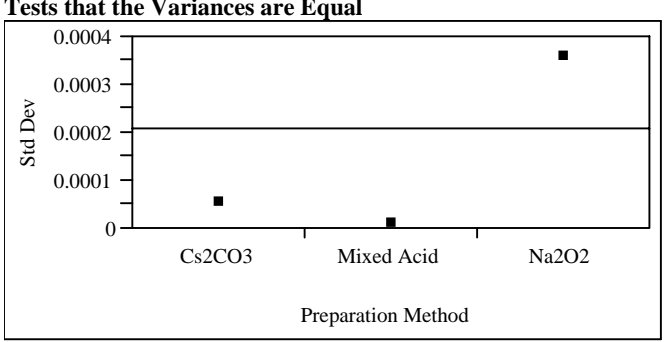

$\begin{array}{lrrrr}\text { Level } & \text { Count } & \text { Std Dev } & \begin{array}{r}\text { MeanAbsDif to } \\ \text { Mean }\end{array} & \begin{array}{r}\text { MeanAbsDif to } \\ \text { Median }\end{array} \\ \text { Cs2CO3 } & 3 & 0.0000528 & 0.0000406 & 0.0000305 \\ \text { Mixed Acid } & 3 & 0.0000114 & 0.0000076 & 0.0000114 \\ \text { Na2O2 } & 3 & 0.0003597 & 0.0002551 & 0.0003313 \\ \text { Test } & \text { F Ratio } & \text { DFNum } & \text { DFDen } & \text { Prob }>\text { F } \\ \text { O'Brien[.5] } & 1.7377 & 2 & 6 & 0.2539 \\ \text { Brown- } & 27.0497 & 2 & 6 & 0.0010 \\ \text { Forsythe } & & & & \\ \text { Levene } & 5.0575 & 2 & 6 & 0.0516 \\ \text { Bartlett } & 6.1443 & 2 & . & 0.0021 \\ \text { Warning: Small sample sizes. Use Caution. } & & \end{array}$

Welch Anova testing Means Equal, allowing Std Devs Not Equal

F Ratio DFNum DFDen Prob $>$ F

$\begin{array}{llll}1912.0793 & 2 & 2.7884 & <.0001\end{array}$ 


\section{Exhibit B3. Statistical Comparisons of Chemical Composition Measurements of ARG-1 Samples in Radioactive Set by Dissolution Method for Each Oxide (Concentrations in Wt\% Oxides)}

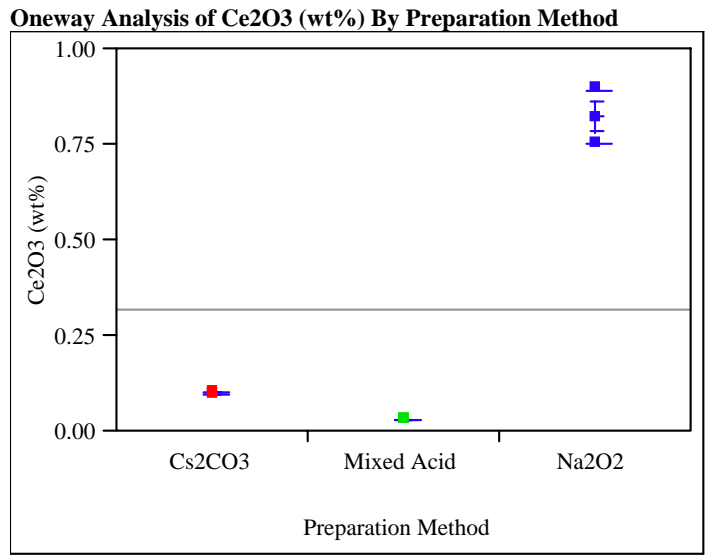

Means Comparisons

Comparisons for all pairs using Tukey-Kramer HSD q* Alpha

Abs(Dif)-LSD Na2O2 Cs2CO3 Mixed Acid

$\begin{array}{llll}\mathrm{Na} 2 \mathrm{O} 2 & -0.10179 & 0.62031 & 0.68901\end{array}$

Cs2CO3 $\quad 0.62031-0.10179 \quad-0.03310$

Mixed Acid $\quad 0.68901 \quad-0.03310 \quad-0.10179$

Positive values show pairs of means that are significantly different.

Level

$\mathrm{Na} 2 \mathrm{O} 2$

Cs2CO3

Mean

A 0.82264303

Levels not connected by same letter are significantly different

Tests that the Variances are Equal

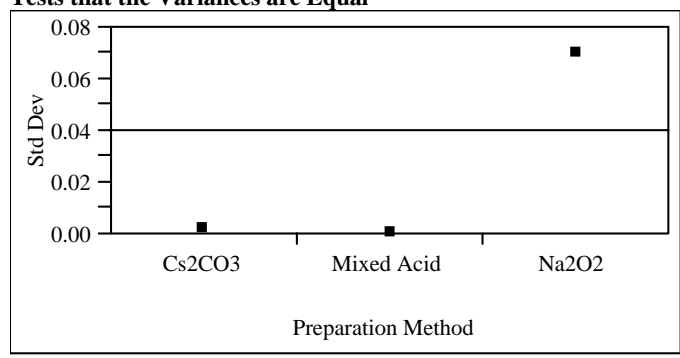

\begin{tabular}{|c|c|c|c|c|}
\hline Level & Count & Std Dev & $\begin{array}{r}\text { MeanAbsDif to } \\
\text { Mean }\end{array}$ & $\begin{array}{r}\text { MeanAbsDif } \\
\text { Medi }\end{array}$ \\
\hline $\mathrm{Cs} 2 \mathrm{CO} 3$ & 3 & 0.0016906 & 0.0013014 & 0.00097 \\
\hline Mixed Acid & 3 & 0.0003226 & 0.0002212 & 0.0003 \\
\hline $\mathrm{Na} 2 \mathrm{O} 2$ & 3 & 0.0703593 & 0.0481534 & 0.06832 \\
\hline Test & F Ratio & DFNum & DFDen & Prob \\
\hline O'Brien[.5] & 1.7767 & 2 & 6 & 0.24 \\
\hline $\begin{array}{l}\text { Brown- } \\
\text { Forsythe }\end{array}$ & 282.8394 & 2 & 6 & $<.00$ \\
\hline Levene & 4.5772 & 2 & 6 & $0.0 €$ \\
\hline Bartlett & 12.2181 & 2 & . & $<.00$ \\
\hline
\end{tabular}

Warning: Small sample sizes. Use Caution.

Welch Anova testing Means Equal, allowing Std Devs Not Equal

F Ratio DFNum DFDen Prob $>$ F

$\begin{array}{llll}2076.4965 & 2 & 2.7603 & <.0001\end{array}$

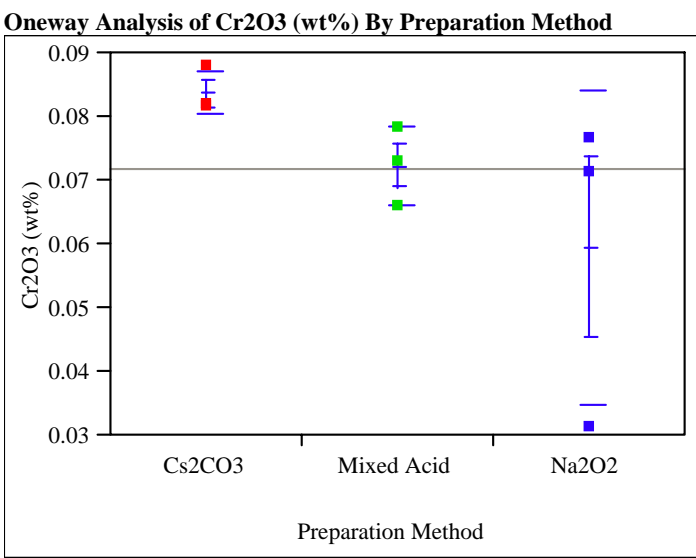

Means Comparisons

Comparisons for all pairs using Tukey-Kramer HSD q* Alpha

$3.06815 \quad 0.05$

Abs(Dif)-LSD Cs2CO3 Mixed Acid Na2O2

$\begin{array}{llll}\text { Cs2CO3 } & -0.03716 & -0.02576 & -0.01314\end{array}$

Mixed Acid $\quad-0.02576 \quad-0.03716-0.02455$

$\mathrm{Na2O} 2 \quad-0.01314 \quad-0.02455-0.03716$

Positive values show pairs of means that are significantly different.

Level

Cs2CO3 A 0.08365224

Mixed Acid A 0.07225176

$\mathrm{Na} 2 \mathrm{O} 2 \quad$ A 0.05963328

Levels not connected by same letter are significantly different

Tests that the Variances are Equal

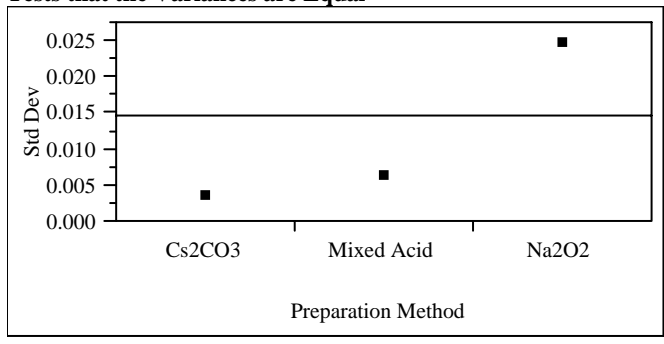

$\begin{array}{lrrrr}\text { Level } & \text { Count } & \text { Std Dev } & \begin{array}{r}\text { MeanAbsDif to } \\ \text { Mean }\end{array} & \begin{array}{r}\text { MeanAbsDif to } \\ \text { Median }\end{array} \\ \text { Cs2CO3 } & 3 & 0.0035028 & 0.0026958 & 0.0020950 \\ \text { Mixed Acid } & 3 & 0.0061671 & 0.0043198 & 0.0057977 \\ \text { Na2O2 } & 3 & 0.0246967 & 0.0189034 & 0.0168084 \\ \text { Test } & \text { F Ratio } & \text { DFNum } & \text { DFDen } & \text { Prob > F } \\ \text { O'Brien[.5] } & 1.6296 & 2 & 6 & 0.2721 \\ \text { Brown- } & 1.2771 & 2 & 6 & 0.3451 \\ \text { Forsythe } & & & & \\ \text { Levene } & 8.4025 & 2 & 6 & 0.0182 \\ \text { Bartlett } & 2.9643 & 2 & . & 0.0516\end{array}$

Warning: Small sample sizes. Use Caution.

Welch Anova testing Means Equal, allowing Std Devs Not Equal

F Ratio DFNum DFDen Prob > F

$\begin{array}{llll}4.1349 & 2 & 3.3073 & 0.1260\end{array}$ 


\section{Exhibit B3. Statistical Comparisons of Chemical Composition Measurements of ARG-1 Samples in Radioactive Set by Dissolution Method for Each Oxide (Concentrations in Wt\% Oxides)}

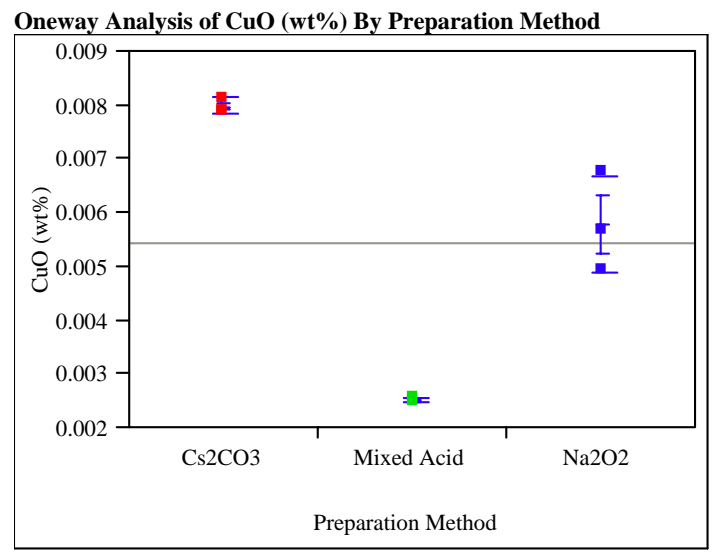

Means Comparisons

Comparisons for all pairs using Tukey-Kramer HSD

$\begin{array}{lrrr}q^{*} & \text { Alpha } & & \\ 3.06815 & 0.05 & & \\ \text { Abs(Dif)-LSD } & \text { Cs2CO3 } & \text { Na2O2 } & \text { Mixed Acid } \\ \text { Cs2CO3 } & -0.00133 & 0.00084 & 0.00411 \\ \text { Na2O2 } & 0.00084 & -0.00133 & 0.00194 \\ \text { Mixed Acid } & 0.00411 & 0.00194 & -0.00133\end{array}$

Positive values show pairs of means that are significantly different.

$\begin{array}{lrrr}\text { Level } & & & \text { Mean } \\ \text { Cs2CO3 } & \text { A } & & 0.00796979 \\ \text { Na2O2 } & & \text { B } & 0.00579166 \\ \text { Mixed Acid } & & \text { C } & 0.00252029\end{array}$

Levels not connected by same letter are significantly different Tests that the Variances are Equal

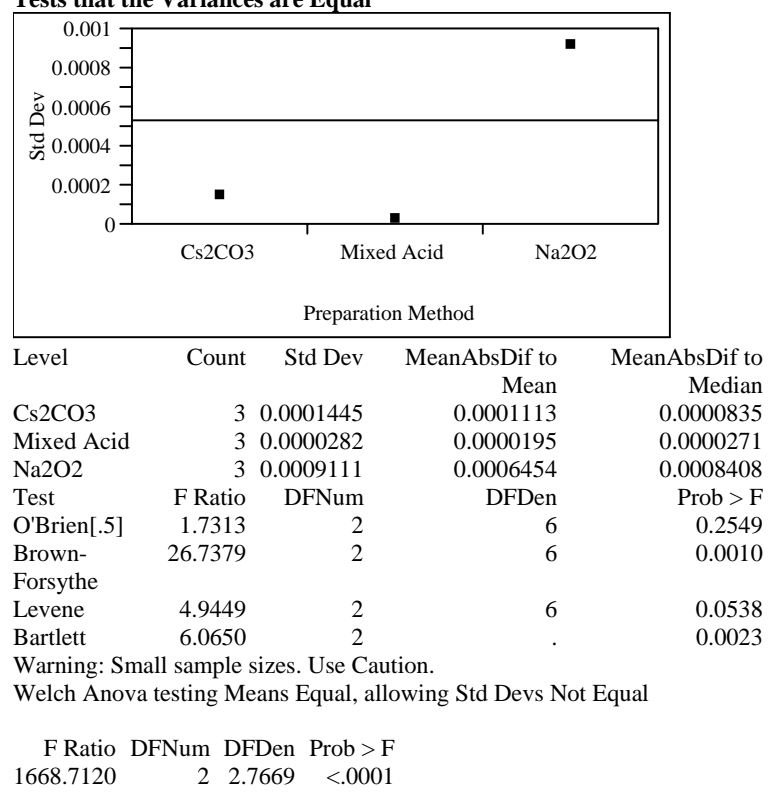

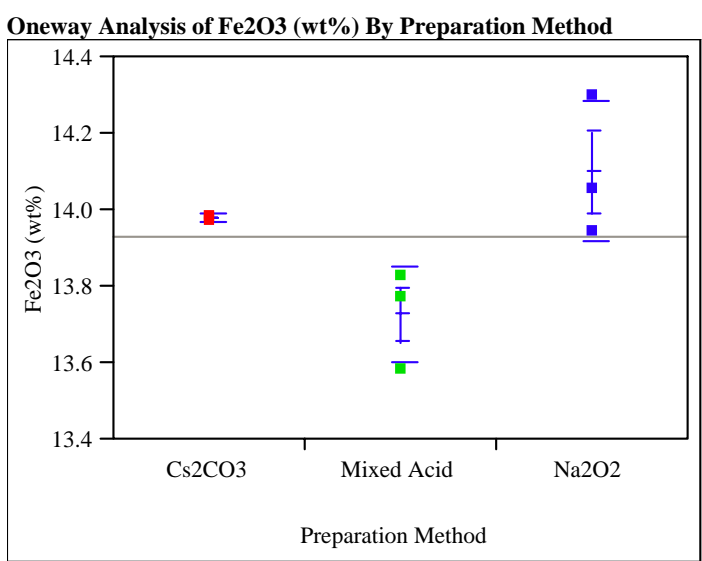

Means Comparisons

Comparisons for all pairs using Tukey-Kramer HSD q* Alpha

$3.06815 \quad 0.05$

Abs(Dif)-LSD Na2O2 Cs2CO3 Mixed Acid

$\begin{array}{llll}\mathrm{Na} 2 \mathrm{O} 2 & -0.32190 & -0.20276 & 0.04982\end{array}$

$\begin{array}{llll}\mathrm{Cs} 2 \mathrm{CO} 3 & -0.20276 & -0.32190 & -0.06932\end{array}$

Mixed Acid $\quad 0.04982 \quad-0.06932 \quad-0.32190$

Positive values show pairs of means that are significantly different.

$\begin{array}{llrr}\text { Level } & & & \text { Mean } \\ \text { Na2O2 } & \text { A } & & 14.096842 \\ \text { Cs2CO3 } & \text { A } & \text { B } & 13.977700 \\ \text { Mixed Acid } & & \text { B } & 13.725120\end{array}$

Levels not connected by same letter are significantly different Tests that the Variances are Equal

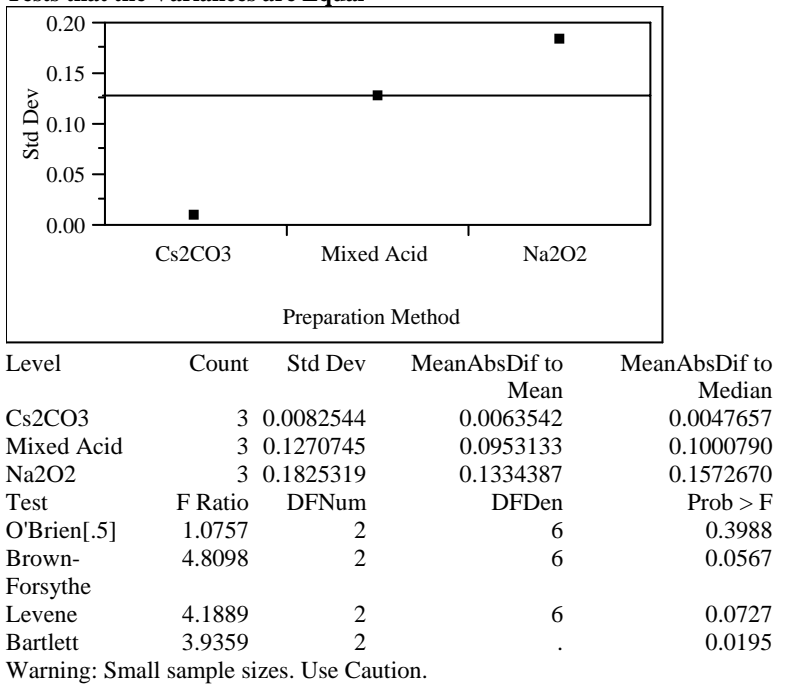

Welch Anova testing Means Equal, allowing Std Devs Not Equal

F Ratio DFNum DFDen Prob $>$ F

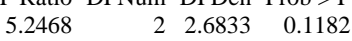




\section{Exhibit B3. Statistical Comparisons of Chemical Composition Measurements of ARG-1 Samples in Radioactive Set by Dissolution Method for Each Oxide (Concentrations in Wt\% Oxides)}

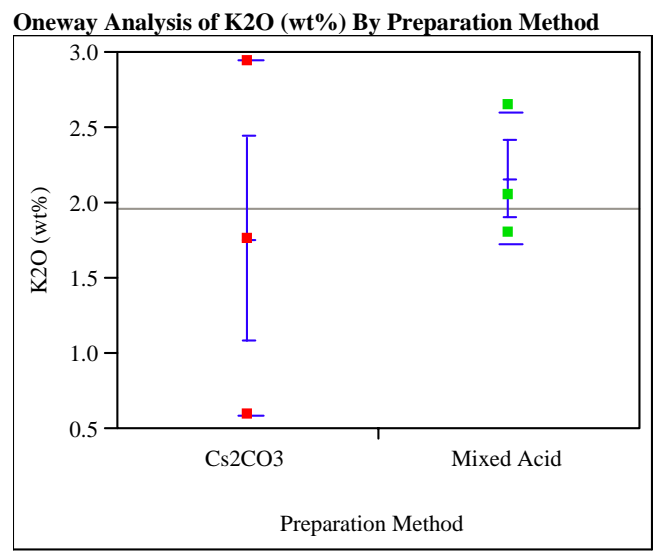

Missing Rows

3Means Comparisons

Comparisons for all pairs using Tukey-Kramer HSD

$$
\begin{array}{rr}
\text { q* }^{*} & \text { Alpha } \\
2.77646 & 0.05
\end{array}
$$

Abs(Dif)-LSD Mixed Acid Cs2CO3

$\begin{array}{lll}\text { Mixed Acid } & -2.0092 & -1.6081\end{array}$

$\begin{array}{lll}\mathrm{Cs} 2 \mathrm{CO} 3 & -1.6081 & -2.0092\end{array}$

Positive values show pairs of means that are significantly different.

Level - Level Difference Lower CL Upper CL

Mixed Acid Cs2CO3 $0.4011318 \quad-1.60808 \quad 2.410345$

Tests that the Variances are Equal

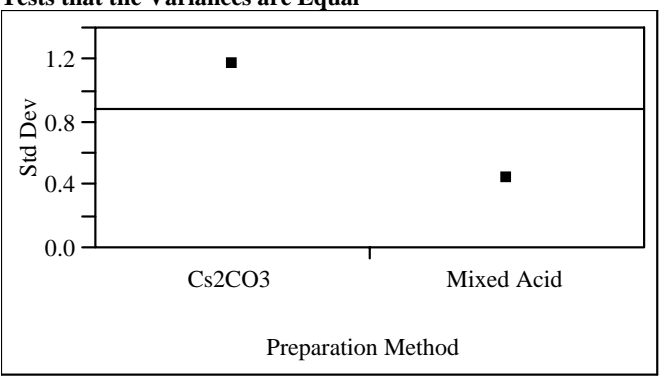

$\begin{array}{lrrrr}\text { Level } & \text { Count } & \text { Std Dev } & \begin{array}{r}\text { MeanAbsDif to } \\ \text { Mean }\end{array} & \begin{array}{r}\text { MeanAbsDif to } \\ \text { Median }\end{array} \\ \text { Cs2CO3 } & 3 & 1.173889 & 0.7840608 & 1.171674 \\ \text { Mixed Acid } & 3 & 0.439363 & 0.3239036 & 0.369411 \\ \text { Test } & \text { F Ratio } & \text { DFNum } & \text { DFDen } & \text { Prob > F } \\ \text { O'Brien[.5] } & 1.2893 & 1 & 4 & 0.3196 \\ \text { Brown- } & 47.3992 & 1 & 4 & 0.0023 \\ \text { Forsythe } & & & & \\ \text { Levene } & 1.2923 & 1 & 4 & 0.3191 \\ \text { Bartlett } & 1.3463 & 1 & . & 0.2459 \\ \text { F Test 2-sided } & 7.1385 & 2 & 2 & 0.2457 \\ \text { Warn }\end{array}$

Warning: Small sample sizes. Use Caution.

Welch Anova testing Means Equal, allowing Std Devs Not Equal

F Ratio DFNum DFDen Prob $>$ F

$\begin{array}{llll}0.3073 & 1 & 2.5496 & 0.6242\end{array}$

t Test

0.5543

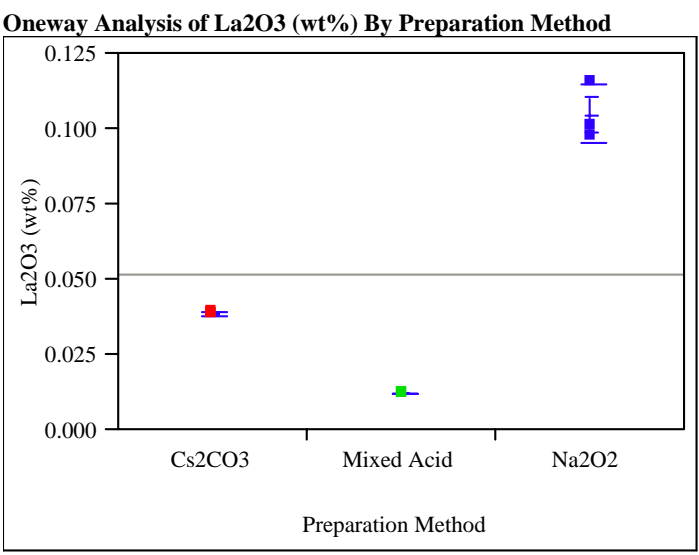

Means Comparisons

Comparisons for all pairs using Tukey-Kramer HSD $\begin{array}{rr}q^{*} & \text { Alpha } \\ 3.06815 & 0.05\end{array}$

Abs(Dif)-LSD Na2O2 Cs2CO3 Mixed Acid

$\begin{array}{llll}\mathrm{Na} 2 \mathrm{O} 2 & -0.01388 & 0.05215 & 0.07865\end{array}$

$\begin{array}{llll}\mathrm{Cs} 2 \mathrm{CO} 3 & 0.05215 & -0.01388 & 0.01262\end{array}$

Mixed Acid $\quad 0.07865 \quad 0.01262 \quad-0.01388$

Positive values show pairs of means that are significantly different

$\begin{array}{lrrr}\text { Level } & & & \text { Mean } \\ \text { Na2O2 } & \text { A } & & 0.10480923 \\ \text { Cs2CO3 } & & \text { B } & 0.03878059 \\ \text { Mixed Acid } & & \text { C } & 0.01227531\end{array}$

Levels not connected by same letter are significantly different Tests that the Variances are Equal

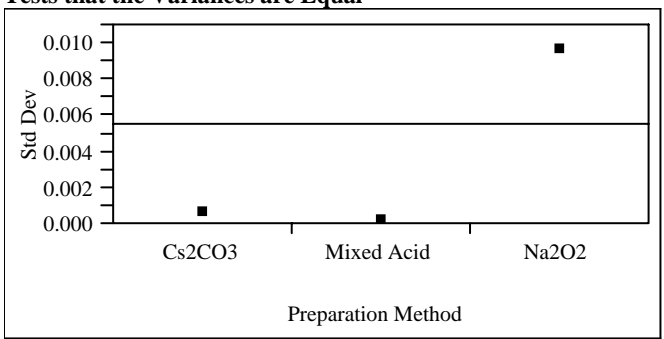

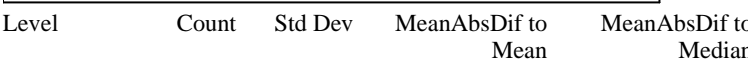

$\begin{array}{lllll}\mathrm{Cs} 2 \mathrm{CO} 3 & 3 & 0.0006433 & 0.0004952 & 0.0003714\end{array}$

$\begin{array}{lllll}\text { Mixed Acid } & 3 & 0.0001221 & 0.0000912 & 0.0000977\end{array}$

$\begin{array}{lllll}\mathrm{Na} 2 \mathrm{O} 2 & 3 & 0.0095756 & 0.0072192 & 0.0073495\end{array}$

Test $\quad$ F Ratio DFNum $\quad$ DFDen $\quad$ Prob $>$ F

$\begin{array}{lllll}\text { O'Brien[.5] } & 1.7695 & 2 & 6 & 0.2489\end{array}$

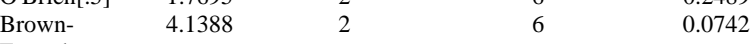

Forsythe $\quad 10.6359-6.0106$

$\begin{array}{rrrrr}\text { Levene } & 10.6359 & 2 & 6 & 0.0106 \\ \text { Bartlett } & 8.8722 & 2 & . & 0.0001\end{array}$

Warning: Small sample sizes. Use Caution.

Welch Anova testing Means Equal, allowing Std Devs Not Equal

F Ratio DFNum DFDen Prob $>$ F

$\begin{array}{llll}2090.4731 & 2 & 2.7597 & <.0001\end{array}$ 


\section{Exhibit B3. Statistical Comparisons of Chemical Composition Measurements of ARG-1 Samples in Radioactive Set by Dissolution Method for Each Oxide (Concentrations in Wt\% Oxides)}

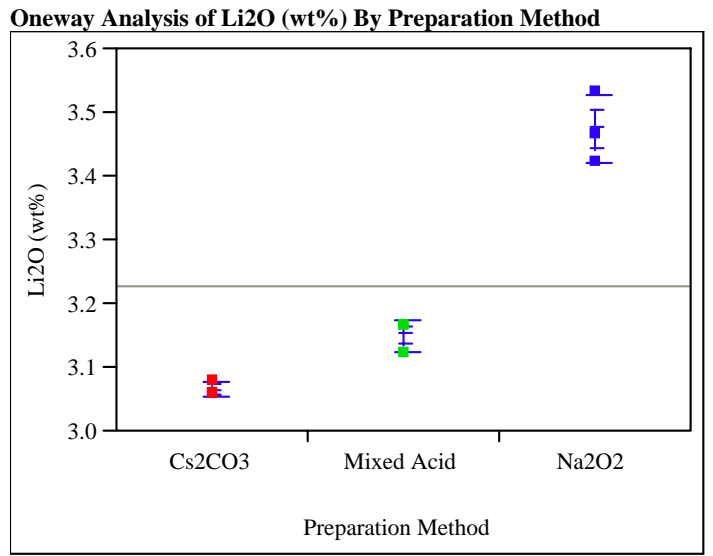

Means Comparisons

Comparisons for all pairs using Tukey-Kramer HSD q* Alpha

Abs(Dif)-LSD Na2O2 Mixed Acid Cs2CO3

$\begin{array}{llll}\mathrm{Na} 2 \mathrm{O} 2 & -0.08807 & 0.23486 & 0.32098\end{array}$

Mixed Acid $\quad 0.23486 \quad-0.08807-0.00196$

Cs2CO3 $\quad 0.32098 \quad-0.00196-0.08807$

Positive values show pairs of means that are significantly different.

Level Mean

$\mathrm{Na2O} 2 \quad$ A 3.4733453

Mixed Acid B 3.1504103

Cs2CO3 B 3.0642943

Levels not connected by same letter are significantly different

Tests that the Variances are Equal

\begin{tabular}{lrrrr|}
\hline \\
\hline 5
\end{tabular}

Warning: Small sample sizes. Use Caution.

Welch Anova testing Means Equal, allowing Std Devs Not Equal

F Ratio DFNum DFDen Prob $>$ F

$\begin{array}{rrrr}74.5191 & 2 & 3.2764 & 0.0019\end{array}$

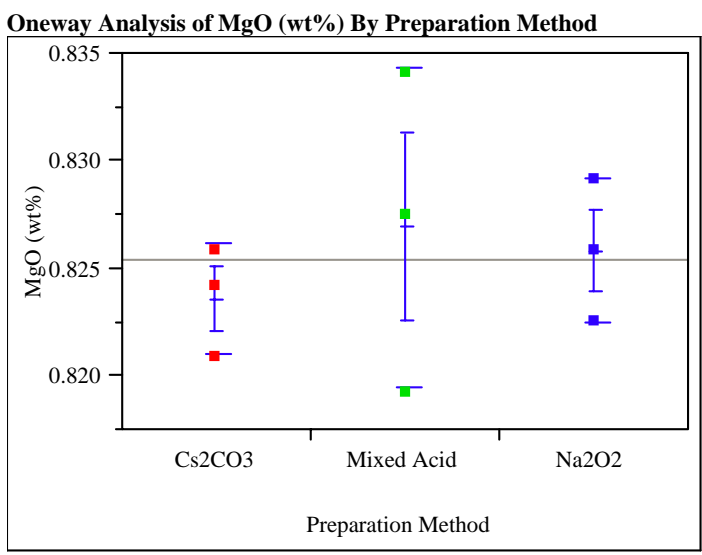

Means Comparisons

Comparisons for all pairs using Tukey-Kramer HSD

\begin{tabular}{|c|c|c|c|}
\hline$q^{*}$ & Alpha & & \\
\hline 3.06815 & 0.05 & & \\
\hline $\mathrm{SD}$ & Mixed Acid & $\mathrm{Na} 2 \mathrm{O} 2$ & Cs2CO3 \\
\hline & -0.01239 & -0.01128 & -0.00907 \\
\hline & -0.0 & -0.0 & -0.01017 \\
\hline & -0.00907 & -0.01017 & -0.01239 \\
\hline
\end{tabular}

Positive values show pairs of means that are significantly different.

Level Mean

Mixed Acid A 0.82693893

$\mathrm{Na2O} 2$ A 0.82583340

Cs2CO3 A 0.82362233

Levels not connected by same letter are significantly different Tests that the Variances are Equal

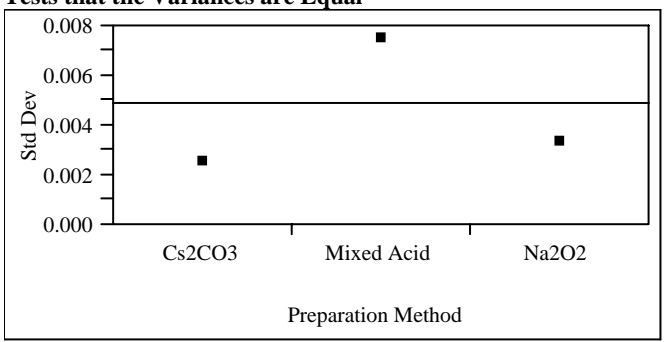

\begin{tabular}{|c|c|c|c|c|}
\hline Level & Count & Std Dev & $\begin{array}{r}\text { MeanAbsDif to } \\
\text { Mean }\end{array}$ & $\begin{array}{r}\text { MeanAbsDif to } \\
\text { Median }\end{array}$ \\
\hline Cs2CO3 & 3 & 0.0025331 & 0.0018426 & 0.0022111 \\
\hline Mixed Acid & 3 & 0.0074777 & 0.0051592 & 0.0071860 \\
\hline $\mathrm{Na} 2 \mathrm{O} 2$ & 3 & 0.0033166 & 0.0022111 & 0.0033166 \\
\hline Test & F Ratio & DFNum & DFDen & Prob $>$ F \\
\hline O'Brien[.5] & 1.2132 & 2 & 6 & 0.3610 \\
\hline $\begin{array}{l}\text { Brown- } \\
\text { Forsythe }\end{array}$ & 33.5000 & 2 & 6 & 0.0006 \\
\hline Levene & 1.4175 & 2 & 6 & 0.3132 \\
\hline Bartlett & 1.0706 & 2 & 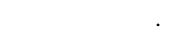 & 0.3428 \\
\hline
\end{tabular}

Welch Anova testing Means Equal, allowing Std Devs Not Equal

F Ratio DFNum DFDen Prob $>$ F

$\begin{array}{llll}0.4880 & 2 & 3.6297 & 0.6491\end{array}$ 


\section{Exhibit B3. Statistical Comparisons of Chemical Composition Measurements of ARG-1 Samples in Radioactive Set by Dissolution Method for Each Oxide (Concentrations in Wt\% Oxides)}

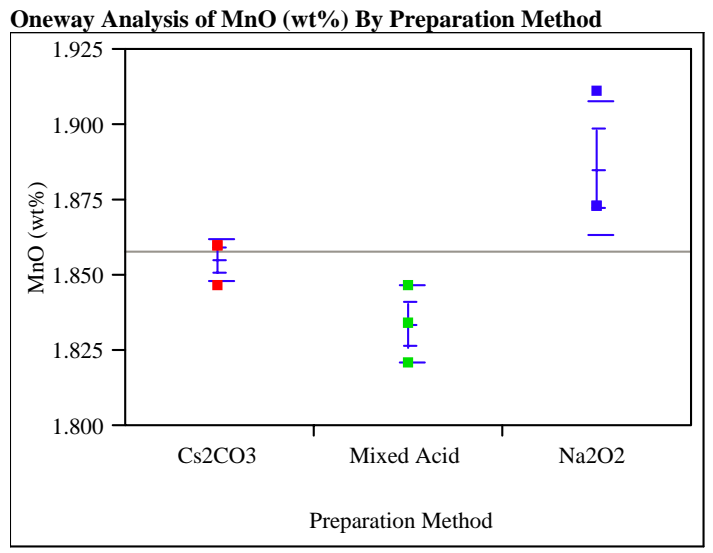

Means Comparisons

Comparisons for all pairs using Tukey-Kramer HSD

$$
\begin{array}{rr}
\text { q*}^{*} & \text { Alpha } \\
3.06815 & 0.05
\end{array}
$$

Abs(Dif)-LSD Na2O2 Cs2CO3 Mixed Acid

$\begin{array}{llll}\mathrm{Na} 2 \mathrm{O} 2 & -0.03888 & -0.00875 & 0.01277\end{array}$

$\begin{array}{llll}\mathrm{Cs} 2 \mathrm{CO} 3 & -0.00875 & -0.03888 & -0.01736\end{array}$

Mixed Acid $\quad 0.01277 \quad-0.01736 \quad-0.03888$

Positive values show pairs of means that are significantly different.

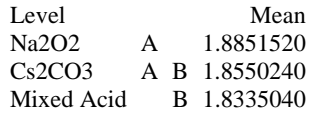

Levels not connected by same letter are significantly different Tests that the Variances are Equal

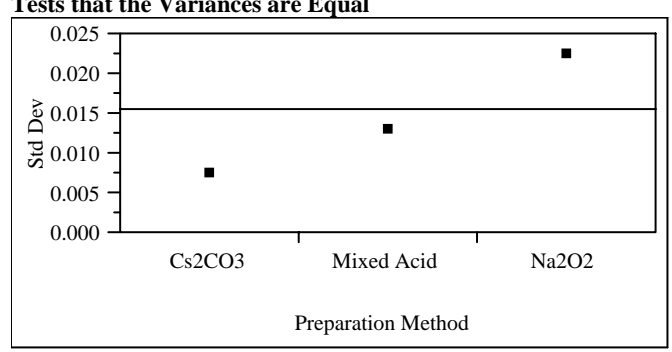

\begin{tabular}{|c|c|c|c|c|}
\hline Level & Count & Std Dev & $\begin{array}{r}\text { MeanAbsDif to } \\
\text { Mean }\end{array}$ & $\begin{array}{r}\text { MeanAbsDif to } \\
\text { Median }\end{array}$ \\
\hline Cs2CO3 & 3 & 0.0074547 & 0.0057387 & 0.0043040 \\
\hline Mixed Acid & 3 & 0.0129120 & 0.0086080 & 0.0129120 \\
\hline $\mathrm{Na} 2 \mathrm{O} 2$ & 3 & 0.0223642 & 0.0172160 & 0.0129120 \\
\hline Test & F Ratio & DFNum & DFDen & Prob $>$ F \\
\hline O'Brien[.5] & 1.0159 & 2 & 6 & 0.4169 \\
\hline $\begin{array}{l}\text { Brown- } \\
\text { Forsythe }\end{array}$ & 0.4000 & 2 & 6 & 0.6870 \\
\hline Levene & 2.7368 & 2 & 6 & 0.143 \\
\hline Bartlett & 0.9026 & 2 & . & 0.40 \\
\hline
\end{tabular}

Warning: Small sample sizes. Use Caution.

Welch Anova testing Means Equal, allowing Std Devs Not Equal

F Ratio DFNum DFDen Prob $>$ F

$\begin{array}{llll}5.5484 & 2 & 3.4667 & 0.0831\end{array}$

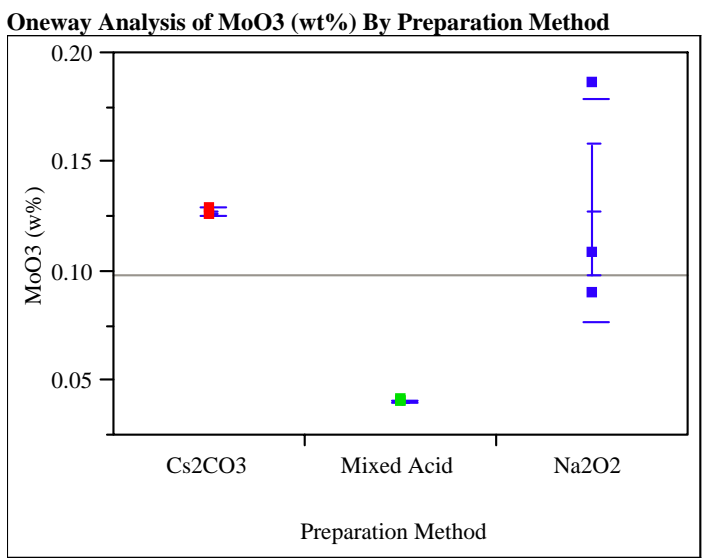

Means Comparisons

Comparisons for all pairs using Tukey-Kramer HSD q* Alpha

$3.06815 \quad 0.05$

Abs(Dif)-LSD Na2O2 Cs2CO3 Mixed Acid

$\begin{array}{llll}\mathrm{Na} 2 \mathrm{O} 2 & -0.07387 & -0.07287 & 0.01398\end{array}$

$\begin{array}{llll}\mathrm{Cs} 2 \mathrm{CO} 3 & -0.07287 & -0.07387 & 0.01298\end{array}$

$\begin{array}{llll}\text { Mixed Acid } \quad 0.01398 & 0.01298 & -0.07387\end{array}$

Positive values show pairs of means that are significantly different.

Level

$\begin{array}{lll} & \text { A } & 0.12702540\end{array}$

Mixed Acid B 0.04018303

Levels not connected by same letter are significantly different

Tests that the Variances are Equal

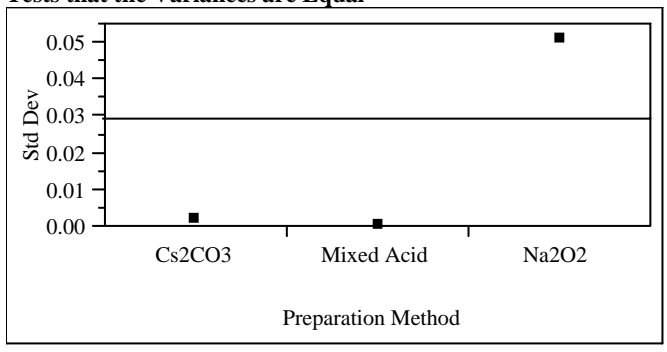

Level Count Std Dev MeanAbsDif to MeanAbsDif to

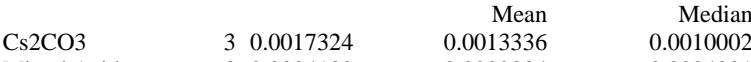

$\begin{array}{llll}\text { Mixed Acid } & 30.0004132 & 0.0002834 & 0.0004001\end{array}$

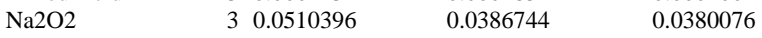

Test F Ratio DFNum DFDen Prob $>$ F

$\begin{array}{lllll}\text { O'Brien[.5] } & 1.7756 & 2 & 6 & 0.2479\end{array}$

$\begin{array}{lllll}\text { Brown- } & 3.4702 & 2 & 6 & 0.0997\end{array}$

$\begin{array}{llll}\text { Forsythe } & & & \\ \text { Levene } & 11.8939 & 2 & 6\end{array}$

$\begin{array}{llll}\text { Bartlett } & 10.7240 & 2 & <.0001\end{array}$

Warning: Small sample sizes. Use Caution.

Welch Anova testing Means Equal, allowing Std Devs Not Equal

F Ratio DFNum DFDen Prob $>$ F

$\begin{array}{rrrr}2885.7648 & 2 & 2.8099 & <.0001\end{array}$ 


\section{Exhibit B3. Statistical Comparisons of Chemical Composition Measurements of ARG-1 Samples in Radioactive Set by Dissolution Method for Each Oxide (Concentrations in Wt\% Oxides)}
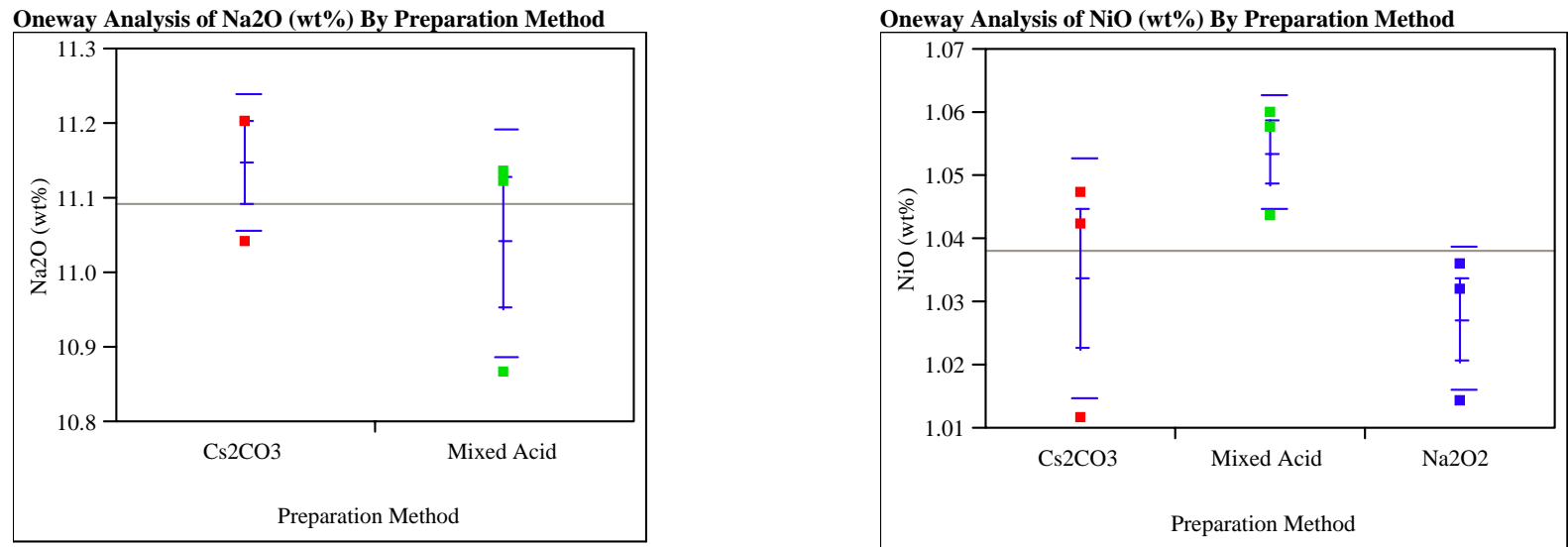

Missing Rows

3Means Comparison

Comparisons for all pairs using Tukey-Kramer HSD

$$
\text { q* Alpha }
$$

Abs(Dif)-LSD Cs2CO3 Mixed Acid

$\begin{array}{lll}\text { Cs2CO3 } & -0.28585 & -0.17801\end{array}$

Mixed Acid $\quad-0.17801 \quad-0.28585$

Positive values show pairs of means that are significantly different.

Level - Level Difference Lower CL Upper CL

Cs2CO3 Mixed Acid $0.1078400 \quad-0.1780110 .3936907$

Tests that the Variances are Equal

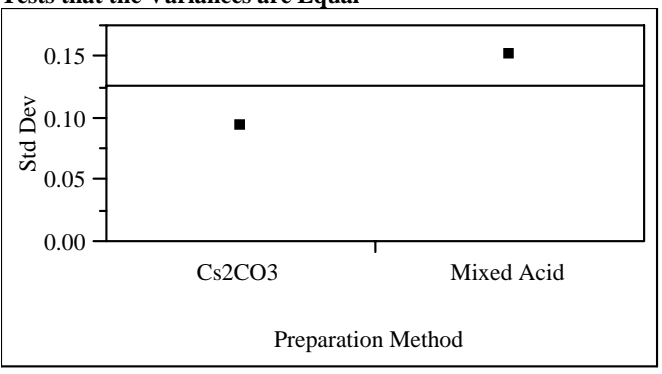

$\begin{array}{lrrrr}\text { Level } & \text { Count } & \text { Std Dev } & \begin{array}{r}\text { MeanAbsDif to } \\ \text { Mean }\end{array} & \begin{array}{r}\text { MeanAbsif to } \\ \text { Median }\end{array} \\ \text { Cs2CO3 } & 3 & 0.0933922 & 0.0718933 & 0.0539200 \\ \text { Mixed Acid } & 3 & 0.1519119 & \begin{array}{r}0.1168267 \\ \text { DFDen }\end{array} & 0.0943600 \\ \text { Test } & \text { F } & \text { DFNum } & & \text { Prob }>\text { F } \\ & \text { Ratio } & & 4 & 0.4812 \\ \text { O'Brien[.5] } & 0.6019 & 1 & 4 & 0.6987 \\ \text { Brown- } & 0.1731 & 1 & & \\ \text { Forsythe } & & & 4 & 0.2629 \\ \text { Levene } & 1.6949 & 1 & . & 0.5459 \\ \text { Bartlett } & 0.3646 & 1 & 2 & 0.5486 \\ \text { F Test 2-sided } & 2.6458 & 2 & & \end{array}$

Warning: Small sample sizes. Use Caution.

Welch Anova testing Means Equal, allowing Std Devs Not Equal

F Ratio DFNum DFDen Prob $>$ F

$\begin{array}{llll}1.0971 & 1 & 3.3228 & 0.3650\end{array}$

t Test

1.0474

Means Comparisons

Comparisons for all pairs using Tukey-Kramer HSD q* Alpha

Abs(Dif)-LSD Mixed Acid Cs2CO3 Na2O2

$\begin{array}{llll}\text { Mixed Acid } \quad-0.03495 & -0.01502 & -0.00865\end{array}$

Cs2CO3 $\quad-0.01502-0.03495-0.02859$

$\mathrm{Na} 2 \mathrm{O} 2 \quad-0.00865-0.02859-0.03495$

Positive values show pairs of means that are significantly different.

Level

Mean

Mixed Acid A 1.0536300

Cs2CO3 A 1.0336942

Na2O2 A 1.0273317

Levels not connected by same letter are significantly different

Tests that the Variances are Equal

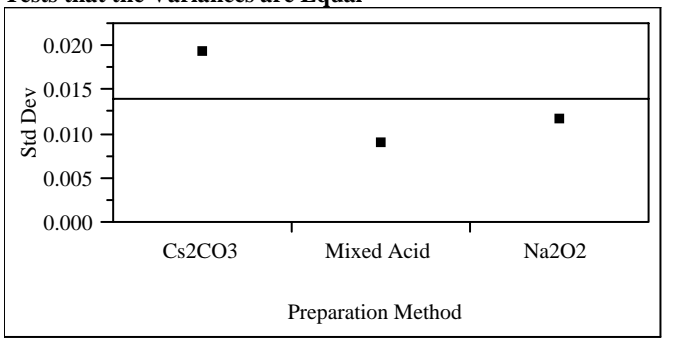

Level Count Std Dev MeanAbsDif to MeanAbsDif to

Cs2CO3

$0.0147044 \quad 0.0135733$

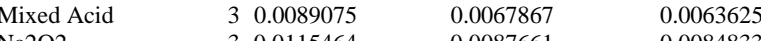

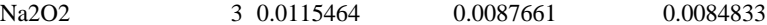

$\begin{array}{llll}\text { Test } & \text { F Ratio DFNum } & \text { DFDen } & \text { Prob }>\text { F }\end{array}$

$\begin{array}{lrrrr}\text { O'Brien[.5] } & 0.7949 & 2 & 6 & 0.4940\end{array}$

$\begin{array}{lllll}\text { Brown- } & 0.3804 & 2 & 6 & 0.6990\end{array}$

$\begin{array}{lllll}\text { Forsythe } & & & 6 & 0.2123 \\ \text { Levene } & 2.0287 & 2 & 6 & 0.5971\end{array}$

Bartlett $\quad 0.5157 \quad 2 \quad .0 .5971$

Warning: Small sample sizes. Use Caution.

Welch Anova testing Means Equal, allowing Std Devs Not Equal

F Ratio DFNum DFDen Prob > F

$\begin{array}{llll}4.4492 & 2 & 3.7352 & 0.1027\end{array}$ 


\section{Exhibit B3. Statistical Comparisons of Chemical Composition Measurements of ARG-1 Samples in Radioactive Set by Dissolution Method for Each Oxide (Concentrations in Wt\% Oxides)}

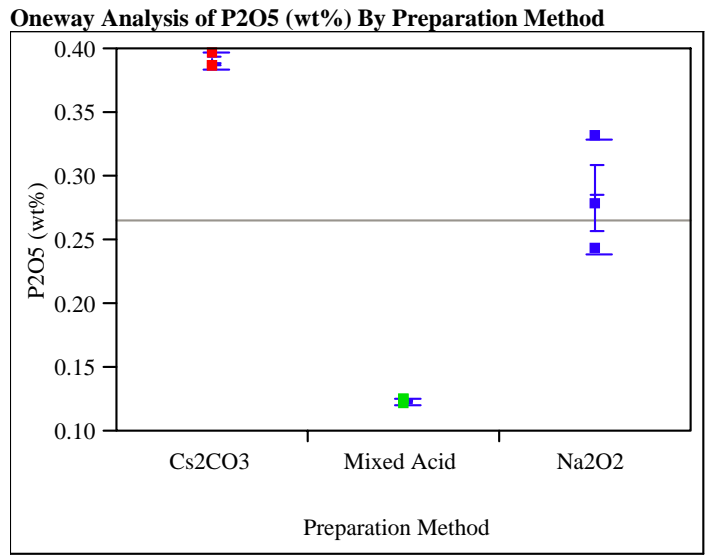

Means Comparisons

Comparisons for all pairs using Tukey-Kramer HSD $\mathrm{q}^{*} \quad$ Alpha

Abs(Dif)-LSD Cs2CO3 Na2O2 Mixed Acid

$\begin{array}{llll}\mathrm{Cs} 2 \mathrm{CO} 3 & -0.06569 & 0.04047 & 0.20049\end{array}$

$\mathrm{Na2O} \quad 0.04047-0.06569-0.09432$

Mixed Acid $\quad 0.20049 \quad 0.09432 \quad-0.06569$

Positive values show pairs of means that are significantly different.

$\begin{array}{lrrr}\text { Level } & & & \text { Mean } \\ \text { Cs2CO3 } & \text { A } & & 0.38953800 \\ \text { Na2O2 } & & \text { B } & 0.28336980 \\ \text { Mixed Acid } & & \text { C } & 0.12335370\end{array}$

Levels not connected by same letter are significantly different

Tests that the Variances are Equal

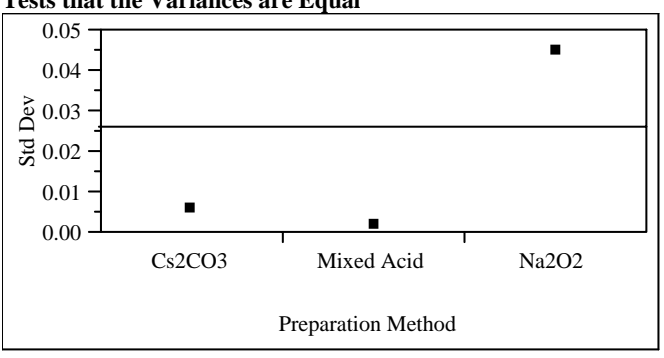

$\begin{array}{lrrrr}\text { Level } & \text { Count } & \text { Std Dev } & \begin{array}{r}\text { MeanAbsDif to } \\ \text { Mean }\end{array} & \begin{array}{r}\text { MeanAbsDif to } \\ \text { Median }\end{array} \\ \text { Cs2CO3 } & 3 & 0.0059532 & 0.0045828 & 0.0034371 \\ \text { Mixed Acid } & 3 & 0.0017501 & 0.0012730 & 0.0015276 \\ \text { Na2O2 } & 3 & 0.0449946 & 0.0318250 & 0.0416271 \\ \text { Test } & \text { F Ratio } & \text { DFNum } & \text { DFDen } & \text { Prob > F } \\ \text { O'Brien[.5] } & 1.7439 & 2 & 6 & 0.2529 \\ \text { Brown- } & 31.1391 & 2 & 6 & 0.0007 \\ \text { Forsythe } & & & & \\ \text { Levene } & 4.9620 & 2 & 6 & 0.0535 \\ \text { Bartlett } & 5.9724 & 2 & . & 0.0025 \\ \text { Wat } & 5.94 & & \end{array}$

Warning: Small sample sizes. Use Caution.

Welch Anova testing Means Equal, allowing Std Devs Not Equal

F Ratio DFNum DFDen Prob $>\mathrm{F}$

$\begin{array}{rrrr}2253.1616 & 2 & 2.881 & <.0001\end{array}$

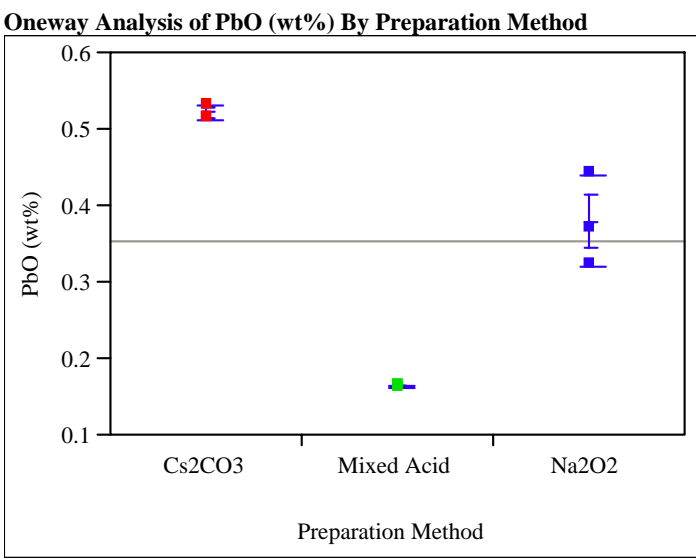

Means Comparisons

Comparisons for all pairs using Tukey-Kramer HSD q* Alpha

$3.06815 \quad 0.05$

Abs(Dif)-LSD Cs2CO3 Na2O2 Mixed Acid

$\begin{array}{llll}\text { Cs2CO3 } & -0.08767 & 0.05434 & 0.26889\end{array}$

$\begin{array}{llll}\mathrm{Na} 2 \mathrm{O} 2 & 0.05434 & -0.08767 & 0.12687\end{array}$

Mixed Acid $\quad 0.26889 \quad 0.12687 \quad-0.08767$

Positive values show pairs of means that are significantly different.

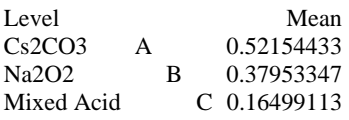

Levels not connected by same letter are significantly different

Tests that the Variances are Equal

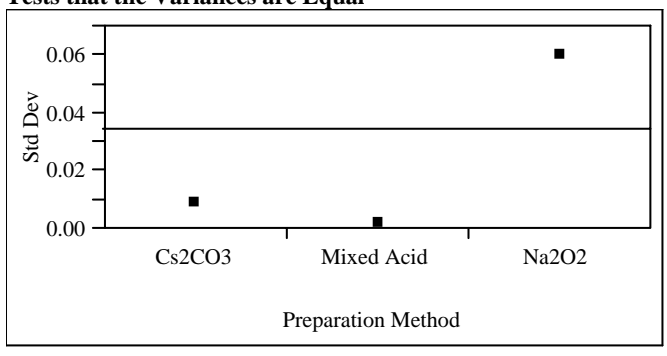

$\begin{array}{lrrrr}\text { Level } & \text { Count } & \text { Std Dev } & \begin{array}{r}\text { MeanAbsDif to } \\ \text { Mean }\end{array} & \begin{array}{r}\text { MeanAbsDif to } \\ \text { Median }\end{array} \\ \text { Cs2CO3 } & 3 & 0.0087069 & 0.0067026 & 0.0050269 \\ \text { Mixed Acid } & 3 & 0.0016455 & 0.0011969 & 0.0014363 \\ \text { Na2O2 } & 3 & 0.0599623 & 0.0424896 & 0.0552963 \\ \text { Test } & \text { F Ratio } & \text { DFNum } & \text { DFDen } & \text { Prob > F } \\ \text { O'Brien[.5] } & 1.7389 & 2 & 6 & 0.2537 \\ \text { Brown- } & 28.1615 & 2 & 6 & 0.0009 \\ \text { Forsythe } & & & & \\ \text { Levene } & 5.0470 & 2 & 6 & 0.0518 \\ \text { Bartlett } & 6.3978 & 2 & . & 0.0017\end{array}$

Warning: Small sample sizes. Use Caution.

Welch Anova testing Means Equal, allowing Std Devs Not Equal

F Ratio DFNum DFDen Prob $>$ F $\begin{array}{rrrr}1969.9802 & 2 & 2.7604 & <.0001\end{array}$ 


\section{Exhibit B3. Statistical Comparisons of Chemical Composition Measurements of ARG-1 Samples in Radioactive Set by Dissolution Method for Each Oxide (Concentrations in Wt\% Oxides)}

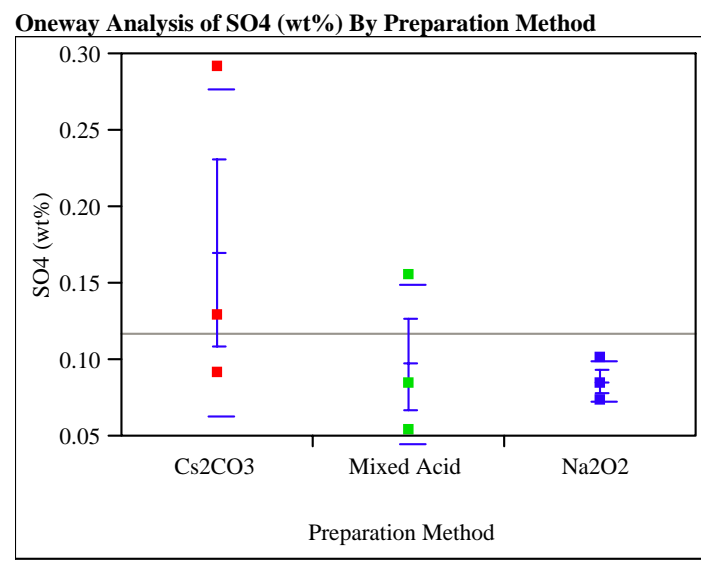

Means Comparisons

Comparisons for all pairs using Tukey-Kramer HSD $\mathrm{q}^{*}$ Alpha

Abs(Dif)-LSD Cs2CO3 Mixed Acid Na2O2

$\begin{array}{llll}\text { Cs2CO3 } & -0.17173 & -0.09913 & -0.08780\end{array}$

Mixed Acid $\quad-0.09913 \quad-0.17173-0.16040$

$\mathrm{Na2O} 2 \quad-0.08780 \quad-0.16040-0.17173$

Positive values show pairs of means that are significantly different.

Level Mean

Cs2CO3 A 0.16986753

Mixed Acid A 0.09726689

$\mathrm{Na} 2 \mathrm{O} 2$ A 0.08593240

Levels not connected by same letter are significantly different

Tests that the Variances are Equa

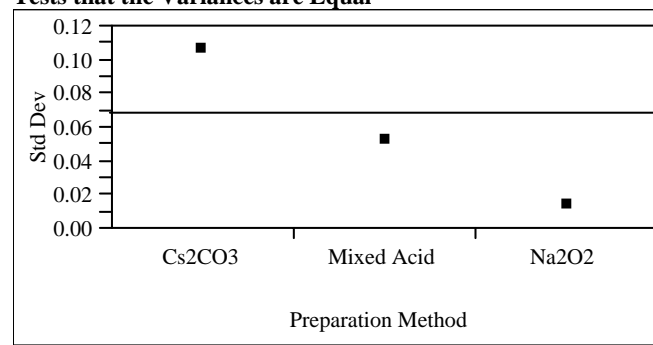

\begin{tabular}{|c|c|c|c|c|}
\hline Level & Count & Std Dev & $\begin{array}{r}\text { MeanAbsDif to } \\
\text { Mean }\end{array}$ & $\begin{array}{r}\text { MeanAbsDif to } \\
\text { Median }\end{array}$ \\
\hline Cs2CO3 & 3 & 0.1060477 & 0.0802901 & 0.0793913 \\
\hline Mixed Acid & 3 & 0.0516481 & 0.0380146 & 0.0436403 \\
\hline $\mathrm{Na} 2 \mathrm{O} 2$ & 3 & 0.0135813 & 0.0096202 & 0.0125328 \\
\hline Test & F Ratio & DFNum & DFDen & Prob $>F$ \\
\hline O'Brien[.5] & 1.3445 & 2 & 6 & 0.325 \\
\hline $\begin{array}{l}\text { Brown- } \\
\text { Forsythe }\end{array}$ & 1.7983 & 2 & 6 & 0.244 \\
\hline Levene & 5.3640 & 2 & 6 & \\
\hline Bartlett & 2.3985 & 2 & . & $0.0 \mathrm{~s}$ \\
\hline
\end{tabular}

Warning: Small sample sizes. Use Caution.

Welch Anova testing Means Equal, allowing Std Devs Not Equal

F Ratio DFNum DFDen Prob $>$ F

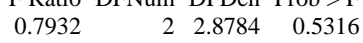

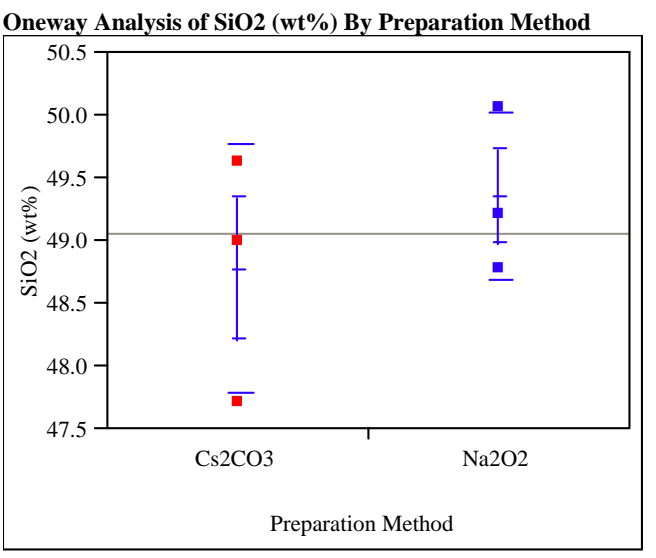

Missing Rows

3Means Comparisons

Comparisons for all pairs using Tukey-Kramer HSD q* Alpha

Abs(Dif)-LSD Na2O2 Cs2CO3

$\mathrm{Na} 2 \mathrm{O} 2 \quad-1.8887-1.3182$

Cs2CO3 $\quad-1.3182-1.8887$

Positive values show pairs of means that are significantly different Level - Level Difference Lower CL Upper CL

Na2O2 Cs2CO3 $0.5704800 \quad-1.31822 \quad 2.459176$

Tests that the Variances are Equal

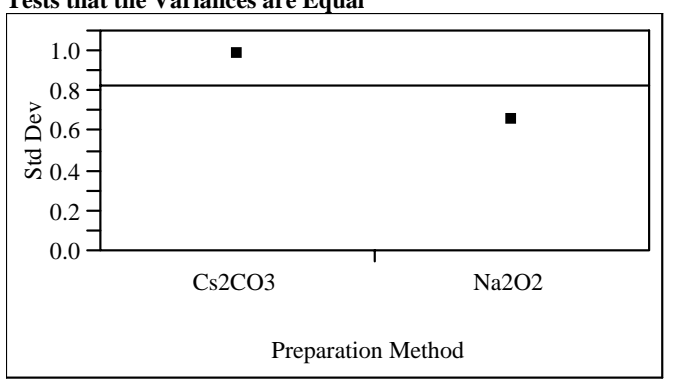

$\begin{array}{lrrrr}\text { Level } & \text { Count } & \text { Std Dev } & \begin{array}{r}\text { MeanAbsDif to } \\ \text { Mean }\end{array} & \begin{array}{r}\text { MeanAbsif to } \\ \text { Median }\end{array} \\ \text { Cs2CO3 } & 3 & 0.9803504 & 0.7131000 & 0.8557200 \\ \text { Na2O2 } & 3 & 0.6535669 & 0.4754000 & 0.5704800 \\ \text { Test } & \text { F Ratio } & \text { DFNum } & \text { DFDen } & \text { Prob }>\text { F } \\ \text { O'Brien[.5] } & 0.4582 & 1 & 4 & 0.5356 \\ \text { Brown- } & 1.2308 & 1 & 4 & 0.3295 \\ \text { Forsythe } & & & & \\ \text { Levene } & 0.5917 & 1 & 4 & 0.4847 \\ \text { Bartlett } & 0.2561 & 1 & . & 0.6128 \\ \text { F Test 2-sided } & 2.2500 & 2 & 2 & 0.6154 \\ \text { Wat }\end{array}$

Warning: Small sample sizes. Use Caution.

Welch Anova testing Means Equal, allowing Std Devs Not Equal

F Ratio DFNum DFDen Prob $>$ F

$\begin{array}{llll}0.7033 & 1 & 3.4845 & 0.4553\end{array}$

t Test

0.8386 


\section{Exhibit B3. Statistical Comparisons of Chemical Composition Measurements of ARG-1 Samples in Radioactive Set by Dissolution Method for Each Oxide (Concentrations in Wt\% Oxides)}

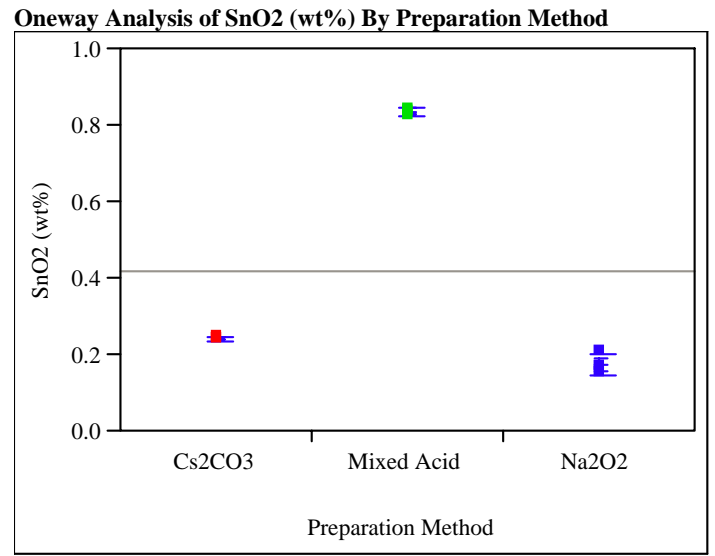

Means Comparisons

Comparisons for all pairs using Tukey-Kramer HSD q* Alpha

Abs(Dif)-LSD Mixed Acid Cs2CO3 Na2O2

$\begin{array}{llll}\text { Mixed Acid } & -0.04303 & 0.54924 & 0.61547\end{array}$

Cs2CO3 $\quad 0.54924-0.04303 \quad 0.02321$

$\begin{array}{llll}\mathrm{Na} 2 \mathrm{O} 2 & 0.61547 & 0.02321 & -0.04303\end{array}$

Positive values show pairs of means that are significantly different.

Level Mean

Mixed Acid A 0.83455040

Cs2CO3 B 0.24228200

$\mathrm{Na} 2 \mathrm{O} 2 \quad \mathrm{C} \quad 0.17605120$

Levels not connected by same letter are significantly different

Tests that the Variances are Equal

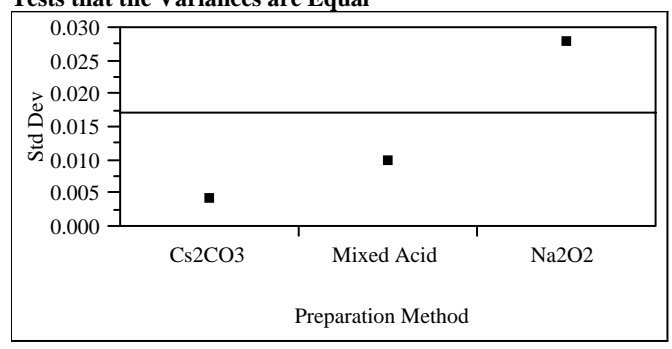

$\begin{array}{lrrrr}\text { Level } & \text { Count } & \text { Std Dev } & \begin{array}{r}\text { MeanAbsDif to } \\ \text { Mean }\end{array} & \begin{array}{r}\text { MeanAbsif to } \\ \text { Median }\end{array} \\ \text { Cs2CO3 } & 3 & 0.0040315 & 0.0031035 & 0.0023276 \\ \text { Mixed Acid } & 3 & 0.0096967 & 0.0070533 & 0.0084640 \\ \text { Na2O2 } & 3 & 0.0278324 & 0.0197493 & 0.0256036 \\ \text { Test } & \text { F Ratio } & \text { DFNum } & \text { DFDen } & \text { Prob > F } \\ \text { O'Brien[.5] } & 1.5240 & 2 & 6 & 0.2916 \\ \text { Brown- } & 16.7543 & 2 & 6 & 0.0035 \\ \text { Forsythe } & & & & \\ \text { Levene } & 3.2297 & 2 & 6 & 0.1117 \\ \text { Bartlett } & 2.5171 & 2 & . & 0.0807 \\ \text { Wart } & 2.75 & & \end{array}$

Warning: Small sample sizes. Use Caution.

Welch Anova testing Means Equal, allowing Std Devs Not Equal

F Ratio DFNum DFDen Prob $>$ F

$3962.6763 \quad 2 \quad 3.0955<.0001$

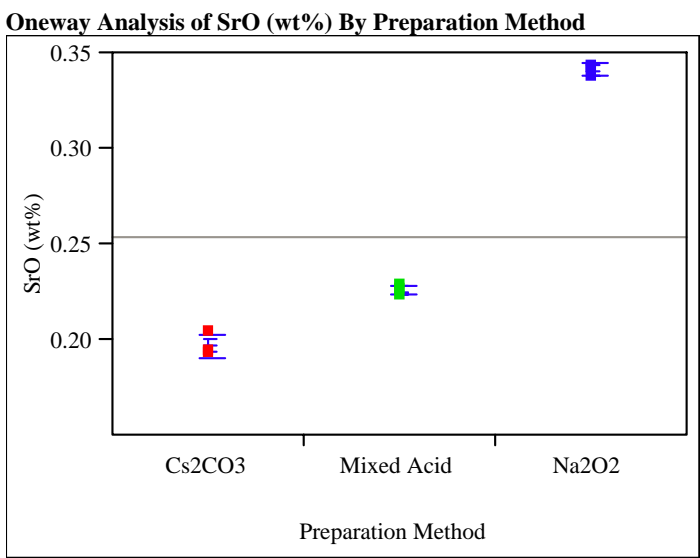

Means Comparisons

Comparisons for all pairs using Tukey-Kramer HSD q* Alpha

$3.06815 \quad 0.05$

Abs(Dif)-LSD Na2O2 Mixed Acid Cs2CO3

$\begin{array}{llll}\mathrm{Na} 2 \mathrm{O} 2 & -0.01050 & 0.10461 & 0.13339\end{array}$

Mixed Acid $\quad 0.10461 \quad-0.01050 \quad 0.01828$

$\begin{array}{llll}\text { Cs2CO3 } & 0.13339 & 0.01828 & -0.01050\end{array}$

Positive values show pairs of means that are significantly different.

$\begin{array}{lrrr}\text { Level } & & & \text { Mean } \\ \text { Na2O2 } & \text { A } & & 0.34058880 \\ \text { Mixed Acid } & & \text { B } & 0.22548240 \\ \text { Cs2CO3 } & & \text { C } & 0.19670580\end{array}$

Levels not connected by same letter are significantly different Tests that the Variances are Equal

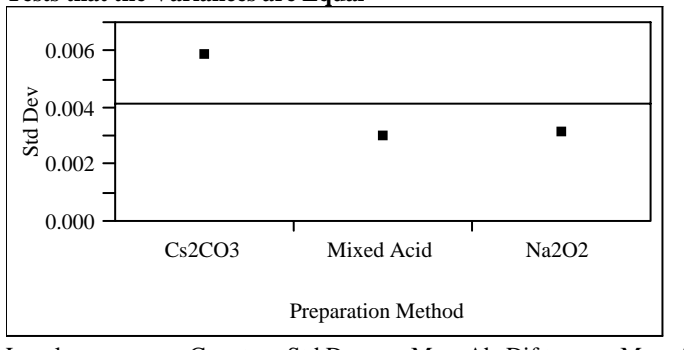

$\begin{array}{lrrrr}\text { Level } & \text { Count } & \text { Std Dev } & \begin{array}{r}\text { MeanAbsDif to } \\ \text { Mean }\end{array} & \begin{array}{r}\text { MeanAbsDif to } \\ \text { Median }\end{array} \\ \text { Cs2CO3 } & 3 & 0.0058336 & 0.0044676 & 0.0039420 \\ \text { Mixed Acid } & 3 & 0.0029761 & 0.0021024 & 0.0027594 \\ \text { Na2O2 } & 3 & 0.0031289 & 0.0023652 & 0.0023652 \\ \text { Test } & \text { F Ratio } & \text { DFNum } & \text { DFDen } & \text { Prob > F } \\ \text { O'Brien[.5] } & 0.8154 & 2 & 6 & 0.4861 \\ \text { Brown- } & 0.2203 & 2 & 6 & 0.8085 \\ \text { Forsythe } & & & & \\ \text { Levene } & 1.9597 & 2 & 6 & 0.2213 \\ \text { Bartlett } & 0.4966 & 2 & . & 0.6086\end{array}$

Warning: Small sample sizes. Use Caution.

Welch Anova testing Means Equal, allowing Std Devs Not Equal

F Ratio DFNum DFDen Prob $>$ F

$\begin{array}{llll}1136.6107 & 2 & 3.8029 & <.0001\end{array}$ 
Westinghouse Savannah River Company

WSRC-TR-2005-00396

Savannah River National Laboratory

Rev. 0

Analytical Development/Statistical Consulting Sections

Page 115 of 140

\section{Exhibit B3. Statistical Comparisons of Chemical Composition Measurements of ARG-1 Samples in Radioactive Set by Dissolution Method for Each Oxide (Concentrations in Wt\% Oxides)}

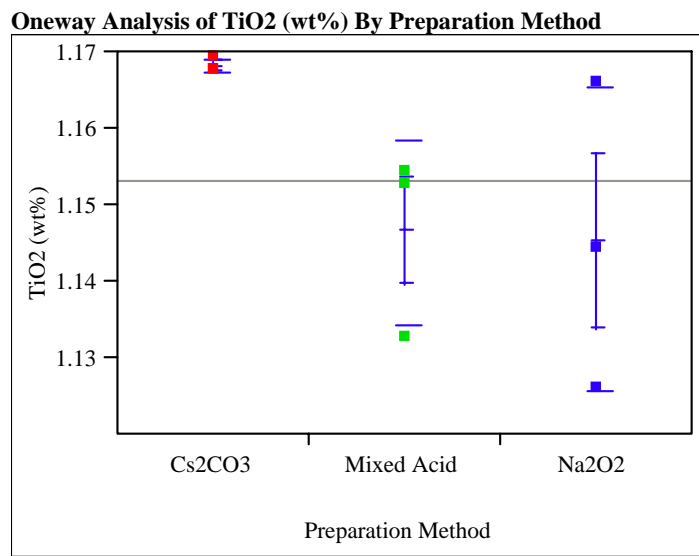

Means Comparisons

Comparisons for all pairs using Tukey-Kramer HSD q Alpha

Abs(Dif)-LSD Cs2CO3 Mixed Acid Na2O2

$\begin{array}{llll}\text { Cs2CO3 } & -0.03386 & -0.01218 & -0.01107\end{array}$

Mixed Acid $\quad-0.01218 \quad-0.03386-0.03275$

$\mathrm{Na2O} \quad-0.01107 \quad-0.03275-0.03386$

Positive values show pairs of means that are significantly different.

Level

Cs2CO3 A 1.1681560

Mixed Acid A 1.1464720

$\mathrm{Na} 2 \mathrm{O} 2 \quad$ A 1.1453600

Levels not connected by same letter are significantly different

Tests that the Variances are Equal

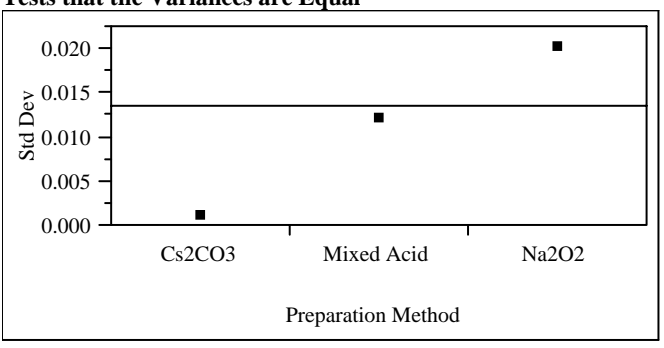

$\begin{array}{lrrrr}\text { Level } & \text { Count } & \text { Std Dev } & \begin{array}{r}\text { MeanAbsDif to } \\ \text { Mean }\end{array} & \begin{array}{r}\text { MeanAbsif to } \\ \text { Median }\end{array} \\ \text { Cs2CO3 } & 3 & 0.0009630 & 0.0007413 & 0.0005560 \\ \text { Mixed Acid } & 3 & 0.0120666 & 0.0092667 & 0.0077840 \\ \text { Na2O2 } & 3 & 0.0200392 & 0.0137147 & 0.0194600 \\ \text { Test } & \text { F Ratio } & \text { DFNum } & \text { DFDen } & \text { Prob }>\text { F } \\ \text { O'Brien[.5] } & 1.2031 & 2 & 6 & 0.3636 \\ \text { Brown- } & 7.0079 & 2 & 6 & 0.0269 \\ \text { Forsythe } & & & & \\ \text { Levene } & 2.8692 & 2 & 6 & 0.1335 \\ \text { Bartlett } & 3.8640 & 2 & . & 0.0210 \\ \text { Want } & 2.0 & & \end{array}$

Warning: Small sample sizes. Use Caution.

Welch Anova testing Means Equal, allowing Std Devs Not Equal

F Ratio DFNum DFDen Prob $>$ F

$\begin{array}{llll}5.3906 & 2 & 2.6896 & 0.1146\end{array}$

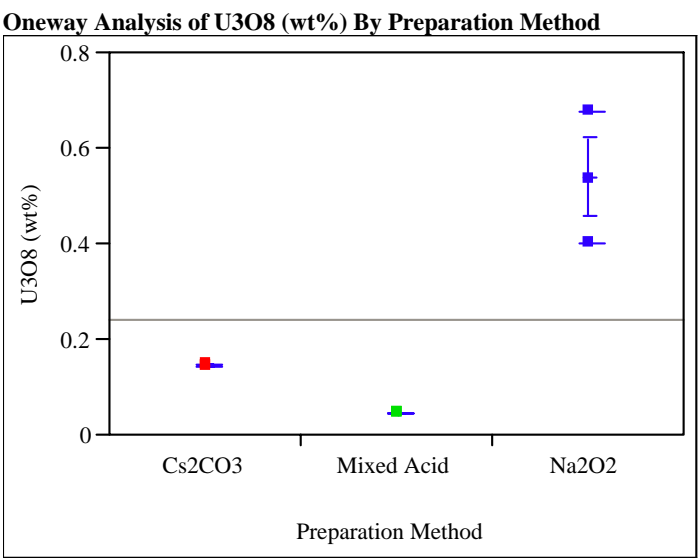

Means Comparisons

Comparisons for all pairs using Tukey-Kramer HSD $\mathrm{q}^{*}$ Alpha

$3.06815 \quad 0.05$

Abs(Dif)-LSD Na2O2 Cs2CO3 Mixed Acid

Na2O2 $\quad-0.19874 \quad 0.19275 \quad 0.29322$

$\begin{array}{llll}\mathrm{Cs} 2 \mathrm{CO} 3 & 0.19275 & -0.19874 & -0.09827\end{array}$

Mixed Acid $\quad 0.29322 \quad-0.09827 \quad-0.19874$

Positive values show pairs of means that are significantly different.

Level

B 0.14700693

Mixed Acid B 0.04653909

Levels not connected by same letter are significantly different Tests that the Variances are Equal

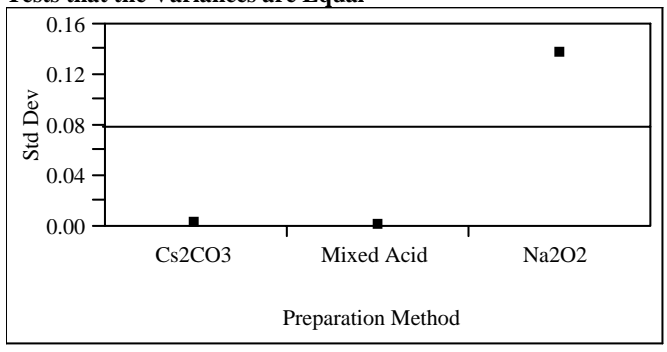

Level Count Std Dev MeanAbsDif to MeanAbsDif to

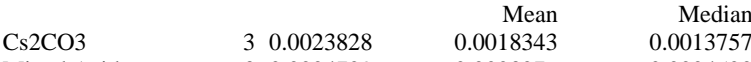

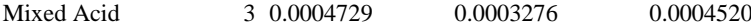

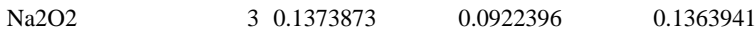

Test F Ratio DFNum DFDen $\quad$ Prob $>$ F

$\begin{array}{lllll}\text { O'Brien[.5] } & 1.7772 & 2 & 6 & 0.2476\end{array}$

$\begin{array}{lllll}\text { Brown- } & 3188.5399 & 2 & 6 & <.0001\end{array}$

$\begin{array}{lllll}\text { Forsythe } & & & & \\ \text { Levene } & 4.0785 & 2 & 6 & 0.0761\end{array}$

$\begin{array}{llll}\text { Bartlett } & 13.2197 & 2 & <.0001\end{array}$

Warning: Small sample sizes. Use Caution.

Welch Anova testing Means Equal, allowing Std Devs Not Equal

F Ratio DFNum DFDen Prob $>$ F

$\begin{array}{rrrr}2082.7694 & 2 & 2.7676 & <.0001\end{array}$ 


\section{Exhibit B3. Statistical Comparisons of Chemical Composition Measurements of ARG-1 Samples in Radioactive Set by Dissolution Method for Each Oxide (Concentrations in Wt\% Oxides)}

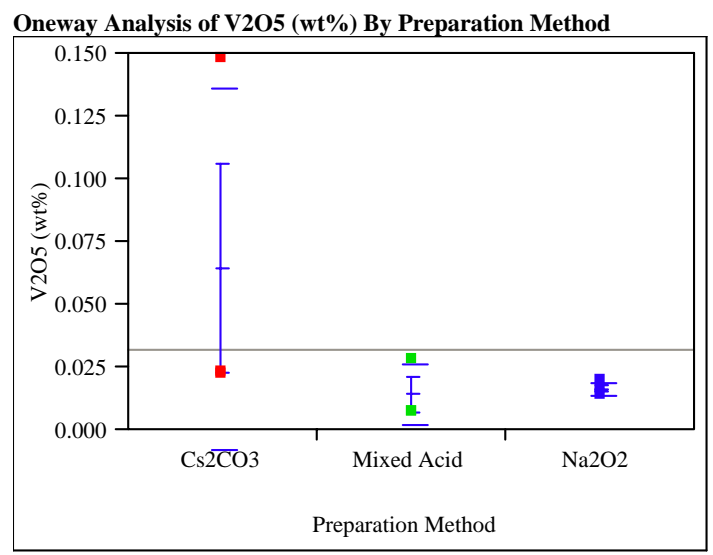

Means Comparisons

Comparisons for all pairs using Tukey-Kramer HSD

$$
\begin{array}{rr}
q^{*} & \text { Alpha } \\
3.06815 & 0.05
\end{array}
$$

Abs(Dif)-LSD Cs2CO3 Na2O2 Mixed Acid

Cs2CO3 $\quad-0.10592-0.05795 \quad-0.05566$

$\mathrm{Na} 2 \mathrm{O} 2 \quad-0.05795-0.10592 \quad-0.10362$

Mixed Acid $\quad-0.05566 \quad-0.10362 \quad-0.10592$

Positive values show pairs of means that are significantly different.

Level Mean

Cs2CO3 A 0.06447547

$\mathrm{Na} 2 \mathrm{O} 2 \quad$ A 0.01651310

Mixed Acid A 0.01421912

Levels not connected by same letter are significantly different Tests that the Variances are Equal

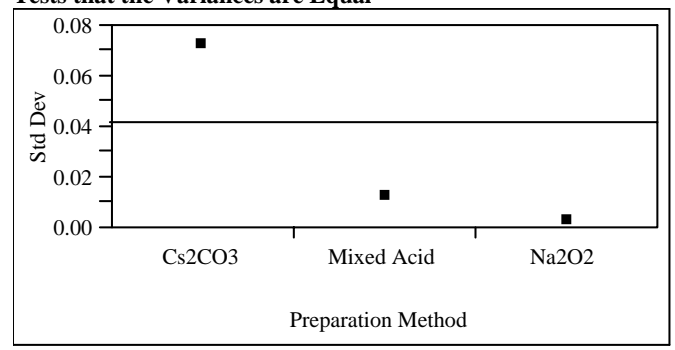

$\begin{array}{lrrrr}\text { Level } & \text { Count } & \text { Std Dev } & \begin{array}{r}\text { MeanAbsDif to } \\ \text { Mean }\end{array} & \begin{array}{r}\text { MeanAbsDif to } \\ \text { Median }\end{array} \\ \text { Cs2CO3 } & 3 & 0.0721744 & 0.0555594 & 0.0419820 \\ \text { Mixed Acid } & 3 & 0.0121132 & 0.0093247 & 0.0070248 \\ \text { Na2O2 } & 3 & 0.0026069 & 0.0018447 & 0.0024100 \\ \text { Test } & \text { F Ratio } & \text { DFNum } & \text { DFDen } & \text { Prob > F } \\ \text { O'Brien[.5] } & 1.7254 & 2 & 6 & 0.2559 \\ \text { Brown- } & 0.7985 & 2 & 6 & 0.4926 \\ \text { Forsythe } & & & & \\ \text { Levene } & 12.7636 & 2 & 6 & 0.0069 \\ \text { Bartlett } & 5.7295 & 2 & . & 0.0032\end{array}$

Warning: Small sample sizes. Use Caution.

Welch Anova testing Means Equal, allowing Std Devs Not Equal

F Ratio DFNum DFDen Prob $>$ F

$\begin{array}{llll}0.5777 & 2 & 2.7878 & 0.6167\end{array}$

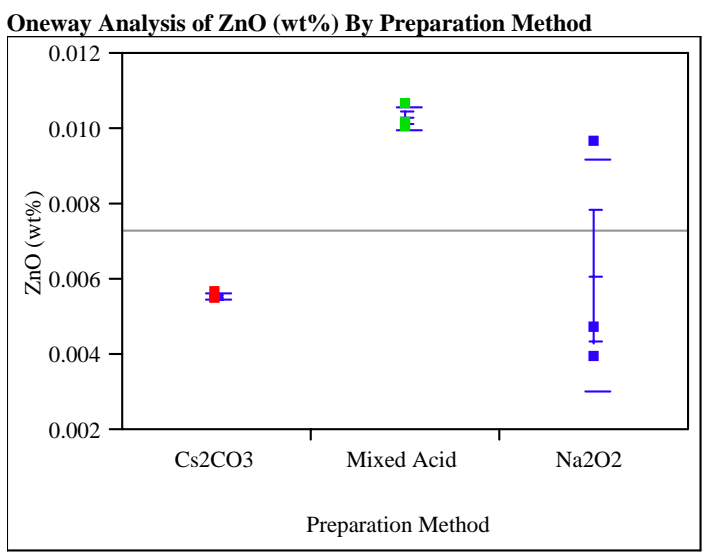

Means Comparisons

Comparisons for all pairs using Tukey-Kramer HSD

\begin{tabular}{lrrr}
\multicolumn{1}{c}{$\mathrm{q}^{*}$} & Alpha & & \\
3.06815 & 0.05 & & \\
Abs(Dif)-LSD & Mixed Acid & $\mathrm{Na} 2 \mathrm{O} 2$ & $\mathrm{Cs} 2 \mathrm{CO} 3$ \\
Mixed Acid & -0.00449 & -0.00031 & 0.00024 \\
Na2O2 & -0.00031 & -0.00449 & -0.00394 \\
Cs2CO3 & 0.00024 & -0.00394 & -0.00449
\end{tabular}

Positive values show pairs of means that are significantly different

$\begin{array}{lrrr}\text { Level } & & \text { Mean } \\ \text { Mixed Acid } & \text { A } & & 0.01028205 \\ \text { Na2O2 } & \text { A } & \text { B } & 0.00609537 \\ \text { Cs2CO3 } & & \text { B } & 0.00554351\end{array}$

Levels not connected by same letter are significantly different Tests that the Variances are Equal

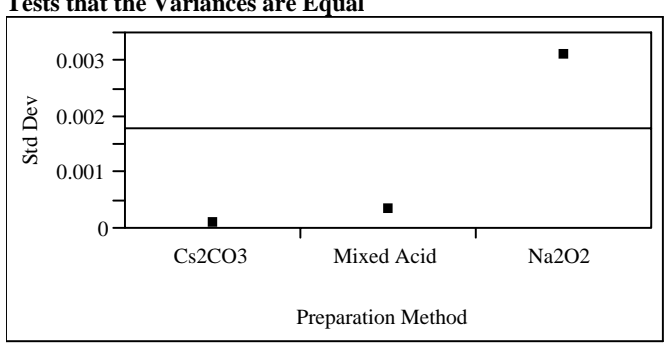

$\begin{array}{lrrrr}\text { Level } & \text { Count } & \text { Std Dev } & \begin{array}{r}\text { MeanAbsDif to } \\ \text { Mean }\end{array} & \begin{array}{r}\text { MeanAbsDif to } \\ \text { Median }\end{array} \\ \text { Cs2CO3 } & 3 & 0.0000934 & 0.0000719 & 0.0000539 \\ \text { Mixed Acid } & 3 & 0.0003176 & 0.0002407 & 0.0002365 \\ \text { Na2O2 } & 3 & 0.0030894 & 0.0023596 & 0.0021556 \\ \text { Test } & \text { F Ratio } & \text { DFNum } & \text { DFDen } & \text { Prob > F } \\ \text { O'Brien[.5] } & 1.7573 & 2 & 6 & 0.2508 \\ \text { Brown- } & 2.1034 & 2 & 6 & 0.2031 \\ \text { Forsythe } & & & & \\ \text { Levene } & 12.1108 & 2 & 6 & 0.0078 \\ \text { Bartlett } & 6.7789 & 2 & . & 0.0011\end{array}$

Warning: Small sample sizes. Use Caution.

Welch Anova testing Means Equal, allowing Std Devs Not Equal

F Ratio DFNum DFDen Prob $>$ F

$\begin{array}{rrrr}249.5345 & 2 & 2.8798 & 0.0006\end{array}$ 


\section{Exhibit B3. Statistical Comparisons of Chemical Composition Measurements of ARG-1 Samples in Radioactive Set by Dissolution Method for Each Oxide (Concentrations in Wt\% Oxides)}

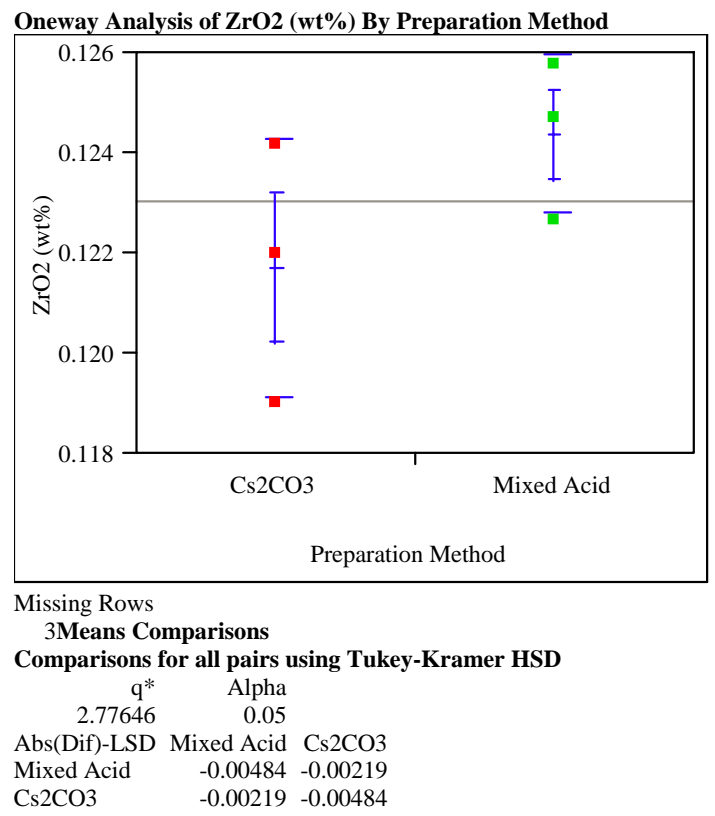

Positive values show pairs of means that are significantly different.

Level - Level Difference Lower CL Upper CL

Mixed Acid Cs2CO3 $0.0026566 \quad-0.002187 \quad 0.0075000$

Tests that the Variances are Equal

\begin{tabular}{l|r|r|r|}
\hline \\
\cline { 2 - 4 }
\end{tabular}


Westinghouse Savannah River Company

WSRC-TR-2005-00396

Savannah River National Laboratory

Rev. 0

Analytical Development/Statistical Consulting Sections

Page 118 of 140

Exhibit B4. Chemical Composition Measurements by Type of Prepared Sample by Oxide for DWPF Radioactive Glass

(Concentrations are in wt\%.)

Oneway Analysis of Ag2O (wt \%) By Preparation Method

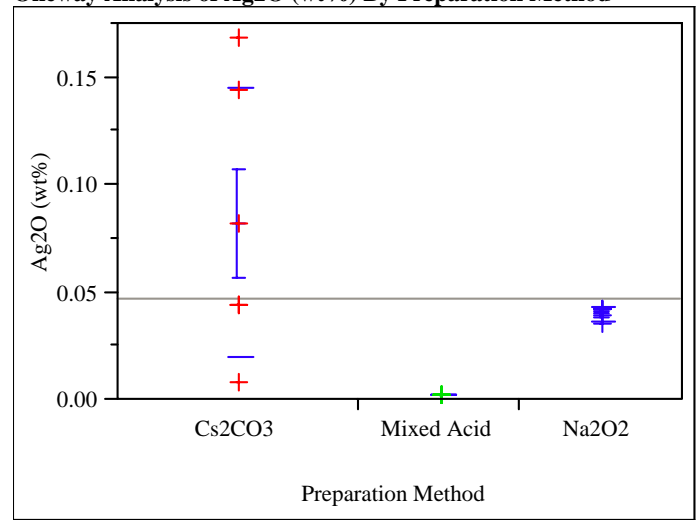

Means Comparisons

Comparisons for all pairs using Tukey-Kramer HSD

$\mathrm{q}^{*}$ Alpha

Abs(Dif)-LSD Cs2CO3 Na2O2 Mixed Acid

$\begin{array}{lllr}\mathrm{Cs} 2 \mathrm{CO} 3 & -0.06578 & -0.03138 & 0.00626\end{array}$

$\begin{array}{llll}\mathrm{Na} 2 \mathrm{O} 2 & -0.03138 & -0.08056 & -0.04292\end{array}$

Mixed Acid $\quad 0.00626 \quad-0.04292 \quad-0.08056$

Positive values show pairs of means that are significantly different.

Level Mean

Cs2CO3 A 0.08212259

$\mathrm{Na} 2 \mathrm{O} 2 \quad$ A $\quad$ B 0.03996024

Mixed Acid B 0.00232027

Levels not connected by same letter are significantly different Tests that the Variances are Equal

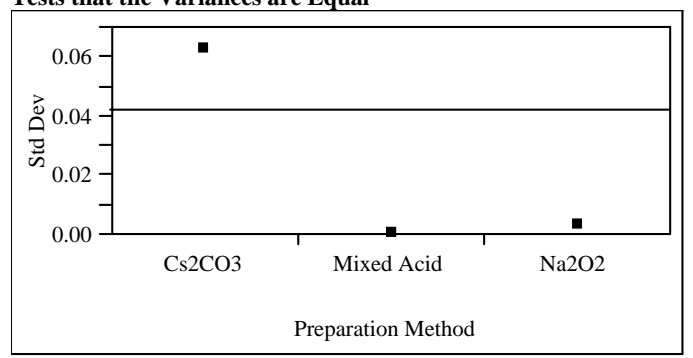

$\begin{array}{lrrrr}\text { Level } & \text { Count } & \text { Std Dev } & \begin{array}{r}\text { MeanAbsDif to } \\ \text { Mean }\end{array} & \begin{array}{r}\text { MeanAbsDif to } \\ \text { Median }\end{array} \\ \text { Cs2CO3 } & 6 & 0.0625171 & 0.0495385 & 0.0495385 \\ \text { Mixed Acid } & 4 & 0.0000661 & 0.0000483 & 0.0000483 \\ \text { Na2O2 } & 4 & 0.0032309 & 0.0024169 & 0.0024169 \\ \text { Test } & \text { F Ratio } & \text { DFNum } & \text { DFDen } & \text { Prob }>\text { F } \\ \text { O'Brien[.5] } & 3.9053 & 2 & 11 & 0.0523 \\ \text { Brown- } & 6.3852 & 2 & 11 & 0.0144 \\ \text { Forsythe } & & & & \\ \text { Levene } & 9.1315 & 2 & 11 & 0.0046 \\ \text { Bartlett } & 22.2419 & 2 & . & <.0001\end{array}$

Warning: Small sample sizes. Use Caution.

Welch Anova testing Means Equal, allowing Std Devs Not Equal

F Ratio DFNum DFDen Prob $>$ F

$\begin{array}{llll}243.7299 & 2 & 5.0026 & <.0001\end{array}$

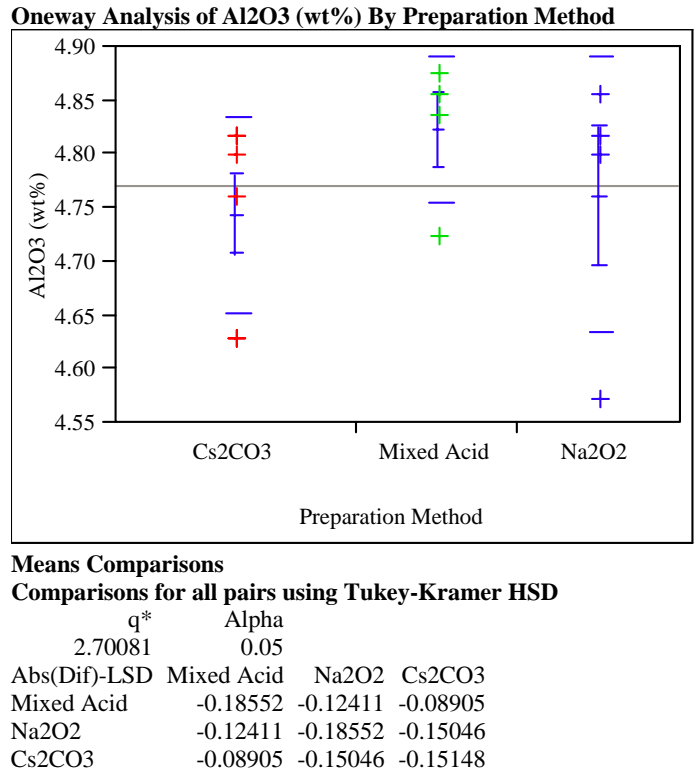

Positive values show pairs of means that are significantly different.

$$
\begin{array}{lrr}
\text { Level } & & \text { Mean } \\
\text { Mixed Acid } & \text { A } & 4.8229487 \\
\text { Na2O2 } & \text { A } & 4.7615400 \\
\text { Cs2CO3 } & \text { A } & 4.7426450
\end{array}
$$

\begin{tabular}{|c|c|c|c|c|}
\hline Level & Count & Std Dev & $\begin{array}{r}\text { MeanAbsDif to } \\
\text { Mean }\end{array}$ & $\begin{array}{r}\text { MeanAbsDif to } \\
\text { Median }\end{array}$ \\
\hline Cs2CO3 & 6 & 0.0902224 & 0.0755800 & 0.0692817 \\
\hline Mixed Acid & 4 & 0.0679082 & 0.0495994 & 0.042513 \\
\hline $\mathrm{Na} 2 \mathrm{O} 2$ & 4 & 0.1281521 & 0.0944750 & 0.075580 \\
\hline Test & F Ratio & DFNum & DFDen & Prob > \\
\hline O'Brien[.5] & 0.6546 & 2 & 11 & 0.538 \\
\hline $\begin{array}{l}\text { Brown- } \\
\text { Forsythe }\end{array}$ & 0.2166 & 2 & 11 & 0.808 \\
\hline Levene & 0.9334 & 2 & 11 & 0.42 \\
\hline Bartlett & 0.5424 & 2 & . & 0.581 \\
\hline
\end{tabular}

Levels not connected by same letter are significantly different Tests that the Variances are Equal

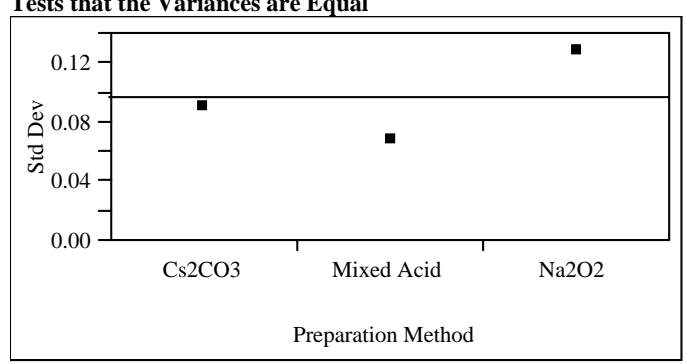

Warning: Small sample sizes. Use Caution.

Welch Anova testing Means Equal, allowing Std Devs Not Equal

F Ratio DFNum DFDen Prob $>$ F

$\begin{array}{llll}1.2210 & 2 & 6.3888 & 0.3556\end{array}$ 
Westinghouse Savannah River Company

WSRC-TR-2005-00396

Savannah River National Laboratory

Rev. 0

Analytical Development/Statistical Consulting Sections

Page 119 of 140

Exhibit B4. Chemical Composition Measurements by Type of Prepared Sample by Oxide for DWPF Radioactive Glass

(Concentrations are in wt\%.)

Oneway Analysis of B2O3 (wt \%) By Preparation Method

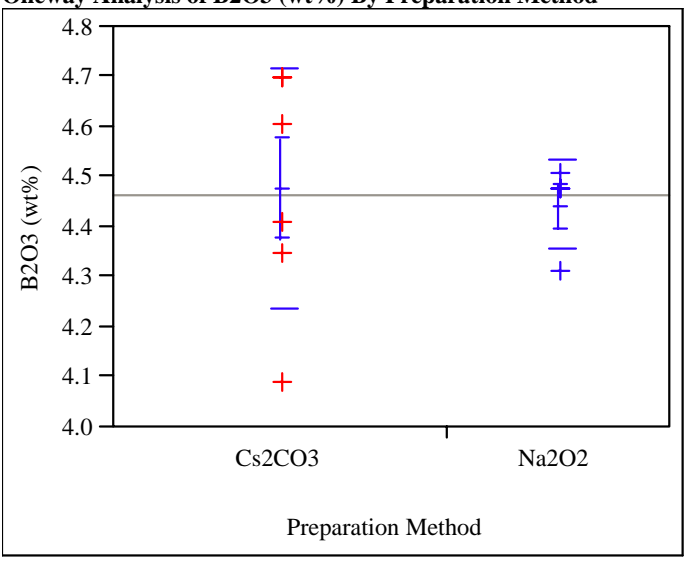

Missing Rows

4Means Comparisons

Comparisons for all pairs using Tukey-Kramer HSD

$$
\text { Alpha }
$$

Abs(Dif)-LSD Cs2CO3 Na2O2

$\begin{array}{lll}\mathrm{Cs} 2 \mathrm{CO} 3 & -0.26251 & -0.26129 \\ \mathrm{Na} 2 \mathrm{O} 2 & -0.26129 & -0.32151\end{array}$$$
\text { Na202- }-0.26129-0.32151
$$

Positive values show pairs of means that are significantly different.

Level - Level Difference Lower CL Upper CL

$\begin{array}{lllll}\mathrm{Cs} 2 \mathrm{CO} 3 & \mathrm{Na} 2 \mathrm{O} 2 & 0.0321990 & -0.261294 & 0.3256924\end{array}$

Tests that the Variances are Equal

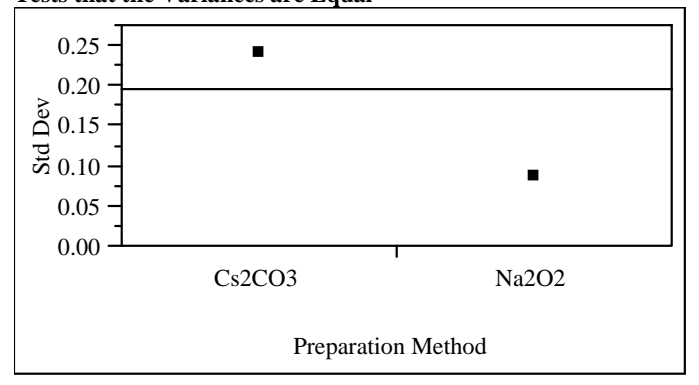

\begin{tabular}{|c|c|c|c|c|}
\hline Level & Count & Std Dev & $\begin{array}{r}\text { MeanAbsDif to } \\
\text { Mean }\end{array}$ & $\begin{array}{r}\text { MeanAbsDif to } \\
\text { Median }\end{array}$ \\
\hline Cs2CO3 & 6 & 0.2400932 & 0.1931940 & 0.1931940 \\
\hline $\mathrm{Na} 2 \mathrm{O} 2$ & 4 & 0.0871953 & 0.0643980 & 0.0482985 \\
\hline Test & F Ratio & DFNum & DFDen & Prob $>$ F \\
\hline O'Brien[.5] & 1.8290 & 1 & 8 & 0.2132 \\
\hline $\begin{array}{l}\text { Brown- } \\
\text { Forsythe }\end{array}$ & 4.5741 & 1 & 8 & 0.0649 \\
\hline Levene & 4.5176 & 1 & 8 & 0.0663 \\
\hline Bartlett & 2.5759 & 1 & . & 0.1085 \\
\hline F Test 2-sided & 7.5818 & 5 & 3 & 0.1261 \\
\hline
\end{tabular}

Warning: Small sample sizes. Use Caution.

Welch Anova testing Means Equal, allowing Std Devs Not Equal

F Ratio DFNum DFDen Prob $>$ F

$\begin{array}{llll}0.0901 & 1 & 6.7348 & 0.7731\end{array}$

t Test

0.3002

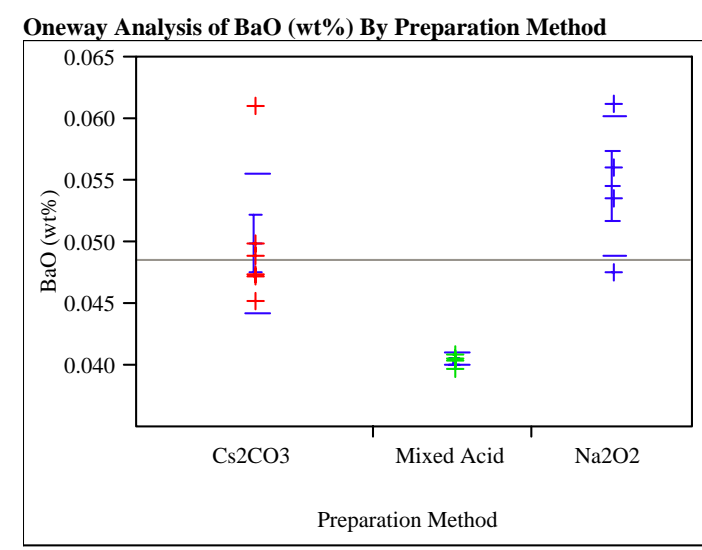

Means Comparisons

Comparisons for all pairs using Tukey-Kramer HSD $\begin{array}{rr}q^{*} & \text { Alpha } \\ 2.70081 & 0.05\end{array}$

Abs(Dif)-LSD Na2O2 Cs2CO3 Mixed Acid

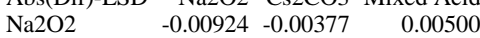

$\begin{array}{llll}\mathrm{Cs} 2 \mathrm{CO} 3 & -0.00377 & -0.00754 & 0.00114\end{array}$

Mixed Acid $\quad 0.00500 \quad 0.00114 \quad-0.00924$

Positive values show pairs of means that are significantly different.

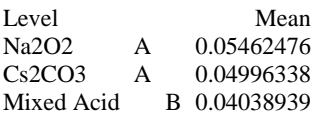

Levels not connected by same letter are significantly different Tests that the Variances are Equal

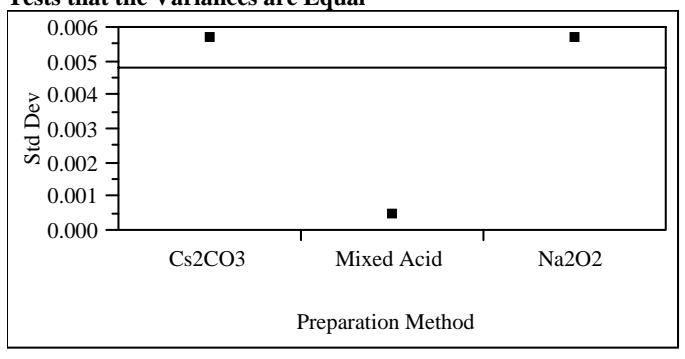

\begin{tabular}{|c|c|c|c|c|}
\hline Level & Count & Std Dev & $\begin{array}{r}\text { MeanAbsDif to } \\
\text { Mean }\end{array}$ & $\begin{array}{r}\text { MeanAbsDif to } \\
\text { Median }\end{array}$ \\
\hline Cs2CO3 & 6 & 0.0056635 & 0.0037031 & 0.0033309 \\
\hline Mixed Acid & 4 & 0.0004682 & 0.0003210 & 0.0003070 \\
\hline $\mathrm{Na} 2 \mathrm{O} 2$ & 4 & 0.0056663 & 0.0040473 & 0.0040473 \\
\hline Test & F Ratio & DFNum & DFDen & Prob $>$ F \\
\hline O'Brien[.5] & 0.5999 & 2 & 11 & 0.5659 \\
\hline $\begin{array}{l}\text { Brown- } \\
\text { Forsythe }\end{array}$ & 1.2299 & 2 & 11 & 0.3296 \\
\hline Levene & 1.7980 & 2 & 11 & 0.2110 \\
\hline Bartlett & 5.0846 & 2 & & 0.0062 \\
\hline
\end{tabular}

Warning: Small sample sizes. Use Caution.

Welch Anova testing Means Equal, allowing Std Devs Not Equal

F Ratio DFNum DFDen Prob $>$ F

$\begin{array}{rrrr}18.4347 & 2 & 5.0798 & 0.0047\end{array}$ 
Westinghouse Savannah River Company

WSRC-TR-2005-00396

Savannah River National Laboratory

Rev. 0

Analytical Development/Statistical Consulting Sections

Page 120 of 140

Exhibit B4. Chemical Composition Measurements by Type of Prepared Sample by Oxide for DWPF Radioactive Glass

(Concentrations are in wt\%.)

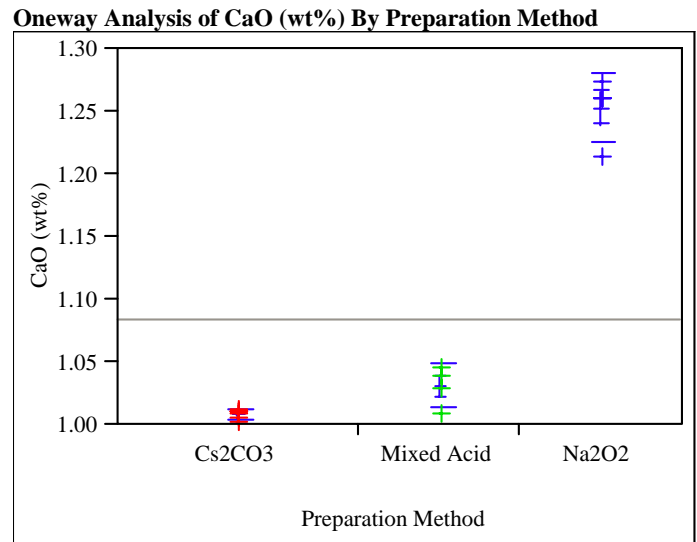

Means Comparisons

Comparisons for all pairs using Tukey-Kramer HSD

$$
\text { q* Alpha }
$$

Abs(Dif)-LSD Na2O2 Mixed Acid Cs2CO3

$\begin{array}{llll}\mathrm{Na} 2 \mathrm{O} 2 & -0.03134 & 0.19008 & 0.21543\end{array}$

$\begin{array}{llll}\text { Mixed Acid } \quad 0.19008 & -0.03134 & -0.00599\end{array}$

$\begin{array}{llll}\mathrm{Cs} 2 \mathrm{CO} 3 & 0.21543 \quad-0.00599 & -0.02559\end{array}$

Positive values show pairs of means that are significantly different.

Level Mean

$\mathrm{Na} 2 \mathrm{O} 2 \quad$ A 1.2526338

Mixed Acid B 1.0312104

Cs2CO3 B 1.0085900

Levels not connected by same letter are significantly different

Tests that the Variances are Equal

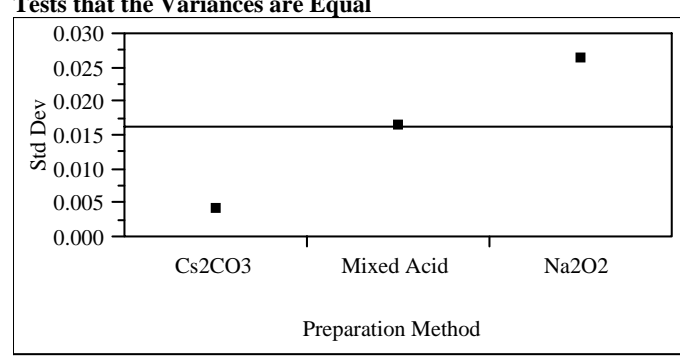

\begin{tabular}{|c|c|c|c|c|}
\hline Level & Count & Std Dev & $\begin{array}{r}\text { MeanAbsDif to } \\
\text { Mean }\end{array}$ & $\begin{array}{r}\text { MeanAbsDif to } \\
\text { Median }\end{array}$ \\
\hline Cs2CO3 & 6 & 0.0040953 & 0.0031093 & 0.0030316 \\
\hline Mixed Acid & 4 & 0.0164369 & 0.0118932 & 0.0118932 \\
\hline $\mathrm{Na} 2 \mathrm{O} 2$ & 4 & 0.0262606 & 0.0190641 & 0.0150414 \\
\hline Test & F Ratio & DFNum & DFDen & Prob $>$ F \\
\hline O'Brien[.5] & 1.4985 & 2 & 11 & 0.2657 \\
\hline $\begin{array}{l}\text { Brown- } \\
\text { Forsythe }\end{array}$ & 1.2355 & 2 & 11 & 0.3281 \\
\hline Levene & 3.8962 & 2 & 11 & 0.0526 \\
\hline Bartlett & 4.8940 & 2 & . & 0.0075 \\
\hline
\end{tabular}

Warning: Small sample sizes. Use Caution.

Welch Anova testing Means Equal, allowing Std Devs Not Equal

F Ratio DFNum DFDen Prob > F

$\begin{array}{rrrr}148.9471 & 2 & 4.2225 & 0.0001\end{array}$

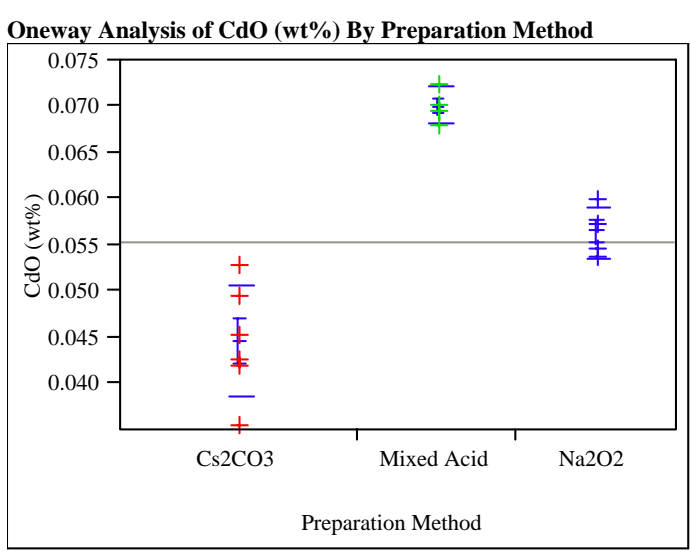

$$
\text { Means Comparisons }
$$

Comparisons for all pairs using Tukey-Kramer HSD

$$
\begin{array}{rr}
q^{*} & \text { Alpha } \\
2.70081 & 0.05
\end{array}
$$

Abs(Dif)-LSD Mixed Acid Na2O2 Cs2CO3

$\begin{array}{llll}\text { Mixed Acid } & -0.00848 & 0.00514 & 0.01767\end{array}$

$\begin{array}{llll}\mathrm{Na} 2 \mathrm{O} 2 & 0.00514 & -0.00848 & 0.00404\end{array}$

$\begin{array}{llll}\text { Cs2CO3 } & 0.01767 & 0.00404 & -0.00692\end{array}$

Positive values show pairs of means that are significantly different.

$\begin{array}{lrrr}\text { Level } & & \text { Mean } \\ \text { Mixed Acid A } & & 0.07005155 \\ \text { Na2O2 } & \text { B } & 0.05642962 \\ \text { Cs2CO3 } & & \text { C } & 0.04464489\end{array}$

Levels not connected by same letter are significantly different

\begin{tabular}{|c|c|c|c|c|}
\hline Level & Count & Std Dev & $\begin{array}{r}\text { MeanAbsDif to } \\
\text { Mean }\end{array}$ & $\begin{array}{r}\text { MeanAbsDif to } \\
\text { Median }\end{array}$ \\
\hline Cs2CO3 & 6 & 0.0060589 & 0.0045502 & 0.0045502 \\
\hline Mixed Acid & 4 & 0.0018475 & 0.0012851 & 0.0012851 \\
\hline $\mathrm{Na} 2 \mathrm{O} 2$ & 4 & 0.0027699 & 0.0021704 & 0.0021704 \\
\hline Test & F Ratio & DFNum & DFDen & Prob $>F$ \\
\hline O'Brien[.5] & 1.6021 & 2 & 11 & 0.2451 \\
\hline $\begin{array}{l}\text { Brown- } \\
\text { Forsythe }\end{array}$ & 2.2311 & 2 & 11 & 0.1537 \\
\hline Levene & 2.3659 & 2 & 11 & 0.1398 \\
\hline Bartlett & 2.2054 & 2 & . & 0.1102 \\
\hline
\end{tabular}
Tests that the Variances are Equal

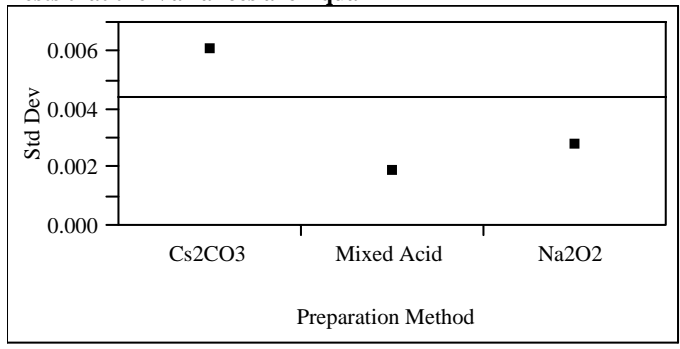

Warning: Small sample sizes. Use Caution.

Welch Anova testing Means Equal, allowing Std Devs Not Equal

F Ratio DFNum DFDen Prob $>$ F

$\begin{array}{rrrr}61.1406 & 2 & 6.9426 & <.0001\end{array}$ 
Westinghouse Savannah River Company

WSRC-TR-2005-00396

Savannah River National Laboratory

Rev. 0

Analytical Development/Statistical Consulting Sections

Page 121 of 140

Exhibit B4. Chemical Composition Measurements by Type of Prepared Sample by Oxide for DWPF Radioactive Glass

(Concentrations are in wt\%.)

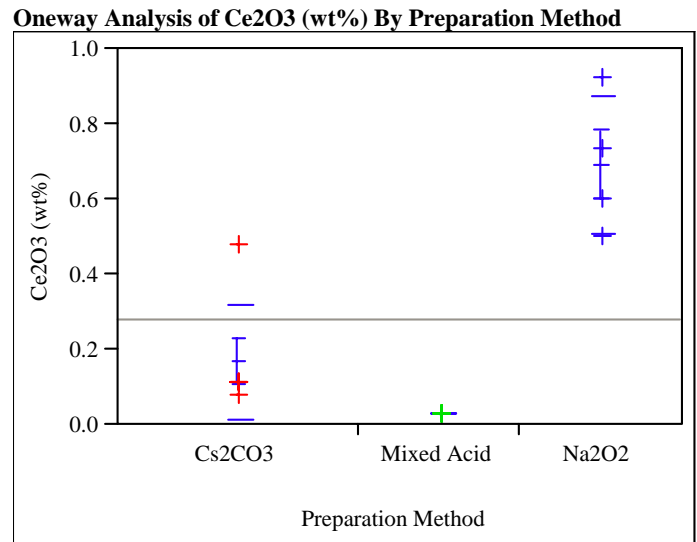

Means Comparisons

Comparisons for all pairs using Tukey-Kramer HSD

$$
\begin{array}{rr}
\text { q* Alpha } \\
70081 & 0.05
\end{array}
$$

Abs(Dif)-LSD Na2O2 Cs2CO3 Mixed Acid

$\begin{array}{llll}\mathrm{Na} 2 \mathrm{O} 2 & -0.26764 & 0.27876 & 0.39297\end{array}$

$\begin{array}{llll}\mathrm{Cs} 2 \mathrm{CO} 3 & 0.27876 & -0.21853 & -0.10679\end{array}$

Mixed Acid $\quad 0.39297 \quad-0.10679 \quad-0.26764$

Positive values show pairs of means that are significantly different.

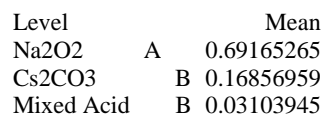

Levels not connected by same letter are significantly different

Tests that the Variances are Equal

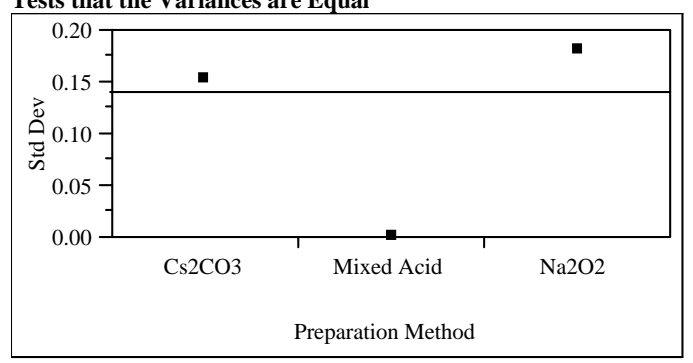

$\begin{array}{lrrrr}\text { Level } & \text { Count } & \text { Std Dev } & \begin{array}{r}\text { MeanAbsDif to } \\ \text { Mean }\end{array} & \begin{array}{r}\text { MeanAbsDif to } \\ \text { Median }\end{array} \\ \text { Cs2CO3 } & 6 & 0.1527594 & 0.1034974 & 0.0676426 \\ \text { Mixed Acid } & 4 & 0.0008856 & 0.0006442 & 0.0006442 \\ \text { Na2O2 } & 4 & 0.1819918 & 0.1382134 & 0.1382134 \\ \text { Test } & \text { F Ratio } & \text { DFNum } & \text { DFDen } & \text { Prob > F } \\ \text { O'Brien[.5] } & 0.7189 & 2 & 11 & 0.5088 \\ \text { Brown- } & 1.5694 & 2 & 11 & 0.2514 \\ \text { Forsythe } & & & & \\ \text { Levene } & 3.0529 & 2 & 11 & 0.0882 \\ \text { Bartlett } & 12.3773 & 2 & . & <.0001 \\ \text { Warn } & & 2 & & \end{array}$

Warning: Small sample sizes. Use Caution.

Welch Anova testing Means Equal, allowing Std Devs Not Equal

F Ratio DFNum DFDen Prob $>$ F

$\begin{array}{llll}25.3968 & 2 & 5.0003 & 0.0024\end{array}$

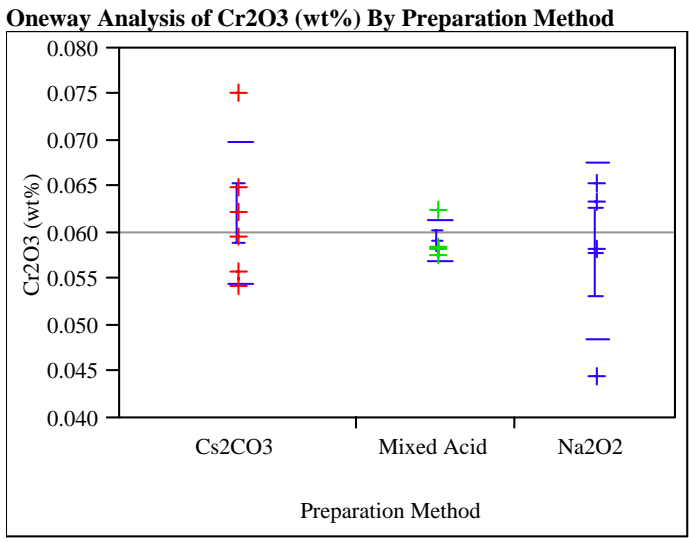

Means Comparisons

Comparisons for all pairs using Tukey-Kramer HSD

$$
\begin{array}{rr}
\mathrm{q}^{*} & \text { Alpha } \\
2.70081 & 0.05
\end{array}
$$

Abs(Dif)-LSD Cs2CO3 Mixed Acid Na2O2

$\begin{array}{llll}\text { Cs2CO3 } & -0.01120 & -0.00973 & -0.00838\end{array}$

Mixed Acid $\quad-0.00973 \quad-0.01372-0.01237$

$\begin{array}{llll}\mathrm{Na} 2 \mathrm{O} 2 & -0.00838 & -0.01237 & -0.01372\end{array}$

Positive values show pairs of means that are significantly different.

Level Mean

Cs2CO3 A 0.06209364

Mixed Acid A 0.05930442

$\mathrm{Na2O}$ A 0.05795244

Levels not connected by same letter are significantly different Tests that the Variances are Equal

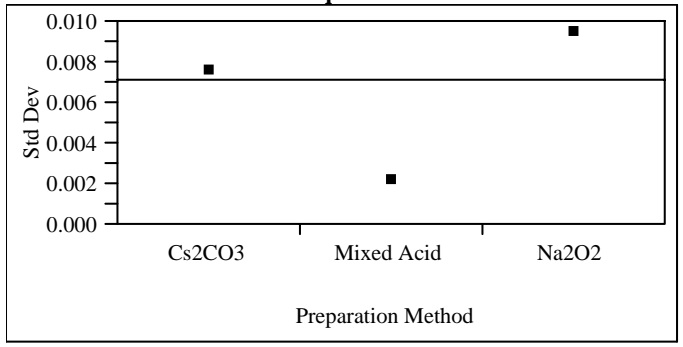

$\begin{array}{lrrrr}\text { Level } & \text { Count } & \text { Std Dev } & \begin{array}{r}\text { MeanAbsDif to } \\ \text { Mean }\end{array} & \begin{array}{r}\text { MeanAbsDif to } \\ \text { Median }\end{array} \\ \text { Cs2CO3 } & 6 & 0.0075809 & 0.0054810 & 0.0054810 \\ \text { Mixed Acid } & 4 & 0.0021985 & 0.0016260 & 0.0012789 \\ \text { Na2O2 } & 4 & 0.0094108 & 0.0066868 & 0.0065041 \\ \text { Test } & \text { F Ratio } & \text { DFNum } & \text { DFDen } & \text { Prob > F } \\ \text { O'Brien[.5] } & 0.8732 & 2 & 11 & 0.4447 \\ \text { Brown- } & 1.3623 & 2 & 11 & 0.2961 \\ \text { Forsythe } & & & & \\ \text { Levene } & 1.5911 & 2 & 11 & 0.2472 \\ \text { Bartlett } & 2.1888 & 2 & . & 0.1121\end{array}$

Warning: Small sample sizes. Use Caution.

Welch Anova testing Means Equal, allowing Std Devs Not Equal

F Ratio DFNum DFDen Prob $>$ F

$\begin{array}{llll}0.3761 & 2 & 5.6667 & 0.7025\end{array}$ 
Westinghouse Savannah River Company

WSRC-TR-2005-00396

Savannah River National Laboratory

Rev. 0

Analytical Development/Statistical Consulting Sections

Page 122 of 140

Exhibit B4. Chemical Composition Measurements by Type of Prepared Sample by Oxide for DWPF Radioactive Glass

(Concentrations are in wt\%.)

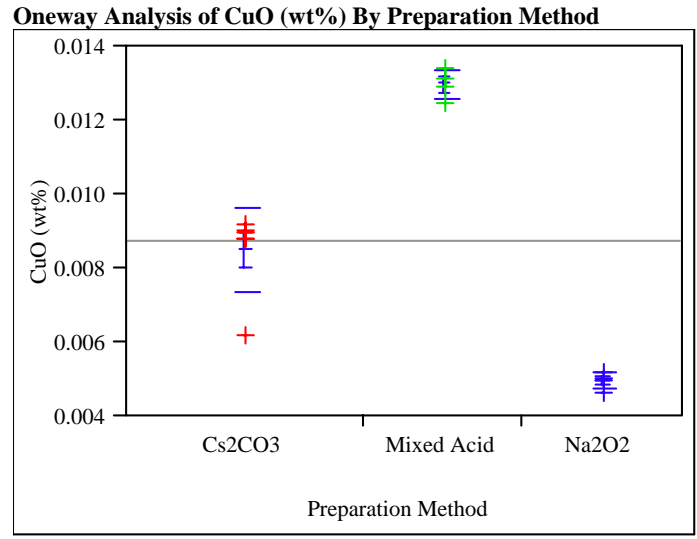

Means Comparisons

Comparisons for all pairs using Tukey-Kramer HSD

$$
\begin{array}{rr}
\mathrm{q}^{*} & \text { Alpha } \\
2.70081 & 0.05
\end{array}
$$

Abs(Dif)-LSD Mixed Acid Cs2CO3 Na2O2

$\begin{array}{llll}\text { Mixed Acid } & -0.00154 & 0.00307 & 0.00648\end{array}$

$\begin{array}{llll}\mathrm{Cs} 2 \mathrm{CO} 3 & 0.00307 & -0.00126 & 0.00214\end{array}$

$\begin{array}{llll}\mathrm{Na} 2 \mathrm{O} 2 & 0.00648 & 0.00214 & -0.00154\end{array}$

Positive values show pairs of means that are significantly different.

$\begin{array}{lrrr}\text { Level } & & & \text { Mean } \\ \text { Mixed Acid A } & & 0.01297178 \\ \text { Cs2CO3 } & \text { B } & 0.00850077 \\ \text { Na2O2 } & & \text { C } & 0.00495556\end{array}$

$\mathrm{Na} 2 \mathrm{O} 2 \quad \mathrm{C} \quad 0.00495556$

Levels not connected by same letter are significantly different Tests that the Variances are Equal

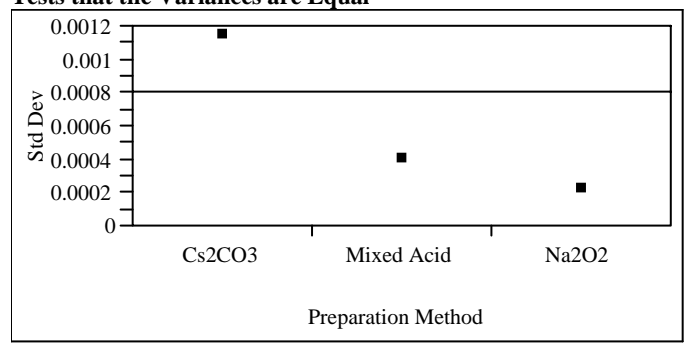

$\begin{array}{lrrrr}\text { Level } & \text { Count } & \text { Std Dev } & \begin{array}{r}\text { MeanAbsDif to } \\ \text { Mean }\end{array} & \begin{array}{r}\text { MeanAbsDif to } \\ \text { Median }\end{array} \\ \text { Cs2CO3 } & 6 & 0.0011405 & 0.0007702 & 0.0005539 \\ \text { Mixed Acid } & 4 & 0.0004004 & 0.0002973 & 0.0002973 \\ \text { Na2O2 } & 4 & 0.0002258 & 0.0001588 & 0.0001424 \\ \text { Test } & \text { F Ratio } & \text { DFNum } & \text { DFDen } & \text { Prob > F } \\ \text { O'Brien[.5] } & 0.6780 & 2 & 11 & 0.5277 \\ \text { Brown- } & 0.4098 & 2 & 11 & 0.6735 \\ \text { Forsythe } & & & & \\ \text { Levene } & 1.8417 & 2 & 11 & 0.2042 \\ \text { Bartlett } & 3.6969 & 2 & . & 0.0248\end{array}$

Warning: Small sample sizes. Use Caution.

Welch Anova testing Means Equal, allowing Std Devs Not Equal

F Ratio DFNum DFDen Prob $>$ F

$\begin{array}{llll}557.5837 & 2 & 6.5779 & <.0001\end{array}$

Oneway Analysis of Fe2O3 (wt\%) By Preparation Method

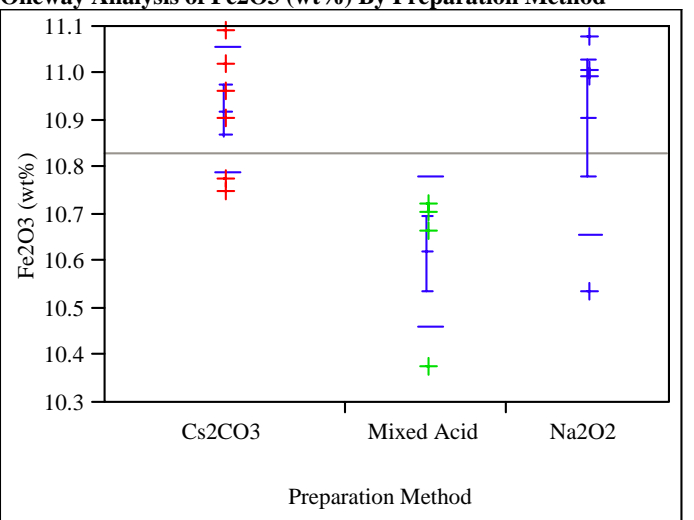

Means Comparisons

Comparisons for all pairs using Tukey-Kramer HSD $q^{*} \quad$ Alpha

Abs(Dif)-LSD Cs2CO3 Na2O2 Mixed Acid

Cs2CO3 $\quad-0.27993 \quad-0.29748 \quad-0.01154$

$\begin{array}{llll}\mathrm{Na} 2 \mathrm{O} 2 & -0.29748 & -0.34284 & -0.05690\end{array}$

Mixed Acid $\quad-0.01154 \quad-0.05690 \quad-0.34284$

Positive values show pairs of means that are significantly different.

Level Mean

Cs2CO3 A 10.920525

$\mathrm{Na} 2 \mathrm{O} 2 \quad$ A 10.905037

Mixed Acid A 10.619097

Levels not connected by same letter are significantly different Tests that the Variances are Equal

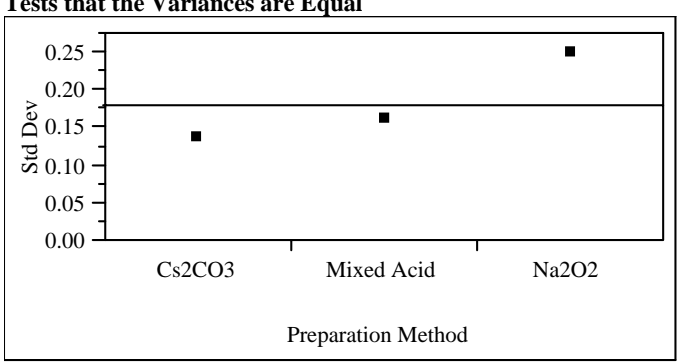

\begin{tabular}{|c|c|c|c|c|}
\hline Level & Count & Std Dev & $\begin{array}{r}\text { MeanAbsDif to } \\
\text { Mean }\end{array}$ & $\begin{array}{r}\text { MeanAbsDif to } \\
\text { Median }\end{array}$ \\
\hline Cs2CO3 & 6 & 0.1351552 & 0.1072275 & 0.1072275 \\
\hline Mixed Acid & 4 & 0.1614886 & 0.1197374 & 0.0965048 \\
\hline $\mathrm{Na} 2 \mathrm{O} 2$ & 4 & 0.2482839 & 0.1840739 & 0.1393957 \\
\hline Test & F Ratio & DFNum & DFDen & Prob > \\
\hline O'Brien[.5] & 0.7037 & 2 & 11 & 0.51 \\
\hline $\begin{array}{l}\text { Brown- } \\
\text { Forsythe }\end{array}$ & 0.0983 & 2 & 11 & 0.907 \\
\hline Levene & 0.8978 & 2 & 11 & 0.4353 \\
\hline Bartlett & 0.6766 & 2 & . & 0.5083 \\
\hline
\end{tabular}

Warning: Small sample sizes. Use Caution.

Welch Anova testing Means Equal, allowing Std Devs Not Equal

F Ratio DFNum DFDen Prob $>$ F

$\begin{array}{llll}4.4228 & 2 & 5.7555 & 0.0686\end{array}$ 
Westinghouse Savannah River Company

WSRC-TR-2005-00396

Savannah River National Laboratory

Rev. 0

Analytical Development/Statistical Consulting Sections

Page 123 of 140

Exhibit B4. Chemical Composition Measurements by Type of Prepared Sample by Oxide for DWPF Radioactive Glass

(Concentrations are in wt\%.)

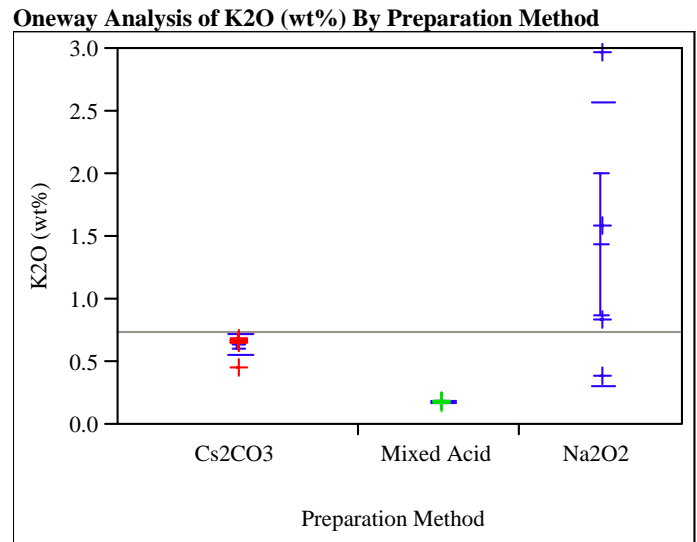

Means Comparisons

Comparisons for all pairs using Tukey-Kramer HSD q* Alpha

$2.70081 \quad 0.05$

Abs(Dif)-LSD Na2O2 Cs2CO3 Mixed Acid

$\mathrm{Na} 2 \mathrm{O} 2 \quad-1.1356 \quad-0.2271 \quad 0.1271$

$\begin{array}{llll}\mathrm{C} 2 \mathrm{CO} 3 & -0.2271 & -0.9272 & -0.5835\end{array}$

Mixed Acid $\quad 0.1271 \quad-0.5835 \quad-1.1356$

Positive values show pairs of means that are significantly different.

Level Mean

$\mathrm{Na} 2 \mathrm{O} 2 \quad$ A 1.4470258

Cs2CO3 A B 0.6374342

Mixed Acid B 0.1843038

Levels not connected by same letter are significantly different

Tests that the Variances are Equal

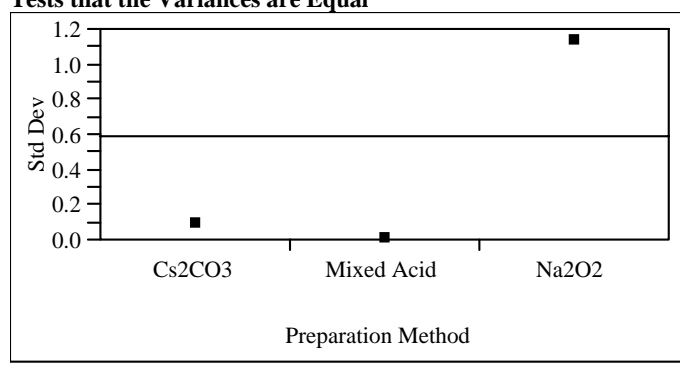

$\begin{array}{lrrrr}\text { Level } & \text { Count } & \text { Std Dev } & \begin{array}{r}\text { MeanAbsDif to } \\ \text { Mean }\end{array} & \begin{array}{r}\text { MeanAbsif to } \\ \text { Median }\end{array} \\ \text { Cs2CO3 } & 6 & 0.085553 & 0.0578877 & 0.0411572 \\ \text { Mixed Acid } & 4 & 0.005274 & 0.0039150 & 0.0039150 \\ \text { Na2O2 } & 4 & 1.133269 & 0.8356913 & 0.8356913 \\ \text { Test } & \text { F Ratio } & \text { DFNum } & \text { DFDen } & \text { Prob }>\text { F } \\ \text { O'Brien[.5] } & 2.9471 & 2 & 11 & 0.0944 \\ \text { Brown- } & 7.8625 & 2 & 11 & 0.0076 \\ \text { Forsythe } & & & & \\ \text { Levene } & 9.3693 & 2 & 11 & 0.0042 \\ \text { Bartlett } & 19.4237 & 2 & . & <.0001\end{array}$

Warning: Small sample sizes. Use Caution.

Welch Anova testing Means Equal, allowing Std Devs Not Equal

F Ratio DFNum DFDen Prob $>$ F

$\begin{array}{llll}76.0556 & 2 & 5.0213 & 0.0002\end{array}$

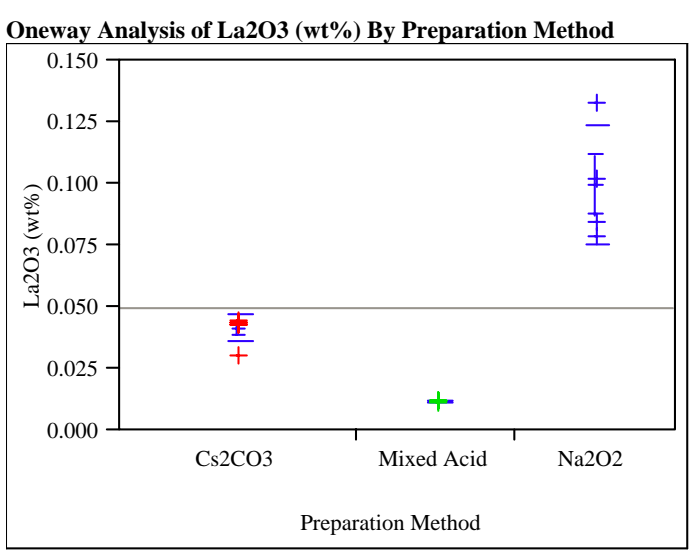

Means Comparisons

Comparisons for all pairs using Tukey-Kramer HSD

$\begin{array}{rr}q^{*} & \text { Alpha } \\ 2.70081 & 0.05\end{array}$

Abs(Dif)-LSD Na2O2 Cs2CO3 Mixed Acid

$\begin{array}{llll}\mathrm{Na} 2 \mathrm{O} 2 & -0.02513 & 0.03505 & 0.06229\end{array}$

$\begin{array}{llll}\mathrm{Cs} 2 \mathrm{CO} 3 & 0.03505 & -0.02052 & 0.00650\end{array}$

$\begin{array}{llll}\text { Mixed Acid } \quad 0.06229 & 0.00650 & -0.02513\end{array}$

Positive values show pairs of means that are significantly different.

$\begin{array}{lrrr}\text { Level } & & & \text { Mean } \\ \text { Na2O2 } & \text { A } & & 0.09939480 \\ \text { Cs2CO3 } & \text { B } & 0.04140961 \\ \text { Mixed Acid } & & \text { C } & 0.01197722\end{array}$

Levels not connected by same letter are significantly different Tests that the Variances are Equal

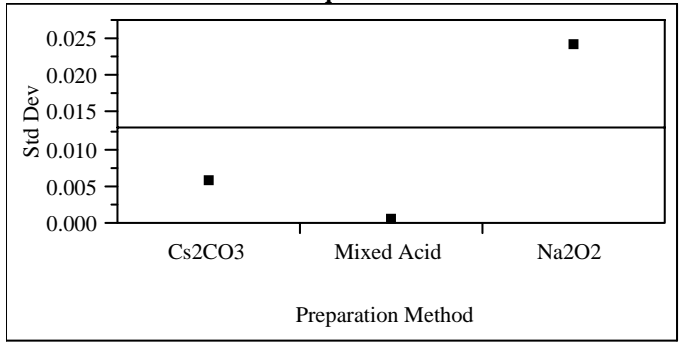

\begin{tabular}{|c|c|c|c|c|}
\hline Level & Count & Std Dev & $\begin{array}{r}\text { MeanAbsDif to } \\
\text { Mean }\end{array}$ & $\begin{array}{r}\text { MeanAbsDif to } \\
\text { Median }\end{array}$ \\
\hline Cs2CO3 & 6 & 0.0055540 & 0.0037562 & 0.0026681 \\
\hline Mixed Acid & 4 & 0.0003465 & 0.0002492 & 0.0002492 \\
\hline $\mathrm{Na} 2 \mathrm{O} 2$ & 4 & 0.0241488 & 0.0177093 & 0.0177093 \\
\hline Test & F Ratio & DFNum & DFDen & Prob $>$ F \\
\hline O'Brien[.5] & 2.6541 & 2 & 11 & 0.1147 \\
\hline $\begin{array}{l}\text { Brown- } \\
\text { Forsythe }\end{array}$ & 5.2537 & 2 & 11 & 0.0250 \\
\hline Levene & 6.8493 & 2 & 11 & 0.0117 \\
\hline Bartlett & 11.8663 & 2 & . & $<.0001$ \\
\hline
\end{tabular}

Warning: Small sample sizes. Use Caution.

Welch Anova testing Means Equal, allowing Std Devs Not Equal

F Ratio DFNum DFDen Prob $>$ F

$\begin{array}{llrr}96.9879 & 2 & 5.023 & <.0001\end{array}$ 
Exhibit B4. Chemical Composition Measurements by Type of Prepared Sample by Oxide for DWPF Radioactive Glass

(Concentrations are in wt\%.)

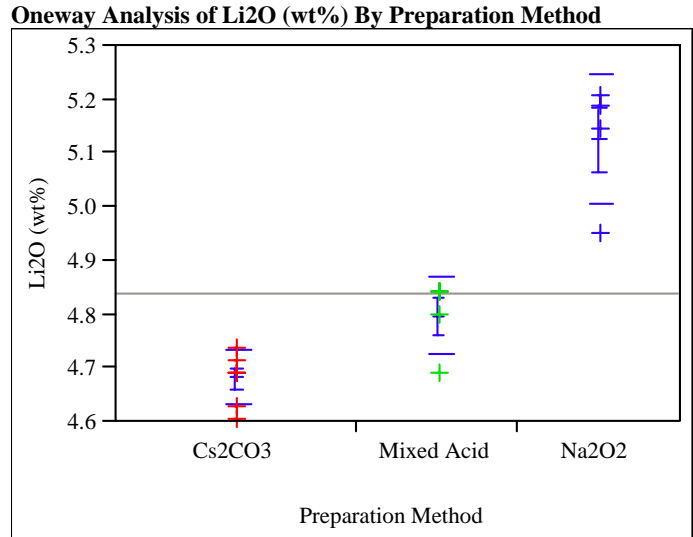

Means Comparisons

Comparisons for all pairs using Tukey-Kramer HSD

$$
\begin{array}{rr}
\text { q* } & \text { Alpha } \\
2.70081 & 0.05
\end{array}
$$

Abs(Dif)-LSD Na2O2 Mixed Acid Cs2CO3

$\begin{array}{llrr}\mathrm{Na} 2 \mathrm{O} 2 & -0.15187 & 0.17645 & 0.30629\end{array}$

$\begin{array}{llll}\text { Mixed Acid } \quad 0.17645 \quad-0.15187 & -0.02202\end{array}$

$\begin{array}{llll}\mathrm{Cs} 2 \mathrm{CO} 3 & 0.30629 & -0.02202 & -0.12400\end{array}$

Positive values show pairs of means that are significantly different.

$\begin{array}{lrr}\text { Level } & & \text { Mean } \\ \text { Na2O2 } & \text { A } & 5.1239020 \\ \text { Mixed Acid } & \text { B } & 4.7955847 \\ \text { Cs2CO3 } & \text { B } & 4.6789693\end{array}$

Levels not connected by same letter are significantly different Tests that the Variances are Equal

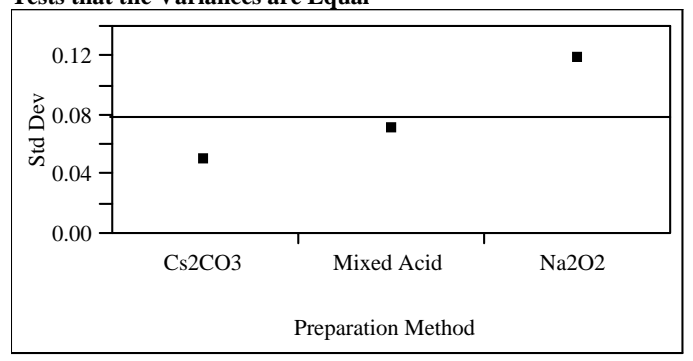

\begin{tabular}{|c|c|c|c|c|}
\hline Level & Count & Std Dev & $\begin{array}{r}\text { MeanAbsDif to } \\
\text { Mean }\end{array}$ & $\begin{array}{r}\text { MeanAbsDif to } \\
\text { Median }\end{array}$ \\
\hline Cs2CO3 & 6 & 0.0503367 & 0.0406659 & 0.0358817 \\
\hline Mixed Acid & 4 & 0.0711326 & 0.0511314 & 0.0484402 \\
\hline $\mathrm{Na} 2 \mathrm{O} 2$ & 4 & 0.1179192 & 0.0861160 & 0.0753515 \\
\hline Test & F Ratio & DFNum & DFDen & Prob $>$ F \\
\hline O'Brien[.5] & 1.0759 & 2 & 11 & 0.3743 \\
\hline $\begin{array}{l}\text { Brown- } \\
\text { Forsythe }\end{array}$ & 0.5020 & 2 & 11 & 0.6185 \\
\hline Levene & 1.4448 & 2 & 11 & 0.277 \\
\hline Bartlett & 1.2744 & 2 & . & 0.2796 \\
\hline
\end{tabular}

Warning: Small sample sizes. Use Caution.

Welch Anova testing Means Equal, allowing Std Devs Not Equal

F Ratio DFNum DFDen Prob $>$ F

$\begin{array}{llll}23.8492 & 2 & 5.3598 & 0.0021\end{array}$

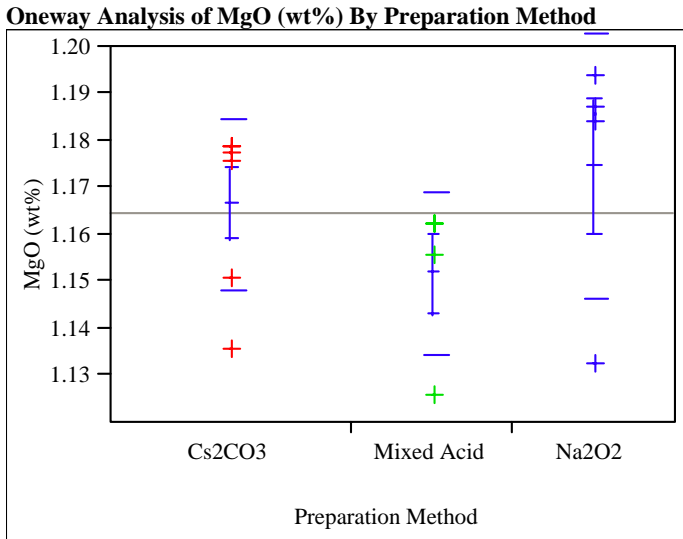

Means Comparisons

Comparisons for all pairs using Tukey-Kramer HSD $\mathrm{q}^{*} \quad$ Alpha

$2.70081 \quad 0.05$

Abs(Dif)-LSD Na2O2 Cs2CO3 Mixed Acid

$\mathrm{Na2O} 2 \quad-0.04070-0.02900 \quad-0.01790$

$\begin{array}{llll}\text { Cs2CO3 } & -0.02900 & -0.03323 & -0.02251\end{array}$

$\begin{array}{lll}\text { Mixed Acid } \quad-0.01790 & -0.02251 \quad-0.04070\end{array}$

Positive values show pairs of means that are significantly different.

Level

1.1744910

Mixed Acid A 1.1516893

Levels not connected by same letter are significantly different Tests that the Variances are Equal

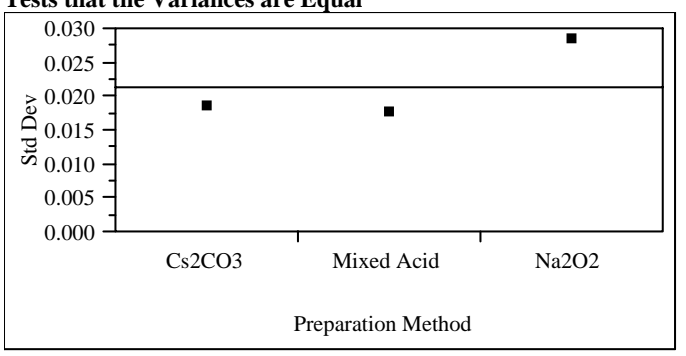

$\begin{array}{lrrrr}\text { Level } & \text { Count } & \text { Std Dev } & \begin{array}{r}\text { MeanAbsDif to } \\ \text { Mean }\end{array} & \begin{array}{r}\text { MeanAbsDif to } \\ \text { Median }\end{array} \\ \text { Cs2CO3 } & 6 & 0.0184263 & 0.0152932 & 0.0121609 \\ \text { Mixed Acid } & 4 & 0.0174187 & 0.0128518 & 0.0107790 \\ \text { Na2O2 } & 4 & 0.0282195 & 0.0209360 & 0.0161684 \\ \text { Test } & \text { F Ratio } & \text { DFNum } & \text { DFDen } & \text { Prob > F } \\ \text { O'Brien[.5] } & 0.5227 & 2 & 11 & 0.6070 \\ \text { Brown- } & 0.0891 & 2 & 11 & 0.9154 \\ \text { Forsythe } & & & & \\ \text { Levene } & 0.6504 & 2 & 11 & 0.5408 \\ \text { Bartlett } & 0.4349 & 2 & . & 0.6473\end{array}$

Warning: Small sample sizes. Use Caution.

Welch Anova testing Means Equal, allowing Std Devs Not Equal

F Ratio DFNum DFDen Prob $>$ F

$\begin{array}{llll}1.1362 & 2 & 6.1813 & 0.3800\end{array}$ 
Westinghouse Savannah River Company

WSRC-TR-2005-00396

Savannah River National Laboratory

Rev. 0

Analytical Development/Statistical Consulting Sections

Page 125 of 140

Exhibit B4. Chemical Composition Measurements by Type of Prepared Sample by Oxide for DWPF Radioactive Glass

(Concentrations are in wt\%.)
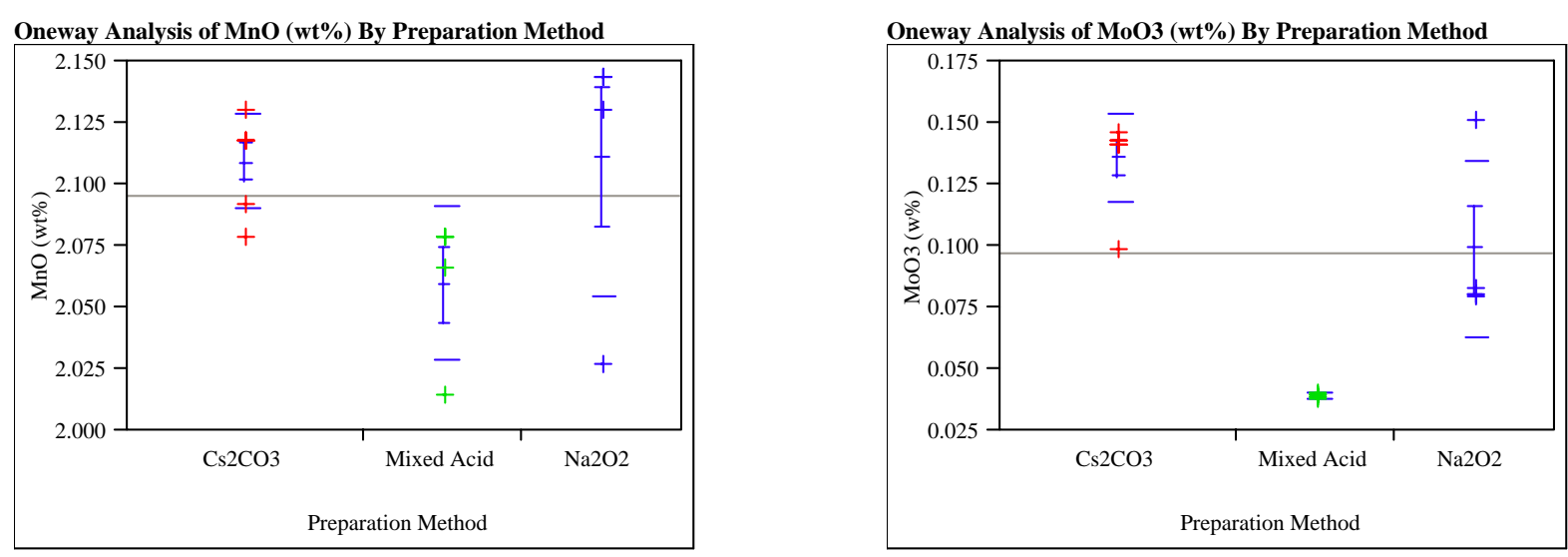

Means Comparisons

Comparisons for all pairs using Tukey-Kramer HSD

$$
\begin{array}{rr}
q^{*} & \text { Alpha } \\
2.70081 & 0.05
\end{array}
$$

Abs(Dif)-LSD Na2O2 Cs2CO3 Mixed Acid

$\mathrm{Na} 2 \mathrm{O} 2 \quad-0.06868-0.06054 \quad-0.01703$

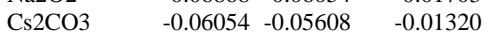

Mixed Acid $\quad-0.01703 \quad-0.01320 \quad-0.06868$

Positive values show pairs of means that are significantly different.

Level Mean

$\mathrm{Na} 2 \mathrm{O} 2$ A 2.1111120

Cs2CO3 A 2.1089600

Mixed Acid A 2.0594640

Levels not connected by same letter are significantly different Tests that the Variances are Equal

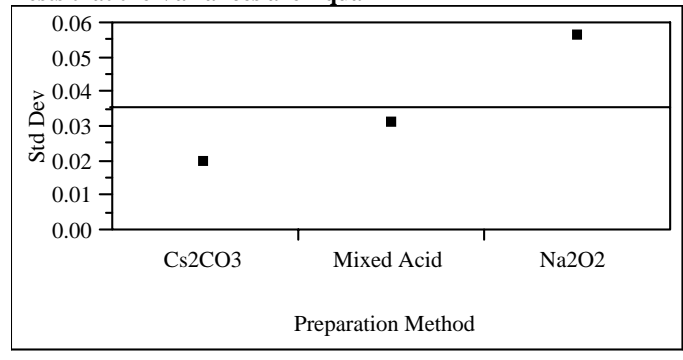

\begin{tabular}{|c|c|c|c|c|}
\hline Level & Count & Std Dev & $\begin{array}{r}\text { MeanAbsDif to } \\
\text { Mean }\end{array}$ & $\begin{array}{r}\text { MeanAbsDif to } \\
\text { Median }\end{array}$ \\
\hline Cs2CO3 & 6 & 0.0194396 & 0.0157813 & 0.0129120 \\
\hline Mixed Acid & 4 & 0.0307367 & 0.0225960 & 0.0193680 \\
\hline $\mathrm{Na} 2 \mathrm{O} 2$ & 4 & 0.0562821 & 0.0419640 & 0.0322800 \\
\hline Test & F Ratio & DFNum & DFDen & Prob $>$ F \\
\hline O'Brien[.5] & 1.2031 & 2 & 11 & 0.3369 \\
\hline $\begin{array}{l}\text { Brown- } \\
\text { Forsythe }\end{array}$ & 0.4391 & 2 & 11 & 0.6555 \\
\hline Levene & 2.5394 & 2 & 11 & 0.1240 \\
\hline Bartlett & 1.9511 & 2 & . & 0.1421 \\
\hline
\end{tabular}

Warning: Small sample sizes. Use Caution.

Welch Anova testing Means Equal, allowing Std Devs Not Equal

F Ratio DFNum DFDen Prob $>$ F

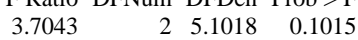

Means Comparisons

Comparisons for all pairs using Tukey-Kramer HSD $\begin{array}{rr}q^{*} & \text { Alpha } \\ 2.70081 & 0.05\end{array}$

Abs(Dif)-LSD Cs2CO3 Na2O2 Mixed Acid

$\begin{array}{llll}\mathrm{Cs} 2 \mathrm{CO} 3 & -0.03453 & -0.00141 & 0.05785\end{array}$

$\mathrm{Na2O} 2 \quad-0.00141-0.04229 \quad 0.01698$

$\begin{array}{llll}\text { Mixed Acid } & 0.05785 & 0.01698 & -0.04229\end{array}$

Positive values show pairs of means that are significantly different.

Level

$\mathrm{Cs} 2 \mathrm{CO} 3 \mathrm{~A}-0.13565213$

Cs2CO3 A 0.13565213

$\mathrm{Na} 2 \mathrm{O} 2$ A 0.09845719

Mixed Acid ～B 0.03919534

Levels not connected by same letter are significantly different Tests that the Variances are Equal

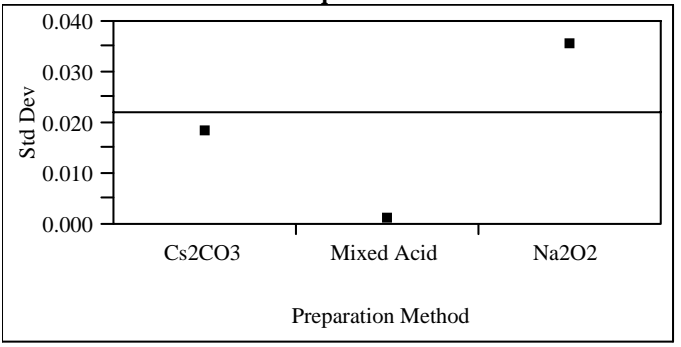

\begin{tabular}{|c|c|c|c|c|}
\hline Level & Count & Std Dev & $\begin{array}{r}\text { MeanAbsDif to } \\
\text { Mean }\end{array}$ & $\begin{array}{r}\text { MeanAbsDif to } \\
\text { Median }\end{array}$ \\
\hline Cs2CO3 & 6 & 0.0180499 & 0.0122108 & 0.0086267 \\
\hline Mixed Acid & 4 & 0.0010982 & 0.0007877 & 0.0007877 \\
\hline $\mathrm{Na} 2 \mathrm{O} 2$ & 4 & 0.0354050 & 0.0265366 & 0.0185662 \\
\hline Test & F Ratio & DFNum & DFDen & Prob $>F$ \\
\hline O'Brien[.5] & 1.2178 & 2 & 11 & 0.3329 \\
\hline $\begin{array}{l}\text { Brown- } \\
\text { Forsythe }\end{array}$ & 0.7017 & 2 & 11 & 0.5166 \\
\hline Levene & 4.3665 & 2 & 11 & 0.0402 \\
\hline Bartlett & 7.6376 & 2 & . & 0.0005 \\
\hline
\end{tabular}

Warning: Small sample sizes. Use Caution.

Welch Anova testing Means Equal, allowing Std Devs Not Equal

F Ratio DFNum DFDen Prob $>$ F

$\begin{array}{lrrr}80.0761 & 2 & 5.0266 & 0.0002\end{array}$ 
Westinghouse Savannah River Company

WSRC-TR-2005-00396

Savannah River National Laboratory

Rev. 0

Analytical Development/Statistical Consulting Sections

Page 126 of 140

Exhibit B4. Chemical Composition Measurements by Type of Prepared Sample by Oxide for DWPF Radioactive Glass

(Concentrations are in wt\%.)

Oneway Analysis of Na2O (wt\%) By Preparation Method

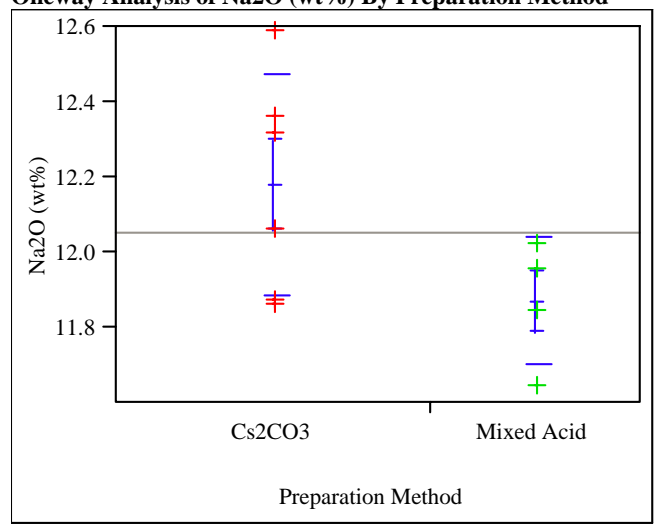

Missing Rows

4Means Comparisons

Comparisons for all pairs using Tukey-Kramer HSD

$$
\begin{array}{rr}
q^{*} & \text { Alpha } \\
2.30593 & 0.05
\end{array}
$$

Abs(Dif)-LSD Cs2CO3 Mixed Acid

Cs2CO3 $\quad-0.33589-0.06550$

Mixed Acid $\quad-0.06550 \quad-0.41139$

Positive values show pairs of means that are significantly different.

Level - Level Difference Lower CL Upper CL

Cs2CO3 Mixed Acid $0.3100400 \quad-0.0655020 .6855815$

Tests that the Variances are Equal

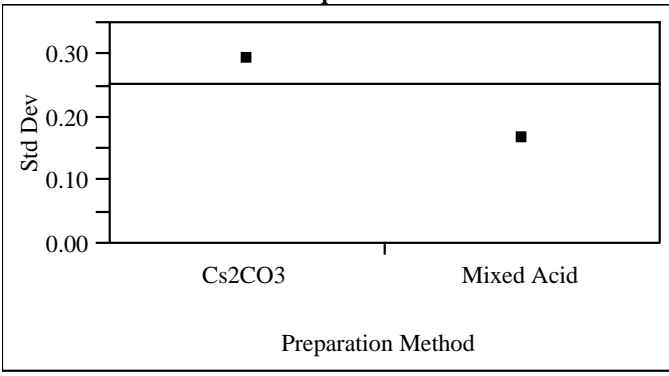

$\begin{array}{lrrrr}\text { Level } & \text { Count } & \text { Std Dev } & \begin{array}{r}\text { MeanAbsDif to } \\ \text { Mean }\end{array} & \begin{array}{r}\text { MeanAbsif to } \\ \text { Median }\end{array} \\ \text { Cs2CO3 } & 6 & 0.2924570 & 0.2448867 & 0.2448867 \\ \text { Mixed Acid } & 4 & 0.1649121 & 0.1213200 & 0.1213200 \\ \text { Test } & \text { F } & \text { DFNum } & \text { DFDen } & \text { Prob > F } \\ & \text { Ratio } & & & \\ \text { O'Brien[.5] } & 1.7523 & 1 & 8 & 0.2222 \\ \text { Brown- } & 3.0479 & 1 & 8 & 0.1190 \\ \text { Forsythe } & & & & \\ \text { Levene } & 3.2375 & 1 & & 0.1097 \\ \text { Bartlett } & 0.9456 & 1 & 3 & 0.3309 \\ \text { F Test 2-sided } & 3.1450 & 5 & & 0.3745 \\ \text { Warning. Small }\end{array}$

Warning: Small sample sizes. Use Caution.

Welch Anova testing Means Equal, allowing Std Devs Not Equal

F Ratio DFNum DFDen Prob $>$ F

$\begin{array}{llll}4.5656 & 1 & 7.9085 & 0.0655\end{array}$

t Test

2.1367

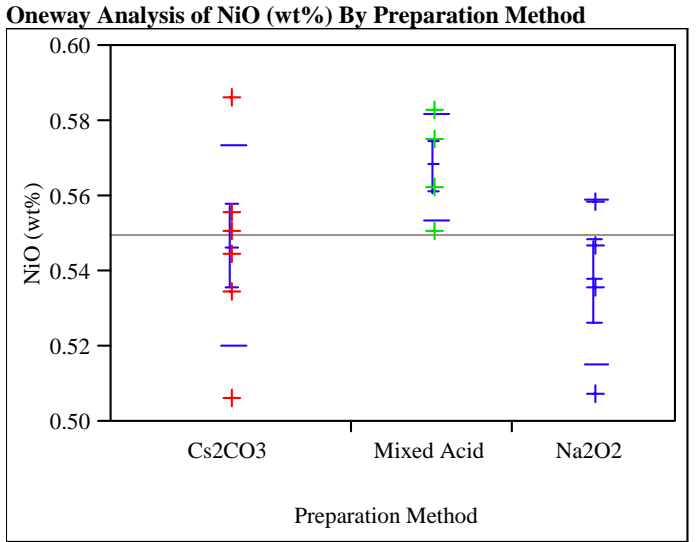

Means Comparisons

Comparisons for all pairs using Tukey-Kramer HSD

$$
\begin{array}{rr}
q^{*} & \text { Alpha } \\
2.70081 & 0.05
\end{array}
$$

Abs(Dif)-LSD Mixed Acid Cs2CO3 Na2O2

Mixed Acid $\quad-0.04268-0.01765-0.01214$

Cs2CO3 $\quad-0.01765-0.03485-0.02974$

$\mathrm{Na2O} 2 \quad-0.01214-0.02974-0.04268$

Positive values show pairs of means that are significantly different.

Level

Mixed Acid A 0.56785312

Cs2CO3 A 0.54653875

$\mathrm{Na} 2 \mathrm{O} 2 \quad$ A 0.53731312

Levels not connected by same letter are significantly different Tests that the Variances are Equal

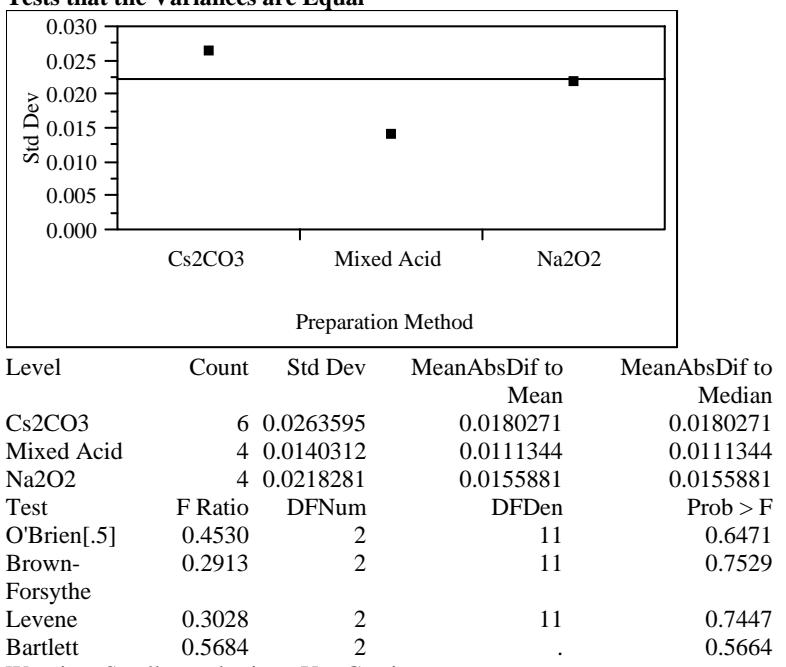

Warning: Small sample sizes. Use Caution.

Welch Anova testing Means Equal, allowing Std Devs Not Equal

F Ratio DFNum DFDen Prob $>$ F

$\begin{array}{llll}2.9896 & 2 & 6.8757 & 0.1163\end{array}$ 
Westinghouse Savannah River Company

WSRC-TR-2005-00396

Savannah River National Laboratory

Rev. 0

Analytical Development/Statistical Consulting Sections

Page 127 of 140

Exhibit B4. Chemical Composition Measurements by Type of Prepared Sample by Oxide for DWPF Radioactive Glass

(Concentrations are in wt\%.)

Oneway Analysis of P2O5 (wt\%) By Preparation Method

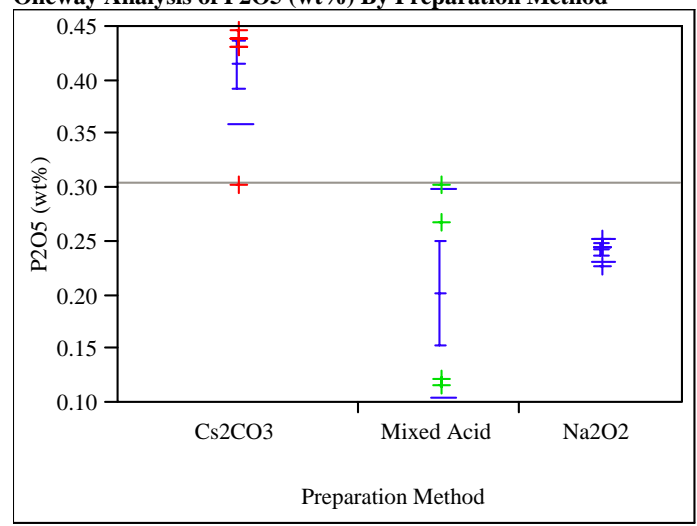

Means Comparisons

Comparisons for all pairs using Tukey-Kramer HSD

q* Alpha

Abs(Dif)-LSD Cs2CO3 Na2O2 Mixed Acid

$\begin{array}{llll}\mathrm{Cs} 2 \mathrm{CO} 3 & -0.09872 & 0.06282 & 0.10291\end{array}$

$\mathrm{Na2O} 2 \quad 0.06282-0.12091 \quad-0.08081$

Mixed Acid $\quad 0.10291 \quad-0.08081 \quad-0.12091$

Positive values show pairs of means that are significantly different.

$\begin{array}{lrr}\text { Level } & & \text { Mean } \\ \text { Cs2CO3 } & \text { A } & 0.41550720 \\ \text { Na2O2 } & \text { B } & 0.24231555 \\ \text { Mixed Acid } & \text { B } & 0.20221605\end{array}$

Levels not connected by same letter are significantly different Tests that the Variances are Equal

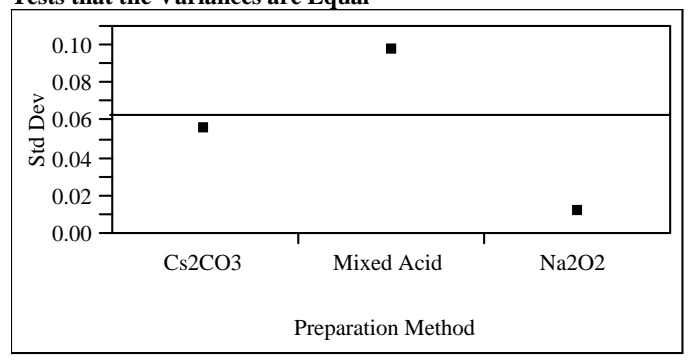

\begin{tabular}{|c|c|c|c|c|}
\hline Level & Count & Std Dev & $\begin{array}{r}\text { MeanAbsDif to } \\
\text { Mean }\end{array}$ & $\begin{array}{r}\text { MeanAbsDif to } \\
\text { Median }\end{array}$ \\
\hline Cs2CO3 & 6 & 0.0556988 & 0.0376808 & 0.0267330 \\
\hline Mixed Acid & 4 & 0.0969748 & 0.0830632 & 0.0830633 \\
\hline $\mathrm{Na} 2 \mathrm{O} 2$ & 4 & 0.0110883 & 0.0077335 & 0.0068742 \\
\hline Test & F Ratio & DFNum & DFDen & Prob $>F$ \\
\hline O'Brien[.5] & 3.6294 & 2 & 11 & 0.0616 \\
\hline $\begin{array}{l}\text { Brown- } \\
\text { Forsythe }\end{array}$ & 4.8049 & 2 & 11 & 0.0316 \\
\hline Levene & 8.2133 & 2 & 11 & 0.0066 \\
\hline Bartlett & 4.0628 & 2 & . & 0.017 \\
\hline
\end{tabular}

Warning: Small sample sizes. Use Caution.

Welch Anova testing Means Equal, allowing Std Devs Not Equal

F Ratio DFNum DFDen Prob $>$ F

$\begin{array}{llll}24.7709 & 2 & 5.2782 & 0.0021\end{array}$

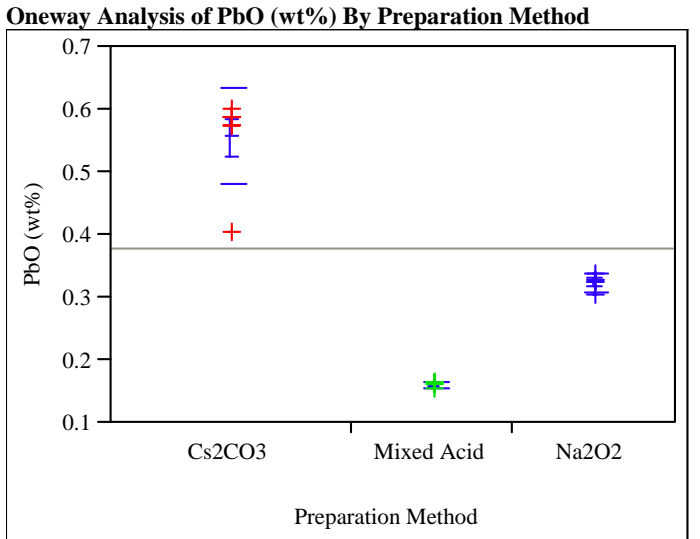

Means Comparisons

Comparisons for all pairs using Tukey-Kramer HSD $q^{*} \quad$ Alpha

bs(Dif)-LSD Cs2CO3 Na2O2 Mixed Acid

$\begin{array}{llll}\text { Cs2CO3 } & -0.07915 & 0.14293 & 0.30653\end{array}$

$\begin{array}{llll}\mathrm{Na} 2 \mathrm{O} 2 & 0.14293 & -0.09693 & 0.06667\end{array}$

$\begin{array}{llll}\text { Mixed Acid } \quad 0.30653 & 0.06667 & -0.09693\end{array}$

Positive values show pairs of means that are significantly different.

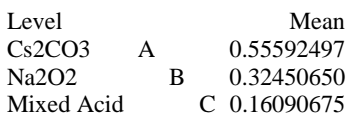

Levels not connected by same letter are significantly different Tests that the Variances are Equal

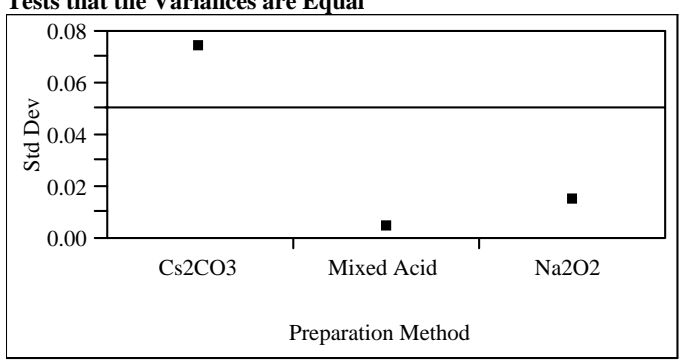

\begin{tabular}{|c|c|c|c|c|}
\hline Level & Count & Std Dev & $\begin{array}{r}\text { MeanAbsDif to } \\
\text { Mean }\end{array}$ & $\begin{array}{r}\text { MeanAbsDif to } \\
\text { Median }\end{array}$ \\
\hline Cs2CO3 & 6 & 0.0743193 & 0.0501197 & 0.0365350 \\
\hline Mixed Acid & 4 & 0.0045992 & 0.0033662 & 0.0033662 \\
\hline $\mathrm{Na} 2 \mathrm{O} 2$ & 4 & 0.0148123 & 0.0103680 & 0.0094255 \\
\hline Test & F Ratio & DFNum & DFDen & Prob > \\
\hline O'Brien[.5] & 0.7723 & 2 & 11 & 0.48 \\
\hline $\begin{array}{l}\text { Brown- } \\
\text { Forsythe }\end{array}$ & 0.7318 & 2 & 11 & 0.50 \\
\hline Levene & 2.8001 & 2 & 11 & 0.1040 \\
\hline Bartlett & 7.9621 & 2 & . & 0.0003 \\
\hline
\end{tabular}

Warning: Small sample sizes. Use Caution.

Welch Anova testing Means Equal, allowing Std Devs Not Equal

F Ratio DFNum DFDen Prob $>$ F

$268.6755 \quad 2 \quad 5.5747<.0001$ 
Westinghouse Savannah River Company

WSRC-TR-2005-00396

Savannah River National Laboratory

Rev. 0

Analytical Development/Statistical Consulting Sections

Page 128 of 140

Exhibit B4. Chemical Composition Measurements by Type of Prepared Sample by Oxide for DWPF Radioactive Glass

(Concentrations are in wt\%.)

Oneway Analysis of SO4 (wt\%) By Preparation Method

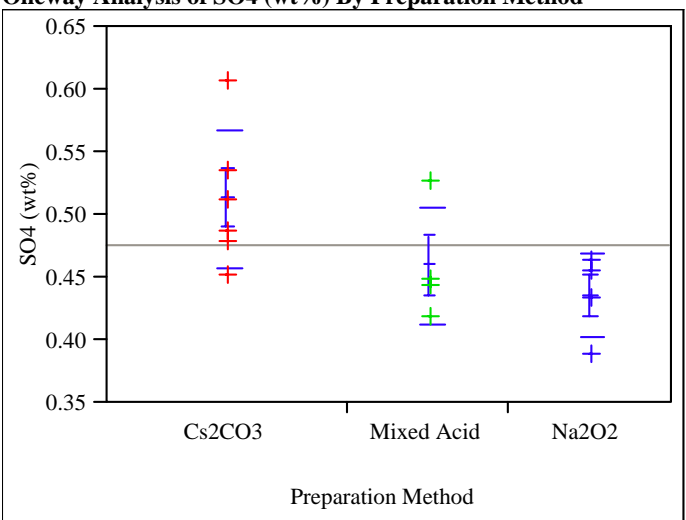

Means Comparisons

Comparisons for all pairs using Tukey-Kramer HSD

$$
\text { q* Alpha }
$$

Abs(Dif)-LSD Cs2CO3 Mixed Acid Na2O2

$\begin{array}{llll}\text { Cs2CO3 } & -0.07422 & -0.03006 & -0.00609\end{array}$

$\begin{array}{llll}\text { Mixed Acid } & -0.03006 & -0.09090 & -0.06694\end{array}$

$\mathrm{Na} 2 \mathrm{O} 2 \quad-0.00609-0.06694-0.09090$

Positive values show pairs of means that are significantly different.

Level Mean

Cs2CO3 A 0.51279822

Mixed Acid A 0.45987065

$\mathrm{Na} 2 \mathrm{O} 2 \quad$ A 0.43590345

Levels not connected by same letter are significantly different Tests that the Variances are Equal

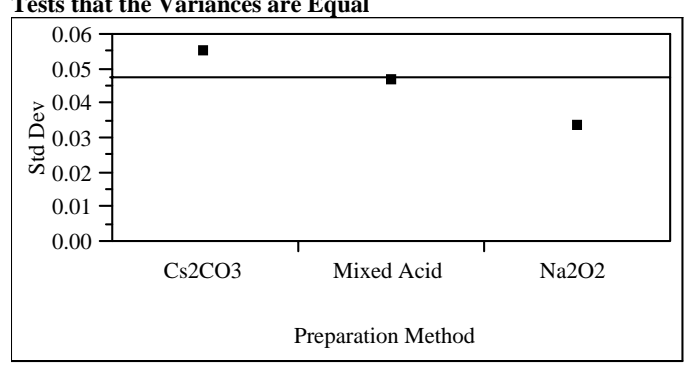

$\begin{array}{lrrrr}\text { Level } & \text { Count } & \text { Std Dev } & \begin{array}{r}\text { MeanAbsDif to } \\ \text { Mean }\end{array} & \begin{array}{r}\text { MeanAbsDif to } \\ \text { Median }\end{array} \\ \text { Cs2CO3 } & 6 & 0.0547985 & 0.0396125 & 0.0394460 \\ \text { Mixed Acid } & 4 & 0.0467655 & 0.0337039 & 0.0284611 \\ \text { Na2O2 } & 4 & 0.0334057 & 0.0239672 & 0.0239672 \\ \text { Test } & \text { F Ratio } & \text { DFNum } & \text { DFDen } & \text { Prob > F } \\ \text { O'Brien[.5] } & 0.3085 & 2 & 11 & 0.7407 \\ \text { Brown- } & 0.2928 & 2 & 11 & 0.7518 \\ \text { Forsythe } & & & & \\ \text { Levene } & 0.3730 & 2 & 11 & 0.6970 \\ \text { Bartlett } & 0.3641 & 2 & . & 0.6948 \\ \text { Warning. } & 2 & & \end{array}$

Warning: Small sample sizes. Use Caution.

Welch Anova testing Means Equal, allowing Std Devs Not Equal

F Ratio DFNum DFDen Prob $>$ F

$\begin{array}{llll}3.4680 & 2 & 6.9319 & 0.0904\end{array}$
Oneway Analysis of SiO2 (wt\%) By Preparation Method

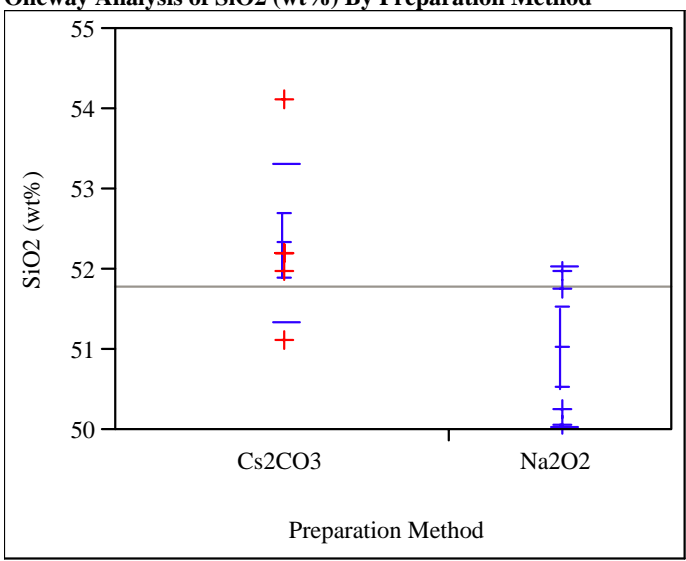

Missing Rows

4Means Comparisons

Comparisons for all pairs using Tukey-Kramer HSD

q* Alpha

Abs(Dif)-LSD Cs2CO3 Na2O2

$\begin{array}{lll}\mathrm{Cs} 2 \mathrm{CO} 3 & -1.3148 & -0.1865\end{array}$

$\mathrm{Na} 2 \mathrm{O} 2 \quad-0.1865-1.6103$

Positive values show pairs of means that are significantly different.

Level - Level Difference Lower CL Upper CL

Cs2CO3 Na2O2 $1.283580 \quad-0.1864602 .753620$

Tests that the Variances are Equal

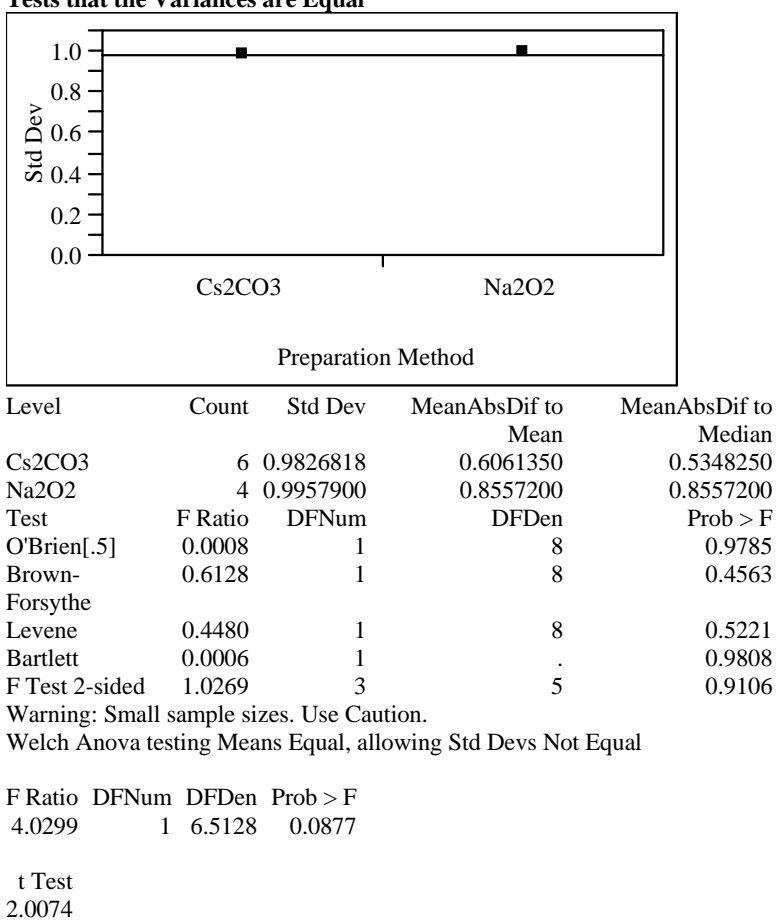


Westinghouse Savannah River Company

WSRC-TR-2005-00396

Savannah River National Laboratory

Rev. 0

Analytical Development/Statistical Consulting Sections

Page 129 of 140

Exhibit B4. Chemical Composition Measurements by Type of Prepared Sample by Oxide for DWPF Radioactive Glass

(Concentrations are in wt\%.)

Oneway Analysis of SnO2 (wt\%) By Preparation Method

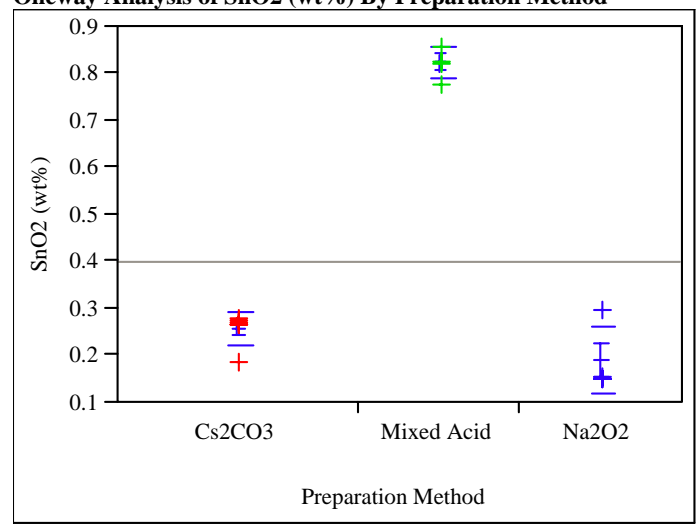

Means Comparisons

Comparisons for all pairs using Tukey-Kramer HSD

q* Alpha

Abs(Dif)-LSD Mixed Acid Cs2CO3 Na2O2

$\begin{array}{llll}\text { Mixed Acid } \quad-0.08994 & 0.48075 & 0.54200\end{array}$

Cs2CO3 $\quad 0.48075-0.07344-0.01302$

$\mathrm{Na} 2 \mathrm{O} 2 \quad 0.54200-0.01302 \quad-0.08994$

Positive values show pairs of means that are significantly different.

Level

Mixed Acid A 0.82143120

Cs2CO3

$\mathrm{Na} 2 \mathrm{O} 2 \quad$ B 0.18948780

Levels not connected by same letter are significantly different Tests that the Variances are Equal

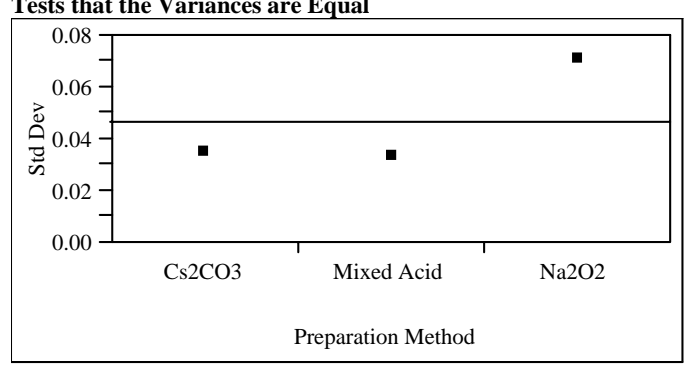

\begin{tabular}{|c|c|c|c|c|}
\hline Level & Count & Std Dev & $\begin{array}{r}\text { MeanAbsDif to } \\
\text { Mean }\end{array}$ & $\begin{array}{r}\text { MeanAbsDif to } \\
\text { Median }\end{array}$ \\
\hline Cs2CO3 & 6 & 0.0345431 & 0.0233465 & 0.0167164 \\
\hline Mixed Acid & 4 & 0.0333658 & 0.0215832 & 0.0215832 \\
\hline $\mathrm{Na} 2 \mathrm{O} 2$ & 4 & 0.0709283 & 0.0531645 & 0.0371358 \\
\hline Test & F Ratio & DFNum & DFDen & Prob $>F$ \\
\hline O'Brien[.5] & 0.9351 & 2 & 11 & 0.4217 \\
\hline $\begin{array}{l}\text { Brown- } \\
\text { Forsythe }\end{array}$ & 0.2708 & 2 & 11 & 0.7677 \\
\hline Levene & 1.8447 & 2 & 11 & 0.2038 \\
\hline Bartlett & 1.2003 & 2 & 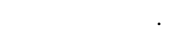 & 0.3011 \\
\hline
\end{tabular}

Warning: Small sample sizes. Use Caution.

Welch Anova testing Means Equal, allowing Std Devs Not Equal

F Ratio DFNum DFDen Prob $>$ F

$\begin{array}{llll}328.5265 & 2 & 5.9215 & <.0001\end{array}$

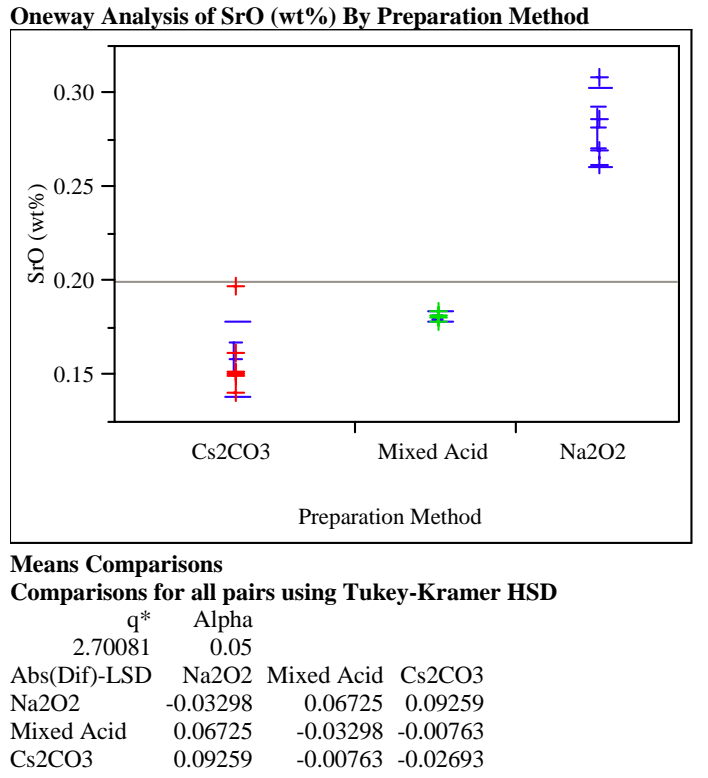

Positive values show pairs of means that are significantly different.

$$
\begin{array}{lrr}
\text { Level } & & \text { Mean } \\
\text { Na2O2 } & \text { A } & 0.28175445 \\
\text { Mixed Acid } & \text { B } & 0.18152910 \\
\text { Cs2CO3 } & \text { B } & 0.15905970
\end{array}
$$

Levels not connected by same letter are significantly different Tests that the Variances are Equal

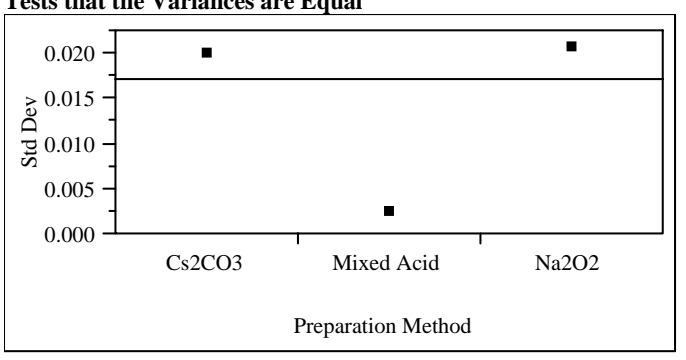

$\begin{array}{lrrrr}\text { Level } & \text { Count } & \text { Std Dev } & \begin{array}{r}\text { MeanAbsDif to } \\ \text { Mean }\end{array} & \begin{array}{r}\text { MeanAbsDif to } \\ \text { Median }\end{array} \\ \text { Cs2CO3 } & 6 & 0.0200101 & 0.0137970 & 0.0116289 \\ \text { Mixed Acid } & 4 & 0.0024618 & 0.0017739 & 0.0017739 \\ \text { Na2O2 } & 4 & 0.0204918 & 0.0156695 & 0.0156695 \\ \text { Test } & \text { F Ratio } & \text { DFNum } & \text { DFDen } & \text { Prob > F } \\ \text { O'Brien[.5] } & 0.6737 & 2 & 11 & 0.5297 \\ \text { Brown- } & 1.2443 & 2 & 11 & 0.3257 \\ \text { Forsythe } & & & & \\ \text { Levene } & 2.2830 & 2 & 11 & 0.1481 \\ \text { Bartlett } & 4.0675 & 2 & . & 0.0171\end{array}$

Warning: Small sample sizes. Use Caution.

Welch Anova testing Means Equal, allowing Std Devs Not Equal

F Ratio DFNum DFDen Prob $>$ F

$\begin{array}{rrrr}45.4818 & 2 & 5.1691 & 0.0005\end{array}$ 
Westinghouse Savannah River Company

WSRC-TR-2005-00396

Savannah River National Laboratory

Rev. 0

Analytical Development/Statistical Consulting Sections

Page 130 of 140

Exhibit B4. Chemical Composition Measurements by Type of Prepared Sample by Oxide for DWPF Radioactive Glass

(Concentrations are in wt\%.)

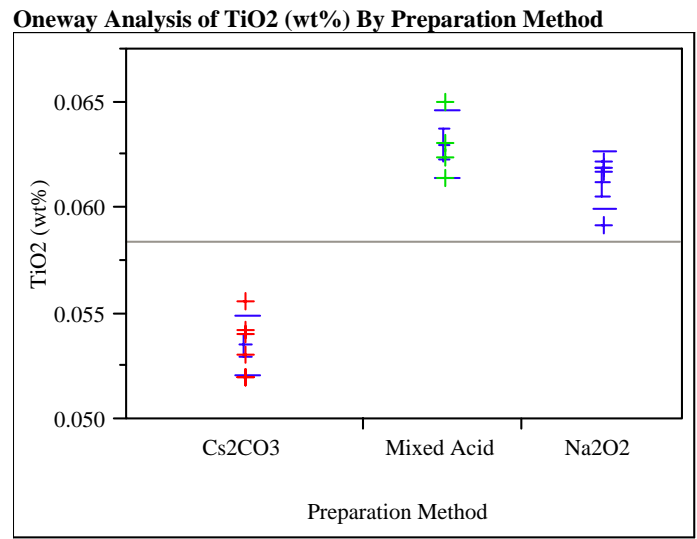

Means Comparisons

Comparisons for all pairs using Tukey-Kramer HSD

$$
\begin{array}{rr}
\mathrm{q}^{*} & \text { Alpha } \\
2.70081 & 0.05
\end{array}
$$

Abs(Dif)-LSD Mixed Acid Na2O2 Cs2CO3

$\begin{array}{llll}\text { Mixed Acid } & -0.00272 & -0.00101 & 0.00700\end{array}$

$\begin{array}{llll}\mathrm{Na} 2 \mathrm{O} 2 & -0.00101 & -0.00272 & 0.00529\end{array}$

$\begin{array}{llll}\mathrm{Cs} 2 \mathrm{CO} 3 & 0.00700 & 0.00529 & -0.00222\end{array}$

Positive values show pairs of means that are significantly different.

$\begin{array}{llr}\text { Level } & & \text { Mean } \\ \text { Mixed Acid A } & 0.06296700 \\ \text { Na2O2 } & \text { A } & 0.06125730 \\ \text { Cs2CO3 } & \text { B } & 0.05348720\end{array}$

Cs2CO3 B 0.05348720

Levels not connected by same letter are significantly different

Tests that the Variances are Equal

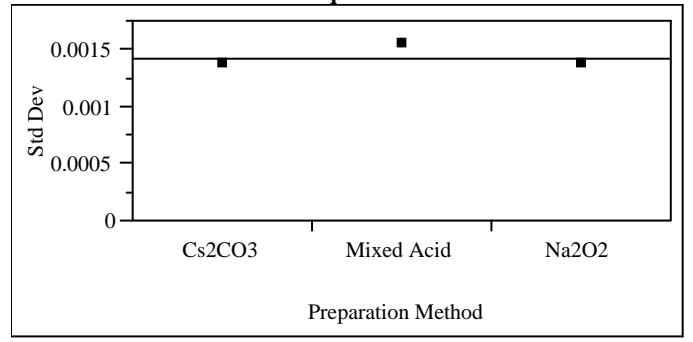

\begin{tabular}{|c|c|c|c|c|}
\hline Level & Count & Std Dev & $\begin{array}{r}\text { MeanAbsDif to } \\
\text { Mean }\end{array}$ & $\begin{array}{r}\text { MeanAbsDif to } \\
\text { Median }\end{array}$ \\
\hline Cs2CO3 & 6 & 0.0013741 & 0.0011120 & 0.0011120 \\
\hline Mixed Acid & 4 & 0.0015498 & 0.0010842 & 0.0010842 \\
\hline $\mathrm{Na} 2 \mathrm{O} 2$ & 4 & 0.0013780 & 0.0010217 & 0.0007923 \\
\hline Test & F Ratio & DFNum & DFDen & Prob $>F$ \\
\hline O'Brien[.5] & 0.0479 & 2 & 11 & 0.9535 \\
\hline $\begin{array}{l}\text { Brown- } \\
\text { Forsythe }\end{array}$ & 0.1645 & 2 & 11 & 0.8503 \\
\hline Levene & 0.0180 & 2 & 11 & 0.9822 \\
\hline Bartlett & 0.0285 & 2 & . & 0.9719 \\
\hline
\end{tabular}

Warning: Small sample sizes. Use Caution.

Welch Anova testing Means Equal, allowing Std Devs Not Equal

F Ratio DFNum DFDen Prob $>$ F

$\begin{array}{llll}58.0297 & 2 & 6.4487 & <.0001\end{array}$

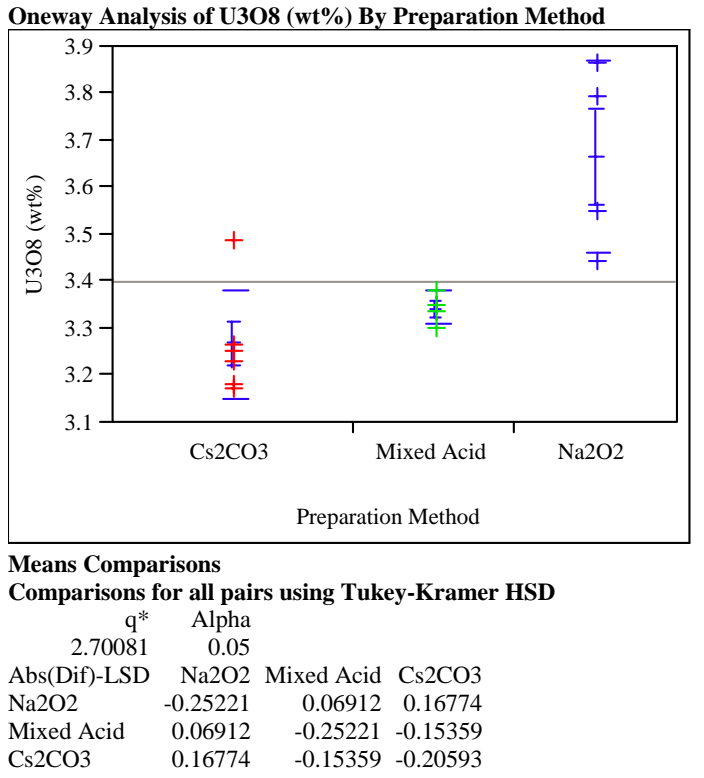

Positive values show pairs of means that are significantly different.

$\begin{array}{lrr}\text { Level } & & \text { Mean } \\ \text { Na2O2 } & \text { A } & 3.6643640 \\ \text { Mixed Acid } & \text { B } & 3.3430320 \\ \text { Cs2CO3 } & \text { B } & 3.2663840\end{array}$

Levels not connected by same letter are significantly different Tests that the Variances are Equal

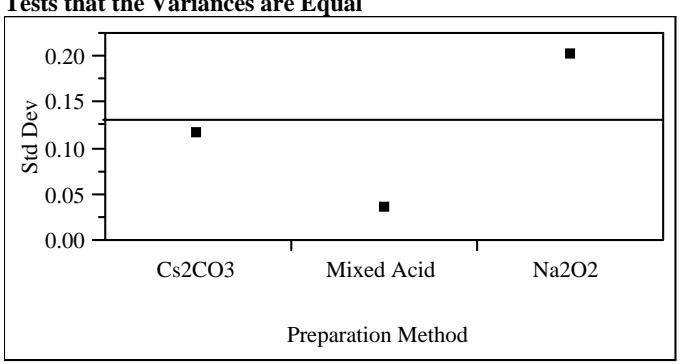

\begin{tabular}{|c|c|c|c|c|}
\hline Level & Count & Std Dev & $\begin{array}{r}\text { MeanAbsDif to } \\
\text { Mean }\end{array}$ & $\begin{array}{r}\text { MeanAbsDif to } \\
\text { Median }\end{array}$ \\
\hline Cs2CO3 & 6 & 0.1160179 & 0.0746827 & 0.0707520 \\
\hline Mixed Acid & 4 & 0.0340406 & 0.0235840 & 0.0235840 \\
\hline $\mathrm{Na} 2 \mathrm{O} 2$ & 4 & 0.2008971 & 0.1680360 & 0.1680360 \\
\hline Test & F Ratio & DFNum & DFDen & Prob $>F$ \\
\hline O'Brien[.5] & 2.9773 & 2 & 11 & 0.0926 \\
\hline $\begin{array}{l}\text { Brown- } \\
\text { Forsythe }\end{array}$ & 4.7984 & 2 & 11 & 0.0318 \\
\hline Levene & 5.5053 & 2 & 11 & 0.0220 \\
\hline Bartlett & 3.0608 & 2 & . & 0.0468 \\
\hline
\end{tabular}

Warning: Small sample sizes. Use Caution.

Welch Anova testing Means Equal, allowing Std Devs Not Equal

F Ratio DFNum DFDen Prob $>$ F

$\begin{array}{llll}5.7356 & 2 & 5.5431 & 0.0447\end{array}$ 
Westinghouse Savannah River Company

WSRC-TR-2005-00396

Savannah River National Laboratory

Rev. 0

Analytical Development/Statistical Consulting Sections

Page 131 of 140

Exhibit B4. Chemical Composition Measurements by Type of Prepared Sample by Oxide for DWPF Radioactive Glass

(Concentrations are in wt\%.)

Oneway Analysis of V2O5 (wt\%) By Preparation Method

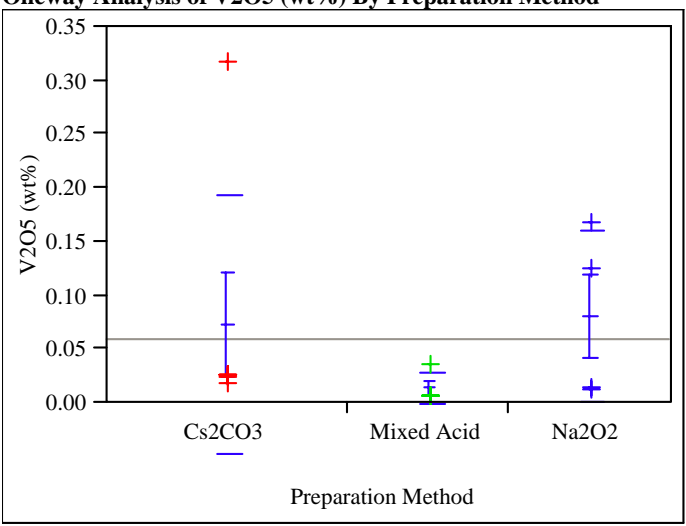

Means Comparisons

Comparisons for all pairs using Tukey-Kramer HSD

q* Alpha

0.05

Abs(Dif)-LSD Na2O2 Cs2CO3 Mixed Acid

$\mathrm{Na} 2 \mathrm{O} 2 \quad-0.17395-0.15114 \quad-0.10734$

$\begin{array}{llll}\mathrm{Cs} 2 \mathrm{CO} 3 & -0.15114 & -0.14203 & -0.09984\end{array}$

Mixed Acid $\quad-0.10734 \quad-0.09984 \quad-0.17395$

Positive values show pairs of means that are significantly different.

Level Mean

$\mathrm{Na} 2 \mathrm{O} 2$ A 0.08066872

Cs2CO3 A 0.07301468

Mixed Acid A 0.01405622

Levels not connected by same letter are significantly different Tests that the Variances are Equal

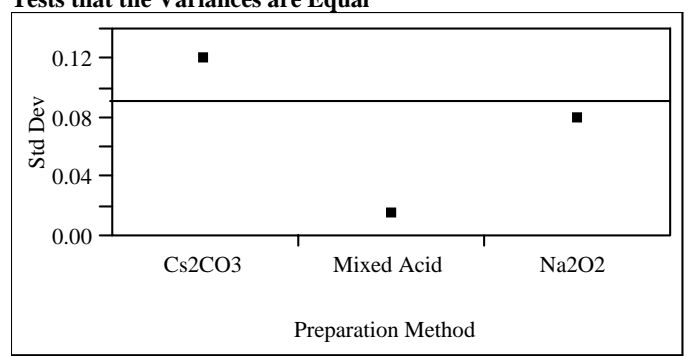

$\begin{array}{lrrrr}\text { Level } & \text { Count } & \text { Std Dev } & \begin{array}{r}\text { MeanAbsDif to } \\ \text { Mean }\end{array} & \begin{array}{r}\text { MeanAbsDif to } \\ \text { Median }\end{array} \\ \text { Cs2CO3 } & 6 & 0.1199460 & 0.0815836 & 0.0501939 \\ \text { Mixed Acid } & 4 & 0.0141948 & 0.0106454 & 0.0071877 \\ \text { Na2O2 } & 4 & 0.0789978 & 0.0666995 & 0.0666995 \\ \text { Test } & \text { F Ratio } & \text { DFNum } & \text { DFDen } & \text { Prob > F } \\ \text { O'Brien[.5] } & 0.5430 & 2 & 11 & 0.5958 \\ \text { Brown- } & 0.5853 & 2 & 11 & 0.5734 \\ \text { Forsythe } & & & & \\ \text { Levene } & 2.0946 & 2 & 11 & 0.1695 \\ \text { Bartlett } & 4.0978 & 2 & . & 0.0166\end{array}$

Warning: Small sample sizes. Use Caution.

Welch Anova testing Means Equal, allowing Std Devs Not Equal

F Ratio DFNum DFDen Prob $>$ F

$\begin{array}{llll}1.8096 & 2 & 5.2684 & 0.2522\end{array}$

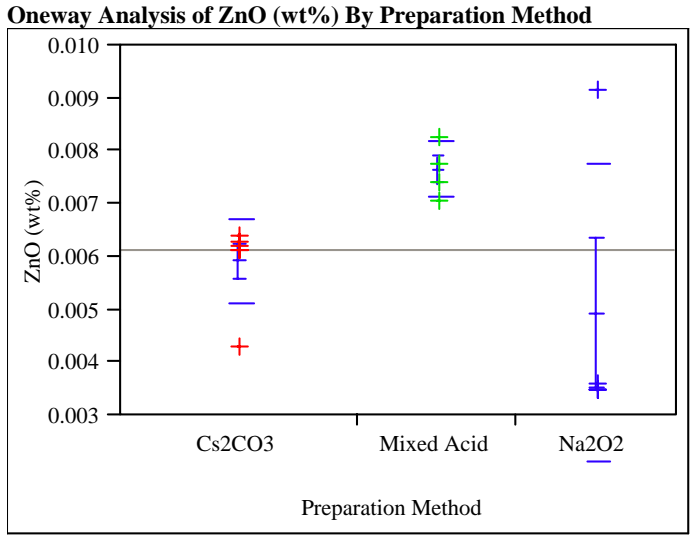

Means Comparisons

Comparisons for all pairs using Tukey-Kramer HSD

$$
\begin{array}{rr}
q^{*} & \text { Alpha } \\
2.70081 & 0.05
\end{array}
$$

Abs(Dif)-LSD Mixed Acid Cs2CO3 Na2O2

Mixed Acid $\quad-0.00304-0.00106-0.00035$

$\begin{array}{llll}\mathrm{Cs} 2 \mathrm{CO} 3 & -0.00106 & -0.00248 & -0.00179\end{array}$

$\mathrm{Na2O} 2 \quad-0.00035-0.00179-0.00304$

Positive values show pairs of means that are significantly different.

Level

Cs2CO3 A 0.00591902

$\mathrm{Na} 2 \mathrm{O} 2$ A 0.00493719

Levels not connected by same letter are significantly different Tests that the Variances are Equal

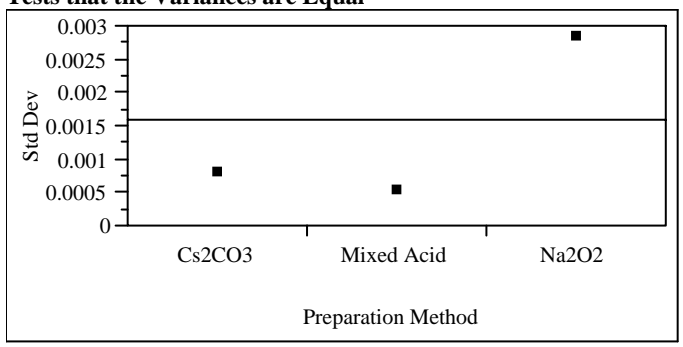

$\begin{array}{lrrrr}\text { Level } & \text { Count } & \text { Std Dev } & \begin{array}{r}\text { MeanAbsDif to } \\ \text { Mean }\end{array} & \begin{array}{r}\text { MeanAbsDif to } \\ \text { Median }\end{array} \\ \text { Cs2CO3 } & 6 & 0.0007961 & 0.0005373 & 0.0003880 \\ \text { Mixed Acid } & 4 & 0.0005125 & 0.0003890 & 0.0003890 \\ \text { Na2O2 } & 4 & 0.0028252 & 0.0021185 & 0.0014517 \\ \text { Test } & \text { F Ratio } & \text { DFNum } & \text { DFDen } & \text { Prob > F } \\ \text { O'Brien[.5] } & 1.6381 & 2 & 11 & 0.2384 \\ \text { Brown- } & 0.6813 & 2 & 11 & 0.5261 \\ \text { Forsythe } & & & & \\ \text { Levene } & 5.5936 & 2 & 11 & 0.0211 \\ \text { Bartlett } & 4.5600 & 2 & . & 0.0105\end{array}$

Warning: Small sample sizes. Use Caution.

Welch Anova testing Means Equal, allowing Std Devs Not Equal

F Ratio DFNum DFDen Prob $>$ F

$\begin{array}{llll}8.6326 & 2 & 5.9217 & 0.0176\end{array}$ 
Westinghouse Savannah River Company

WSRC-TR-2005-00396

Savannah River National Laboratory

Rev. 0

Analytical Development/Statistical Consulting Sections

Page 132 of 140

Exhibit B4. Chemical Composition Measurements by Type of Prepared Sample by Oxide for DWPF Radioactive Glass

(Concentrations are in wt\%.)

Oneway Analysis of ZrO2 (wt\%) By Preparation Method

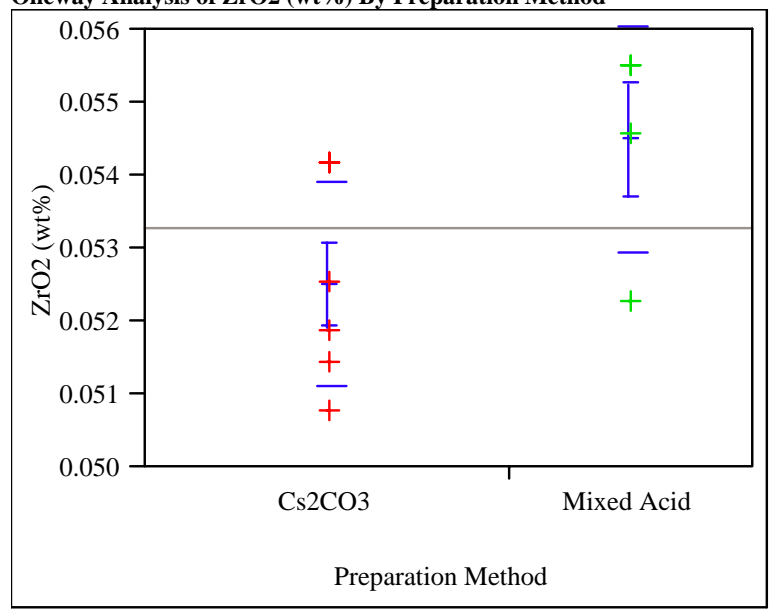

Missing Rows

4Means Comparisons

Comparisons for all pairs using Tukey-Kramer HSD

$$
\begin{array}{rr}
q^{*} & \text { Alpha } \\
2.30593 & 0.05
\end{array}
$$

Abs(Dif)-LSD Mixed Acid Cs2CO3

Mixed Acid $\quad-0.00237 \quad-0.00020$

Cs2CO3 $\quad-0.00020-0.00194$

Positive values show pairs of means that are significantly different.

Level - Level Difference Lower CL Upper CL

Mixed Acid Cs2CO3 0.0019699 -0.000198 0.0041379

Tests that the Variances are Equal

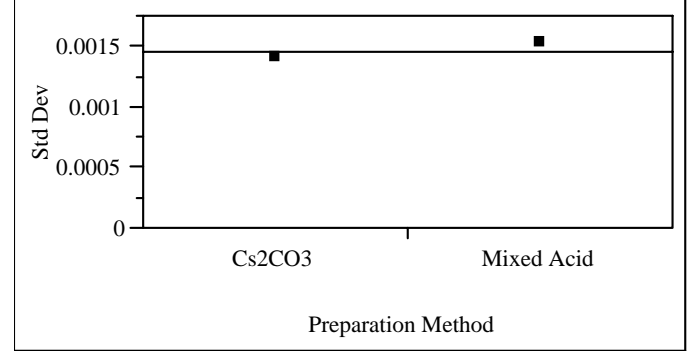

$\begin{array}{lrrrr}\text { Level } & \text { Count } & \text { Std Dev } & \begin{array}{r}\text { MeanAbsDif to } \\ \text { Mean }\end{array} & \begin{array}{r}\text { MeanAbsDif to } \\ \text { Median }\end{array} \\ \text { Cs2CO3 } & 6 & 0.0014107 & 0.0011257 & 0.0011257 \\ \text { Mixed Acid } & 4 & 0.0015297 & 0.0010975 & 0.0010469 \\ \text { Test } & \text { F Ratio } & \text { DFNum } & \text { DFDen } & \text { Prob > F } \\ \text { O'Brien[.5] } & 0.0444 & 1 & 8 & 0.8383 \\ \text { Brown- } & 0.0175 & 1 & 8 & 0.8981 \\ \text { Forsythe } & & & & \\ \text { Levene } & 0.0033 & 1 & 8 & 0.9553 \\ \text { Bartlett } & 0.0219 & 1 & . & 0.8823 \\ \text { F Test 2-sided } & 1.1759 & 3 & 5 & 0.8132 \\ \text { W } & & & \end{array}$

Warning: Small sample sizes. Use Caution.

Welch Anova testing Means Equal, allowing Std Devs Not Equal

F Ratio DFNum DFDen Prob $>$ F

$\begin{array}{llll}4.2331 & 1 & 6.1751 & 0.0840\end{array}$

t Test

2.0575 


\section{APPENDIX C. SUPPORTING ANALYTICAL PROCEDURES}


Rev. 0

Analytical Development/Statistical Consulting Sections

This page intentionally left blank. 
Procedure for Measuring Wt.\% Solids by Drying at $115{ }^{\circ} \mathrm{C}$ and Wt. \% Vitrified Solids at $1100{ }^{\circ} \mathrm{C}$ followed by $\mathrm{Cs}_{2} \mathrm{CO}_{3}$ Fusion Digestion of the Resulting Glass Wafers

General Comment: The following procedure conveniently combines the measurements of wt. \% solids SME samples, after both drying to constant weight at $115^{\circ} \mathrm{C}$ and after vitrifying the sample at $1100{ }^{\circ} \mathrm{C}$, with the digestion of the resulting glass wafer. The manipulator operations required are straightforward, but a clean, dry work area must be maintained in order to achieve accurate weight measurements.

\section{Steps 1-22 pertain to measuring the wt. \% solids of SME samples after drying at $115^{\circ} \mathrm{C}$}

1. Preheat the drying oven to $115^{\circ} \mathrm{C}$ and the muffle furnace to $1100{ }^{\circ} \mathrm{C}$.

2. Calibrate the cell balance and record the calibration data in the cell balance logbook.

3. Tare the balance with nothing on the balance pan.

4. Place a clean, dry $50 \mathrm{~mL}$ Pt crucible on the balance pan. Record the weight of the empty $50 \mathrm{~mL}$ Pt crucible in the Data Table.

5. Place the $50 \mathrm{~mL}$ Pt crucible in a clean, dry area convenient to the mixer used for mixing the SME sample.

6. Uncap the $250 \mathrm{~mL}$ SME sample bottle and place the stirring shaft at the prescribed depth.

7. Turn on the stirring motor and slowly increase the rpm until the prescribed maximum rpm is attained.

8. Mix the SME at the prescribed rpm for two minutes.

9. Using a plastic slurry transfer pipette that has $1 \frac{1}{1} 4$ inch of the tip cut off, transfer about a pipette full (about $1 \mathrm{~mL}$ ) to the $50 \mathrm{~mL}$ Pt crucible.

10. Place the $50 \mathrm{~mL}$ Pt crucible on the balance pan and record the weight of the $50 \mathrm{~mL} \mathrm{Pt}$ crucible + SME sample.

11. Subtract the weight of the empty $50 \mathrm{~mL}$ Pt crucible (Step 4) from the weight of the 50 $\mathrm{mL}$ Pt crucible + SME sample (Step 10) and record the wet weight of SME sample in the Data Table.

12. Repeat for all of the required replicate determinations. 
13. Place the crucibles in the drying oven and close the oven door.

14. Monitor the oven temperature. When the oven temperature re-equilibrates to $115 \pm 5^{\circ} \mathrm{C}$, record this time in the Data Table.

15. Heat for 1 hour after the oven temperature re-equilibrates to $115 \pm 5^{\circ} \mathrm{C}$ and then remove the crucibles and place them on clean, dry surface convenient to the cell balance. Record the time that the crucibles are removed from the oven and record this time in the Data Table.

16. After cooling for 5 minutes, weigh each of the crucibles as quickly as possible to minimize re-absorption of moisture from the air (if a dessicator is used, then the crucibles will be allowed to cool in it for 5-10 minutes before weighing). Record the weight of the $50 \mathrm{~mL}$ crucible + dry SME sample after $1^{\text {st }}$ drying step in the Data Table.

17. Place the crucibles back in the drying oven. Monitor the time and record the time that the drying oven re-equilibrates to $115^{\circ} \mathrm{C}$.

18. Heat for 30 minutes or for the length of time prescribed by the Task Supervisor. Record the time that the crucibles are removed from the oven in the Data Table.

19. After cooling for 5 minutes, weigh each of the crucibles as quickly as possible to minimize re-absorption of moisture from the air (if a dessicator is used, then the crucibles will be allowed to cool in it for 5-10 minutes before weighing). Record the weight of the $50 \mathrm{~mL}$ crucible + dry SME sample after $2^{\text {nd }}$ drying step in the Data Table.

20. Compare the weights of crucible + dry SME sample after the $1^{\text {st }}$ and $2^{\text {nd }}$ drying step (Steps 16 and 19) and record the difference in weights in the Data Table. If the weights differ by no more than $10 \mathrm{mg}(0.010 \mathrm{~g})$, the dry weight is considered to be constant and weight of $50 \mathrm{~mL}$ crucible + dry SME sample after the $2^{\text {nd }}$ drying step (Step 19) is used to calculate wt. \% solids.

If the weights differ by more than $0.010 \mathrm{~g}$, consult with the Task Supervisor.

21. Subtract the weight of the $50 \mathrm{~mL}$ Pt crucible (Step 4) from the weight of the $50 \mathrm{~mL} \mathrm{Pt}$ crucible + dry SME after $2^{\text {nd }}$ drying step (Step 19) and record the weight of SME sample after drying at $115^{\circ} \mathrm{C}$ in the Data Table.

22. Divide the weight of the SME sample after drying at $115^{\circ} \mathrm{C}$ (Step 21) by the wet weight of SME sample (Step 11) and multiply this quotient by 100 to obtain the wt. \% SME solids after drying at $115^{\circ} \mathrm{C}$. Record this result in the Data Table. 


\section{Steps 23-29 pertain to vitrifying the dried SME sample and measuring the wt. \% vitrified solids}

23. Place the $50 \mathrm{~mL}$ Pt crucibles (the number of crucibles will be determined by the size of the muffle furnace) in the muffle furnace. Close the door.

24. Monitor the temperature. Record the time that the furnace re-equilibrates to $1100{ }^{\circ} \mathrm{C}$.

25. Heat the crucibles for 30 minutes after the muffle furnace temperature re-equilibrates to $1100{ }^{\circ} \mathrm{C}$. Record the time that the samples are removed from the furnace and the elapsed time of heating at $1100^{\circ} \mathrm{C}$.

26. Remove the crucibles and place them on a dry, clean Kevlar board.

27. After cooling for 10 minutes, weigh each of the crucibles and record the weight of $50 \mathrm{~mL}$ Pt crucibles + vitrified SME sample in the Data Sheet.

28. Subtract the weight of the $50 \mathrm{~mL}$ Pt crucible + vitrified SME (Step 27) from the weight of $50 \mathrm{~mL}$ Pt crucible (Step 4) and record this remainder as the weight of vitrified SME sample.

29. Divide the weight of the vitrified SME sample (Step 28) by the wet weight of SME sample (Step 11) and multiply this quotient by 100 to obtain the wt. \% SME solids after vitrifying at $1100^{\circ} \mathrm{C}$. Record this result in the Data Table.

Comment: This procedure specifies only one heating and weighing step because 30 minutes at $1100{ }^{\circ} \mathrm{C}$ produces a stable glass wafer for which an accurate weight for both the wt. \% vitrified solids and the $\mathrm{Cs}_{2} \mathrm{CO}_{3}$ fusion digestion procedure can be obtained. Prolonged heating beyond 30 minutes or using two heating and weighing cycles will unnecessarily add both time and handling steps to the procedure.

\section{Steps 30-42 pertain to the $\mathrm{Cs}_{2} \mathrm{CO}_{3}$ fusion digestion of the glass wafer formed by vitrifying the SME sample}

30. Add the pre-measured 6-7 g portion (depending on the average weight of the glass wafer following the SME sample vitrification step-this procedure assumes that a 0.6-0.7 g glass wafer is formed) of $\mathrm{Cs}_{2} \mathrm{CO}_{3}$ to the $50 \mathrm{~mL}$ Pt crucible containing the vitrified SME sample. The required grade of $\mathrm{Cs}_{2} \mathrm{CO}_{3}$ is $99.994 \%$ minimum purity ( Alfa Puratronic ${ }^{\circledR}$ grade is recommended).

31. Place the $50 \mathrm{~mL}$ Pt crucibles in the furnace and heat for 10 minutes after the temperature re-equilibrates to $1100^{\circ} \mathrm{C}$.

32. Remove the crucibles from the furnace and let them cool for 5 minutes. 
33. Drop the first crucible into a wide-mouth bottle (either $500 \mathrm{~mL}$ or $1000 \mathrm{~mL}$ can be used) that has been pre-charged with $100 \mathrm{~mL}$ of $3 \% \mathrm{H}_{2} \mathrm{O}_{2}$ solution. [The $\mathrm{H}_{2} \mathrm{O}_{2}$ solution was prepared by diluting $10 \mathrm{~mL}$ of $30 \% \mathrm{H}_{2} \mathrm{O}_{2}$ solution to $100 \mathrm{~mL}$ in a plastic volumetric flask, then transferring all of this solution to the wide-mouth bottle.] Swirl the widemouth bottle to tilt the crucible on its side to promote contact of the flux residue with the $3 \% \mathrm{H}_{2} \mathrm{O}_{2}$ solution.

Comment: The $100 \mathrm{~mL}$ of $3 \% \mathrm{H}_{2} \mathrm{O}_{2}$ is enough solution to ensure adequate contact with the flux residue, but small enough volume that the decomposition of the $\mathrm{H}_{2} \mathrm{O}_{2}$ warms the solution. It is believed that the warm solution helps to dissolve the caustic flux residue and that the resulting warm caustic solution helps to dissolve any highcesium glass that forms from attack of the cesium carbonate on the glass sample.

34. The decomposition of $\mathrm{H}_{2} \mathrm{O}_{2}$ solution in strong caustic solution will create a warm, frothy solution that dissolves the flux residue in the bottom of the Pt crucible, leaving metal oxides and hydrous oxides that are insoluble in highly caustic solution. Let this reaction take place for 15 minutes. The fizzing should stop within this time, indicating that $\mathrm{H}_{2} \mathrm{O}_{2}$ decomposition is complete.

35. Add $250 \mathrm{~mL}$ de-ionized water that has been pre-measured in a $250 \mathrm{~mL}$ plastic volumetric flask.

Comment: The $250 \mathrm{~mL}$ de-ionized water is added at this point, before the acid addition, to dilute the mixture and minimize the potential for silicates to irreversibly precipitate upon addition of strong acid.

36. Swirl the wide-mouth bottle gently to mix the water with the strong caustic solution.

37. Slowly add $100 \mathrm{~mL}$ of approximately 7.8 $\mathrm{M} \mathrm{HNO}_{3}$ solution. [The $\mathrm{HNO}_{3}$ solution was prepared by adding $25 \mathrm{~mL}$ of de-ionized water to a $100 \mathrm{~mL}$ plastic volumetric flask, then slowly adding $50 \mathrm{~mL}$ concentrated $\mathrm{HNO}_{3}$. After the solution cooled, de-ionized water was added to the mark on the volumetric flask. The volumetric flask was capped and the solution mixed by inverting the flask several times.] Add about $15 \mathrm{~mL}$ of the $7.8 \mathrm{M}$ $\mathrm{HNO}_{3}$ solution and then swirl the wide-mouth bottle. Continue addition of the $7.8 \mathrm{M}$ $\mathrm{HNO}_{3}$ solution until all of it has been added. Set aside for 15 minutes.

Comment: Slow, step-wise acid addition is specified for two reasons: (1) to avoid loss of solution (by overflowing the wide-mouth bottle-only a potential problem if $\mathbf{5 0 0}$ $\mathrm{mL}$ wide-mouth bottles are used rather than $1000 \mathrm{~mL}$ bottles) from excess evolution of $\mathrm{CO}_{2}$ that could occur from too rapid decomposition of carbonate brought on by acid addition; and (2) to minimize the possibility of silicates precipitating upon rapid addition of strong acid solution.

38. Add 3-5 drops of $30 \% \mathrm{H}_{2} \mathrm{O}_{2}$ solution. 
39. Cap the bottle and mix well.

Comment: No insolubles should be apparent at this point. If insoluble particles are apparent, notify the Task Supervisor and await further instructions.

A tell-tale gray color may be apparent after the digestion. This gray color is usually the result of minute amounts of the Pt or Pt-Au from attack of the cesium carbonate on the crucible at high temperature. The particles are extremely small and in such low concentration that filtration is not necessary before the serial dilution or the ICP-AES analysis. The particles do not affect the ICP-AES performance.

40. Perform a serial dilution as directed by the Task Supervisor and transfer the sample bottles to the ICP-AES laboratory.

41. Record the total dilution volume of the digestion + serial dilution performed in the cells.

42. After the ICP-AES analyses are completed satisfactorily, discard the acid solution as directed by the Task Supervisor. Rinse thoroughly the wide-mouth bottles and $50 \mathrm{~mL} \mathrm{Pt}$ crucibles with de-ionized water and allow them to dry in a clean area before the next SME sample batch. 


\begin{tabular}{|c|c|c|}
\hline \multicolumn{3}{|c|}{$\begin{array}{l}\text { Data Table for SME Samples } \\
\text { Wt. \% Solids at } 115^{\circ} \mathrm{C} \text { Drying } \\
\text { Wt. \% Vitrified Solids at } 1100{ }^{\circ} \mathrm{C} \\
\mathrm{Cs}_{2} \mathrm{CO}_{3} \text { Fusion Digestion }\end{array}$} \\
\hline $\begin{array}{l}\text { Procedure } \\
\text { Step \# }\end{array}$ & Measurement Operation & Measurement \\
\hline 4 & Wt. of empty $50 \mathrm{~mL}$ Pt crucible & g \\
\hline 10 & Wt. of $50 \mathrm{~mL}$ Pt crucible + wet SME sample & g \\
\hline 11 & Wt. of wet SME sample & g \\
\hline 14 & $\begin{array}{l}\text { Military Time that oven re-equilbrates to } 115^{\circ} \mathrm{C}\left(1^{\text {st }}\right. \\
\text { drying step) }\end{array}$ & hr. \\
\hline 15 & $\begin{array}{l}\text { Military Time that samples are removed from oven ( } 1^{\text {st }} \\
\text { drying step); Elapsed Time of } 1^{\text {st }} \text { drying step }\end{array}$ & $\begin{array}{l}\text { hr. } \\
\text { hr. }\end{array}$ \\
\hline 16 & $\begin{array}{l}\text { Wt. of } 50 \mathrm{~mL} \text { Pt crucible + dry SME sample after } 1^{\text {st }} \\
\text { drying step }\end{array}$ & g \\
\hline 17 & $\begin{array}{l}\text { Military Time that oven re-equilbrates to } 115^{\circ} \mathrm{C}\left(2^{\text {nd }}\right. \\
\text { drying step) }\end{array}$ & hr. \\
\hline 18 & $\begin{array}{l}\text { Military Time that samples are removed from oven ( } 2^{\text {nd }} \\
\text { drying step); Elapsed Time of } 2^{\text {nd }} \text { drying step }\end{array}$ & hr. \\
\hline 19 & $\begin{array}{l}\text { Wt. of } 50 \mathrm{~mL} \text { Pt crucible + dry SME sample after } 2^{\text {nd }} \\
\text { drying step }\end{array}$ & g \\
\hline 20 & $\begin{array}{l}\text { Difference in } 50 \mathrm{~mL} \text { Pt crucible + dry SME sample after } \\
1^{\text {st }} \text { and } 2^{\text {nd }} \text { drying steps }\end{array}$ & g \\
\hline 21 & Wt. of dry SME sample & g \\
\hline 22 & Wt. \% of SME sample after drying at $115^{\circ} \mathrm{C}$ & $\%$ \\
\hline 24 & Military Time that furnace re-equilbrates to $1100^{\circ} \mathrm{C}$ & hr. \\
\hline 25 & $\begin{array}{l}\text { Military Time that samples are removed from furnace; } \\
\text { elapsed time for heating at } 1100^{\circ} \mathrm{C} \text {. }\end{array}$ & $\begin{array}{l}\text { hr. } \\
\text { hr. }\end{array}$ \\
\hline 27 & Wt. of $50 \mathrm{~mL}$ Pt crucible + vitrified SME sample & g \\
\hline 28 & Wt. of vitrified SME sample (glass wafer) & g \\
\hline 29 & $\begin{array}{l}\text { Wt. \% of vitrified SME sample after vitrifying } \\
\text { at } 1100^{\circ} \mathrm{C}\end{array}$ & $\%$ \\
\hline 41 & $\begin{array}{l}\text { Total volume of dilution (initial fusion digest + serial } \\
\text { dilution performed in cells). }\end{array}$ & $\mathbf{m L}$ \\
\hline
\end{tabular}

